\title{
AN OBJECTIVE METHOD TO ANALYZE OCEANIC VARICLINES AND THEIR STATISTICAL RELATION TO SURFACE WINDS
}

\section{DOSÉ́ MANUEL PINTO BASTOS SALDANHA}


LIBRAPY

NATAL POSIGRADUMTE SCHOOL

MONTFRFY MAT.TF. 93940 




\section{United States}

\section{Naval Postgraduate School}
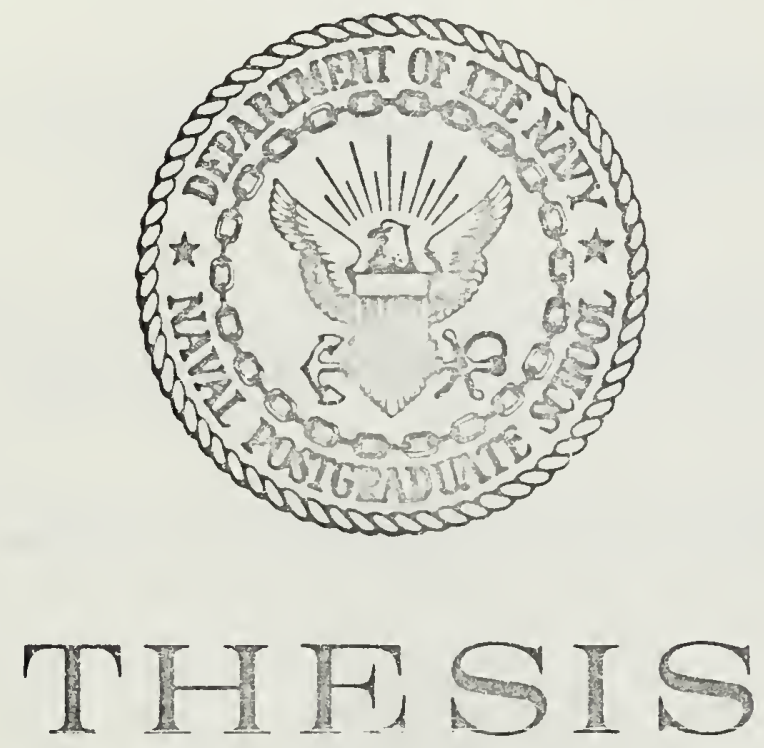

AN OBJECTIVE METHOD TO ANALYZE OCEANIC VARICLINES AND THEIR STATISTICAL RELATION TO SURFACE WINDS

by

José Manuel Pinto Bastos Saldanha

Thesis Advisors: Dale Leipper/Noel Boston

September 1971

Approved for public release; distribution uilimited. 
An Objective Method to Analyze Oceanic Variclines and

Their Statistical Relation to Surface Winds

by

Jose Manuel Pinto Bastos Saldanha

Lieutenant, Portuguese Navy

Submitted in partial fulfillment of the requirements for the degree of

MASTER OF SCIENCE IN OCEANOGRAPHY

from the

NAVAL POSTGRADUATE SCHOOL

September 1971 


\section{ABSTRACT}

Oceanographic and meteorologic data, namely expendable or mechanical bathythermograph traces and wind reports have been systematically collected for years and every day more observations are made.

To handle such a large amount of information, it is advantageous to set up a computerized model which is: automatic, (2) performs an objective analysis of XBT/BT traces to identify the most significant thermal features, such as thermoclines and inversions, (3) restricts the human participation to an initial stage dealing with organization of data available and selection of proper values for the parameters of the modei, ano $\{4 j$ preserves ine initiai information.

A FORTRAN IV program takes into consideration the above requirements to: (1) perform objective analyses of XBT/BT digital traces, (2) process wind information, (3) study the statistical characteristics of the original and processed data, and (4) plot the generated fields.

The digital model was tested and its application may be extended to the study of any environmental variable continuously distributed along a vertical or horizontal axis. 


\section{TABLE OF CONTENTS}

I. OBJECTIVE OF THE THESIS . . . . . . . . . 13

II. OBJECTIVE ANALYSIS OF DIGITAL TRACES . . . . . 16

A. REVIEW OF PRESENT IDEAS . . . . . . . . 16

1. Introduction ......... . 16

2. Evaluation of Boston's Concepts . . . . 18

a. Significant Features in the

Thermal structure . . . . . . 18

(1) Primary Features . . . . . 20

(2) Secondary Features . . . . 20

b. Analytical Techniques .... . . 20

(1) The Gaussian Distribution . . 20

(2) The Finite Difference Scheme • . 21

c. Application of Analytical

Techniques to the Thermal

structure . . . . . . . . . 21

(1) The Gaussian Thermocline . . . 21

(2) The Non-Gaussian Thermocline . 22

(3) Transients . . . . . . . 22

(4) Multiple Thermoclines . . . . 22

(5) Inversions . . . . . . . 24

d. Remarks about Boston's Concepts . . 24

B. AN OBJECTIVE MODEL . . . . . . . . 27

1. Significant Features in a Two

Dimensional Trace . . . . . . . . 28

a. Trend . . . . . . . . . 28

b. Varicline . . . . . . . . . 28

c. Inversion . . . . . . . . . 30 
2. The Finite Difference Technique . . . . 31

a. The Preparation of the Mesh . . . . 31

b. Finite Difference Schemes . . . . 34

(1) First Finite Difference of $\mathrm{V}$. . . 34

(2) First Finite Difference of S . . 34

(3) Second Finite Difference of S . . 34

(4) Third Finite Difference of S . . 37

(5) Higher order Finite Differences of S . . . . . 37

3. Two Dimensional Objective Analysis . • . 37 a. Trend . . . . . . . . . . 37

(1) Variation . . . . . . . 39

(2) Extreme Values . . . . . . 39

b. Varicline . . . . . . . . . 39

c. Inversion .......... 40

d. Complex Type .. . . . . . . . 40

4. Tri-dimensional objective Analysis . . . 42

5. The Problem of Filtering . . . . . . 42

a. The Mesh ......... . . 46

b. Significant Features . . . . . . 48

c. A COMPUTER PROGRAM . . . . . . . . . . . . 49

1. Description . . . . . . . . . . 49

a. The Basic Computer Model . . . . . 49

(1) "START" to "NORDER = 1" . . . . 50

(2) "DO 460" Loop . . . . . . . 50

(3) "IKONTR" to "IKONTR $=0 "$ " . . . 51

(4) "IERIOD ..." to "CALL STATCS" •. . 51

(5) "ILONTR" to "ILONTR $=0$. . . . 51 
(6) "ItItL6" to "PLTMIT" . . . . . 51

(7) "ICSEC" to "STORAGE" . . . . . 52

b. Other Subroutines Available . . . 52

(1) Subroutine TREND . . . . . 52

(2) Subroutine INVERS . . . . . 52

(3) Subroutine PROPAR ...... 52

(4) Subroutine ITRATE . . . . . 52

(5) Subroutine DRAW . . . . . . 53

2. Input . . . . . . . . . . . 53

a. Main Program . . . . . . . 53

b. Subroutine THERMC . . . . . . 53

c. Subroutine PROPAR ....... . 53

d. Subroutine ITRATE ....... 53

3. Outpnt . . . . . . . . . 53

a. Printouts ......... . 54

b. Plots ........... . 54

(1) PLOTIT . . . . . . . . . 54

(2) PLOTBT . . . . . . . . . 54

(3) $\mathrm{PLOTFT} \mathrm{.} \mathrm{.} \mathrm{.} \mathrm{.} \mathrm{.} \mathrm{.} \mathrm{.} \mathrm{.} \mathrm{.} 54$

(4) PLTMIT . . . . . . . . . 54

(5) RLINE and STATCS . . . . . 56

(6) WINDOO, WINDO1, WINDO2, WINDO3, WINDO5, WINDIO . . . . . 56

(7) DRAW .......... . 56

c. Storage in Disk . . . . . . 56

4. Operation . . . . . . . . . 56

a. Characteristics of the Basic

Program .......... 56 
(1) Source Language . . . . . 56

(2) Subroutines and Functions Subprograms .. . . . . 59

(3) Core .......... . . 59

(4) Time . . . . . . . . 59

(5) Disk . . . . . . . . 60

b. Equipment . . . . . . . 60

III. APPLICATION OF THE OBJECTIVE MODEL . . . . . 61

IV. CONCLUSIONS . . . . . . . . . . . 63

V. RECOMMENDATIONS .............. . . 64

APPENDIX A - Flow Diagram Symbols . . . . . . . . 65

APPENDIX B - Flow Diagram of the FORTRAN IV
Basic Computer Program . . . . . . 66

APPENDIX C - Flow Diagram of the Thermocline Analysis - 69

APPENDi: I - Flow Diagram of the Trend Analysis • • 71

APPENDIX E - Flow Diagram of the Inversion Analysis • 75

APPENDIX F - FORTRAN IV Basic Program for IBM 360/OS • 79

APPENDIX G - FORTRAN IV Subprogram TREND for
IBM 360/OS . . . . . . . . . . . . . . . . . 181

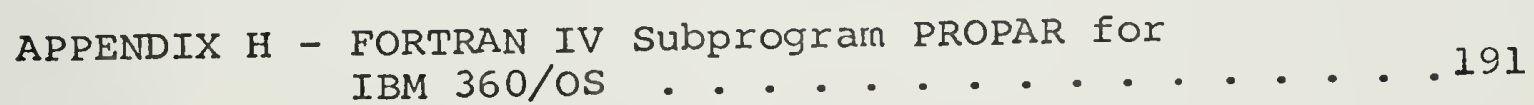

APPENDIX I - FORTRAN IV Subprogram ITRATE for
IBM 360/OS . . . . . . . . . . 193

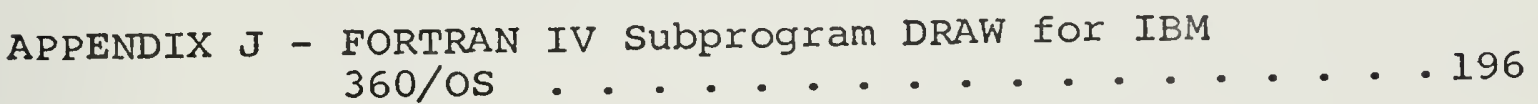

APPENDIX $K$ - Modification to the FORTRAN IV Basic
Computer Program (APPENDIX F) to Include the Subprogram PROPAR (APPENDIX I) . . . 197

APPENDIX L - Modification to the FORTRAN IV Basic Computer Program (APPENDIX F) to Include the Subprogram ITRATE (APPENDIX J) . . . 199

APPENDIX $M$ - Modification to the FORTRAN IV Bi sic

Computer Program (APPENDIX F) to Include

the Subprogram TREND (APPENDIX G) ....201 
APPENDIX N - Job Control Language (JCL) for the Head Part of the FORTRAN IV

Basic Computer Program (APPENDIX F) • • 202

APPENDIX O - Job Control Language (JCL) for the Last Part of the FORTRAN IV Basic

Computer Program (APPENDIX F) . . . . 203

APPENDIX P - Job Control Language (JCL) for the Last Part of the FORTRAN IV Basic

Computer Program (APPENDIX F) . . . . . 204

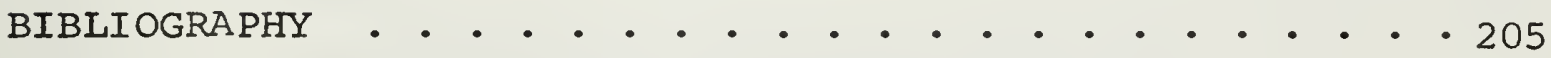

INITIAL DISTRIBUTION LIST . . . . . . . . . 206

FORM DD 1473 . . . . . . . . . . . . . . 209 


\section{LIST OF FIGURES}

Figure

Page

1

Eastern North Atlantic Ocean . . . . . 14

2

The Gaussian Thermoclj.ne and

its Frequency Function [Boston, 1966]

A Non-Gaussian Thermocline Showing

Top, Center, and Bottom as

Determined by Finite Difference

Procedure [Boston, 1966] . . . . . . 23

4

Thermal structure with One, Two,

and Three Thermoclines [Boston, 1966] • . 25

5

Inversion Illustrating Main

Features [Boston, 1966]

6

A Two-dimensional Trace . . . . . . . . 29

7

The Mosh

8

The Linear Interpolation of a

Digital Trace: A. Digital Trace,

B. Mesh (increment $\mathrm{V}=2$ arbitrary

units), C. Mesh (increment $V=1$ arbitrary unit).

9 The Mesh and Its components:

A. Segments of the Mesh,

B. Length Components,

C. Variable Components

10

Schemes of the Second Finite Differences:

A. Constant slope, B. Increasing

Slope, C. Decreasing Slope . . . . . . 36

11 Schemes of the Third Finite Differences:

A. Constant Slope, B. Successive

Increase of the Slope, C. Successive

Decrease of the Slope, D. Successively

Decreasing and Increasing the Slope,

E. Successively Increasing and Decreasing

the Slope . . . . . . . . . . . . 

12 Computer output plot of a Digital Trace showing the Tops (squares) and the Bottoms (triangles) of

Analyzed Variclines. The control of the conditions limiting the bottom of a varicline allows: A. identification of all the variclines present, and $B$. definition of the significant features) . . .

13 Tri-dimensional Analysis: A. The Tri-dimensional Distribution,

B. A New Trace, C. The Projection of Two-dimensional Traces Along a

15 Computer output Plot of the Linear Field of Isotherms for one Cruise or the Average Linear Field of Isotherms for a Given Number of Cruises . . . . . . . . . . . . . . . .

16 Computer output Plots of :

A. The Irterpoiatca Digital Treces

Along a Cross section for a Given

Cruise, and $B$. The Fifth order

Least Square Polynomial Approximation of the Same Digital Traces . . . . . . . .

17 Computer output Plots of the Linear Field of Isopleths Defining the Top $(T)$, the Mean Depth (M), and the Bottom (B) of the Three Shallowest Thermoclines for Different Values of the Filtering parameters

18 Computer output plot of the Least Square Regression Lines and the Scatter Diagram (t) for Two Given Parameters: Variable 1 and Variable 2 . .

19 Computer output Plot of the Wind Vector and its Components (along and perpendicular to the direction of the near coastline) Measured at Same station and Day of XBT Observation and one, Two, Three, Five and Ten Days Before . . . . . 


\section{TABLE OF SYMBOLS AND ABBREVIATIONS}

BT

D

e

k

L

m

n

$\pi$

$S$

$S(b)$

$S(V)$

$S_{b I}$

$s_{b V}$

$\mathrm{S}_{\mathrm{CV}}$

seI

$s_{t I}$

$s_{t V}$

$\Delta S_{I}$

$\Delta \mathrm{S}_{\mathrm{V}}$

$\sigma$

Mechanical Bathythermograph

Time variable

$2.718281 \ldots$

Any positive integer

Second length variable for tri-dimensional distribution

Median of a Gaussian distribution

Any positive integer

3.14159 ...

Length variable for two-dimensional distribution

Value of the length variable at location $b$ of $a$ given distribution

Length variabie as a function of the environmental variable

Value of the length variable at bottom of an inversion

Value of the length variable at bottom of a varicline

Value of the length variable at center of a varicline

Value of the length variable at point of symmetry of an inversion

Value of the length variable at top of an inversion

Value of the length variable at top of a varicline

Range (length) of an inversion

Range (length) of a varicline

Standard deviation 
Temperature variable (environmental variable)

$\mathrm{T}_{\mathrm{n}}$

$\Delta \mathrm{T}_{\mathrm{T}}$

$\mathrm{V}$

$\mathrm{V}_{\mathrm{n}}$

$\mathrm{v}\left(\mathrm{v}_{\mathrm{n}}\right)$

$\mathrm{V}(\mathrm{a})$

$\Delta \mathrm{V}$

$\Delta \mathrm{V}_{I}$

$\Delta \mathrm{V}_{\mathrm{V}}$

XBT

$\mathrm{z}$

$\mathrm{Z}(\mathrm{T})$

$\mathrm{z}_{\mathrm{bT}}$

$\mathrm{Z}_{\mathrm{CT}}$

$\mathrm{z}_{\mathrm{tT}}$

Nth value of temperature read off a trace

Thermal magnitude of a thermocline

Environmental variable

Nth value of $V$ read off a trace

Nth value of $V$ read off a trace

Value of $\mathrm{V}$ at location a of a given distribution

Magnitude of the mesh increment

Variable magnitude of an inversion

Variable magnitude of a varicline

Expendable Bathythermograph

Depth (length variable) increases downwards from the sea surface

Depth as a function of temperature

Depth of the bottom of a thermocline

Depth of the center of a thermocline

Depth of the top of a thermocline 


\section{ACKNOWLEDGEMENT}

This research was conducted under the advice of $\mathrm{Dr}$. Dale F. Leipper, Chairman of the Department of Oceanography, Dr. Taivo Laevastu of the Fleet Numerical Weather Central, Dr. Noel Boston of the Department of Oceanography, and Dr. Kenneth Davidson of the Department of Meteorology.

The project required utilization of the Computer Facility of the Naval Postgraduate School and environmental data from the Fleet Numerical Weather Central in Monterey, whose staffs were particularly helpful and dedicated.

I would like to express my sincere thanks to my advisors, faculty and members of the above facilities, classmates and students, to everyone who really helped me to develop numan and environmental understanding. 



\section{OBJECTIVE OF THE THESIS}

The study of oceanographic phenomena occurring in an area west of the Strait of Gibraltar from Cabo Finisterra (in Europe) to Canary Islands (in Africa) (Figure 1) based on climatological information, constituted the initial purpose of this thesis.

Later, the availability of oceanographic and meteorologic data from Fleet Numerical Weather Central (in Monterey), broadened the descriptive nature of the above intention giving opportunity to investigate the variations of the oceanic thermal structure taking place in the upper layers.

The large amount of accessible data, namely expendable bathythermograph traces and surface uncorrected geostrophic wind fields, were processed using a digital model and presented in a form simple to analyze. Initially, the model was set up to study the distribution of temperature with depth; soon, it became evident that it could be applied to other studies dealing with the continuous vertical distribution of any environmental parameter (sound velocity, salinity, light extinction, etc.).

The lack of information about air-sea temperature differences made impossible the transformation of the uncorrected geostrophic wind into "friction velocity" and cut short the last part of the study, which was to establish the statistical interrelation between the ccrrected wind information and the thermal features given by the objective analy is of traces. 


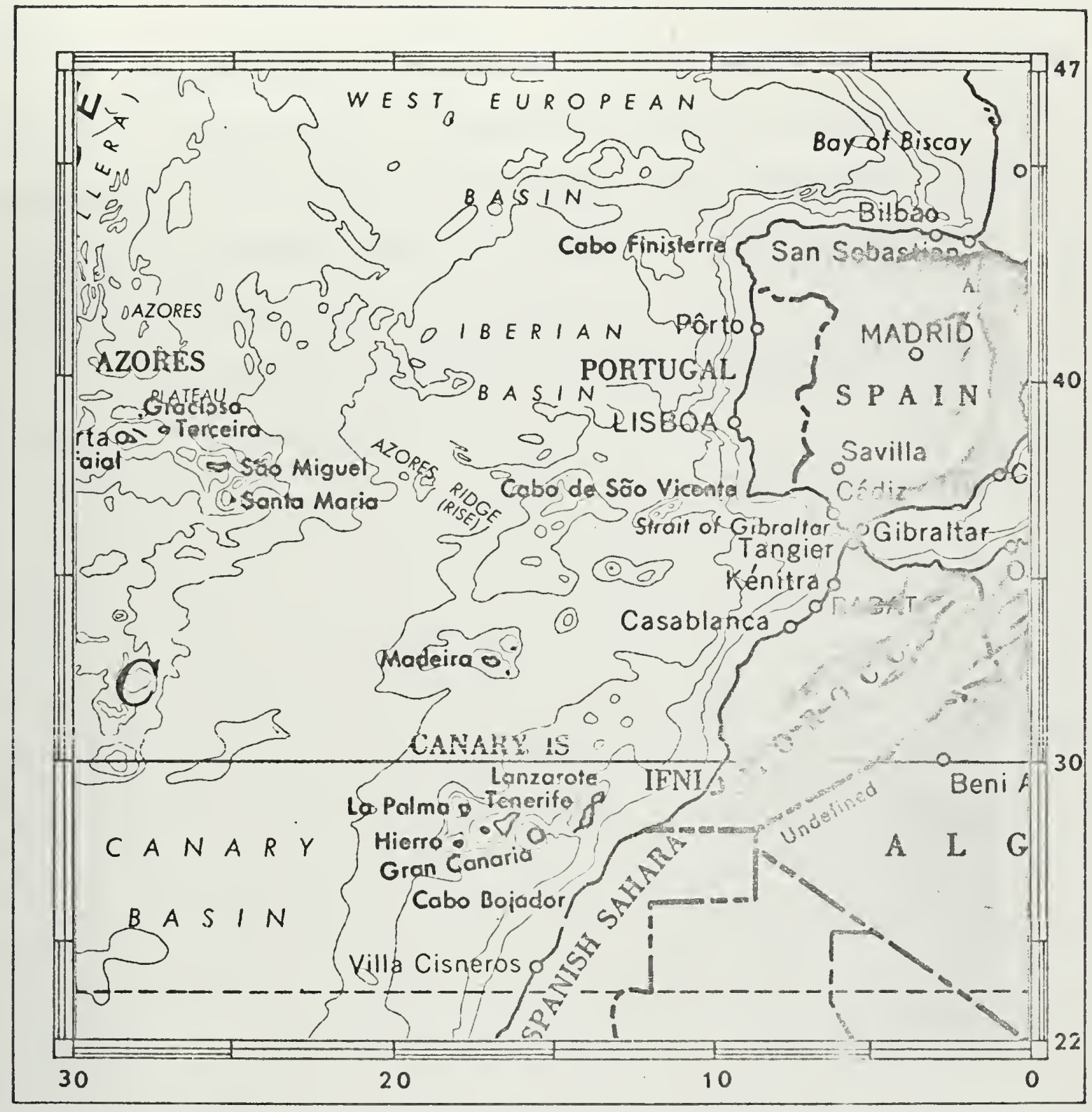

Figure 1. Eastern North Atlantic Ocean 

The new objective of the thesis then is defined in two parts :

a. To develop a digital model to analyze the continuous vertical distribution of any environmental parameter, to process the wind data and to present the generated information in a tractable form to reveal geophysical phenomena and their statistical connections.

b. To test the capability of the computer program by means of data available. 



\section{OBJECTIVE ANALYSIS OF DIGITAL TRACES}

\section{A. REVIEW OF PRESENT IDEAS}

\section{Introduction}

The mechanical bathythermograph, BT, first introduced by Rossby and Montgmory [1935] and developed by Spilhaus [1938] provided one of the first successful attempts to obtain a continuous graphical record of an oceanic variable, namely the distribution of temperature with depth. The expendable bathythermograph, XBT, introduced by Francis and Campbell [1955] increased the continuous thermal record to a maximum depth of 457 meters. More sophisticated devices with greater depth ranges have been used to record continuousiy the vertical äistribution of salinity, temperaluie asu sound velocity as discussed by Magruder [1970]. Recently, Tucker and Shepard [1971] presented results from continuous registration of light transmission versus depth.

Continuous horizontal distribution of current speed, temperature, bathymetry and gravity are among the other variables that have been measured with sensors which are carried or towed in one direction at the sea surface or along other horizontal reference planes below or above the sea surface.

It is important to note that although these distributions are usually labelled as temperature (or other variable) versus depth (or other horizontal or vertical axis), the independent variable is the depth or the 
successive positions where the sensor measures the oceanographic variable. A significant characteristic of a continuous distribution of a given variable along a length axis compared to time distributions is the absence of regularity or law of repetition, which means that distinct phenomena or different intensities of the same phenomenon are taking place along the sensed axis and makes impossible the application of time series analysis techniques.

The small cost of some equipment, namely BTs, their reliability and easy maintenance, together with a recording operation that does not require great ability from an observer led to widespread use; an immediate consequence was enormous accumulation of BT traces.

Recent development in automatic processing techniques of bathythermographic data is due to LaFond's [1951] pioneering efforts to establish a standardized method for processing BT traces and in predicting the need for automatic tecinology. Sauer [1964] described the Bathythermograph Card processor which automatically transposes the BT record to an aperture card. Sauer and Hope [1967] gave a detailed account of the "BT Digitizer" operation which produces semiautomatic digitization of BT aperture caras. Dale and Stevens [1970] presented the Fleet Numerical Weather Central technique to digitize the XBT traces by the definition of the linear portions of the curve.

The analysis of a continuous record, BT or XBT, requires an acute visual sense, experience and superior 

understanding of oceanographic processes. The researcher looks for characteristic geometric features of the trace; usually he follows the evolution of one feature throughout a set of records to assure consistency of reasoning. The human system is slow, inaccurate for subtle variations, reactive to routine procedures and subjective.

Efforts towards an automatic analysis of BT traces have been described among others by Boston [1966] who identified the most significant features in the thermal structure of oceanic upper layers and derived mathematical schemes to define the depth of the mixed layer, single and multiple thermoclines, transients and inversions. The mathematical schemes are based on: (1) an assumption of Ganssian nr Normal distribution of temperature as a function of depth (Figure 2), and (2) a finite difference method. Grosfils [1968] translated into a computer program the schemes above outlined and. Denham [1969] applied the same principles to a digital analysis of internal waves. Boston initially developed and applied his method while examining nearshore internal waves.

\section{Evaluation of Boston's Concepts}

The first part of this thesis generaiizes some of Boston's [1966] concepts and their presentation. An evaluation will outline and hlep to understand the purpose of the present research.

a. Significant Features in the Thermal structure Boston defined the significant fe-tures of the thermal structure obtainable with a BT within the upper 300 meters o: the ocean as 


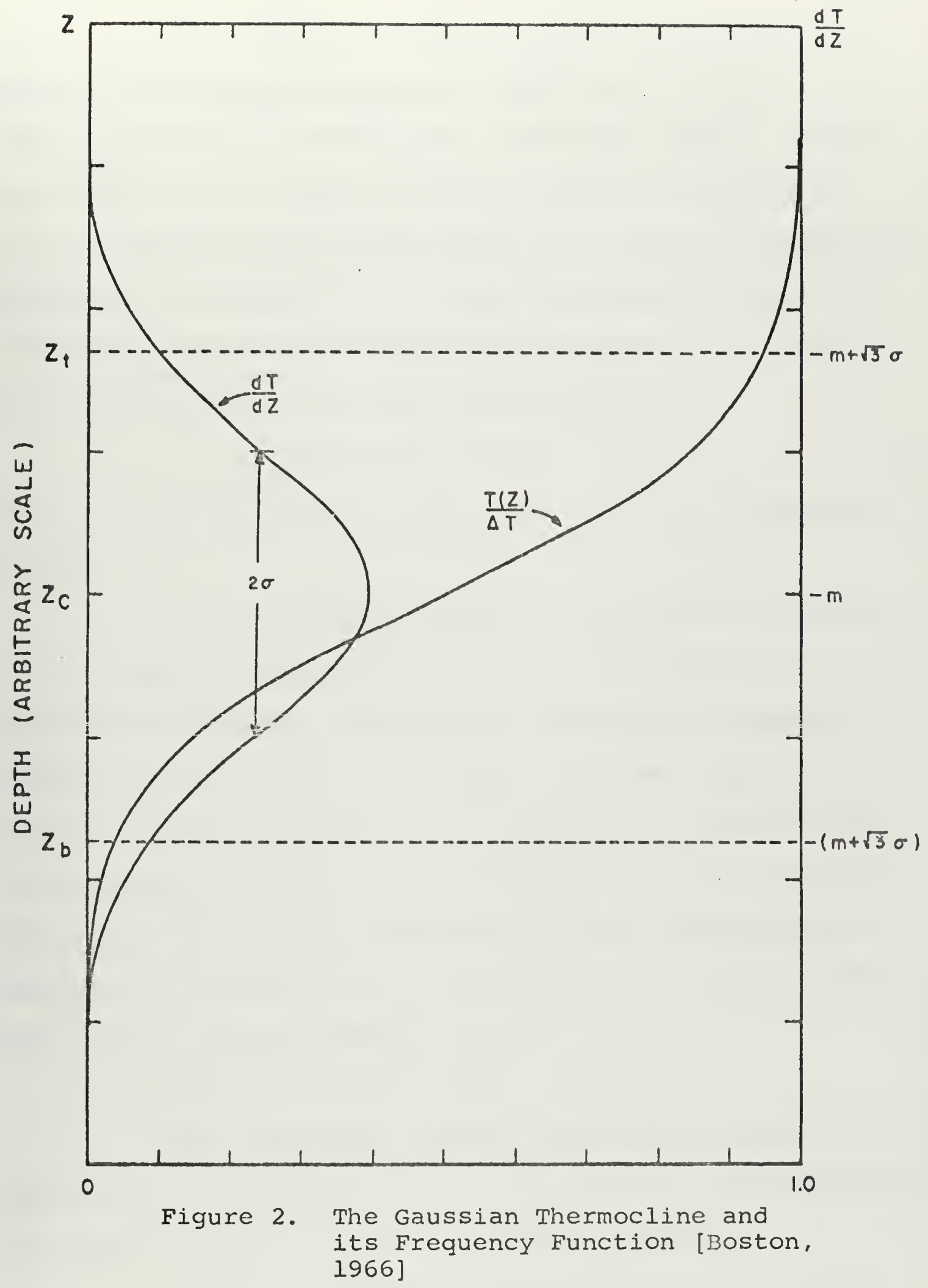



(1) Primary features or those characteristics common to most oceanic regions. The thermal structure is simply approximated by an upper isothermal layer, a thermocline and a lower region of nearly constant temperature. Three parameters describe this type of structure, namely (a) the depth of the mixed layer (upper isothermal), $\mathrm{z}_{t \mathrm{~T}^{\prime}}$ (b) the depth of the "bottom" of the thermocline, $\mathrm{z}_{\mathrm{bT}}$ ' and (c) the thermal magnitude of the thermocline is

$$
\Delta T_{T}=T_{t T}-T_{b T}
$$

if $\mathrm{T}_{t \mathrm{~T}}$ is the temperature at $\mathrm{z}_{\mathrm{tT}}$ and $\mathrm{T}_{\mathrm{bT}}$ is the temperature at $\mathrm{z}_{\mathrm{bT}}$.

(2) Secondary features are variations occurring in the primary structure at any point and classified as (a) tiansients or theriai fluctüticna less than one degree Celsius and localized at the upper isothermal layer, (b) multiple thermoclines which are the result of heating and mixing conditions and (c) inversions or a type of structure occurring below the main thermocline where the temperature decreases with depth to a minimum, then increases and sometimes regains the decreasing trend.

\section{b. Analytical Techniques}

The analytical methods developed by Boston are based on (1) a Gaussian or Normal distribution of temperature with depth, and (2) a finite difference approach.

(1) The Gaussian distribution is described by the well-known frequency function; Figure 2, 



$$
\frac{d T(z)}{d z}=\frac{1}{\sqrt{2} \sigma \pi} e^{-\frac{(z-m)^{2}}{2 \sigma^{2}}}
$$

where $\sigma$ and $m$ are constants. The function (2) has (a) symmetry about $z=m,(b)$ points of inflection at $z=m \pm \sigma$ and (c) maximum rate of change at $z=m \pm \sigma \sqrt{3}$. Application of the Gaussian distribution requires that the central moments of odd order be zero.

(2) The finite difference scheme implies the substitution of analog trace $z\left(T_{n}\right)=f\left(T_{n}\right)$ by a discrete set of successive positions $\left(T_{n}, z\left(T_{n}\right)\right)$ obtained through a constant increment, $\Delta T$, of the independent variable, $T_{n}$. The first finite difference of $\mathrm{z}\left(\mathrm{T}_{n}\right)$ will be defined as

$$
\Delta z\left(T_{n}\right)=z\left(w_{n+1}\right)=z\left(T_{n}\right)
$$

and the second finite difference as

$$
\Delta^{2} \mathrm{z}\left(\mathrm{T}_{\mathrm{n}}\right)=\Delta \mathrm{Z}\left(\mathrm{T}_{\mathrm{n}+\mathrm{I}}\right)-\Delta \mathrm{z}\left(\mathrm{T}_{\mathrm{n}}\right)
$$

The value of the increment $\Delta T$ will vary according to the desired accuracy of the scheme.

c. Application of the Analytical Techniques to the Thermal structure

The above concepts may be applied to the analysis of the thermal structure. It is assumed that the BT trace is replaced by a discrete set of ordered points $(T, Z)$ from the surface and taken at equal thermal increment, $\Delta \mathrm{T}$.

(1) The Gaussian Thermocline. In accordance with the Normal or Gaussian distribution of temperature with 
depth, T $\left(\frac{z-m}{\sigma}\right),($ a) the center of the thermocline is located at

$$
\mathrm{z}_{\mathrm{CT}}=-\mathrm{m}
$$

(b) the top and the bottom of the thermocline are considered to be at the points of the maximum rate of change of the slope of the $T\left(\frac{z-m}{\sigma}\right)$ curve or at

$$
\begin{aligned}
& z_{t T}=-m+\sigma \sqrt{3} \\
& z_{b T}=-m-\sigma \sqrt{3}
\end{aligned}
$$

(2) The Non-Gaussian Thermocline. The analysis of the non-Gaussian thermocline makes use of the variation in sign of the second finite difference scheme, equation (4), to find the point of inflection of the $(T, Z)$ curve. This point identifies tne center of Lite thermocline, $z_{c m}$. The top of the thermocline, $\mathrm{z}_{\mathrm{tT}}$ ' is determined using the technique outlined in ( 1 ) and generating an artificial Gaussian distribution around $z_{C T}$ by reflection of the upper part of the trace $\left(z \leqslant z_{C} T\right)$. The bottom of the thermocline, $z_{b T^{\prime}}$ is obtained in the same way but making use of the lower part of the $(T, Z)$ curve, $\left(Z \geq Z_{C T}\right)$. Figure 3 shows the successive steps of this analysis.

(3) Transients are recognized by the sign changes of the second finite difference, $\Delta^{2} \mathrm{z}\left(\mathrm{T}_{\mathrm{n}}\right)$. A sign change from positive to negative signifies the end of one transient and the beginning of the next one.

(4) Multiple Thermoclines. An examination of the sign changes of the second finite differe ce, as made in 



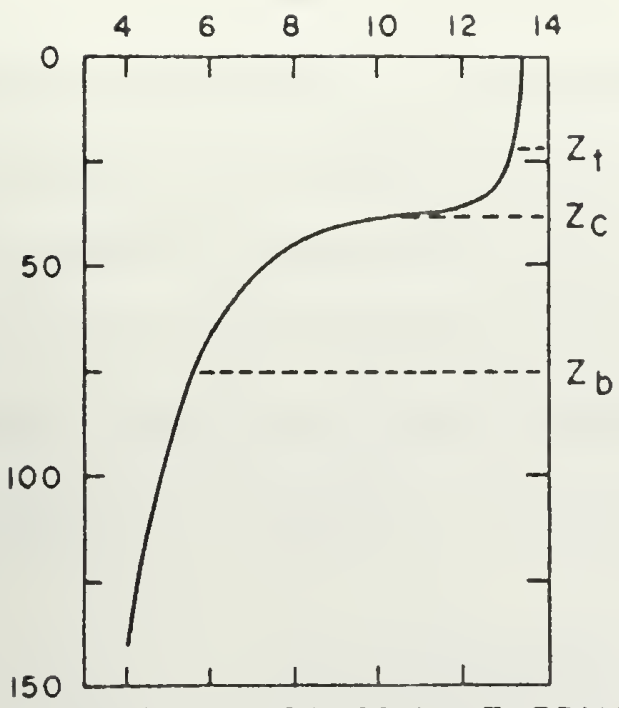

o. A NON-GAUSSIAN THERMOCLINE SHOWING TOP, CENTER AND BOTTOM AS DETERMINED BY FINITE DIFFERENCE PROCEDURE

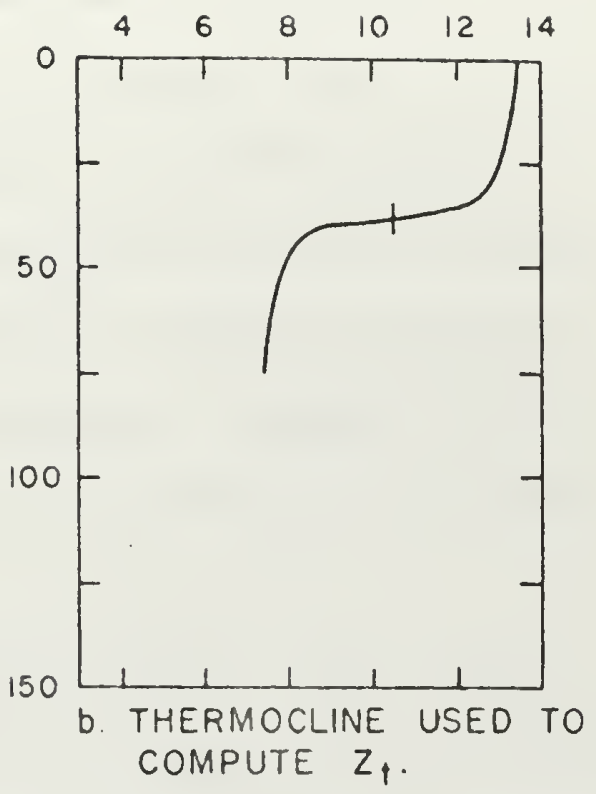

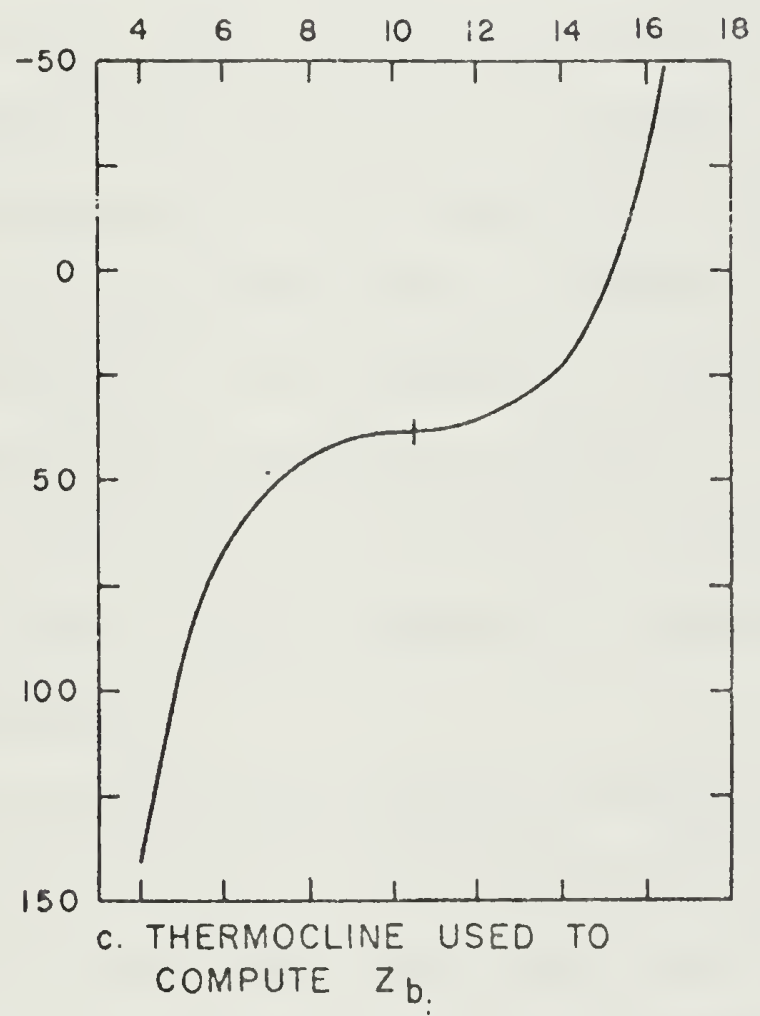

Figure 3. A Non-Gaussian Thermocline Showing Top, Center, and Bottom as Determined by Finite Difference Procedure [Boston, 1966] 

(2), will reveal the points of inflection of the $(T, Z)$ curve. These correspond to the centers of the thermoclines. The top and the bottom of each thermocline are found applying the method explained in (2). The separation between thermoclines is determined by the location of successive points where the first finite difference attains a maximum value. Examples of multiple thermoclines are illustrated in Figure 4.

(5) Inversions. The sign change of the first finite difference of the $(T, Z)$ curve indicates the existence of an inversion. The upper and lower limits of any inversion delineate a continuum where the representative trace is double-valued (same temperature at two depths) but outside the ragivis the distribution of temnerature with depth is single-valued (one temperature and one depth). The main features investigated by this method are shown in Figure 5 .

d. Remarks about Boston's Concepts

(1) The problem of automatic processing of bathythermograph data is well stated and represents a remarkable contribution.

(2) The objective digital analysis of the thermal structure requires application of several methods, each one is independent from the others and each describes a distinct thermal feature. More general objective schemes to analyze other features in the thermal structure would be even more useful and might be extended to the objective study of other oceanographic variables with vertical or horizontal axial distributions. 


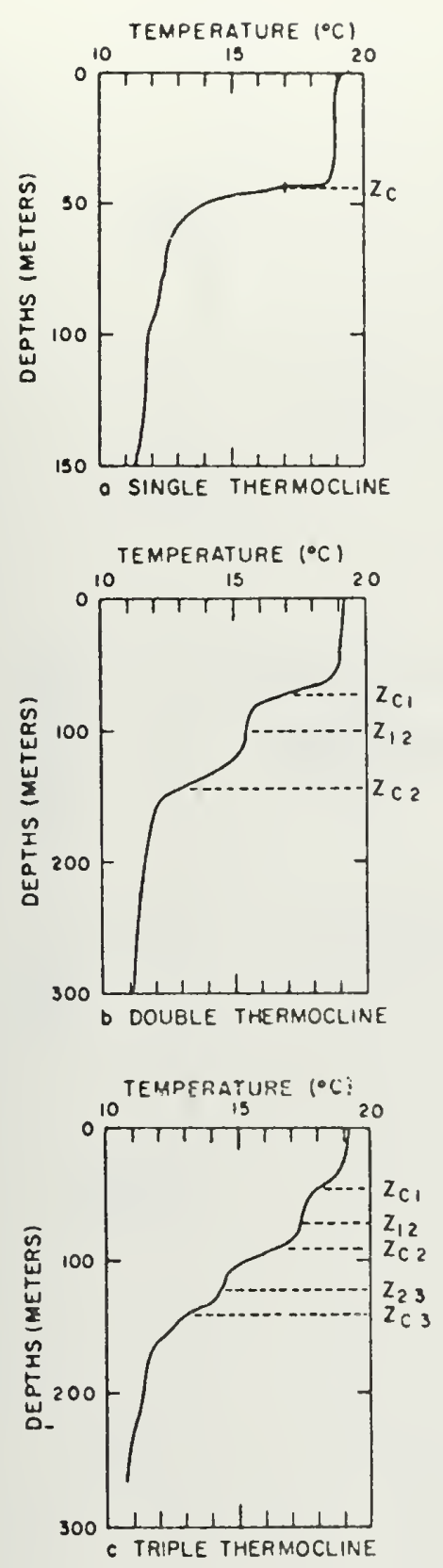

IABLE IV. Multiple thermoctine ond lyeito

\begin{tabular}{|c|c|c|c|c|}
\hline$n$ & $T_{n}$ & $z\left(T_{n}\right)$ & $\Delta z\left(T_{n}\right)$ & $S^{2} 2\left(T_{n}\right)$ \\
\hline 1 & 19 & 5 & \multirow{2}{*}{$\begin{array}{r}39 \\
0\end{array}$} & \multirow[b]{2}{*}{.39} \\
\hline 2 & 18 & 44 & & \\
\hline 3 & 17 & \multicolumn{3}{|c|}{ 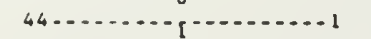 } \\
\hline 4 & 16 & 45 & l & 0 \\
\hline 3 & 13 & 46 & \multirow{2}{*}{4} & 3 \\
\hline 6 & 14 & 30 & & 4 \\
\hline 7 & 13 & 58 & \multirow[t]{2}{*}{32} & \multirow[t]{2}{*}{24} \\
\hline 8 & 12 & 90 & & \\
\hline
\end{tabular}

Iable IV(b)

\begin{tabular}{|c|c|c|c|c|}
\hline$n$ & $I_{n}$ & $z\left(I_{n}\right)$ & $\Delta z\left(I_{n}\right)$ & $\Delta^{2} z\left(I_{n}\right)$ \\
\hline 1 & 19 & 40 & 28 & \\
\hline 2 & 18 & 68 & 4 & -24 \\
\hline 3 & 17 & $72=$ & $\cdots-\cdot$ & $=. .4$ \\
\hline 4 & 16 & 80 & $40 \ldots$ & $\ldots 32$ \\
\hline 3 & is & 120 & 14 & -26 \\
\hline 6 & 14 & 134 & 10 & .4 \\
\hline 7 & 13 & $144 \ldots$ & $10^{\cdots}$ & $\ldots .0$ \\
\hline 8 & 12 & 154 & & \\
\hline
\end{tabular}

Iable IV $(c)$.

\begin{tabular}{|c|c|c|c|c|}
\hline$n$ & $I_{n}$ & $2\left(T_{n}\right)$ & $\therefore 2\left(I_{n}\right)$ & $\Delta^{2} z\left(I_{n}\right)$ \\
\hline 1 & 19.0 & 28 & 10 & \\
\hline 2 & 18.5 & 38 & 6 & .4 \\
\hline 3 & 18.0 & $44 \ldots$ & - $[1] \cdots$ & $\ldots-6$ \\
\hline 4 & 17.3 & 36 & $30 \ldots$ & $\ldots 18$ \\
\hline$s$ & 17.0 & 86 & 4 & -26 \\
\hline 6 & 16.5 & $90 \ldots$ & $\cdots 4_{4} \cdots$ & $\ldots-0$ \\
\hline 7 & 16.0 & 94 & 4 & 0 \\
\hline 8 & 15.5 & 98 & 4 & 0 \\
\hline 9 & 15.0 & 102 & 10 & 6 \\
\hline 10 & 14.5 & 112 & $18 \ldots$ & $\ldots$ \\
\hline 11 & 14.0 & 130 & 6 & -12 \\
\hline 12 & 13.5 & 136 & 4 & -2 \\
\hline 13 & 13.0 & $140 \ldots$ & $\cdots 10^{-\cdots}$ & $\ldots--6$ \\
\hline 14 & 12.5 & 150 & 10 & 0 \\
\hline 15 & 12.0 & 160 & 20 & 10 \\
\hline 16 & 11.5 & 180 & so & 30 \\
\hline 17 & 11.0 & 230 & & \\
\hline
\end{tabular}

Figure 4. Thermal Structure with one, Two, and Three Thermoclines [Boston, 1966] 



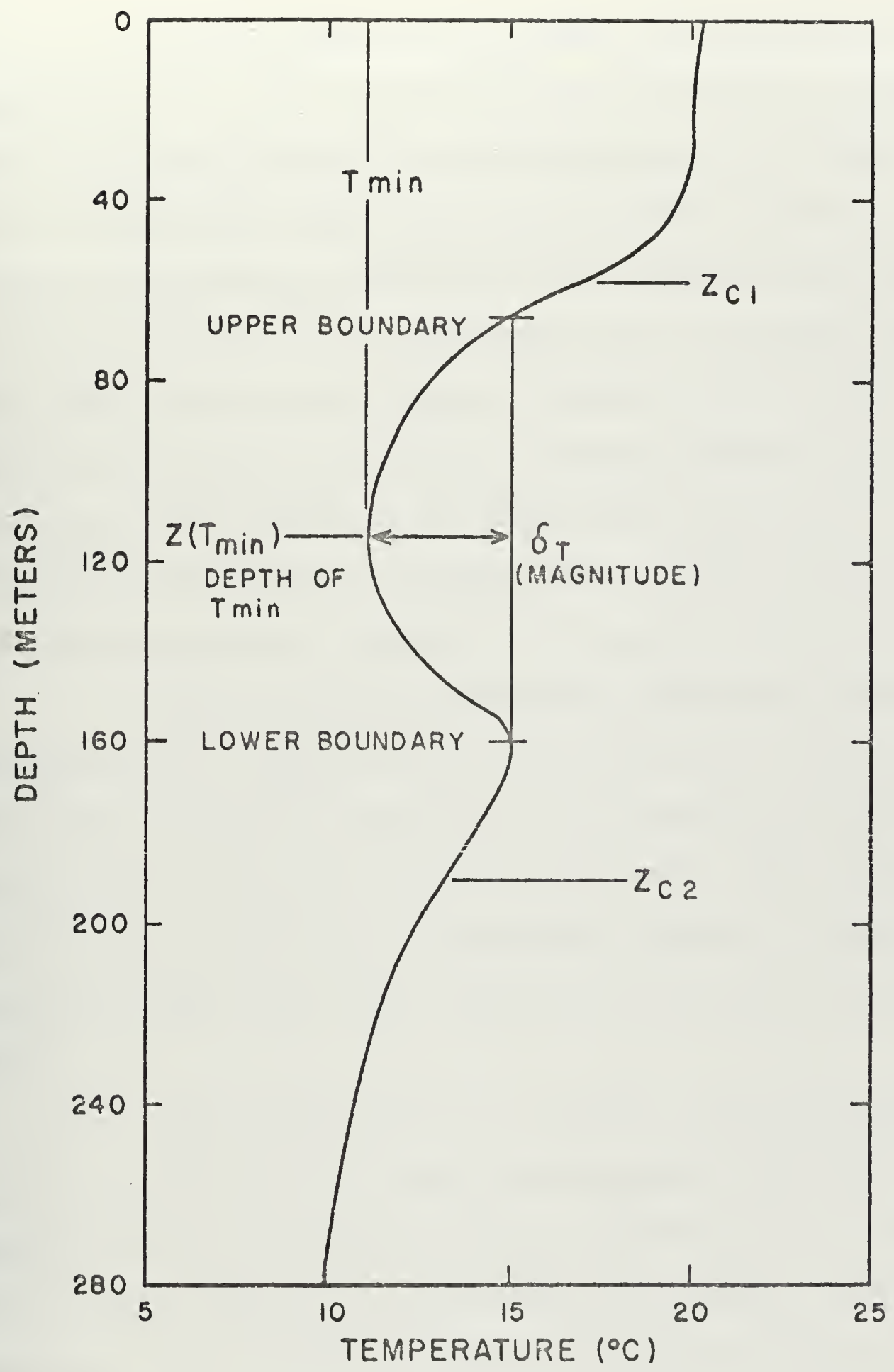

FIGURE 5. Inversion Illustrating Main Features [Boston, 1966] 

(3) The Gaussian or normal distribution of temperature with depth constitutes an interesting investigation. Its application to the definition of a thermocline is restricted depending upon whether or not a condition of symmetry around the first moment does exist. If this provision is not fulfilled there is not any thermal normal distribution with depth. However, in this case of non-Gaussian thermocline the top and the bottom of the thermocline are determined by means of an artificial reflected construction of normal distribution around the center which distorts slightly the initial information.

(4) The finite difference technique explores conveniently the behavior of first and second order schemes which give a familiar feeling for fluctuations of slope and points of inflection of the original trace. The development and study of higher order schemes may indicate interesting variations associated with significant features of any curve.

(5) The digitization of the analog trace by the selection of depths at uniform temperature intervals appears to be advantageous because the data is in a form where it may readily be processed by the finite difference technique. The thermal increment should be chosen as small as possible but compatible with the accuracy of the sensor.

\section{B. AN OBJECTIVE MODEL}

The primary purpose of this chapter is the presentation and development of general concepts which may be utilized 
in the objective analysis of any given two-dimensional trace.

\section{Significant Features in a Two-dimensional Trace}

A two-dimensional trace presents the distribution of any oceanographic variable, $\mathrm{V}$, with depth or along a horizontal axis, 5 , as shown in Figure 6. A pecularity of the trace consists in the continuous increase in $S$ with fluctuations in $V$. Such curves $(V, S)$ show three general characteristics, namely:

(1) trend,

(2) varicline, and inversion.

\section{a. Trend}

The trend indicates the general direction of development of the curve $(V, S)$ in Figure $6:$ ( $(1)$ between the boginning of the remurr, $a$; and $a$, the variable $V$ is decreasing at different rates to the left of the figure; (2) $g-h$, the variable $V$ is increasing and the trace is directed to the right; and. (3) the final position of the curve between $\mathrm{h}$ and $i$ again shows a decrease of $\mathrm{V}$.

\section{b. Varicline}

Varicline was derived from the composition of the words: relative "variation" of the slope or "inclination"; it identifies portions of the curve where the slope increases relative to the neighbor regions. In Figure 6 , parts b - c and $d$ - e of the curve are variclines. Considering varicline $d-e$, the limiting parameter values are (1) the top, b，

$$
s_{t V}=s(d)
$$





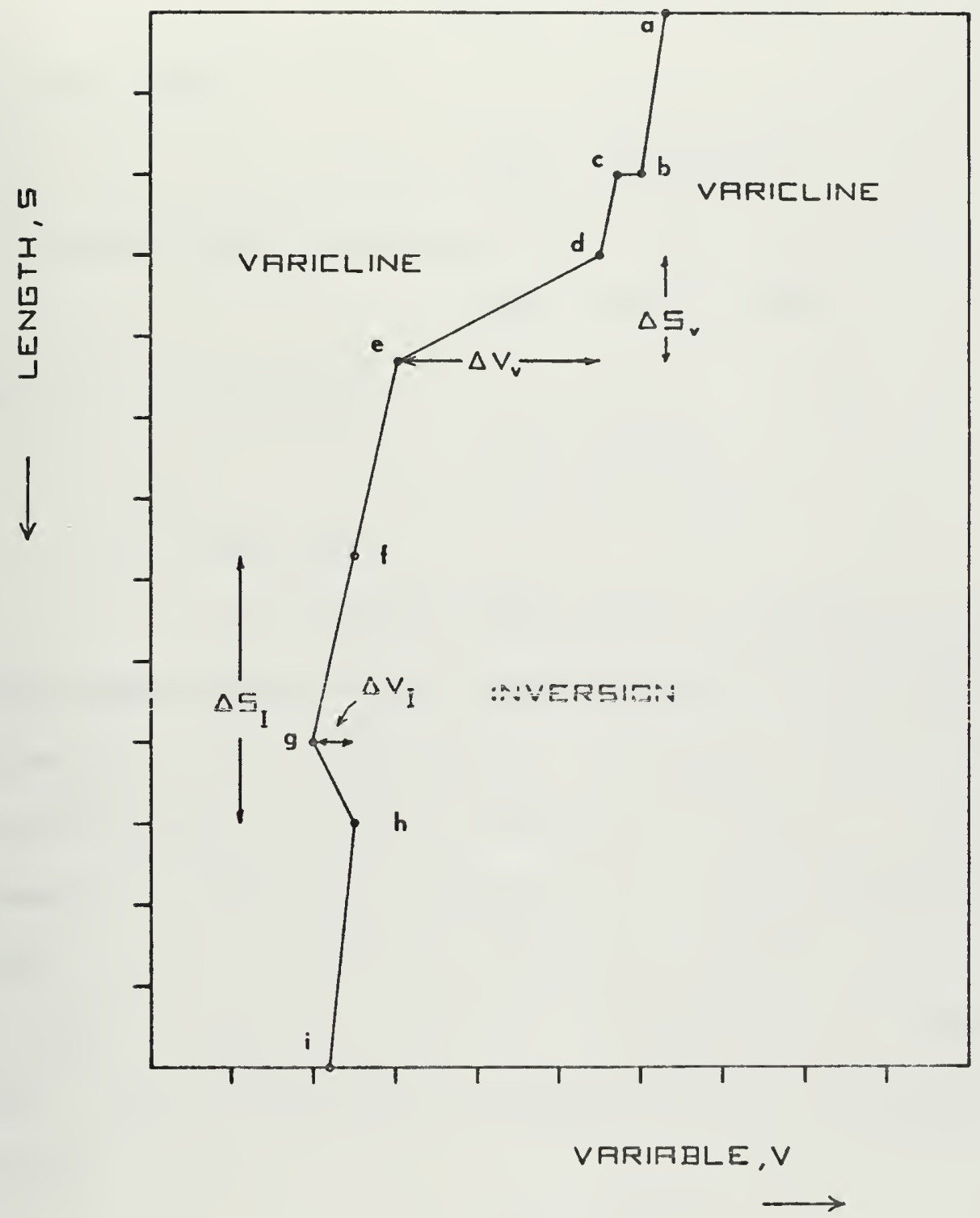

Figure 6. A Two-dimensional Trace 

(2) the bottom, e,

$$
s_{b V}=s(e)
$$

(3) the range, $\Delta \mathrm{S}_{\mathrm{V}}$ '

$$
\Delta s_{V}=s(e)-s(d)
$$

(4) the center,

$$
s_{C V}=s(d)+\Delta s / 2
$$

(5) the variable magnitude, $\Delta V_{V}$,

$$
\Delta V_{V}=V(e)-V(d)
$$

and (6) the gradient,

$$
G_{V}=\Delta V_{V} / \Delta S_{V}
$$

c. Inversion

An inversion identifies a segment of the curve $(V, s):$ has there axists an apronimately symmetrical distribution of the oceanographic variable, $V$ with length $S$ around a point, namely location $g$ in Figure 6 . The point of symmetry is found by examination of the variation of the trend: it is a relative maximum or minimum of $V$. Referring to Figure 6 again, the point $g$ constitutes a relative minimum. The most important parameters of an inversion are (1) the top, f,

$$
s_{t I}=s(f)
$$

(2) the bottom, $h$,

$$
\mathrm{s}_{\mathrm{bI}}=\mathrm{s}(\mathrm{h})
$$

(3) the location of the extreme value of $V$ in the inversion, g,

$$
s_{e I}=s(g)
$$



(4) the variable magnitude, $\Delta V_{I}$

$$
\begin{aligned}
\Delta V_{I} & =|V(g)-V(f)| \\
& =|V(g)-V(h)|
\end{aligned}
$$

and (5) the range, $\Delta S_{I^{\prime}}$

$$
\Delta S_{I}=S(h)-S(f)
$$

\section{The Finite Difference Technique}

The finite difference technique investigates the variation of a two-dimensional distribution. The original analog record is previously digitized according to semiautomatic procedures. The digital trace consitutes an ordered and discrete set of positions.

a. The Preparation of the Mesh

The finite difference technique requires an organized two-dimensional distribution or mesh such that the values of one variable $V$, chosen as independent, are read at constant interval $\Delta V$ together with the correspondent values of the other variable $S(V)$, dependent, Figure 7 . The magnitude of the increment $\Delta V$ is directly related to the accuracy of the record and it is unrealistic to select a small but incompatible value. The digital trace may present the structure as a satisfactory mesh with values at constant intervals; if not, it must be altered accordingly. Assuming that the digital trace has a linear variation, the mesh points will be obtained by a simple linear interpolation. However, the selected constant interval may introduce an alteration to the original trace and $\Delta V$ must be small enough as shown in Figure 8. 



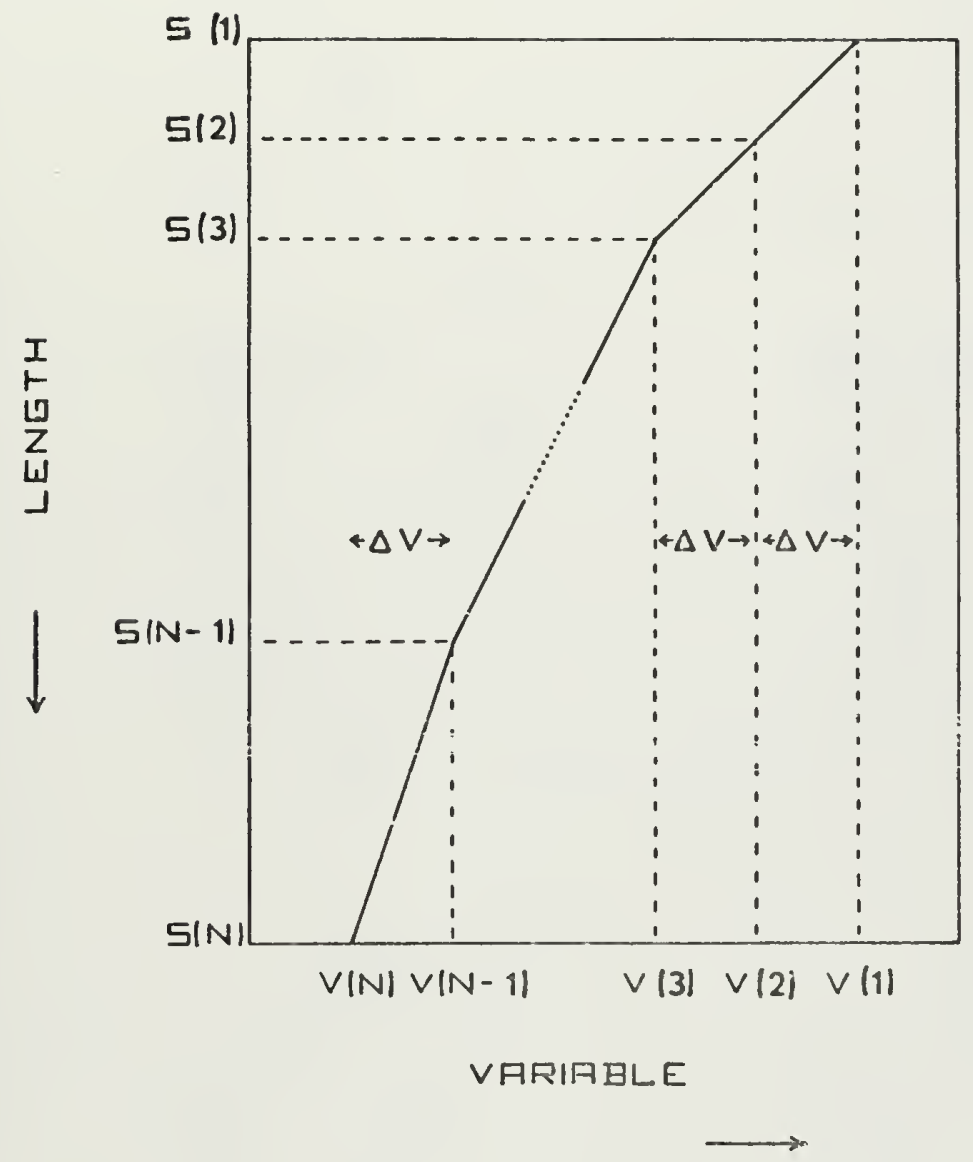

Figure 7. The Mesh 

A.
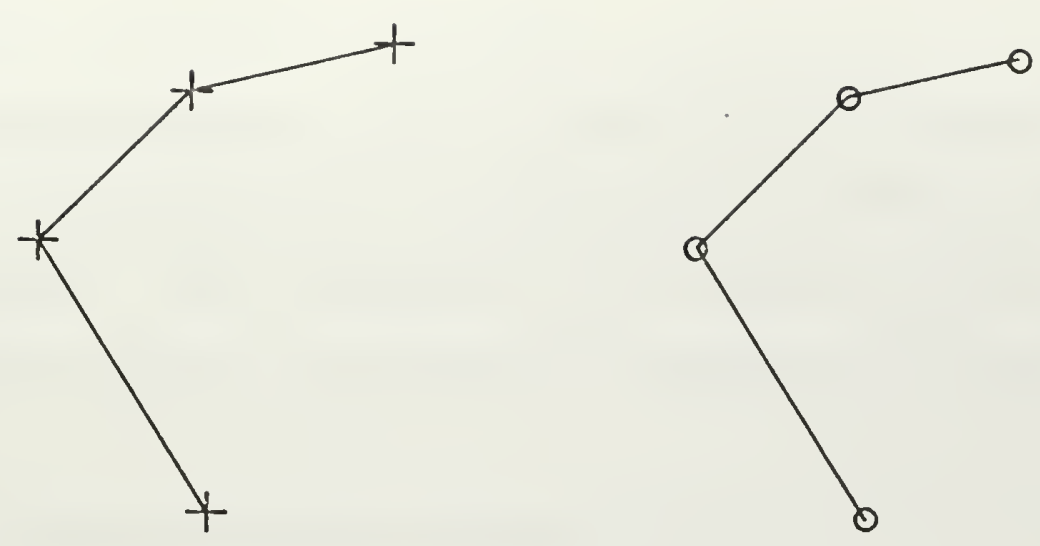

B.

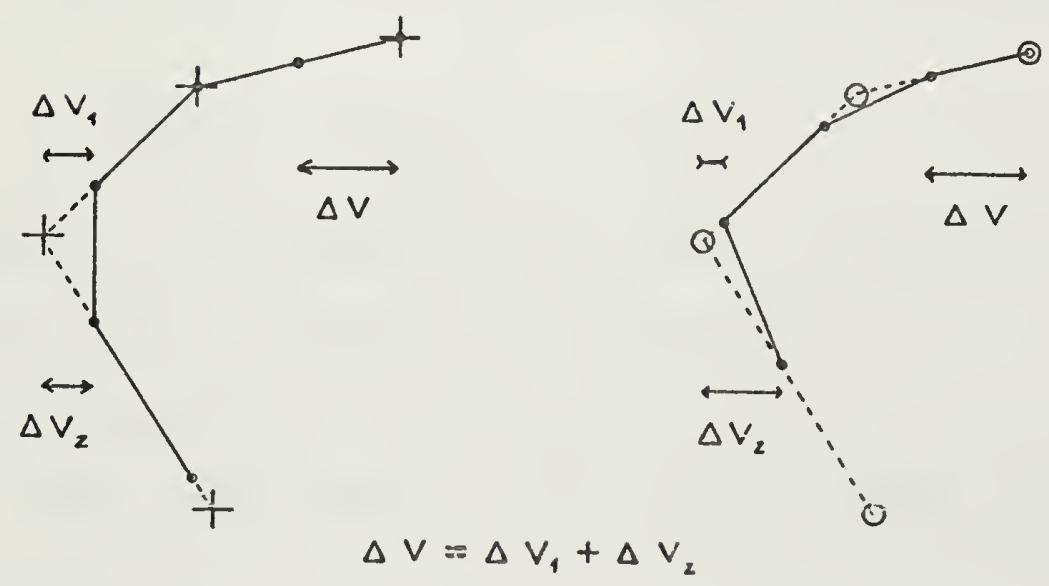

c.
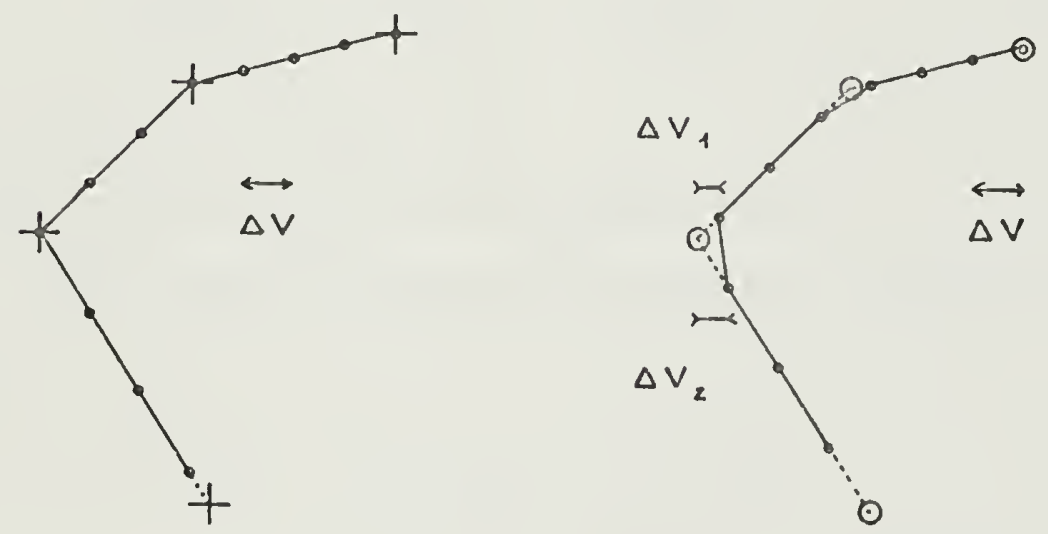

Figure 8. The Linear Interpolation of a Digital Trace: A. Digital Trace, B. Mesh (increment $V=2$ arbitrary units), C. Mesh (increment $V=1$ arbitrary unit) 



\section{b. Finite Difference Schemes}

A mesh consists of an ordered set of points at fixed intervals of variable and two consecutive positions define one linear segment. Each segment is specified by (a) the length component and (b) the variable component as illustrated in Figure $9 \mathrm{~A}$.

\section{(1) First Finite Difference of $\mathrm{V}$ The first finite difference of $V\left(V_{n}\right)$ is} defined as

$$
\Delta v\left(v_{n}\right)=v\left(v_{n+1}\right)-v\left(v_{n}\right)
$$

and may be positive (pointing to the right) or negative (directed to the left), as shown in Figure $9 \mathrm{C}$.

$$
\begin{aligned}
& \text { (2) First Finite Difference of } s \\
& \text { The Ilrst finice aiifference of } s\left(V_{n}\right) \text { is }
\end{aligned}
$$

stated as

$$
\Delta s\left(V_{n}\right)=s\left(V_{n+1}\right)-s\left(V_{n}\right)
$$

and is always positive, Figure $9 \mathrm{~B}$.

(3) Second Finite Difference of. $\mathrm{S}$

The second finite difference of $s\left(V_{n}\right)$ is

described as

$$
\begin{aligned}
\Delta^{2} s\left(V_{n}\right) & =\Delta s\left(v_{n+1}\right)-\Delta s\left(V_{n}\right) \\
& =s\left(v_{n+2}\right)-2 s\left(V_{n+1}\right)+s\left(V_{n}\right)
\end{aligned}
$$

and employs three points, $n, n+1, n+2$, from the mesh. The three points define two linear segments, whose relative arrangement will be related to the negative, positive or null value of $\Delta^{2} s\left(V_{n}\right)$, Figure 10 

A.

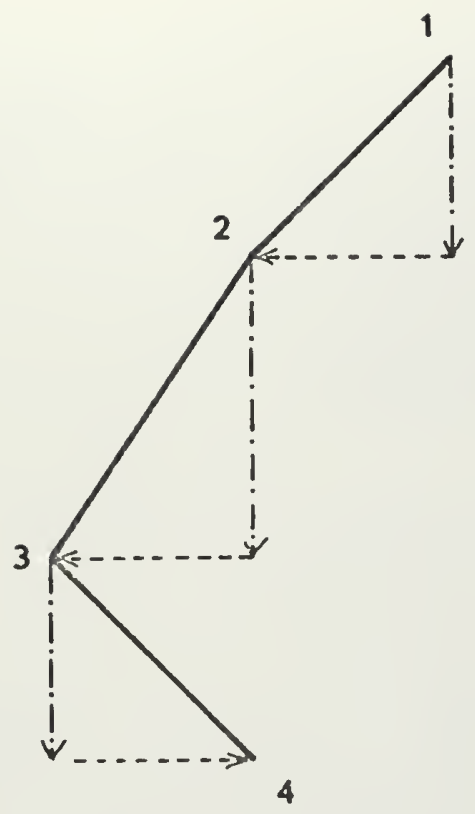

B.
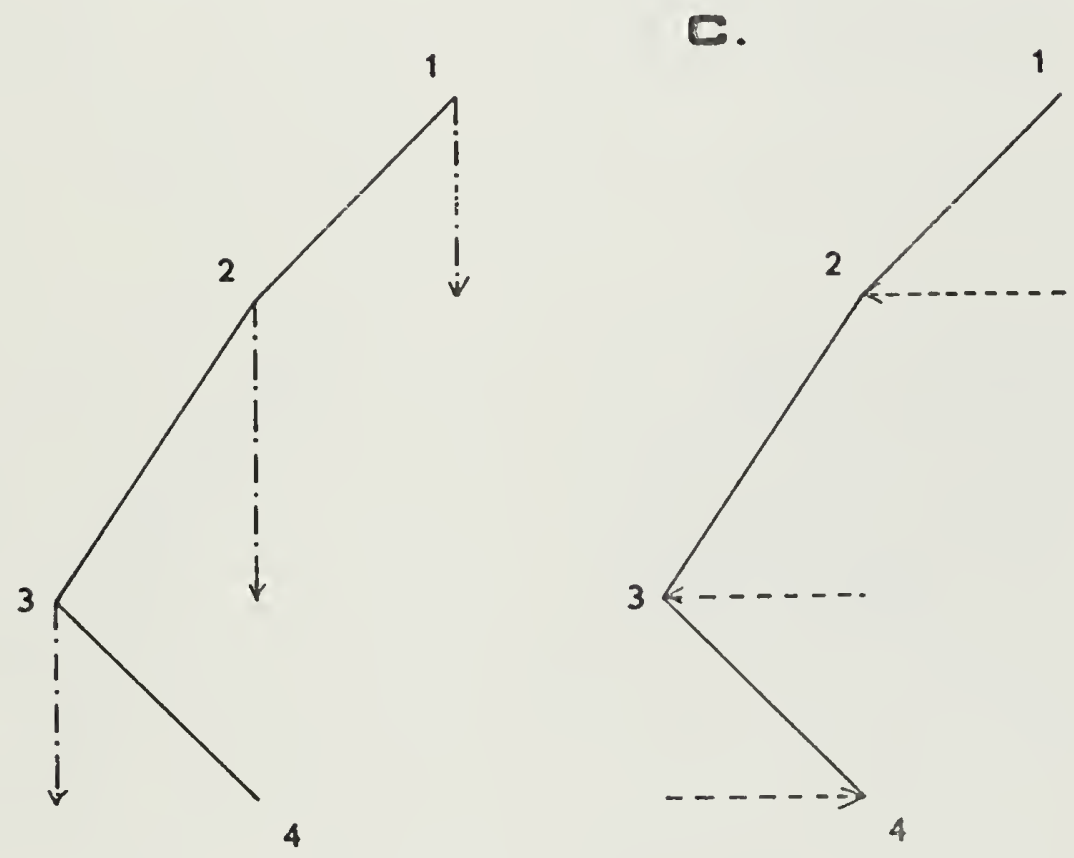

Figure 9. The Mesh and Its components:

A. Segments of the Mesh

B. Length Components

C. Variable Components 

A.

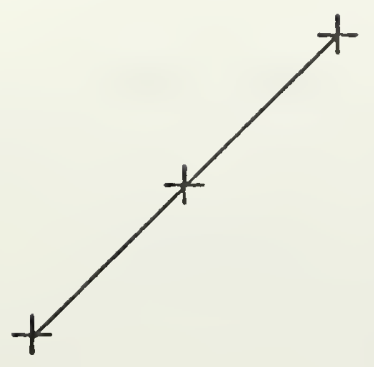

\begin{tabular}{|c|c|c|c|}
\hline & $5 \mathrm{IV}$ & $\Delta$ & $\Delta^{2}$ \\
\hline$N$ & 10 & \multirow{2}{*}{15} & \multirow{3}{*}{0} \\
\hline$N+1$ & 25 & & \\
\hline$N+2$ & 40 & 15 & \\
\hline
\end{tabular}

B.

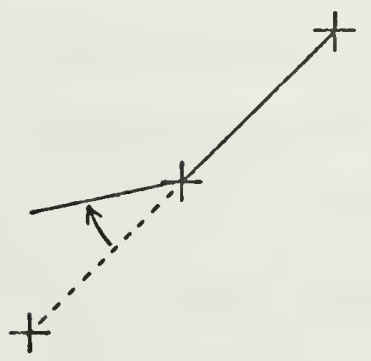

\begin{tabular}{|c|c|c|c|}
\cline { 2 - 3 } \multicolumn{1}{c|}{} & $S(V)$ & $\Delta$ & $\Delta^{2}$ \\
\hline$N$ & 10 & \multirow{2}{*}{15} & \\
\cline { 2 - 2 }+1 & 25 & -12 \\
\cline { 1 - 2 }$N+2$ & 28 & 3 & \\
\hline
\end{tabular}

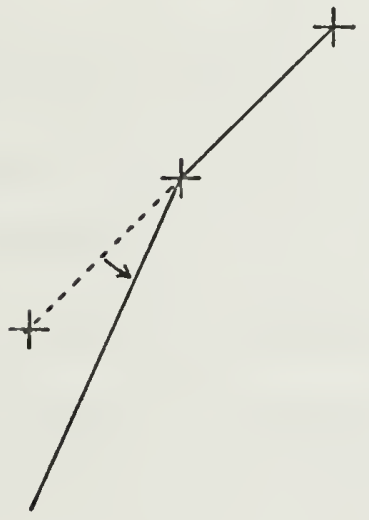

\begin{tabular}{|c|c|c|c|}
\hline & $5(V)$ & $\Delta$ & $\Delta^{2}$ \\
\hline$N$ & 10 & \multirow{2}{*}{15} & \multirow{3}{*}{18} \\
\hline$N+1$ & 25 & & \\
\hline$N+2$ & 58 & 33 & \\
\hline
\end{tabular}

Figure 10. Schemes of the Second Finite Differences: A. Constant Slope, B. Increasing Slope, C. Decreasing Slope 



$$
\begin{aligned}
& \text { (4) Third Finite Difference of } s \\
& \text { The third finite difference of } S\left(V_{n}\right) \text { is }
\end{aligned}
$$

specified as

$$
\begin{aligned}
\Delta^{3} S\left(v_{n}\right)= & \Delta^{2} s\left(v_{n+1}\right)-\Delta^{2} s\left(v_{n}\right) \\
= & \Delta s\left(v_{n+2}\right)-2 \Delta s\left(v_{n+1}\right)+\Delta s\left(v_{n}\right) \\
= & s\left(v_{n+3}\right)-3 s\left(v_{n+2}\right)-3 s\left(v_{n+1}\right) \\
& +s\left(v_{n}\right)
\end{aligned}
$$

and requires four points, $n, n^{+1}, n^{+2}, n^{+3}$, from the mesh. The $\Delta^{3} S\left(V_{n}\right)$ summarizes the relative disposition between three linear segments, defined by four points. Five basic schemes of the $\Delta^{3} S\left(V_{n}\right)$ are presented in Figure 11 .

(5) Higher Order Finite Difference of $\mathrm{S}$ Higher order finite difference of $S$,

$$
\Delta^{k} S\left(v_{n}\right)=\Delta^{k-1} S\left(v_{n+1}\right)-\Delta^{k-1} S\left(v_{n}\right)
$$

will demand more points from the mesh, namely the number of the order plus one and concurrently the analysis of the relative structure will increase in complexity. The development of the finite difference schemes up to the third order seems to constitute adequate advancement for the operational needs in the present research.

3. Two-dimensional objective Analysis

The finite difference schemes above introduced, will be applied to the significant features of a two-dimensional trace and illustrated by schematic diagrams.

a. Trend

As explained before, the trend shcis the tendency of the independent variable, $V$, which is investigated by the 

A.

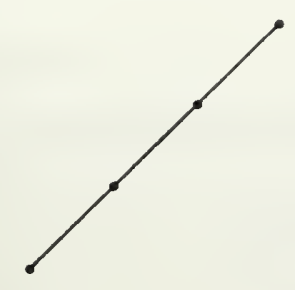

\begin{tabular}{|c|c|c|c|c|}
\hline & $5 \mid \mathrm{VI}$ & $\Delta$ & $\Delta^{2}$ & $\Delta^{\prime}$ \\
\hline$N$ & 10 & 10 & \multirow{2}{*}{0} & \multirow{4}{*}{0} \\
\hline$N+1$ & 20 & \multirow{2}{*}{10} & & \\
\hline$N+2$ & 30 & & \multirow{2}{*}{0} & \\
\hline$N+3$ & 40 & 10 & & \\
\hline
\end{tabular}

B.

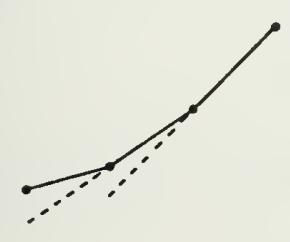

\begin{tabular}{|c|c|c|c|c|}
\hline & $5(V)$ & $\Delta$ & $\Delta^{2}$ & $\Delta^{\prime}$ \\
\hline$N$ & 10 & 10 & \multirow{2}{*}{-3} & \multirow{4}{*}{-1} \\
\hline$N+1$ & 20 & \multirow{2}{*}{7} & & \\
\hline$N+2$ & 27 & & \multirow{2}{*}{-4} & \\
\hline$N+3$ & 30 & 3 & & \\
\hline
\end{tabular}

E.

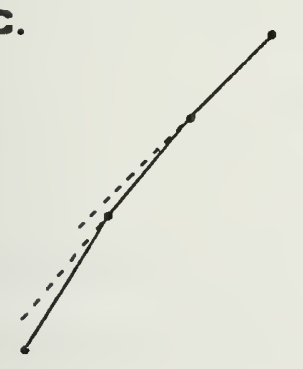

\begin{tabular}{|c|c|c|c|c|}
\hline & $5 \mathrm{IV} !$ & $\Delta$ & $\Delta^{2}$ & $\Delta^{\prime}$ \\
\hline$N$ & 10 & 10 & \multirow{2}{*}{2} & \multirow{4}{*}{2} \\
\hline$N+1$ & 20 & & & \\
\hline$N+2$ & 32 & & \multirow{2}{*}{4} & \\
\hline$N+3$ & 48 & 16 & & \\
\hline
\end{tabular}

D.

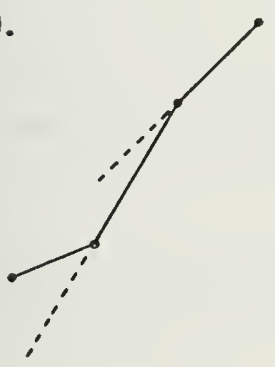

\begin{tabular}{|c|c|c|c|c|}
\hline & $5 \mid \mathrm{V})$ & $\Delta$ & $\Delta^{2}$ & $\Delta^{\prime}$ \\
\hline$N$ & 10 & 10 & \multirow{2}{*}{7} & \multirow{4}{*}{-20} \\
\hline$N+1$ & 20 & \multirow{2}{*}{17} & & \\
\hline$N+2$ & 37 & & \multirow{2}{*}{-13} & \\
\hline$N+3$ & 41 & 4 & & \\
\hline
\end{tabular}

E.

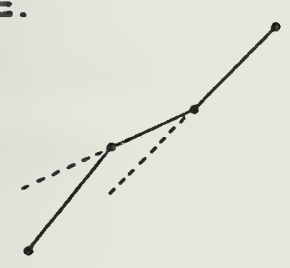

\begin{tabular}{|c|c|c|c|c|}
\cline { 2 - 3 } \multicolumn{1}{c|}{} & $5(V)$ & $\Delta$ & $\Delta^{2}$ & $\Delta^{2}$ \\
\hline$N$ & 10 & 10 & & \\
\cline { 2 - 2 }+1 & 20 & -5 & \multirow{2}{*}{5} & 12 \\
\cline { 1 - 2 }$N+2$ & 25 & 7 & \\
\cline { 1 - 2 }$N+3$ & 37 & 12 & & \\
\hline
\end{tabular}

Figure 11. Schemes of the Third Finite Differences: A. Constant Slope, B. Successive Increase of the Slope, C. Successive Decrease of the Slope, D. Successively Decreasing and Increasing the Slope, E. Successively Increasing and Decreasing the Slope 

use of its first finite difference, $\Delta V\left(V_{n}\right)$ expressed in (20). Assuming that $V$ increases to the right, as shown in Figure 6, the analysis of $V$ may be summarized as follows:

(1) Variation

(a) $\Delta V\left(V_{n}\right)<0: V_{n+1}<V_{n}, V$ is decreasing or directed to the left of the graph.

(b) $\Delta v\left(v_{n}\right)=0: v_{n+1}=v_{n}, v$ is constant or parallel to the length axis.

(c) $\Delta V\left(V_{n}\right)>0: V_{n+1}>V_{n}, V$ is increasing or tending to the right.

\section{(2) Extreme values}

The change in sign of the first finite difference of $V$ indicates that the trend has changed direction eñoj definos an extrame feature:

(a) $\mathrm{V}\left(\mathrm{V}_{\mathrm{n}+1}\right)<0$ and $\mathrm{V}\left(\mathrm{V}_{\mathrm{n}}\right)>0$ : relative maximum at point $n^{+1}$

(b) $\quad \mathrm{V}\left(\mathrm{V}_{\mathrm{n}+1}\right)>0$ and $\mathrm{V}\left(\mathrm{V}_{\mathrm{n}}\right)<0$ : relative minimum at point $n+1$

(c) The largest and the least of the $V$ values above found with the values of $\mathrm{V}$ at the beginning and end of the trace give the absolute maximum and minimum of the trace.

\section{b. Varicline}

The basic concept of the objective varicline is presented in Figure IIE composed of three segments and, although their relative arrangement is unchangeable the middle part can be divided into more than one segment. The analysis of the characteristics, 

(1) $\Delta^{2} V\left(V_{n}\right)<0$ : top of varicline at point $n^{+1}$.

(2) $\Delta^{2} v\left(V_{n+1}\right)>0$ : base or bottom of varicline at point $n^{+2}$ is indicated in Figure 11.

\section{c. Inversion}

The analysis of the trend indicates whether or not there are maxima and minima present in the record. The maxima and minima (excluding the values of $\mathrm{V}$ in the beginning and the end of the trace) evidence areas of approximate symmetry of $\mathrm{V}$ around the extrema. Referring to Figure 6 the analysis of the trend gave (a) the absolute minimum at g, (b) a relative maximum at $h$, and (c) the absolute maximum value in $\mathrm{V}$ was found at $\mathrm{a}$, the beginning of the trace. It is evident that locations $f$ and $h$ define a region of approximate symmetry of $\mathrm{v}$ around the relative minimum $q$. An inversion is characterized by a portion of the $(V, S)$ curve where the trend changes sign, illustrated by the layer $g$ - $h$ in Figure 6. The limits of the approximate symmetry are established by comparison of the variable magnitudes, $\Delta V^{\prime} s$ (17 or 18) determined using the point of symmetry of the extrema in consideration, $g$, and the adjacent positions defining extreme values in $V$, namely $a$ and $h$. The least $\Delta V$ is chosen as the variable magnitude of the inversion.

\section{d. Complex type}

The complex type of trace combines the basic investigations of trend, variclines and inversions. The method of procedure is outlined in three stages:

(1) objective analysis of the trend. 


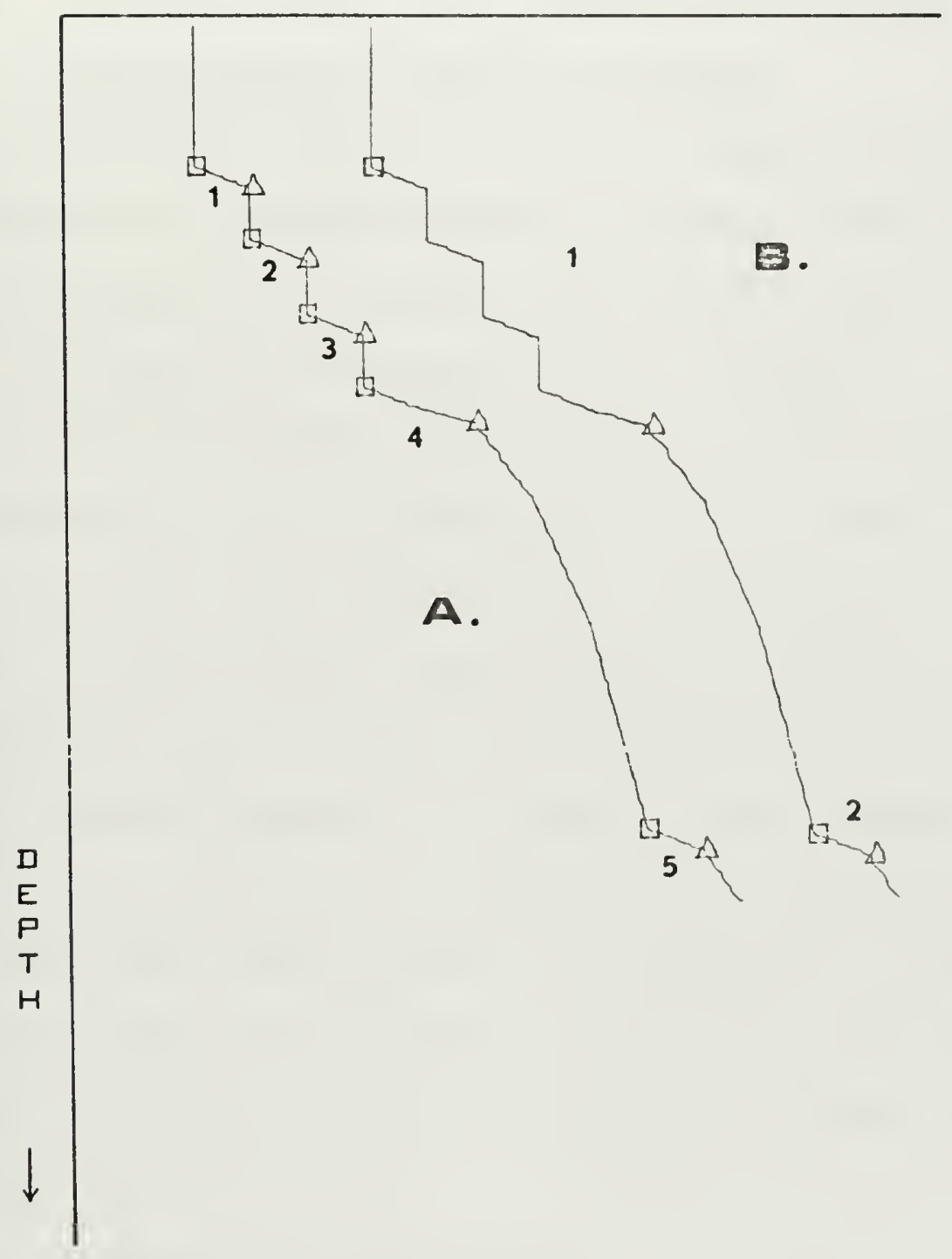

Figure 12. Computer Output plot of a Digital Trace Showing the Tops (squares) and the Bottoms (triangles) of Analyzed Variclines. (The control of the conditions limiting the bottom of a varicline allows: A. identification of all the variclines present, and B. definition of the significart features) 
(2) objective analysis of inversions.

(3) Objective analysis of variclines, which may be conducted throughout the entire trace or be restricted to those portions outside the symmetrical regions.

\section{Tri-dimensional Objective Analysis}

The two-dimensional objective analysis studies the distribution of a given variable $\mathrm{V}$ along a length axis $\mathrm{s}$. The availability of a collection of traces spread out in time, D, or along a horizontal, L, or vertical, S, direction illustrated in Figure 13, makes possible the investigation of a tri-dimensional distribution of $\mathrm{V}$ in (a) length $(\mathrm{V}, \mathrm{S}, \mathrm{L})$ and in (b) length and time $(V, S, D)$. An objective analysis of this tri-dimensional distribution is possible if the law of́ variation of $\mathrm{V}$ is known ox tha interval lin time oi length) between consecutive traces is small enough to assume a linear interpolation or digitization of the new trace. One of the above requirements is fundamental to generate a mesh and to apply the concepts of the two-dimensional objective analysis to each one of the new traces, shown in Figure 13B.

\section{The Problem of Filtering}

Usually a trace shows a complicated form with small variations superimposed on a general pattern. The small fluctuations may have physical significance or may simply represent an artificial response of the sensor; however, it may be impossible to distinguish whether a regular feature is valid or invalid. The most common pproach 
A.

LENETH,L DR TIME, D

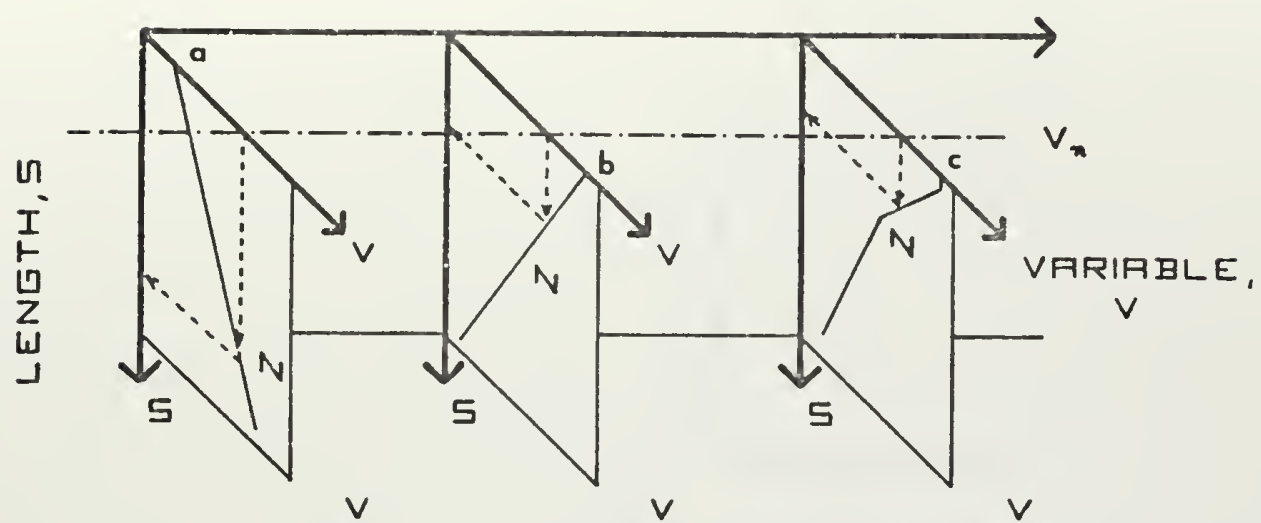

B.

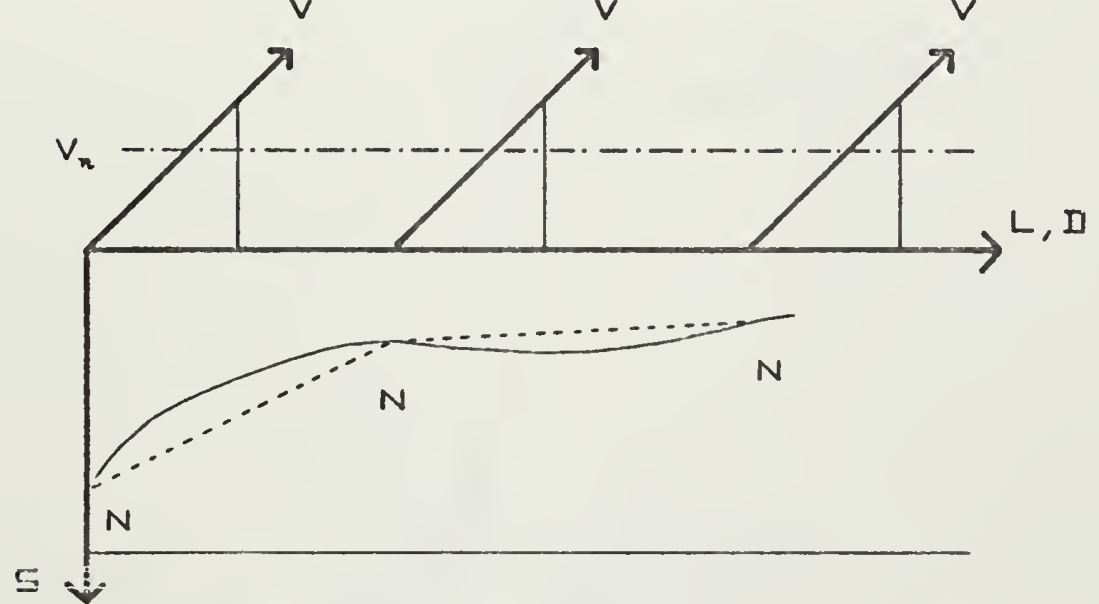

c.

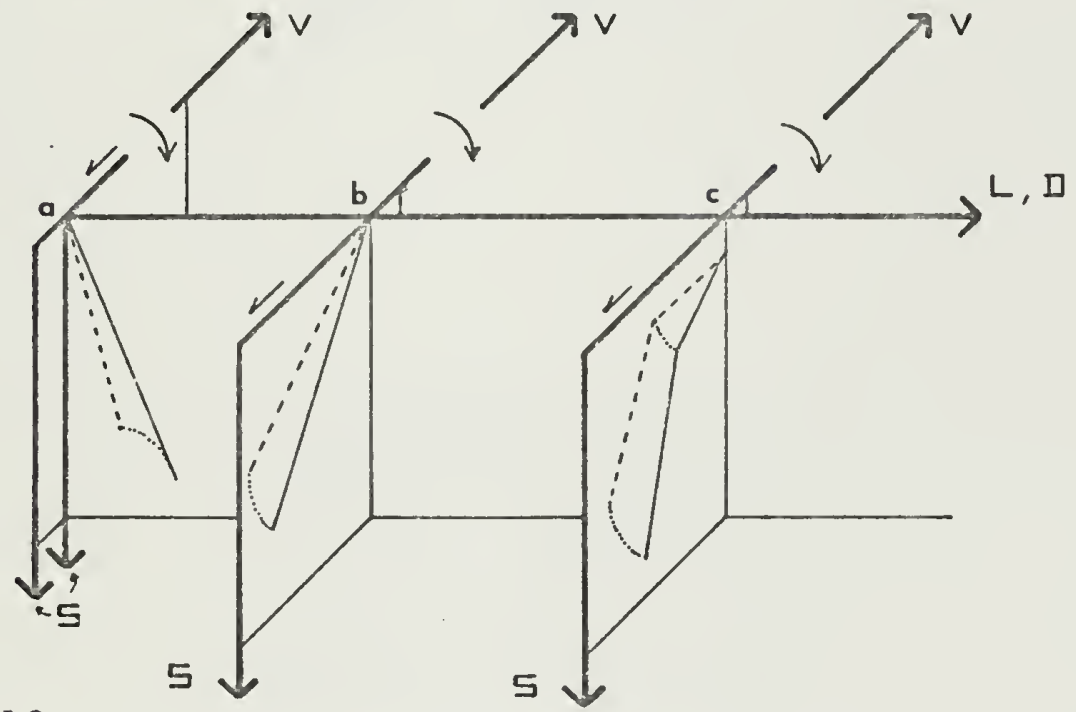

Figure 13. Tri-aimensional Analysis: A. The Tri-dimensional Distribution, B. A New Trace, C. The Projection of Two-dimensional Traces Along a Given Axis 

TEMPERATURE

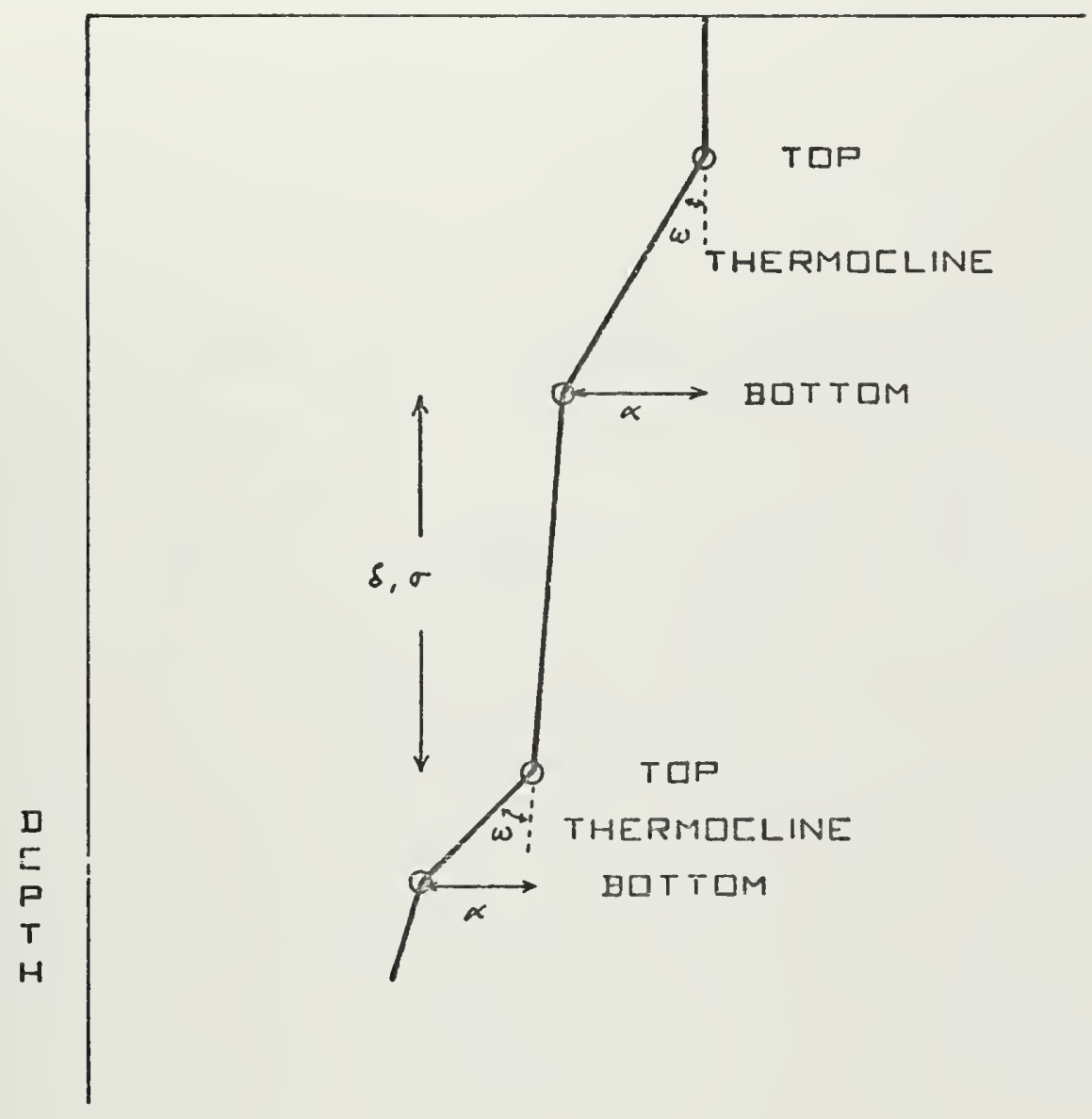

\begin{tabular}{|l|l|}
\hline \multicolumn{2}{|c|}{ FILTERING STEPS } \\
\hline 1 & $\delta$ or $\sigma, \theta=\tan \omega$ \\
\hline 2 & $\alpha$ or $\beta=\tan \omega$ \\
\hline
\end{tabular}

Figure 14. The Filtering Parameters of Variclines 

$\times$ XT STATIONS RLDNG CRUSS SECTION AXIS $\rightarrow$

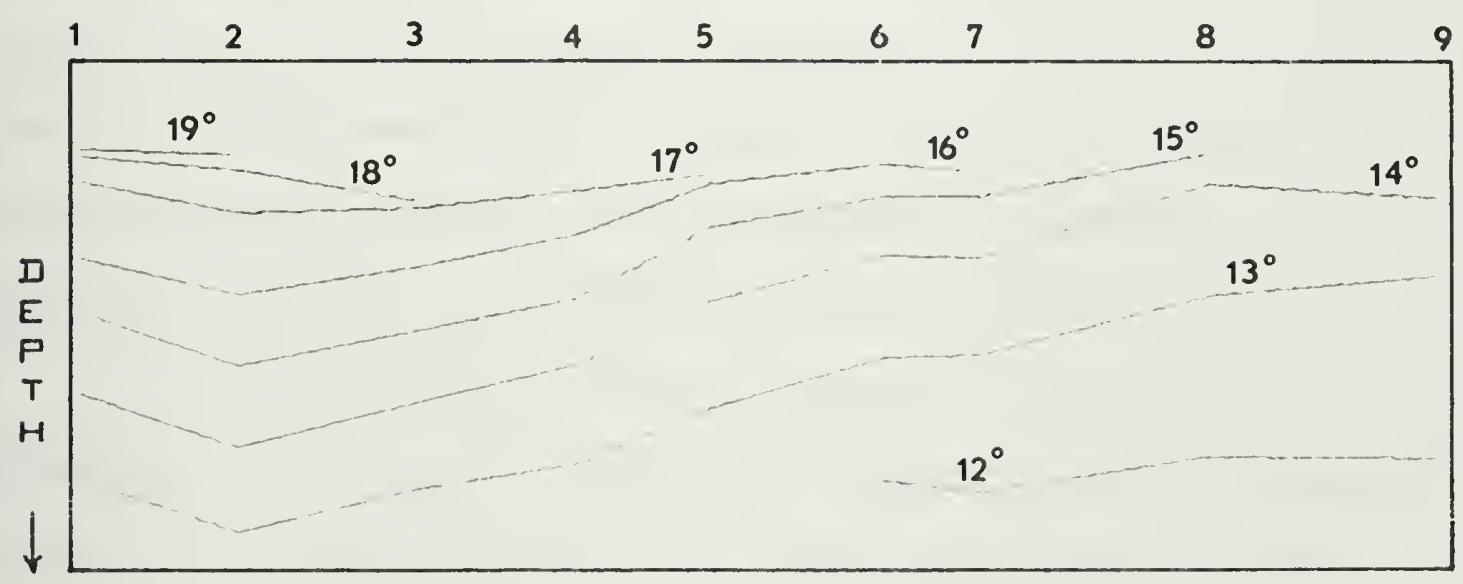

Figure 15. Computer output Plot of the Linear Field of Isotherms for one Cruise or the Average Linear Field of Isotherms for a Given Number of Cruises 

consists in the identification of the general pattern of the trace and this outlines a problem of scale: if the investigator is interested in microstructure, his main efforts are concentrated in the improvement of the accuracy of the sensor. The technique of suppressing the small features has not found definite and complete solution. It reveals a compromise established between the need to avoid small fluctuations and the unfortunate results when those variations were physically meaningful and their filtering has introduced artificial values. The best approach requires a good understanding of the geophysical phenomena and a suitable application of the filtering methods.

In this thesis study, analytical filters such as the binomial expansion and the least square polynomial apprcximation were used to smooth the generated mesh but they introduced severe modifications which (as presented in Figure 16B) completely deformed the original information, and their further application was avoided.

The discussion of the filtering capaility of the finite difference technique constitutes the purpose of the present chapter.

\section{a. The Mesh}

The generated mesh depends upon the selection of the constant increment, $\Delta \mathrm{V}:$ (a) a large value erases the smal.I features but introduces distortion in the digital trace, and (b) a small value (compatible with the accuracy of the sensor) keeps the details and reproduces closely the di ital trace, as shown in Figure 8. 



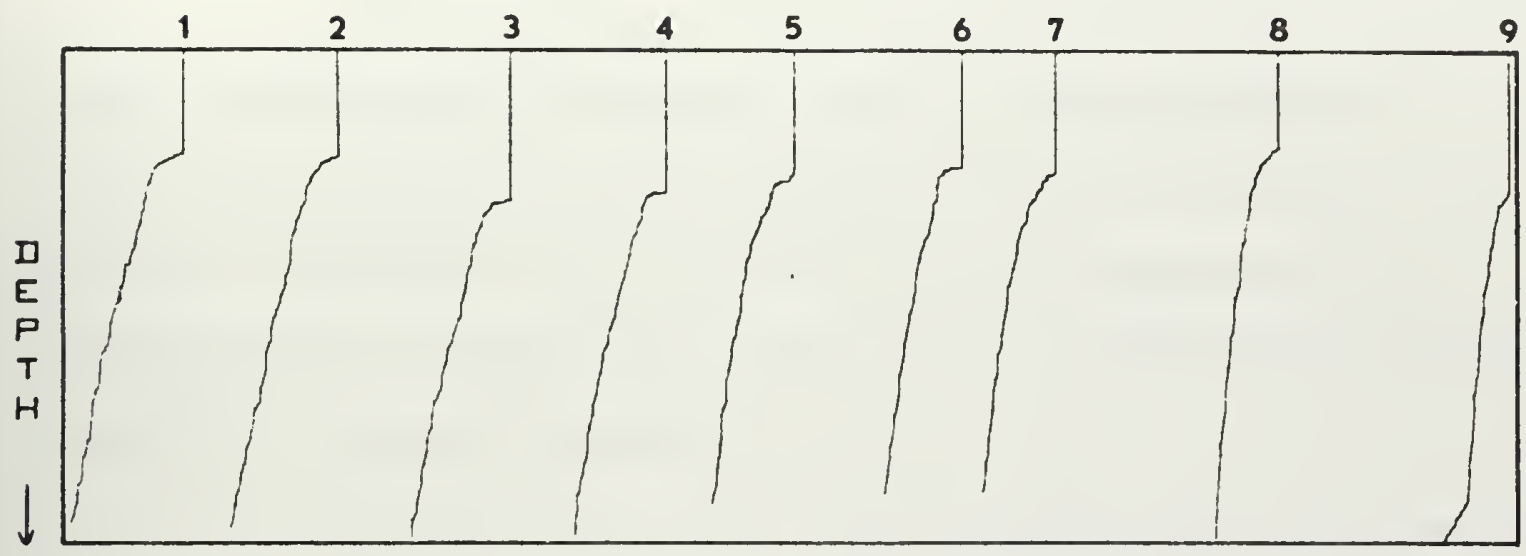

B.

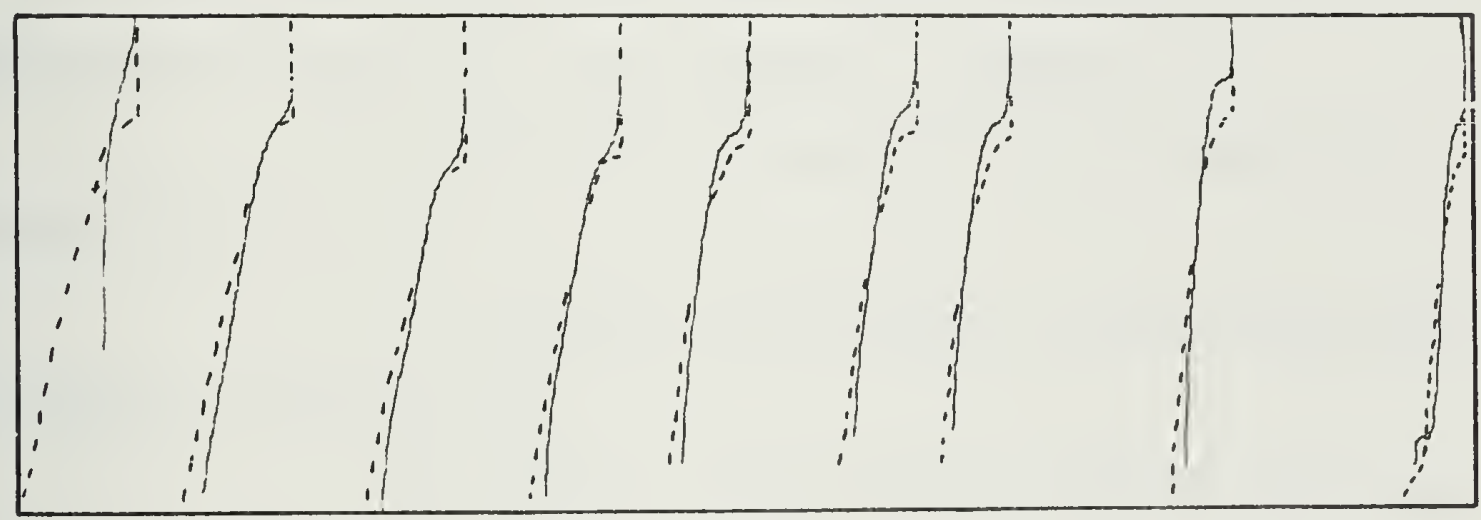

5TH. DEGREE POL. APP. (FULL)

Figure 16. Computer output Plots of: A. The Interpolated Digital Traces Along a Cross section for a Given Cruise, and $B$. The Fifth Order Least Square Polynomial Approximation of the same Digital Traces 


\section{b. Significant Features}

Generically, significant features like inversions and variclines may be filtered out by specification of filtering parameters or the minimum values of (a) the defining parameters $(8-11)$ and (b) the separation in length between consecutive and identical features (bottom of one and the top of the next).

Specifically, the filtering of variclines may be obtained by :

(1) The conditions limiting the top and the bottom of variclines (especially the bottom) and expressed in II.B.3.b., may be modified and extended to more points of the mesh above and below the feature resulting in its more clear definition, as evidenced in Figure 12.

(2) The filtering parameters illustrated in

Figure 14:

(a) ALPHA or the minimum variable magnitude limiting the varicline.

(b) BETA or the minimum gradient defining the varicline.

(c) DELTA or the minimum separation in length between consecutive variclines.

(d) THETA or the minimum gradient selecting the variclines to be merged, using simultaneously the SIGMA parameter.

(e) SIGMA or the maximum separation in length between variclines to be merged and already desined by the THETA parameter. 


\section{A COMPUTER PROGRAM}

The investigation of the vertical distribution of temperature in the upper oceanic layers can provide a digital model which applies the concepts of objective analysis described above.

Usually the data available is too non-uniformly distributed in time and length to make possible the use of a tri-dimensional objective analysis; however, a two-dimensional type may be utilized to examine each trace and to plot the result of the investigation (of all the traces obtained in a cruise or passage along a cross section) in a way which allows a subjective analysis.

An introduction of the model, its characteristics and cocration is summarized in the noxt presentation.

\section{Description}

a. The Basic Computer Model

The computer model is organized in two parts:

(a) the main program, and (b) the subroutines. The main program presents in a logical way a sequence of events concerning the execution of the job. This arrangement is a compromise between the space available in the computer memory and the least time of execution. The subroutines deal with specific stages of that execution.

The following description outlines the major operations of the model and their implementation must be monitored closely by the use of the flow diagram presented in Appendix B, with the symbols explained in Jpendix A. 


\section{(1) "START" to "NORDER = $1 "$}

The computer program receives the general information about the number identifying the cross section in study (ICSEC), the number of cruises per cross section (ICRUI), the number (ISO) and the values (TI) of isotherms, the mesh interval (DTW) and the number (NBT) and location (x) of stations for the cross section.

(2) "DO 460" Loop

The computer program processes the complete set of information about the thermal structure and wind field for a given cross section, cruise after cruise:

(a) "ITITLE'S" reads the titles of the plots drawn per cruise.

(b) "DO 250" loop reads the incation (ISTA), the number (NOP) and pairs of points ( $\mathrm{ZO}, \mathrm{TO})$ defining each digital trace in the cruise and processes the information about the depth of each isothermal value (GTVALU or ARTFTl). This scheme applies to all digital traces of a cruise.

(c) "CALL PLOTIT" receives the above generated data for the whole cruise and plots the field of isotherms (TI).

(d) "DO 350" loop reads the location (ISTA), the number (NOP) and pairs of points $(\mathrm{ZO}, \mathrm{TO})$ defining each digital trace in the cruise and prepares the mesh for that trace (LINTPS), plots the obtained mesh (PLOTBT) and analyzes it to identify thermoclines (THERMC).

(e) "CALL PLOTFT" receives the above generated data for the whole cruise and plots the field of thermocline isopleths. 

(f) "DO 450" loop reads the location (ISTA) and the wind observations (WIND); determines the components of the wind along and perpendicular to the next coastline at the day of XBT/BT drop and one day before (WINDOI), two days before (WINDO2), three days before (WINDO3), five days before (WINDO5) and ten days before (WIND10); plots the wind and its components.

(g) "NPLOT $=3 "$ to "460" completes the drawing of the wind information for a cruise and sets in the process for a new cruise.

(3) "IKONTR" to "IKONTR = 0"

This step controls the execution of the remaining part of the program. If the IKONTR value is zero the joh ends.

(4) "IERIOD ..." to "CALL STATCS"

The computer program reads the required information to start the statistical study (STATCS) between any two parameters $(\operatorname{KEK}=1, \operatorname{KEK}=2)$ and to plot the scatter diagram and the least square regression lines in the same graph (ITITL5). The continuation of the statistical study relies on the value of $\mathrm{KRK}$ read if different from zero.

(5) "ILONTR" to "ILONTR = 0 "

This part controls the execution of the last part of the program. If the ILONTR value is zero, the job ends.

(6) "ITITL6" to "PLTMIT"

The program receives the gene: ated data of the whole cross section and plots the average field of isotherns (PLTMIT) with a printed label (ITITL6). 



\section{(7) "ICSEC" to "STORAGE"}

The program indicates that the study of the cross section (ICSEC) is completed and ends the job by the storage in a disk of the original and generated information (STORAGE) .

b. Other Subroutines Available

Other subroutines are available to improve or to modify the operation of the basic model but keeping its main structure.

\section{(1) Subroutine TREND}

The subroutine TREND, Appendixes D, G and M, analyzes the digital trace using the finite difference technique to locate the maxima and the minima.

\section{(2) Subroutine INVERS}

The subroutine INVERS, Appendixes E, G and $M$, determines the inversion parameters for each minimum identified by subroutine TREND.

\section{(3) Subroutine PROPAR}

The subroutine PROPAR, Appendixes $H$ and $K$, computes the friction velocity, roughness parameter and stability length from wind speed and air-sea temperature difference data.

\section{(4) Subroutine ITRATE}

The subroutine ITRATE, Appendixes I and L, calculates the friction velocity, inflow angle, roughness parameter and stability length from the large scale synoptic parameters. 



\section{(5) Subroutine DRAW}

The subroutine DRAW, Appendix J, neutralizes

the "IBM subroutine DRAW" avoiding output plots.

\section{Input}

The form of input to this program consists in data

deck.

a. Main Program

The input to the main program is identified by the symbol READ shown in Appendix A. The flow diagram of the main program, Appendix B, gives the ordered sequence of the data deck. The format of individual input is specified in the computer program, Appendix F.

b. Subroutine THERMC

The subroutine THERMC requires the insertion of chosen values for the filtering parameters (ALPHA, BETA, DELTA, SIGMA, THETA) before running the program.

c. Subroutine PROPAR

The subroutine PROPAR requires special data described in Appendix $H$, which must be read together with the wind information as shown in Appendix $\mathrm{K}$.

d. Subroutine ITRATE

The subroutine ITRATE needs large scale synoptic information pictured in Appendix I, which must be read together with the wind data as presented in Appendix L.

\section{Output}

The forms of putput from this program are (1)

printouts, (2) plots, and (3) storage in disk. 

a. Printouts

The printouts are identified by the symbol

PRINTOUT, in Appendix A. The flow diagrams show the occurrence of printouts and their formats are specified in the computer programs.

The inclusion of the DUMMY card, Appendix P.a. neutralizes this form of output.

\section{b. Plots}

The basic program presents six types of plots which appear according to the execution of the following subroutines:

\section{(1) PLOTIT}

The subroutine PLOTIT plots the linear field of ispthorms per crilise, as shown in Figure 15.

\section{(2) PLOTBT}

The subroutine PLOTBT plots the interpolated digital traces obtained in a given cruise, Figure 16.

\section{(3) PLOTFT}

The subroutine PLOTFT plots the linear field of isopleths defining the top, the mean depth and the bottom of the thermoclines analyzed from the interpolated digital traces of a given cruise, Figure 17.

\section{(4) PLTMIT}

The subroutine PLTMIT plots the average linear field of isotherms for a specified number of cruises, Figure 15. 



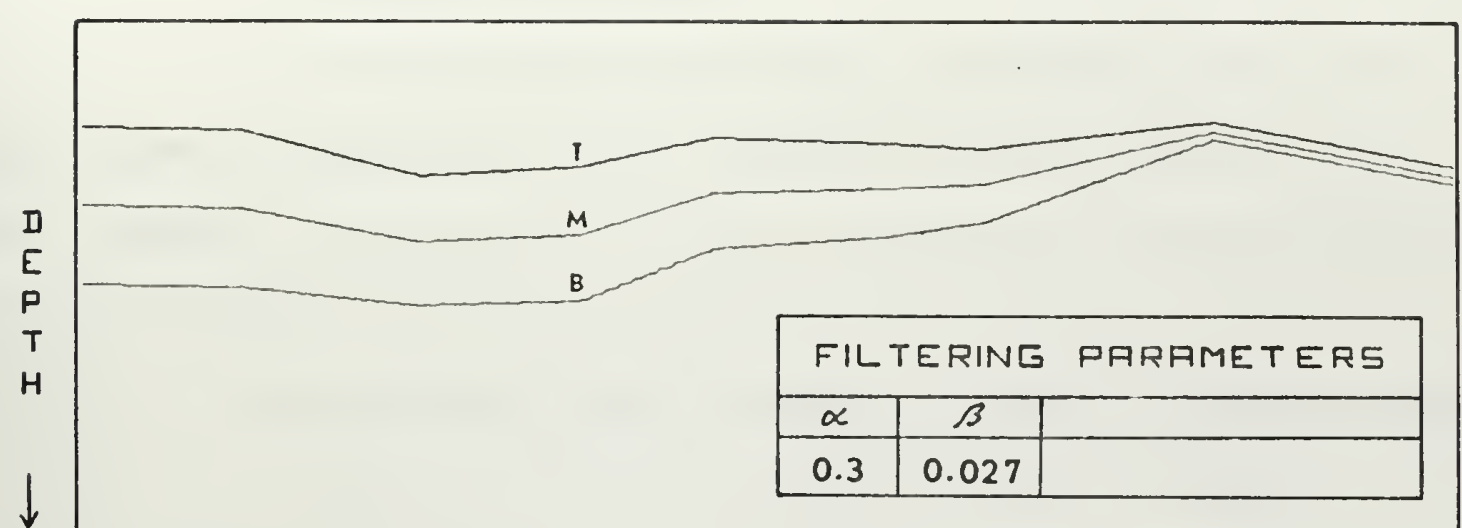

B.

CROSS SECTION FXIS $\rightarrow$

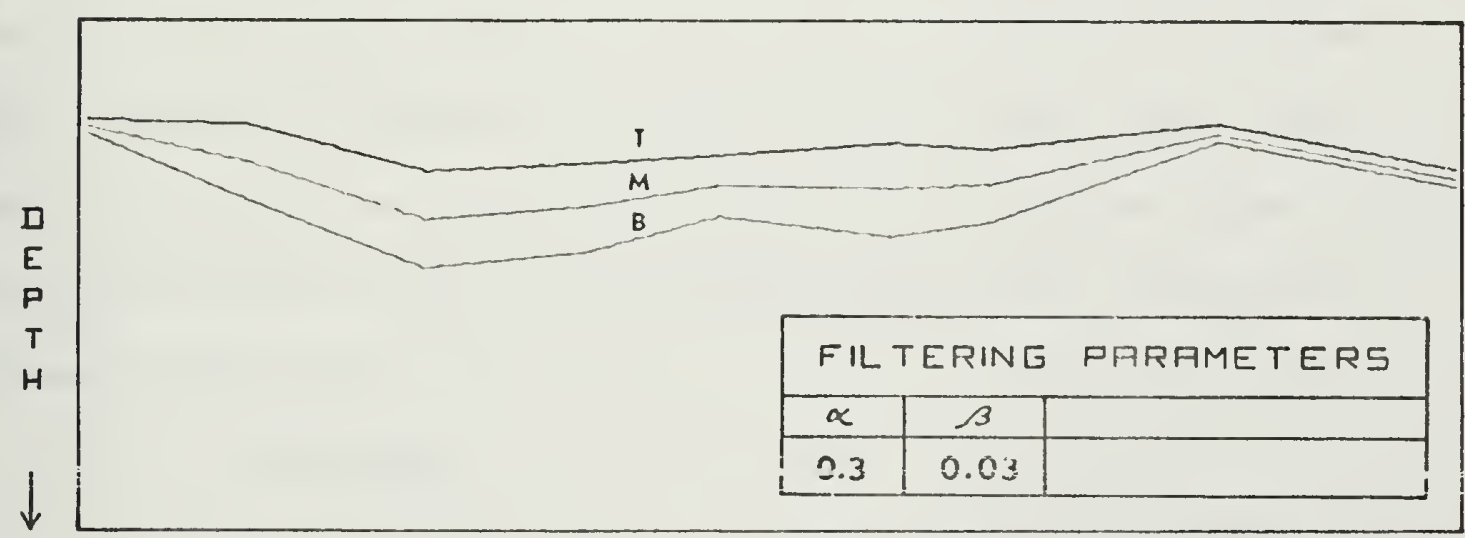

CRDSS SELTION RXIS $\rightarrow$

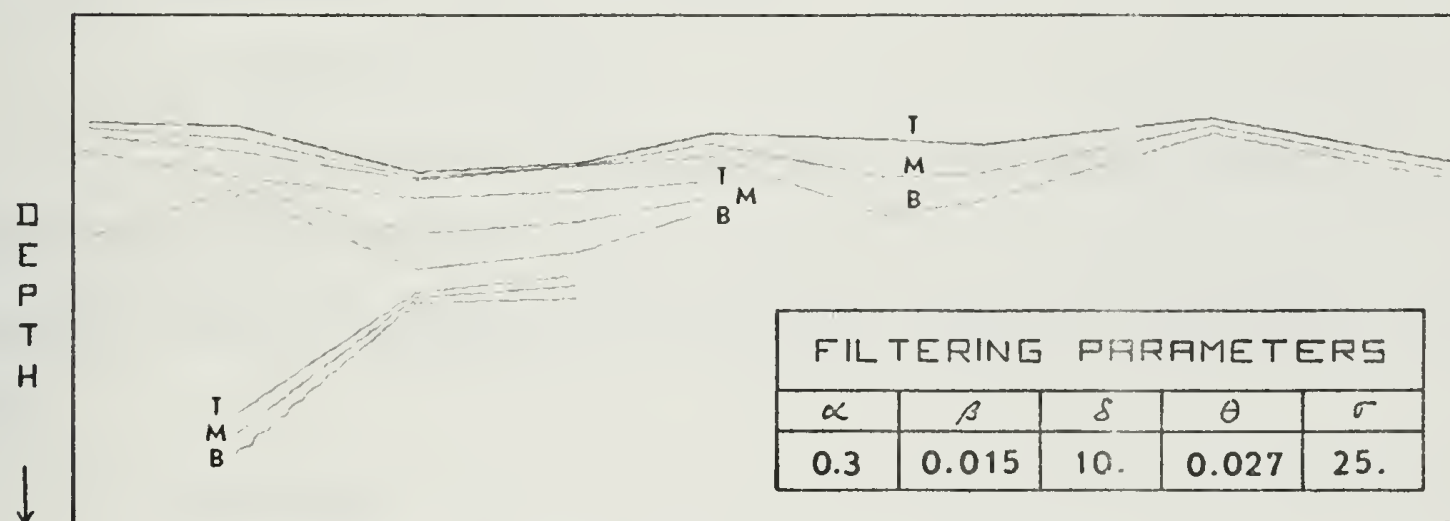

Figure 17. Computer output plots of the Linear Field of Isopleths Defining the Top (T), the Mean Depth (M), and the Bottom (B) of the Three Shallowest Thermoclines for Different Values of the Filtering parameters 



\section{(5) RLINE and STATCS}

The subroutines RLINE and STATCS plot the least square regression lines (RLINE) and the scatter diagram (STATCS) in the same graph to illustrate the statistical relation between two given variables, Figure 18.

(6) WINDOO, WINDO1, WINDO2, WINDO3, WINDO5, WIND10 The subroutines WINDOO, WINDO1, WINDO2, WINDO3, WINDO5 and WIND10 plot the wind vector and its components (along and perpendicular to the direction of the near coastline) measured at given station and day of XBT observation and at one, two, three, five and ten days before. The drawings are made in the same diagram for a specific cruise, Figure 19.

\section{(7) DRALE}

The insertion of the subroutine DRAW, Appendix $J$, will avoid this type of output.

\section{c. Storage in Disk}

The initial and processed data are stored in allocated space of disk depending upon the inclusion of the Job Control Language instructions shown in Appendix O.b, $c, d$.

4. Operation

a. Characteristics of the Basic Program

\section{(1) Source Language}

The computer program is written in FORTRAN IV $(H)$ and the calculations are performed in real single precision arithmetic. 
VARIFELE 1

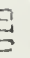

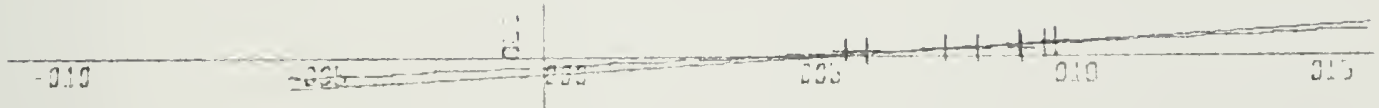

VARIAELE 2

Figure 18. Computer output Plot of the Least Square Regression Lines and the Scatter Diagram (t) for Two Given parameters: Variable 1 and Variable 2 

XBT STATIONS RLING CRDSS SELTIDN AXIS

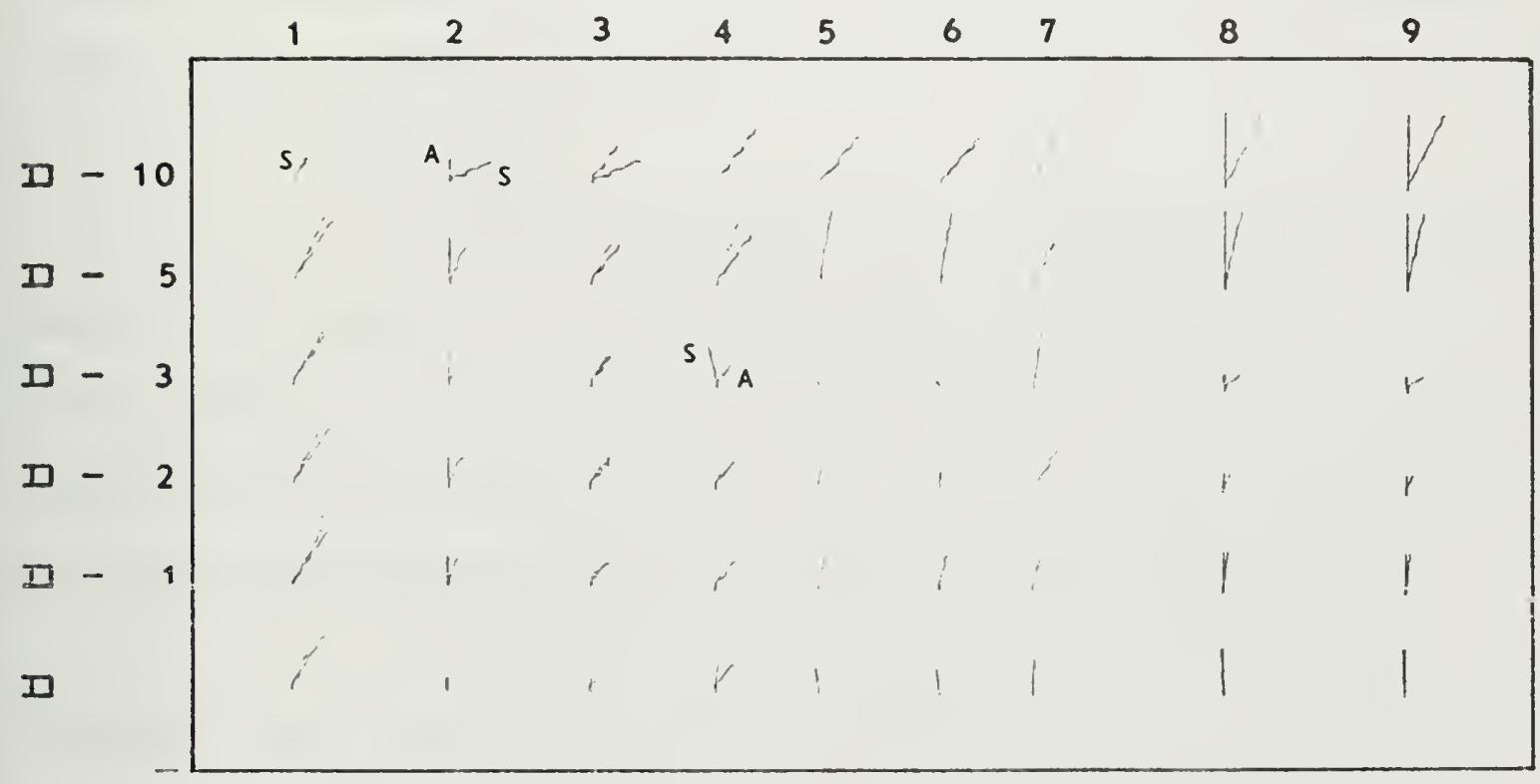

D - DAY DF XBT $\square$ - DERVATION

$S$ - WINDVELDLITY

A - VECTDR COMPDNENT DF S ALONG THENEAR

Figure 19. Computer output Plot of the Wind Vector and its Components (along and perpendicular to the direction of the near coastline) Measured at Same station and Day of XBT Observation and one, Two, Three, Five and Ten Days Before 



\section{(2) Subroutines and Functions Subprograms}

The computer program uses subroutines and functions subprograms that had been compiled or been added to a library under OS/MVT. If the source deck of subroutine MISR had not been precompiled, it might be accessed (in Naval Postgraduate School Computer Facility) by concatenation in the FORTRAN compile step using the Job Control Language cards shown in Appendix N.d,e.

\section{(3) Core}

The basic computer program processes information for twenty cruises, each containing twelve stations and requires $288 \mathrm{~K}$ in core dimension, demanding the FORTRAN compilation step $150 \mathrm{~K}$ which must be requested by means of the Job Control Language card oresented in Appondix N.b.

The space available is divided in five "COMMON" labelled areas which enables (a) the exchange of information between the main program, and (b) an economic and profitable use of the computer memory.

\section{(4) $\underline{\text { Time }}$}

The time required to process (without statistical study) a set of seventeen cruises, each with nine stations, is about three minutes and thirty-four seconds. This time is distributed by (a) the compilation (one minute and forty-one seconds), (b) the linkage (three seconds), and (c) the execution (one minute and fifty seconds). 



\section{(5) Disk}

The storage of the initial and processed

information in a disk is obtained by the use of the Job Control Language cards shown in Appendix o.b,c,d. The requested space in disk corresponds to one cylinder or IBM 2314 .

b. Equipment

The basic equipment used was:

(1) IBM 360/Model 67, OS - MVT with a

(2) CALCOMP Plotter, Model 765. 



\section{APPLICATION OF THE OBJECTIVE MODEL}

The computer model was tested by the use of bathythermographic and wind information available from Fleet Numerical Weather central for the geographical area shown in Figure 1.

The XBTs were taken during three years of passages between Canary Islands and Ferrol (Bay of Biscay) and Canary Islands and Barcelona (Mediterranean Sea) by Spanish ships. The XBT traces, in digital form and FORTRAN format compatible with this program, were organized in three cross sections, each one describing the normal routing of the survey ships and containing ten stations. In total, about 750 XBT traces were ready for further processing. Values for the filtering parameters of the thermocline analysis were previously chosen, namely

$$
\begin{aligned}
& \text { ALPHA }=0.3^{\circ} \mathrm{C} \\
& \text { BETA }=0.015^{\circ} \mathrm{C} / \text { meter } \\
& \text { DELTA }-10.0 \text { meters } \\
& \text { THETA }=0.027^{\circ} \mathrm{C} / \text { meter } \\
& \text { SIGMA }=25.0 \text { meters }
\end{aligned}
$$

and applicable to all seasons and stations of the area. Finally, the objective analysis of the bathythermograph information was performed.

For each station of the cross sections, geostrophic wind data were computed from surface pressure analysis in FNWC. The wind obtained was not corrected for instability due to lack of information concerning the air-sea te jerature 

difference and according to Cardone [1969], such correction is fundamental to assure reliable wind data, specifically "friction velocity". Without this transformation, further handling of wind was decided to be inappropriate.

The final part of the application of the model, which is a statistical study between the wind and the thermal fields, as well as the computation of the first, second, third and fourth statistical moments (to infer known statistical distributions) of each field for a given station of the area could not be physically concluded due to the lack of proper wind data. Otherwise, the difficulties pointed out the importance of valid wind information and motivated the inclusion of subroutines, developed by Cardone[1969], to calculate "friction rolocity" from large sinale synoptic information (ITRATE) or ship reports (PROPAR). 



\section{CONCLUSIONS}

A FORTRAN IV program was developed and tested to handle large amounts of wind and bathythermographic information in an automatic way without distorting the initial data and restricting the human participation to a preparation phase dealing with organization of the information available and selection of values for the parameters of the model. The program performs objective analysis of XBT/BT digital traces, processes wind information, studies the statistical characteristics of the original and processed data and plots the generated fields.

The objective analysis of XBT/BT traces makes use of finite difference schemes which requires preceeding transformation of the analog trace into digital form. The same concepts may be extended to analyze the distribution of any environmental variable (similar to the bathythermograph) along vertical or horizontal directions. 



\section{RECOMMENDATIONS}

It is recommended that bathythermograph data be analyzed by an objective method before any subjective interpretations are made.

The objective analysis of XBT/BT traces may be performed aboard ships by the use of digital computers requiring a small memory but, the pre-digitization of the analog trace constitutes a fundamental prerequisite for processing. 



\section{APPENDIX A}

FLOW DIAGRAM SYMBOLS

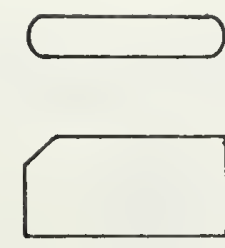

$$
\text { LIMITS }
$$

RERD

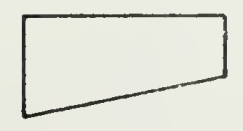

PRINT DUT

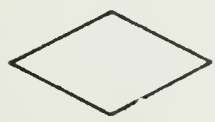

DECISION

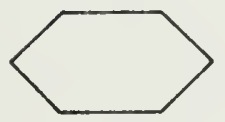

LDRP

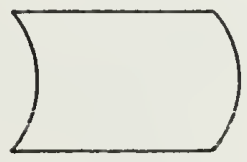

STDRAGE IN DISK

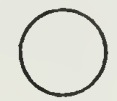

LONNEETION

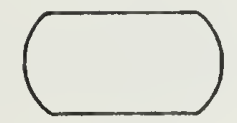

SUEROLTINE IR FUNETION

END DF LDQP

PRDEESS 



\section{APPENDIX B}

FLOW DIAGRAM OF THE FORTRAN

IV BASIC COMPUTER PROGRAM

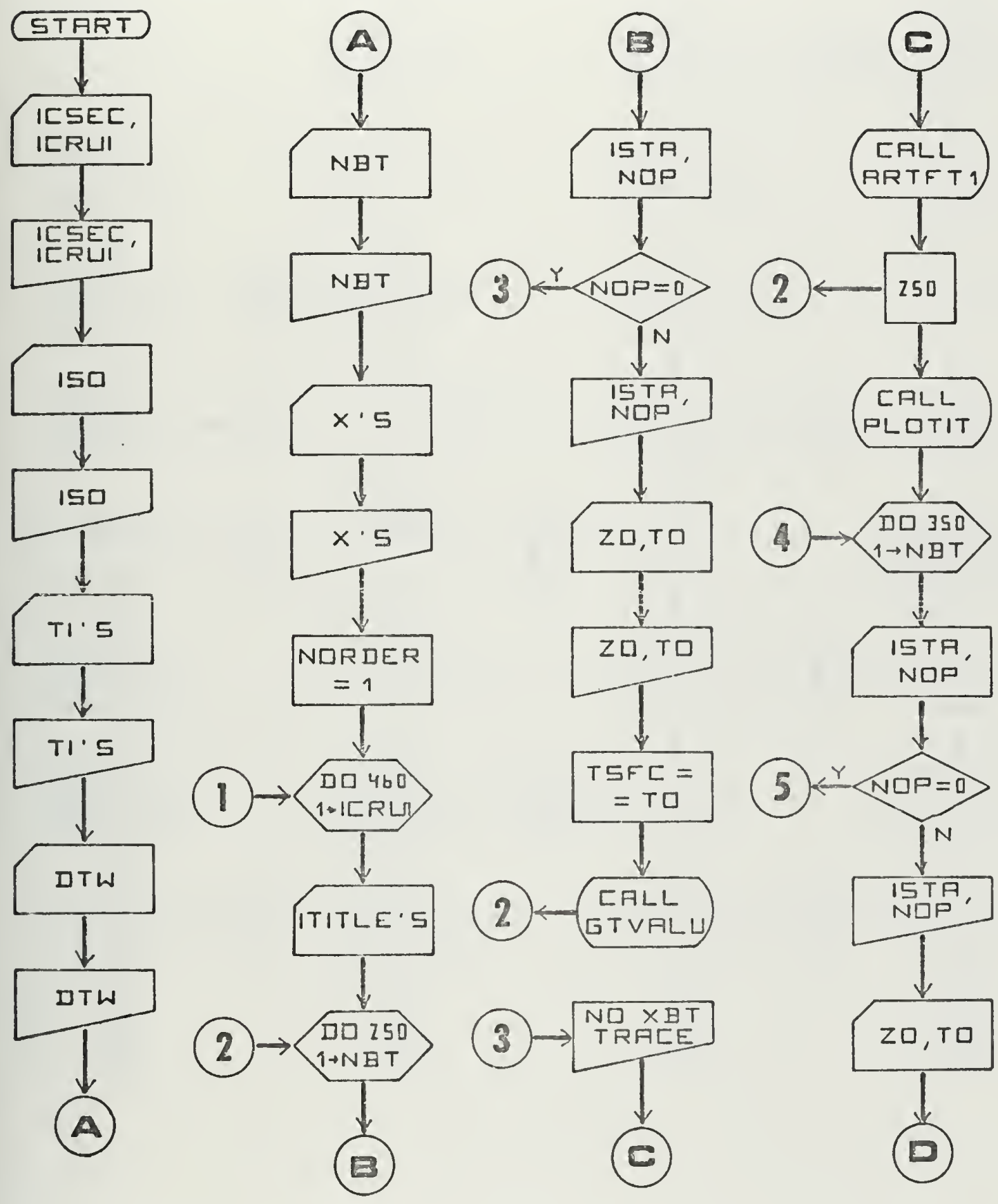





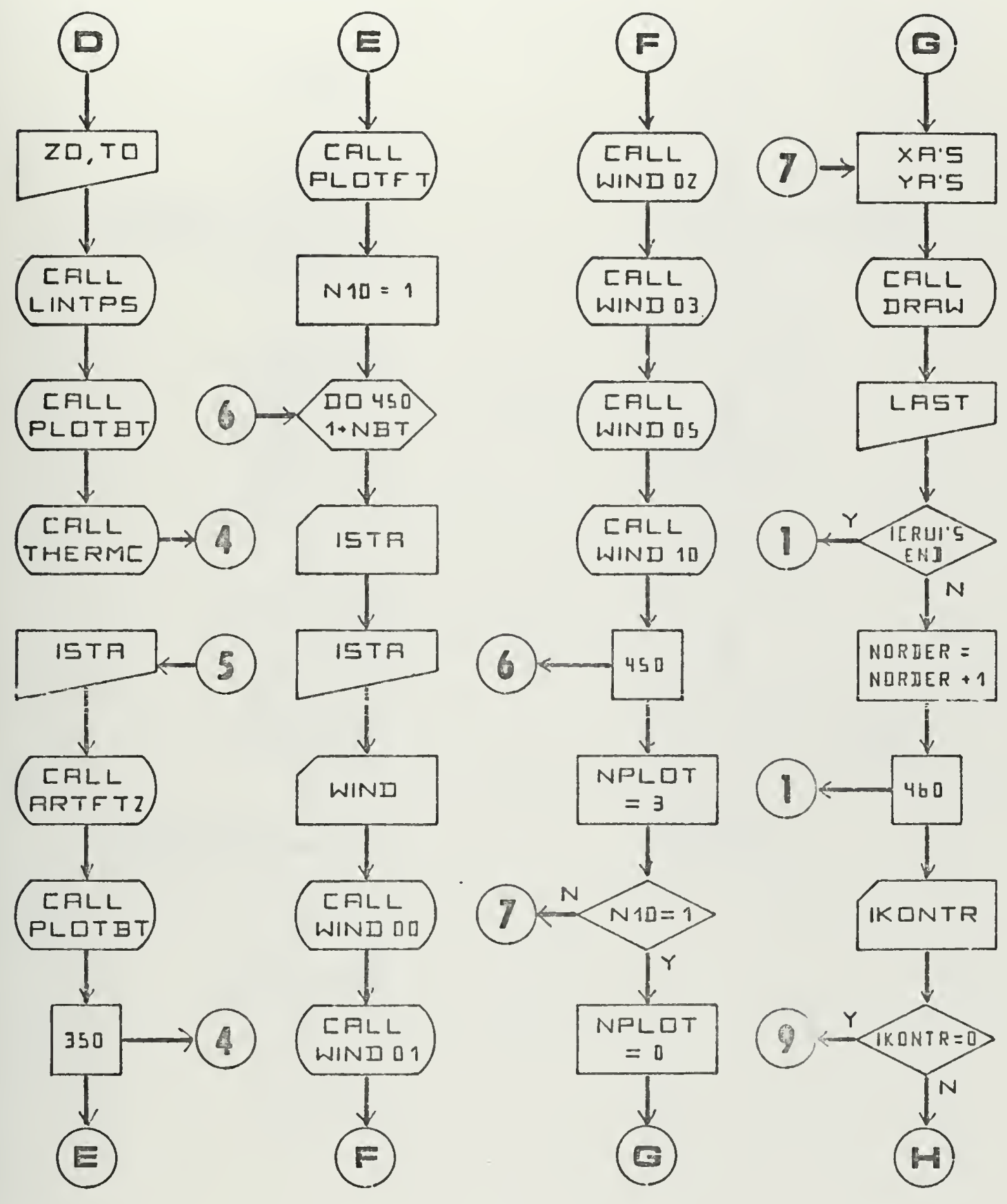



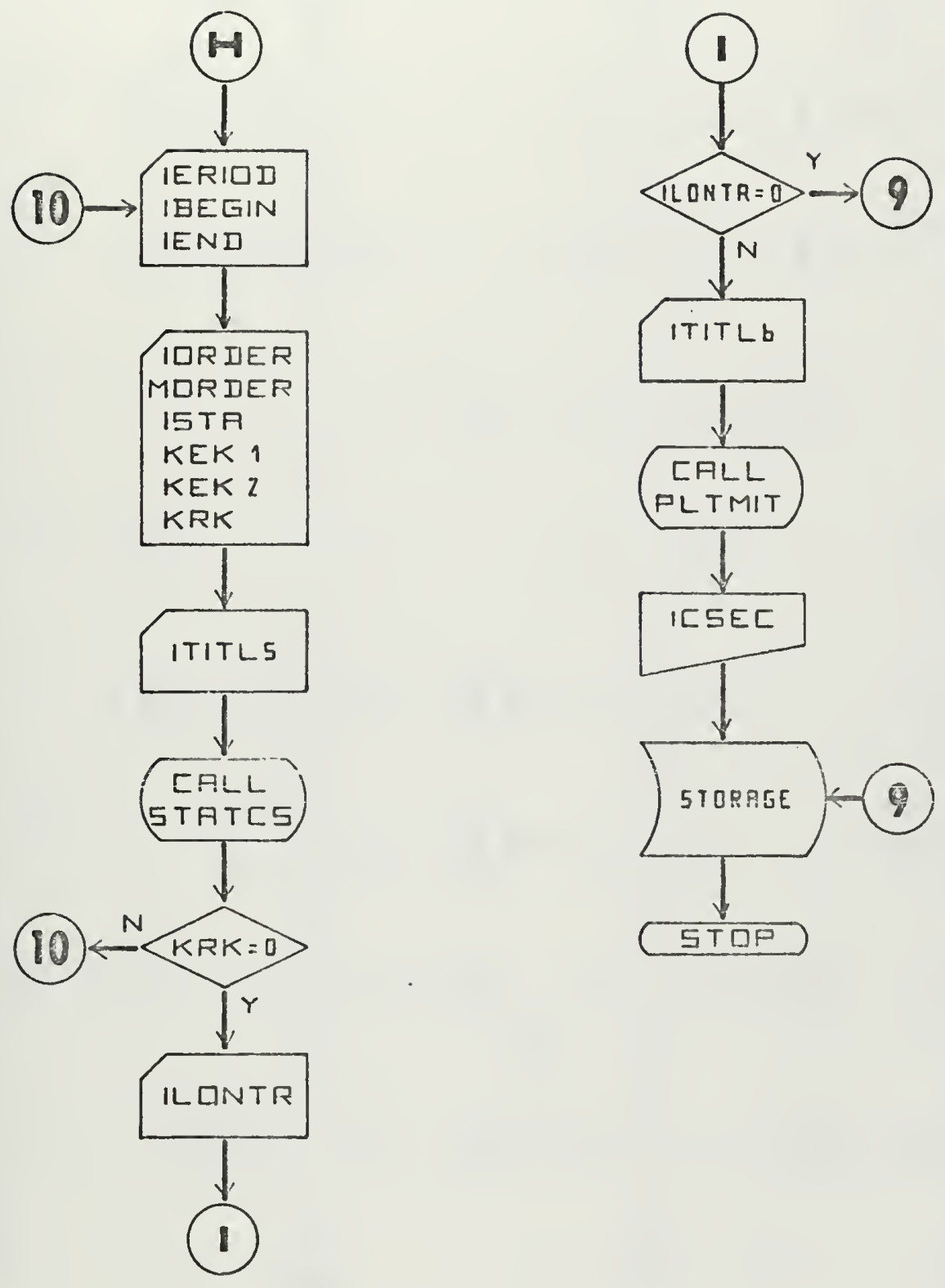



\section{APPENDIX C}

FLOW DIAGRAM OF THE THERMOCLINE ANALYSIS

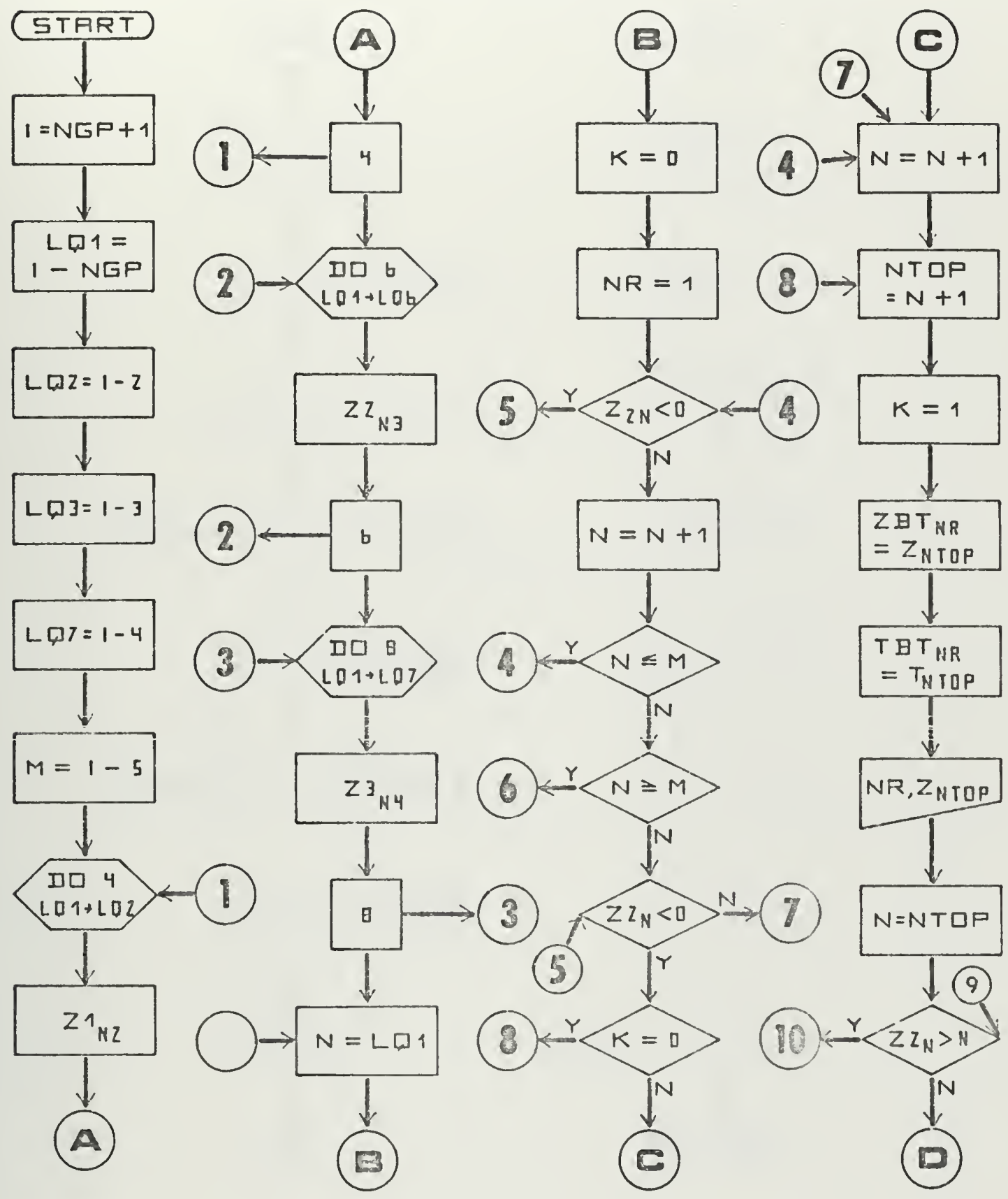



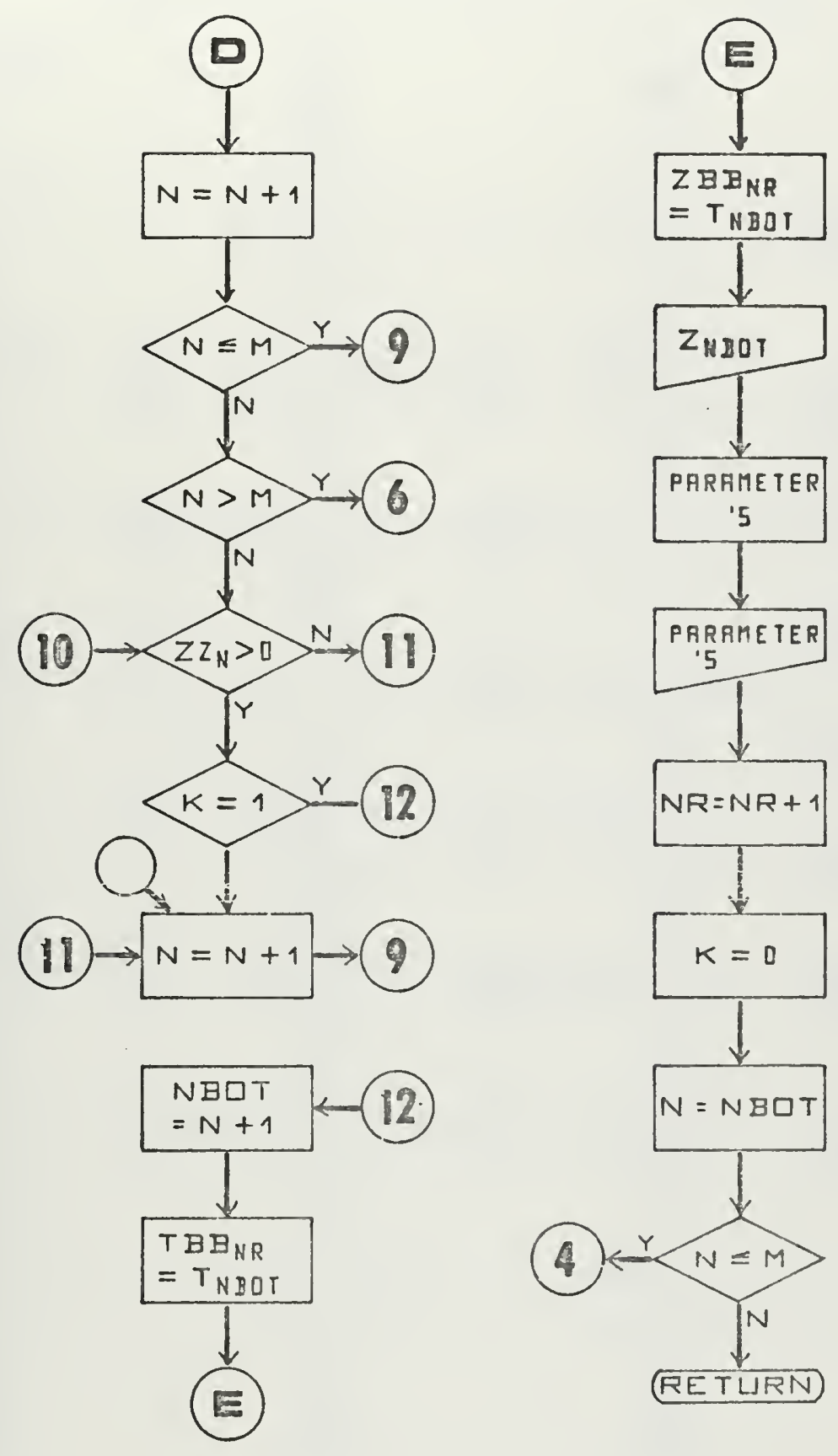

FLOW DIAGRAM OF THE TREND ANALYSIS

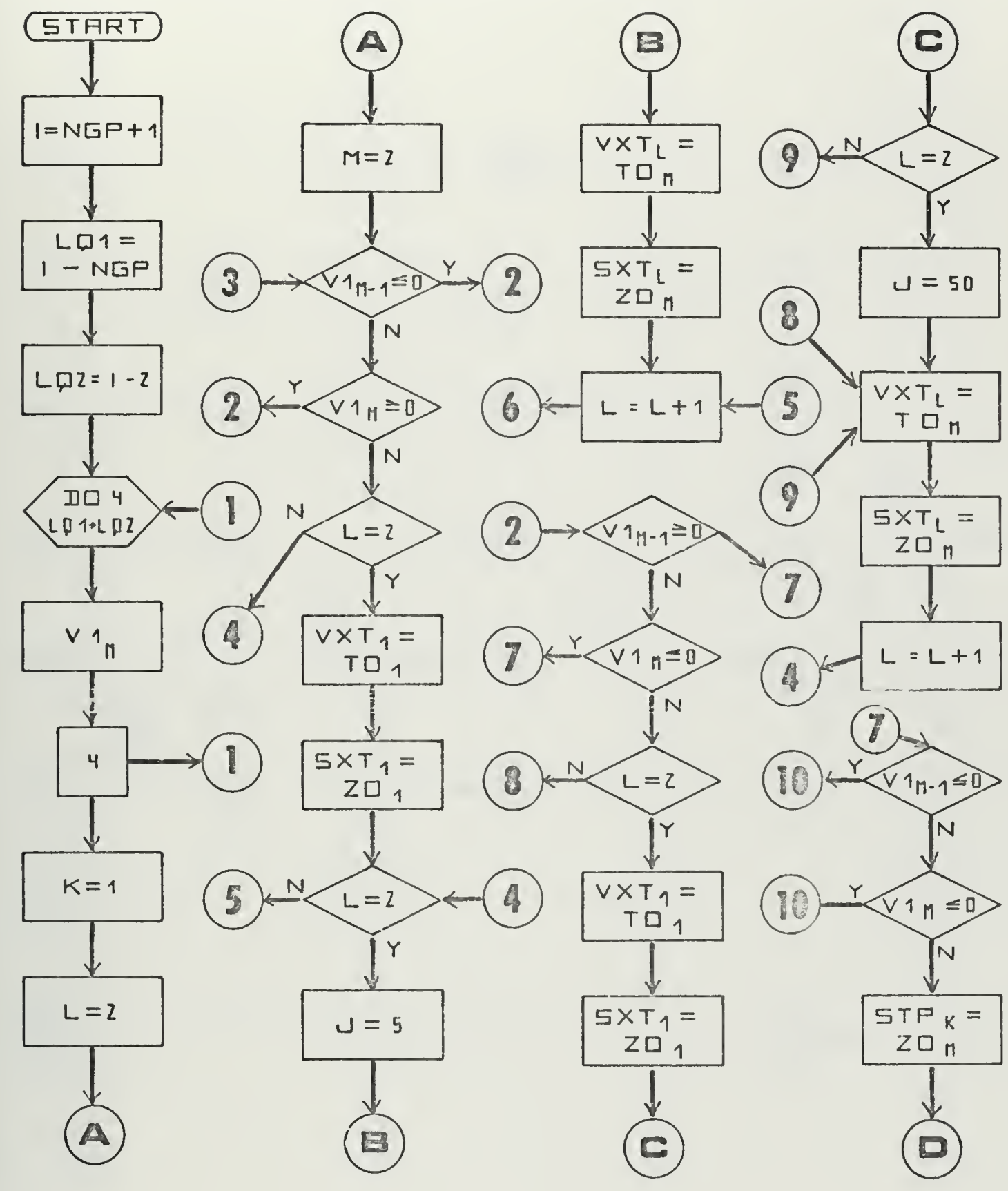





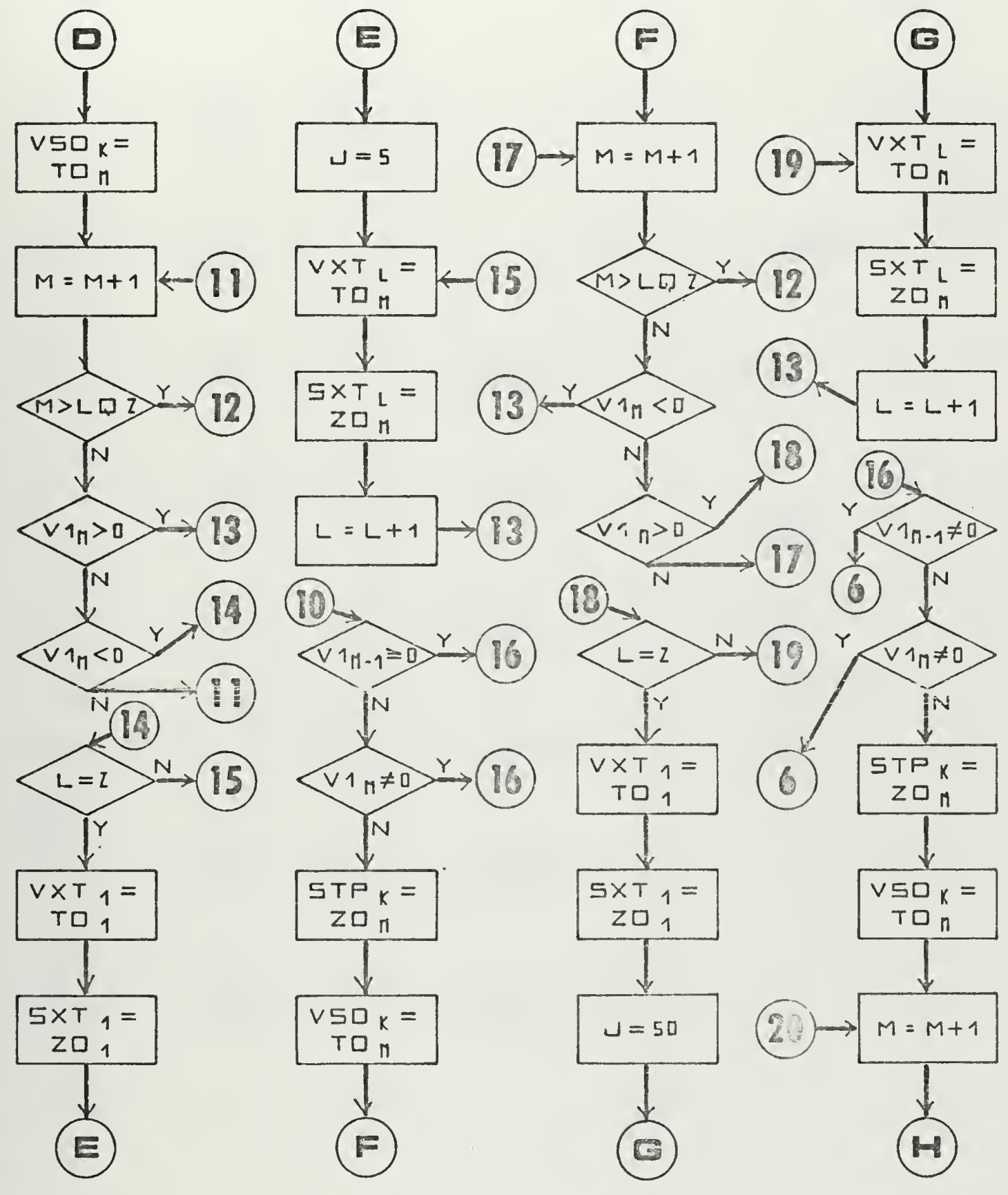




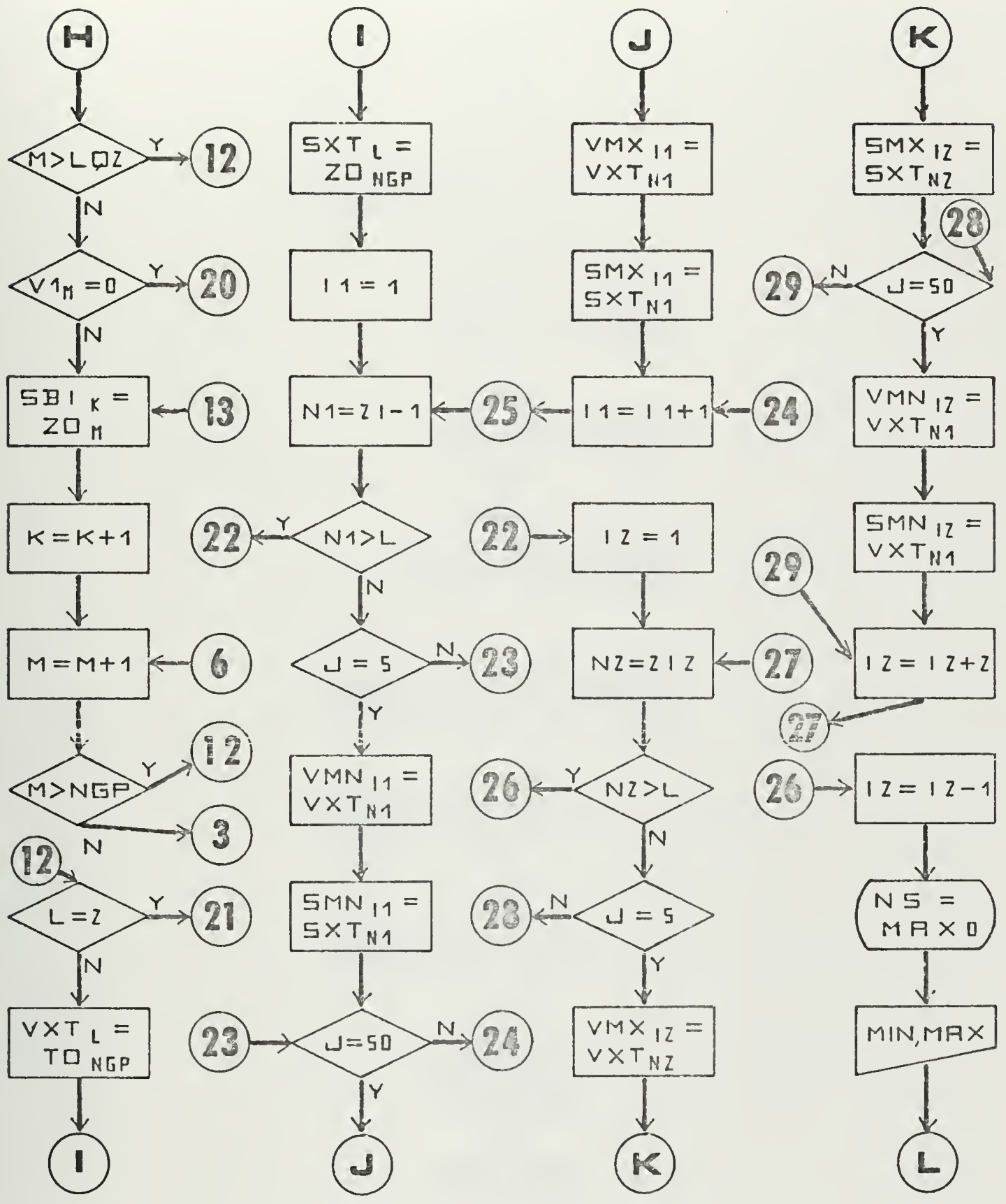



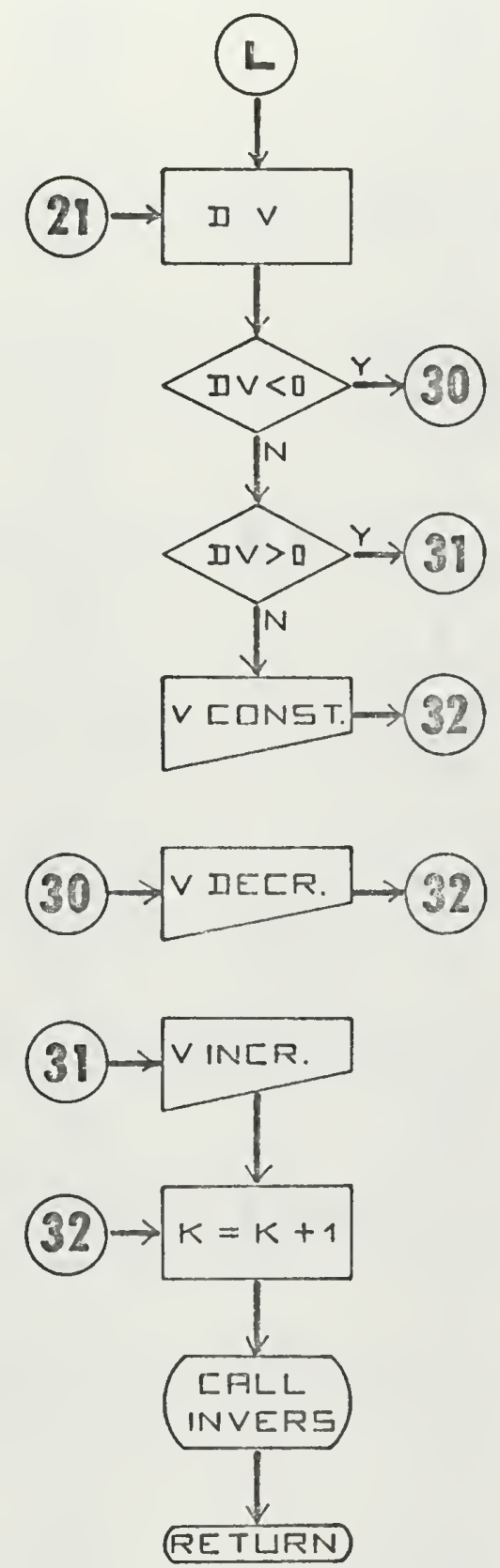



\section{APPENDIX E}

FLOW DIAGRAM OF THE INVERSION ANALYSIS

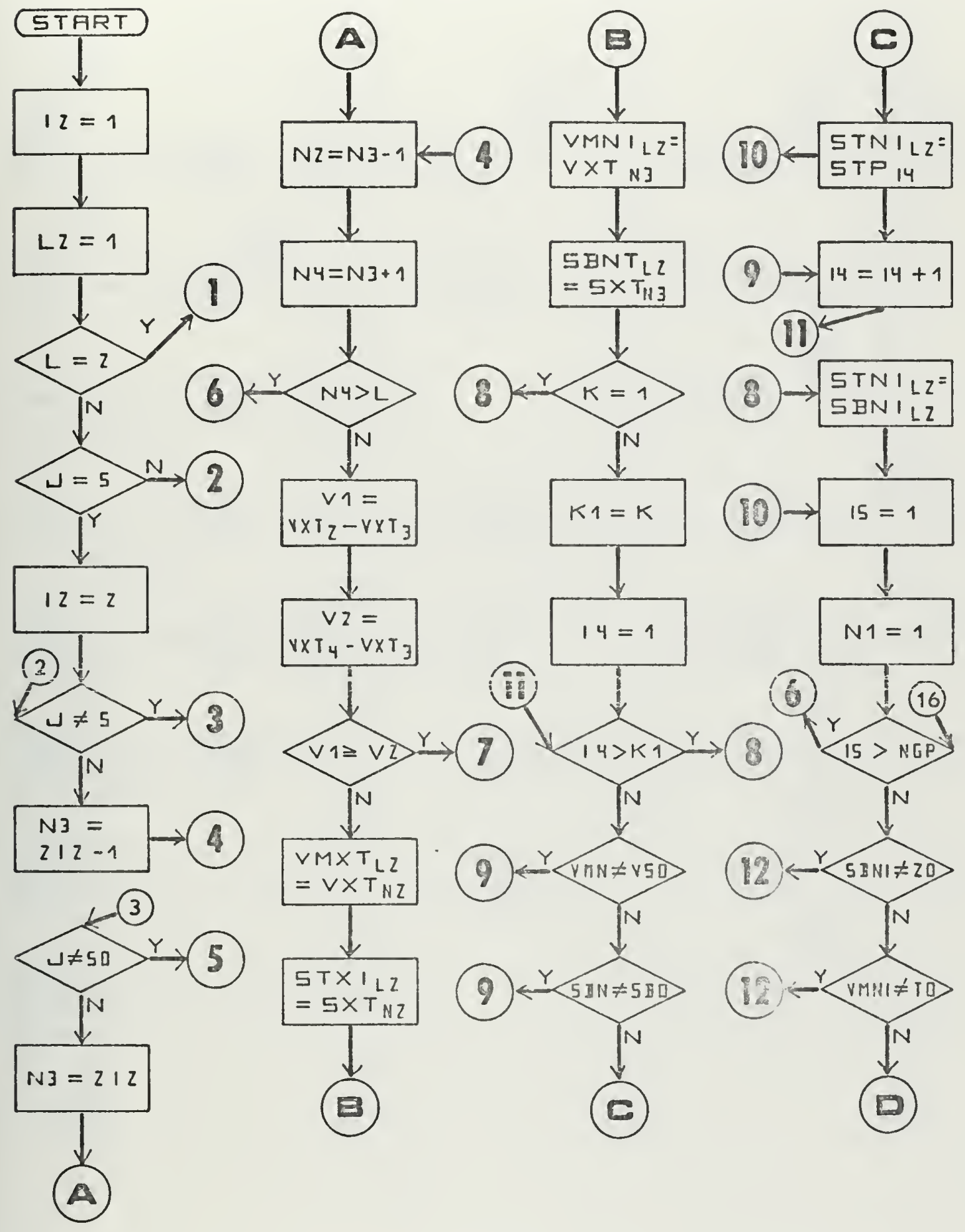




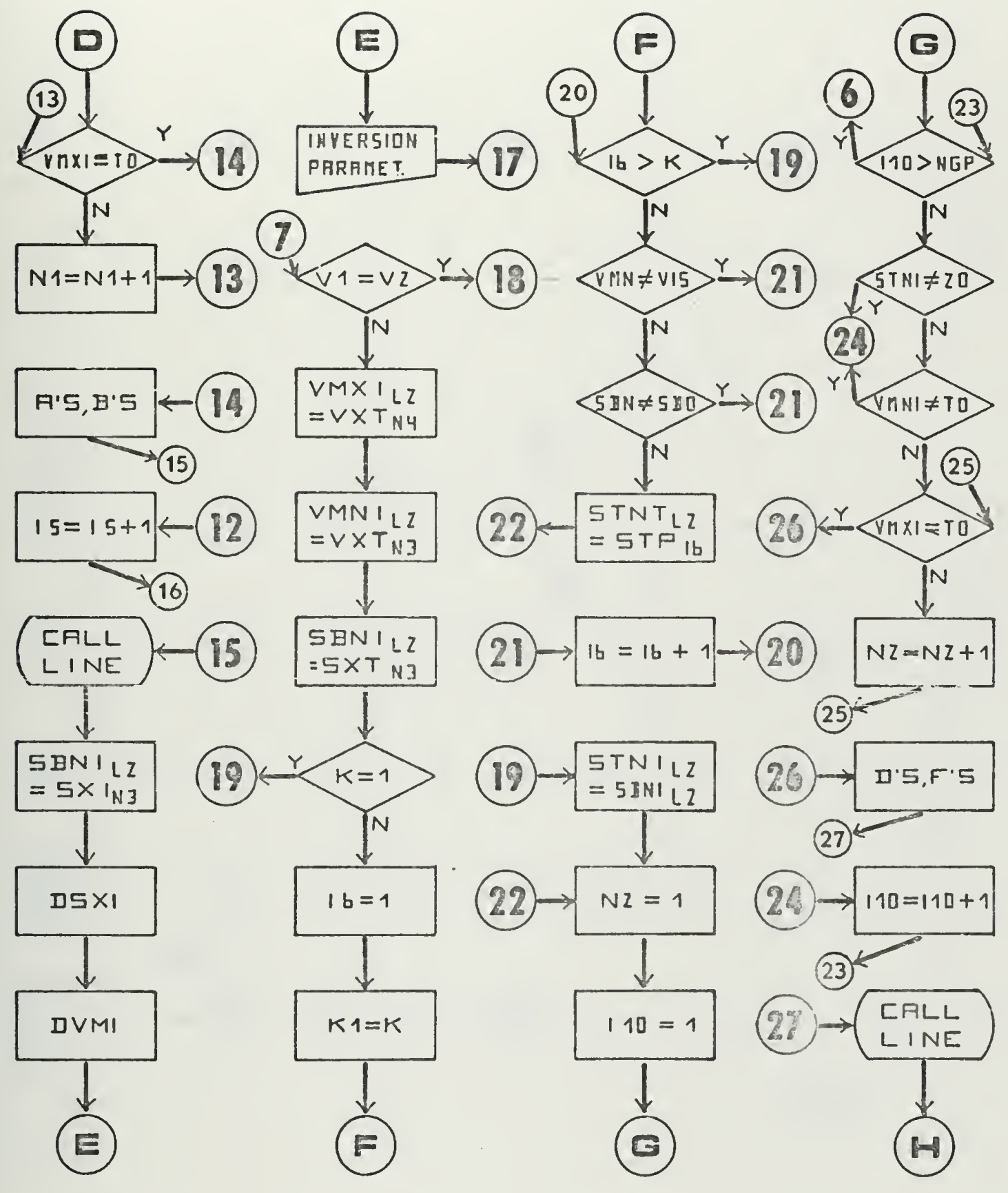





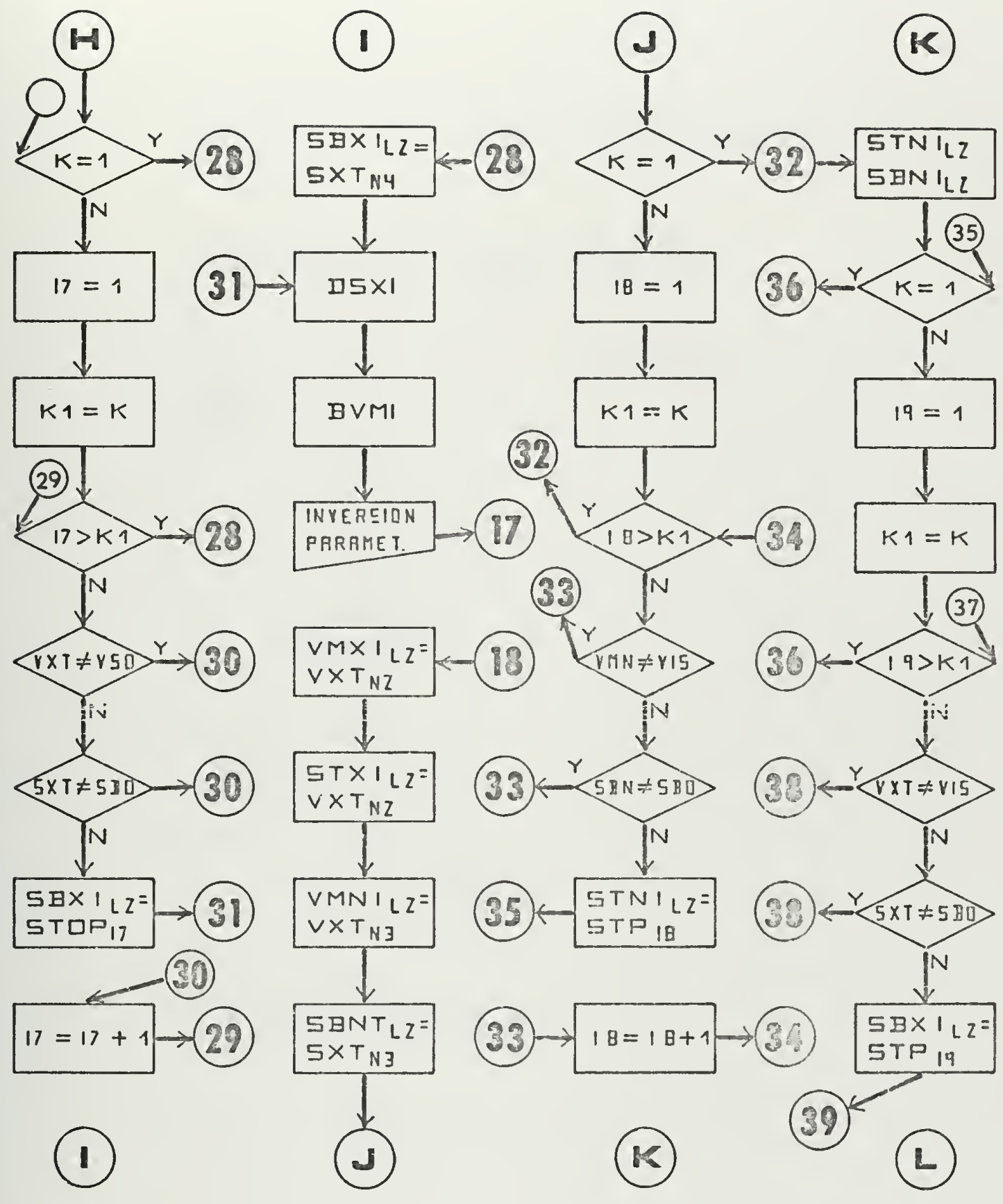


(L)
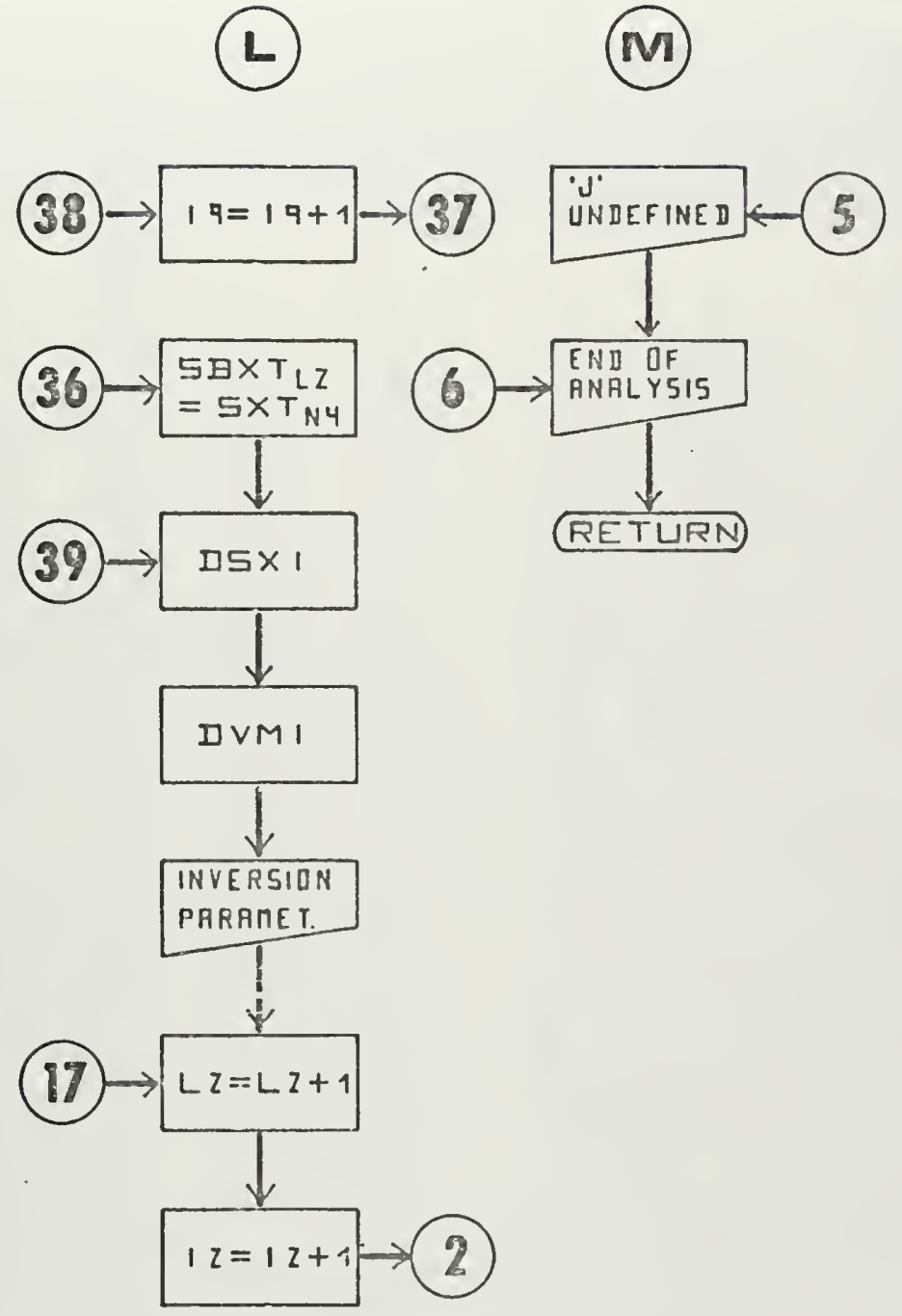

(1) $\rightarrow$ INVERSIONS $\rightarrow$ (6)

(M) 



\section{FORTRAN IV BASIC PROGRAM FOR IBM 360/OS}

$00002000=00.300000000000000 .0000000000000000000000$

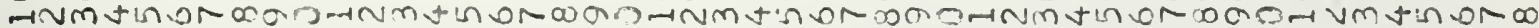

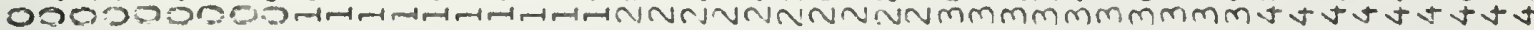

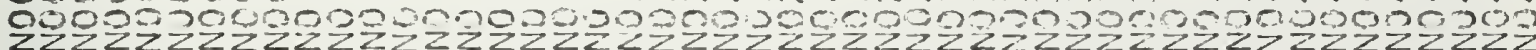

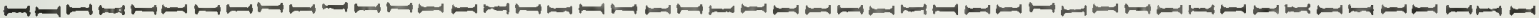

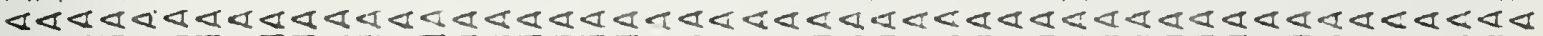

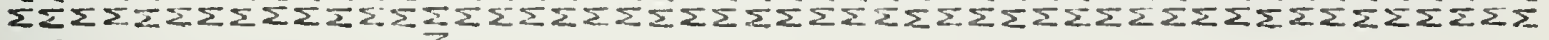

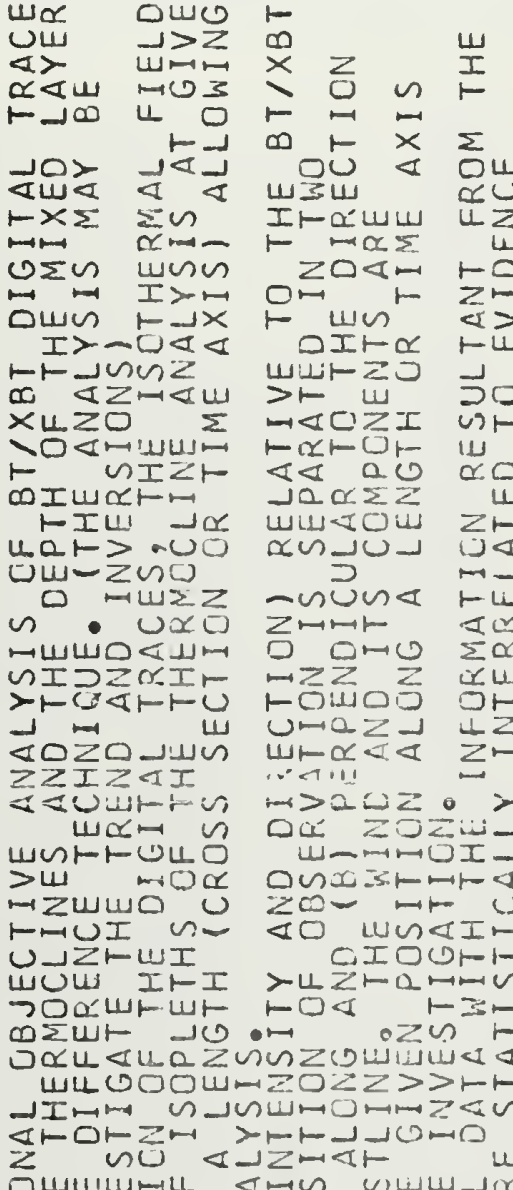

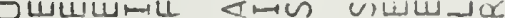

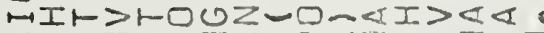

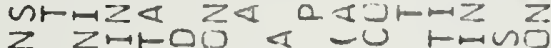

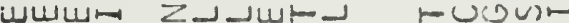

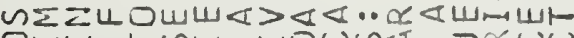
On

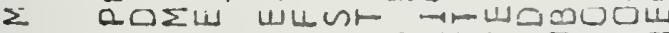

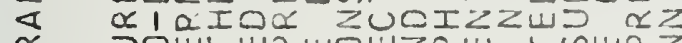

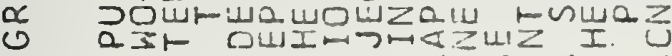

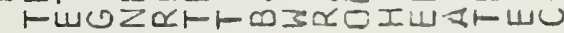

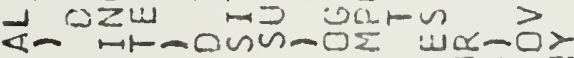

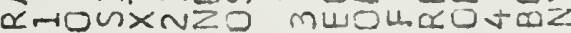
แ-トDய$\sum$ U)

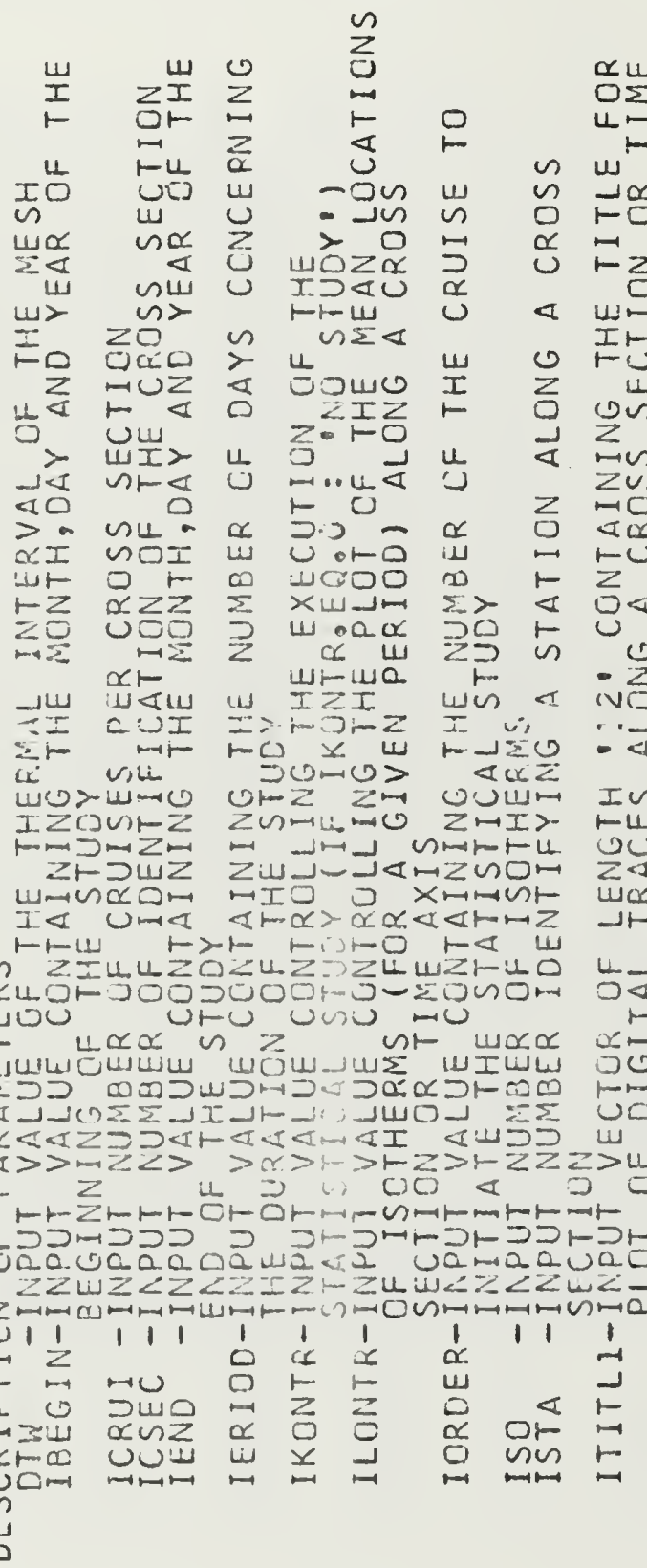

工⺊

$=0$

Dn $\sum_{\infty \infty}^{\infty}$ Zus $\square \square$ \& iv

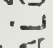
In -U $\sum<$ uor נr

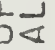
5 ) $\rightarrow-1$

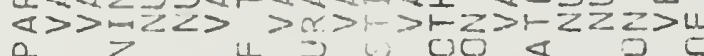
A

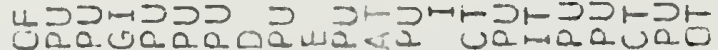

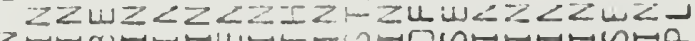

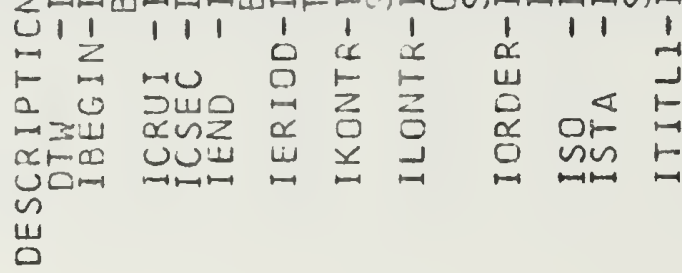



0009020900120005000000530000003000000006000000000

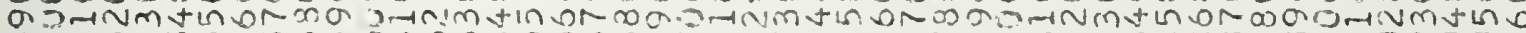

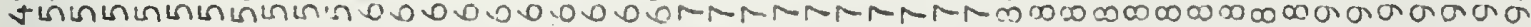

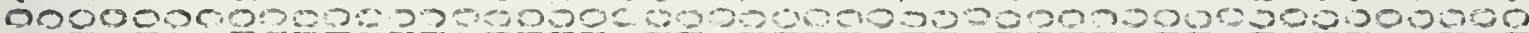
$Z Z Z Z Z Z Z Z Z Z Z Z Z Z Z Z Z Z Z Z Z Z Z Z Z Z Z Z Z Z Z Z Z Z Z Z Z Z Z Z Z Z Z Z Z Z Z Z Z$ $<<<<<<<<<<<<<<<<<<<<<<<<\varangle<<<1<<<<<<<<<<<<<<<<<<<$

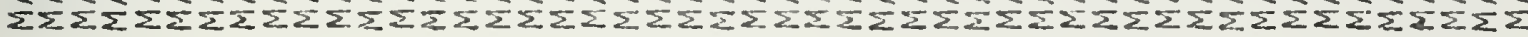

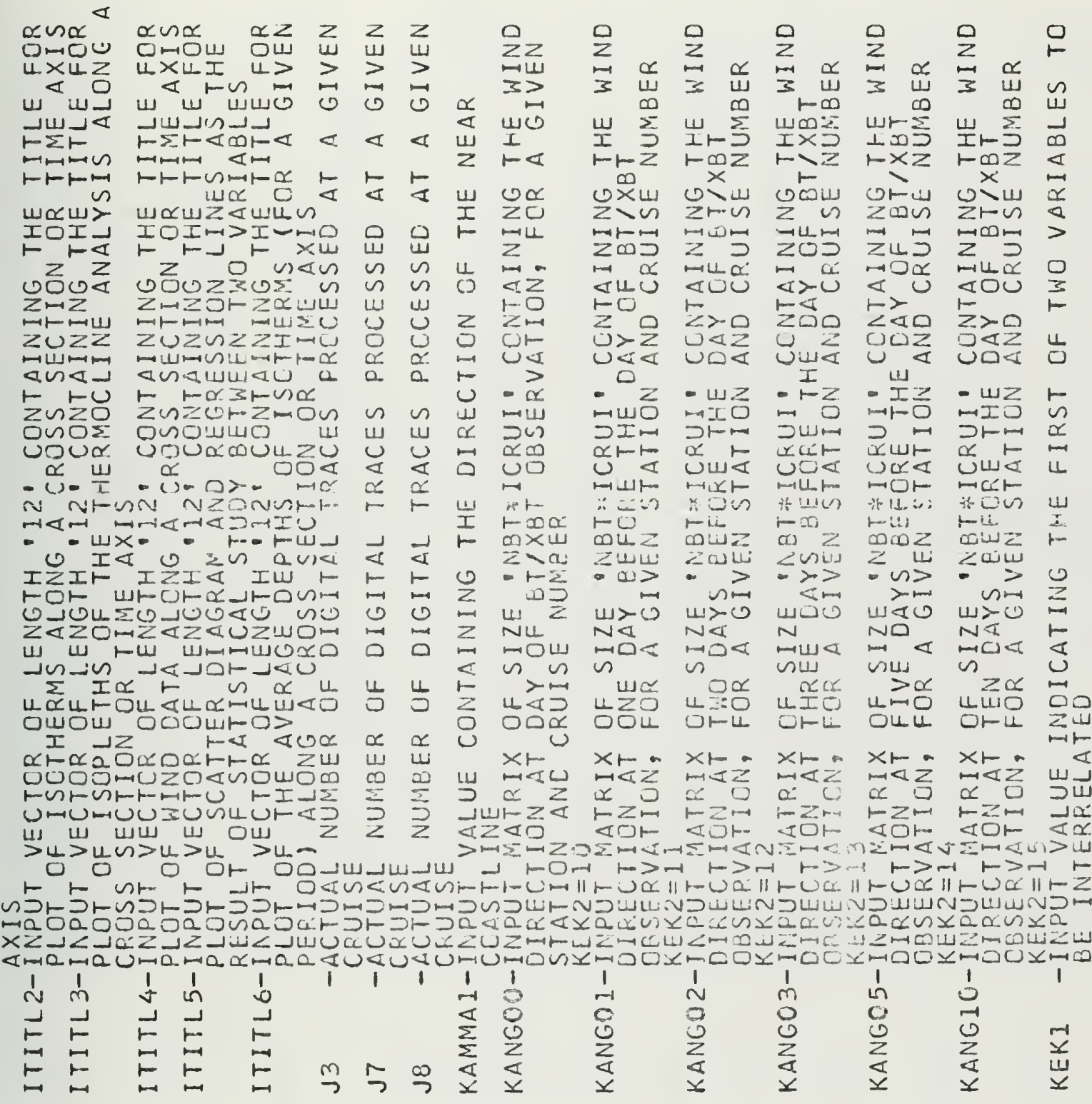



0000000600000000000200000000000230200000000000000 - $\infty 00 \mathrm{n}$ -

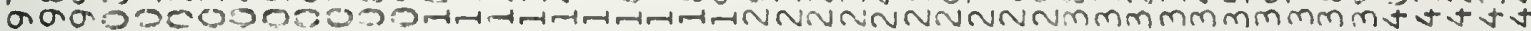
QSC -1 -1 -

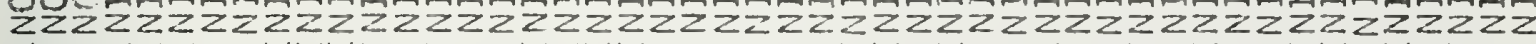

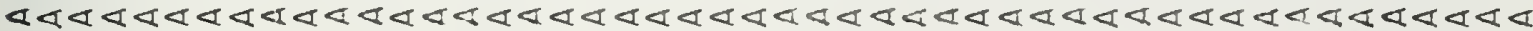

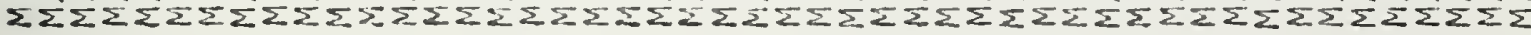





tᄂ

I

(1)

in

m

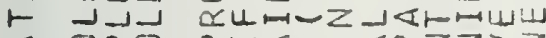

\& $000 \circ 0, \varangle \quad \cdots<0 \geq Z>3$

トトトடல

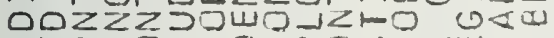

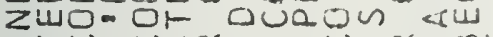

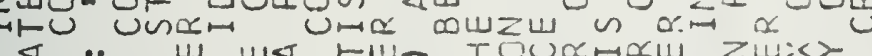

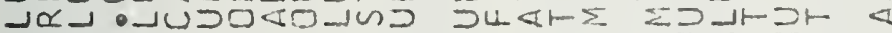

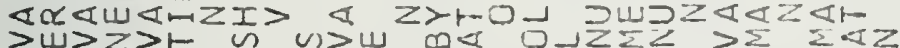

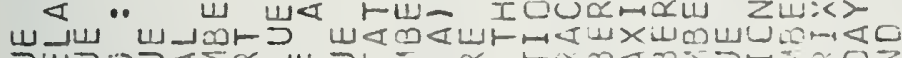

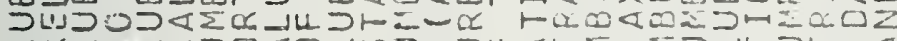

$2 \sum_{0=1}$

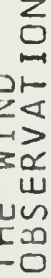

เW

అक

$\geq 0$

Z1

$-\infty$

$\tau \times$

cos

- $\begin{aligned} & u \\ & -0 \sum\end{aligned}$

D> 4

व $U$

U०

다드

化

$\infty$

osino

$\angle-0$ zख

- -2 -

шर

NOZ

แ z

NuS

认ト

$\rightleftarrows \infty$

$z>$

<क $\alpha$

ॠu

J0

$>2$

- a

$\rightarrow$ u

c.

$\cup<0$

a -1

जIञ

is

ULE U๐

1105
Or

ur un orin udir

$x . z$ un

$\mapsto z Z$ -

xढ口 $\propto x>$

⺊- $>$-Ur

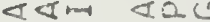

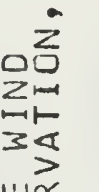

넝

IU

ஸ๐

$\geq$

$\sum_{1-\infty}$

Zo

$\mapsto x$

다

$<\infty u$

$\checkmark \mathrm{CO}$

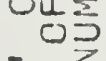

$\because>$

वरा

$\cup \mapsto$

띠

* I

$\infty$

Zயu

- 0 z

U. 11

NU?

$\rightarrow \infty C$

()

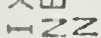

$\because 22$ $\vdash r \frac{x}{11}$

$1<<$

앤인

zo zo

s>m $\stackrel{m}{m}$

ए๘ U

ID

I $\mathscr{1}$ I

I

गम० एल

$2 z \quad z 0$

जயष

ZCC

$\mapsto \infty$

$<\infty 1$

DC

In w

- 0

$\mapsto z$

כயய

다구

U๐口

$\rightarrow 0$

$x^{2} \leq 1=$

$\infty$

$z-\alpha$

$-1-0$

un

யय

NO -

(n) 0 जा

UI⺊ U。

CFE Dr

$x 0 \alpha x$

๓Zw

cun ars

$\vdash-100$

r t $<<0<0$

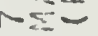
H n-

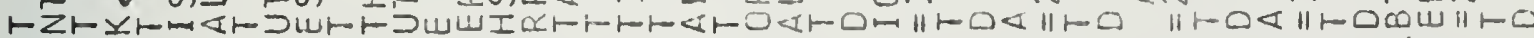

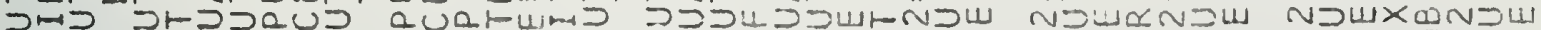

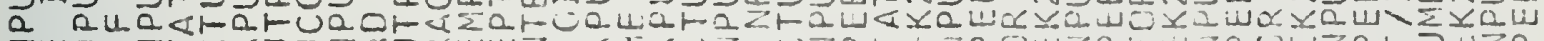

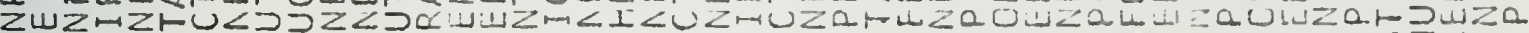

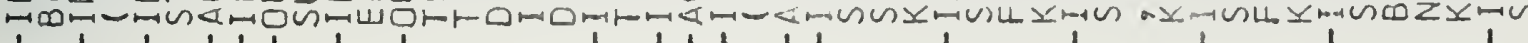

N

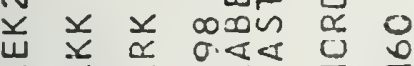

岸文

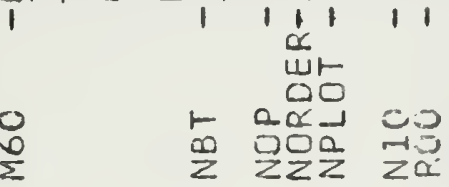

or

0

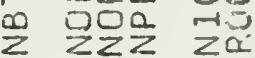

-1
0
0

$N$
0

$\frac{1}{x}$

$\frac{1}{\alpha}$

N

我 



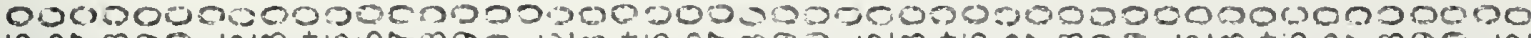

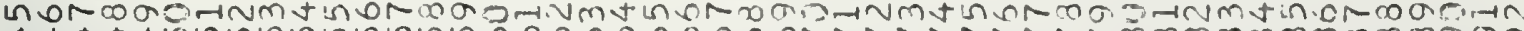

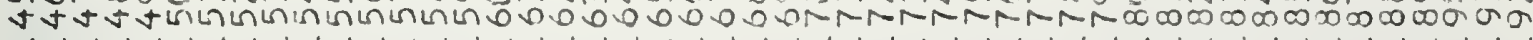

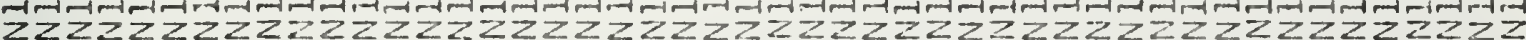

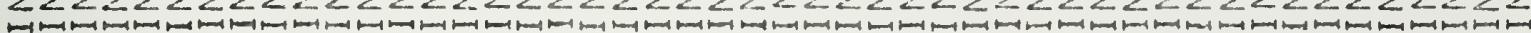

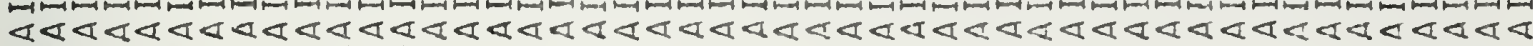

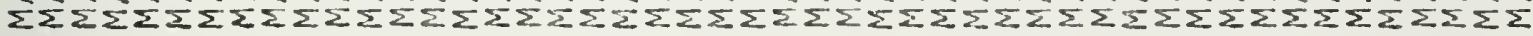

\begin{tabular}{|c|c|c|}
\hline & Z & u \\
\hline$\omega$ & 口- & ט \\
\hline$\sim$ & $z F$ & z \\
\hline 二 & $m<$ & $\varpi>U$ \\
\hline$\supset$ & $3>$ & $3<n$ \\
\hline$\alpha$ & $\alpha$ & on \\
\hline$\omega$ & س山 & $\omega \supset$ \\
\hline & In & エш $\bar{\alpha}$ \\
\hline 0 & $1-\infty$ & トIU \\
\hline$z_{0}$ & 0 & $\vdash$ \\
\hline & $z$ & zuz \\
\hline$z$ & مضi- & $\mapsto \alpha<I$ \\
\hline 0 & $2 x$ & 20 \\
\hline$\mapsto$ & $\rightarrow$ & $-4 Z$ \\
\hline$\vdash$ & $<\leftarrow$ & $<ய 0$ \\
\hline$\varangle$ & $1 \infty \alpha$ & $1-\infty$ \\
\hline- & $z \omega$ & $<1$ \\
\hline$\sim$ & هـ & Oñ \\
\hline & UD $\Sigma$ & $u>r$ \\
\hline Z & 2 & זU \\
\hline Us & $->z$ & -0 \\
\hline$>$ & $m<$ & $\mapsto \quad z$ \\
\hline$m$ & D०س & כow \\
\hline 0 & $\alpha \backsim$ & $\alpha z>$ \\
\hline & ஸய" & Uト \\
\hline$\varangle$ & $-1 T=$ & $\mapsto \quad 0$ \\
\hline & 然1- & $x=$ \\
\hline 0 & कu & $\infty<<$ \\
\hline$u$ & $2 \propto 0$ & $z-\alpha$ \\
\hline a & $-0 z$ & $-1-0$ \\
\hline$z$ & $u<<$ & v) \\
\hline 0 & 7 & யर \\
\hline$\mapsto$ & $\square<$ & Nos $\rightarrow$ \\
\hline$\vdash$ & $\operatorname{lin}_{n \rightarrow 1}$ & $-0 \sum_{0}$ \\
\hline$\varangle$ & $>1$ & win \\
\hline$>$ & $U<<<$ & UIF \\
\hline$\alpha$ & 00ト & UR \\
\hline w & & \\
\hline$n$ & $\times 0$ & $\times 0 \propto$ \\
\hline$\infty$ & $m z Z$ & $m \geq \omega$ \\
\hline$\omega$ & cr-u & $\propto \circlearrowright \backsim$ \\
\hline & & $=-100$ \\
\hline r & $\triangle \vdash \mapsto$ & $\varangle<0$ \\
\hline
\end{tabular}

\begin{tabular}{|c|c|c|c|c|c|}
\hline & & & 2 & $z_{0}^{2}$ & \\
\hline $0<4$ & & U & هus & tr & س \\
\hline $0<4$ & 0 & $\Delta>n$ & $\triangle \backsim$ & Uト & $\Omega \sim$ \\
\hline zon & $z$ & $Z \varangle \mapsto$ & Zயゅ & $z<$ & Z> \\
\hline$\mapsto \quad \mapsto$ & $\rightarrow$ & $-0 D$ & MID & $-1>$ & - \\
\hline ZuD & $\bar{\infty}$ & उ. & $3-\alpha$ & I & $30 \underset{\omega}{\alpha}$ \\
\hline$U F=U$ & $\sum$ & UI & шய & wus & шш \\
\hline I & & IトD & $I \propto 0$ & $I \infty$ & IID \\
\hline -U口 & $1 z$ & F z & $10 Z$ & -0 & $\vdash \vdash Z$ \\
\hline$q z$ & $\stackrel{\vdash}{-}$ & Wर & $4<$ & & 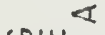 \\
\hline $00<$ & لע مه & $0 \propto$ & ৩U & or & ○ய \\
\hline$\geq u$ & $z \times n$ & $20 z$ & $z \infty \geq$ & 20 & $z \alpha z$ \\
\hline जயUZ & カつッ & $=40$ & $\sim 0$ & $m x$ & -0口 \\
\hline Zळu & 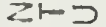 & Zuњ & Znn & $z$ & Zü \\
\hline$m \omega$ & $\rightarrow \infty \bar{\alpha}$ & ロகம & $\mapsto>1$ & $-n t-\alpha$ & ウயレ \\
\hline বUn & $\varangle U$ & $<<$ & $<<<1$ & ४ळய & $<\infty<$ \\
\hline 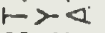 & $1-U$ & Fun & ror & $-\infty$ & $\vdash \vdash$ \\
\hline$\angle \ll \vdash$ & $<60$ & $z>n$ & $z \backsim$ & 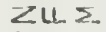 & zun \\
\hline Doun & $0 \quad z$ & $0 \varangle$ & Cथ & ๑ロつ & $0>$ \\
\hline & $v>4$ & $\cup \cup Z$ & ज山z & $\cup \angle$ & $\omega<z$ \\
\hline $0 Z$ & $\varangle$ & H & ๔u & & ฉu \\
\hline - zul & $-0 Z$ & -4> & $-I>$ & - $\varangle \omega$ & $->>$ \\
\hline$M F>$ & $\mapsto 0$ & ஊபோ- & $\mapsto \vdash m$ & $\because D \backsim$ & - \\
\hline$\supset \mapsto$ & Dய以 & $D \alpha 0$ & $\supset 0$ & $D m$ & $D>0$ \\
\hline 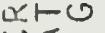 & orIm & $\propto I$ & $\alpha \cdot \vdash$ & 뜩 & $\alpha+n$ \\
\hline$\circlearrowleft<$ & wis & $\cup r-<$ & $\omega<<$ & $\cup I \alpha$ & $\omega u<$ \\
\hline$x=$ & $\because$ uivn & $\therefore-C$ & $\therefore-L C$ & $\because$ & $\ddot{i i}:=$ \\
\hline$\ddot{\mapsto} \vdash \alpha$ & $\ddot{1}-\alpha$ & $\ddot{F}<0$ & $1-1-0$ & $1-\omega 0$ & $1-<0$ \\
\hline$\infty \backsim 0$ & $\infty 0 z$ & $\infty u$ & องu. & $\omega \propto z$ & @ u \\
\hline$z<U$ & Zய山 & $2-$ & $\geq<$ & 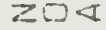 & Z- \\
\hline-0 & - $\omega>$ & $-\vdash m$ & -0 - & -4 & $-1=$ \\
\hline$\omega *$ & $n-1$ & $\sim z$ & $\omega \geq$ & wZ & $n Z$ \\
\hline$\omega z$ & $\omega \omega$ & $\omega<0$ & 110 & யळ0 & $w<0$ \\
\hline NயD & Nus & NOm & Nusnat & $N \varpi$ & ND伍 \\
\hline$\mapsto I \mapsto$ & $\mapsto><$ & 円Uト & "Iト & mus & mur \\
\hline जFE & $\backsim<1$ & $\backsim \varangle$ & $n t-4$ & $\backsim>-<$ & $\sim<$ \\
\hline & $\sigma \alpha$ & $u>$ & $>$ & $\leq \vdash$ & $\omega>$ \\
\hline $110>$ & $\begin{array}{ll}4 & 0\end{array}$ & $\amalg I \sigma$ & $\Perp \varnothing \propto$ & Hon & UIq \\
\hline 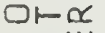 & லயப. & or-u & Oトய & 0 & QFW \\
\hline IIJ & w & $n$ & $n$ & $\omega z$ & $\sim$ \\
\hline x on & XQY - & $\times 00$ & $x \circ \infty$ & $x>$ LIJ & $\times 00$ \\
\hline$\because<0$ & $m$ IZZ & $-2=0$ & ma 0 & トルー $>$ & -200 \\
\hline$\alpha \propto$ & $\alpha F 0$ & $\propto \sigma$ & $c c$ & cunts & $\propto \omega$ \\
\hline トயルト & $10 m$ & $r-d r$ & トயト & 10 & $\vdash-1$ \\
\hline$\varangle 0 \infty$ & สトト & $\varangle<\infty$ & IOD & বட & $\alpha<\infty$ \\
\hline
\end{tabular}

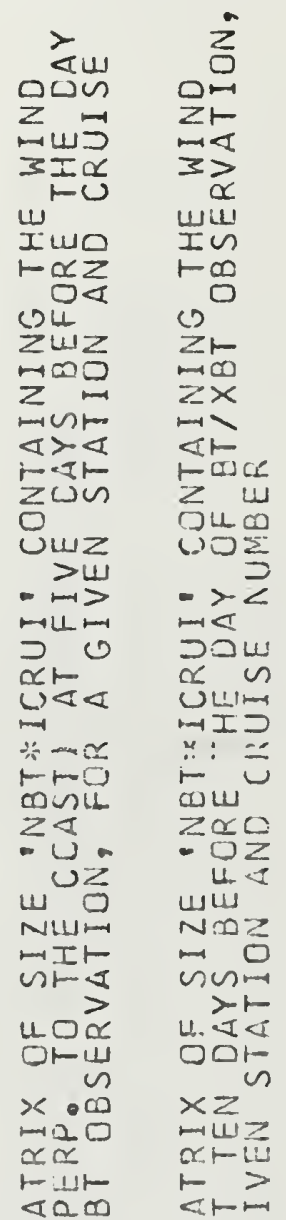
asux

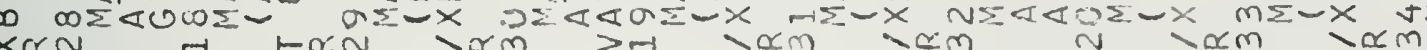

ХưN $\|$ -

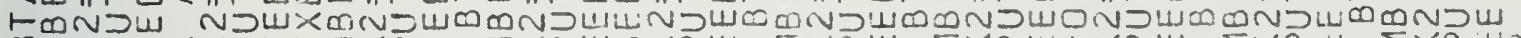

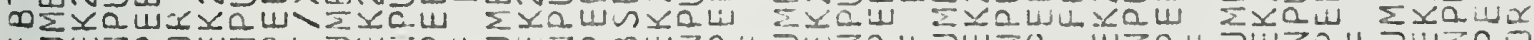

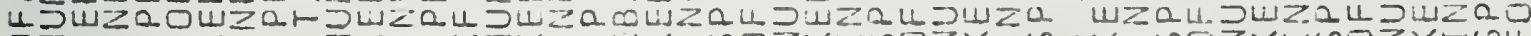

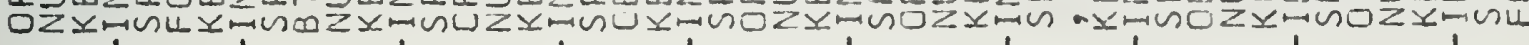
1
$\sum_{0}^{N} \underset{\alpha}{N}$
$\begin{array}{ll}N & m \\ 0 & 0\end{array}$
$\begin{array}{ll}-1 & N \\ 0 & 0\end{array}$
$\begin{array}{lll}N & \text { in } & \text { in } \\ m & 0 & 0\end{array}$
in
$\underset{n-1}{0}$ 

000000000000000000000200000000000005000000000000

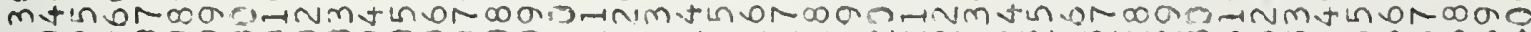

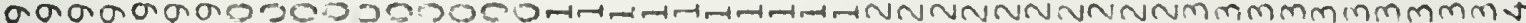
HZZZZZZZZZZZZZZZZZZZZZZZZZZZZZZZZZZZZZZZZZZZZZZZZ

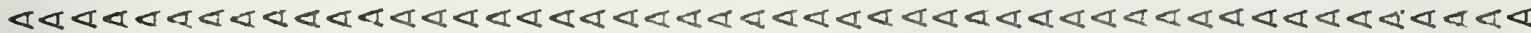

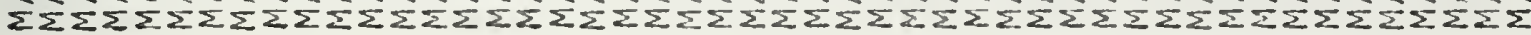

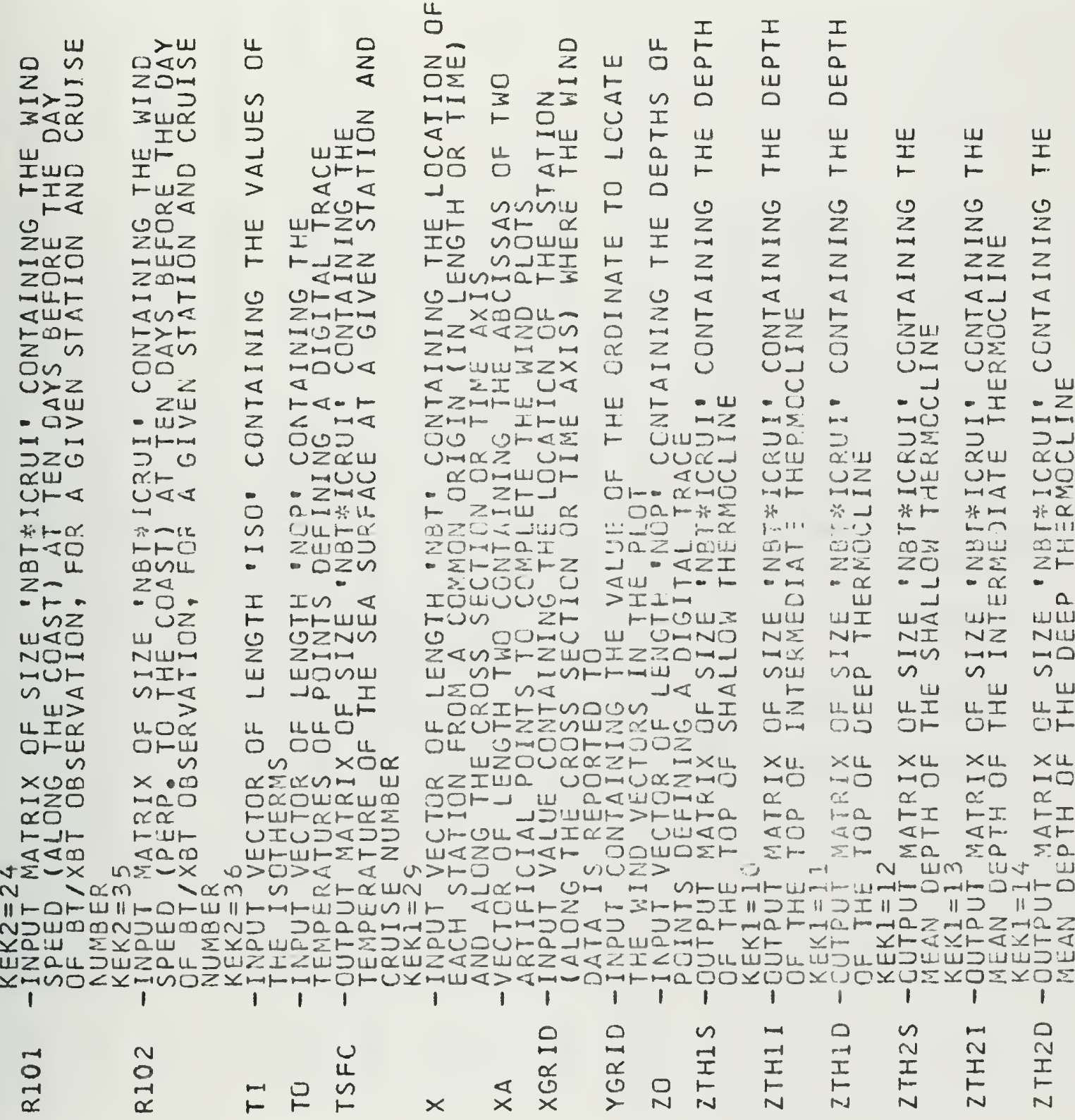


000000050002000000050002000009006020000000000200

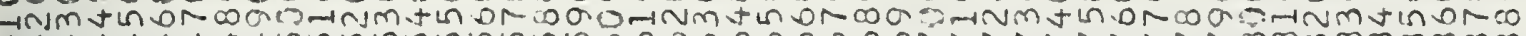

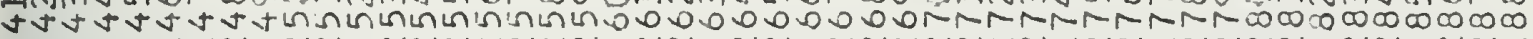

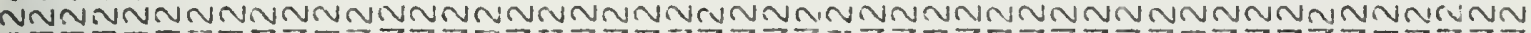
ZZZZZZZZZZZZZZZZZZZZZZZZZZZZZZZZZZZZZZZZZZZZZZZZZZZZ

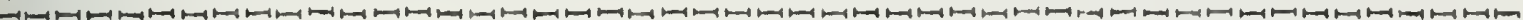

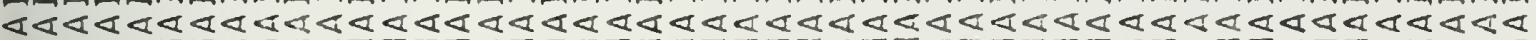

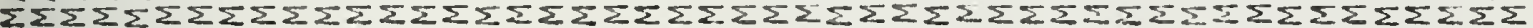

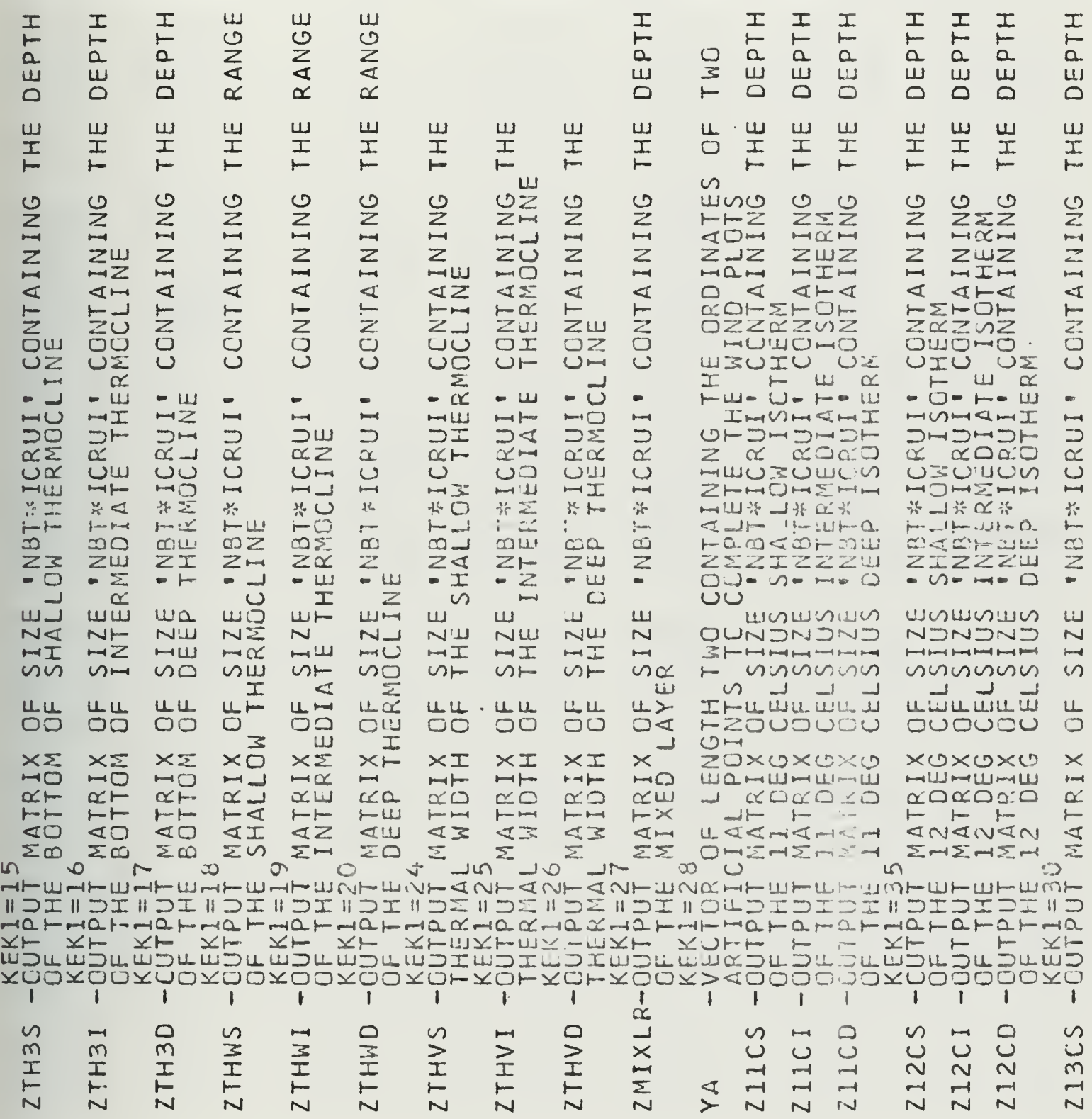


000000000000000000000000000300000000000000000000

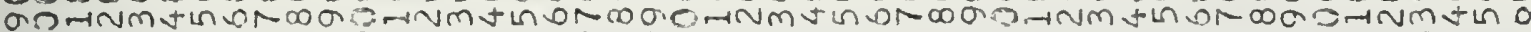

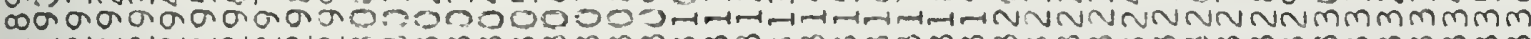

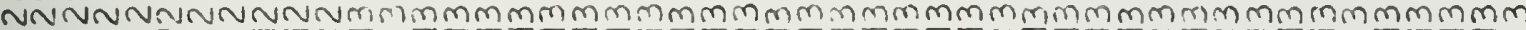
ZZZZZZZZZZZZZZZZZ2ZZZZZZZZZZZZZZZZZZZZZZZZZZZZZZ

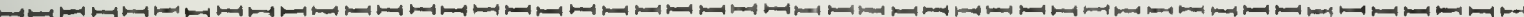

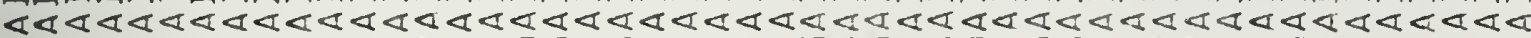

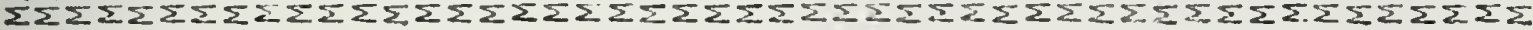

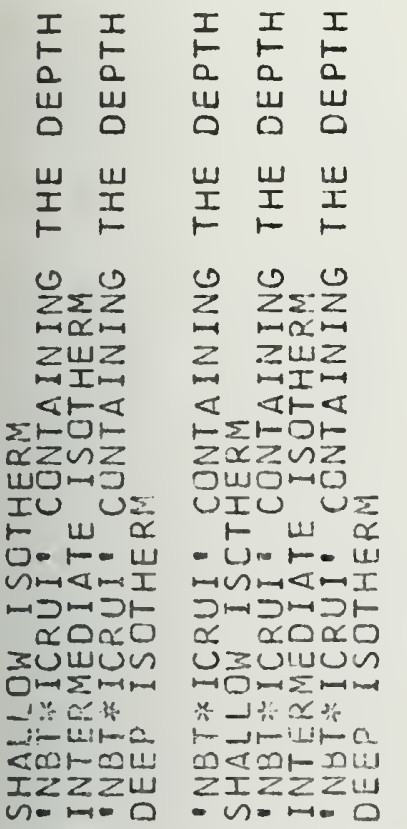

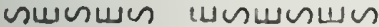
DNDND NDNDND

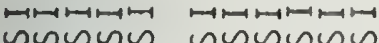
山ا山ـ טouัu

$0 \times 0 \times 0$ யேमயमய oro oro 15 memam m⿺mam

$-1-1$

แயиயル ய oúua

xoxenco ๒யேயாய ㄷऽ이

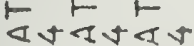

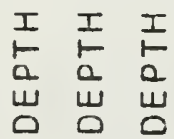

世 س

$I I I$

$\sum_{n=1}^{0} \sum_{i=1}^{0}$

z zwz

$\mapsto \mapsto I$

< \&5<

Zazin

गा10म?

UエU $U$

- 15 d

円以๓<њI

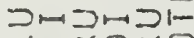

$\propto \approx 0 \propto 0$

ũumun

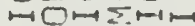

品-

下 $=1-1-\frac{1}{1}$

zIzZzu

- v- mo

Uinunusen NDNDND セロҢҢҢロ แ. யน யน oúdiou

$\times 0 \times 0 \times 10$

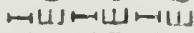

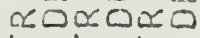
t 5
$\begin{array}{lll}I & I & I \\ E & E & E \\ \omega & \omega & w \\ 0 & 0 & 0\end{array}$

I

000

$\sum_{n=1}^{2} z_{1}^{2}$

$z \geq \omega z$

$\because$ આー

$<$ arb

Za Znz

गUण口心

UIU US F $w \propto$

- U- トL ๓ルロவேI วேつロつト $\therefore \quad 20 \propto 0$ บzบแบง * $] * x_{i}$ it - - i ……

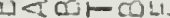
ZIZZZZu

แมงเมตแบ NDNDND

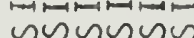

แ. เుய ovouas

$\times 0 \times 0 \times 0$ मUमயयम cट $000 \Omega$ ⺊ $⺊$ $\varangle 0<0<\infty$ $\leq 0 \leq 0<0$ \begin{tabular}{lll}
$I$ & $I$ & $I$ \\
\hline & $\leftarrow$ & $a$
\end{tabular}

- 00

I I I I

0

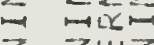
zwz $\rightarrow$ m曰 $<$ वाष 든 Dแ०น०

UEU U U

- O- $1-4$ ロヘロロツI $2 m \square m D F$ a $x \supset x J$ งzuLu $\rightarrow 00 \leq-1$

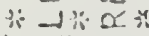
- $-1-1-1-0$

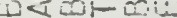

ZIZZZU $-n=n-0$

แnแேnแ⿰ NDNDND ตังunun แயนแ山и Duovo.

$\times 0 \times 0 \times 0$ அயேயम山 $\simeq 0\{0 \times \omega$ $-\vdash \vdash$

$\angle R \leq R<R$ $\sum-1 \sum-\sum-1$

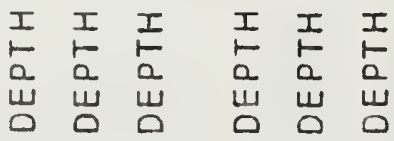

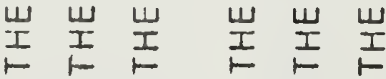

200

$\sum_{i=1} \sum \sum$

z zuz

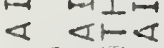

-EトUト

$z \leftarrow z \backsim z$ טगढ口0

UIU Us

Q O- ta

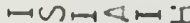

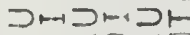
$\alpha \quad x \cap \alpha 0$

טzumun

$x=1-\because n$ if $-x * x$

$-1 !-1.1-a$

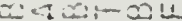

こエこここU

- v)

¿

$=\quad \geq \alpha, 1$

$\sum \sum \omega 2$

$4<1<$

$15 \vdash 0$

$z \alpha z \backsim z$

ดแ⿰七刀口

UIU UI

tw $u$

- U. - U

๓レッனேI

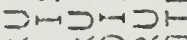

ix 미잉

U.suluU

uㅔ

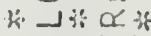

$r-i-i-a$

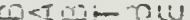

そエンZこU

- Un- -0

แทแกแผ

uinumiun

NDNDND

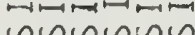

nusunus

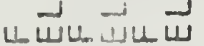

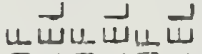
ovousu buguou

×0 $\times 0 \times 0 \times 0 \times 0 \times 0$

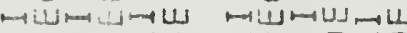

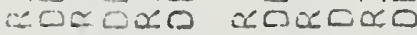

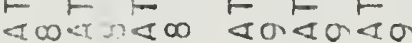
इ

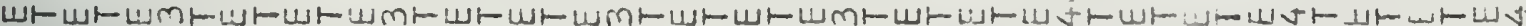

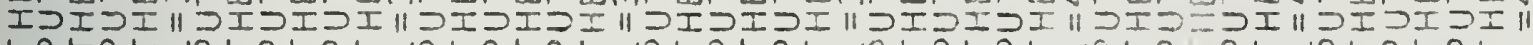

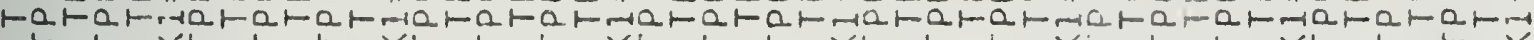

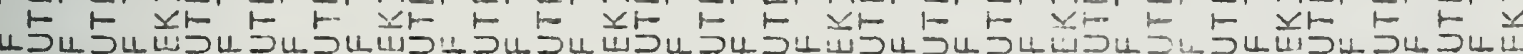
00000x00

$\bigcup_{m}^{m} \bigcup_{-1}^{0}$

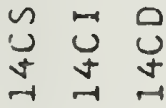

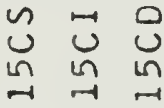

$\bigcup_{0}^{\sim} \bigcup_{0} U_{0}$

$\underset{N}{N} \underset{N}{N} \stackrel{N}{N}$

$\bigcup_{\infty}^{\sim} \underset{\infty}{\infty} \underset{\infty}{\infty}$

$N N N$

$\begin{array}{lll}\sim & \ddots & 0 \\ \sigma & \sigma & \sigma\end{array}$

N $N$ N 


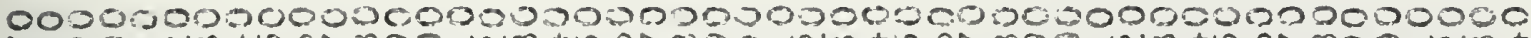

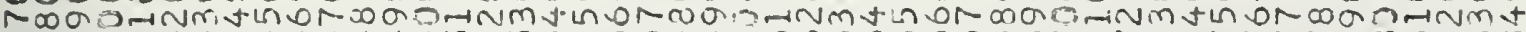

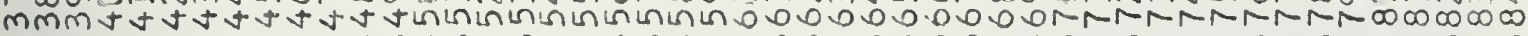

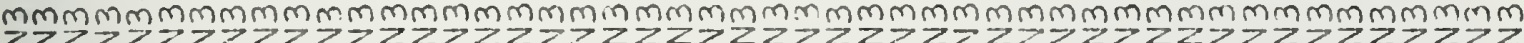

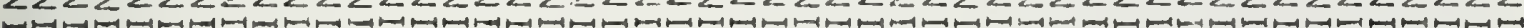

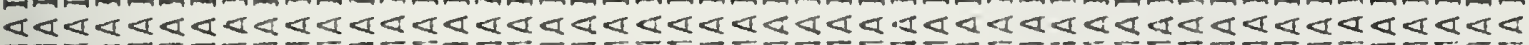

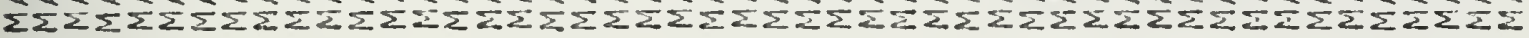

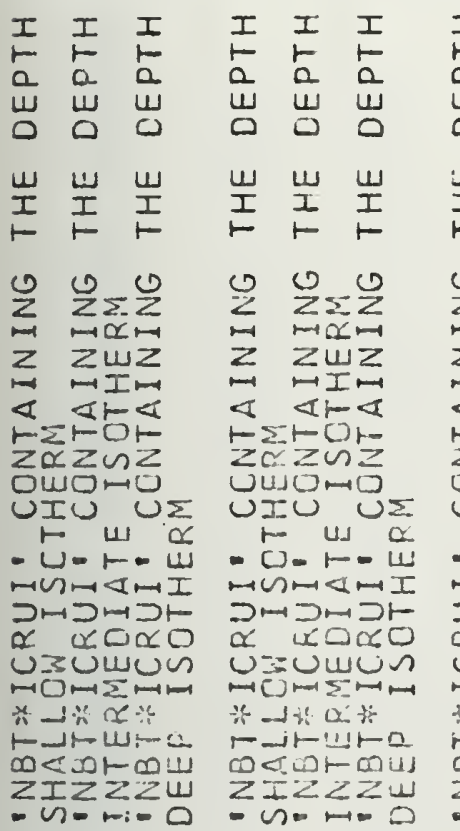

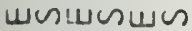

NDNDND

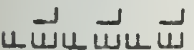

$\times 0 \times 0 \times 0$ 十 $\vdash$

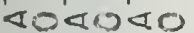
¿NENEN
๓๓口म ouovou

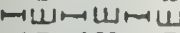
xD๙ण00
NDNDND

nususeses

แ山ய山 ธưủ

$\times 0 \times 0 \times 0$ அயேயேய

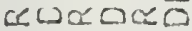
- 5

【一⿻上丨 N N N NEN

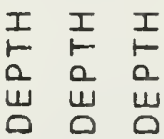

I U U I

$\sum_{n}^{0} \bigcup_{n=1} \sum_{0}^{0}$

z Zwz

$\rightarrow$ 地

\& ए5

ZcZu ๑U⿻尸以

UIU $U$

- U- $-1-u$

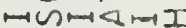

つッコッつト

\& xo⿺辶

Ujumun

fi dic 0 it

r-atliora

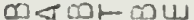

ZIZZZU

- v- $n-0$

แตsusumas

NDNDND ममझझम्नम

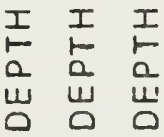

I $\stackrel{U}{I} \underset{F}{I}$

$\sum_{n}^{0} \sum_{m=1}^{0}$

\ $2 \omega \alpha$

- $I I$

$<\quad<i-\alpha$

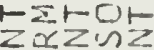

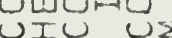

F

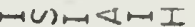

$\alpha \quad x 0 \propto 0$

טzumun

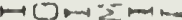

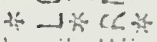

- ji- Lin-a

$0<\cot 20$

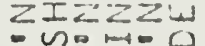

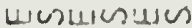

NDNDND

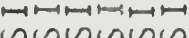

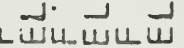
อง

$\times 0 \times 0 \times 0$

-யमแजम xOQD

\section{- $\vdash$}

$\triangle N A N<N$ ¿NENEN

แய山ய山ు ๖งฮบó

xuxoxo

Ш山ேய山ய xoxoxa

$\leftarrow+F$

$\varangle m<m<m$
DMD-DI

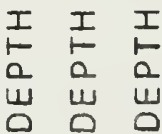

I

Iบ

U $U$

U 4

W U I

I U U I

wz

000

$\sum_{n=1} \sum_{\infty}$

z Zwz

$m$ mIn

$\checkmark<F<$

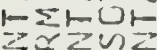

0نU० $\rightarrow 0$

UIU U⿱口口心

- 0- - -

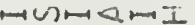

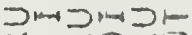

- c có́o

UzuLuU

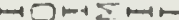

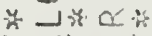

ro-dt-ijt-a

mant-ou

ZIZZE

unsusues

NDNDND

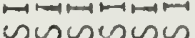

$\rightarrow-1$

50000

roxoxe

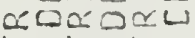

t5 ININEN
내는

$\geq 2 \geq 2$

$\sum_{i=1}^{2} \sum_{1}^{2}$

$z$ zuiz

$\rightarrow$ Im

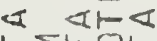

$1 \sum 50$

<uำ

UIU Uะ

L $w \frac{\alpha}{4}$

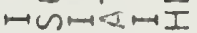

L 1000

บzบแบผ

$\rightarrow$ C) $-5-1$

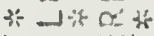

- $2:$ :

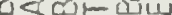

Zエこごてய

$-n=n+0$

wnusnuses

NONONO

(1)

त)

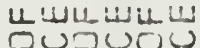

ס

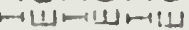

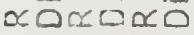

- th

$\varangle \cap<\curvearrowleft<L$ ¿NENEN

$\leftarrow>$ I

0

$\sum$

$z$

-14

40

1

00

Ur

$I$

0 0 Z

a

w

4

ㄴ.

$D \sum_{0}$ $m$
W W ENENEN

Пயாயாய

วッつッつम

ш己 $\underset{1}{\longrightarrow}$ I0 $\geq \frac{1}{0}$

$\sum \sum$ $<0$ 난

OU

- س - U

$\mapsto I \quad m I$.

U० UD

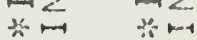

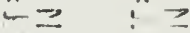

口ir

Z゙U Zu

- - 12

$\mathrm{V}-$

nis

-

$0 \frac{z}{\alpha}$

xis

포

10

$\varangle \backsim$ $\sum m$

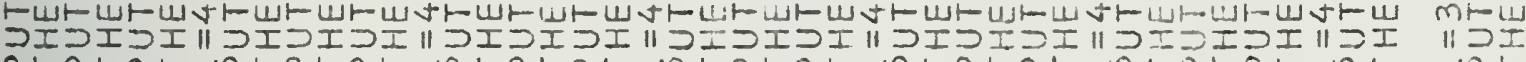
QFa ち

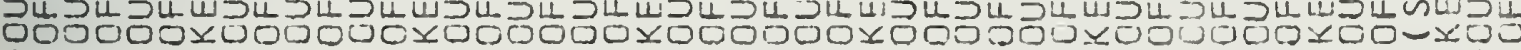

$\begin{array}{lll}v & \ddots & 0 \\ 0 & v & N\end{array}$

$\underset{\sim}{\sim} \underset{\sim}{\sim} \stackrel{U}{v}$

$\begin{array}{lll}\sim & \ddots & 0 \\ \sim & N & N \\ \sim & N & N\end{array}$

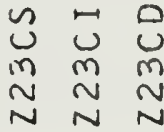

น

$N N N$ $\begin{array}{ll}0 & 0 \\ \sim & \sim \\ \sim & N\end{array}$ 


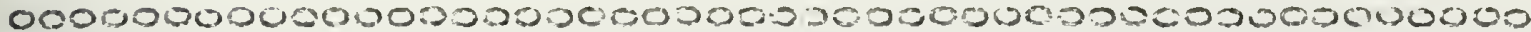

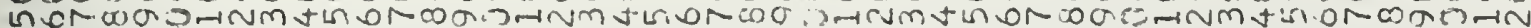

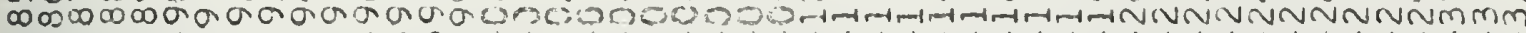

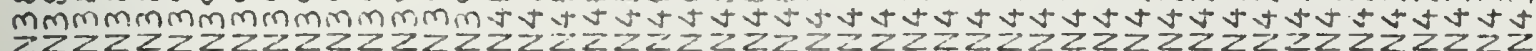

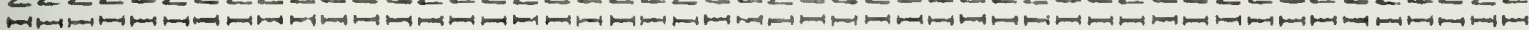

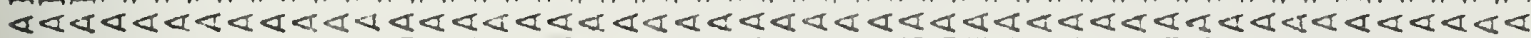

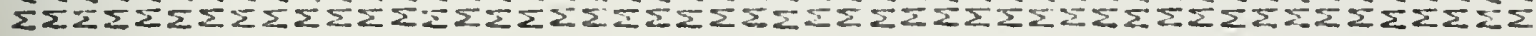

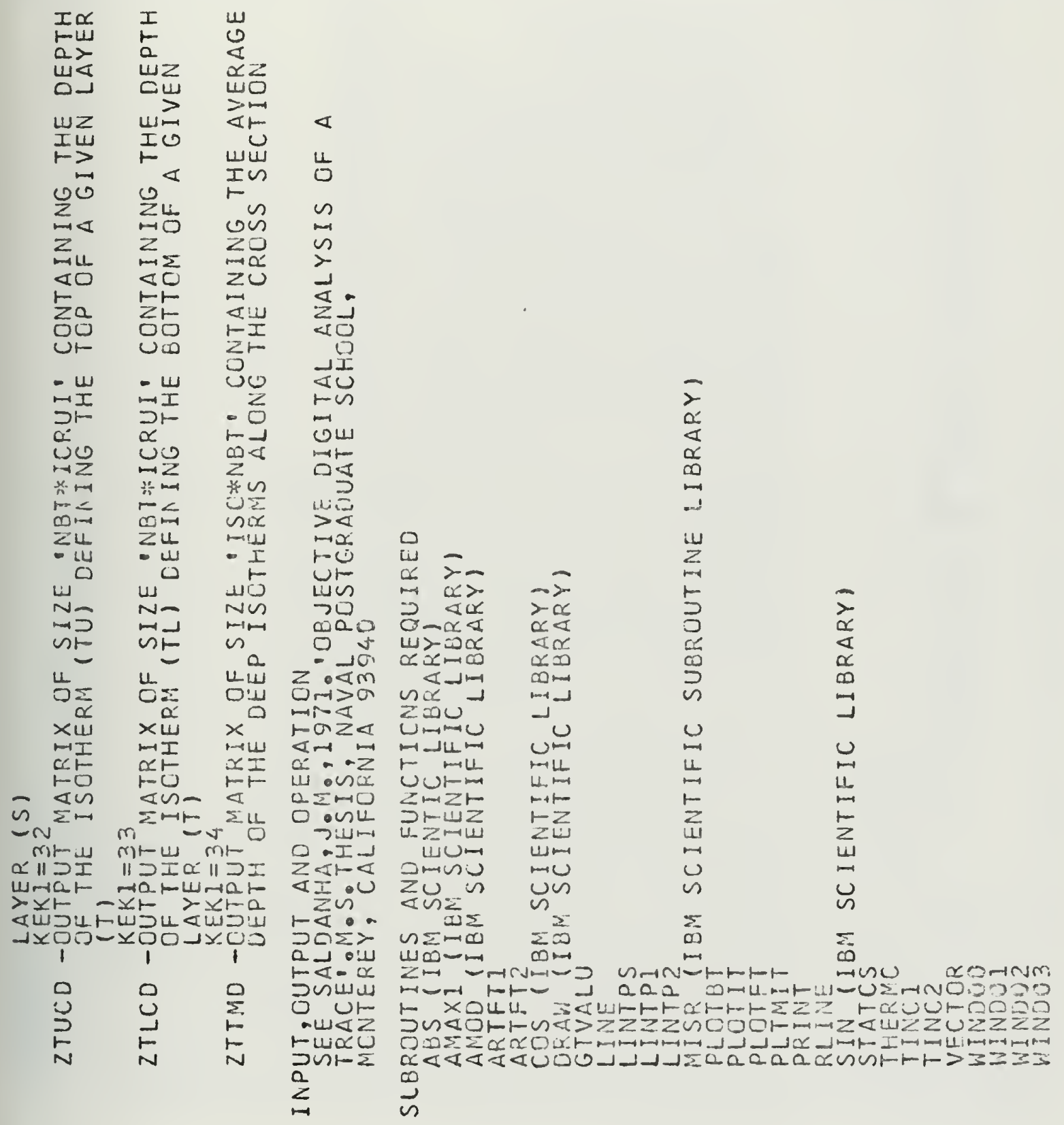


000000000000000000000030000000000000000000000000

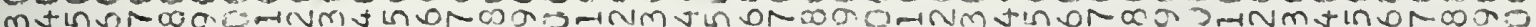

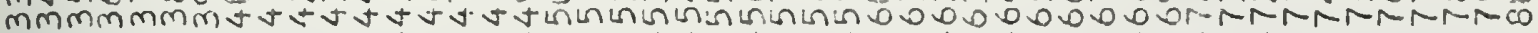

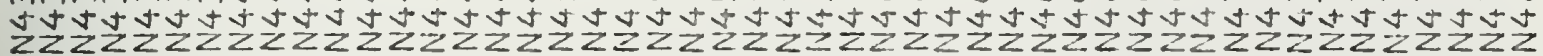

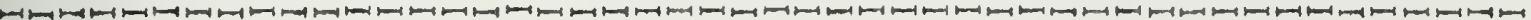

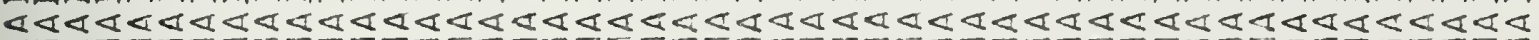

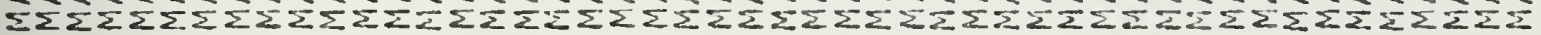

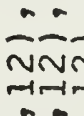

Nin

Cion

Non

บั0-

$N m>$

III

Fト

NNF

$\therefore$.

NNOV

rifir

50

Nive

omen

$m m>$

III

rh. NNF

an aranamana

NONNONONONOA

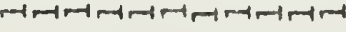

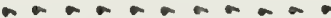

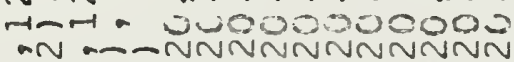
OHDNN-ー- -

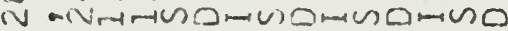
- o- - uuvuluuvuus

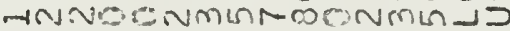

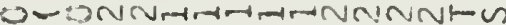
GIRO-NNNNNNNNNNN

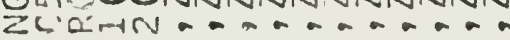

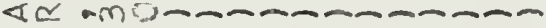

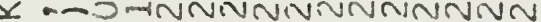

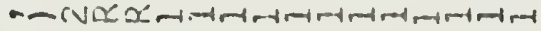

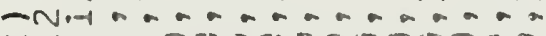

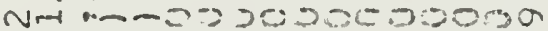
a aNNUTNNNNNNNNNA

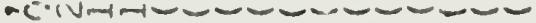

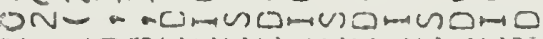

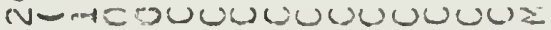
- (1) $5 N$ N mC:3.2- SOONANNNNNNUNNINN $\alpha z \ldots v \in \ldots \ldots \ldots+\ldots \ldots$

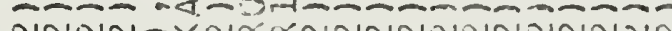

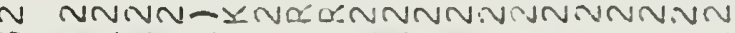

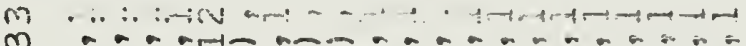

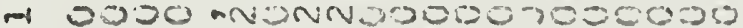

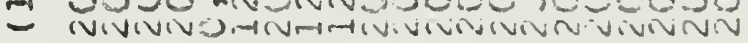

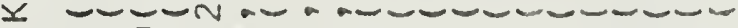

2 munou- ucism nom

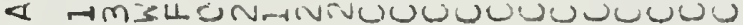

\lrcorner IIIUS-Uー-

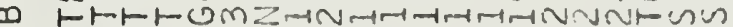

- NNN $Z$ ZOERIINNNNNNNNNNNN

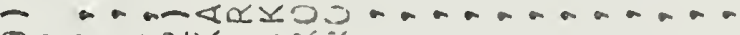

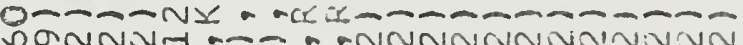

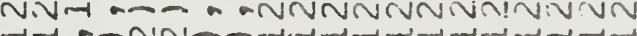
O- a a N

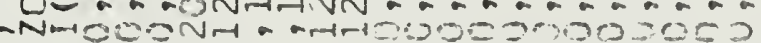

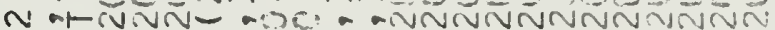
U-a- -

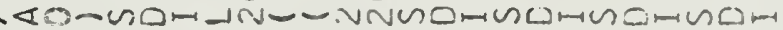

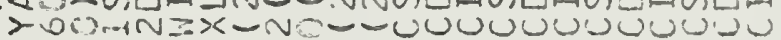

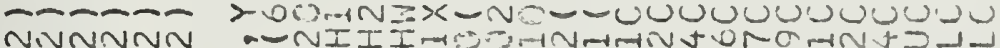

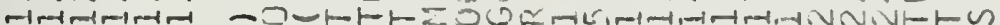

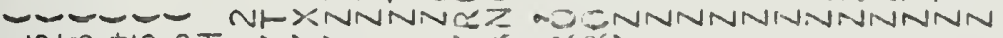

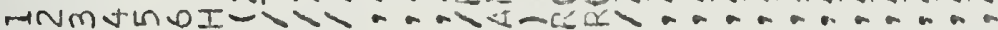

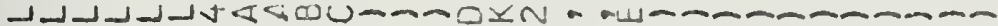
FFमFFF入

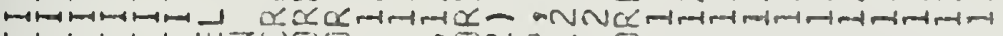

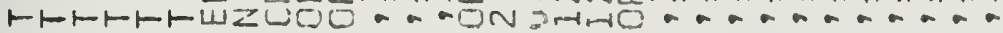
म๓⿴म冂म

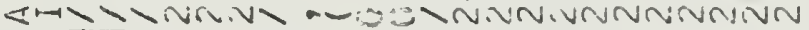

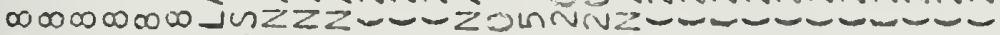

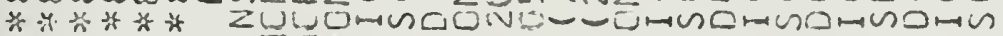

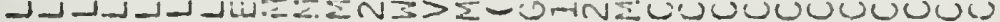

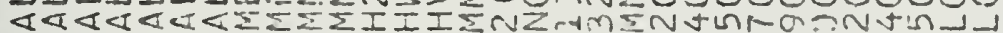

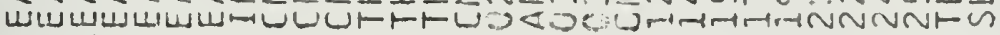

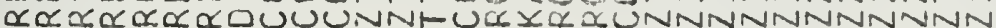

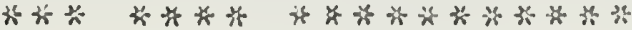

यूn

$n=$

n.

0 ii

ơ

uz

on

แU

$>\backsim$

○一

$F$

$\sim \sum_{0}$

IU

$F$

$\backsim$

แ山

on

खण

ic

$\sum$

$\rightarrow 0$

0

山。

$\infty$

$-$

is

$-6$

mm

$x$

o>

th

$\Rightarrow \quad m \cdots$

$\rightarrow>$

$\checkmark>$

U U人

$\rightarrow \rightarrow$ 光

i

iv Uर

$\backsim$ ज小.

Un

- arath mmmn- -mpHN

a- a- nene

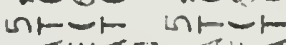
$-<u<2-3 u<$ $0=1 \leq 00 \sum 12$ दर्व

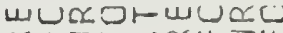

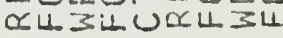

$m m^{*}$ in 
00000000000000.30000000000000520200900003000000000 -

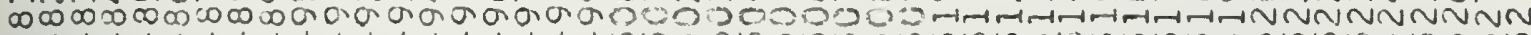

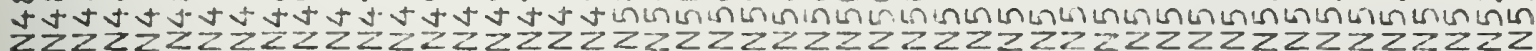

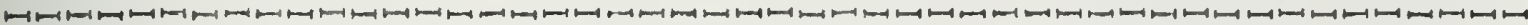

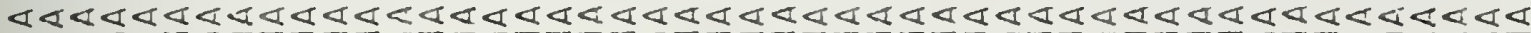

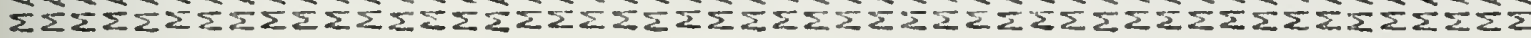

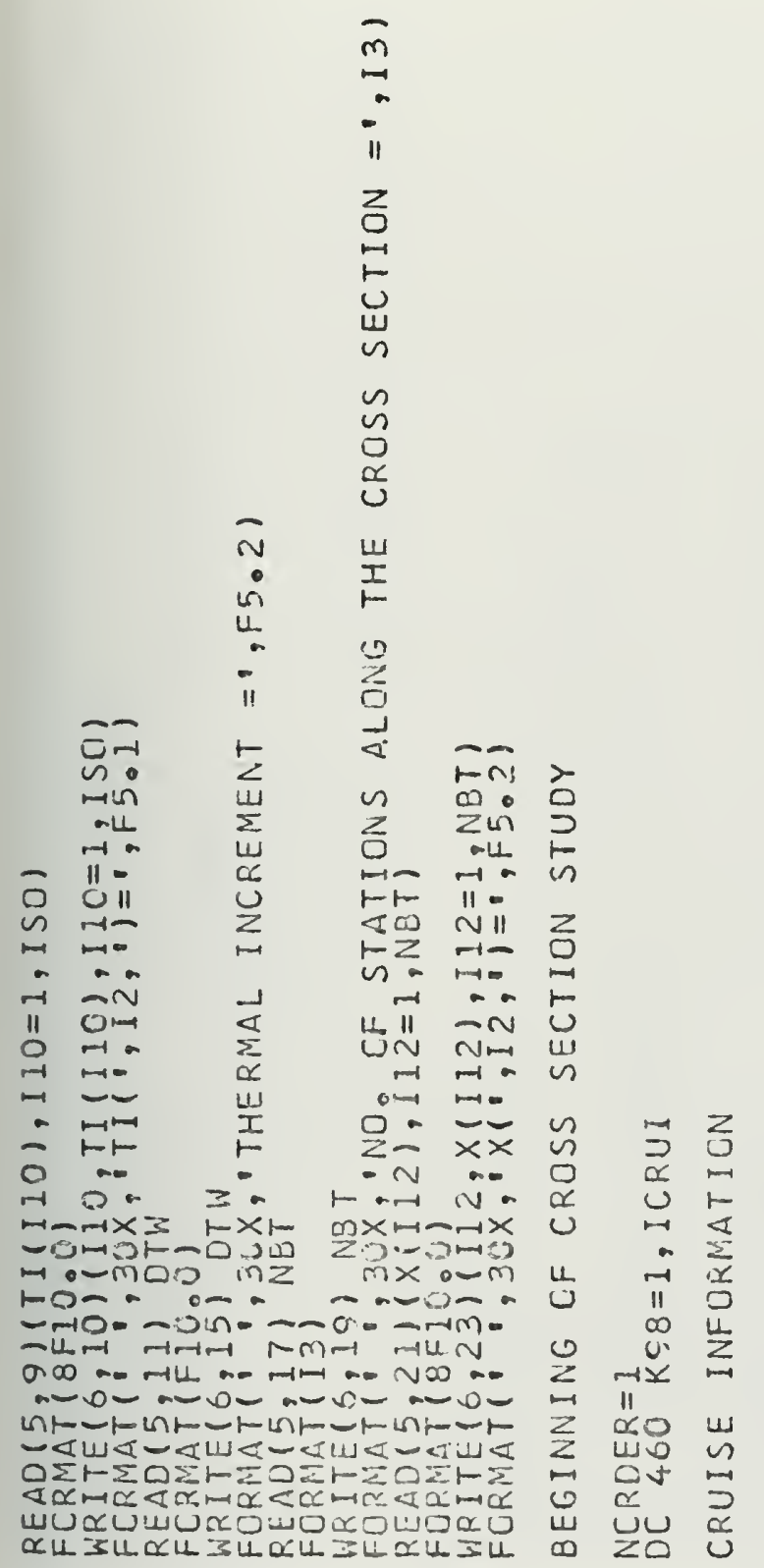

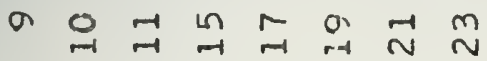

$\stackrel{+\infty}{\times}$

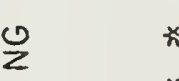

$z$

$\overline{0}$

u $: 0$

$\sim 4 \stackrel{4}{\circ}$

z

"艹

21

u. 0 少

ᄂ $\quad \frac{a}{4} z$

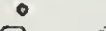

5

○

$\sim$

$\sim \sim$

$\therefore$ m 11

$\sim a \rightarrow$

50.5

-

20

in

- II $\propto ⿻$

-

II นก

$\rightarrow 4$

an

0

$\bar{N} \bar{N} \bar{v} \overrightarrow{0}$

$\rightarrow r \rightarrow r$

$-1+\infty$ in

II II II II

$\rightarrow \mapsto \mapsto$

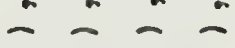

$\mapsto \mapsto \mapsto$

$=\bar{m}-\bar{m}$

$\rightarrow+$

$F F F$

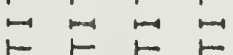

$\leftleftarrows \models$

- - - - -

$-\infty-\infty=\infty-\infty$ Inar Q O I I I NONONOMO

ar a me a เกเடกเ แกเயกเ $-4-0-4-7$ $0 \sum 0 \div 0 \geq 0 \sum$

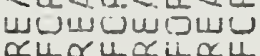

in $N \stackrel{N}{\sim}$

NOZZト

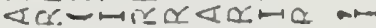

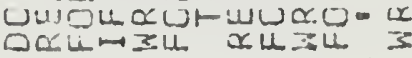

$$
m m^{n} \stackrel{v}{v}
$$

ư טus vus 


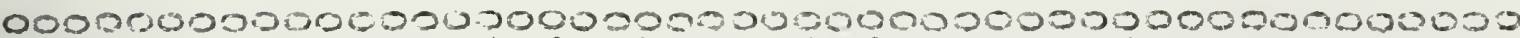

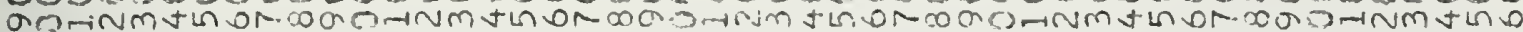

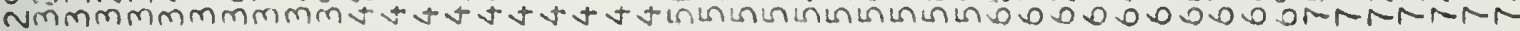

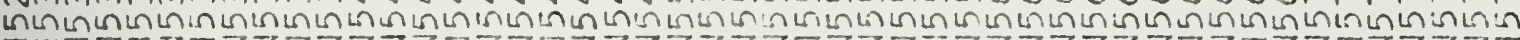
ZZZZZZZZZZZZZZZZZZZZZZZZZZZZZZZZZZZZZZZZZZZZZZZZZ

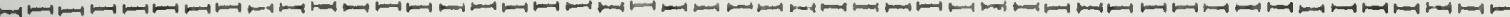

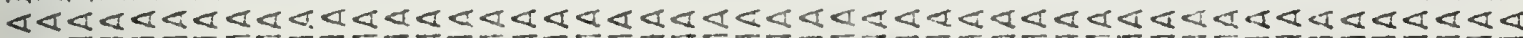

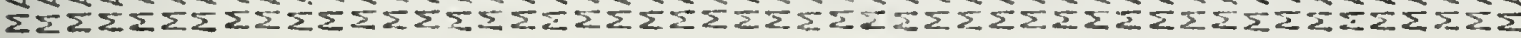

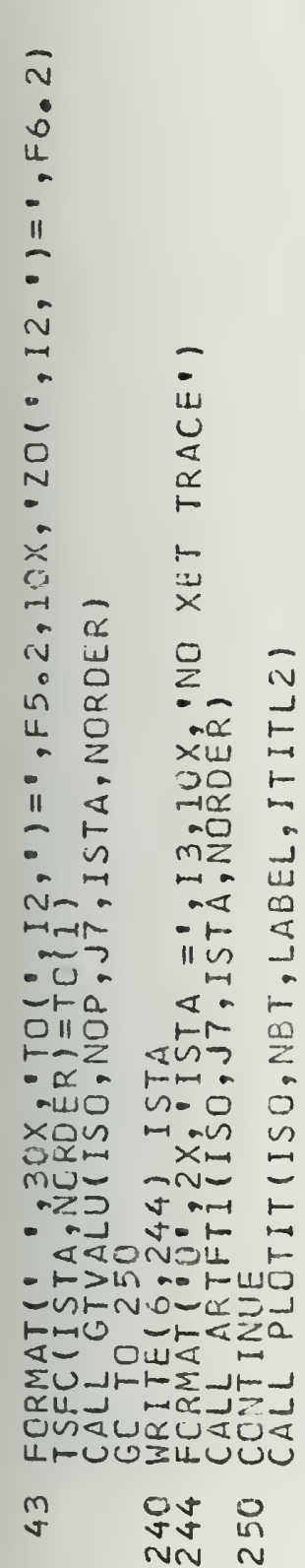

n

$\frac{1}{2}$

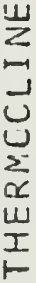

$\stackrel{M}{I}$

2

$\sim$

แง

$\omega$

$\varangle$

1

एक $\quad$ क

- 0 .

$00 \geq 3$

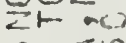

a $<i n$

$1<0$ Orom

aroun

$\sum_{n \rightarrow-1-N} N$

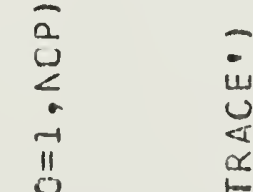

$\rightarrow$ con- - nt-

$11-$ - n-Nmanz

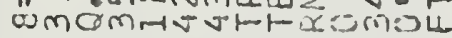

Jinum at a

- $0000 m-1 I m b-\propto$

Drina-nー - JaF -1<

h-Ou-um

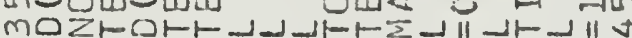

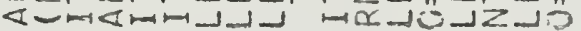

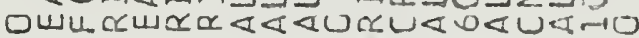

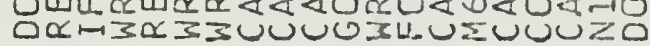

OJ
mm

(s)

m
点

$0 \approx$

$\sum$ in

$\sim n \rightarrow m$

तin -

$\alpha \tilde{\alpha} \leqslant \pm$

- 4 in 0

$-\infty \rightarrow N$

$\varangle \alpha \sim 4$

-0 a

$n<m$

arv or a

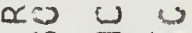

WU ZX。

$0 z \leq x N$

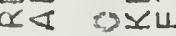

Ox n- no

$\geq a \sim x-107$

- - n स

j) $=2<-1-2$. $3-1-4=$

munte

mżmmaxi

-4 क on -

axca a

- nullixam

" -

दर्व 00 .

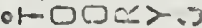

onzzon.

zanz zon

anin-mu

$>$ aroina

ourowom

$\mapsto 0 a \geq 0 \times m$

$-\alpha-x=2$

$<0<1<0$

$1 Z$ - $-x \leq$ 。

$n=n=a+n$

-T- On<I-Uis

$<$ to a a a

1 nxaxur $m$

(n) mo usmencm

M 0 arlu -

- $-\alpha \alpha-00$

- - Dowax.

Mn:M:3mZOUOR

$m n-m-c\llcorner D Z u$

min

जit. un

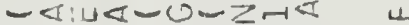

$0 \sum 1-02 \geq m-0 \geq 0$

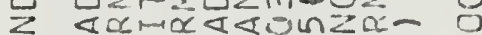

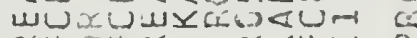

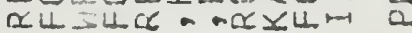
m

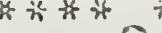
m 
000000000000000000009000000000000300000600000000

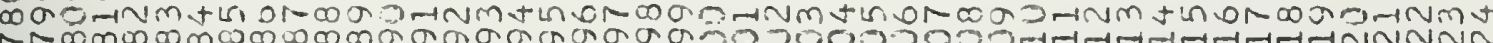
(1)

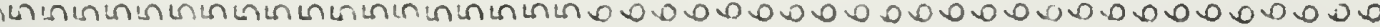

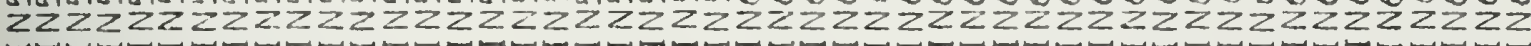

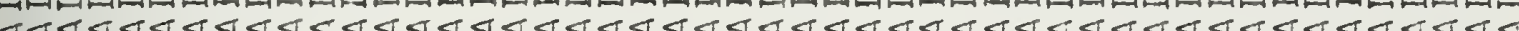

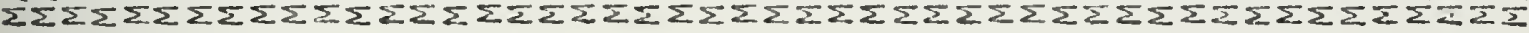

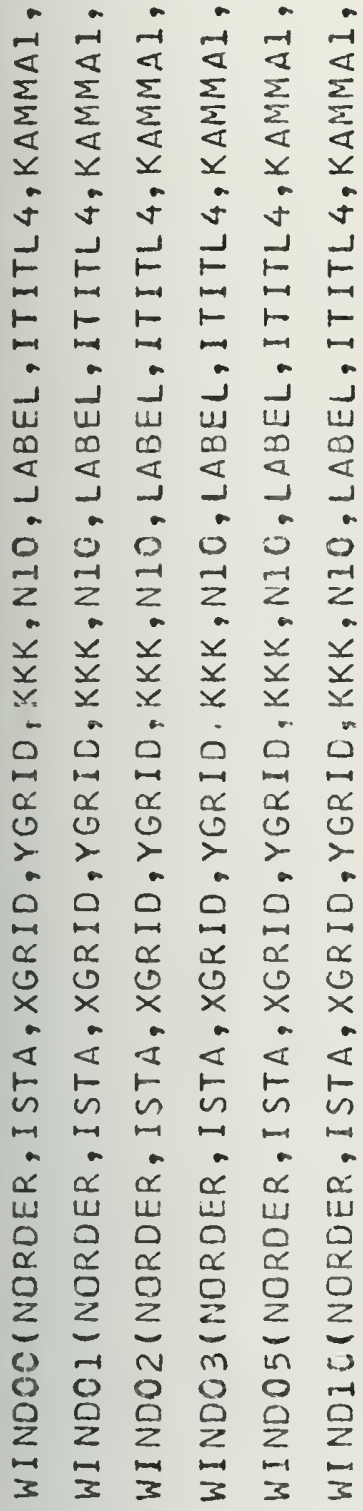

1-1-1-10

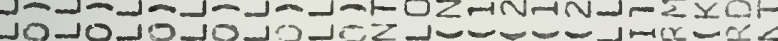

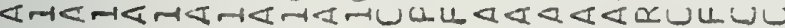

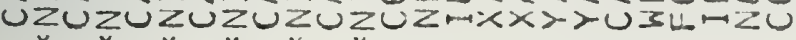
* $\sum_{k} \sum_{i} \sum_{k}$

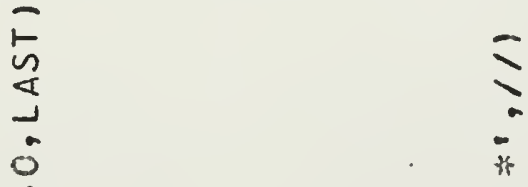

$\frac{2}{5}$

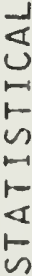

3

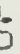

$\infty$

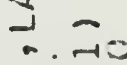

เก $\mapsto 0$

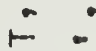

○) 110

$\frac{1}{z} \sqrt{n}$

$\therefore<0$

$>-n=-$

$\rightarrow<1+\infty-1$

<I $-\times \supset+$

$x-50 a$

NR aro

- In- $0 x$

zNvisol

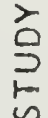$$
\text { 인 }
$$

ก

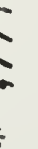




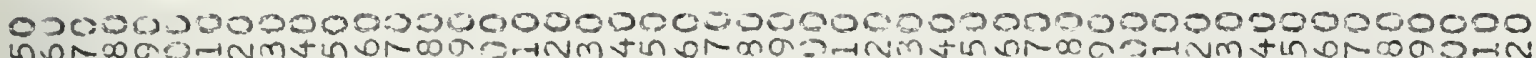

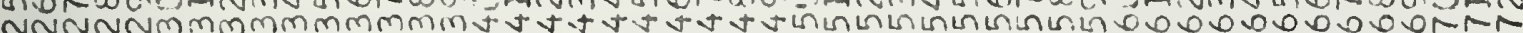
0000000000000000000000000000.00000000000000000000 ZZZZZZZZZZZZZZZZZZZZZZZZZZZZZZZZZZZZZZZZZZZZZZZZZZZZZZZZZZZZZZZZZZZZ

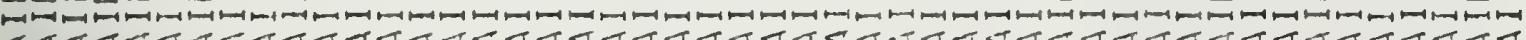

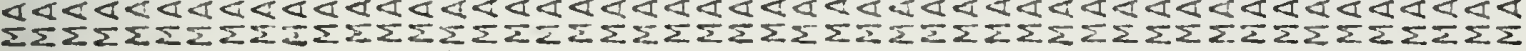

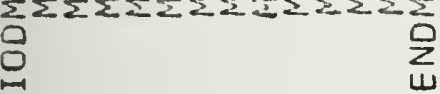

里 2

崖

0

$\Sigma$

$\alpha$

U

00

H $0 \alpha \quad 005$

¿

$\varangle \quad \sum_{0}$

$\backsim$ O.J Ut थ m0 งż- w二-に UW on- 000 ममUनM $0<2$ \& a Z DHFO OH $1 z \cdot \cdots z \cdots-$

जnXut $0 x .<-\varangle$

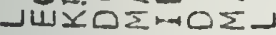
$-\infty \backsim<\alpha \smile<\alpha-$

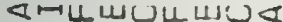

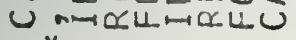
น้

$\begin{array}{ll}N & 0 \\ 0 & 8\end{array}$
u

Un

mCU

\section{DODOO न1 in}

susunususunususuna

\section{IIIIIDO 0}

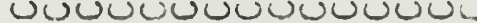

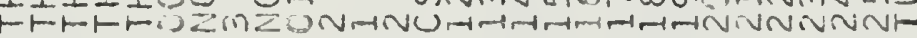

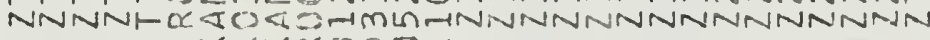

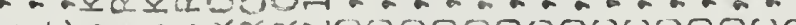

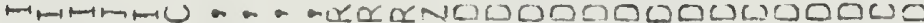

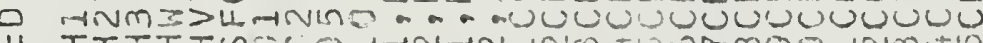

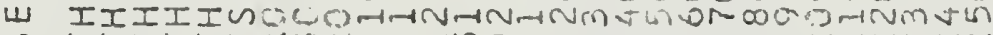
u - - - -

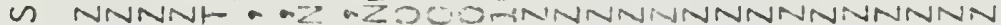

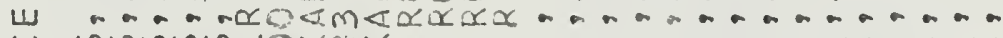
U vunusud TnM $z \geq X 0$ a

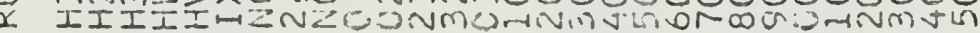

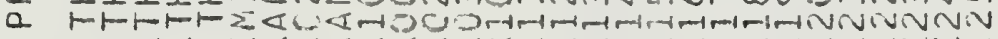

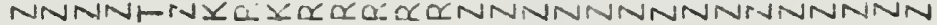

$u$

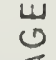

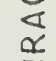

$\widetilde{\alpha}$

is

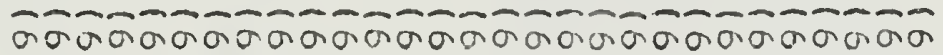

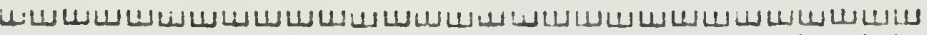

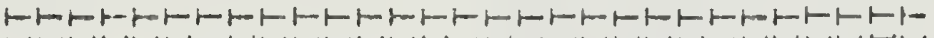
$\alpha \alpha \propto \alpha \alpha \alpha \sigma \alpha \alpha \propto \alpha \alpha \alpha \alpha \alpha \alpha \alpha \propto \alpha \propto \alpha \alpha \alpha \propto \alpha \propto \alpha \propto \alpha$ 喽

0 
30000002002000000000000000006000

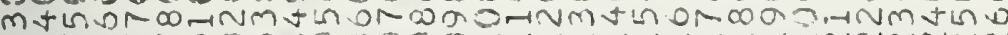

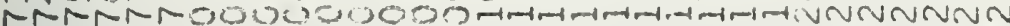
$0000000000050 \times 00001000002.0502000$

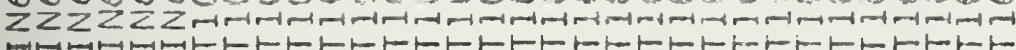

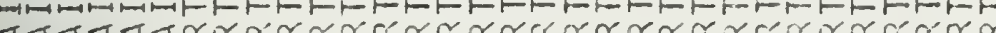

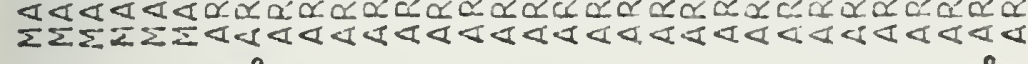

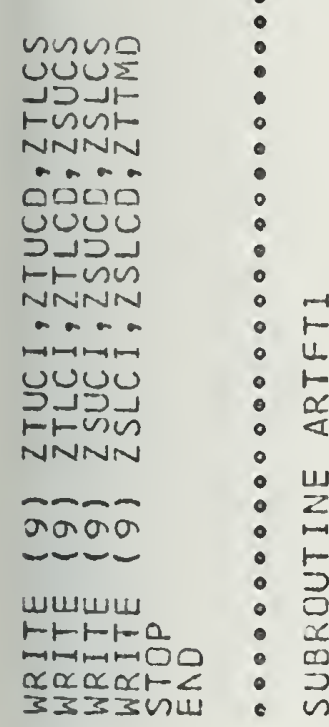

$\sim$

Zu

$\mapsto$

$<1$

¿山

年

$\dddot{4}$

$\sum_{i=1}$

t-

$\vec{\alpha} \alpha$

$\rightarrow 0$

UL

แய山

mu

- $<$

$\frac{\alpha}{<(v)}$

$\rightarrow \infty$

نบ

Ut

âd

$-0$

$\overrightarrow{1}=$

近

$+$

UI

車

$z$

4.

$0 \frac{1}{n-1}$

WU

$\cup$

$\geq 0$

แю

ज

mon

रायड

$1-\alpha$

wबा山

II

แ্-

๑zun

Q

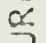

$\frac{a}{2}$ $\sum_{0}^{\sum_{0}^{u}}$

u

40

$=$

8

山

i 0

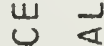

0

$\frac{1}{0}$

U U

U⿺ 丶万

$\propto$

$\frac{x+4}{21}$

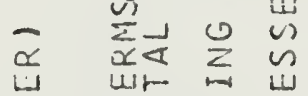

id Ur

Ci Fo u.

2 जo

\& 4 蛋u

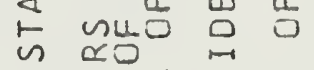

$\mapsto \quad \omega \alpha \propto$

-

$\rightarrow$ Wس

- $4 \sum \sum$

$\infty_{n=1}^{\infty}$

$\rightarrow$

$<之$

-

แつつ๓つト-D

OQFDUU

ZuáLw

Zn<uran $\backsim$

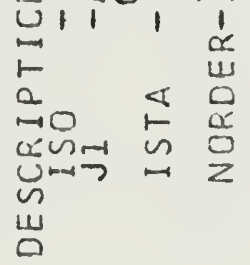

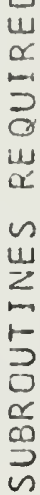

$\sim \leftarrow$
00003000030020

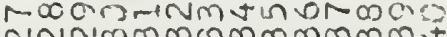
Nis

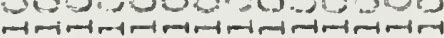
トトトトトートトトレトトルト $\alpha \propto \alpha \propto \alpha \alpha \propto \alpha \propto \alpha \alpha \alpha \alpha \alpha$

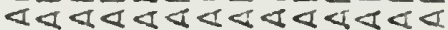

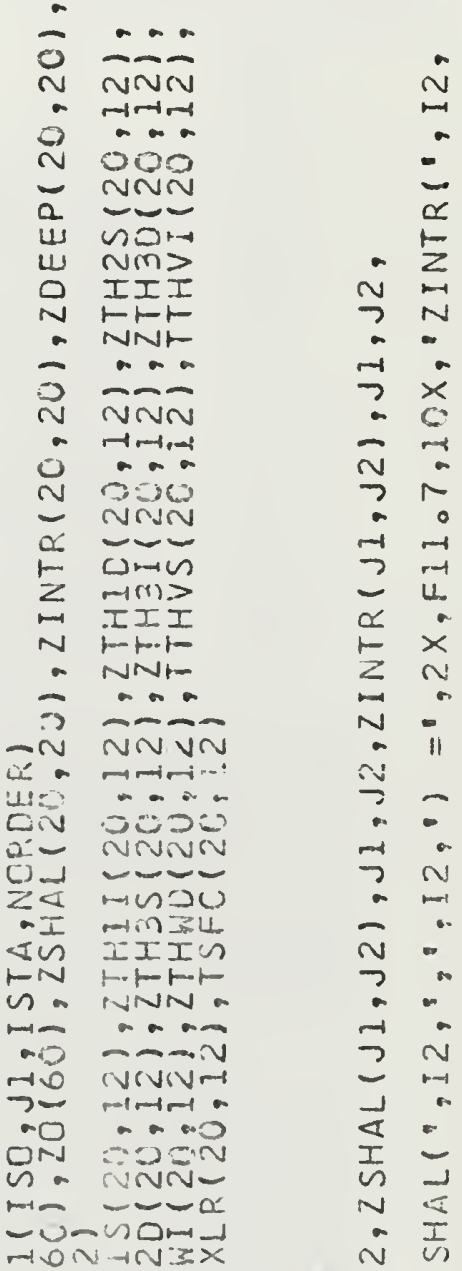
HUNIIEk $000 \mathrm{~N}$

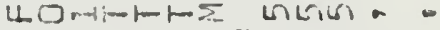
H-MNNNNO 1117 a

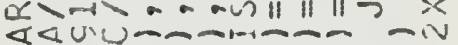
XzonNiv arvivivan a

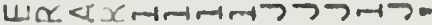
ZU-í a a - n a a o

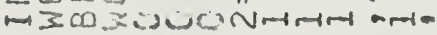

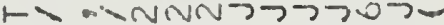

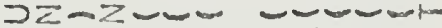

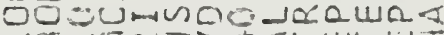

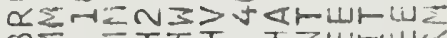
c口E-

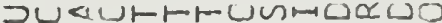
SUNUNHTONNNZNL * in 
000000003050000000200020000000 HNm th

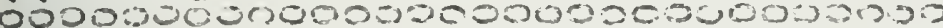

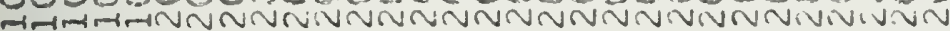

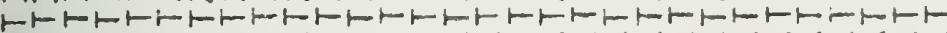

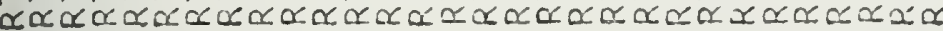

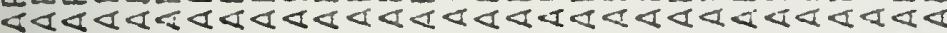

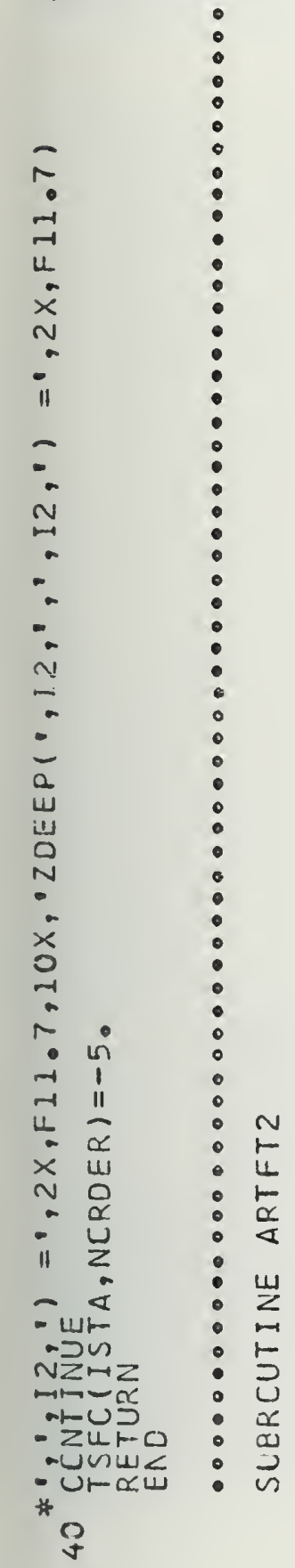

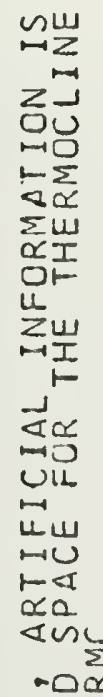

iᄂum

U\}- 1

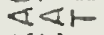

aU

r-OU

$+2$

बर्ग

$\vdash ?$

$\mapsto$ फO

$\circlearrowleft \pm \propto$

$0 \rightarrow$

Zn

$\alpha \mapsto$

$\sum_{10}$

c) 30 $=4$

แL

$\cup 0$

ZOU

$w r z$

n

$\infty 0<$

<U⿺

$1-\infty$

I送

I这

ज ZF

OZயর

Qमण0

$\alpha$

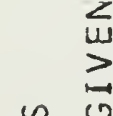

约

$0<$

$\varangle$

z v

$-1$

$n<$

wo

जल

다

art

Uis

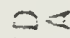

no

Uन

$0>-$

บ>

ou

a.

z

- no:

$\sim \infty$

$\rightarrow$ wo

$\alpha$ แuau

ui. $\geq \sum \infty$

0

a

$z$

-

N

in

$\frac{1}{\alpha}$

$\alpha$ 更i

<

$\vdash$

$\omega \stackrel{\jmath}{\rightleftarrows}$

w<

vu

I. थ

i w
(1) U

- $\vdash ш \alpha$
0000000002000002

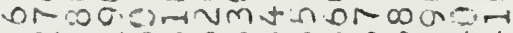

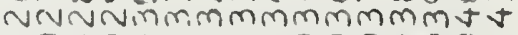
$300.300,3000200303$

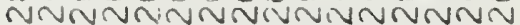

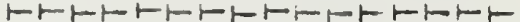
$\propto \alpha \alpha \propto \alpha \alpha \alpha \alpha \alpha \propto \alpha \sigma \alpha \propto \alpha \alpha$

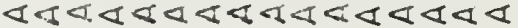

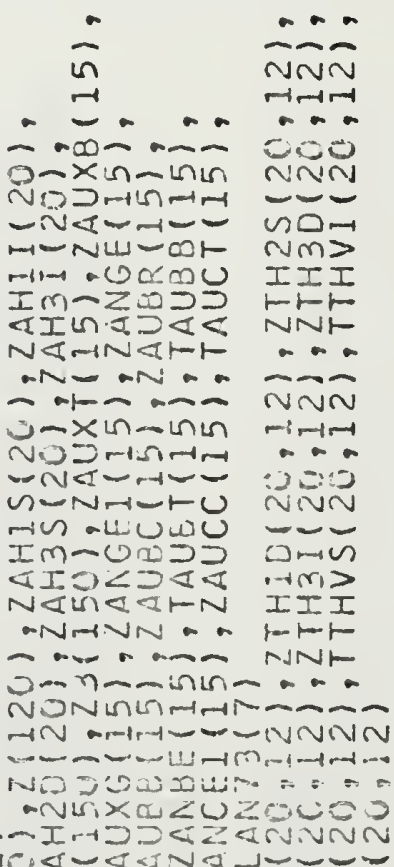
mir<-

TNNELAT ANDMULU

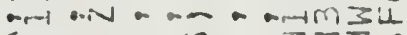
4-O - - in - IIIU

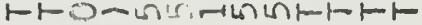

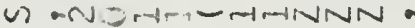
a- L

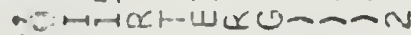
Q O.V-X...JUUU.VNIVH

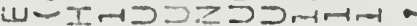
DU 0 रा ants at N Vivt 3600

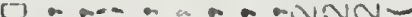
Z-

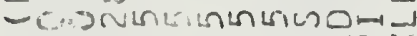
Nen:-retr-indmentive $x$

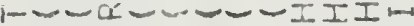

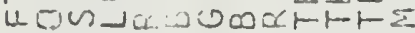

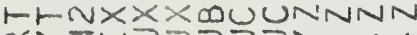

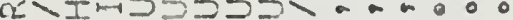

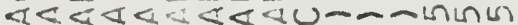

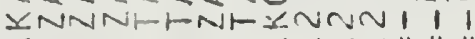

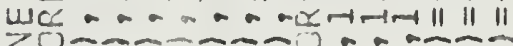


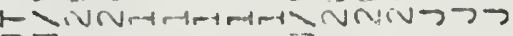
DZ - - - - Z- - - OE

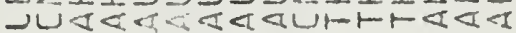
MUNNNIRNTUNNTNNN

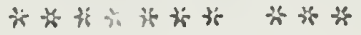


000200000200002000006000020502000000002000000000

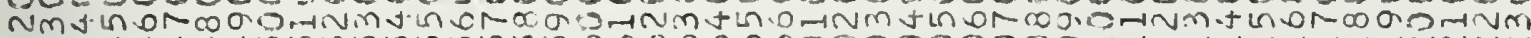

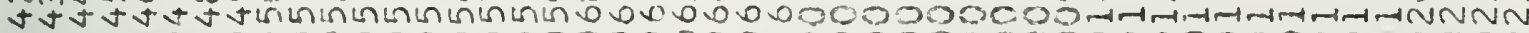

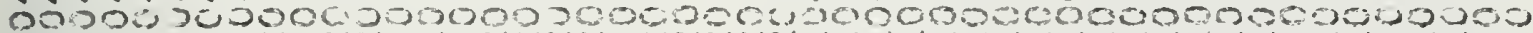

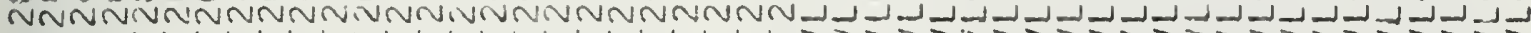

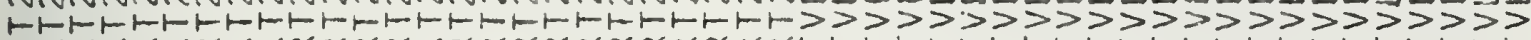

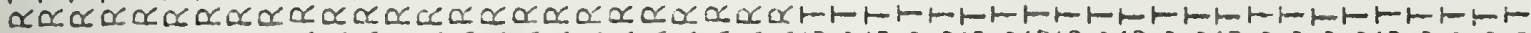

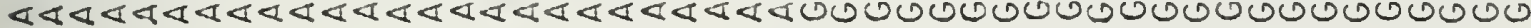

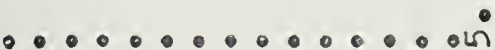

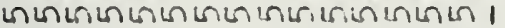
111111111111111 || || || || || || || || || || || || || || ||

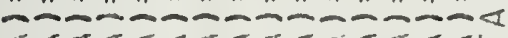
$<<<<<<<<<<<<<<1-$ $1-1-1-1-1-1-1-1-1-1-1-0$

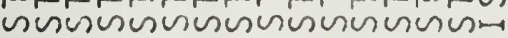

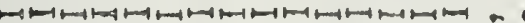

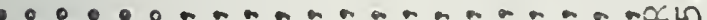

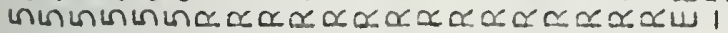

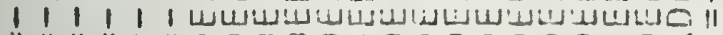

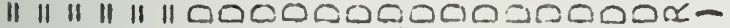

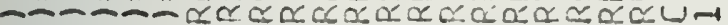

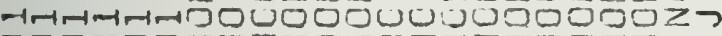

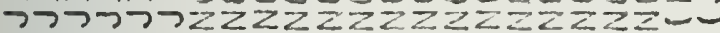

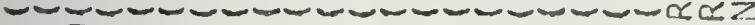

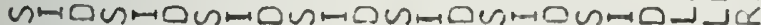

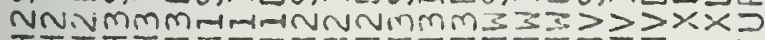
IIIIIIIIIIIIIIIIIIIIII

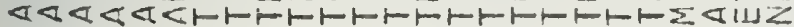

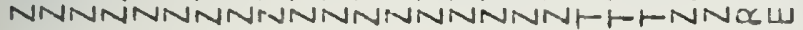

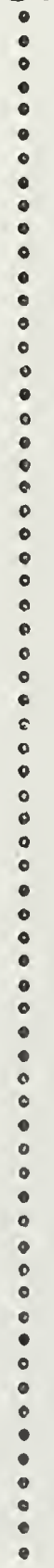

点

$\sum_{i=1} \sum_{0}$

$>0 \rightarrow \cos$

$\Rightarrow$ मயu

0-《

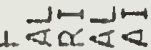

$0 \quad 1>1$

$0>\infty$

Sr $\simeq$

$10<20$

a Uiuk

แ」OZய

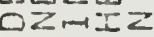

Uन

Uiston

IX $x 山 1$

HW $\Sigma \omega$

$0<ய$

meszun

トோ

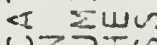

006i=i

Dinton a

$1<<0$

$0<10$

मலOUய

+2 I

U⺊<いト

un w

<นแนเ

$\alpha>n<z$

トZッव⿱㇒

$\rightarrow=0$

$\rightarrow \sigma<$

¿யuแ

$\vdash I>N$

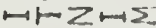

(1) UIF

$\mapsto 0>5 u$

2 our UI

WCगU上

षD vO

J《Zn

Liब 0 . $N>4.0$ $>0 u$

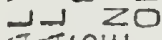

Zsแun $\omega \times x \cup \leq d$

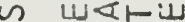

DОIW.

a Fम-

$\stackrel{2}{2}$
4

$4 Z$

따

$\vdash>$

$\vec{\circlearrowleft}$

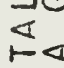

0

$\mapsto<$

0

ष네

on 2

Zu -

Zu

$\ln x<u$

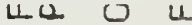

w $\backsim$

on r...

U $<?$

ou is

Nar

ant or ?

no

$\sum r-1$

$\alpha-\alpha$

U.

I.

$1-\infty 0$

O)

जा

$\mapsto 0$

4

in

u

U

0

$a$

a

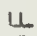

जนL 0 O

w $\alpha$

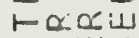

แบบயल

$\sum 000 \Sigma$

$4 \sum \sum 5$

뮐

$<2 Z$

a dس

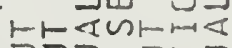

แつつつッつトつ

Q

ZZUCE

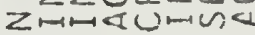
$\omega 11111$

$\mapsto$

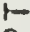

a. $\leftarrow \frac{u}{\alpha}$

$\exists$ mor $\vdash d$

uU Unzo $<$

is

แ 
000.0006000001902060000 thoraon-10Nm NUNNUNMMMInmmmmmm

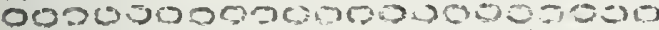

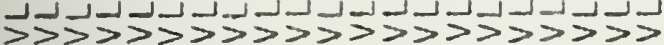

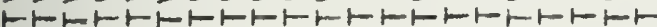
000000000000000000000

\begin{tabular}{|c|c|c|c|}
\hline \multicolumn{2}{|r|}{0} & & $<$ \\
\hline$n$ & \multicolumn{2}{|l|}{ سய } & \\
\hline I шل & \multicolumn{2}{|l|}{$>n$} & $\Perp$ \\
\hline FOQI & \multicolumn{2}{|l|}{ rus } & 0 \\
\hline ator & \multicolumn{2}{|l|}{ Hu } & \\
\hline wz & \multicolumn{2}{|l|}{ ug } & $\sim$ \\
\hline 凹ッய๐ய & \multicolumn{2}{|l|}{$w \propto \alpha$} & $z$ \\
\hline$\alpha \vdash>$ & & 0 \\
\hline யUつ & & & bis \\
\hline - $x<10$ & \multirow{2}{*}{\multicolumn{2}{|c|}{$F U$}} & $\mapsto$ \\
\hline 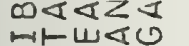 & \multirow{2}{*}{\multicolumn{2}{|c|}{ no }} & $\sim$ \\
\hline$\sim \bar{u}$ & & non & 0 \\
\hline जिIäz & \multicolumn{2}{|l|}{$\Psi_{w}$} & $a$ \\
\hline a जu० & \multirow{2}{*}{\multicolumn{2}{|c|}{$\alpha>$}} & Ш \\
\hline כusw & & & $>$ \\
\hline แล ज๐Z & \multicolumn{2}{|l|}{0} & $\mapsto$ \\
\hline шш - & \multicolumn{2}{|l|}{0} & $\omega$ \\
\hline ஊய山 & \multicolumn{2}{|l|}{ س० } & 5 \\
\hline IDrunus & \multirow{2}{*}{\multicolumn{2}{|c|}{ - }} & $\cup$ \\
\hline$F \quad i n$ & & & LII \\
\hline$\therefore<$ & wס & & $n$ \\
\hline トunz & $\infty z$ & & $z$ \\
\hline বこZuш & $<1$ & & $\square$ \\
\hline$\Delta \searrow \alpha$ & 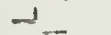 & & $\cup$ \\
\hline$c \omega-i 2$ & $\Sigma$ & & \\
\hline U: $\geq n$ & $\sum \ll$ & & $\frac{9}{3}$ \\
\hline - IUnI & 00 & & $\vdash$ \\
\hline$c c u>r-$ & $\geq 0$ & & \\
\hline utz 2 & $\sum x$ & & $z$ \\
\hline rடாய山ய & $\omega a$ & & ن \\
\hline Un 20 & $u_{-}$ & & 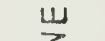 \\
\hline$<0 u-d$ & $-z$ & & 3 \\
\hline$\propto แ 0<\omega$ & $n$ & & 5 \\
\hline$<5>1$ & $n<$ & & 山 \\
\hline 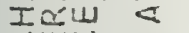 & $w=$ & & $\infty$ \\
\hline லயயலயே & $\sim$ & & \\
\hline $1-z-\omega$ & Du & & $z$ \\
\hline ヘZயலய & & & 0 \\
\hline 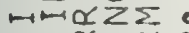 & ด्ए & & $m$ \\
\hline$\alpha=\alpha v$ & $z$ & 0 & F \\
\hline$\Sigma-D \backsim เ \cup u$ & JロI & ш & $\varangle$ \\
\hline$\alpha \infty u \quad \vdash=$ & $1-0$ & $\propto$ & ـ \\
\hline U-৩wZ - & $j \supset \alpha$ & $=$ & $\square$ \\
\hline I $0 I^{\prime} \mapsto<$ & LOU & $D$ & س. \\
\hline $1-1>$ & $a$ & 0 & $\propto u$ \\
\hline 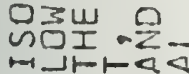 & 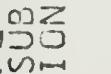 & Us & $\begin{array}{l}w \alpha \\
\qquad \alpha \\
z 5\end{array}$ \\
\hline$\rightarrow$ un & 1 & $\sim$ & $=1$ \\
\hline I $41-130$ & $\sin 4$ & 山 & - \\
\hline UIZ Z X U & $x=-2$ & $z$ & $\propto<$ \\
\hline$\varangle \backsim \supset \omega-1 u$ & $I \mathscr{X}$ & $\curvearrowleft$ & IF \\
\hline w 0 & $1-0<$ & $\vdash \omega$ & ய๓ \\
\hline$m-u r<1$ & -UL & $\supset Z$ & $0=0$ \\
\hline$-1<U \supset I \alpha$ & $N z<$ & $\sqcup \curvearrowleft$ & On- \\
\hline$-\alpha Z n$ & $-\infty 0$ & $\alpha-1$ & I -0 \\
\hline & & 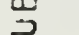 & W \\
\hline & & $\sim$ & $\Sigma$ \\
\hline
\end{tabular}

CDODDD0000000500000000000

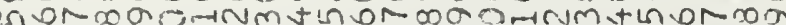

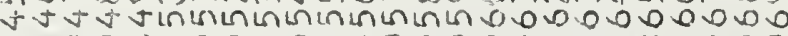

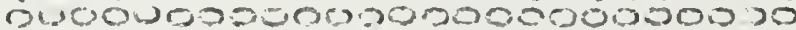

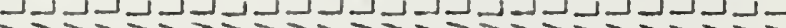

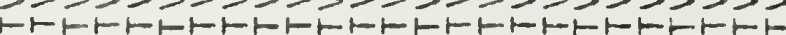

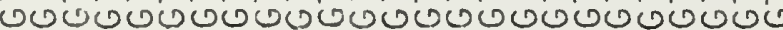

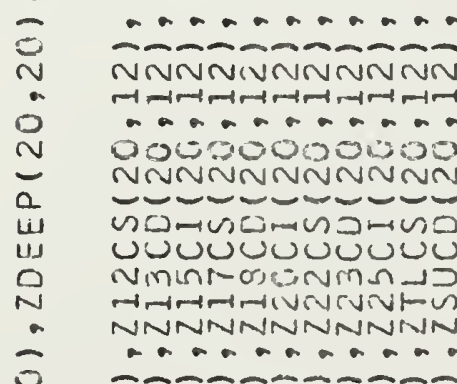

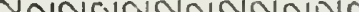

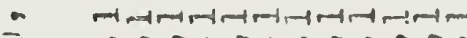
an a a a n a n

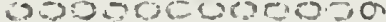
NUNONIVANONONm - -

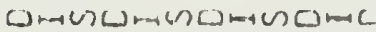

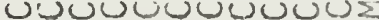
minuा oco 9 -

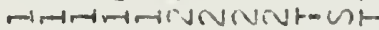
NNNNNNNNNNNN

-

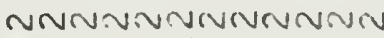

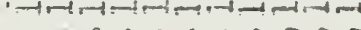

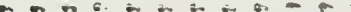
00000000.006 nrvativivinivinan - - - - - - - m(S)

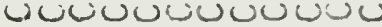
-

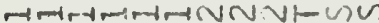
NNNMNINNNNNN - a a a a a a a -200-

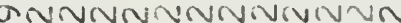

Q 0 an

$Z_{-\infty}$ -

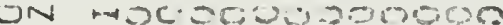

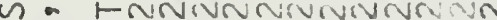

-

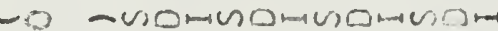

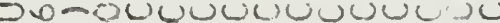
INNm N

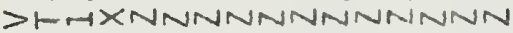

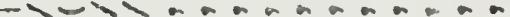

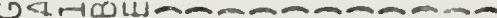

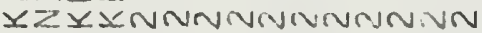

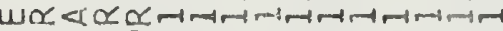

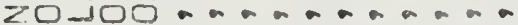

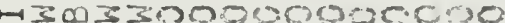

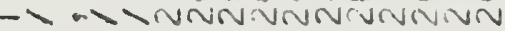

马Z=ZZー- - - - - -

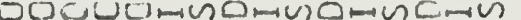

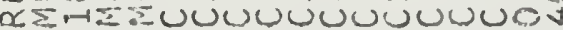

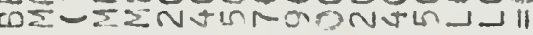

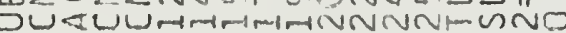

SUNUONNNNNNNNNINNOO H in

Co

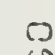

5

1

$0 \cdots$

$-\infty$.

$-a$

110

mor

วแ

om

ming

II 11

लmu

$x \rightarrow 0$ - 
000000300.000000000000000000002000000000000000000

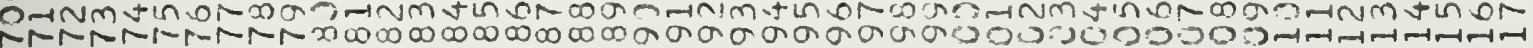

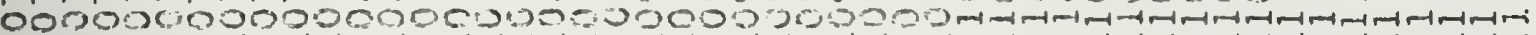

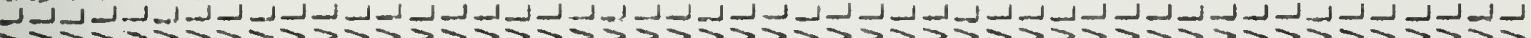
$>>>>>>>>>$

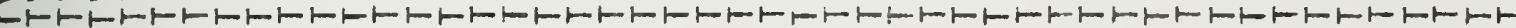

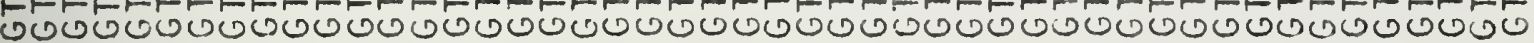

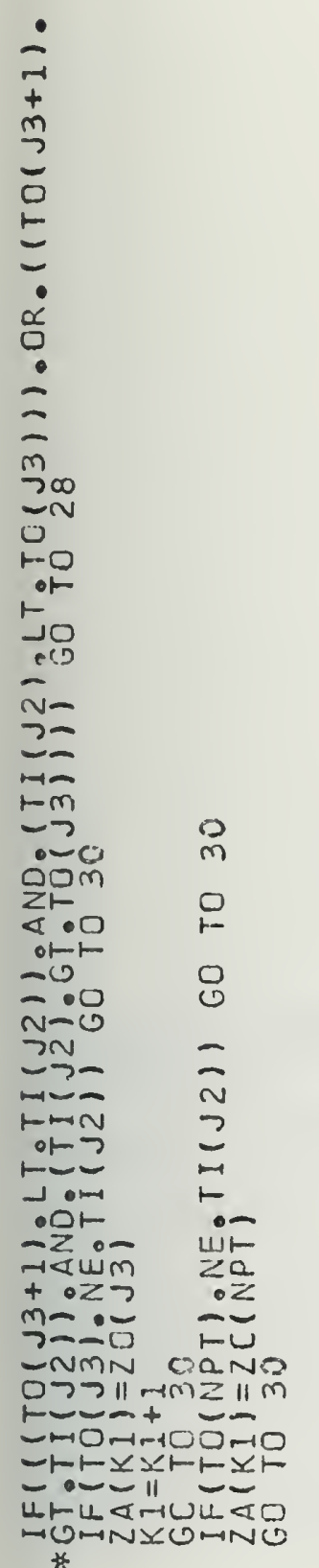

กิ

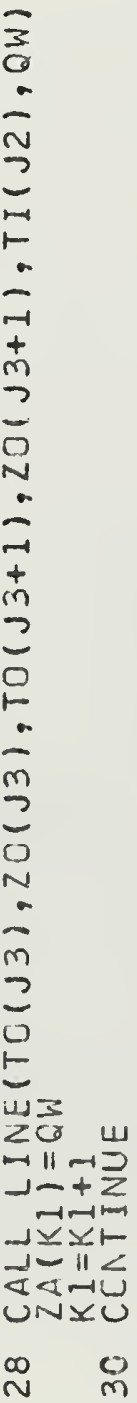

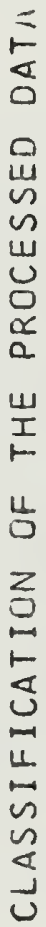

I $N \quad m$

$m$

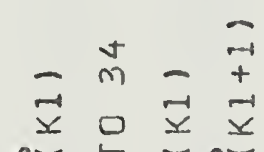
रांय $\ln \ln \ln$ 일 OII 11 -

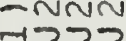
-72.2

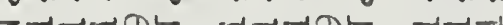

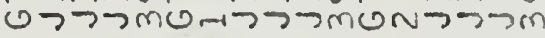
- - 1 1-2 1 1 -

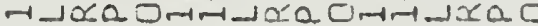

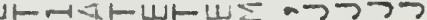

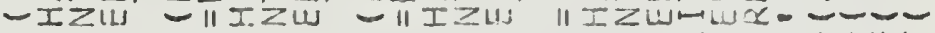

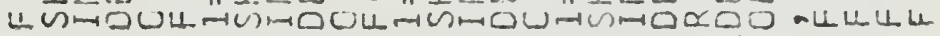

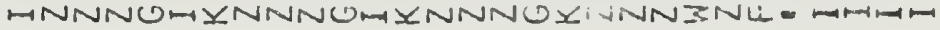
$\stackrel{\sim}{\pi}$
$m$
(1)
$a^{\ddot{x}} \tilde{y}$ 


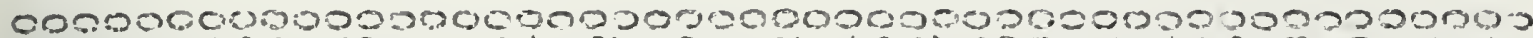

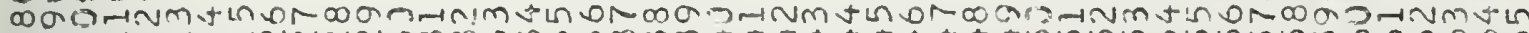

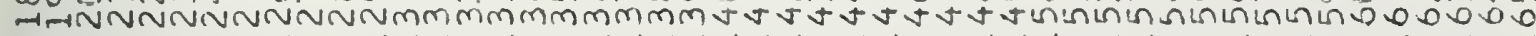

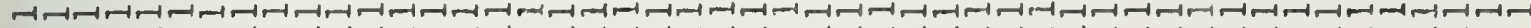

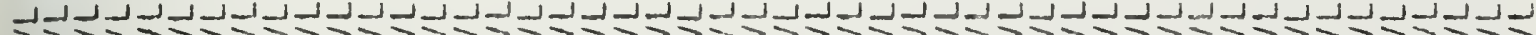

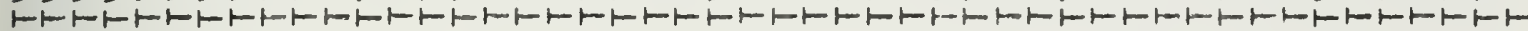

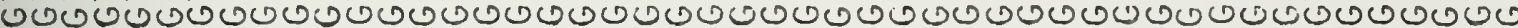

0000000000

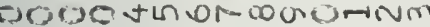

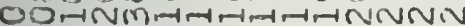
onन-1m-1

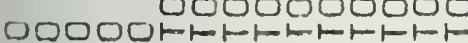
トトトトト

0000000000 D0000000000000 00000

- $-1-\infty-10-10$

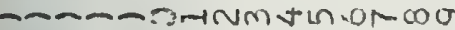

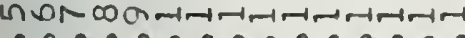

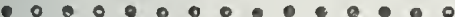

\begin{tabular}{|c|c|c|}
\hline & $\rightarrow-\infty$ & 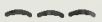 \\
\hline NNT & Niviv & NNT \\
\hline$\supset>$ & $\rightarrow \rightarrow$ & $\rightarrow 7 \rightarrow$ \\
\hline$a-\infty$ & an & $\infty \infty$ \\
\hline$-1-1-1$ & म्नल & r-tr-l \\
\hline$>\rightarrow>$ & $\rightarrow \rightarrow$ & $\longrightarrow>$ \\
\hline & & $-\infty$ \\
\hline$J \propto \Omega$ & $-10<a$ & $-1<22$ \\
\hline 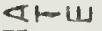 & ar $U$ & $\langle I-\| U$ \\
\hline$I \geq U$ & $I Z U$ & $I Z u$ \\
\hline$n \mapsto 0$ & $n=0$ & 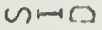 \\
\hline NNW & NNN & NNN \\
\hline$\|$ II $\|$ & II II II & \|\|$\|$ \\
\hline & $\therefore-\infty$ & $-\infty-$ \\
\hline & $\alpha<<$ & $\ll<\alpha$ \\
\hline$-1-1-$ & $1-1-$ & $1-1-1$ \\
\hline nun & inesus & ninen \\
\hline 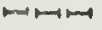 & MேBम & म-1 \\
\hline & $\because-\infty$ & $\sim \infty n$ \\
\hline 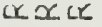 & $x \in, \alpha$ & $\alpha \propto x, \alpha$ \\
\hline u:uUs & U:UUు & Ued ills \\
\hline 020 & 000 & 000 \\
\hline$\sigma \propto \alpha n^{\prime}$ & $\alpha \propto \alpha$ & $\alpha x \not x$ \\
\hline
\end{tabular}

तviño

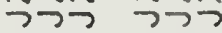

a $\pi_{10}$ $\rightarrow \rightarrow$ - $-1,-1-1$ $\rightarrow \rightarrow-9$ $\sim \sim$ $-\operatorname{lc} a$ 10. \&I-U1 IZU und NMN II II II $-\pi<$ unsos แM-1以

an a

coxes

usus -dura 잔 N) II || || || || || ष्विध बिस tot the unues inves माயम

ar a acc $\alpha r \alpha$ سய:L Luth 000 Q000 ruño TrNiv $\rightarrow \rightarrow \rightarrow \rightarrow$

\section{an} तन्ना mम्न

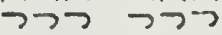

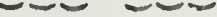
jor acta षाய 단 $I \geq \omega \quad I \geq W$ जmo vmo NNI NNN II || || || || || $20<\frac{1}{10}$ rat int visur visua मा㇒म MAm - on on a

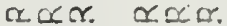

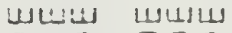
000 a00

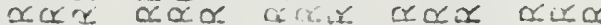

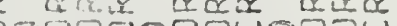

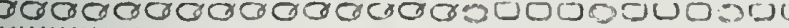

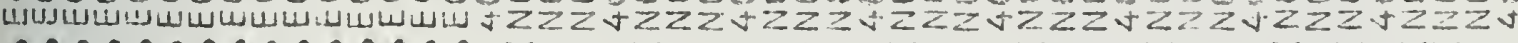

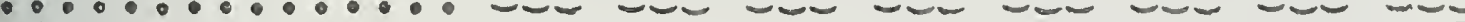

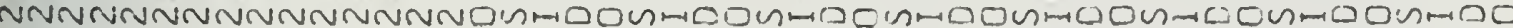

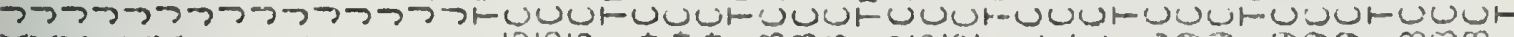

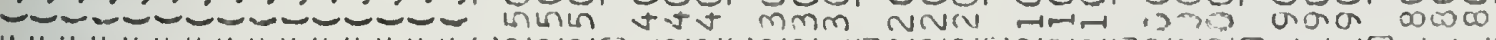

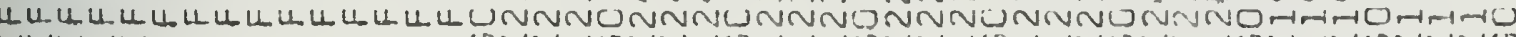

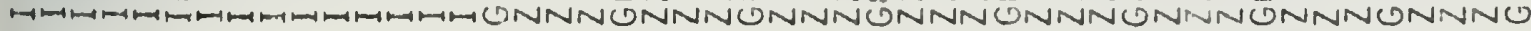

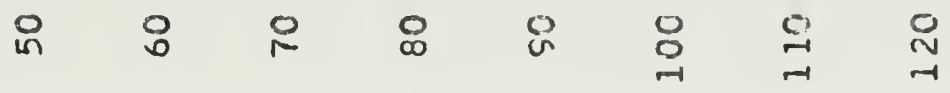




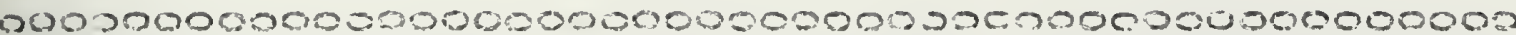

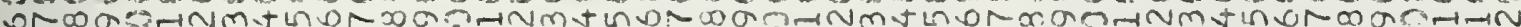

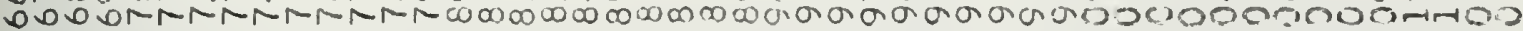

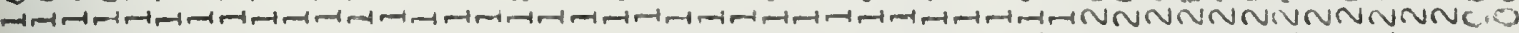

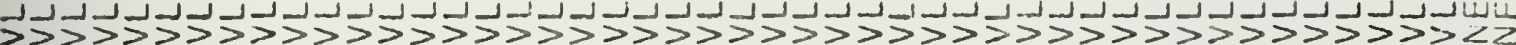

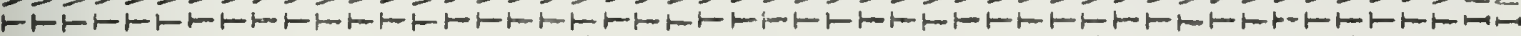

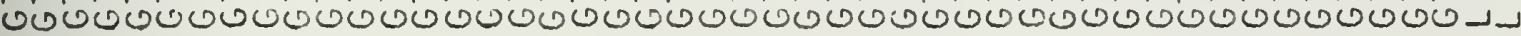

\begin{tabular}{|c|c|c|c|c|c|c|c|c|c|c|}
\hline & $\cdots-$ & 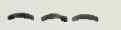 & $m-1$ & $-\infty$ & $m-\infty$ & $-\infty$ & $-m$ & $m-\infty$ & $-\infty-$ & $-\infty-$ \\
\hline NNVIV & Niver & NNN & NNN & NRUN & NNN & Nrurv & NNN & SNRT & rutser & NNN \\
\hline$\neg フ$ & 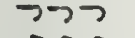 & $\neg \neg$ & $\neg \neg$ & $\neg つ つ$ & $\neg \neg ~$ & $\neg つ フ$ & $\neg \neg$ & $\neg \neg$ & $\neg つ つ$ & $\Rightarrow \neg$ \\
\hline निन्न & $\dot{\vec{n}}$ & min-1-1 & नोमा & निन्निं & $\min _{n=1}^{\infty}$ & min- & जन & $\because \infty$ & $-\infty$ & -aे \\
\hline$\neg \neg$ & 277 & $\neg \neg$ & $\rightarrow 7$ & $\neg \supset$ & $\supset \supset$ & $\neg \longrightarrow$ & 375 & नुन & 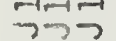 & $37 \rightarrow$ \\
\hline & --2 & & & & & & & & & \\
\hline - $x a$ & 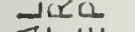 & $-1<0$ & $-x a$ & لـ & 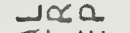 & $-d x a$ & مa & JuL & $\Delta a \Omega$ & 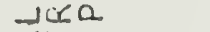 \\
\hline வடトய & 【゙たU & $\Delta k-14$ & ㅁ-U & $\leq-1-U$ & 【⺊ய & $\Delta r-w$ & 【டレய & Qr w L's & ¿t-W & ¿ibu \\
\hline IZய & IZu & IZW & IZW & $I \geq U \mathrm{~J}$ & IZu & IZus & $I Z \omega$ & $I \geq \omega$ & $I \geq U$ & IZU \\
\hline जino & ज๓๓0 & $\sim n 0$ & जino & $n=0$ & ज๓O & $n=0$ & $\omega=0$ & $n=0$ & जmo & vito \\
\hline NNN & NNN & NIN & NNN & NNN & NNN & NNN & NNN & WNiV & Ni-3N & NNN \\
\hline || || || & \|\|$\|$ & || || || & \| il \| & $\|$ || || & \| || || & \| || || & \|\|$\|$ & $\|$ il \| & II || || & \| :I II \\
\hline$-\infty=$ & $=0$ & $=-1$ & $2-1$ & $-2=$ & -2 & -0 & $-\infty$ & $-\infty$ & -2 & $=1$ \\
\hline$<<2$ & $<<<1$ & $<<<$ & $<<<$ & $<17$ & $\varangle<\varangle$ & $<\varangle<$ & $\varangle \ll<$ & 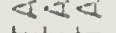 & $2,-1<6$ & $\ll \ll$ \\
\hline thet & Fト & $1-1$ & tht & r-r & トேト & $r-1$ & $5-1$ & rar & $\vdash+\leftarrow$ & トート \\
\hline nusus & nusus & nusu & nesu & sines & nus & vinu & vivius & sines & nun & suses \\
\hline मロー & मேम & ๒ロー & ツロツ & Mmम & ウロロ & サツウ & ேேー & ウロの & ツセカ & 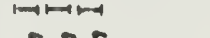 \\
\hline$\hat{\alpha} \dot{\alpha} \dot{\alpha}$ & $\dot{\alpha} \alpha \dot{\alpha}$ & $\dot{\alpha} \alpha \dot{\alpha}$ & $\ddot{c} \ddot{x} \tilde{c}$ & $\dot{x}=\infty$ & $\dot{\alpha} \dot{\alpha} \hat{\alpha}$ & ara & $\dot{\alpha} \alpha \dot{\alpha}$ & $\alpha \dot{\alpha} \alpha$ & $\dot{\alpha} \dot{\alpha}$ & $\hat{c} x^{\infty} \dot{x}$ \\
\hline แлLUய & шபщ & wயयाओ & घแயய & Uisus & แ่แน & แயU & Uisules & แ山س & Uالن & แ山ய \\
\hline 000 & 000 & Q0 & 000 & 000 & Do0 & 000 & 000 & סט & 000 & 000 \\
\hline ๔x. & 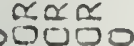 & 舟金品 & ax: & xª & $\stackrel{\alpha x \propto}{0} \propto$ & aic & 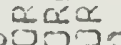 & $a x a$ & $\propto \alpha \alpha$ & $0 \alpha \alpha$ \\
\hline$z z Z=$ & & 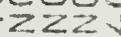 & $-\infty$ & $z z z$ & $=$ & $z z z$ & $-=$ & $z \geq 2$ & $z=z$ & $\geq z \geq 3$ \\
\hline & & & & & & & & & & $-w-z z$ \\
\hline $3 n=$ & Sn & 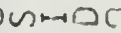 & $n_{m} 0$ & $n \rightarrow \infty$ & $n m 0$ & $\sim \rightarrow C$ & $n m 0$ & $\operatorname{cim} 0$ & $\sim s m 0$ & 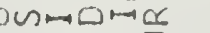 \\
\hline UuWr & & & & & & & & & & u৩uト: \\
\hline 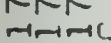 & $\begin{array}{lll}0 & 0 & 0 \\
3-1 & -1 & 0\end{array}$ & भाजी & $\begin{array}{lll}-12 \pi \\
2 \pi\end{array}$ & mmm & N: & 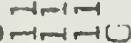 & $\begin{array}{l}1 \\
-10\end{array}$ & $\begin{array}{l}D=D \\
5\end{array}$ & $\Delta-3$ & 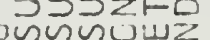 \\
\hline & & & & & & & & & & \\
\hline
\end{tabular}

$m$

$\begin{array}{lllll}0 & c & 0 & 0 & 0 \\ 7 & \text { N } & 0 & \pi & \pi\end{array}$

$\underset{\infty}{\infty}$

in

$\stackrel{O}{\sim}$

$m$ 
0000000500000600700006000500600

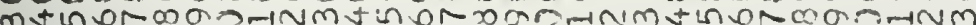

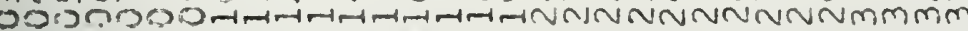
$0000006000000=0000004000090000$.

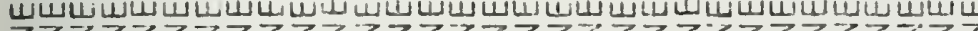

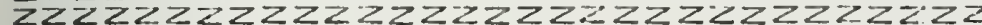

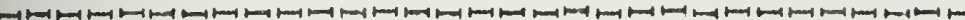

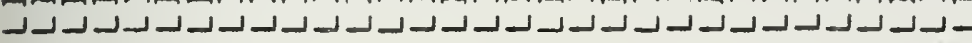

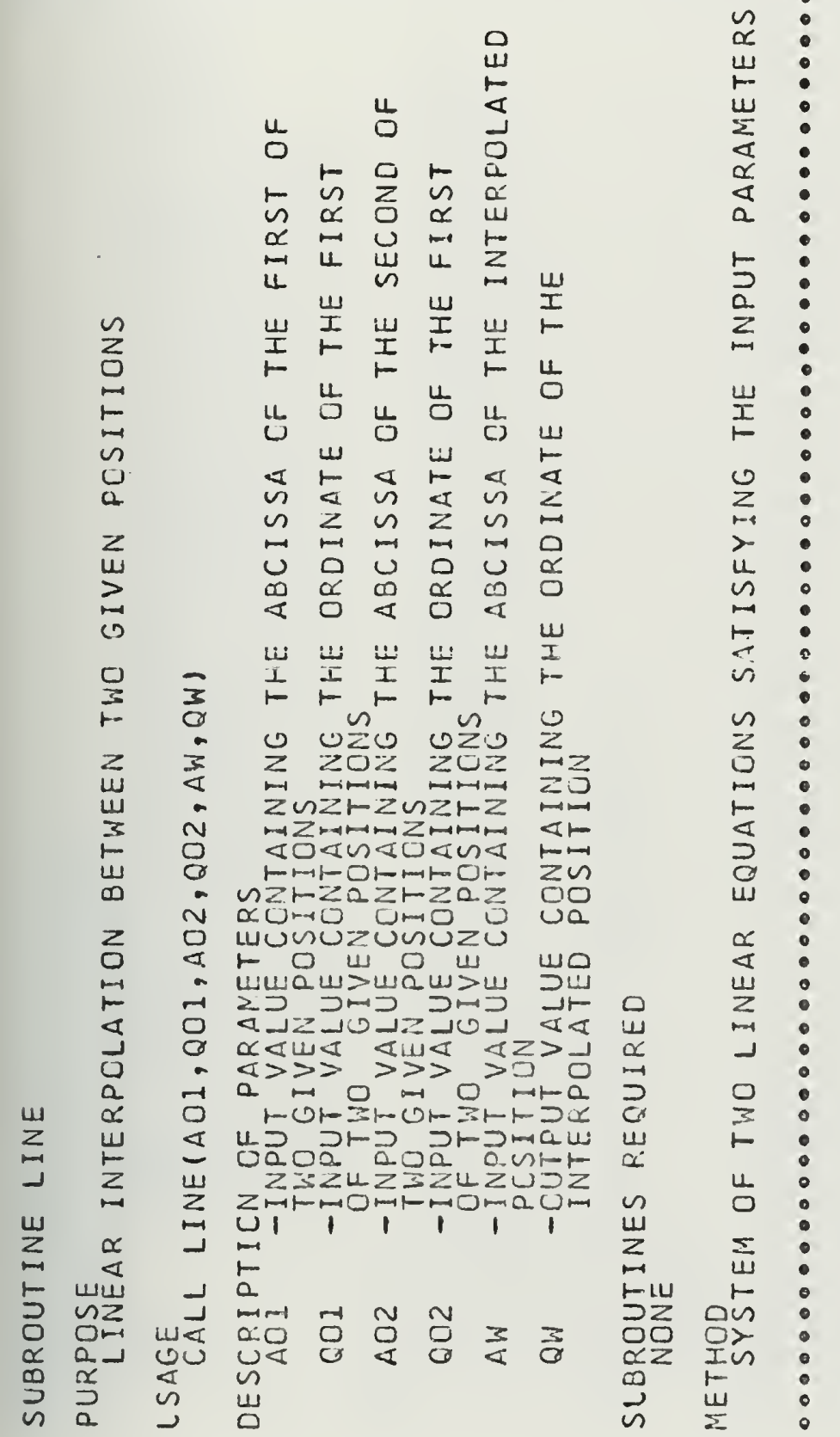

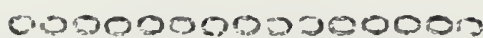
th mmmmmm t.t.5. 000000000003002

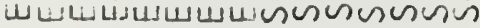

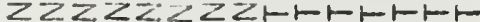
ッッッッッ⿻上ッZZZZZZZ

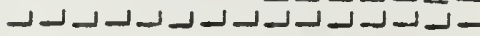

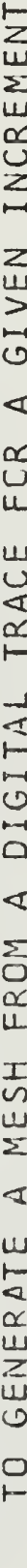
4

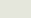


000000000600902000000000300020020

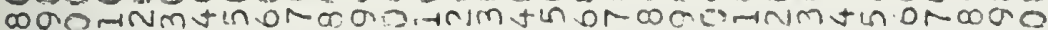

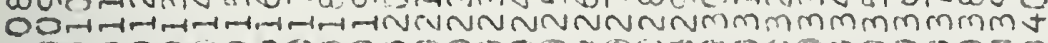

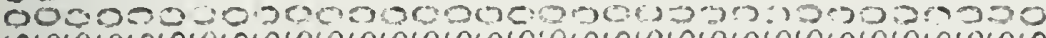

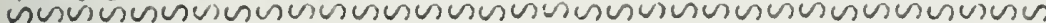

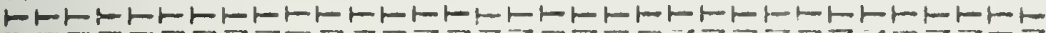
zZZZZZZZZZZZZZZZZZZZZZZZZZZLZZZZZ ג

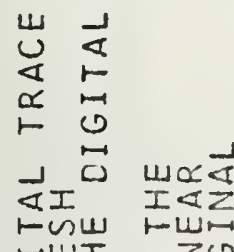

$$
\begin{aligned}
& \text { 㕸I } \\
& \rightarrow-0 \infty-\alpha \\
& \text { ดแ⿰ }-0 \\
& \text { ID. > }
\end{aligned}
$$

200

$\sum_{i=1} u \geq u$

$Z-1-5 u$ Und

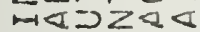

$u>0 w u r u$

wor $\sum \sum \alpha \propto 1-$

ดயดแう0Z

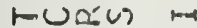

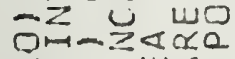
N NमW

- - - c $\alpha$

गद्वाज जा

$1-\Sigma-D \vdash \alpha \omega$

- $\frac{\pi}{0} 0<m z$

Un 25

जIवZणOZ

वுमㄹ แ

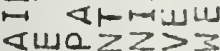

aI OZOF

FUUmous

जu 0 जब

a OLL \&LU

$\omega \square \propto \infty \omega z \omega$

मx u- ISU

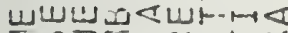

$\sum \infty \supset \geq-\propto \vdash \alpha$

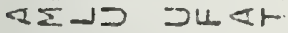

$\propto 2 \varangle z>-1-1$

$\langle z>00<1$

a 5 ane 上たDUU工占

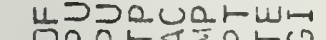
zZつ以

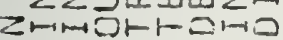

0111

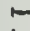

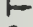

$\exists \quad a$

wa a्रा

Uง UZि

in is

ज
แू

-

UIष

mUULI

Uू क

แงt-v<0

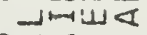

$0<1<$

$\vdash \vdash a<u$

$\mapsto$ uz

《ODC以

Uゃ山く厂

cos $\supset$

* wo

wawa

$\omega I \propto-\infty$

U1

Joñm

UटIடथ

$\infty \rightarrow 1-1+$

$4 \geq 2$

i. $\bigcup \geq$

- U⿺辶万

20002

- $-4 \div=$

$\Sigma \overline{0}>0 \mathrm{~F}$

Uम一-

U.

- No

$\varangle \sim \vdash<0$ जn

nus

UূZய【。

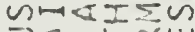

$0=0 z$

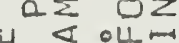

Zo@IZn

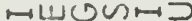

คロ OU 0

DLic $\sum u \alpha$

प0⿱㇒⿻二丿亅八

๙ง

$2 m 1-0$

जu< $<<20$

$0 \leq a<U$

n

$\propto$ IUIU $<\varangle$

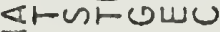
$\Sigma$

u

$\propto$ $\rightarrow$ แग nu

世< 0 -

$\alpha \quad \alpha z z$
0000000200050

Hemtinormoren-1Nm tatatatatatishisin

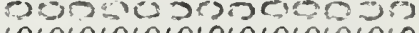
nunununusunesinas

トトトトトトトトトトトトト ZZZZZZZZZZZZZ

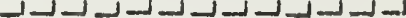

它

แ

$\overline{0}$

$4>$

$\alpha \alpha$

जर्ष

Zo

Un

$\leftarrow$

U

$z m$

난

U4

$\geq \omega$

$\sim \sum$

wam

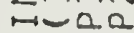

-

JuzZ

00 man

$0<-\downarrow$

車

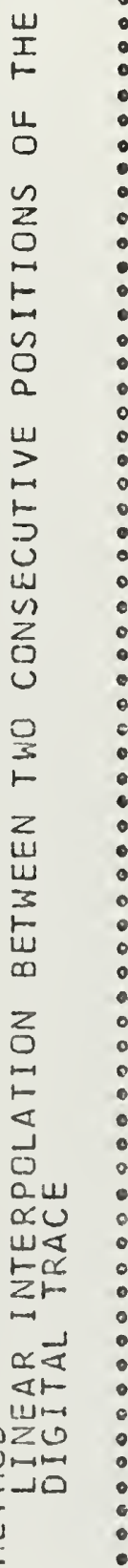

3
0
$z$
5
$z$
$0 u$
$4 u$
20
0

wa

טu

$<\omega$

$\propto$

คU

हit

$\vdash \sum$

$\cup \propto$

WH

0

Uu

$\Psi \propto$

Fr

uा

कu

$x \geq$

U⿺𠃊

ur

Dw

⿰u cs

oI

$\vdash$

ru

2

$x$ Nos

wó on

-

b vivovart a wus

כ2-n- z

Uढ口OQOन॥

o $=m M t N \|$ N

$\infty=I I\|\|-11-$

गwरद दssia

NUNNR-NEZ

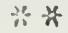

एक

$\omega<\omega$

$$
\text { * }
$$




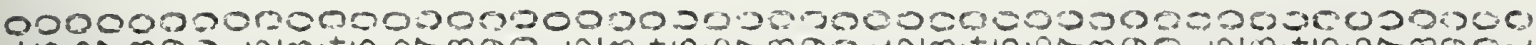

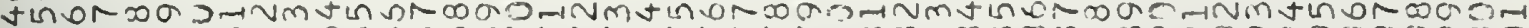

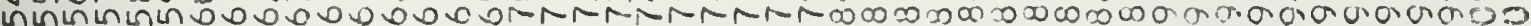

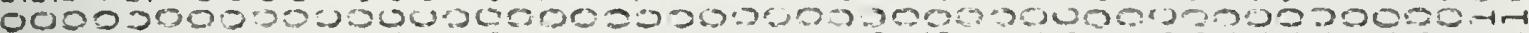

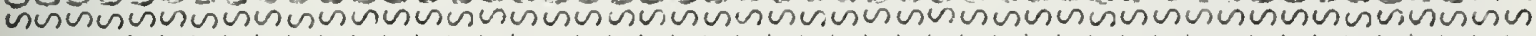

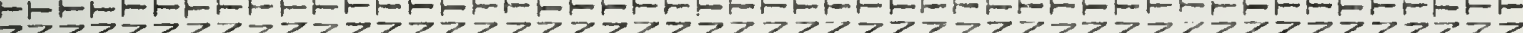

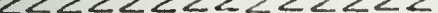

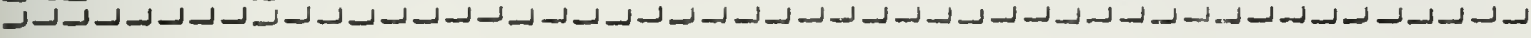

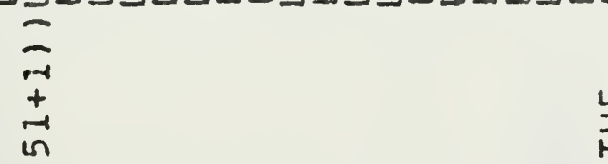

$\underset{F}{\Psi}$

5

$\begin{array}{llll}\pi & & m & 0 \\ \pi & 0 m & m & m \\ + & 0 m & \infty & \infty \\ i & \infty \infty & 0 & 0 \\ \leftarrow & 00 & r & r\end{array}$

㟔

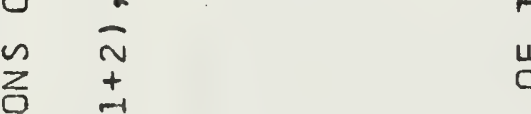

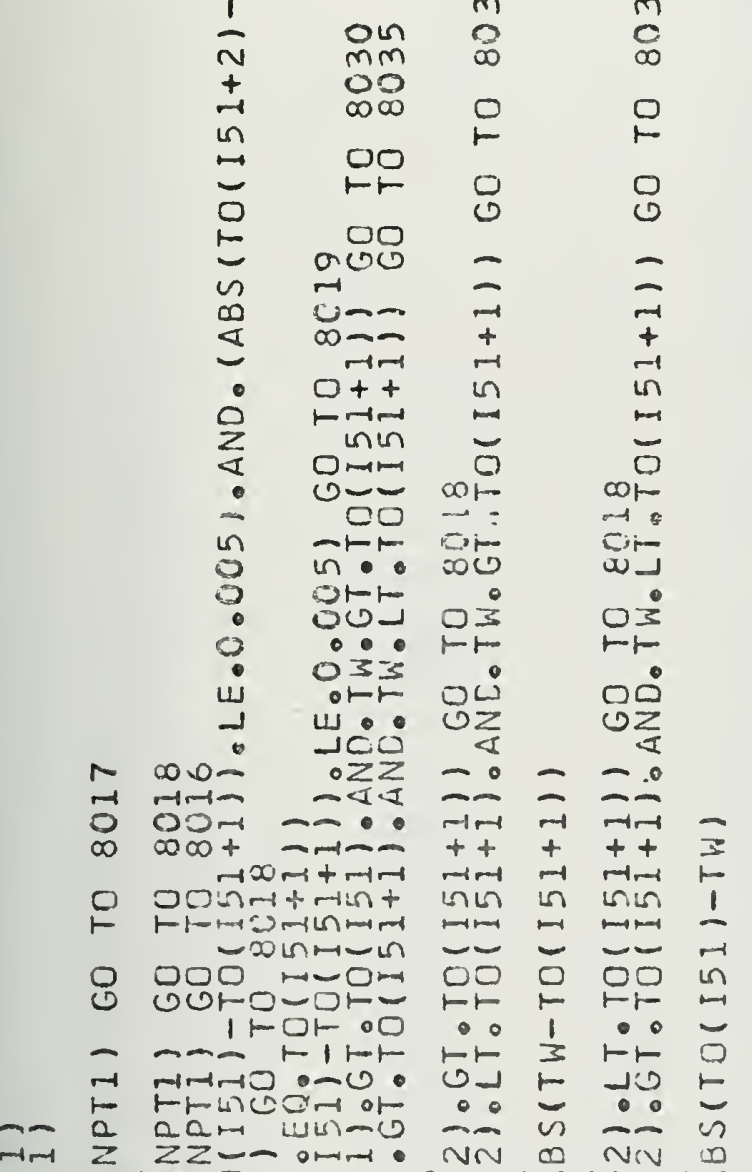

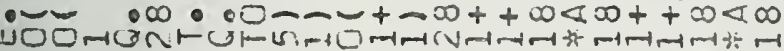

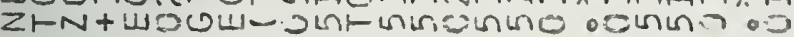
- \|\|$-1$ ०

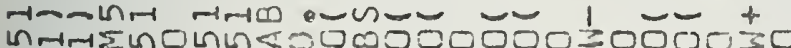

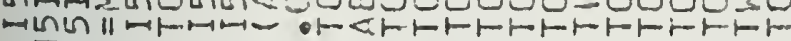
- 2 -

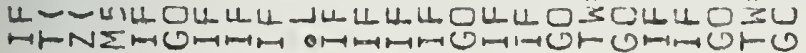
$\stackrel{0}{F}$

in

$\sim$

(

a

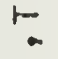

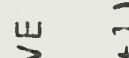

5

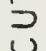

U

is

z

$\frac{0}{5}$

2

药

$+$

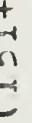

N

-

$+$

in-

$c$
$m$
$\infty$

$m$

tin

$0 \sum$

an

$-3$

iㅏ

步

$\lim _{n \rightarrow \infty}$

-in

ox

Non

$\rightarrow$ in

I $\quad$ in

$\vdash$

U山

OU -3

<. -it-

$z \mathscr{L}$

Ur

$\rightarrow$

0

zN

$\square$

$\infty$

○

$<\frac{1}{2}$

$x 1$

य小心

c:tal stur ง

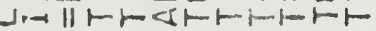
JUII

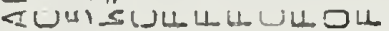

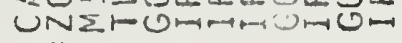
*

$\begin{array}{cccccc}v & * & 0 & N & n & 0 \\ v & & m & m & m & m \\ 0 & & 0 & 0 & 0 & 0\end{array}$

$\begin{array}{llll}\infty & 0 & 0 & N \\ 0 & 0 & m & m \\ 0 & 0 & \infty & \infty\end{array}$




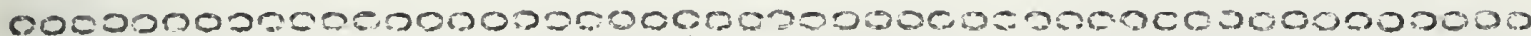

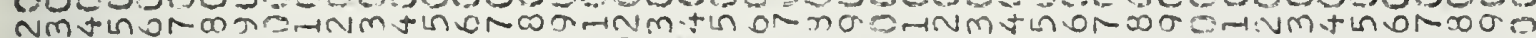

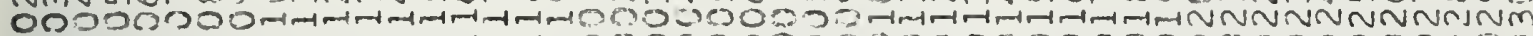

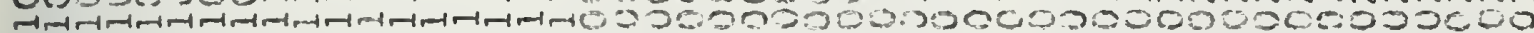

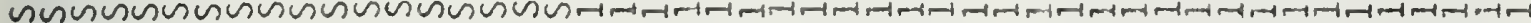

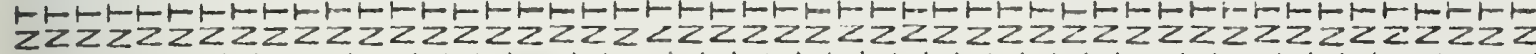

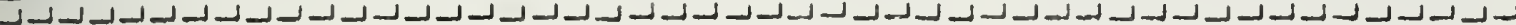

里

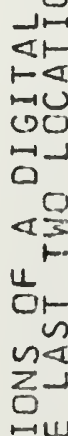

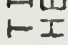

$\lim _{1 \rightarrow-1}$

○o

ar-

Zw

แu山

$>\infty$

$\rightarrow$

Uu

Cis

응

10

$z^{<}$

ய山上

wo

32

wn

$\infty$

IO

แI

$\sum 1$

$\vec{a}$

hr

$\because-1 \frac{1}{2}$

Uण 15

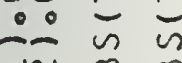

$\pi N \infty \infty \infty \infty$

$\infty \infty++\infty<\infty<\infty$

-1-1-1

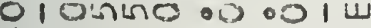

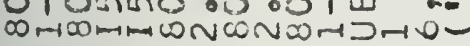
$m$ in $=1$ t in 2 in $-1-z$

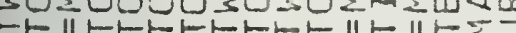

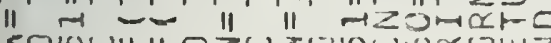

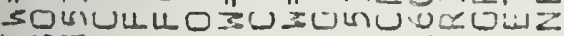

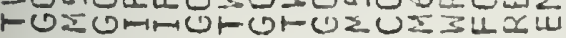
tra $\omega$ $z$.w W Wur

8 जoru $\frac{\alpha}{\alpha} \quad 01-0$

$\supset \stackrel{a}{\square}$

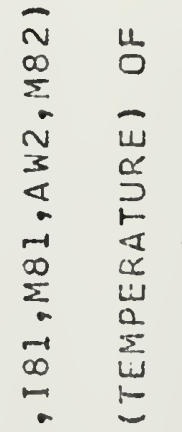

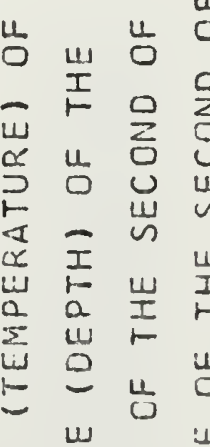

suा-

जiट⿺

nut

mintis

Uito

suru

रु

यूu. : :

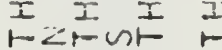

in $\geq$

৩>0ல0

$u$

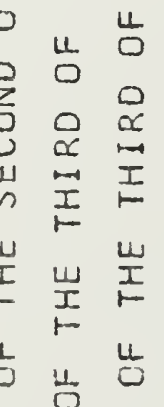

w

w

$\stackrel{\sim}{\perp}$

स다

u

-U⿺𠃊丶万

z

는

I

I

Fin

40

ठ빠

LU 2

¿ $<4$

viuaz

जItL

mit or

u du

口Z৫Z

<Uلt-r U) $1,-1$

山- 65

$1+2$

$$
\infty
$$

znzmZnznznznzzTz

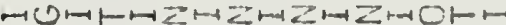
Z ZњZ

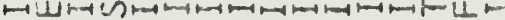
$\varangle U<0<1-<1-<1-<5<1-10-1$

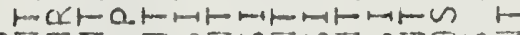

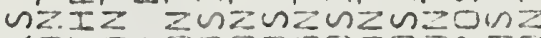

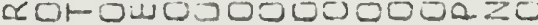
wU Unuavavavau Ju - U $\frac{\alpha}{1}$

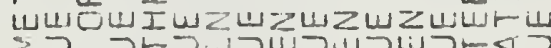

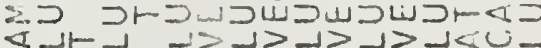

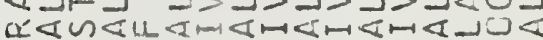
$\alpha>a>0>0>0>0>0>0-1>$ 0 a

une ¿I⁄ 世UI WणU トルトトトル;

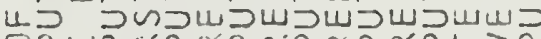

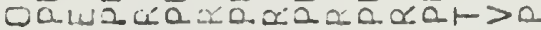
$Z I \geq 121<1 Z 1 Z 1<Z M Z$ $\sum_{0}$ । । । । । ।

$\leftarrow$

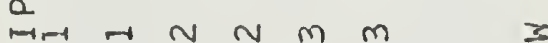
ज过 $\stackrel{\sim}{\sim}$ 
000000000500000200000000005200

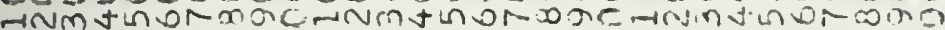

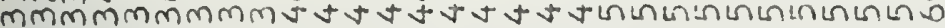
$000000003050030: 300000000000.000$

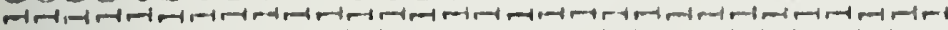

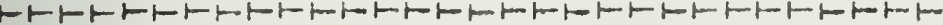
ZZZZZZZZZZZZZZZZZZZZZZZZZZZZZZZ

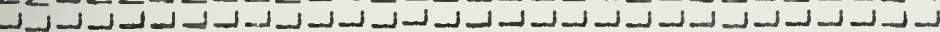

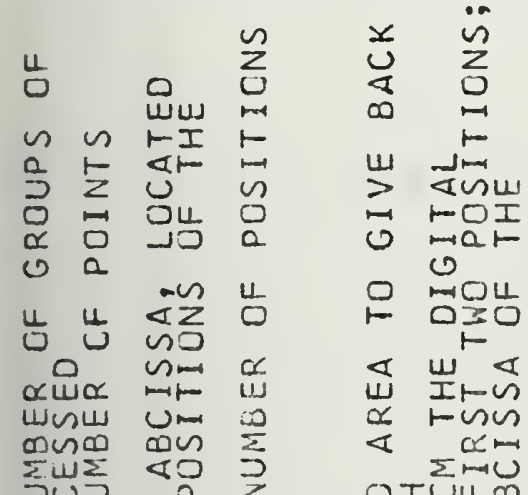

DIUU $\mathrm{m}$ Uino \& -ேயயய $\rightarrow 2+1$ $\infty 0 Z$ <UりZた Stmulu 《rLLI

$-\alpha m 3$

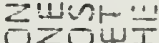

รUa OAF

$\sum_{0} \omega I U$

งแแน

- Ix山n FIU $4 F \omega$ $\sim<0$ जau

Ustumu ง ZงuZ

$\supset \longmapsto \supset \vdash 0$ -4 世上 ZسZOW $\because Z m Q \backsim$

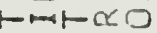
SrDUL ○つ॰

वKKZO

वरका

ग)

$$
\text { u } n+2
$$

$\sim \sim n$ जuा

エIIய

UFमr

$x \rightarrow 0$ -

《ートート

$\sum$

$\sum$

$\propto$
0000000000000000

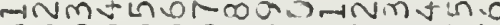
000000000N-NMNMN $000500=200000020$

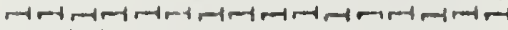

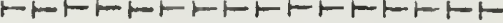
zZzZzZzZzZzZZzzz

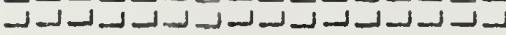

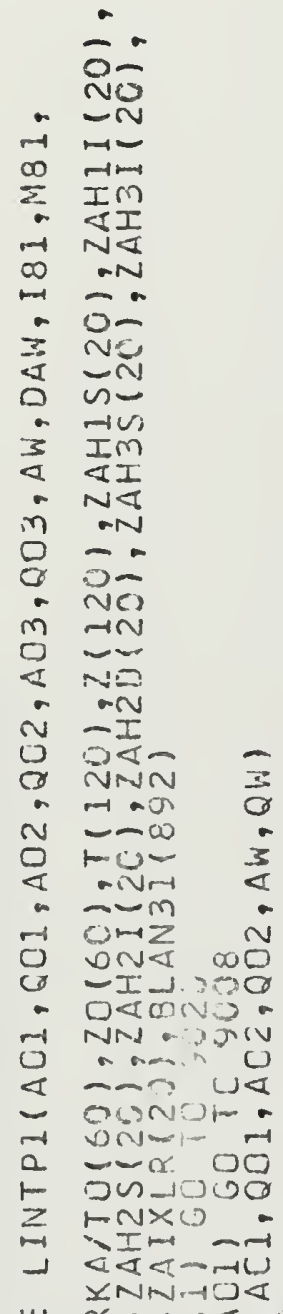

U1 $\alpha \cdots 0<-0$

$z$ U_-

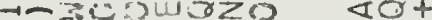
-NDNrV ald $30 \geq-r_{-1} \cdot-1-1-\infty<$

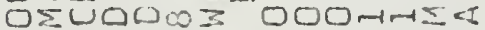

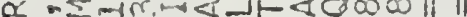
DrviII- $-111155-1 \mathrm{~N}$

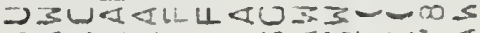

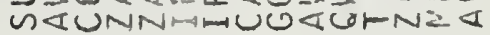
*ै

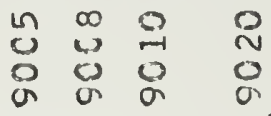


000000000000000000000009000000000702000002000000

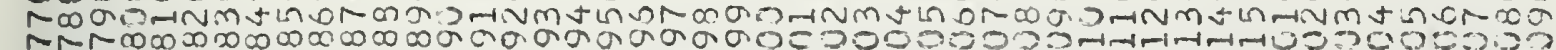

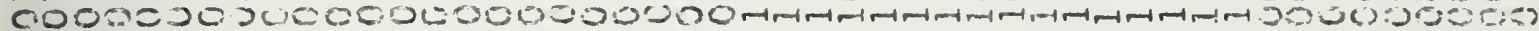
m-

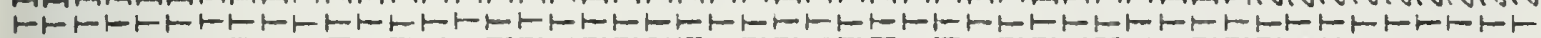
ZZZZZZZZZZZZZZZZZZZZZZZZZZZZZZZZZZZZZZZZZZZZZZZZZZZZZZZZZZZZZZZZZZZZZZZZZZZZZZZZZZZZZZZZZZZZZZZZZZZZZZZZZZZZZZ

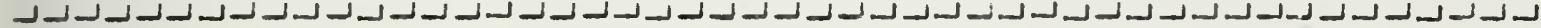

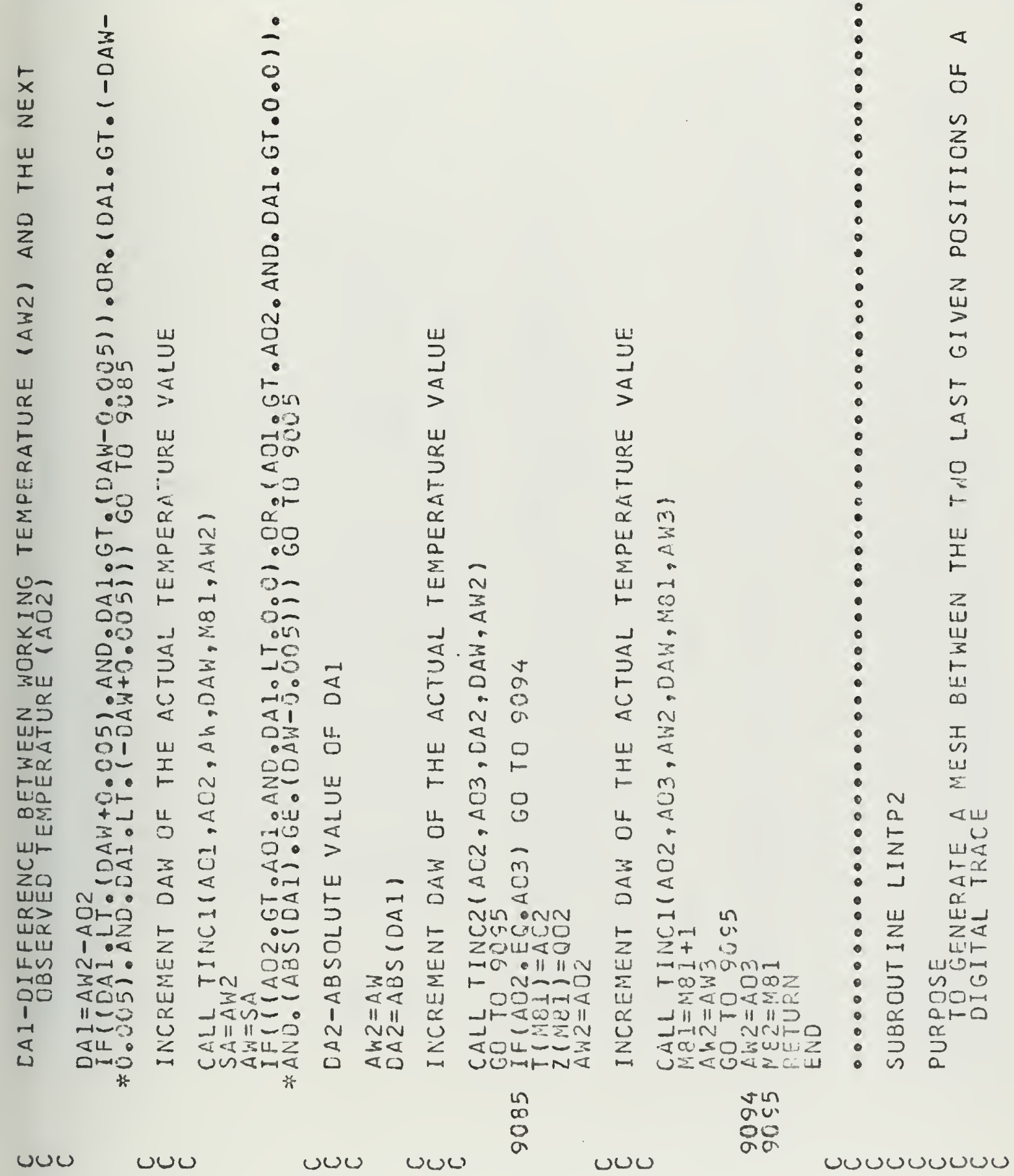


$00000000500=0000030000200000000000000000$

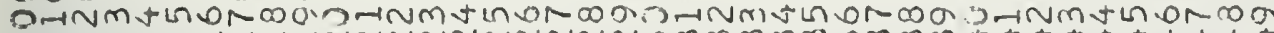

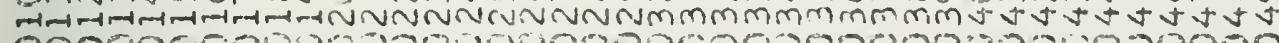

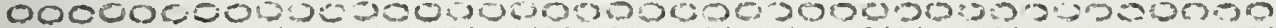

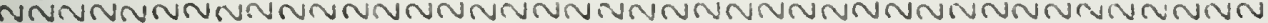

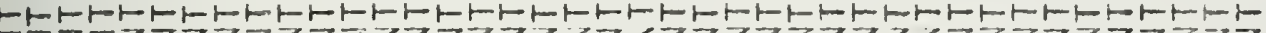
ZZZZZZZZZZZZZZZZZZZZ<ZZZZZZZZZZZZZZZZZZZ

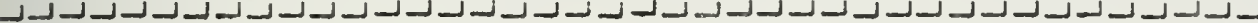

000000 Dringutin แกเกเกเกเกเก $000=20$ Nivivin -トトトトト $z Z Z Z Z Z$ \lrcorner$\lrcorner\lrcorner-\lrcorner-$

\begin{tabular}{|c|c|c|c|c|}
\hline$\frac{1}{0}$ & & & $\underset{I}{\Psi}$ & $\sum_{0}^{n}$ \\
\hline & 0 & 0 & 㟔 & $\bar{s}$ \\
\hline$v$ & $⺊$ & 5 & & $-1<$ \\
\hline & is & $\sim$ & $\vdash$ & $1-U$ \\
\hline & $\alpha$ & 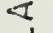 & $\sim$ & $=0$ \\
\hline & $u$ & & 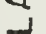 & $<J$ \\
\hline & & ш & & \\
\hline & 岌 & $I$ & I & Wu \\
\hline & 5 & & $\vdash$ & $F-$ \\
\hline 0 & u. & 0 & $u$ & 40 \\
\hline & & $1 \mathrm{~L}$ & & \\
\hline & $\varangle$ & $\vdash$ & $<$ & $\varangle$ \\
\hline$\frac{\delta}{z}$ & $\approx$ & $\frac{\pi}{z}$ & $\tilde{\sim}$ & ヘu \\
\hline & $\mapsto$ & $\leftrightarrow$ & $\mapsto$ & $\rightarrow F$ \\
\hline & $\stackrel{\omega}{\infty}$ & ? & u & $u_{n}$ \\
\hline & & 0 & $<$ & $\alpha$ व \\
\hline & & & & \\
\hline & $I$ & $I$ & $I$ & $I F$ \\
\hline
\end{tabular}

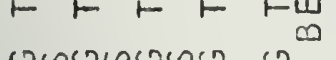

onousone

ZZZアZZZ z

morome $z=$ żozun

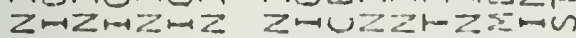

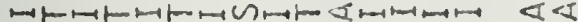

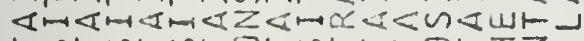

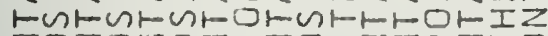

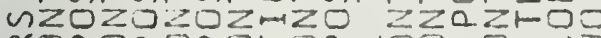

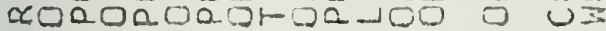

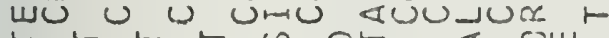
$r+r$ on at \& Ou

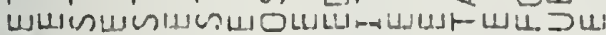

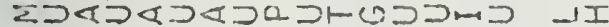

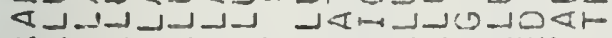

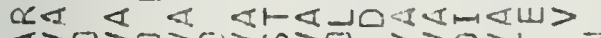
$\varepsilon>0>0>0>n>0>0>1-$

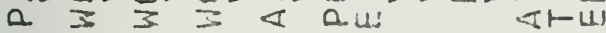

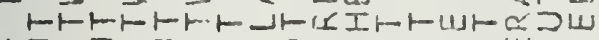

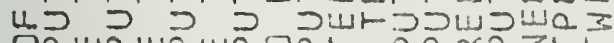

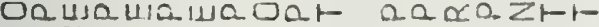
ZIZIZIZ $3 Z Z U Z Z I Z U D U$ ZڤF (3) 1 $-1$

$+a$ $a$ a $N$ N $N$

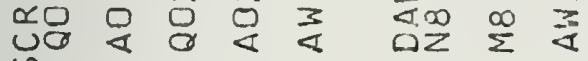
is w $\frac{x}{\infty}$

$\sum_{i=1}^{U}$

ir

$\square \quad \sum$

a $\frac{\alpha}{4}$

เu

\&

OI

uns

- 130

U) $\square$

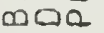

सU I

-

- $\alpha$ \&

zLI -

G.

Eu@

$01-4)$

उus -

- ILi<

$\alpha^{1-I-1}$

$\ln a$

$\operatorname{son} \alpha$

แi-Uய

n?n!

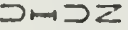

J

ZuZ0

$-2 m 2$

$-515$

ОDত̄u

Gín

$\infty \propto \infty<$

$D$ व $\supset \alpha$ जont

$$
\text { in }
$$

$\sim n$

III

ज展展

$x--0$

$\alpha \mathrm{NON}$

$8-1-\infty$

$\geq$

w

$\alpha$
4

资

F

$\vec{n}$

a

$\stackrel{0}{2}$

$-$

$\stackrel{5}{4}$

논

i

$\stackrel{a}{5}$

us

$\alpha$

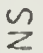

$2 \infty$

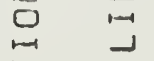

$\frac{1}{2}$

$\sum_{0}$

$\frac{2}{4}$

in $\sum_{\infty}$

$\sum_{\square} \infty$

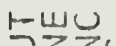

$\sum_{n=1} \sum_{n} \infty$

$\alpha-1+\infty$

品 $z$

湈

5

5

แ

2

5

1

4

0

a. U

(s)

$-\alpha$

Zr

$\rightarrow \vec{\alpha}$

<r

is

$0 \geq 0$

IमD

5

奠

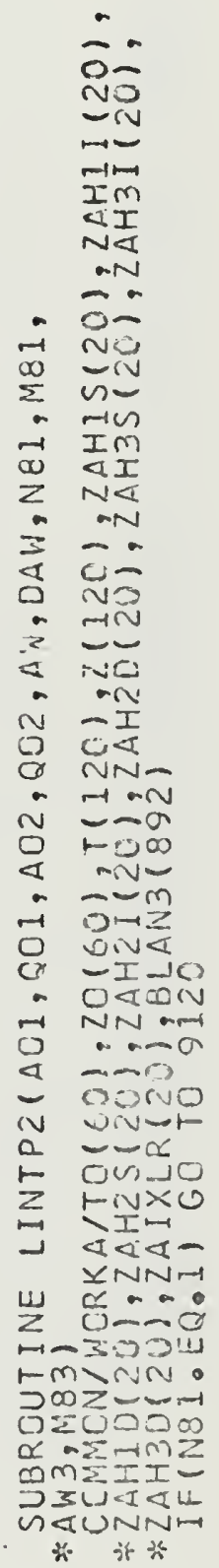


000020400300000000000020000000000005000000000000

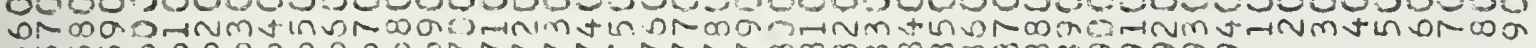

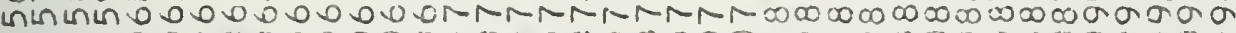

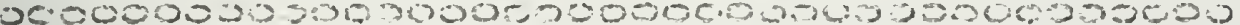

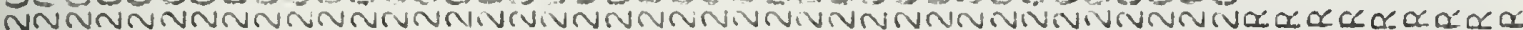

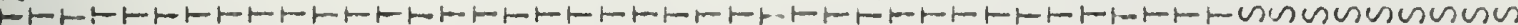

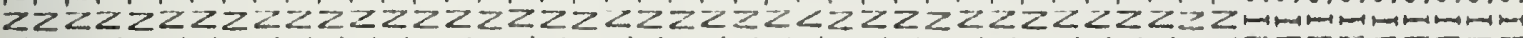

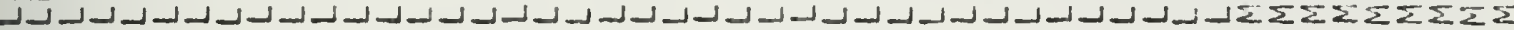

西

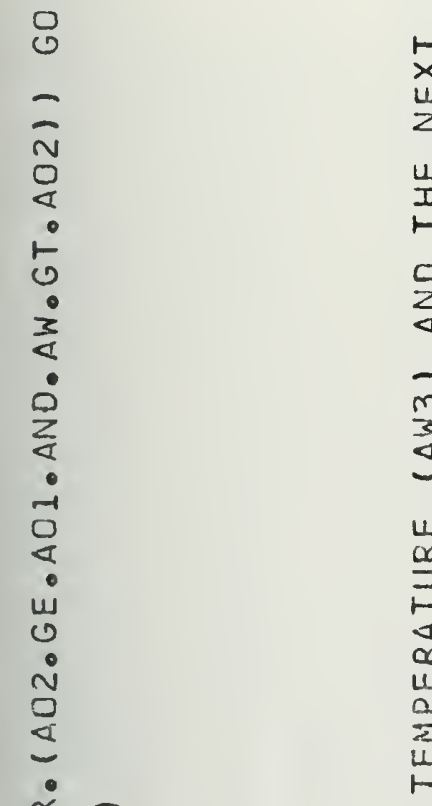

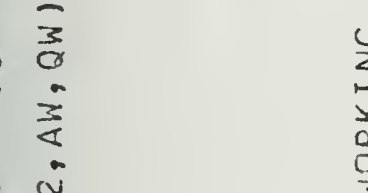

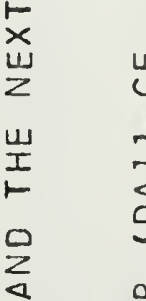

4
0
0
0
0
0
0

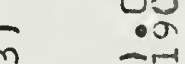

$3 \quad$ in

Gr 2

य 001

$\Rightarrow \quad 10>$

3

它二

$=\ln$

$-3$

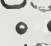

$-10$

or 0

ond o

जं: ल

\& $U$

-

$0<$

0

$-1$

$0<4$

๘ు

wer $z$ zN

$\sum_{m}$ स०ता

$\rightarrow 0$

II $110 .-1$

उद 000 मी

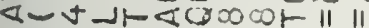

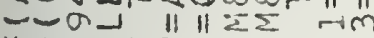

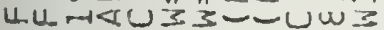

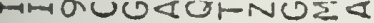

\begin{tabular}{|c|c|c|c|}
\hline น & $\infty$ & 0 & $\operatorname{con}$ \\
\hline 0 & 0 & 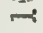 & NN \\
\hline-1 & -1 & -1 & rif \\
\hline$\sigma$ & a & 0 & ona \\
\hline
\end{tabular}

D-

$\mathrm{mt}$

ZN

$\because 0$

$x=$

su

W

0 - $\frac{\infty}{2} \pi-i$

$21 \geq \sum$ in

स० 5 ब

zD

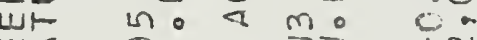

Wय $\quad$ का

मW OU I

wa OO

$\infty 2+0 \quad 02 \quad-m$

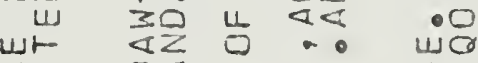

U $0<$

$20=0$

(1)

$\alpha>0$ -

wव $\square-10$

U. (i. I 0 !)

un mmo

$\rightarrow \infty 3$ In

$00 \leq<1$

1

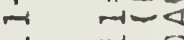

r-

Qu $1<$

பேー

谉
miN 0 .

00 ord

$\leq 400$

milum mo in Novin

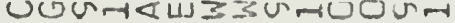

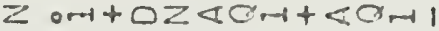

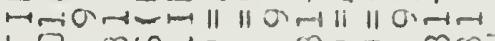
HO

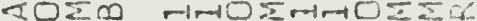

$\lambda-F\|<-110001-\| \infty 001-\|\| D$

J-

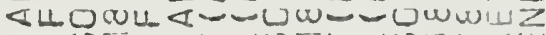

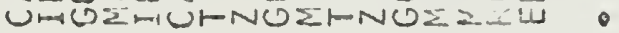
it

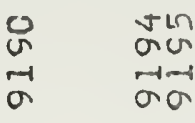

in 4

$\rightarrow \frac{\alpha}{4}$

00

トZu

$\alpha<\alpha$

$\supseteq$ UI

ur

알

¿ะखบ

功?

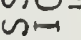

แus

ZLL

झ山之

wous

YUm

$z=$

Z⿱一兀

जsmull

Znu.

ono

"யu

$1-\infty$

$-6 z$

-140

$>x+1$

0 an

ที่

oro

주

IU⿺⿻一𠃋

ond

$\sum \omega$

षே山

ru

nu

usus

.

ตUC

$\alpha \quad z \quad \alpha$

↔ Uuज

$\sum n$

10

แ $ய<a$

$5-105$

गแ०

- maxz

$\supset$ जा $\alpha<$

0 0ujr

a auvu

$\infty \frac{0}{0}$

us 


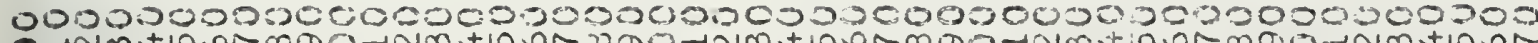

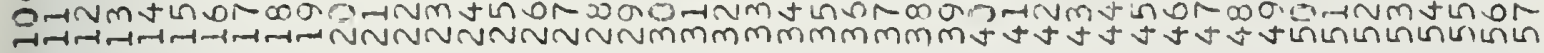

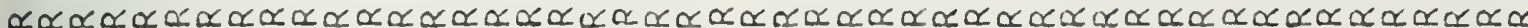

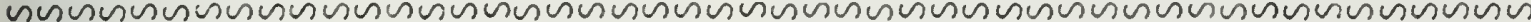

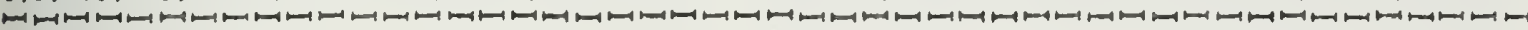

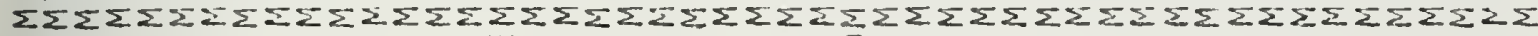
IU.

வUトே

$1-0$

4040

๑U๐ய

onuáa

Zming

मI

$\sim$ जu世

¿ZI造

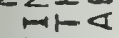

แ>

$I \varangle Z \backsim$

FIロU

nu D

ww $\bullet$

$\sim \supset n>$

UL JU

サட丨u

$\vdash>\mapsto 0$

$z \sim$

Uut

mo<

Ifa。

ari-i: 0

ш >z

ज wzm

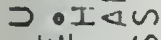

wr $n$

WO

IOOUE

FUZト

↔Zய

Uमயए

inग D.4

vo⿺

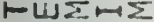

ZহQUU

mSUUI

oZ ur

a 20

ब๓णU

$\leftarrow 40 Z$

ธดแธน

- $a m Z$

nato

けZッ<

$<<x\lrcorner \alpha$

ตเบตแบ

$\sim \sum \propto I$

$\sim$ Uवा

H> $\propto$ OO

$\sum \infty<\cup \omega$

a

wद

ט

4

5<smiles>C1=C[C@@H]2C[C@@H]1O2</smiles>

20

viv

战

$\alpha \leq u$

$\sum \alpha$

डW

Zun

$<0$

$n>\frac{1}{4} \sum_{4} z$

ZZS Ur

$\because 40$ च

1 U.

-

つつつゅ

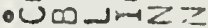

2 $4 \leq 10$

xug

致《つZZ

口I> QDO

Zz

แ IZUOU一

$N \leq<>$

네

$\because$ vII IIF⿵人

¿ u.

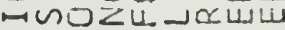

เய ш का ग

$\leq-1 \times-\mu<u$

$>\infty n$ Dn u

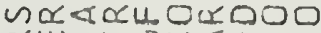

$\propto \amalg \mapsto r-0 \cup<U$

wn $x 1$ >a $x$

$-\infty \pi \Sigma \alpha<$ a

wO> OトZOト

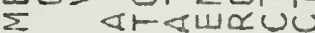

বUHトUல>0LL

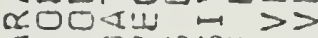

\& o>0லm

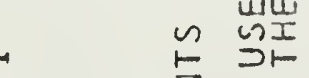

$Z$

때 ज>.

या Zय之

nu Jn $\rightarrow \omega$

जू जu

แง $\geq \sum u$

$1-\square$

a 0

$>0$

stmmo xun

шᄄ口

$x \supset<r$

$\because \geq$

U⿺⿻一⿰冫⿰亅⿱丿丶丶⿱⿰㇒一乂

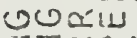

zZax

ルロல0ゅ

ZZUD

\&๐म

vo

Unt

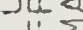

n.u

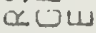

$1-\angle I \geq-m U I$

<ட山r $\mapsto<1$

$00 \sum \Sigma 0 \geq$

טงजu w wu

$=\sum \sum_{1} 0 \times 040$

TI $15=202$

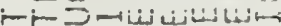

OOCIIUOSC:

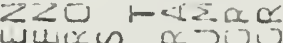

IUト

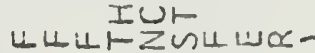

is

$\sim 50$

๙Zயய แ

แ๓ய山IUト

OUL $\frac{001}{2}-10$

งแบแแตแ

$\mapsto \perp \cap \simeq O_{-1}$

ar-we $\propto \circ$

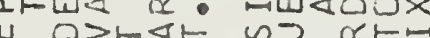

u $a>z$ in $\mu x<\alpha$

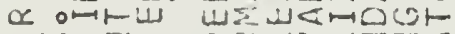

wx zumis $x \alpha>\propto z Z<$

-

$Z+I 0$ I

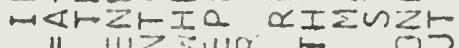

II Uz-山ू

$z$ r.w us tzo

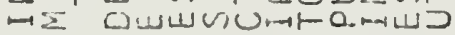

zon I- ImUz $-z \propto C O$

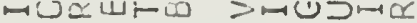

बUயय sक \&ZU

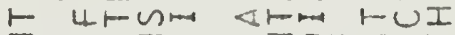

zس山 zuW ZOUZ

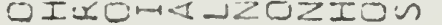

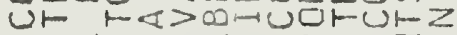

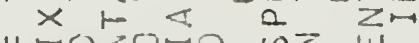

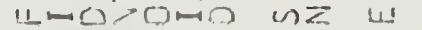

-

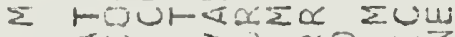

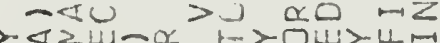

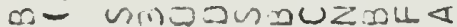
$\backsim \quad \backsim Z Z$ U

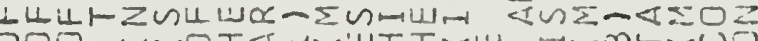
i FJ ZZFFZr

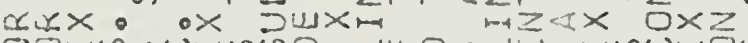

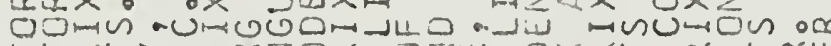
トト w口Z-1

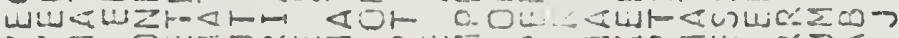

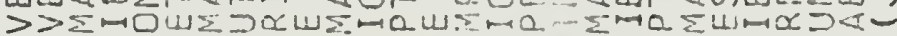
$U \Sigma 0.10$ n- Jathu a $\alpha \propto$ Z $\quad 2$ -

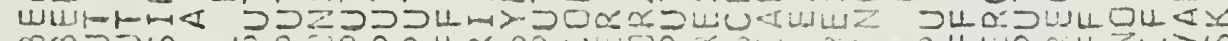

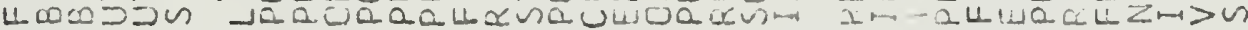

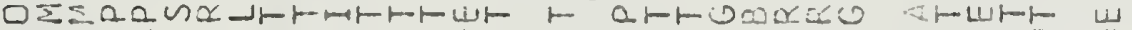

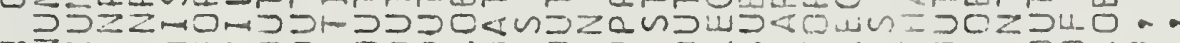

- 2 Z (
$\mapsto 1111$
$\begin{array}{lllll}1 & 1 & 1 & 1\end{array}$

$\vdash$

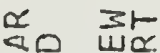

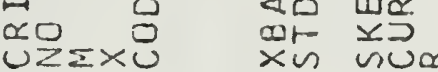

$2 \leq x \omega$

xu vua

U.

U.

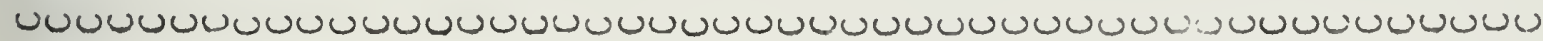


0000000000000009000000

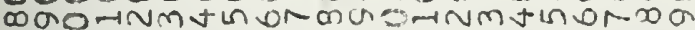

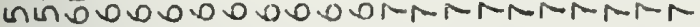

$\propto \alpha \propto \alpha \propto \alpha \propto \alpha \propto \alpha \propto \alpha \propto \alpha \propto \alpha \propto \alpha \propto \alpha \propto \alpha \alpha$ núnúnúnúnúnúnúnúñúños

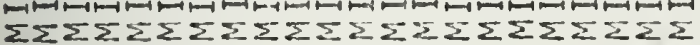
0

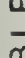
$\sum_{i}^{0+\infty}$ $\propto$

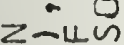

\& $\rightarrow 0-1$

$I=<$

I $F=n$

- $n y \supset x$

- vingle

- ш \&

$-a>4$

虫 $v>-\frac{1}{0}$

1 m-

$\mp$ वषा

usin $\frac{1}{8}$

$\propto \alpha$ o 0

$\alpha \rightarrow \ln$

$\sim$ murnes

oxus

0 in $4<$ \& $I$

$Z N>U G$

$<*$

xं n

- SF-

$\infty-1$ I I

$-0_{1-u}^{-4}$

बru ziul

$0+1 \backsim<$

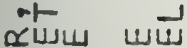

us oro

$4 \quad z-<$

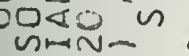

nu $1 \rightarrow 0$ in

U<र $-2 \geq 1$

$\supset>x+<x$

Ju if $\alpha$ iै

<ou. 5 .

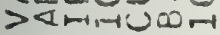

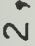

岂

0

$0<$

w1-

$N \leq$

$<0$

0

Zi

《u.

n-

x冋O

$\sum_{1} \leq \alpha$

$\Delta<N$

() 2

$\varangle<0$

o

IDn

जine

ज故

DuD

ivin

EU⿺𠃊⿻一土工

ta. U

nus $\alpha$

th $r$

ous

mW

长

ZzZ, Z

$\geq<-1$

\&.Jn

जo:

z⿺r

mU

, ज先

$\Rightarrow n$ ?

0 - $0<$

वแ⿴ $\alpha$

mo⿺

जou

แU⿺

งฺฺฺ

ㅍㅍ늘

佔F

$\sum_{\text {i. }}$

$\omega$

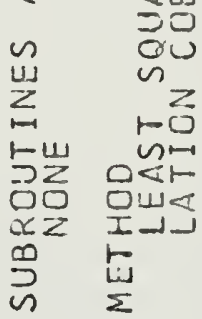

ก

$\alpha$ r

02

xu

$\leq U$

政

10

n.

<1

-

$\Sigma$
000000000000005000030000

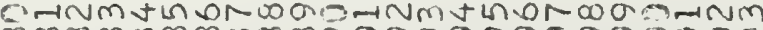

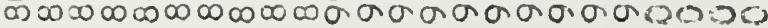
-1-1-1

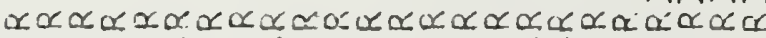

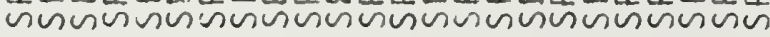

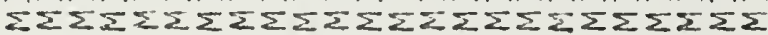

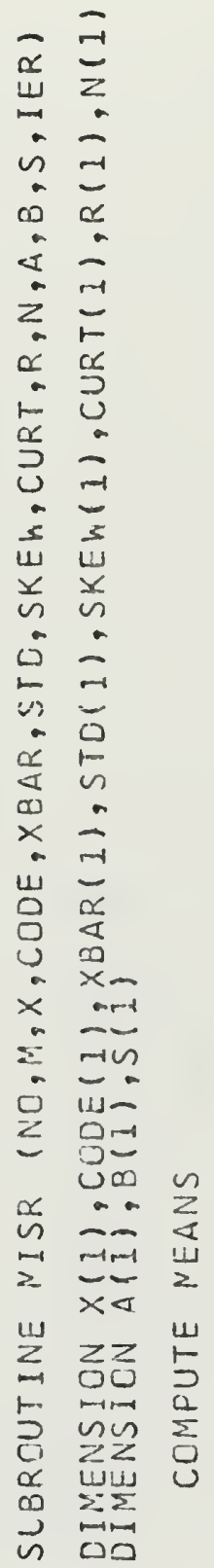

N

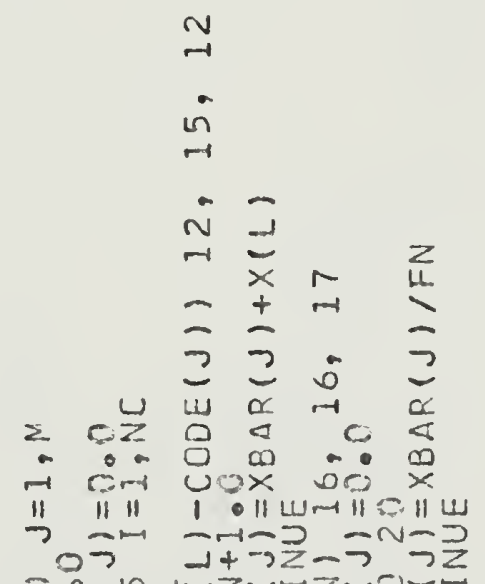

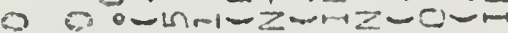
II $N=\alpha \sigma+x u \alpha \vdash u \alpha r-\alpha$

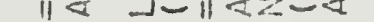

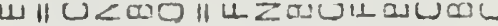
$\rightarrow$ d $0 u \times 0$.

$$
\rightarrow \text { in } \rightarrow \infty
$$




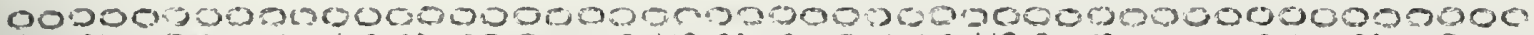
J

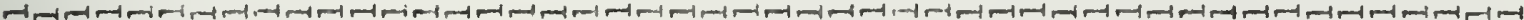

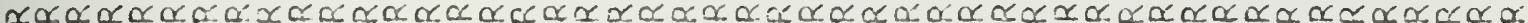

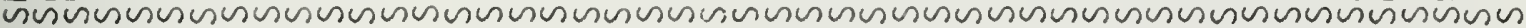

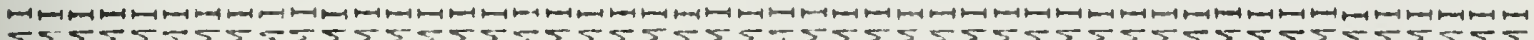

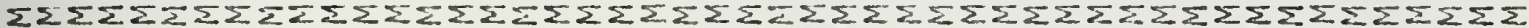





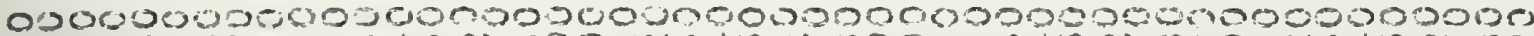

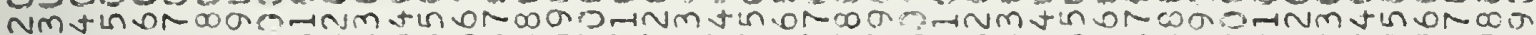

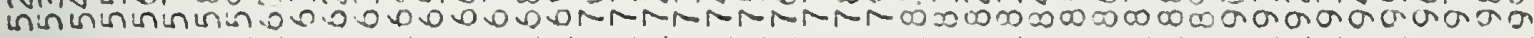

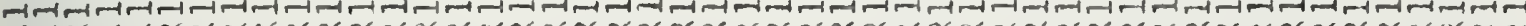

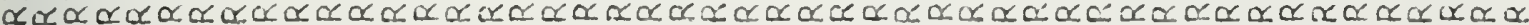

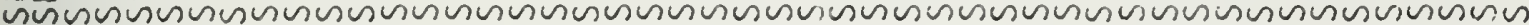

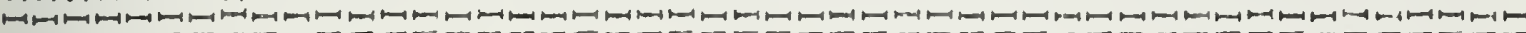

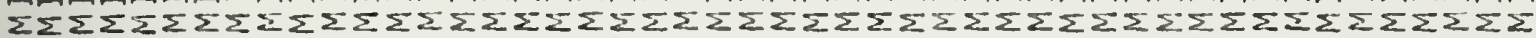

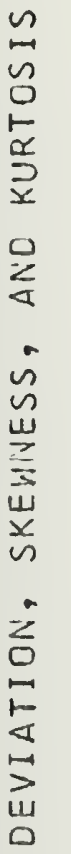

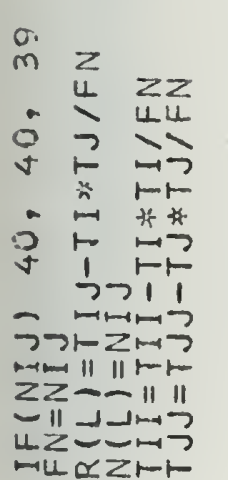

m

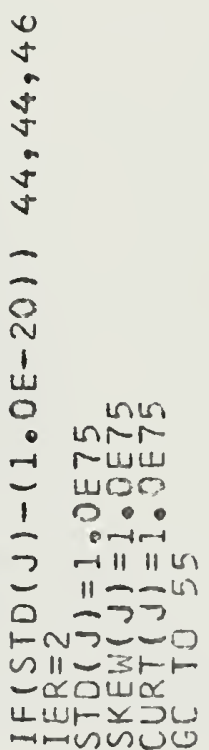

$\underset{2}{\mathrm{a}}$
풍

00

Im

1

는

ars

ज小

舟-

$\frac{x}{x} \bar{c}=$

00

$3=1$

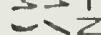

$>-1$

$-2$

zus

$4>2$

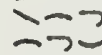

$>0$

ZニトF

$4 z a v$

>แग

-xur

zus $=x$

DII $\|$ un

$10-11$ in

u $\rightarrow 7$

11ーーつด

잔

$x \vec{u} \times \subset$

$0 \times D F u$

tr-

$m$

ㅇำ $\omega$

เ

$\infty$

5

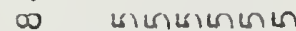
\& intrrar

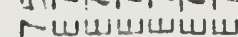
Wrjen:-0

N 0.0 .0

1 वान्नानानान $\rightarrow$ - 11 || || || || $\mid$ -11 - - Z แัน

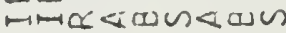

$2 \infty \pi$
$\ln a$ 

000000000000505000200000500000000000000000000006

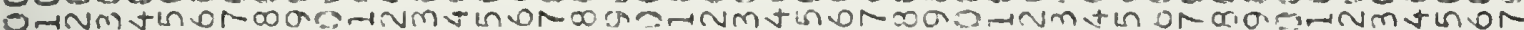

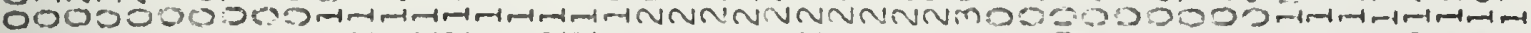

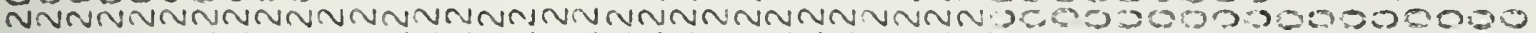

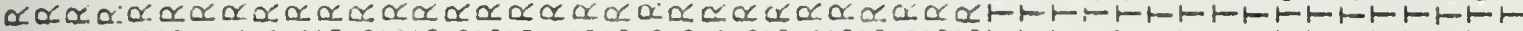

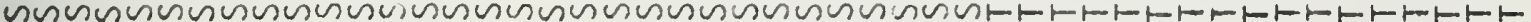

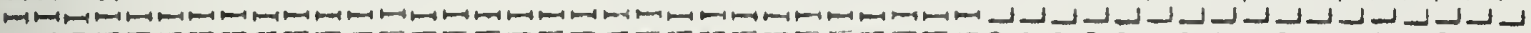

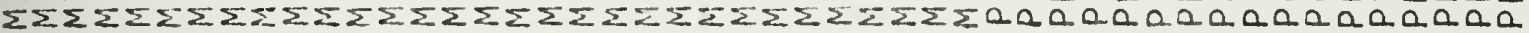

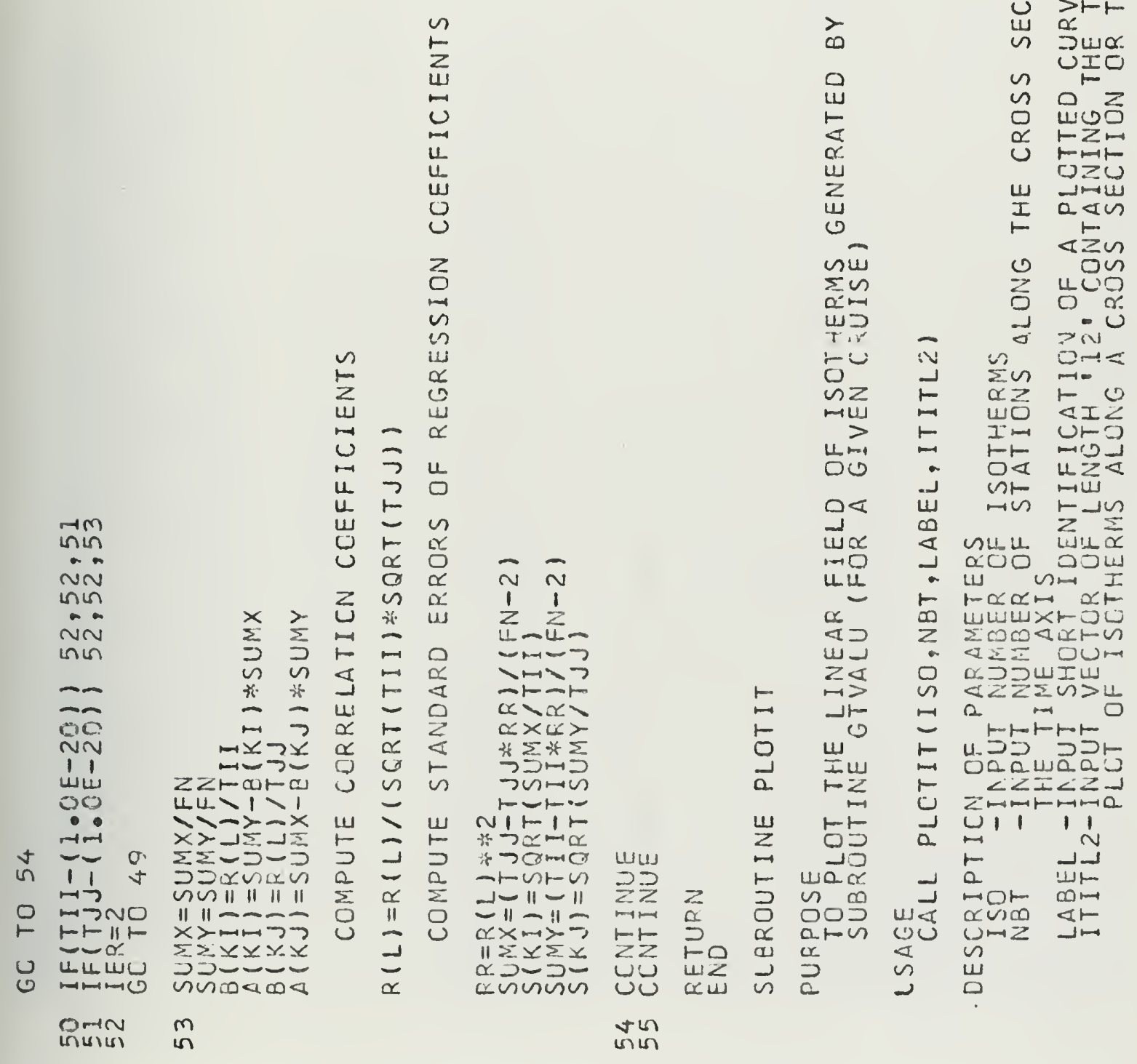


0000000.39300 .2300

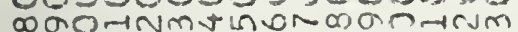

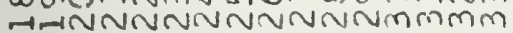
0050900030009000 トトトトトトトトトトトトトトトト トトトトトトトレトトートートトト

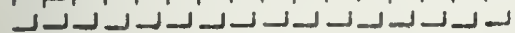
anamarararacar

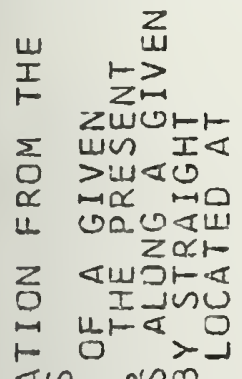

$4 \pi<$ :

$54<\omega 5$

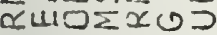

$0 \propto \curvearrowleft \propto \mu z=$

$1<1$ - LUIme

$z<I r-1\rangle$

$\rightarrow 0$ D.

แnumus-

0- ज以Zく

U. IIM ZZ

वயU $40 x$

जா<

गடயயय I

अ] $\rightarrow 0 \ldots$

แ $\alpha U \rightarrow>0$

$\simeq$ - $2-111=2 n$

ZuLU $3 \cdot-2$

İ $\Omega$ OUL

-

वU Imब

nuトแम

U. .0000

$>a$ a $n$ uाno

니단드

யОOトО Оト-

U Ua $-4 L$

wn wa IU

๔Zग० யम

ব] IImI

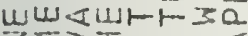

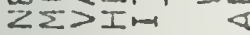

m $\mapsto-1-z v o r$

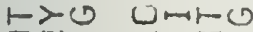

गय - $\approx u$

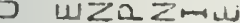

$\alpha \leq 20-000$

करामm Fa

Sortrut w

nODUZUแ>

OOUஈயIm

naxurutus

$\rightarrow 0 \infty \supset v$

I jusonum

जा zviserviu

$x-m-000 Z 0$

$x-<N x \supset \alpha \cdots$

a-s-unu.Ju

$\sum_{\text {U }}$

$\underset{x}{x}$

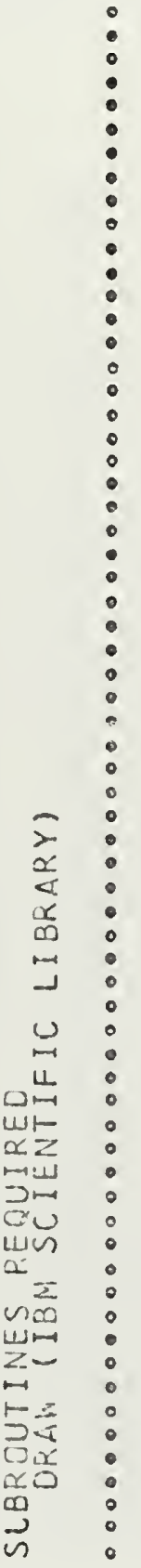

$0050=00025000900000020-300000000$

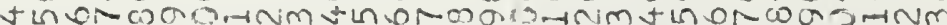

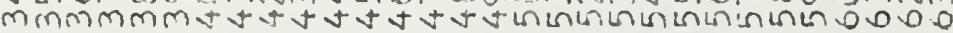

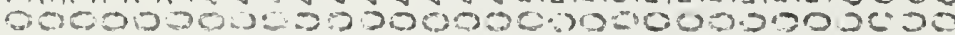

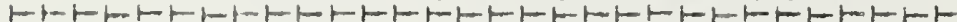

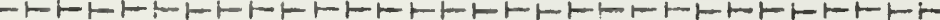

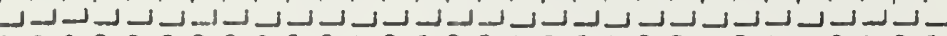

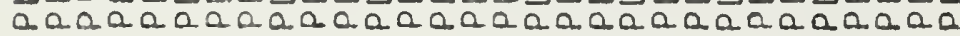

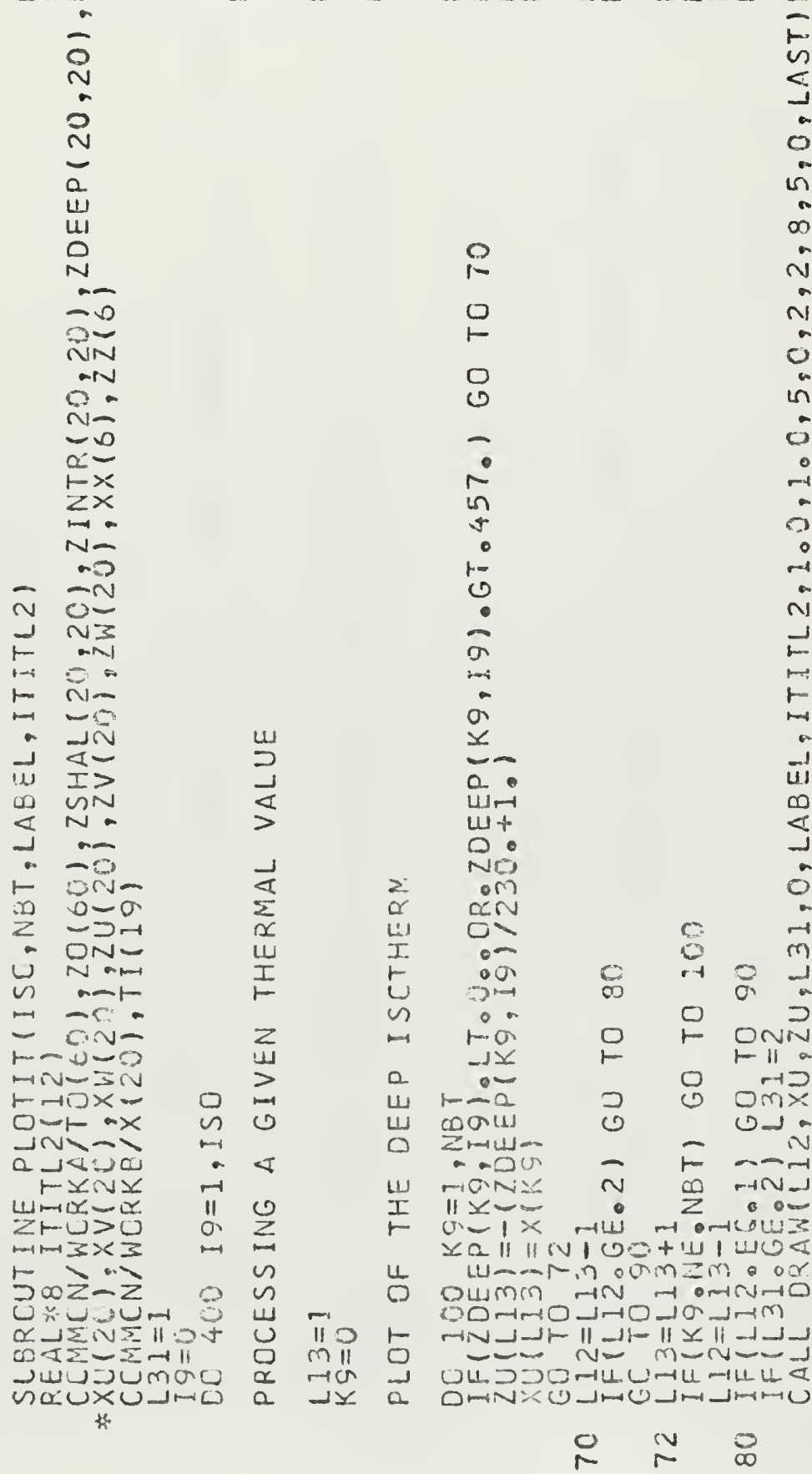


000060020070000000000052000000030000000000000000

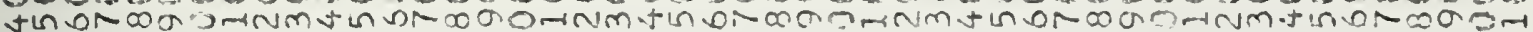

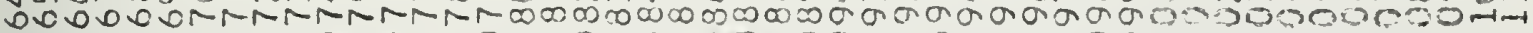

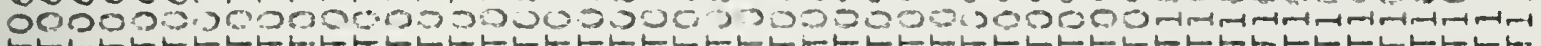

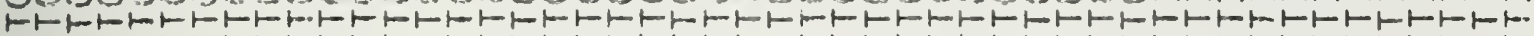

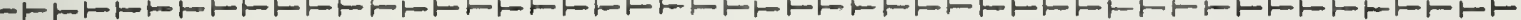

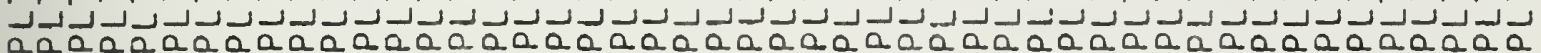

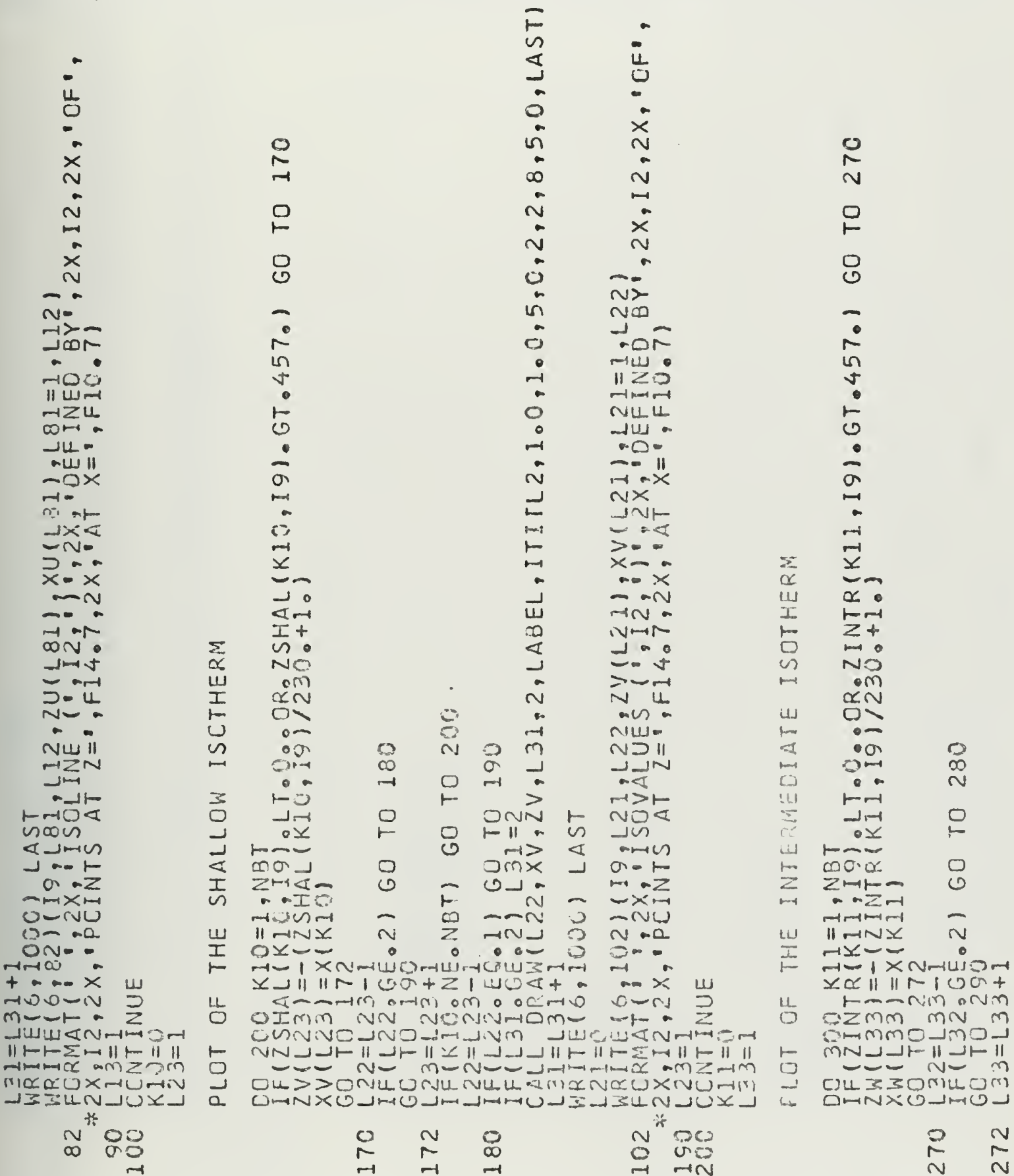

טu 
500000000000000000000000000000000000200000000000

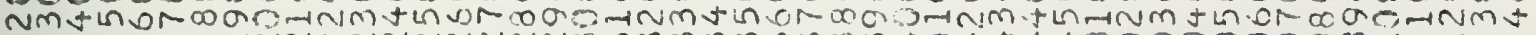

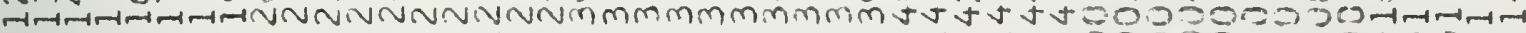

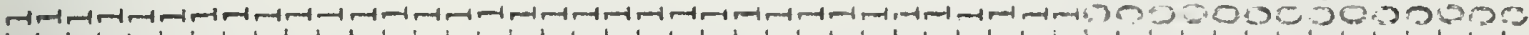

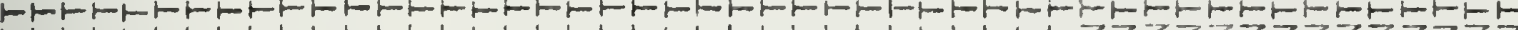

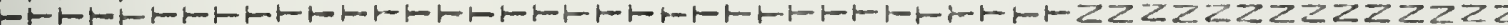

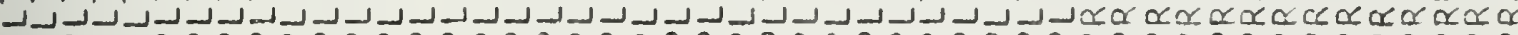

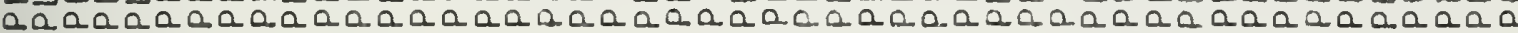

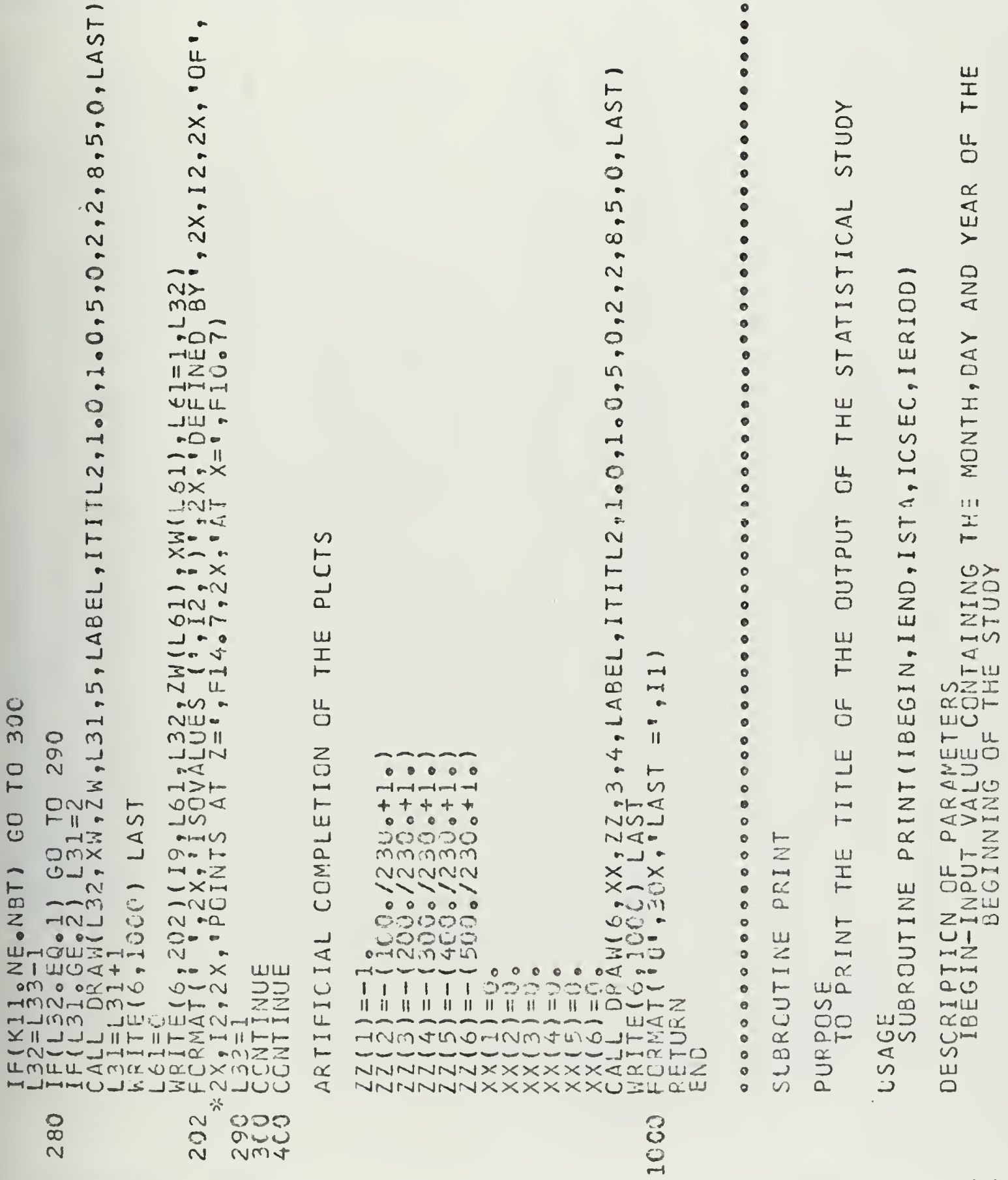


0000000500200 nut 0000.0000 .20000

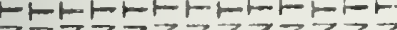

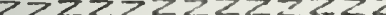
$\alpha \propto \alpha \propto \alpha \propto \alpha \propto \alpha, \alpha \propto \alpha \propto$ ananaanacana

年

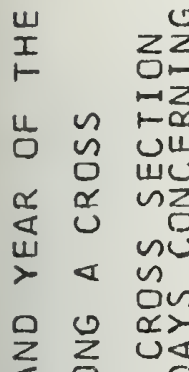

$z_{0} u_{0}$

$>$ \& IU

$<$

- z

I

$\rightleftarrows \vdash$

2

2

\section{2}

\section{r}

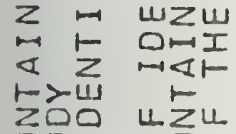

0

ine $U$

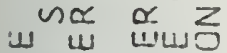

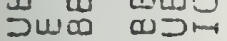

لI: $\Sigma$ एक

$>-\sum>2 \leq a$

${ }_{4}<z<\frac{1}{0}$

$50 \leftleftarrows-5-0$

a Dava

$z=<U<2 I$

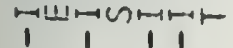

00

0

0

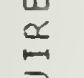

त)

$\omega$

a

u

2

2

in is जo

$-w$
$0 z$
00
$\frac{1}{\infty}$
$D$
$n$

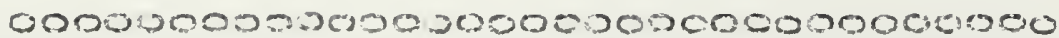

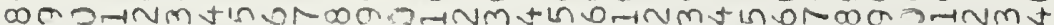

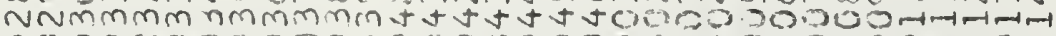

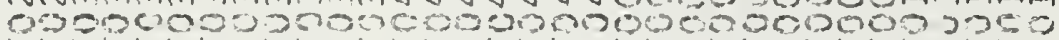

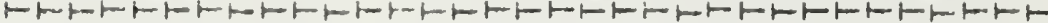
ZZLZZZZZZZZZZZZZZZZZ00000000000000000 $\alpha \alpha \alpha \alpha \alpha \alpha \alpha \alpha \alpha \alpha \sigma \alpha \propto \alpha \alpha \alpha \alpha \alpha \alpha+1-1-1-1-1-1-1-1-1$

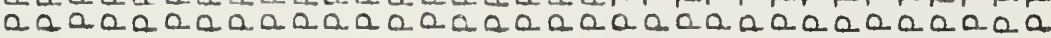

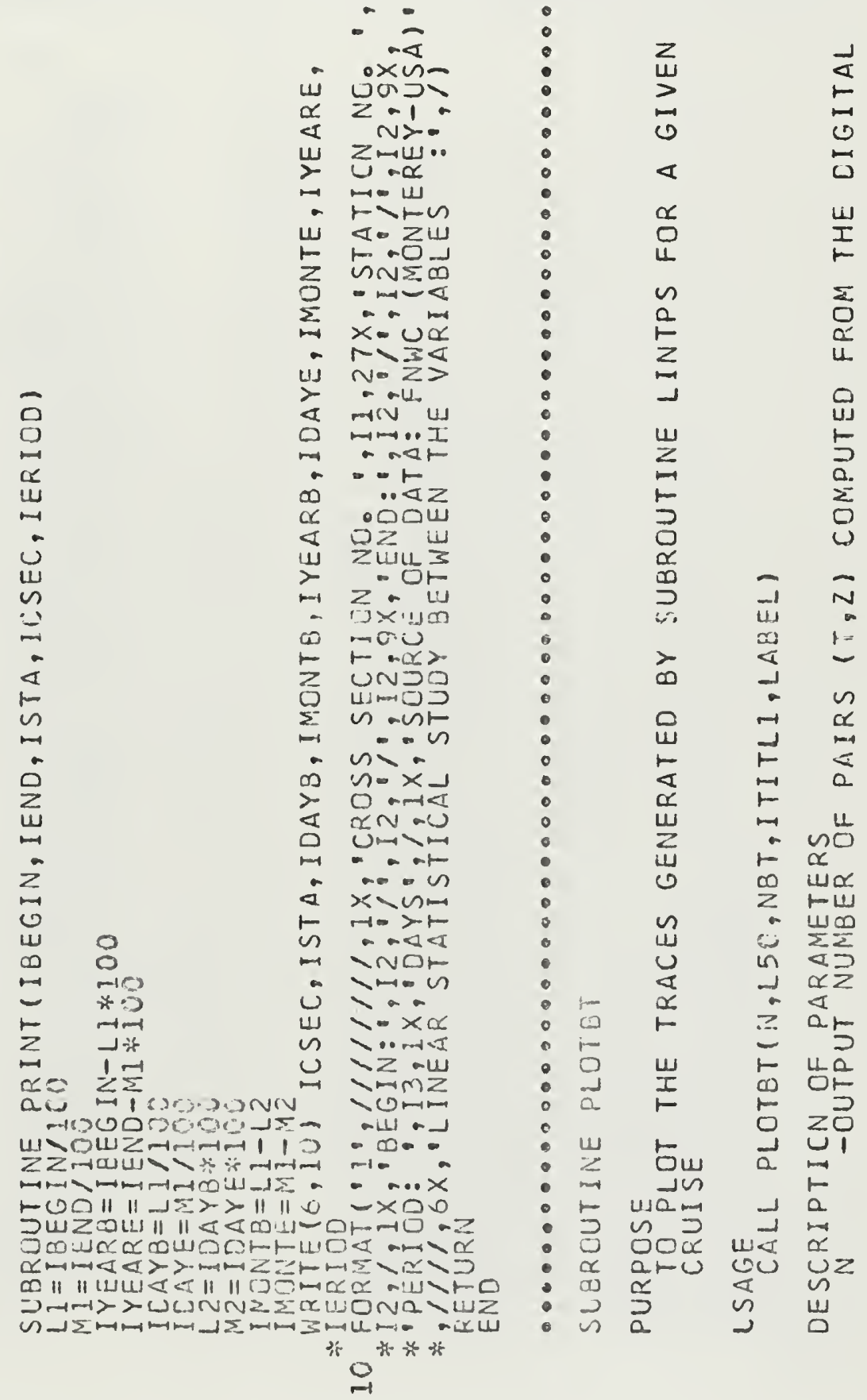


00000005003000030000000500

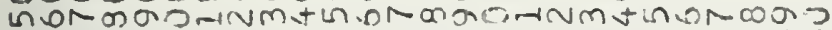

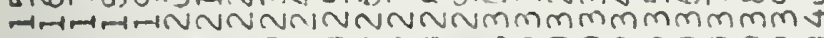

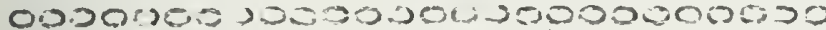

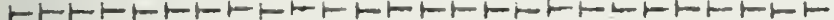
ம

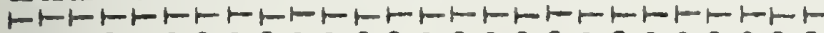

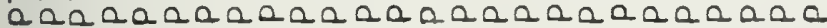

\begin{tabular}{|c|c|c|c|c|}
\hline & $\underset{n}{\underset{1}{Z}}$ & $\frac{\alpha}{0}$ & $\begin{array}{l}x w \\
0 \sum \\
U m\end{array}$ & \\
\hline$w \propto \frac{\vec{\alpha}}{4}$ & 0 & $z$ & $\omega^{5}$ & \\
\hline$I<Z$ & $\varangle$ & 0 & $\amalg \propto$ & \\
\hline$F w=$ & & $m$ & $=0$ & \\
\hline 200 & $\propto$ & $\vdash$ & $\mapsto$ & ש \\
\hline レーロッ & 0 & $\cup$ & $1-z$ & $>$ \\
\hline 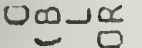 & u. & us & 0 & 이 \\
\hline$\because 0$ & & $\sim$ & $\omega m$ & $?$ \\
\hline 3o & 욤 & $\sim$ & $I 5$ & $\omega$ \\
\hline$F Z I$ & $\sim$ & $n$ & w & 0 \\
\hline $0<0$ - & $\sim$ & 0 & ens & $\omega$ \\
\hline - U & U & $\alpha$ & $\approx$ & $F$ \\
\hline யこし & $\cup$ & $U$ & mes & $\vdash$ \\
\hline Fumu & 0 & & $z \backsim$ & $\omega$ \\
\hline Zをく & $x$ & is & -10 & - \\
\hline$w u,-\omega$ & 1 & 포 & $<\alpha$ & 0 \\
\hline$\sum \alpha \infty-$ & & F & 10 & \\
\hline Wつ0Z & c2 & & $z$ & $<$ \\
\hline$\alpha u=$ & 山 & 0 & 04 & \\
\hline $\begin{array}{l}\text { U⿺辶冋 } \\
z<\alpha a\end{array}$ & $\dot{u}$ & $\frac{z}{0}$ & $\omega_{0}$ & $\frac{U}{0}$ \\
\hline$m \omega<$ & $\alpha$ & - & $-z$ & \\
\hline$\therefore \simeq$ & $\leftarrow$ & $\div$ & no & $\geq$ \\
\hline$\approx \quad n<$ & & & - & 0 \\
\hline गr $\propto u$ & - & $\sim$ & -4 & $m$ \\
\hline ○<मZ & $\varangle$ & $z$ & & 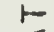 \\
\hline $2<$ & $\vdash$ & 0 & In & 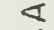 \\
\hline$\angle 002$ & -4 & $m$ & rLu & $\omega$ \\
\hline mz $\quad w$ & 0 & $r$ & U & $\mapsto$ \\
\hline ๒ゅLنய & - & $<$ & $z \bar{z}$ & $u_{-}$ \\
\hline$z Z>3$ & 0 & $\vdash$ & एय & ロ \\
\hline ozor & & $\sim$ & $山$ - & - \\
\hline Um & 4 & & & $z$ \\
\hline$\underset{w}{0<m}$ & 0 & u & $\frac{11}{0} \frac{1}{2}$ & u \\
\hline Фய Zu & $10 x$ & & & $\varpi$ \\
\hline - ID & $\omega$ & $\propto$ & $\alpha=$ & \\
\hline$<u \mid=n<$ & $\infty$ & Шu> & o & $\vdash$ \\
\hline$-c c \quad 1-a$ & & 0 & th $-m$ & $\sigma$ \\
\hline $2 u<1$ & $?$ & 5 & טo & Q \\
\hline $2+0 \frac{1}{0}$ & $z$ & $\geqslant$ & نس لن ل & $I$ \\
\hline aso & & & $>\frac{1}{0}$ & \\
\hline யUIवト & $<v$ & $x-1$ & $\vdash$ & $\infty$ \\
\hline पatam & & & & \\
\hline$\alpha \omega \omega z$ & $u$ & $<3$ & $\langle\langle\omega\rangle$ & $<2$ \\
\hline & & $n-1$ & $m a$ & In \\
\hline & 1 & 1 & 1 & 1 \\
\hline & & & 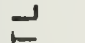 & $\frac{1}{4}$ \\
\hline & $C$ & - & $\mapsto$ & $\infty$ \\
\hline & เก & $\infty$ & $\vdash$ & $<$ \\
\hline & ل & $z$ & -4 & لـ \\
\hline
\end{tabular}

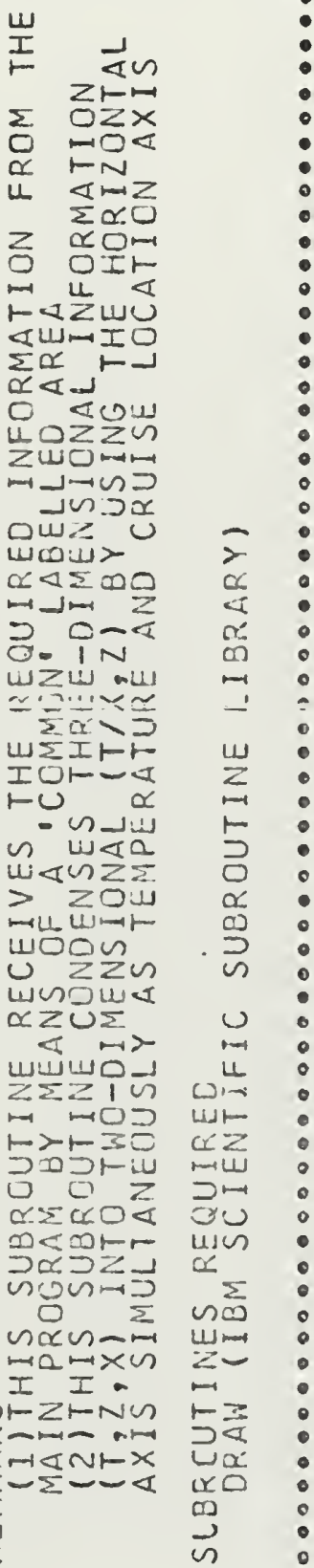

0000000000900000000,3 4nmtin on

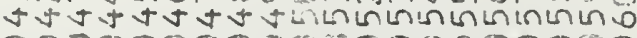

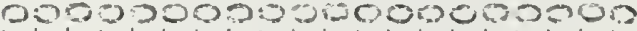

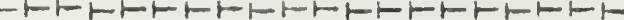

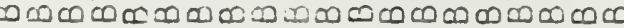
トートトトトトートトトトトトトトトトトトト

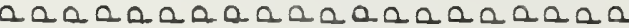

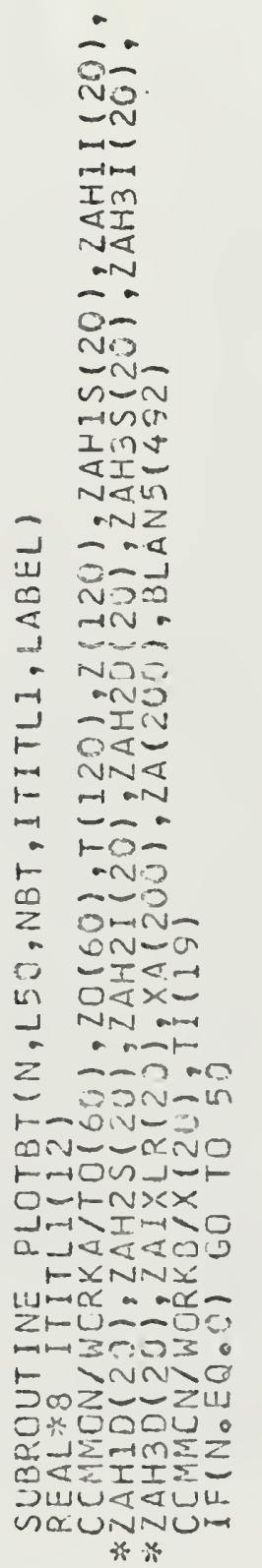

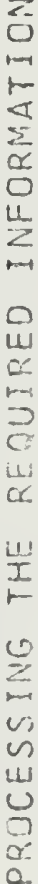

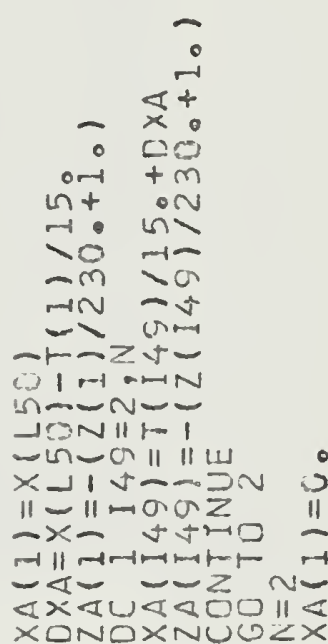

- 1 
00000000000000030000000000000200000000000,2000000

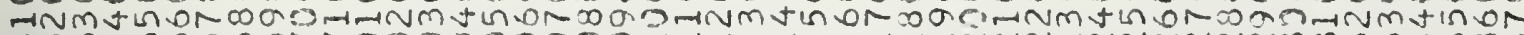

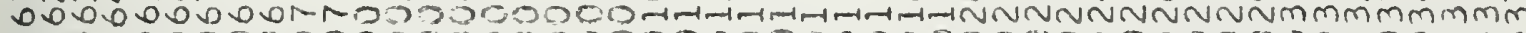

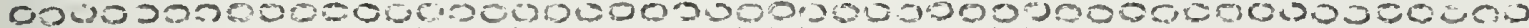

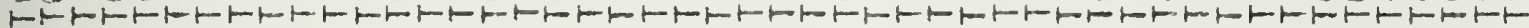

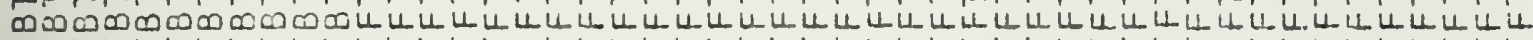

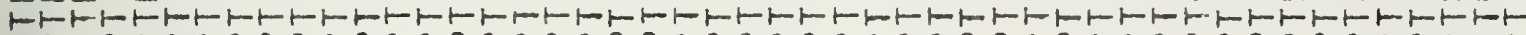

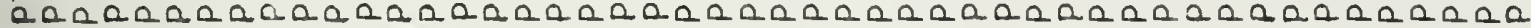

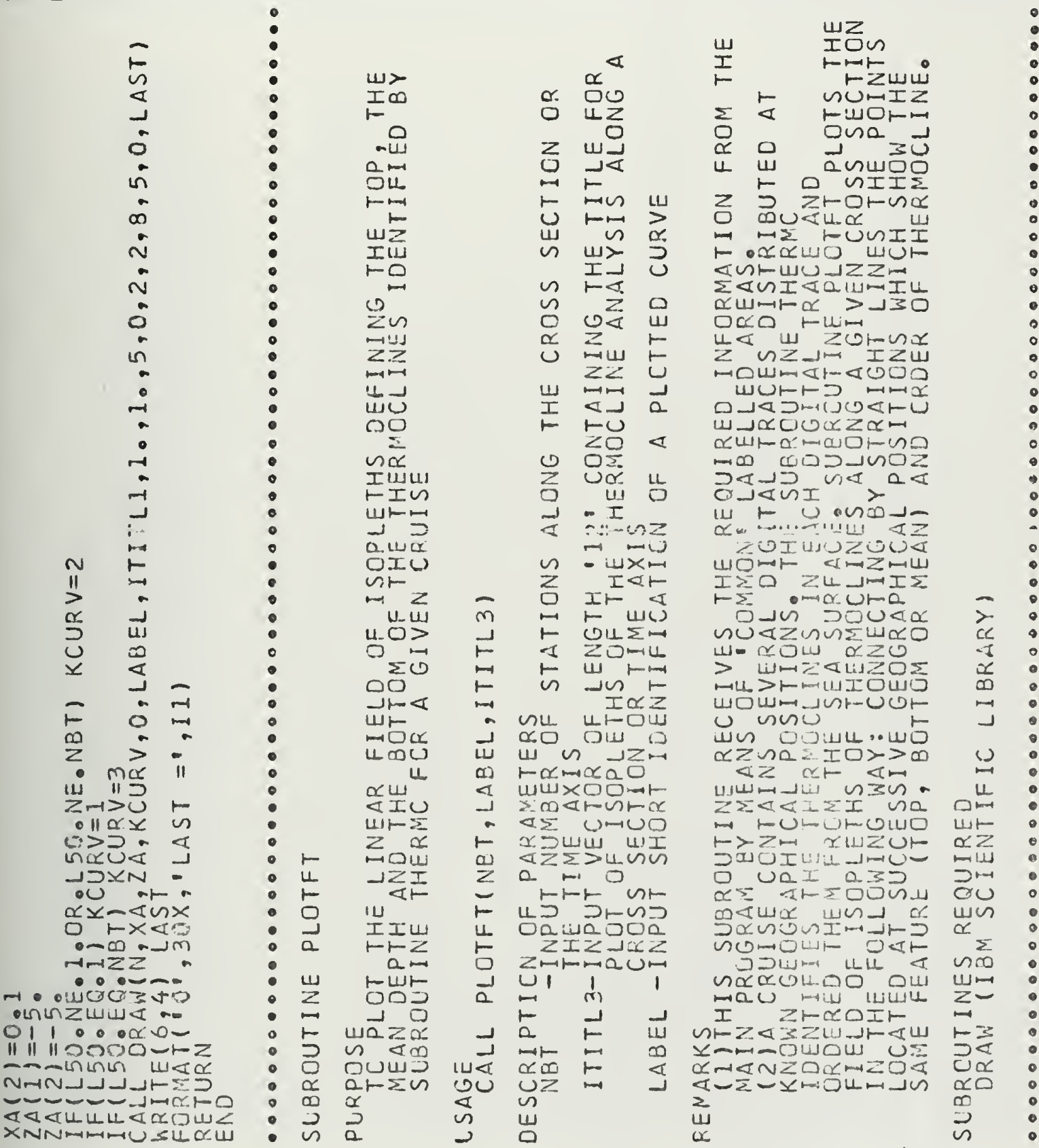

$\sim m s$ 
000002000000000000000000000300000000000003000

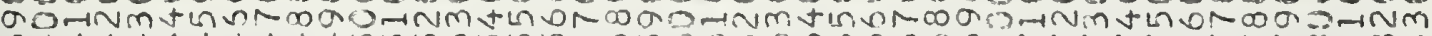

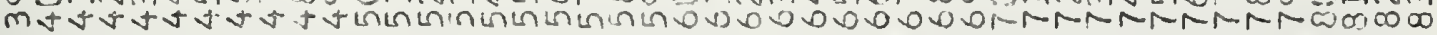

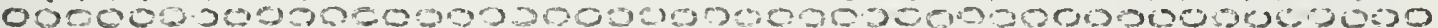

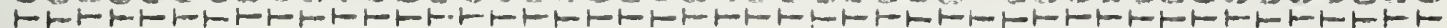

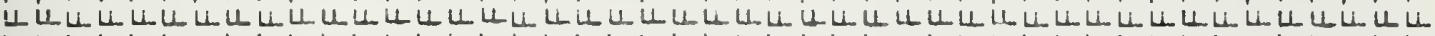

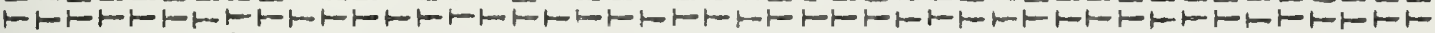

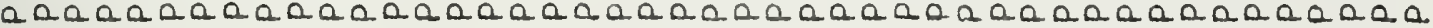

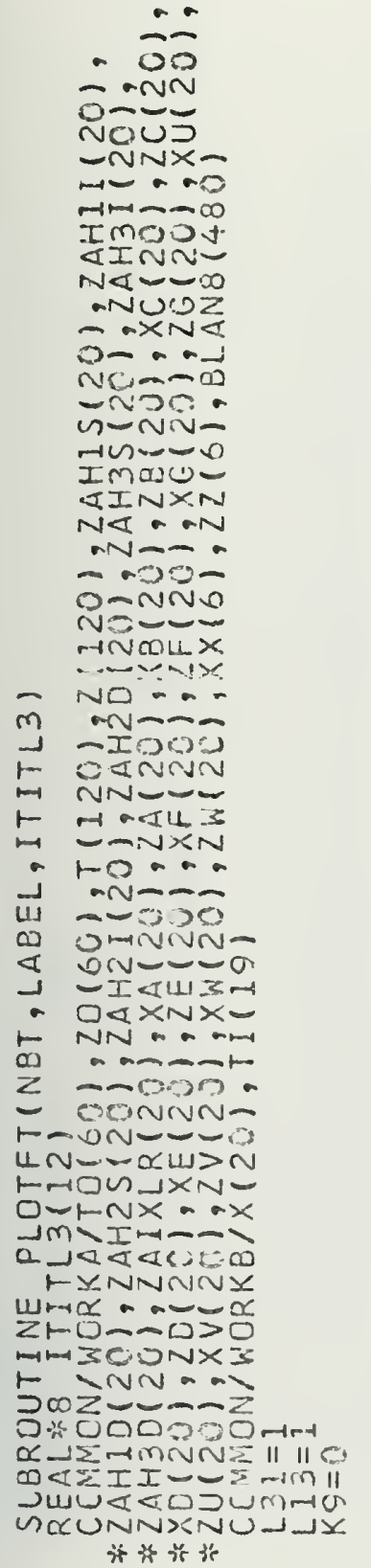

年

a.

岕比

U

0

- in

I

F

$\overbrace{}^{2}$

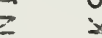

1

$\operatorname{lin}_{-10}$

It

I Nem

w $\alpha 0$

a

a 0

$0 x$

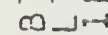

인 $\operatorname{mon} x$

$\| \geq \div$

$x$ z o ONa

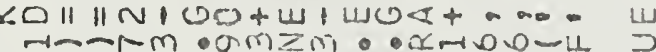

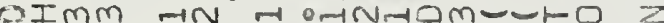

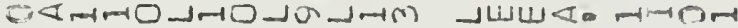

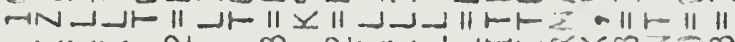

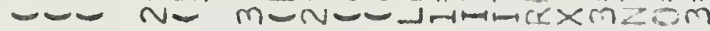

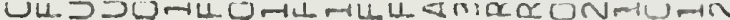

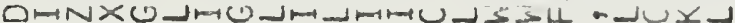

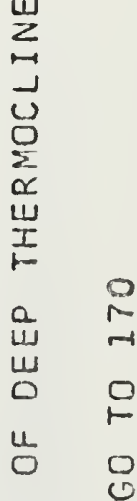

空

r.

号

$\infty$

$\therefore$

i

0

$\sum_{-1}^{2}$

$z$

u.

I

III

o

a :0

只 ग-

a

$0 \rightarrow 0$

$z \cdot m-$

I $-I 0$ जियन II $-1 N Y$ 2x-

$z$ - $z$ Yo\|l m-n DIMm

osnivo NNDJF $\because=$ UnN 
000000000000000002000200000000000009000000000000

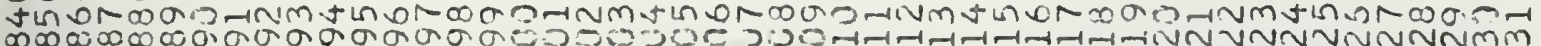

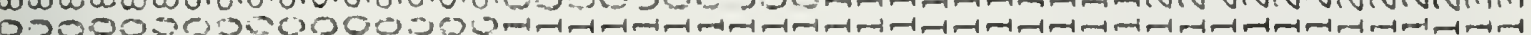

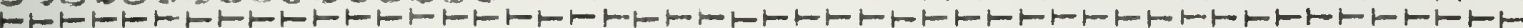

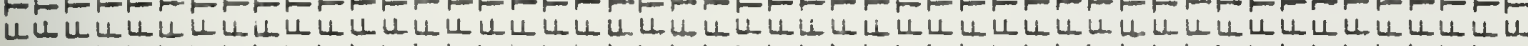

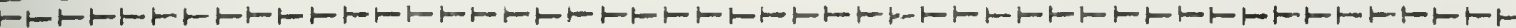

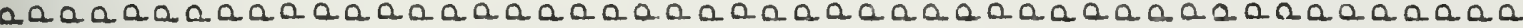

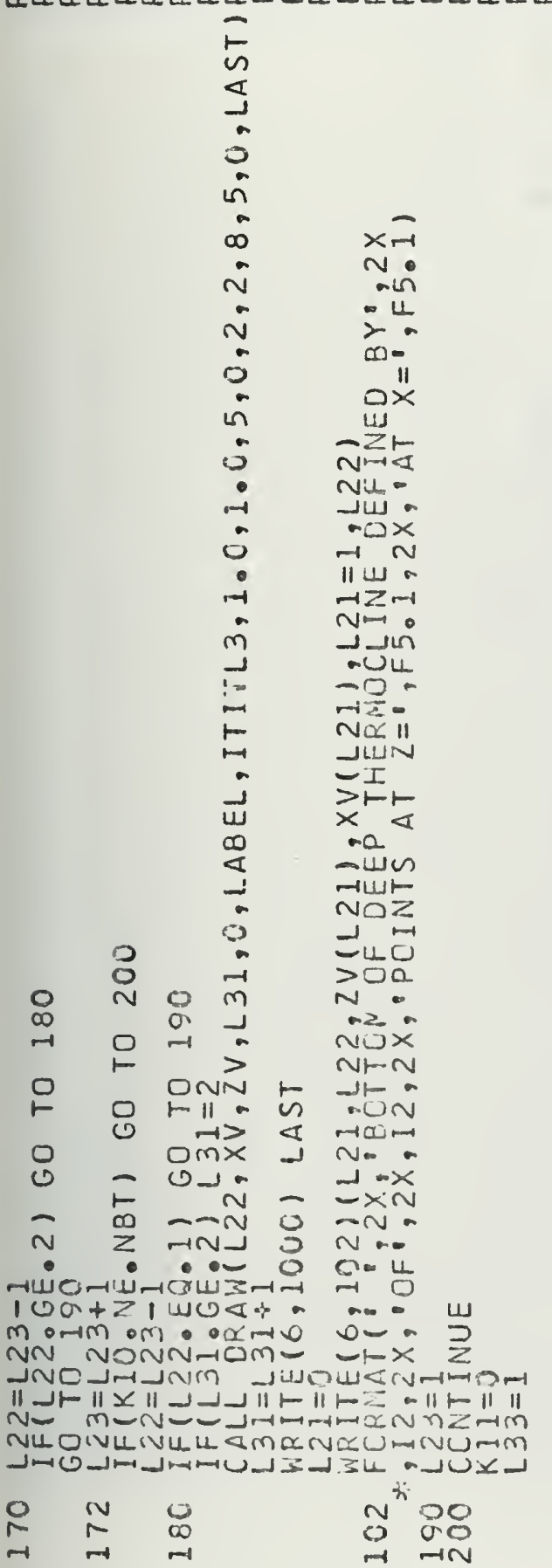

U

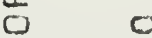

I N

a

19

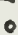

in

W

1

$\ddot{2}$

$z$

1 Na

U. It

I

w

a.

C.

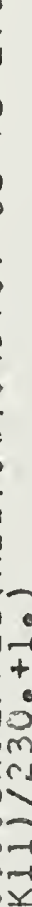

o $\quad 0 x$

m-

$\mathrm{Z} \cdot \mathrm{N}-$

$\frac{w}{1}$

w

-I

-1नखा

II- INY

2

is

I

$\infty$

in

$\infty$

i

N

n

กำ

in

के

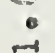

- $2-1$

28

II Uu

$\rightarrow 0$.

$0 \leq-$

गत्रा।

-UN

-I.

$\rightarrow$ it r

$0<$

-1a.

ปயu

उU⿻

xoz

$-m$

$-110$

-100

0

$= \pm x$

$30 \mathrm{~N}$

Nü

- ON

$v \rightarrow$

$m \geq i$

\lrcorner$<x$

aI:

-1:

o- -

$-\dot{x}$

$-x$

Nan

$\rightarrow \infty x$

जै

$\rightarrow$ u

$0-v ?$

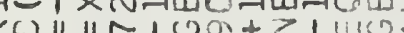
N-N onm on o ox, Ho

Tmm mN mmmNmon-

uर

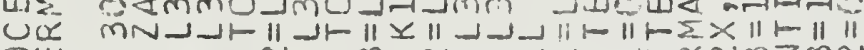

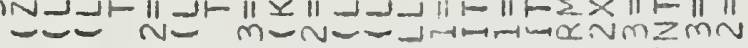

口. OH

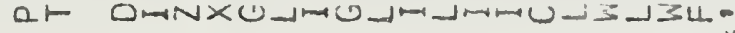

\begin{tabular}{|c|c|c|c|}
\hline 0 & $N$ & 0 & $N$ \\
\hline r & $\Gamma$ & $\infty$ & C. \\
\hline
\end{tabular}




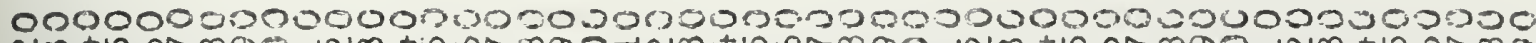

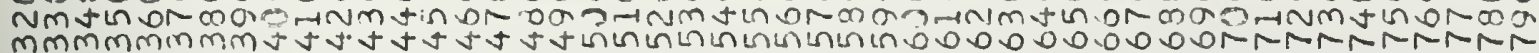

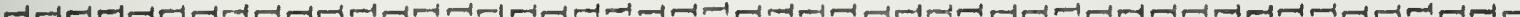

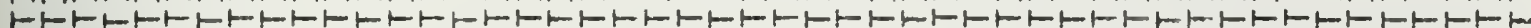

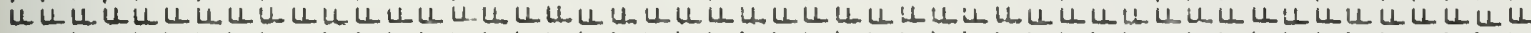

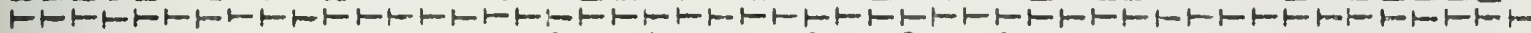

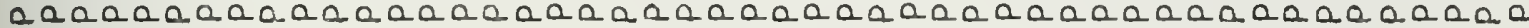

I

$\sum$

닏

F

3

N

0

$\vdash$

0

$\frac{11}{0}$

L

는

$\frac{I}{r}$

L

2

z

m

4

岩

I

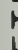

U.

$\sqrt{0}$

vi

I

$I$

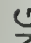

2

$\sim$

$\sim$

บ

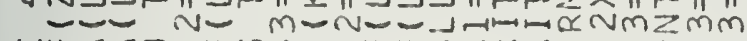

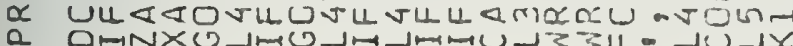

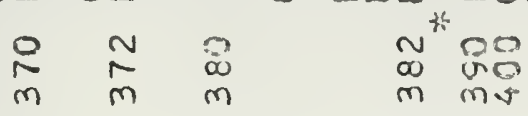

品

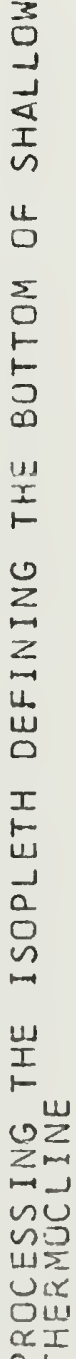

2

$\stackrel{\circ}{\circ}$

미

3

in

n

10

$\circ$

m

Y

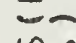

$\mathrm{Mr}^{\mathrm{N}}$

$<$ 过

No

ㅁ

ov

- -

on

- $x$

$1-1$

$\infty$ mus

2 om

$\cdots I m$

il $\rightarrow$ Ny

$m \geq-$

xतrllu un $1110100+z \mid 1100<+\cdots \cdots$ $m-2+m$ osm $m \cdot 00.100-x$

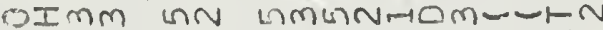

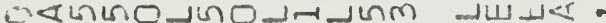

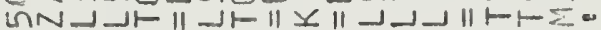

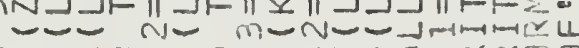

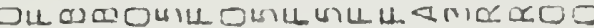

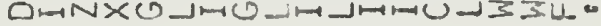

$$
\begin{array}{lllll}
a & j & a & 0 & N \\
0 & N & 0 & N
\end{array}
$$




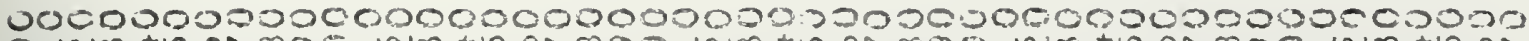

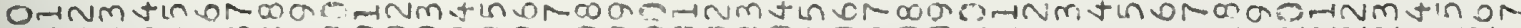

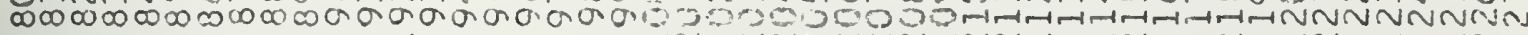

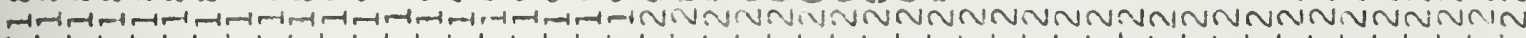

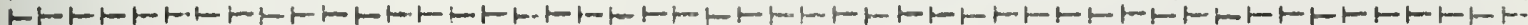

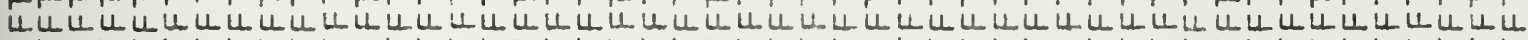

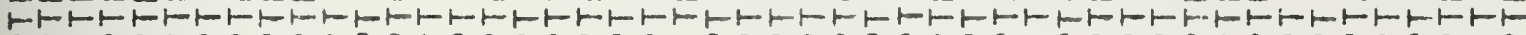

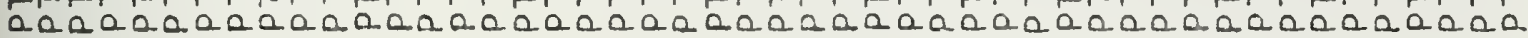

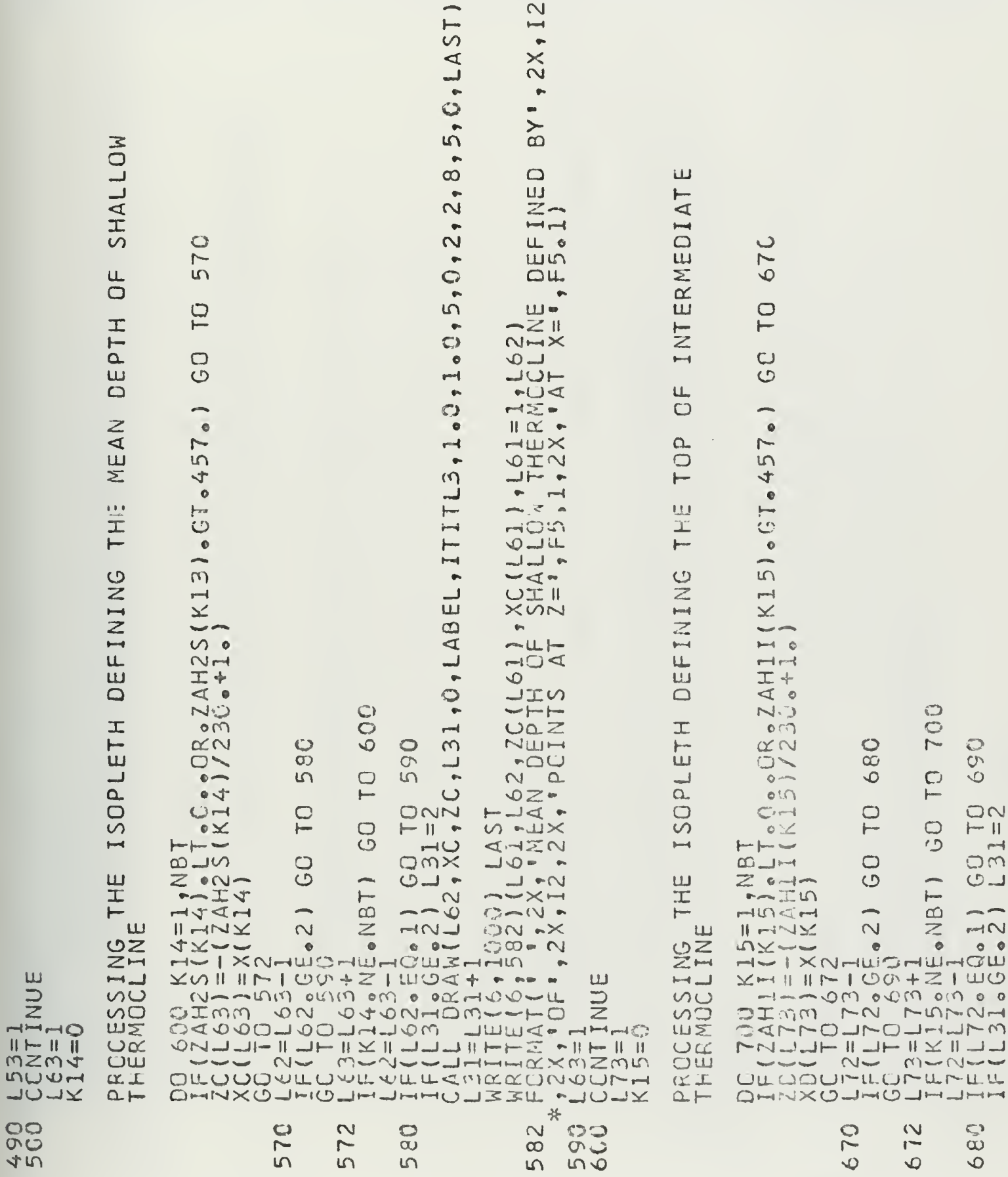

ưu

uwu 
03000000000020000000.2000000000005006030000000000

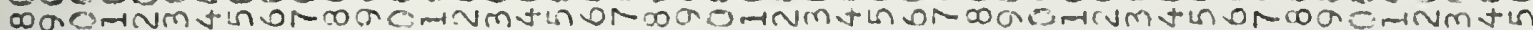

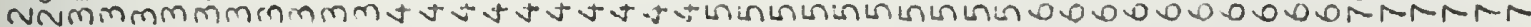

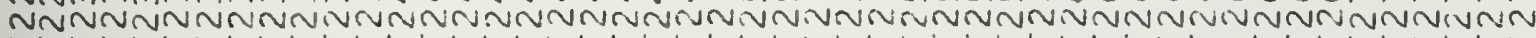

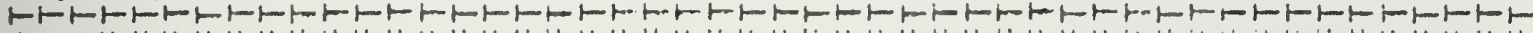

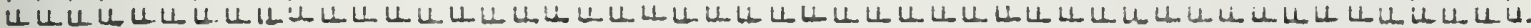

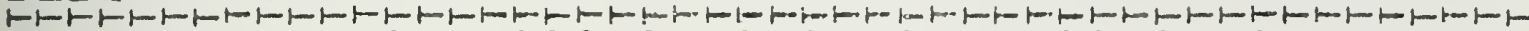

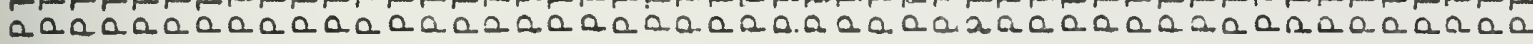

$<$

$\hat{\sim}$

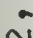

m

$m$

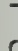

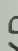

$\times$ <ना

N $- \pm x \sim$

N O-Nm

- 200.2

- $000-x$ $3-1-10 N$ $\varangle+\cdots \cdots$ an-100-

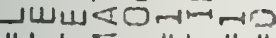

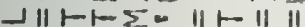

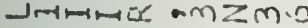
\&inacurwar

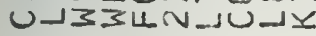
$\begin{array}{ll}* & \\ \infty & 00 \\ 0 & 0\end{array}$

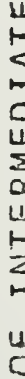

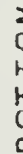

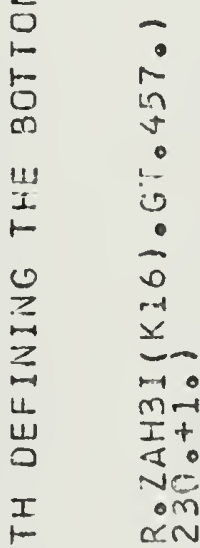

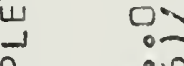

crot

$0 x$

$1-10$

D.jn

I

山

录云

-

जo

Us

पू

at at

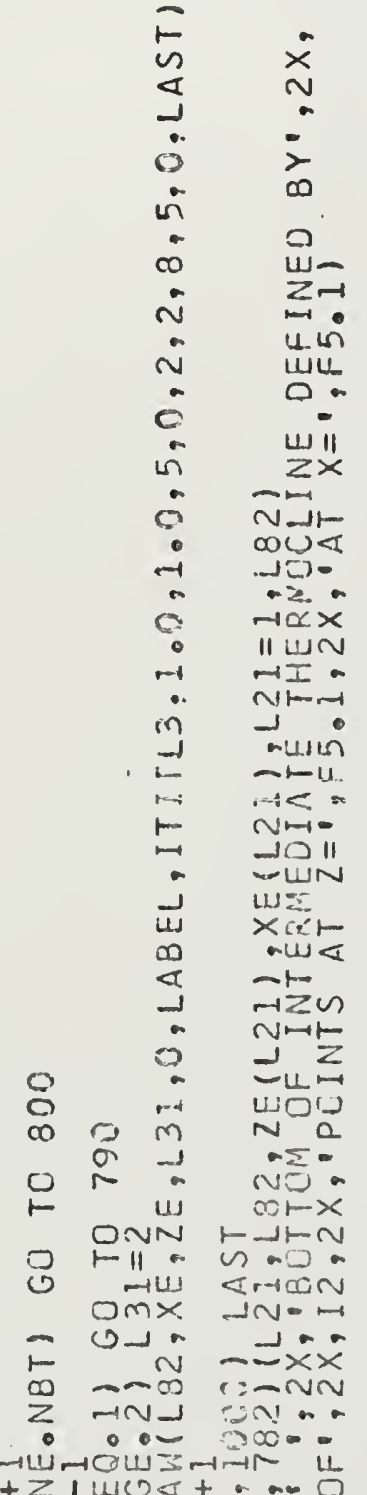

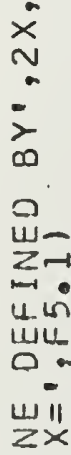

$\sim-4$

$\infty 0<1$

$\rightarrow=0$

$-1 \propto x$

$\| \omega N$

NFr-

auाin

a $-\mathrm{U}$

$\mathrm{Nat}=$

"OIII

U.

aid

ar

¿ Z

Hid

$\rightarrow \infty$

Nivin

$\infty+\infty$

$$
\text { N }
$$

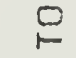

20 in 0

$$
\text { mo< }
$$

$\| \rightarrow N y$

$\widetilde{2}$

$0 x-$

$x=1 \times 20$

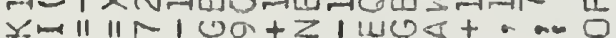

m-n or

Imm mN

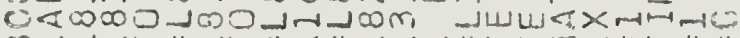

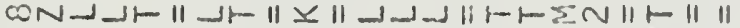

- $N=m=N=-d m m-1, \cdots m<m$

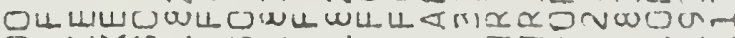

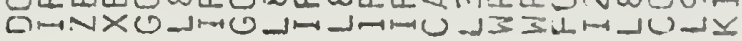

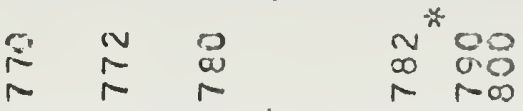

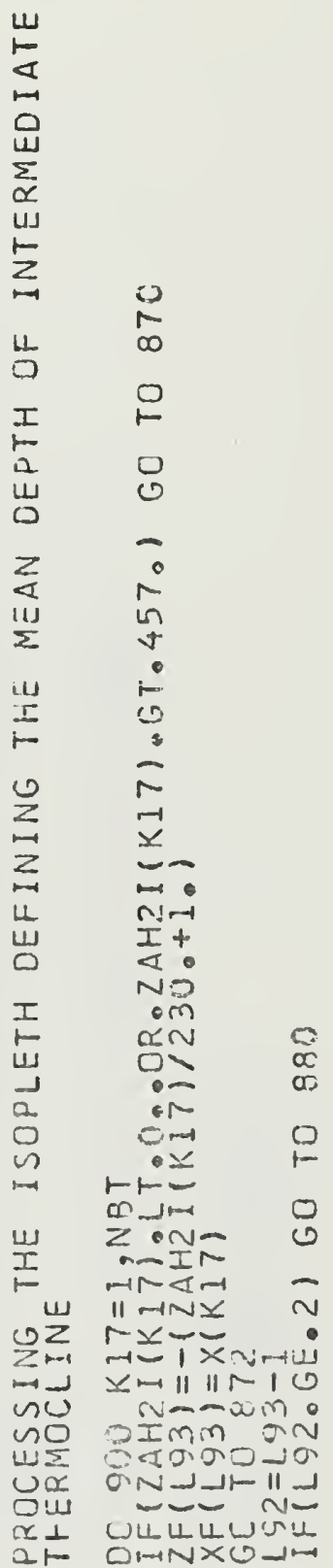

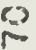


$000000000003000000000000200000000: 200000000005000$

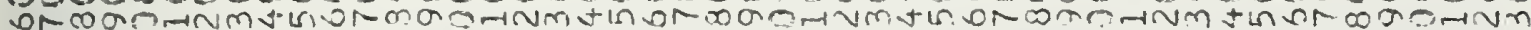

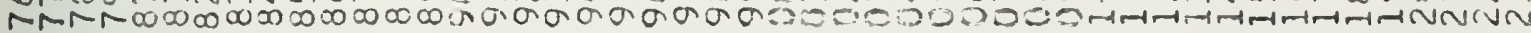

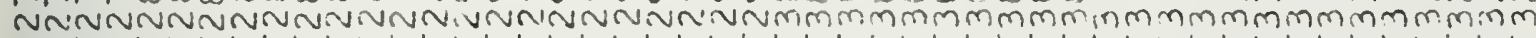

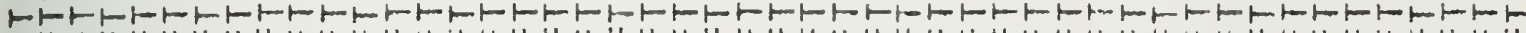

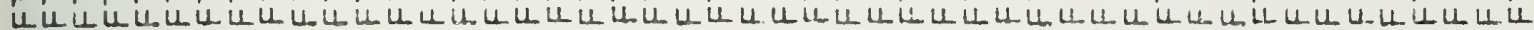

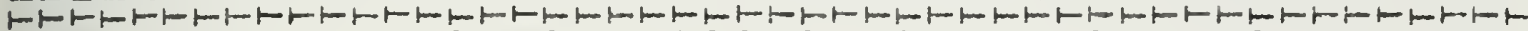

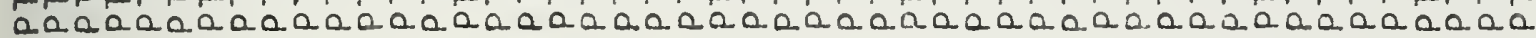

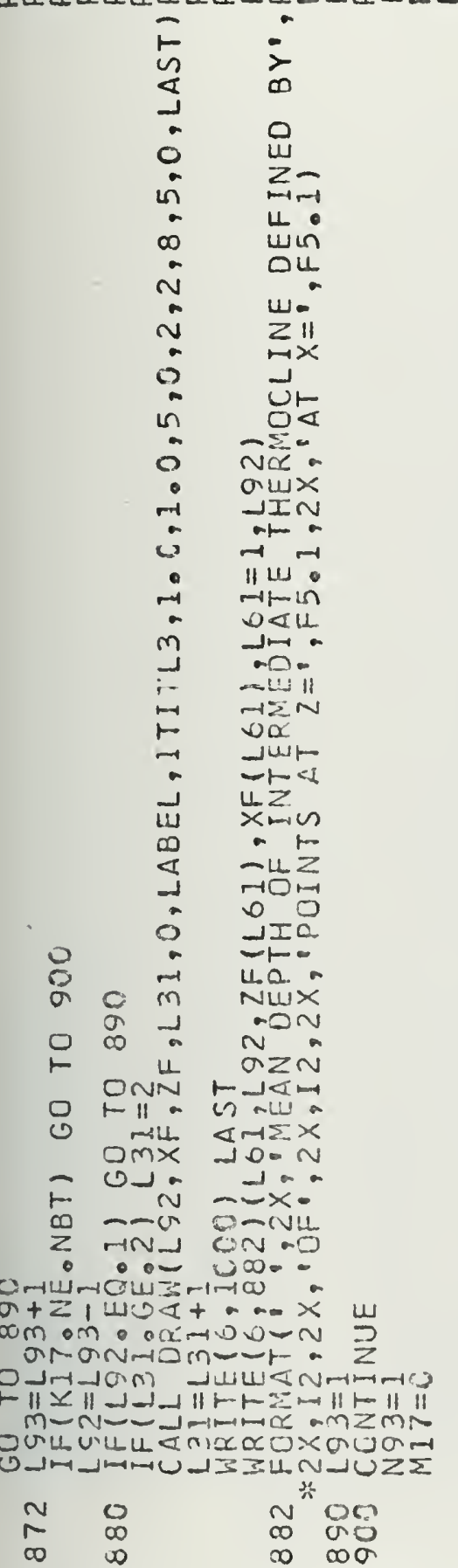

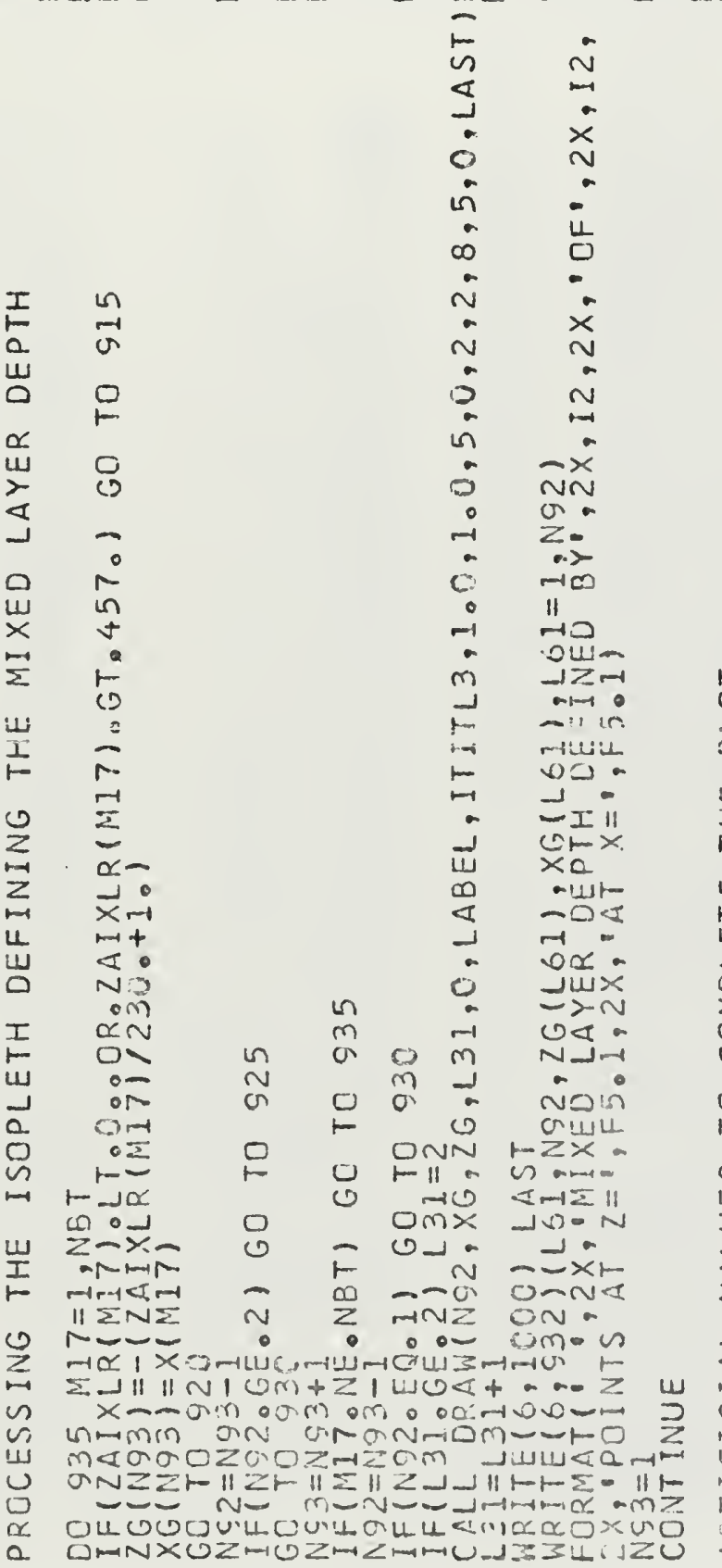

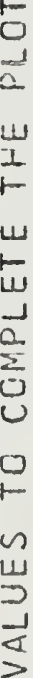



000 -1m-1 $++t+$ 000 क. mmmin vivive $>>$ - 0 - 0 $302 \pi$ C. 36.0 omivmis -1 1 11111 II II || || || - - NNNNN NNNNN

\& $n$ min
m an 


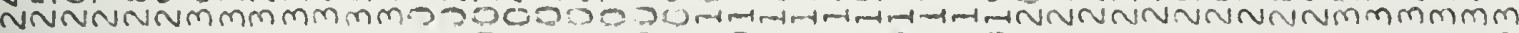

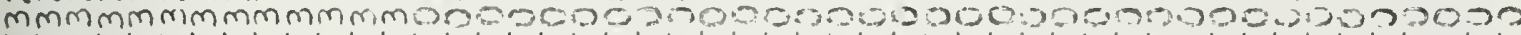

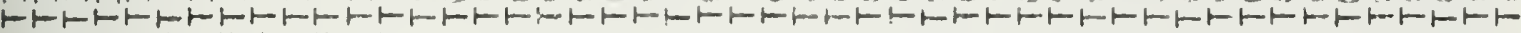

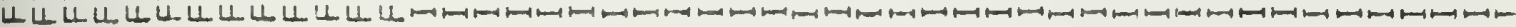

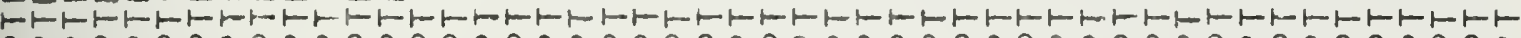

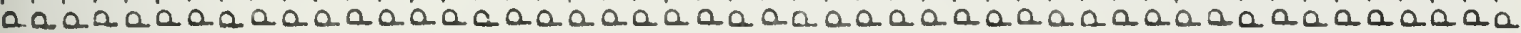

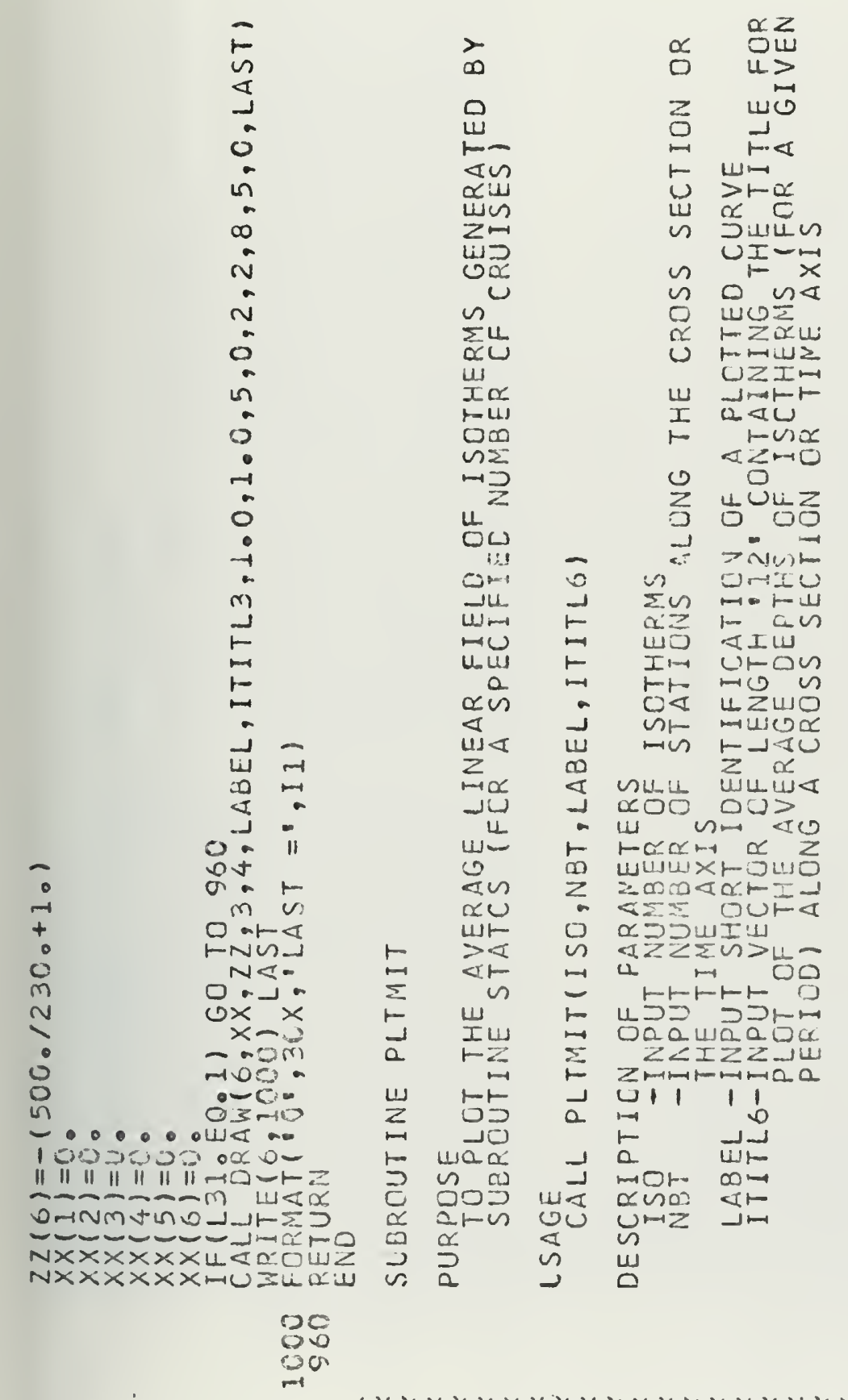

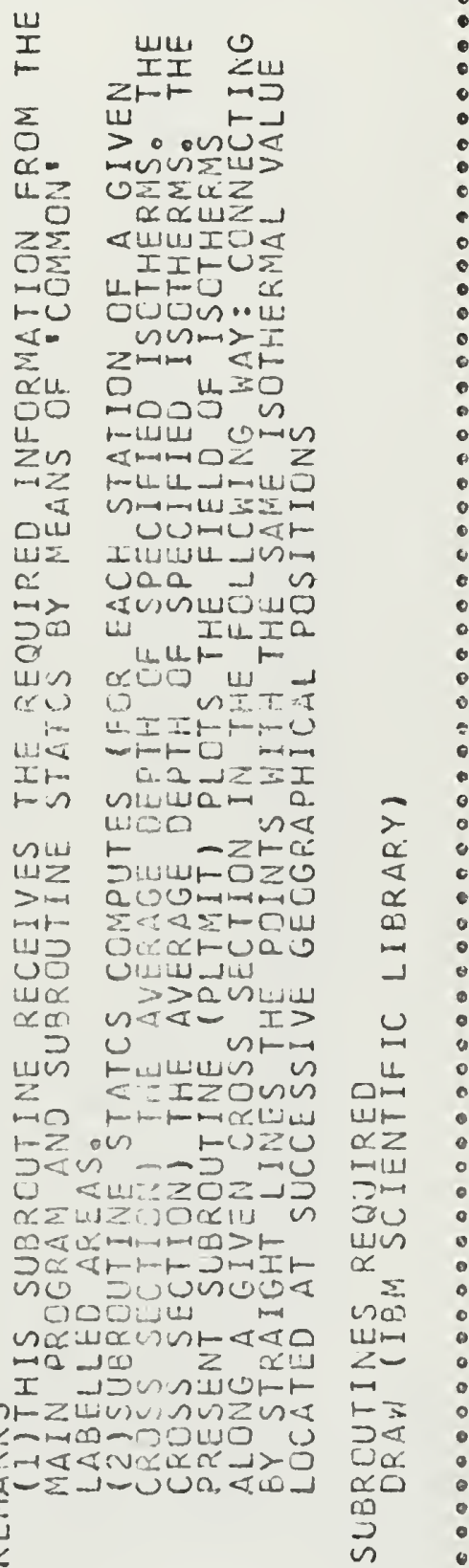

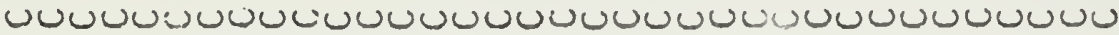


000200090000000000003000000000000000000000000

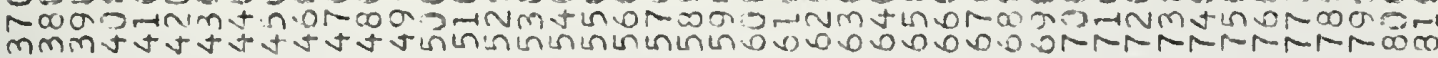
$00030=00 \% 200300000002000000002005000002020200$

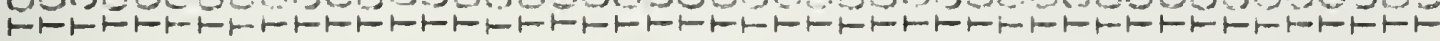

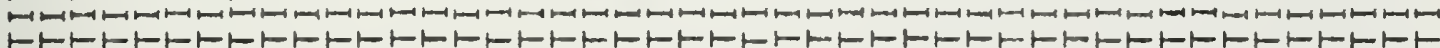

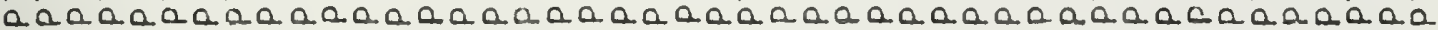

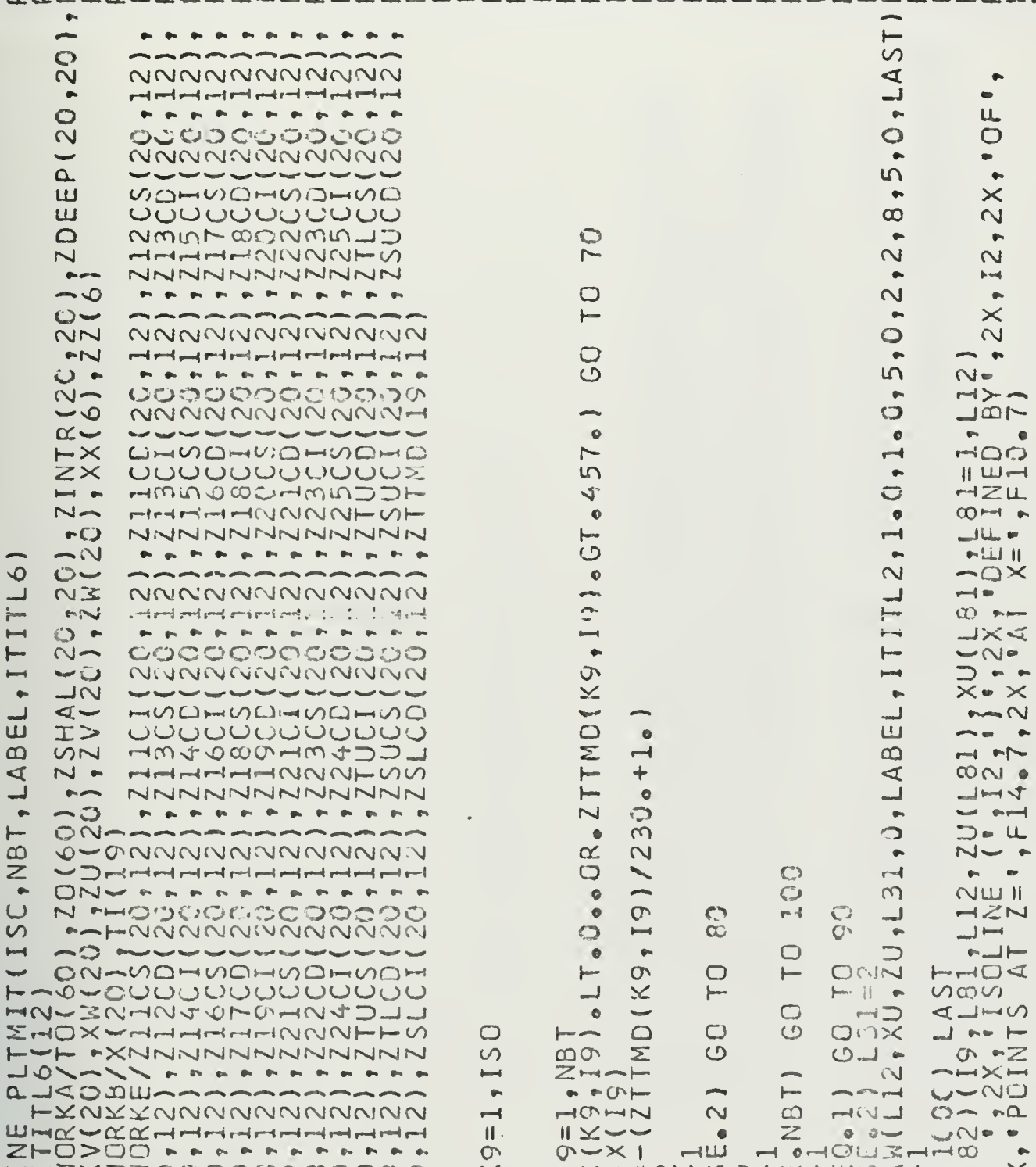

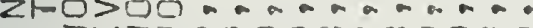
HW $3 \times 3 z 00000000000$

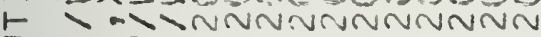

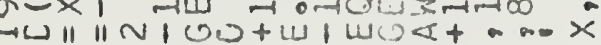
-

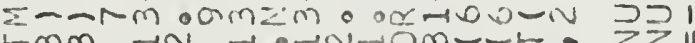

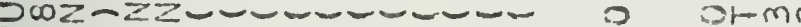
O 0 UUm

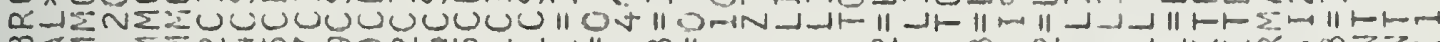

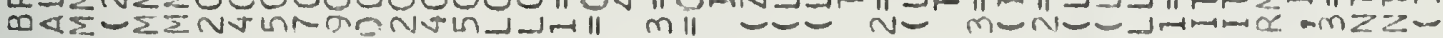
JUU JuUn-1

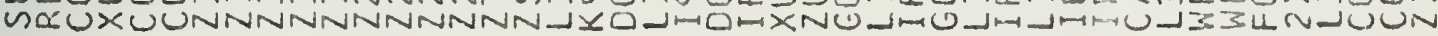

$r \quad \infty \quad \infty \quad \infty$


0000000000900300000000200003909003000002000000

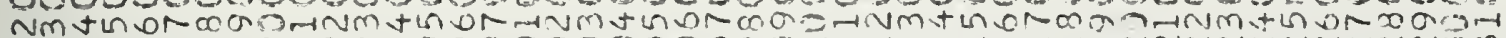

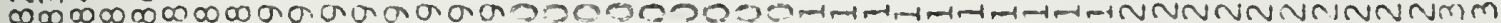
020000000070000000340000003000500.00000000000000

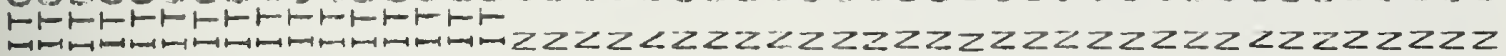

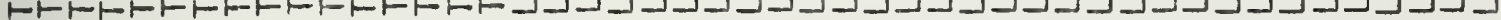

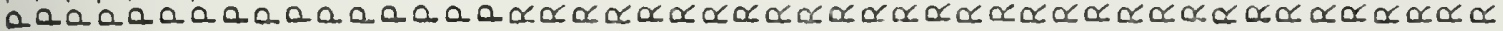

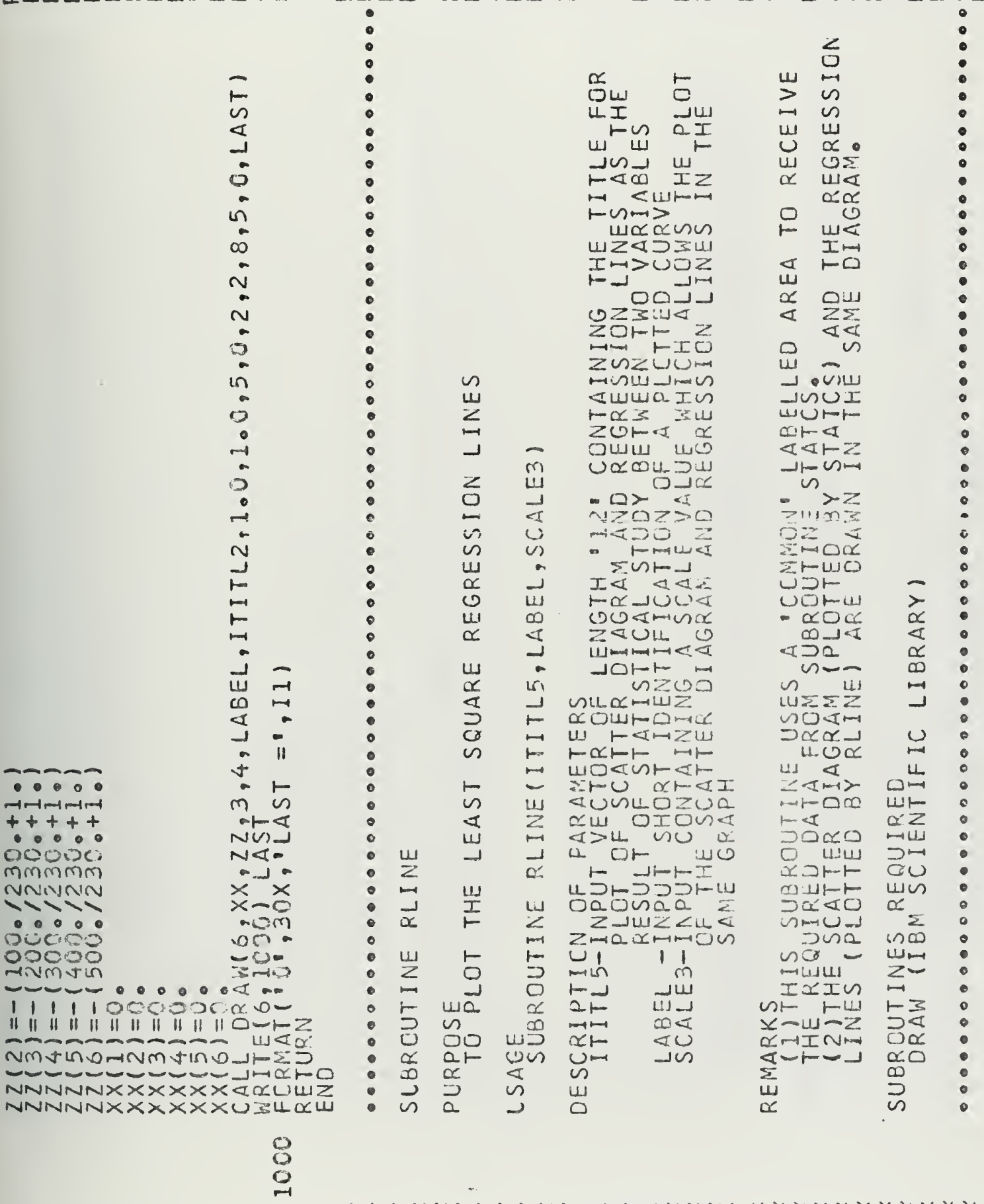




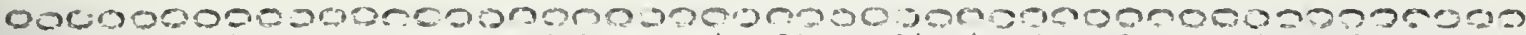

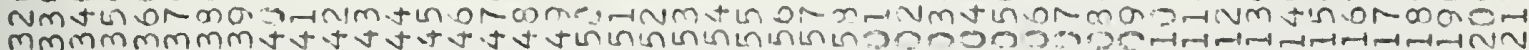

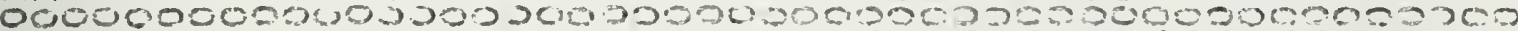

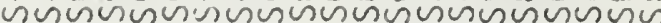

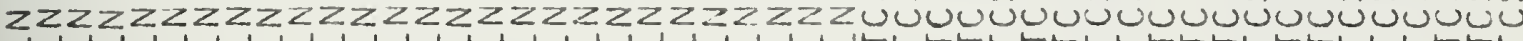

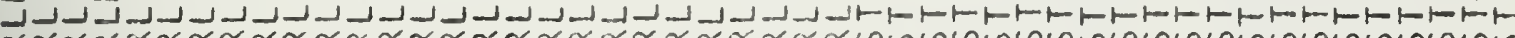

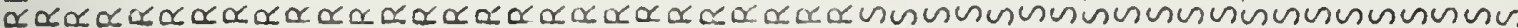

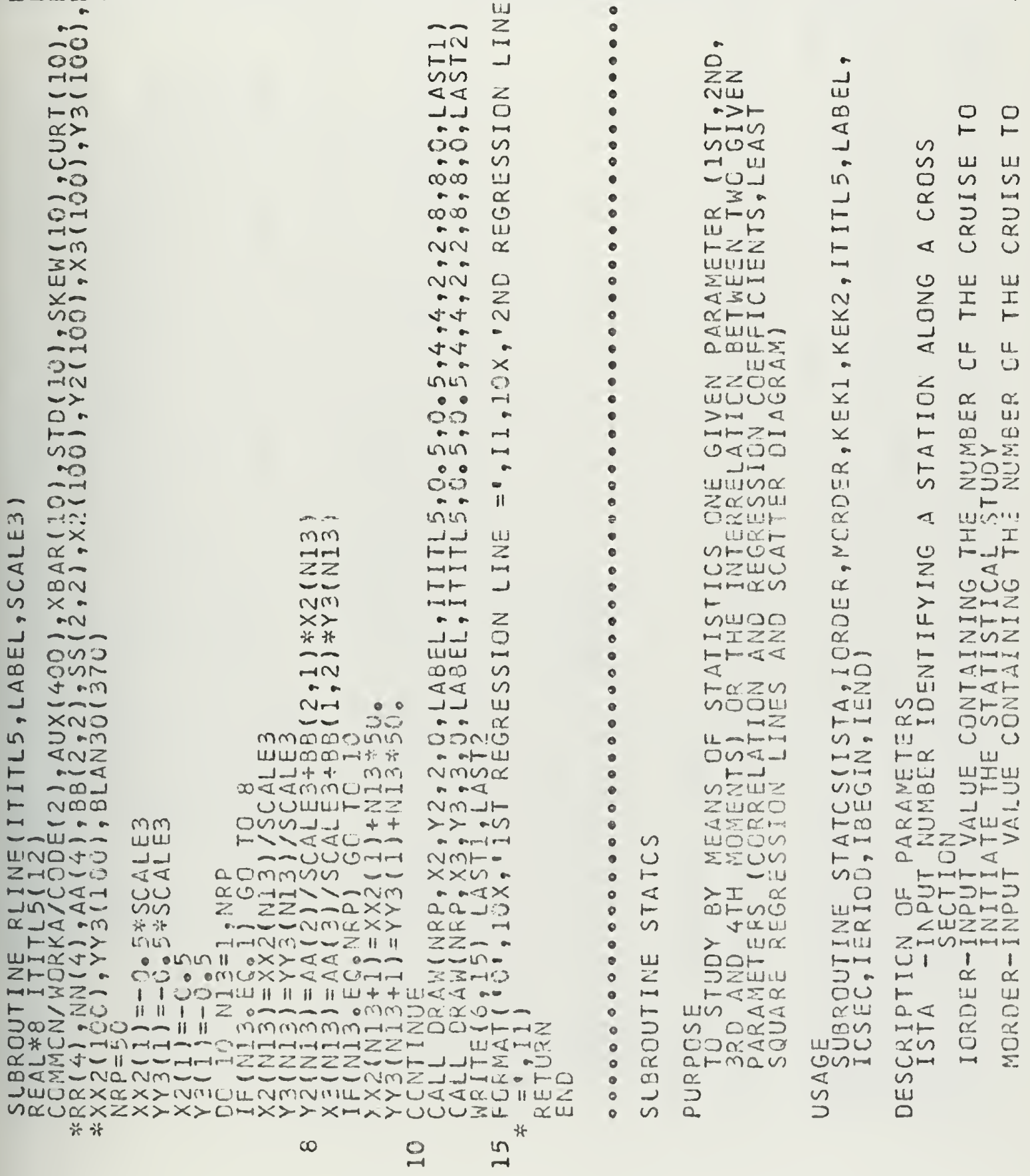



$000000000000=00000030050000005000900000000000000$

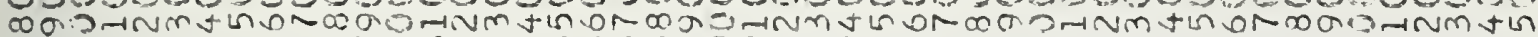

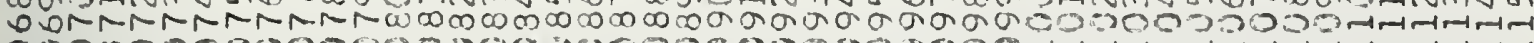

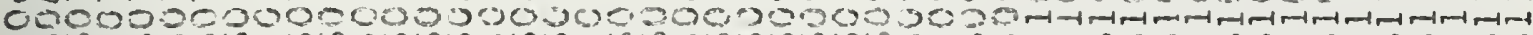

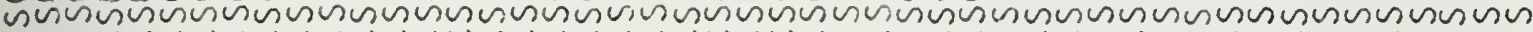

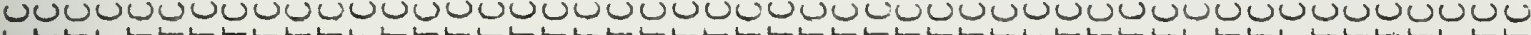

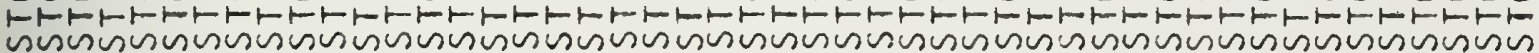

$$
\text { - }
$$

- $N a n$

O

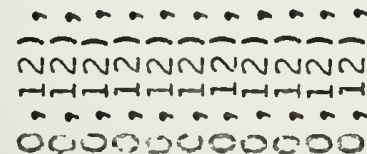

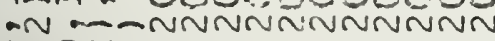

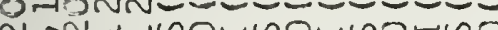

Nan, -or-auunuuvuuuu HNNOBNmInR aran!mLA

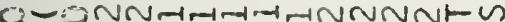
SIDO-NNNNNNNNNNN Z Jornd a. a..........

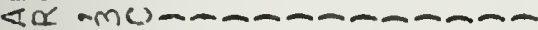

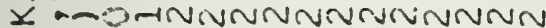

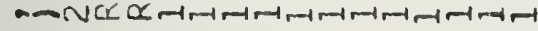

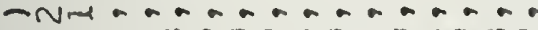

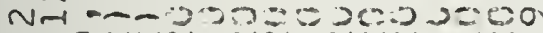

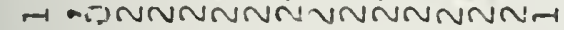

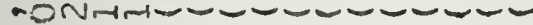

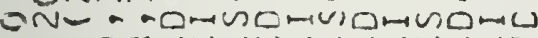

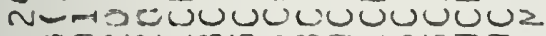
- monNAmLn 0000 on -100- EUONANNNNNNNNNNNN Q $Z$ anO a a a a a a a

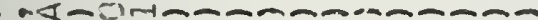

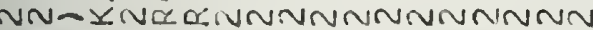

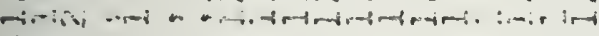
a ara an-a-ararana a a

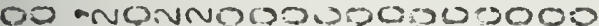

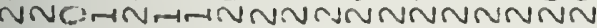
-rva a...-C- - - -

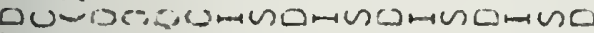
ILONANNUUUUUMUUU UU InO-0ー- -

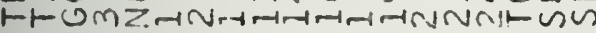
NaZUSININNNNNNNNNNNN

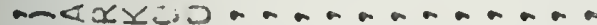

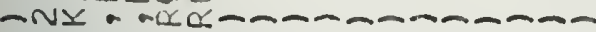

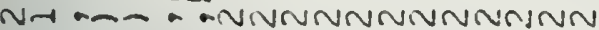

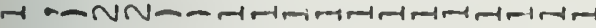

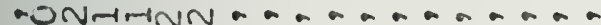

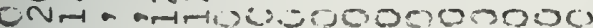

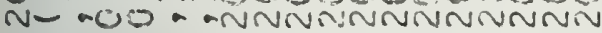

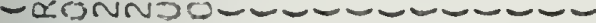

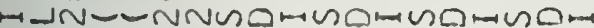

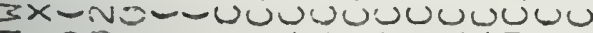

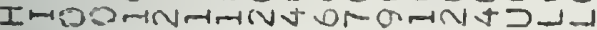

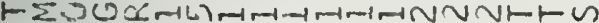
NNEZ ROSNNNNNNNNNNNN

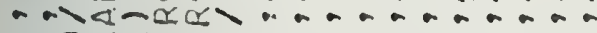
-Dxiva-is-

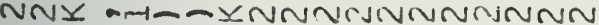

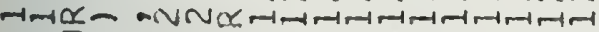
- anderrato a a a a a - a n $003-1 \mathrm{~N}-3.5000050 .000$

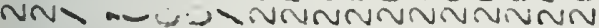
-ZJININZ-W- - - - - -

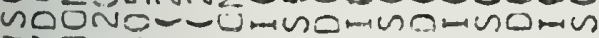

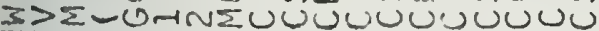

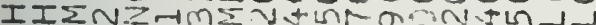

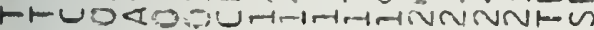
NTUaxaOUUNNNNNNNNNNN

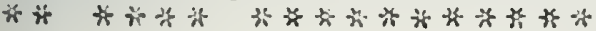

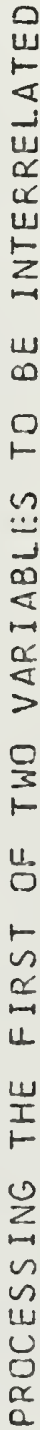

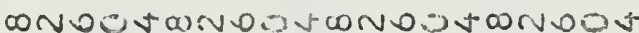

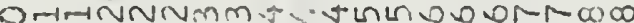
00000000000000000000

OUO00000000000000000 トトトトトトトトトトトトトトトトトトトト

00000000000000000000 00000000000000000000

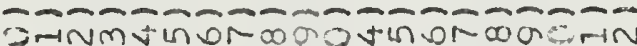

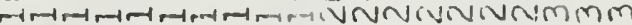

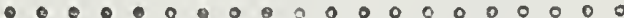

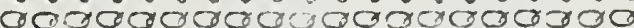
แ山ा

$\because 0.00000000000$

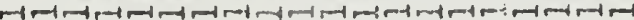

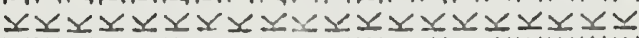

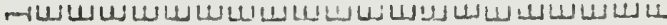

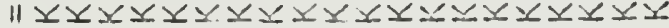
0こーーー-

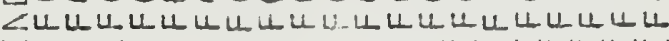

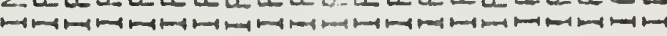




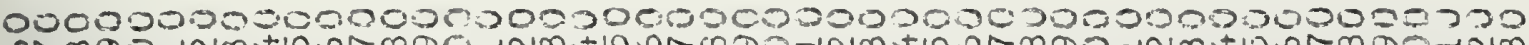

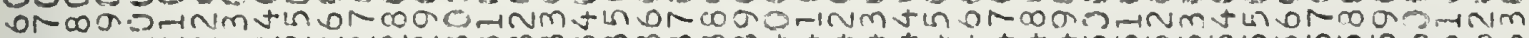

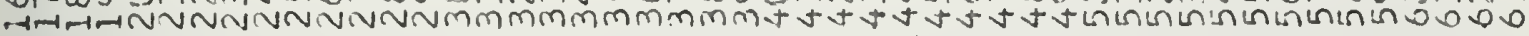

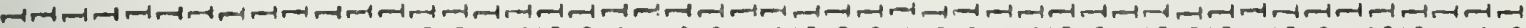

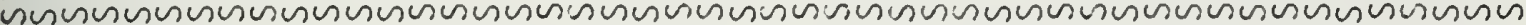

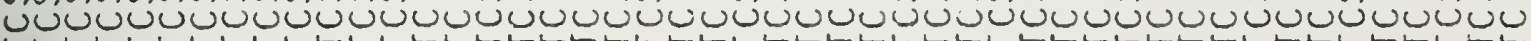

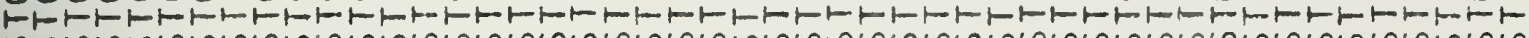

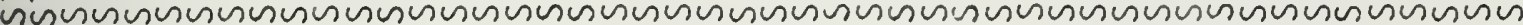

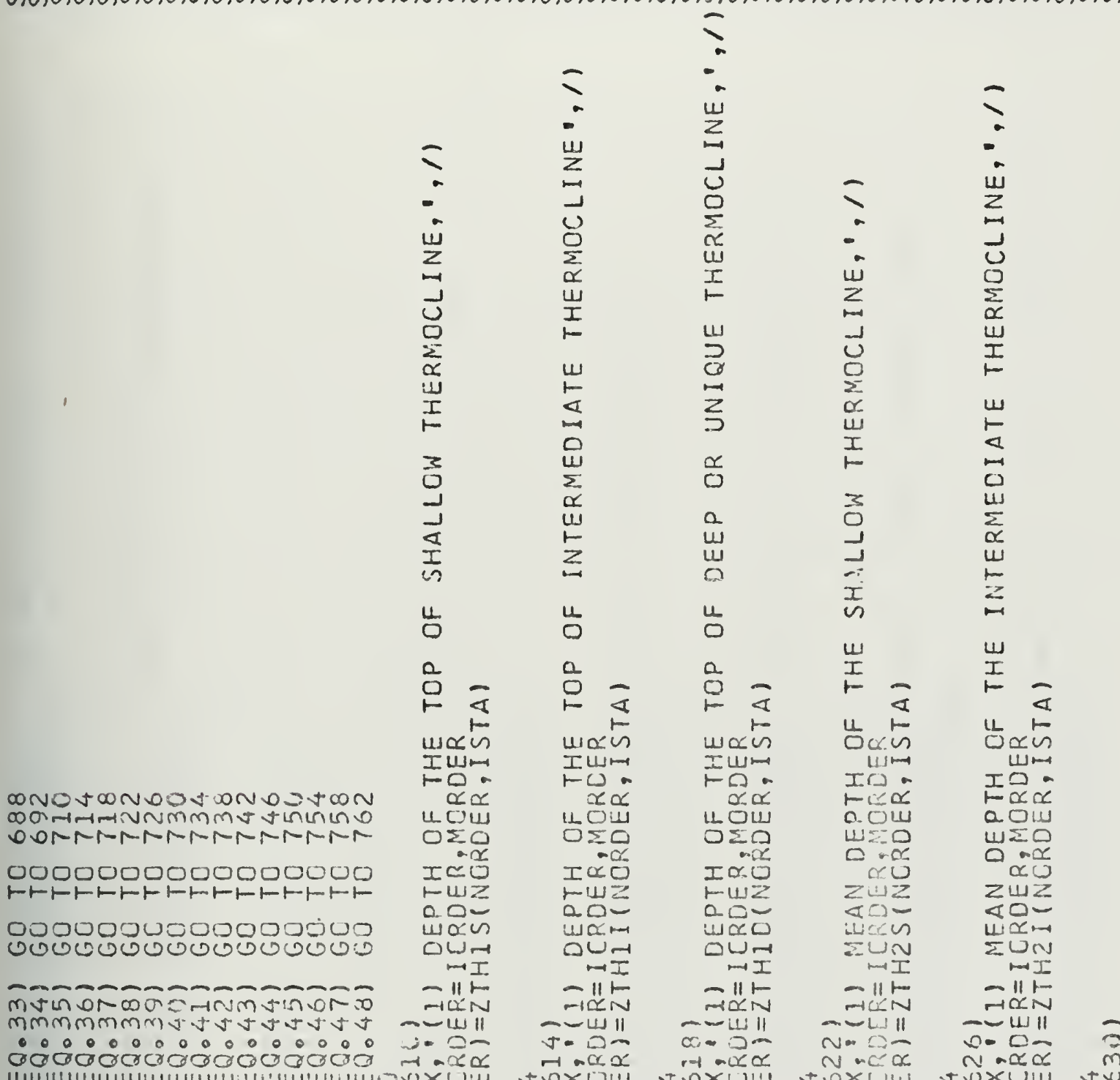

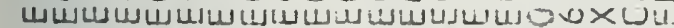
( a

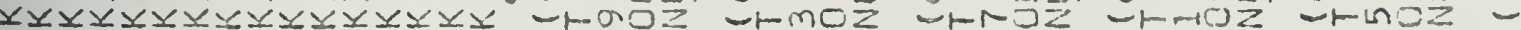

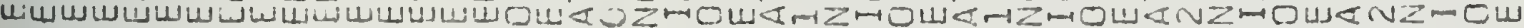

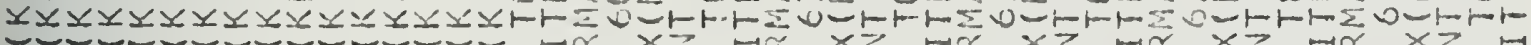

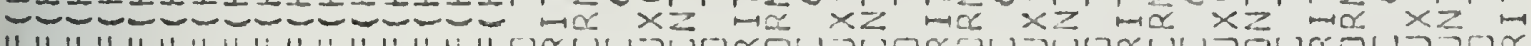

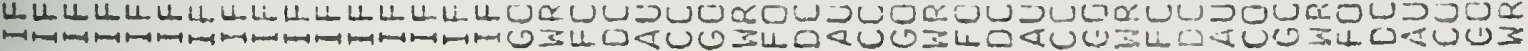

\begin{tabular}{|c|c|c|c|c|c|c|c|c|c|}
\hline$\infty \supset$ & 0 & NT & $m$ & $0 \infty$ & 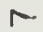 & orv & 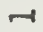 & $\checkmark v$ & in \\
\hline 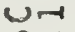 & 0 & नIम & -1 & नीन & -1 & NN & VV & IVN & $N$ \\
\hline ow & 0 & 0.0 & $\sigma$ & 00 & 0 & 0.0 & 0 & Dט & $\infty$ \\
\hline
\end{tabular}




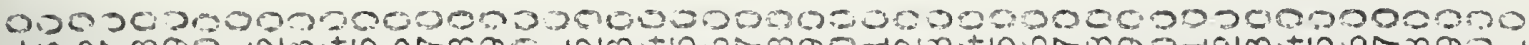

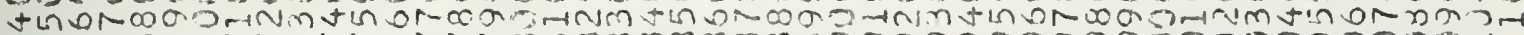

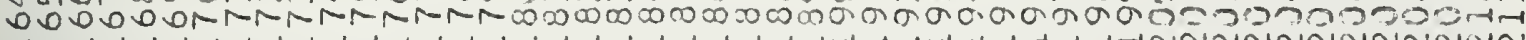

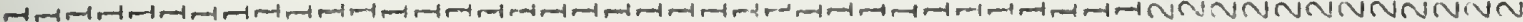

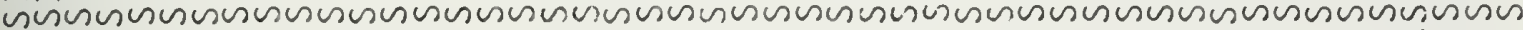

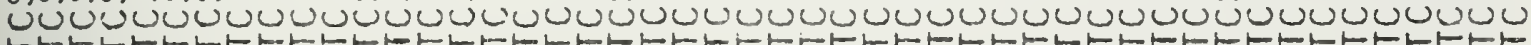

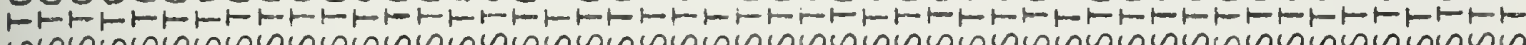

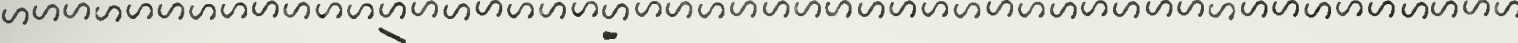

I

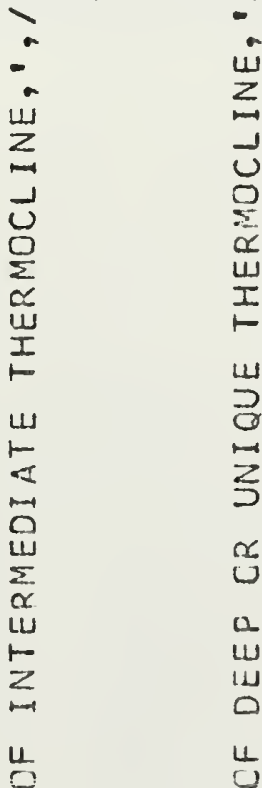

a. $\quad$ u

a $\quad$ u

○

w

$\stackrel{\text { I }}{⺊}$

Uris Lij $n$

ID

$F \propto \alpha$

Q

แะอ

$0-\infty$

aU

Zuz

$<0-$

WळO

IUN

-I

- II

- $\|$

- 0 -

$a \alpha \alpha$

azous ag.zowes ac

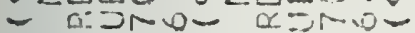
-aロz - -moz -

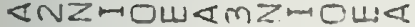

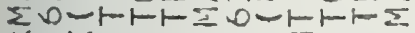
$\propto \times z \mapsto a \times z \mapsto c$

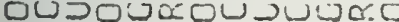

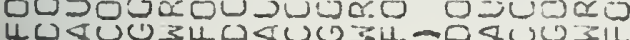

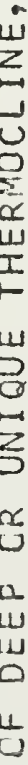

$\Sigma$

C

$\bar{\varepsilon}$

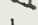

ris

1)

$\propto \propto$

טN

$\sum 0$

$\propto u$

is $Z$

$0=$

Um

$\rightarrow I$

115

II

อา

tad

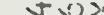
ZQLilo or $\supset$ MOZ - F mŻrowa $0=r-5$

3

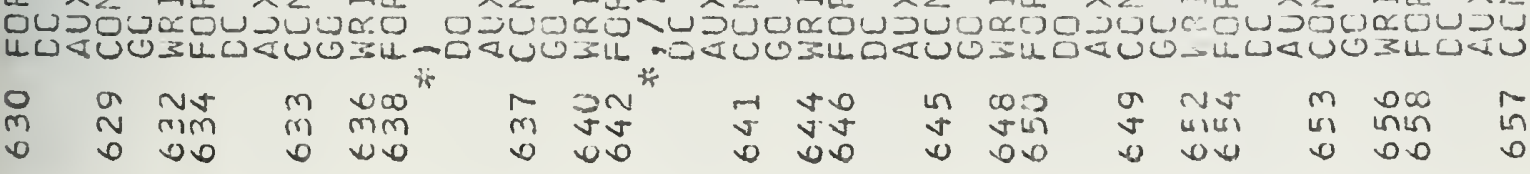
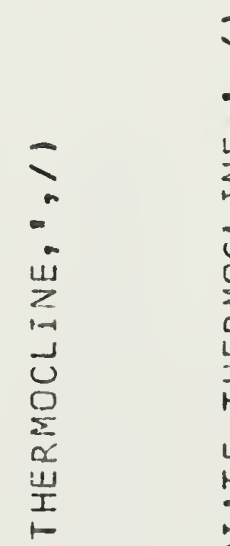

0 <

u) $\alpha \stackrel{5}{n}$

I Wh

$\alpha \propto$

u.

$0 \sum 0$

$\infty x$

I $\amalg Z$

a 02

U

- $\cup(n$

\|F

$=\prod_{1+1}$

Uा

D-

tuxum $+0 \times u \omega$

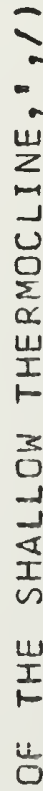

w 15

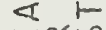
$\varpi \propto \sim$ o.uth $>\alpha \alpha$ ○山 $\checkmark \leq 0$ $\sum \pi 0$ व.सय แบInos $1-U>$ $\rightarrow \mp$ - $111+5$ wat - w W $\infty-0$ in $n x x$ 
000000007200000000000000000000030000000000000000

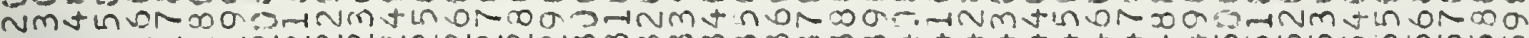

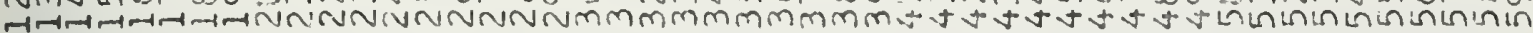

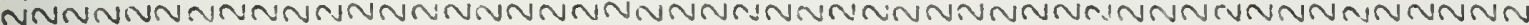

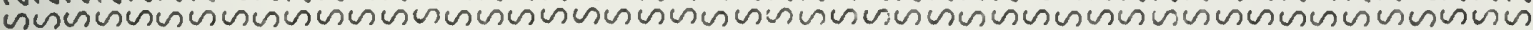

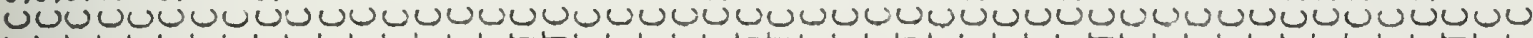

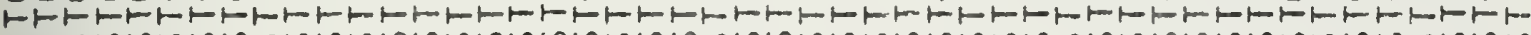

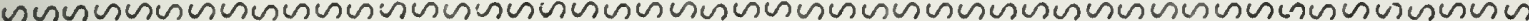

:

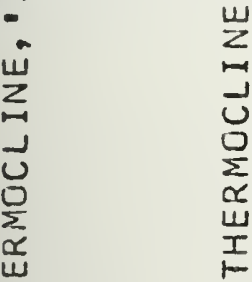

虫

岌岌

㟔范

$z$

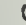

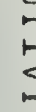

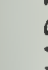

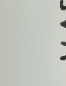

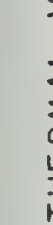

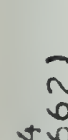$$
\text { tion }
$$

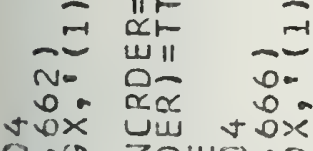
0 -a zour? -o
$\sum_{3}^{\Perp}$

$\stackrel{\propto}{0}$

a

w

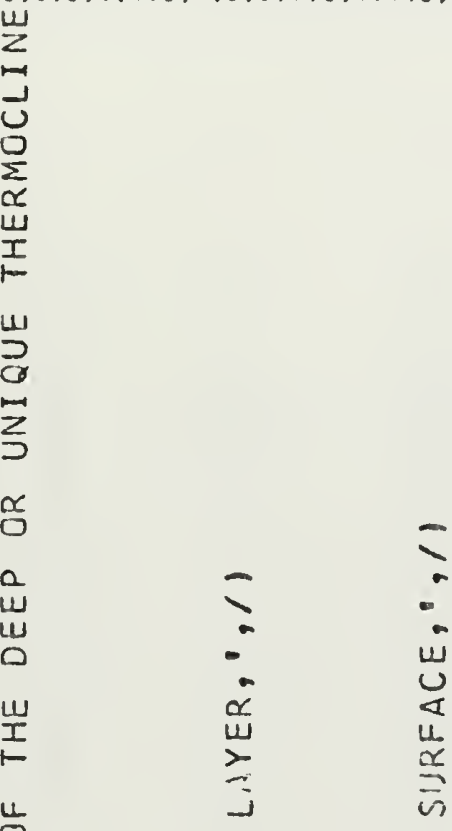

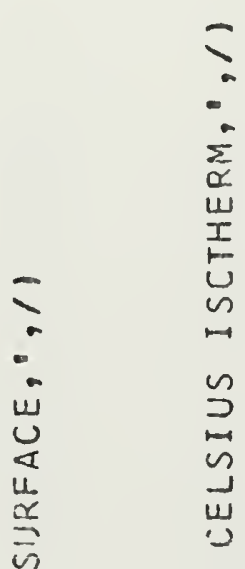

ด

U

$\sum_{1}<$

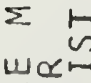

IU.

FOC

든

ए) $\sum 0$

क

I $\propto 2$

-U⿺

a 0 r.

wa-

o $0 x$

$\rightarrow 115$

$\rightarrow \infty N$

-

N- $0=$

$N-\alpha \propto$

品

$<\ll$

แงแผ

$\alpha 2 m$

$20 \alpha$

¿

$\alpha \circ 0$

Uxa

내긴

Wᄄ口

rou

내나

- $11 \mathrm{c}$

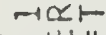

- $-4 \|$

$\checkmark-0-\alpha$

$+\alpha \alpha \alpha$

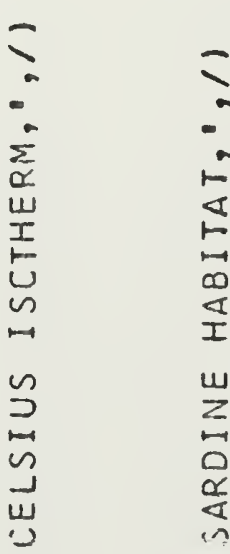

ㅍ

$\infty$

w

出

$\frac{1}{4}$

4

- $-4 \|$

$\infty-\infty \frac{\pi}{2}$

Inxou $5 n \times 4$

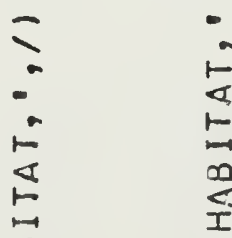

$0_{1}^{0}<$

use

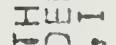

$\propto \propto$

แLㄴ.

$0 \geq 0$

Iccin

I 12

$00=$

ज小ए

ouv

$\pm 0$

- $\|$ n

$-20 \times N$

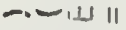

$\mathrm{N}-\mathrm{C}-$

$\infty \sim x \propto$

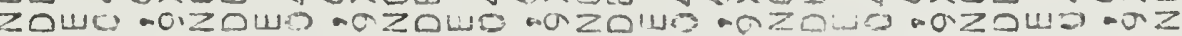

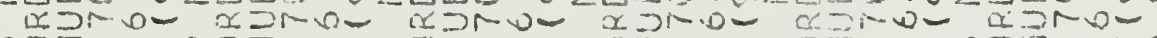
เnOz -

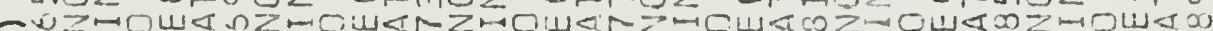
म조

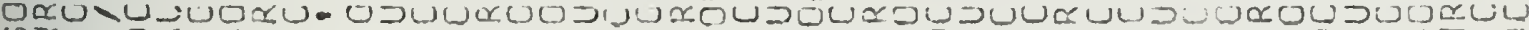

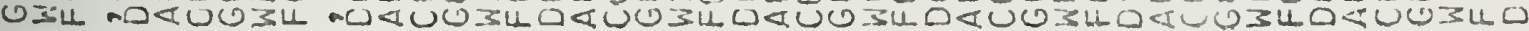

\begin{tabular}{|c|c|c|c|c|c|c|c|c|c|c|c|c|c|c|}
\hline ON & -1 & $\leqslant 0$ & in & cors & un & $\mathrm{NJ}^{+}$ & $m$ & $\infty \infty$ & $r$ & PN & -1 & $\checkmark 0$ & เก & 20 \\
\hline ov & $u$ & $\cup 0$ & $w$ & WN & 0 & $M-N$ & $\Gamma$ & $r-r$ & $r$ & 000 & $\infty$ & $\omega \infty$ & $\omega$ & ioc \\
\hline $0 x$ & $D$ & $\omega$ & $\omega$ & 00 & 0 & 00 & 0 & 00 & 0 & $\emptyset w$ & 0 & 00 & $\omega$ & 00 \\
\hline
\end{tabular}




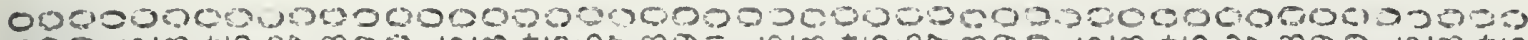

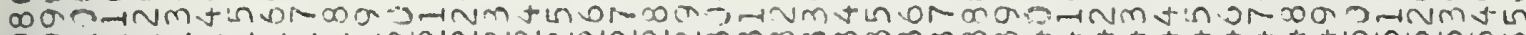

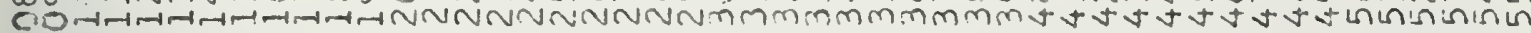

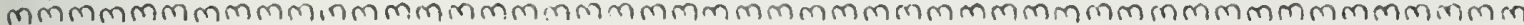

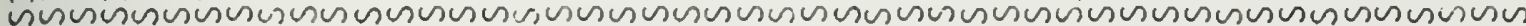

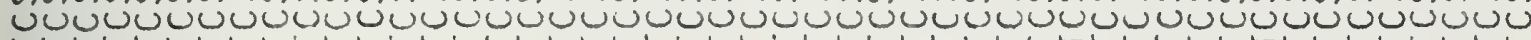

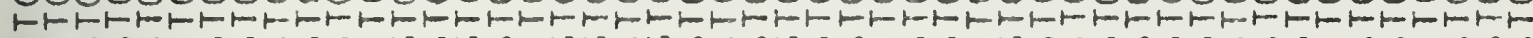

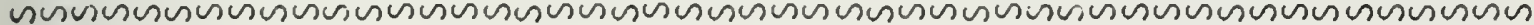

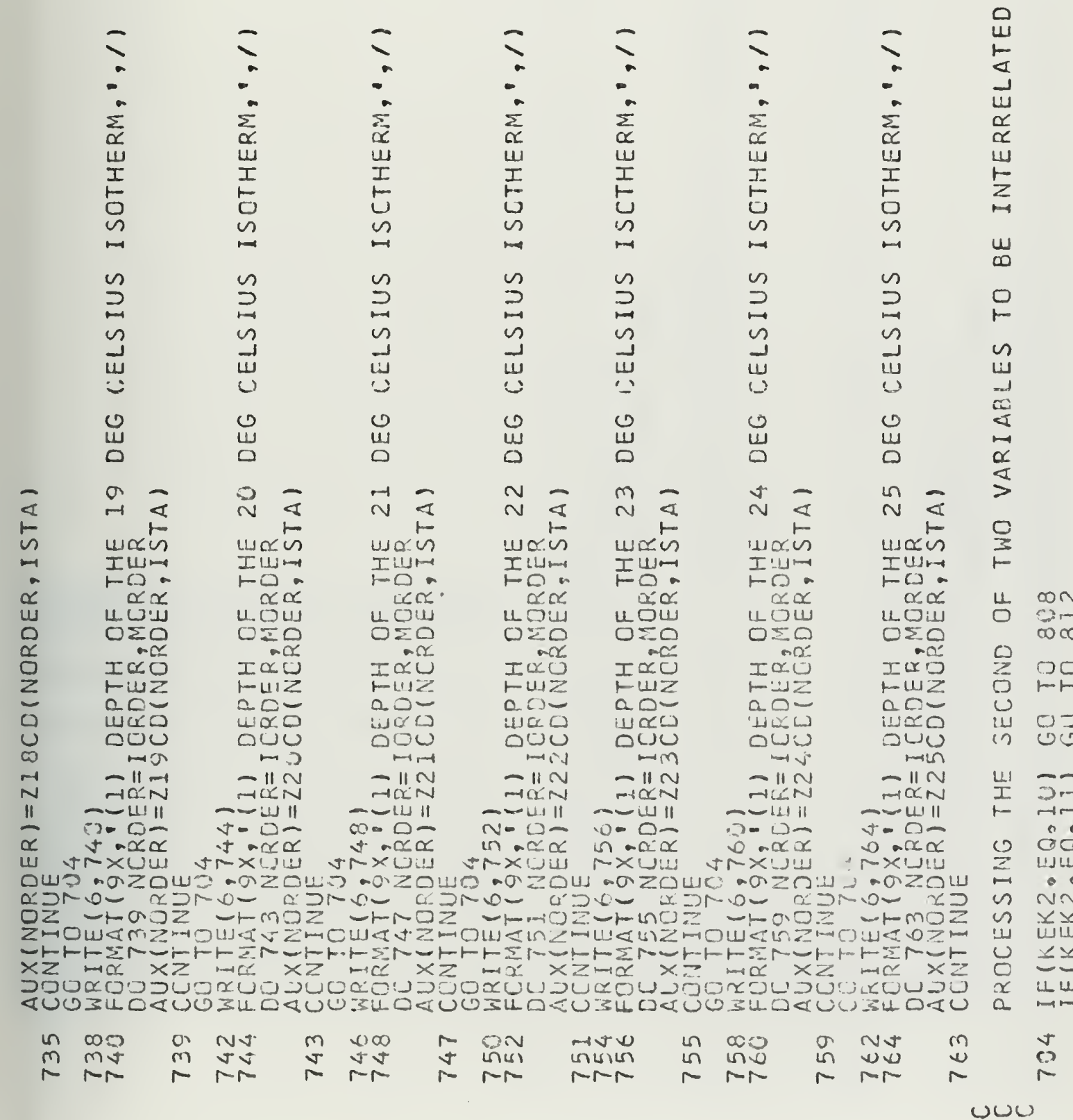


$0000000020000000000000=0000000000090000000000005$

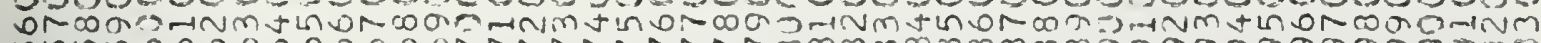

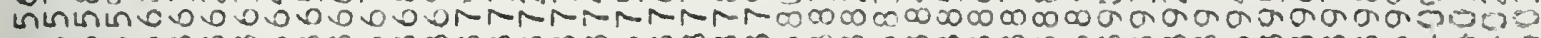

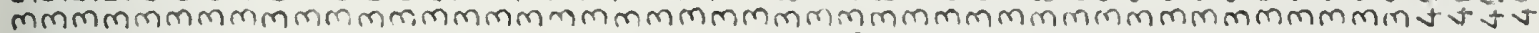

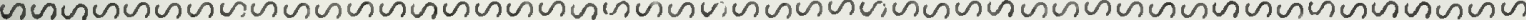

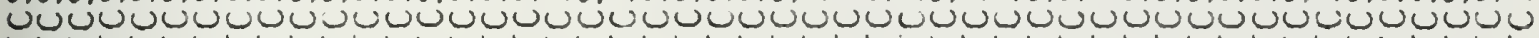

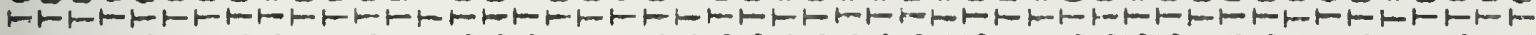

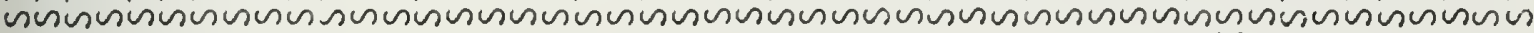

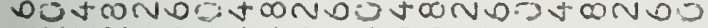

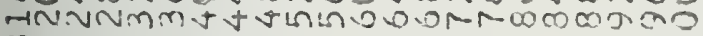
000000000000000000000000000000000 \%

\section{0}

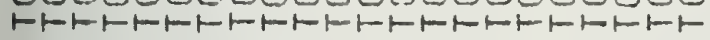

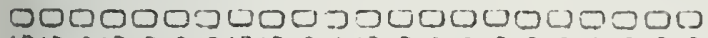
प0O00000000D0000000000

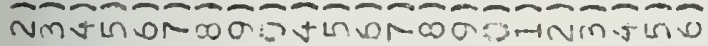

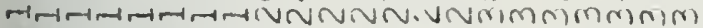
0000000000000000000000

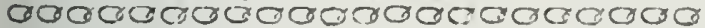
作

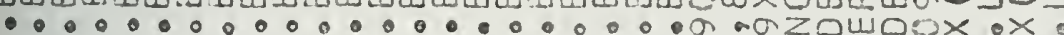

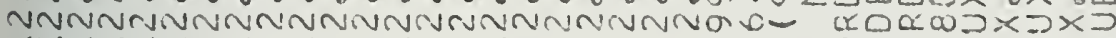

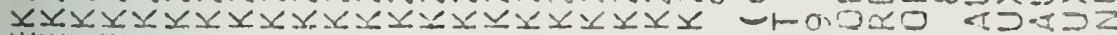

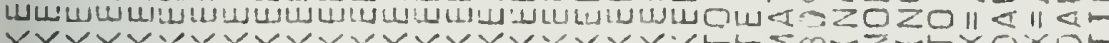

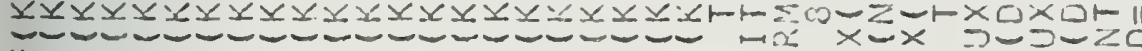

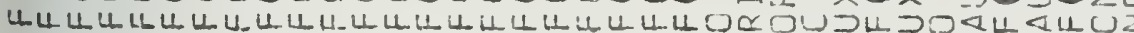

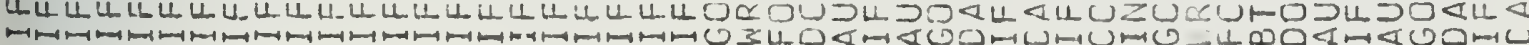

\begin{tabular}{|c|c|c|c|c|}
\hline $20 ?$ & $\infty 01$ & 0 & No & $\mathrm{Vm}$ \\
\hline जन & $05:$ & C) & ${ }^{-1-1}$ & म1न \\
\hline$\infty \subset$ & $\infty \infty$ & $\infty$ & $\infty \infty$ & $\infty_{-1-1}^{\infty \infty}$ \\
\hline
\end{tabular}

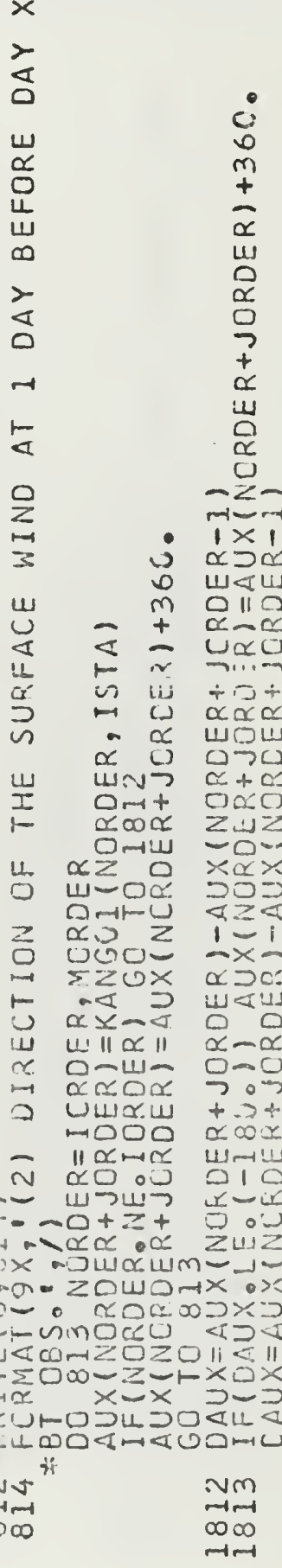




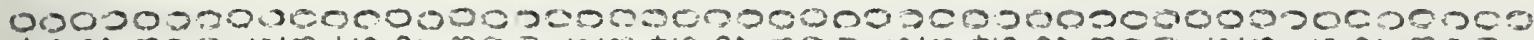

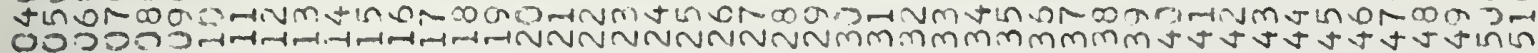

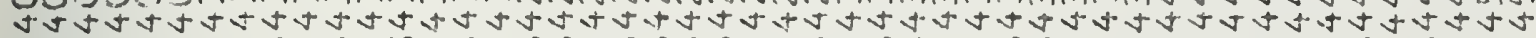

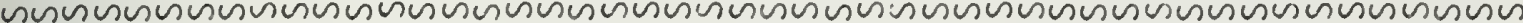

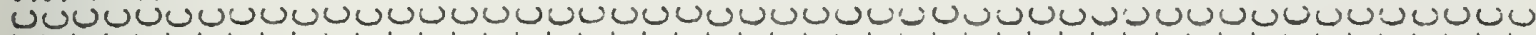

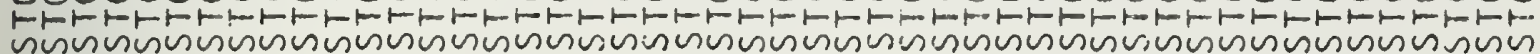

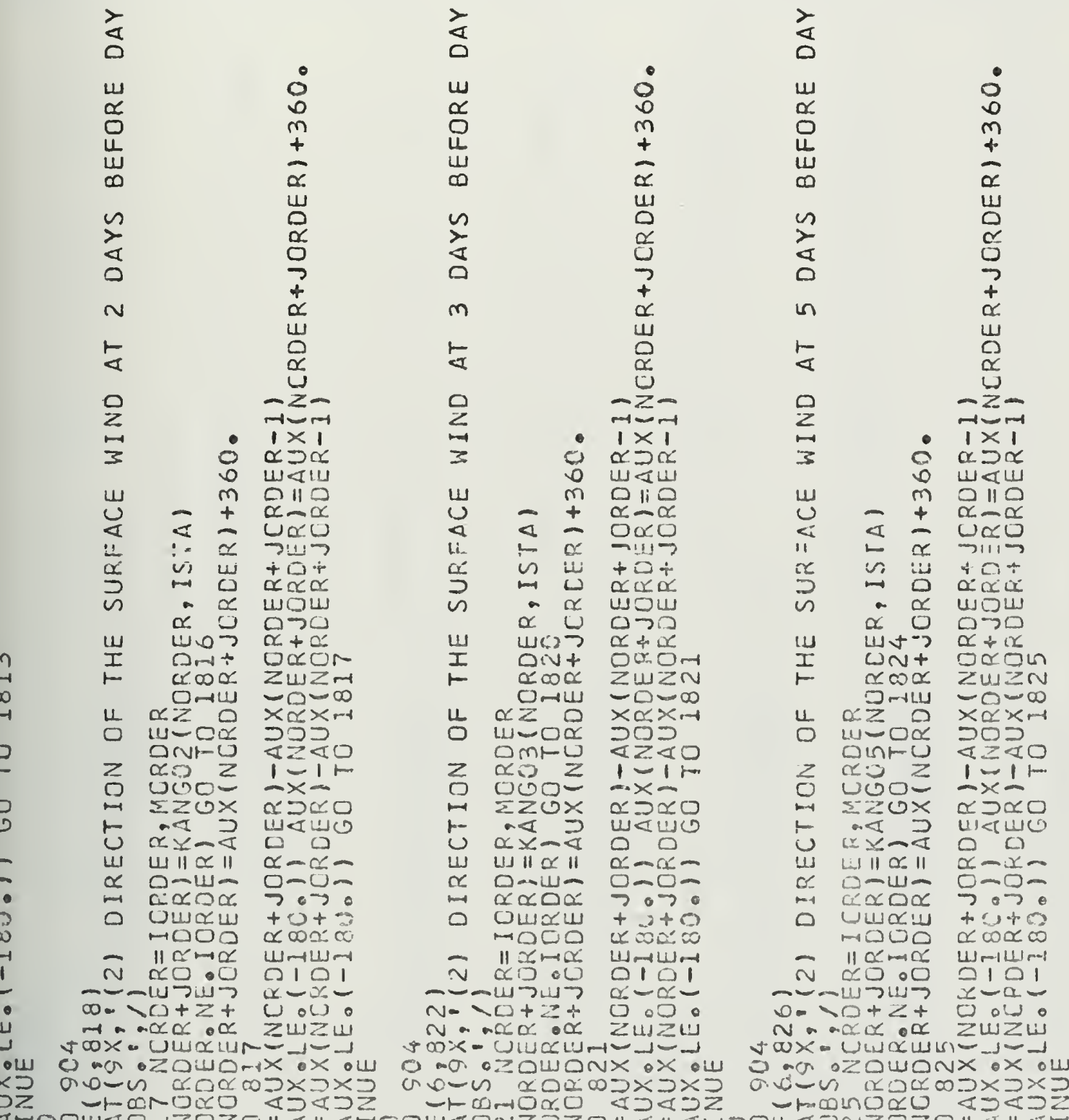

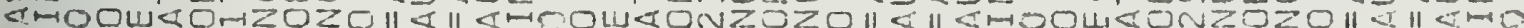

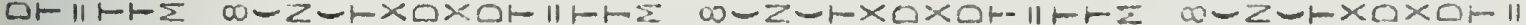

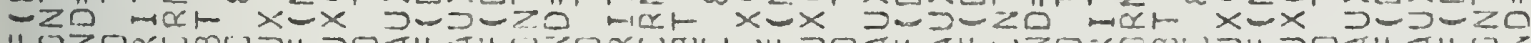

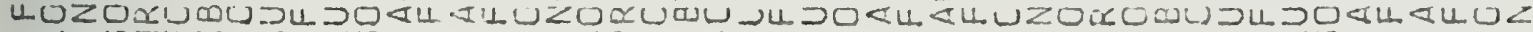

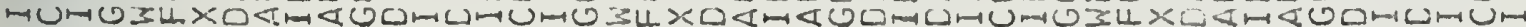

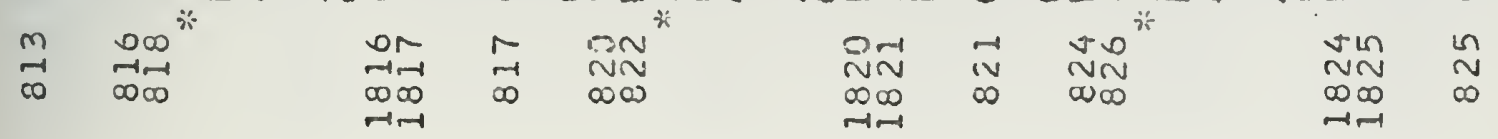


0000093000500000000000000000000.000000000000000300

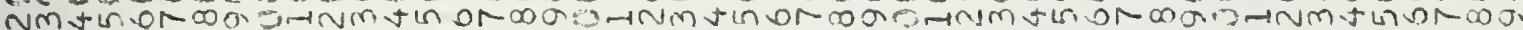

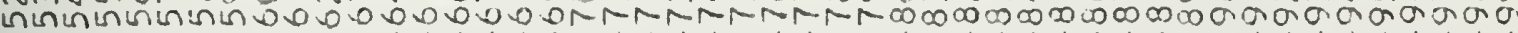

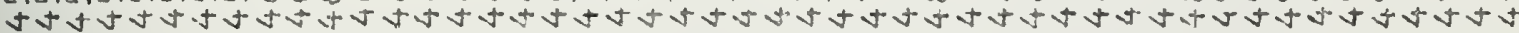

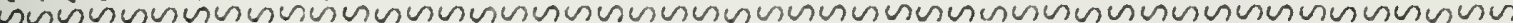

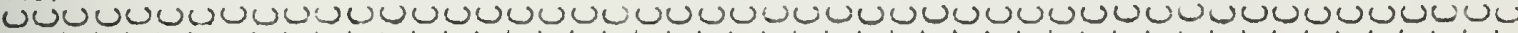

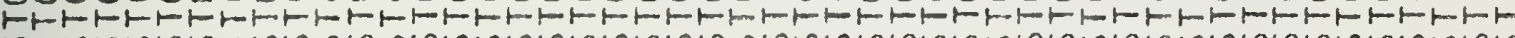

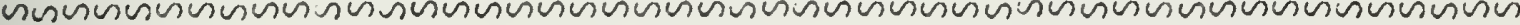

$\frac{1}{0}$

茨

n

$<$

0

$\mathbb{O}_{1}^{0}$

$\leftarrow$

$\sum_{i=1}^{0}$

3

11 0 แयदा

- $m \supseteq 110$

$< \pm \pm \omega \alpha u$

$15 \simeq 741$

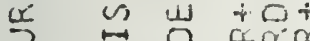

- $\alpha$ cas

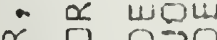

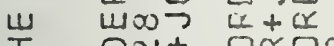

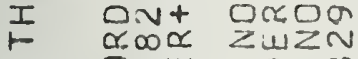

\& $\alpha$ -

u $\alpha z \circ \times a \times n$

प1山

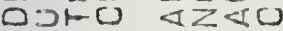

$z$ व $z$

0 บ00$\sum Z 0 x$ कर

$1=11$

$-x-$

$\propto D \propto 0$

世द山心

uा $\propto \|$

एँ \|\|

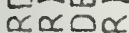

טแल $\propto$ moOO

- $\| \alpha \underline{0}$

Naxo

- $-40+\frac{11}{2}+$

m.n $\alpha \propto \alpha+$

$x-x-$

$9-0-90$

$+0+\infty$

x coó $\infty$

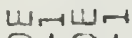

0101

a o. ZuZu

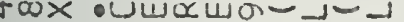

a annzowovx ox ou a $0-\infty$ or $002 x>x$

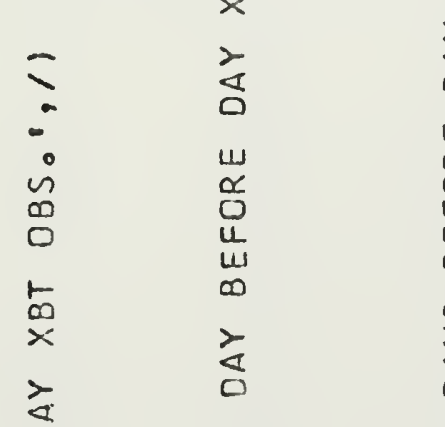

$\frac{1}{0}$

$\frac{1}{0}$

$\frac{1}{0}$

U

인

w

$\alpha$

0

us

$\underset{\infty}{u}$

$\frac{n}{0}$

in

$\frac{1}{2}$

0

$-$

N

m

ก

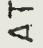

$\leftarrow$

$\leftarrow$

10

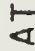

品

2

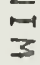

0

$\frac{2}{3}$

$\sum_{i=1}^{0}$

$\bigcup_{0}^{11}$

4

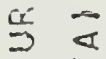

$\rightarrow \infty$

n

I

$u \propto 0$
oud

ous

$>c z$

$10-$

픔

us

$z x \propto$

4ᄂᄂ) II

-OD-

$z \alpha \alpha$

मUu

$\rightarrow 0$

$-11 \alpha$

Noico

- -

$\min a+$

$u$
$a$
$a$

U) $\rightarrow$ U

Uu

느응

$\quad \omega \alpha$

00

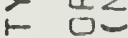

ma $\sum-1$

$\infty \quad 00$

$z \alpha \alpha$

แU แII

$\frac{\omega}{2} \alpha$

wu

-O

त $11 \alpha$

- w

a. $-0+$

$m \leftrightarrow x \propto$

$16 \quad \alpha 0$

प सर

100

m $\sum N$

$\sim$ ल

$z \alpha x$

UI UI

$\leqslant 0-$

$<$ 담

$m 0$

ล $11 x$

$-2-117$

N. $>0+$

ta aco

U1

$\omega$

$<$

$\frac{a}{a}$

4

is

$\frac{W}{5}$

-

$11 \propto 0$

ए w

$>\quad 00$

$1-0=$

in $\sum m$

$\leadsto$ no

$\therefore$ co

-4 ए।

$=\alpha \sigma$

- ৩u

- $\| \alpha$

$\sim \simeq 0$

- - -

$0 .-10+$

$\checkmark$ a c a

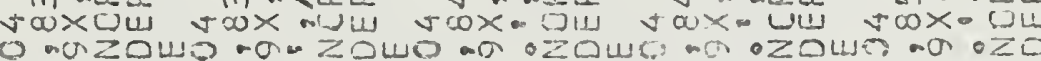

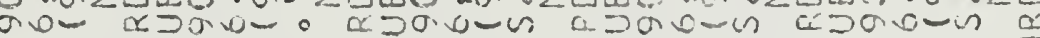
-

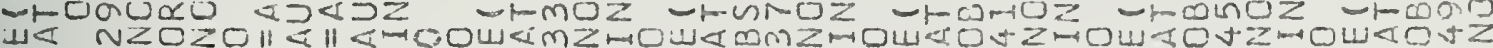
-

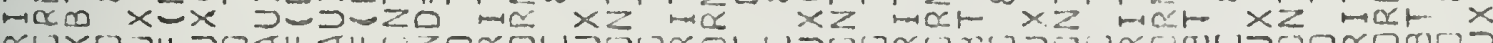

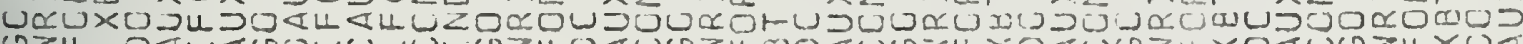

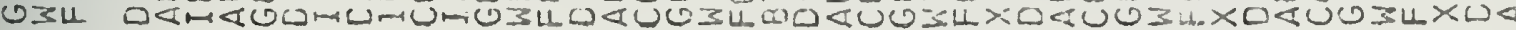

\begin{tabular}{|c|c|c|c|c|c|c|c|c|c|c|c|}
\hline & $\infty \sigma$ & $\sigma$ & No & $m$ & $\cup \infty$ & $N$ & ON & -1 & $\varpi 0$ & in & $\infty ?$ \\
\hline$v m$ & NN & N & mim & (i) & $\mathrm{mm}$ & $m$ & $\leftarrow \checkmark$ & $\checkmark$ & $\checkmark v$ & 5 & जon \\
\hline & $\infty \infty$ & $\infty$ & $\infty \infty$ & $\infty$ & $\infty \infty \infty$ & $\infty$ & $\infty \infty$ & $\infty$ & $\infty 00$ & $\infty$ & $\infty \infty$ \\
\hline
\end{tabular}




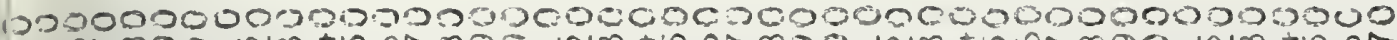

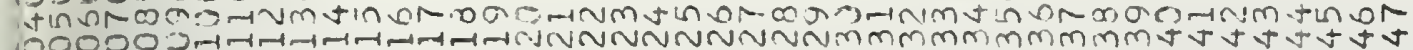
10.

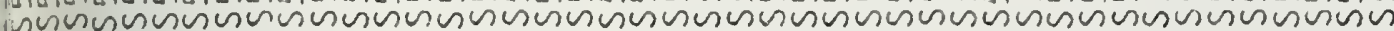

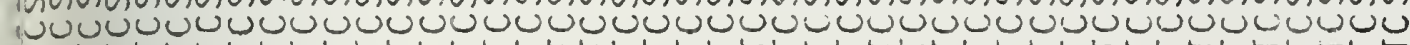

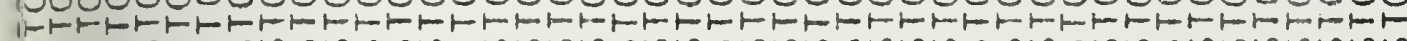

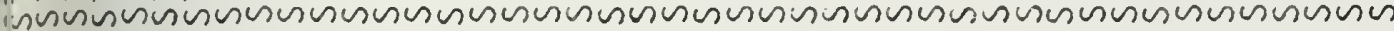

\section{invisunu}
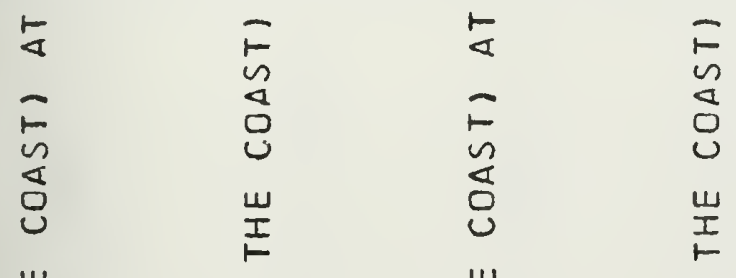

$\stackrel{W}{I}$

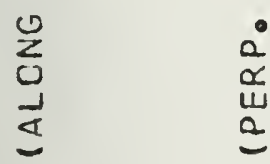

$\underset{1}{\longrightarrow}$

0
$z$
$\frac{1}{2}$

$\sum_{i=1}^{0}$

U

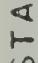

$n$

$\propto$

$\propto 0$

แ०

อ므

느

$0=$

$\leq 0$

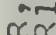

w॥

O-

$\propto \propto \sim$

טய

mo

- $\| \alpha$

$\therefore$

$\because a+$

1. $x x$

$\infty \times 0 \alpha$ $\frac{a}{a}$

$\sum_{3}^{0}$

ш

-

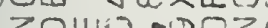

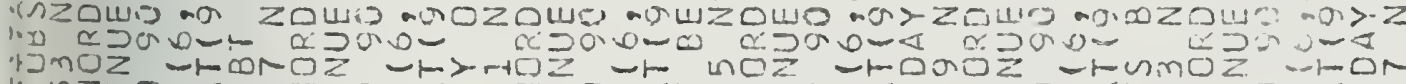

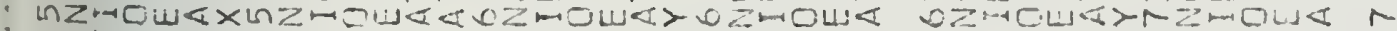

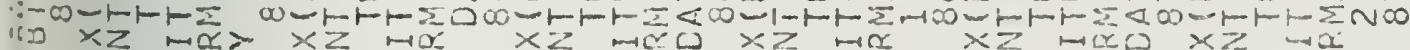

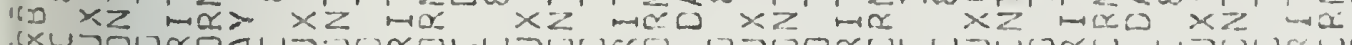

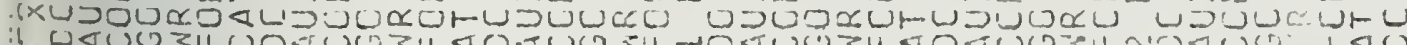
₹Uण_ILFO

\begin{tabular}{|c|c|c|c|c|c|c|c|c|c|c|}
\hline $0 \infty^{*}$ & $r$ & $u N^{3}$ & -1 & $+\infty^{x}$ & in & $\infty=$ & a & $N=$ & $m$ & bळ \\
\hline เท่เก & in & 00 & $\omega$ & $\omega \infty$ & $\omega$ & ON & 0 & $r-1$ & r & $N-N$ \\
\hline$\infty \infty$ & $\infty$ & $\infty \infty$ & $\infty$ & $\omega \infty$ & $\infty$ & $\infty \infty$ & $\infty$ & $\infty \infty \infty$ & $\infty$ & N \\
\hline
\end{tabular}



020000000000009000020000000000000000100002000000

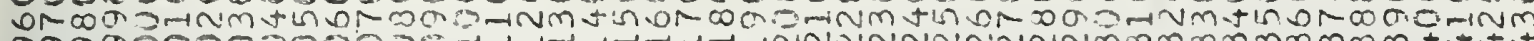

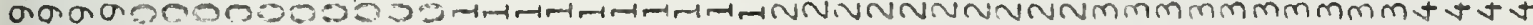
Lnhln 0000000000000000000000000000000000000000000

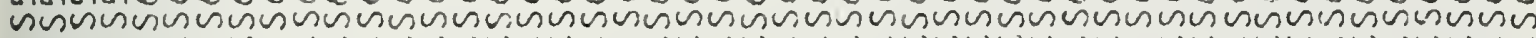

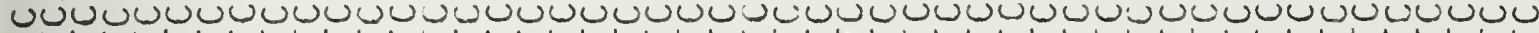

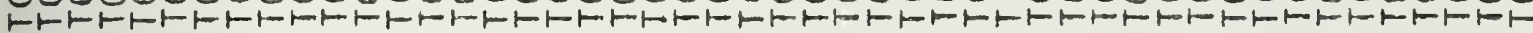

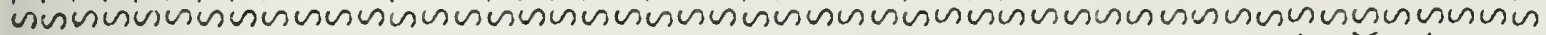

$\approx \tilde{\sim}$

$\tilde{w}$

$\frac{2}{3}$

w $m$

$\frac{x}{n} \lim _{n \rightarrow 1}$

$\rightarrow L$

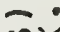

$m x$

$z \mapsto-10$

$0=-1$

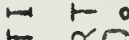

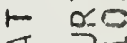

$\lesssim$ U.

$\vec{w}-\dot{x}$

o mo

$\rightarrow$.

$\mapsto-1$

$=0$

$0 \quad 36$

in

- - -

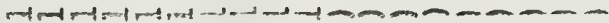

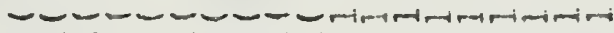

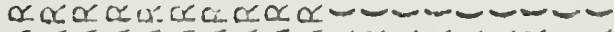

यदर

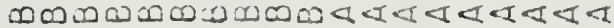

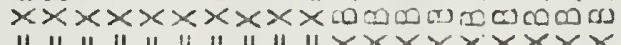
|| || || || || || || || || || $\times \times \times \times \times \times \times \times x$ 近U иงหsunususust-トトトトトトト

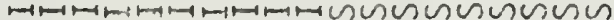

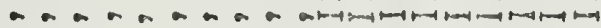
toncoginmomb..........

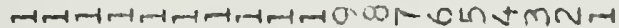

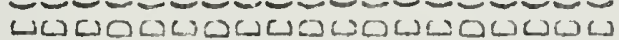

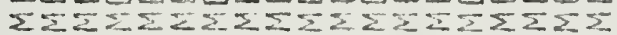

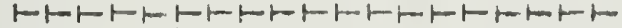
トートトートートートートートートートー

NNNNNNNNNNNNNNNNNNN

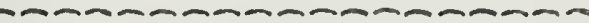

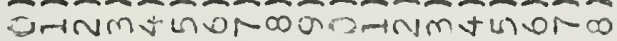

in

$\rightarrow-$

$\bar{m}^{\infty}$

m

z

it $=0$

$\sum 0-$

$1 \mathrm{tu}$

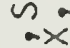

$-\ln$

$n m^{n}$.

य $\rightarrow-$

$\overrightarrow{0}$

\& $x$.

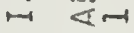

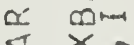

$>+\infty$

- in-

$\rightarrow \rightarrow \infty$

$x \mapsto$ -

- $000000.000 .000000(3-N=$

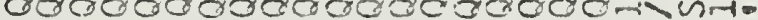

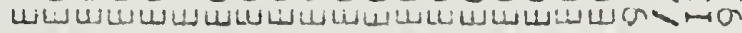

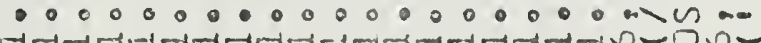

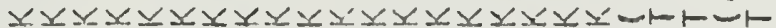

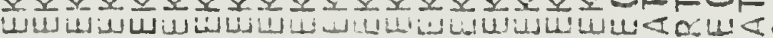

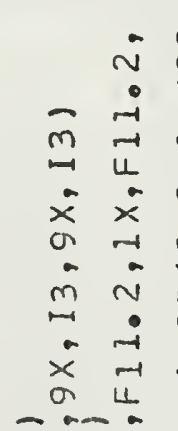

चिलिx

$+m+-$

Nan

mxmo

円心的

zलixन

$\sum \mapsto a u$

an a

$-x-x$

मरना

$+\rightarrow+$

CN N V -

$n=m$

tomi

$-n-z$

zo.xu

Zuख⿰口

- o.

$-11=-1$

$-10-10$

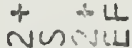

mamo

m小心

-<io

$z a \simeq Z$

$2 \quad 0$

$-0,0$

N vo

$m$ oin

$m: 21 \cdots$

$-\geq-\alpha$

$z-n x$

x 3

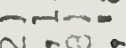

N>mi

orva

n> $\sim$

Q $0-00$

-trow

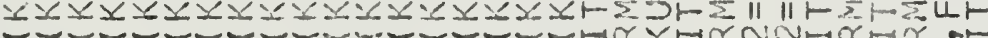

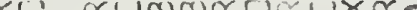
UUUUUUUUUUUUИHUUU

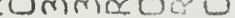

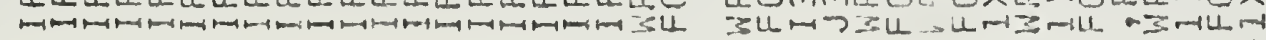


000020020025000003000202000000000200000200000000

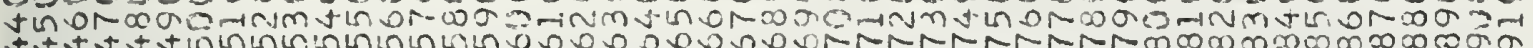
0000000000000000000000000600000000.00000000000000

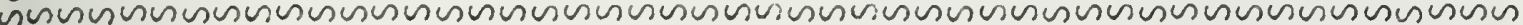

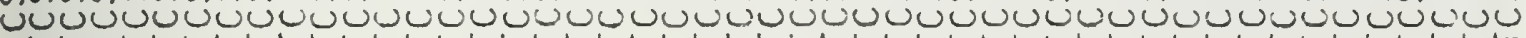

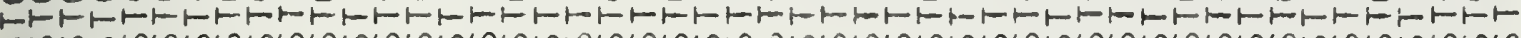

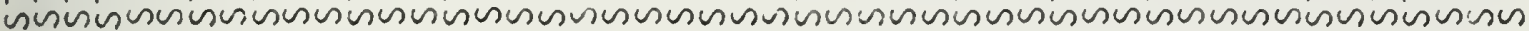

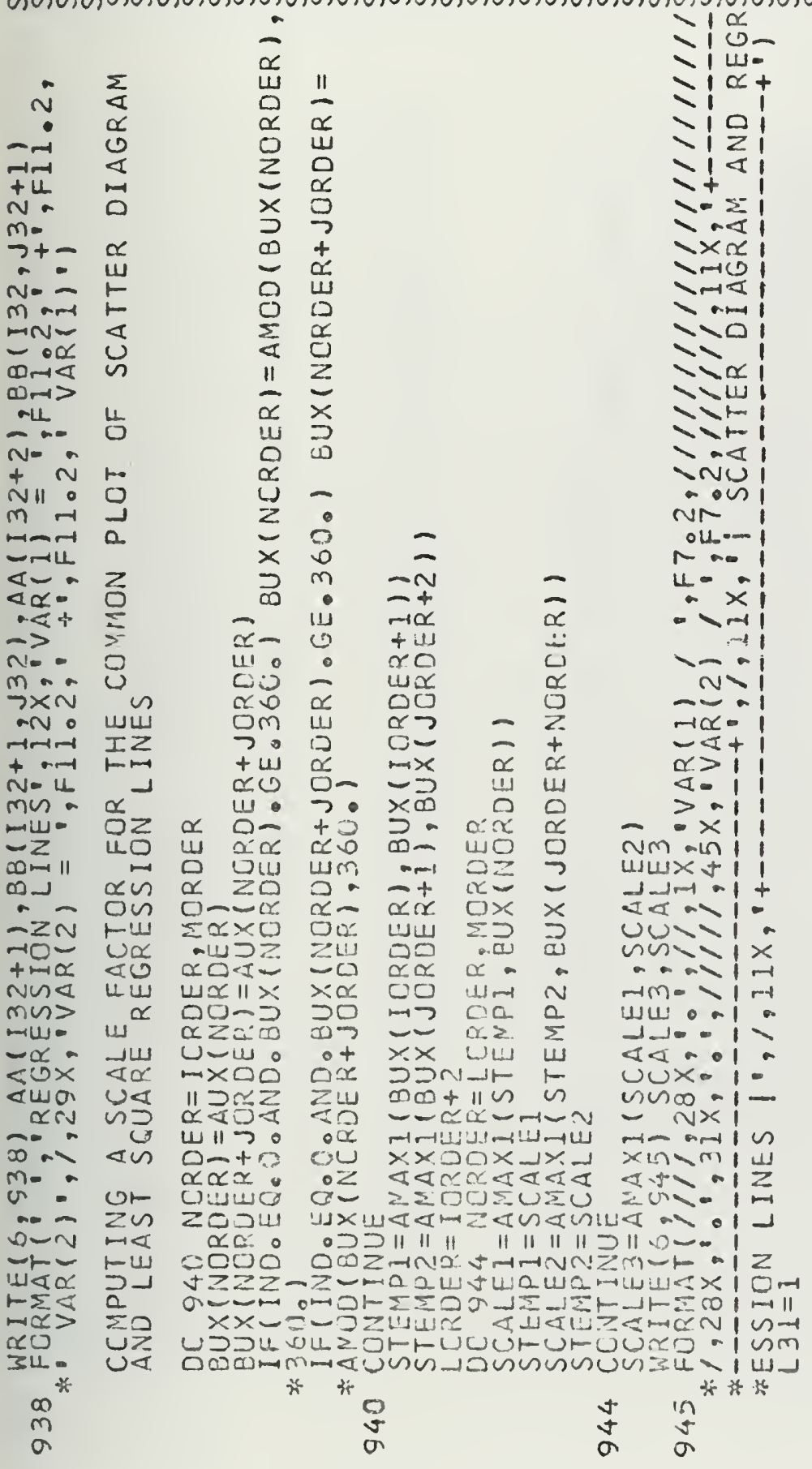

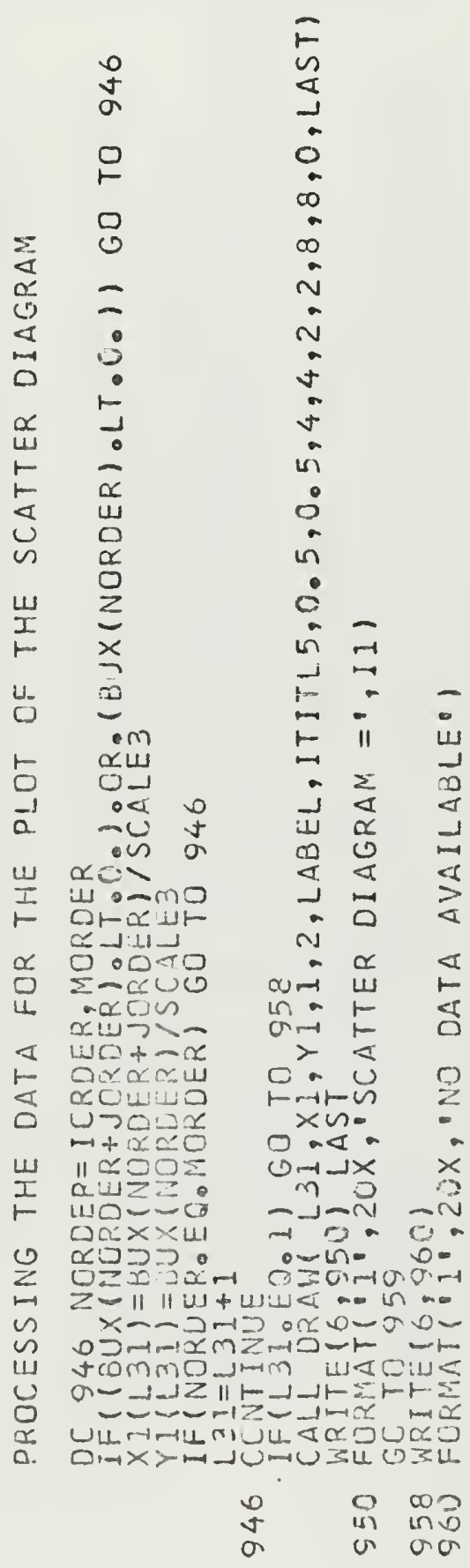

Uư 


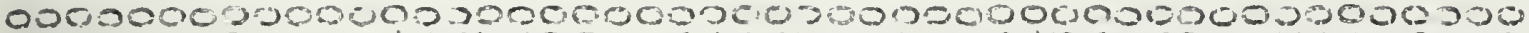

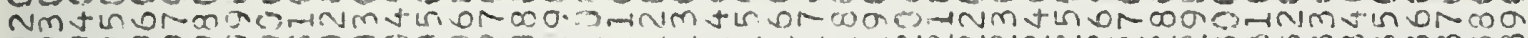

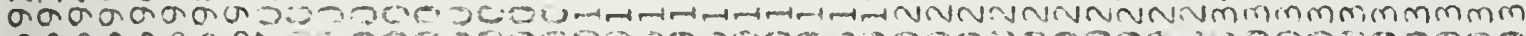

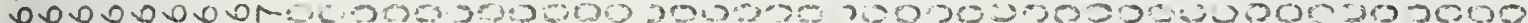

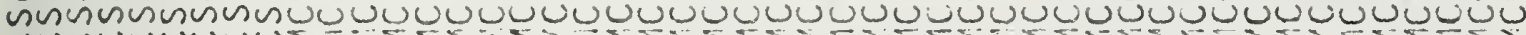

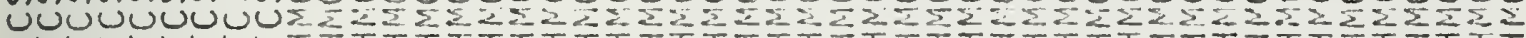

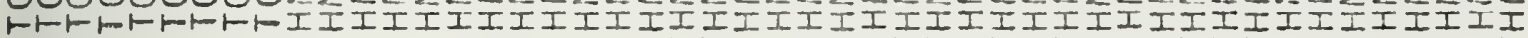

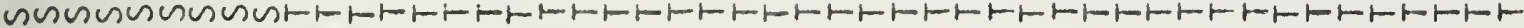

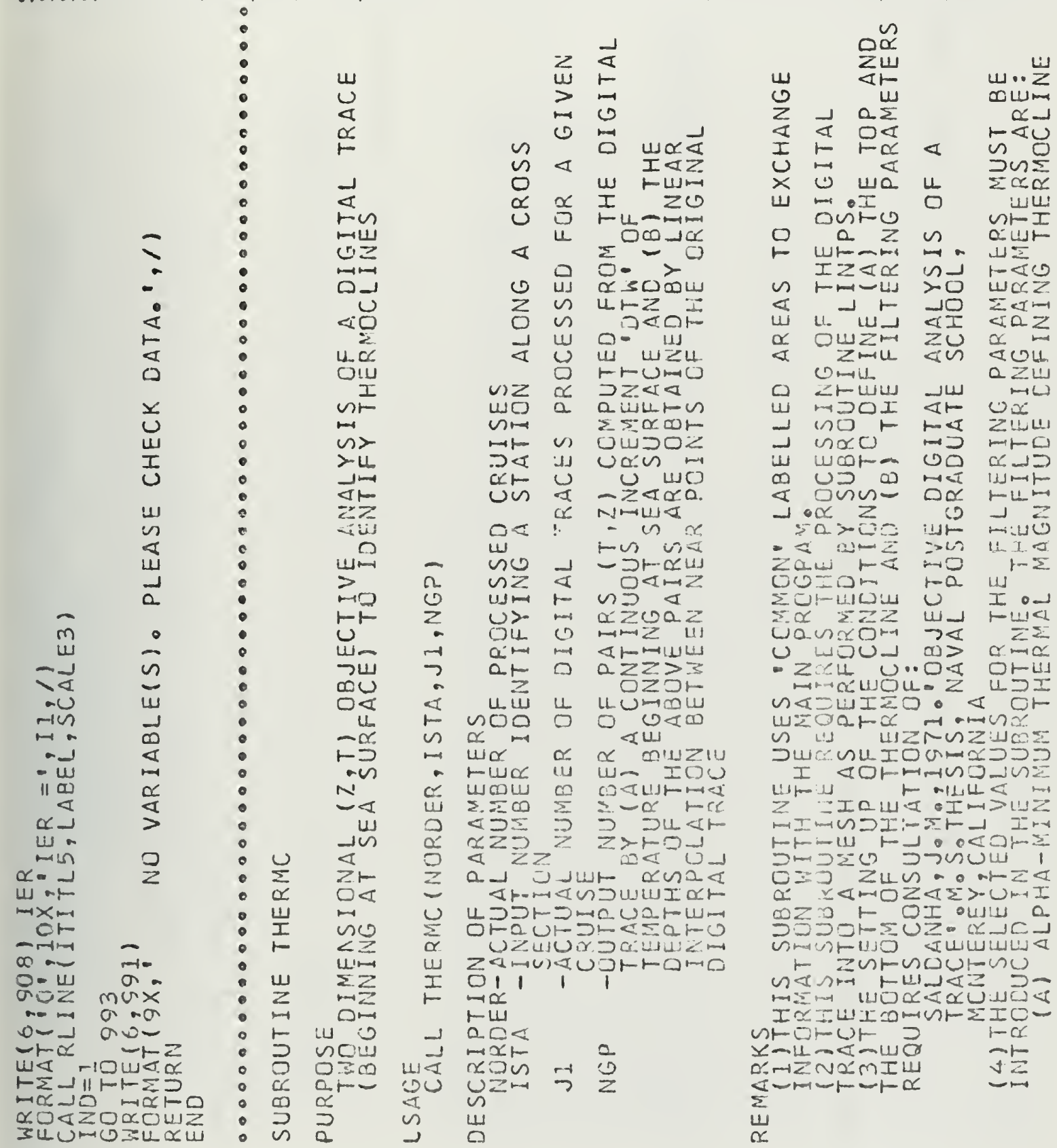

incos 5 Pretm

ana a o.aro 
000000000000000000 omnmtin on tatatatata.tatiminisiniminin $0013010000 \% 320000000$

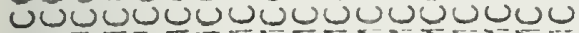

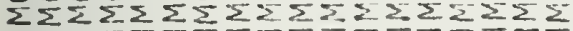

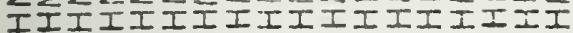
トトトトトトトトトトトトトトトトトー
$00000010300=3002003003230=000$

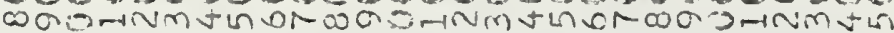

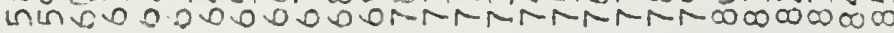
0.2050 .912000050000206300000500

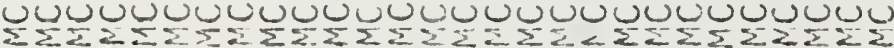

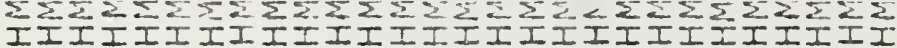

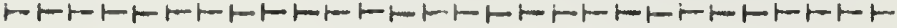

\begin{tabular}{|c|c|c|}
\hline & & \\
\hline - & $\infty \cdots$ & \\
\hline in & NNT & \\
\hline-1 & nth- & \\
\hline$+-\infty$ & $\infty+\infty$ & \\
\hline$-n \infty-\cdots$ & 000 & \\
\hline $0-x \ln =\ln$ & NNT & \\
\hline$r v=3 \supset-i L n-1-1$ & $\sim-\infty$ & \\
\hline$-N<\smile-\infty-$ & งDm & \\
\hline M-NUL-OIL & $r m>$ & \\
\hline 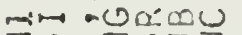 & III & \\
\hline Im-Z0=2 & $1-F$ & \\
\hline IIn< & NNT & $\omega$ \\
\hline NবேनNடト & $a \infty$ & $z$ \\
\hline aN- $-N \cdot a$ & $\cdots$ & $\Rightarrow$ \\
\hline$-a-\infty n-\infty$ & NNN & ــ \\
\hline O-xin-ing & $m-1-1-1$ & 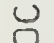 \\
\hline 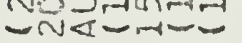 & $a=0$ & $\sum$ \\
\hline
\end{tabular}

n-Naterto men alivose ... IM-U0D \&IC $<D<<<m>$

NGWUदि III

ANFNN a F FF

- - a m- Nitr

- $-m-m$-nin

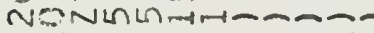

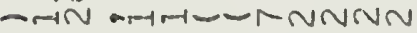

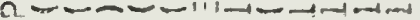

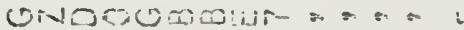

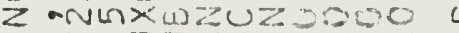

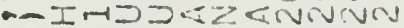

m-1

TNNNHN mUYmeSOS

$a,-1+N \cdot \ldots-a-m i n=4$

<-A m-

-

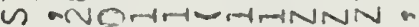

$m=-4=-m$

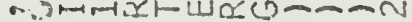

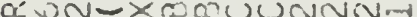

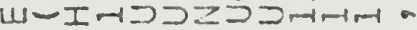

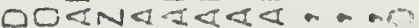

ONN ATNNNI :- EON

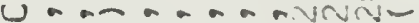

$z-2-2-\cdots-\infty$

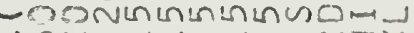

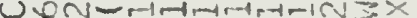

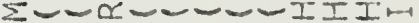

rousdo000RमFE

Ut $N \times x\rangle=0$ UINNNN

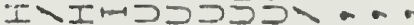

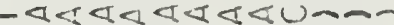

XNNNFFNIYIVNN

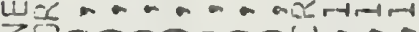

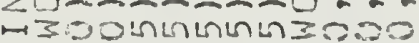

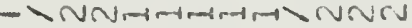
DZー- - - - Z- -

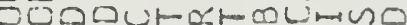
a $\sum-1, n \times x \times \cup u \Sigma N 3=$ ดรエエつつつつつะII

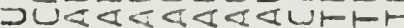
nUNNNEFNIUNN

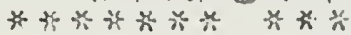

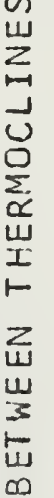



is

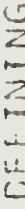

in

5

$\vdash$

$z$

0

2

$\frac{1}{8}$

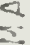

L

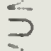

2

2 u

w

2

1

$n$

0

$\underset{1}{0}$

0

$5 \quad$

w

$>$ U

$z$ uा

年

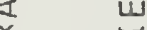

<० 0

a U ₹

wo $\%$

$n \propto$

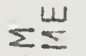

$\sum_{i=1}$ $x$

Z

Ero 5

11

$\varangle<\ll$

$\vdash \vdash$

แ $\frac{3}{1}$

U U I
- 



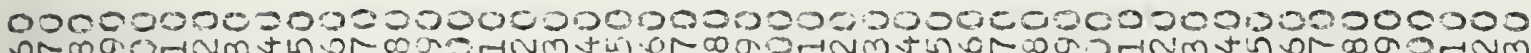

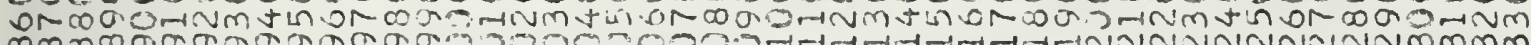

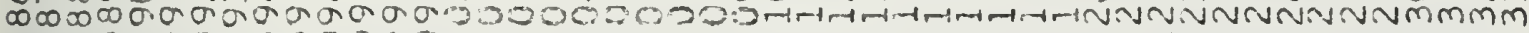

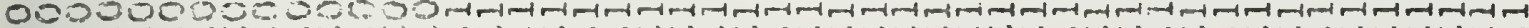

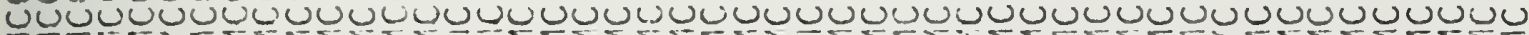

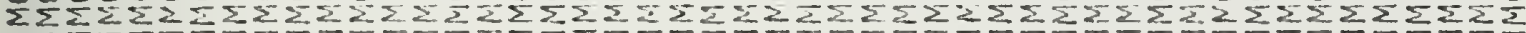
IIIIIIIIIIIIIIIIIIIIIIIIIIIIIIIIIIIIIIIIIIIIIIII

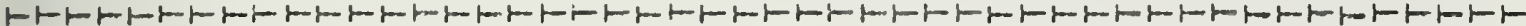

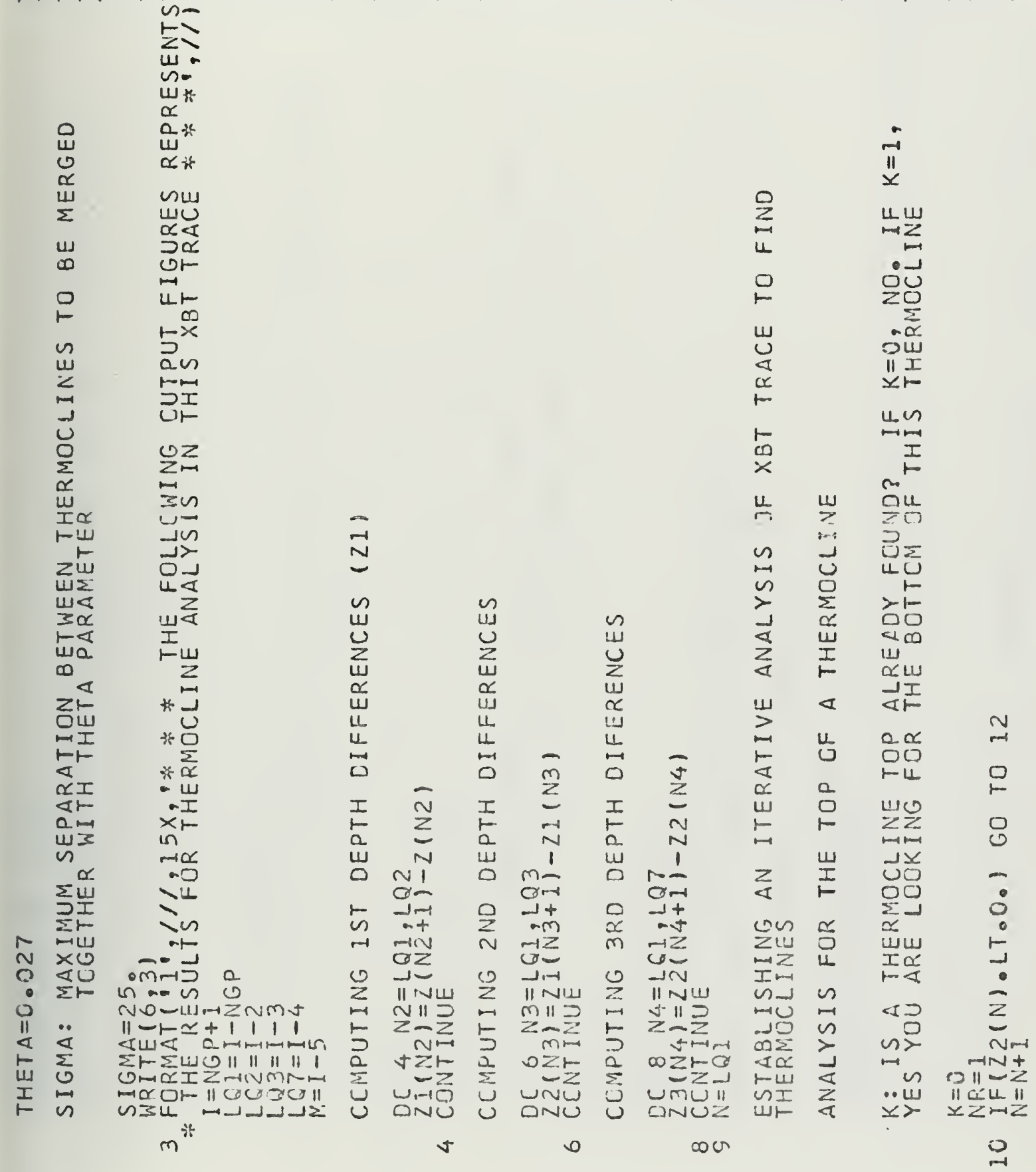


000000000000000000009030030000009000000000000000

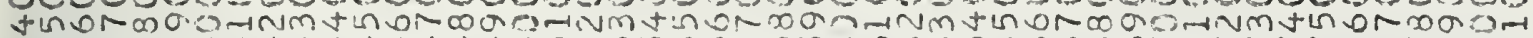

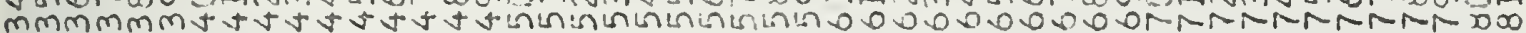
Hली

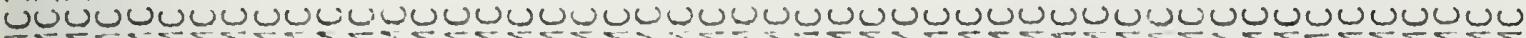

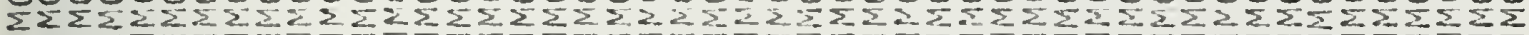
IIIIIIIIIIIIIIIIIIIIIIIIIIIIIIIIIIIIIIIIIIIIIIIIII

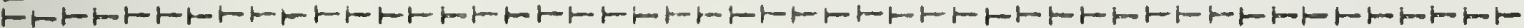

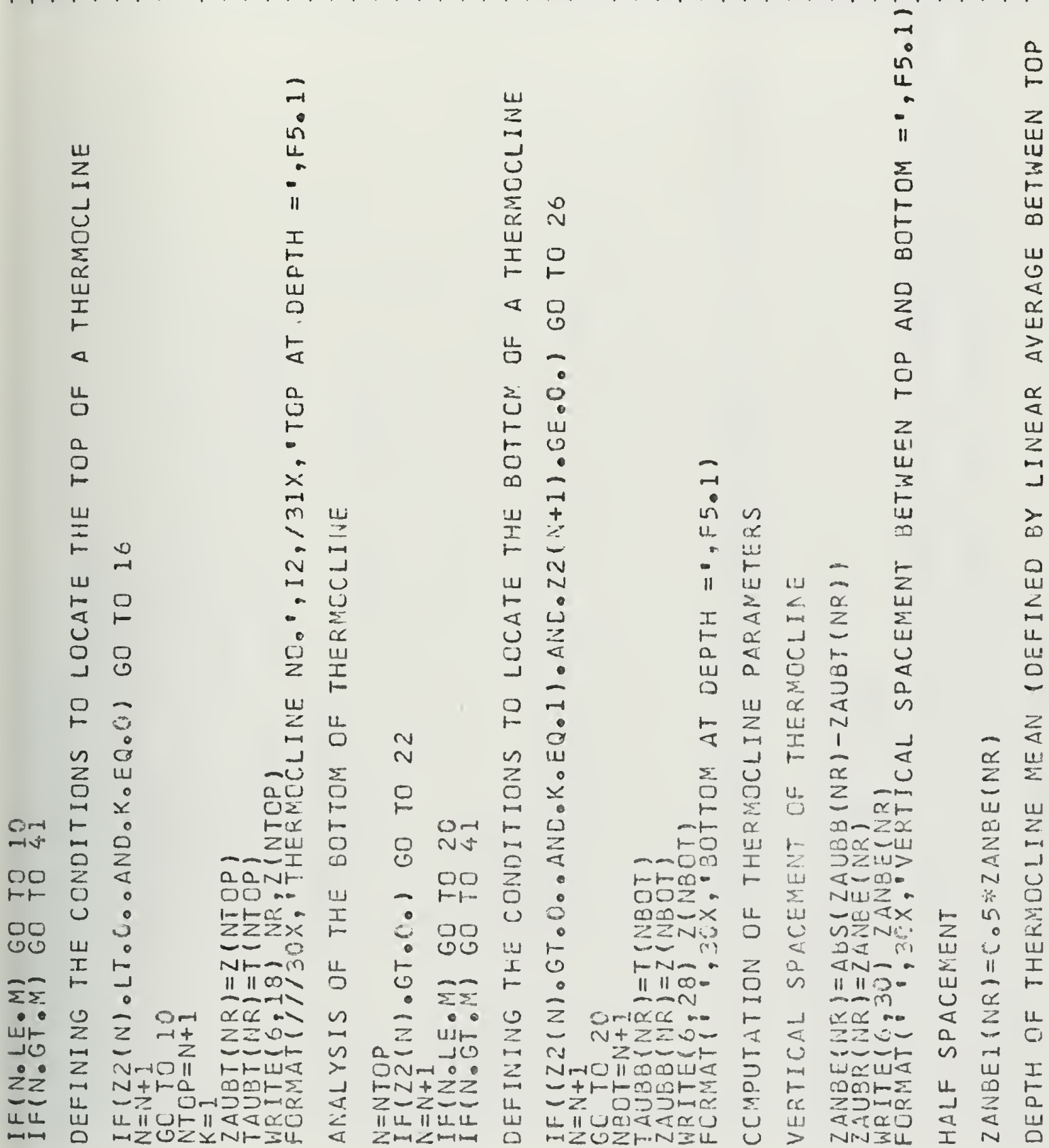

$$
\underset{-1}{N} \quad \infty
$$

궁

Niv ou

$\stackrel{\infty}{\sim}$

m 
0000000090000000020000000000000000000020000002003

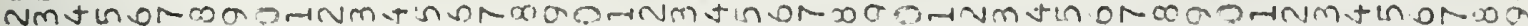

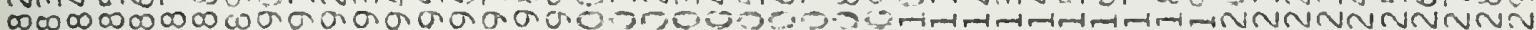

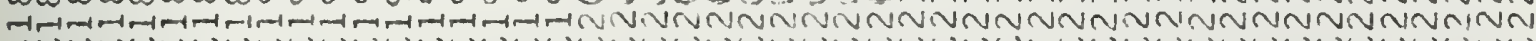

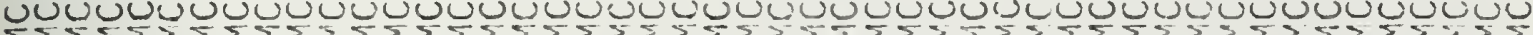

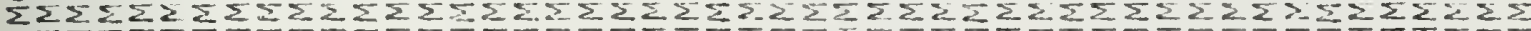
IIIIIIIIIIIIIIIIIIIIIIIIIIIIIIIIIIIIIIIIIIIIIIII

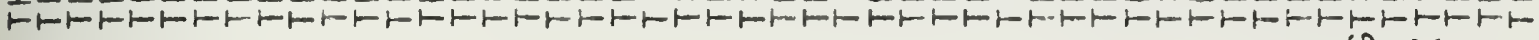

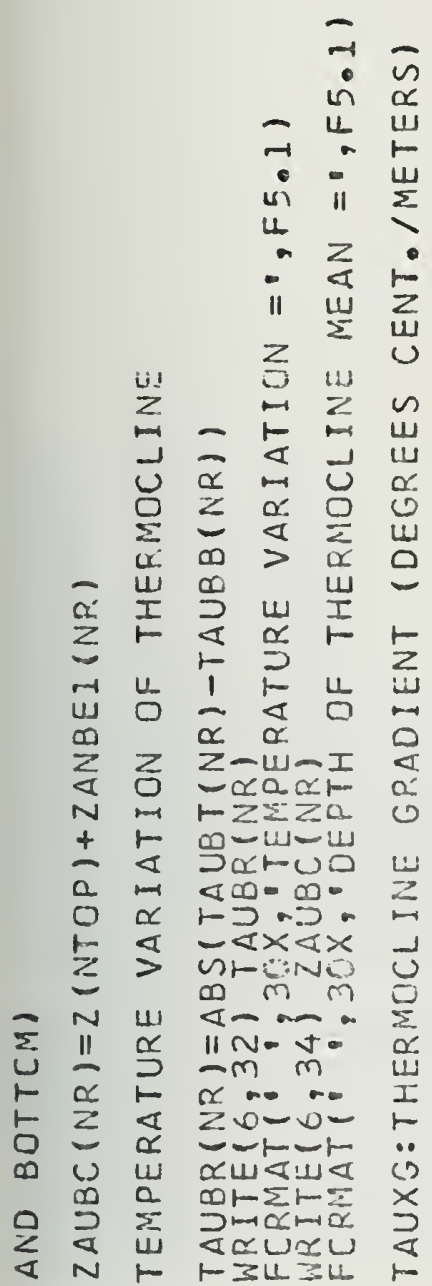

m $m$

0
0
0
11
-1

$\sum_{4}$

แ

0

- 뭉

ㄸ.

$\sum \frac{11}{z}$

w

$\infty \rightarrow$

$2 \omega$

a

$\sqrt{\alpha} \alpha$

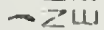

준드

$-\infty$.

$\propto \supset$.

$m<x$

$\sum r$

r-

$110-$

$\frac{1}{4}$

$\geq 0-n$

$-1=+$ or

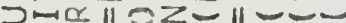

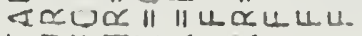

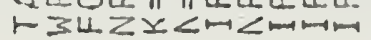

c)

$0 \quad 150$

- $0 \circlearrowleft$

0

- $\sum_{2}^{u}$

$\rightarrow$

III

4

$=$

0

II

崩

i

$z$

뜬

$+\|\|$

$0 \geq 2$

$\sum$ Fin

ar 0

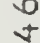

0

1II 11 it OUNA (1) ZZZ

v or

Z

$0=$

$\alpha$

은

$\frac{1}{6}$

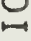

다

w2

$\rightarrow$

of

I

$-\infty$

2

02

C. 2

Cᄂing

n 2

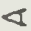

$\infty$

nद

U

$z 2$

$\rightarrow 0$

Uit

$0 \varangle$

2.c

$\therefore<1$

แล

IIU

$r n$

$4-1$

oर

$\geq$

Ur

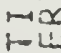

10

$\cup>$

U

யल

nᄂ

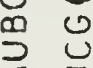

\&

。

4

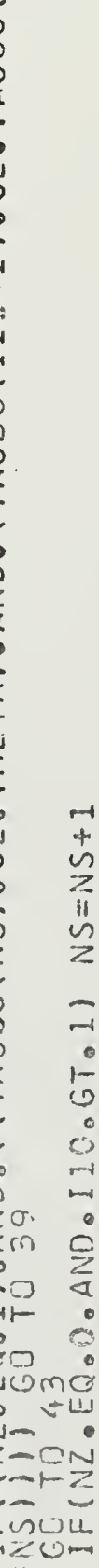

ti 7

索

(r) 



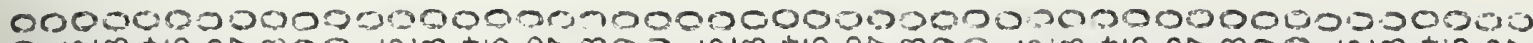
D. HNM mmmmmmmmmis

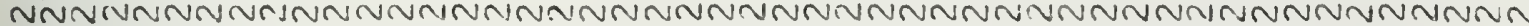

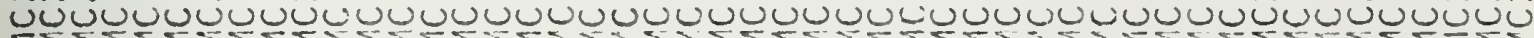

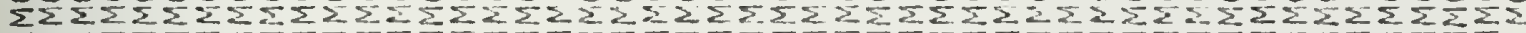

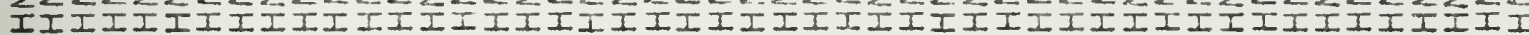
トトトトトトトトトトトトトトトトトトトトトトトトトトトトトトトトトトトトトトトトトトトトトトトト

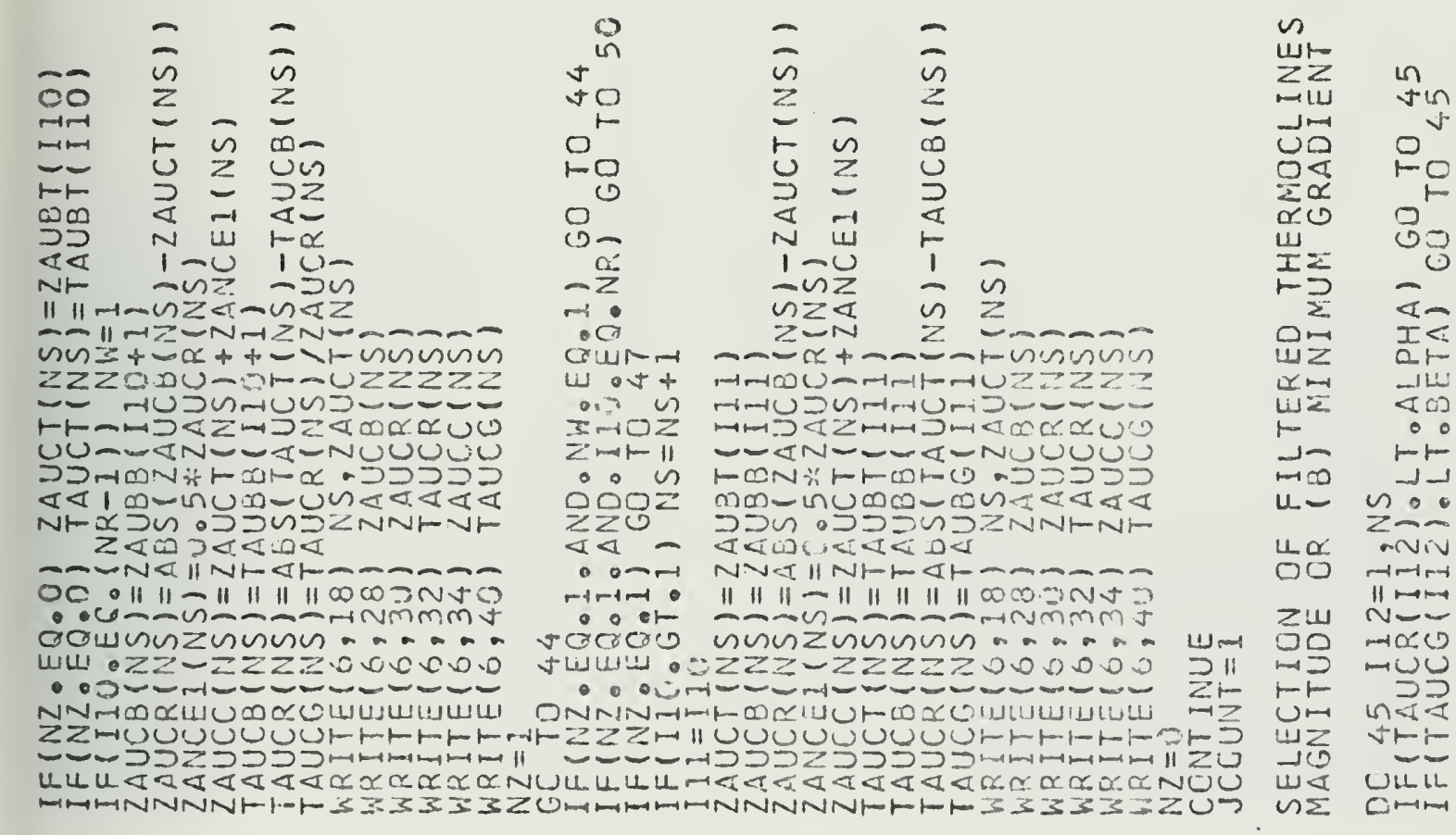

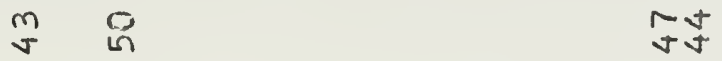




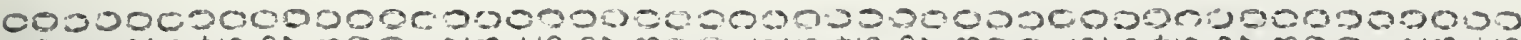

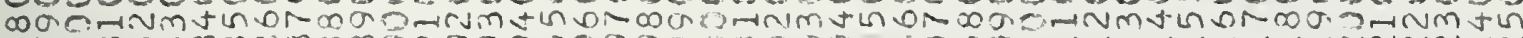

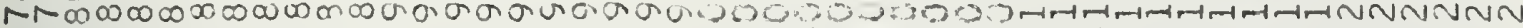

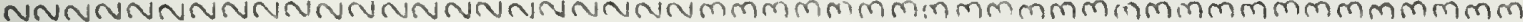

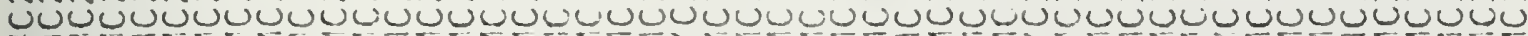

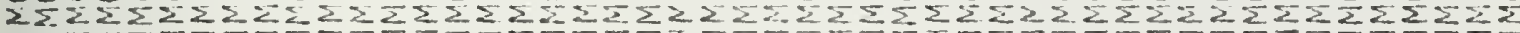

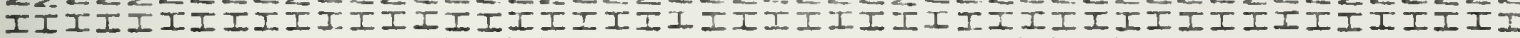

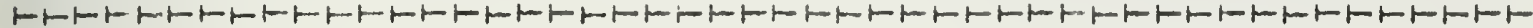

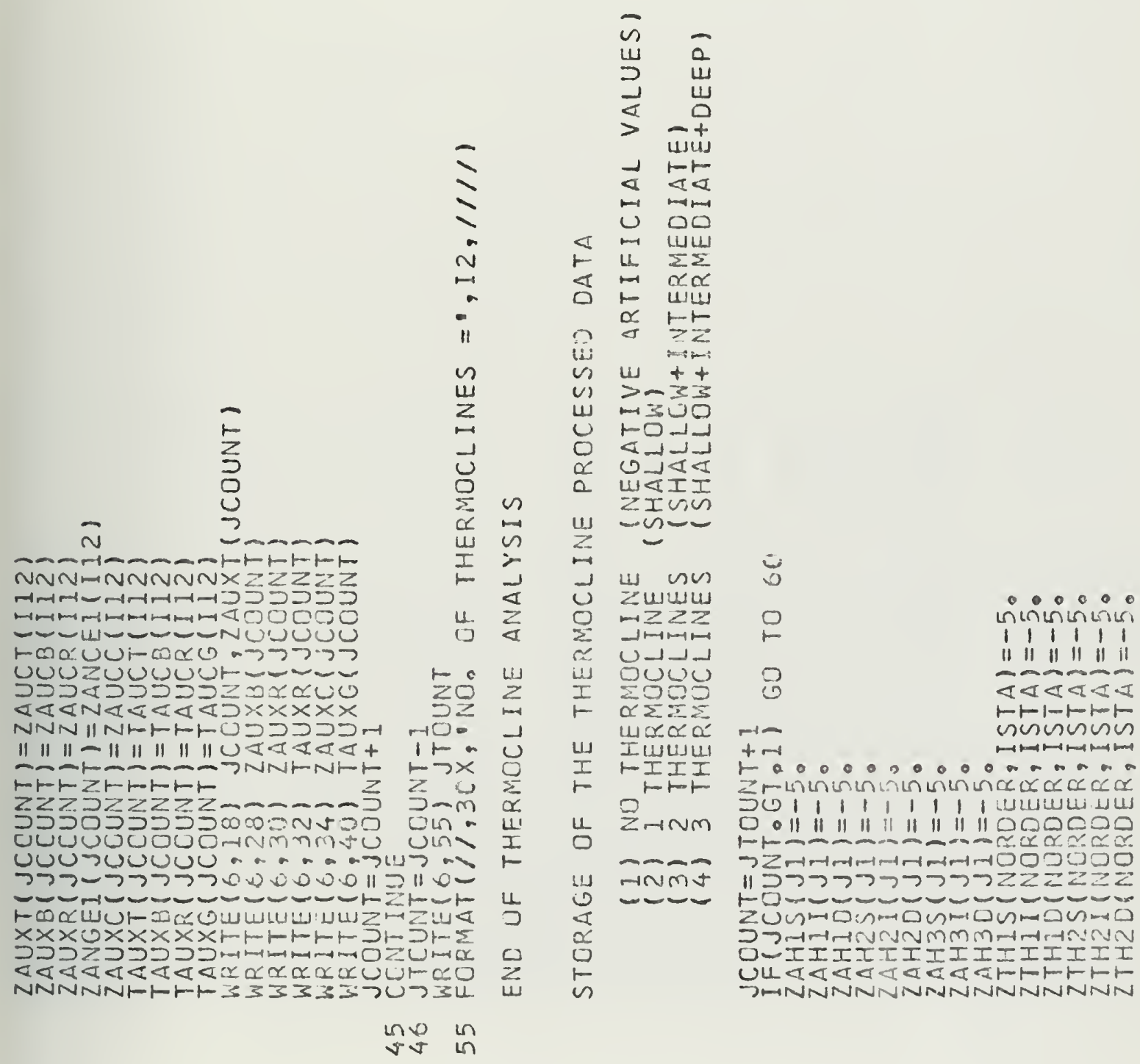

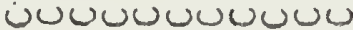




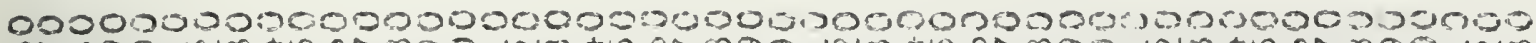

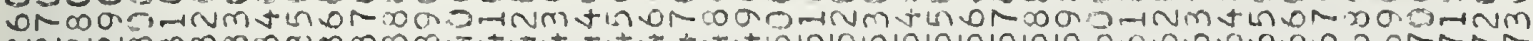

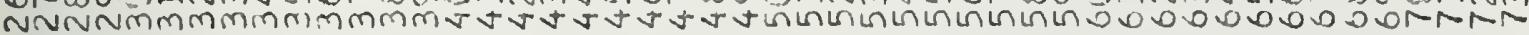

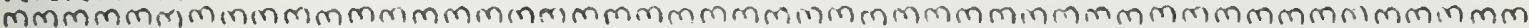

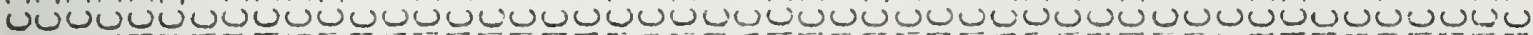

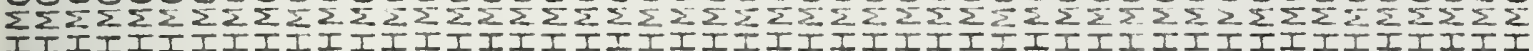
IIIIIIIIIIIIIIIIIIIIIIIIIIIIIII王IIIIIIIIIIIIIXII

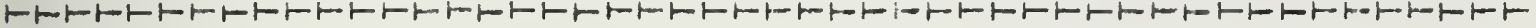

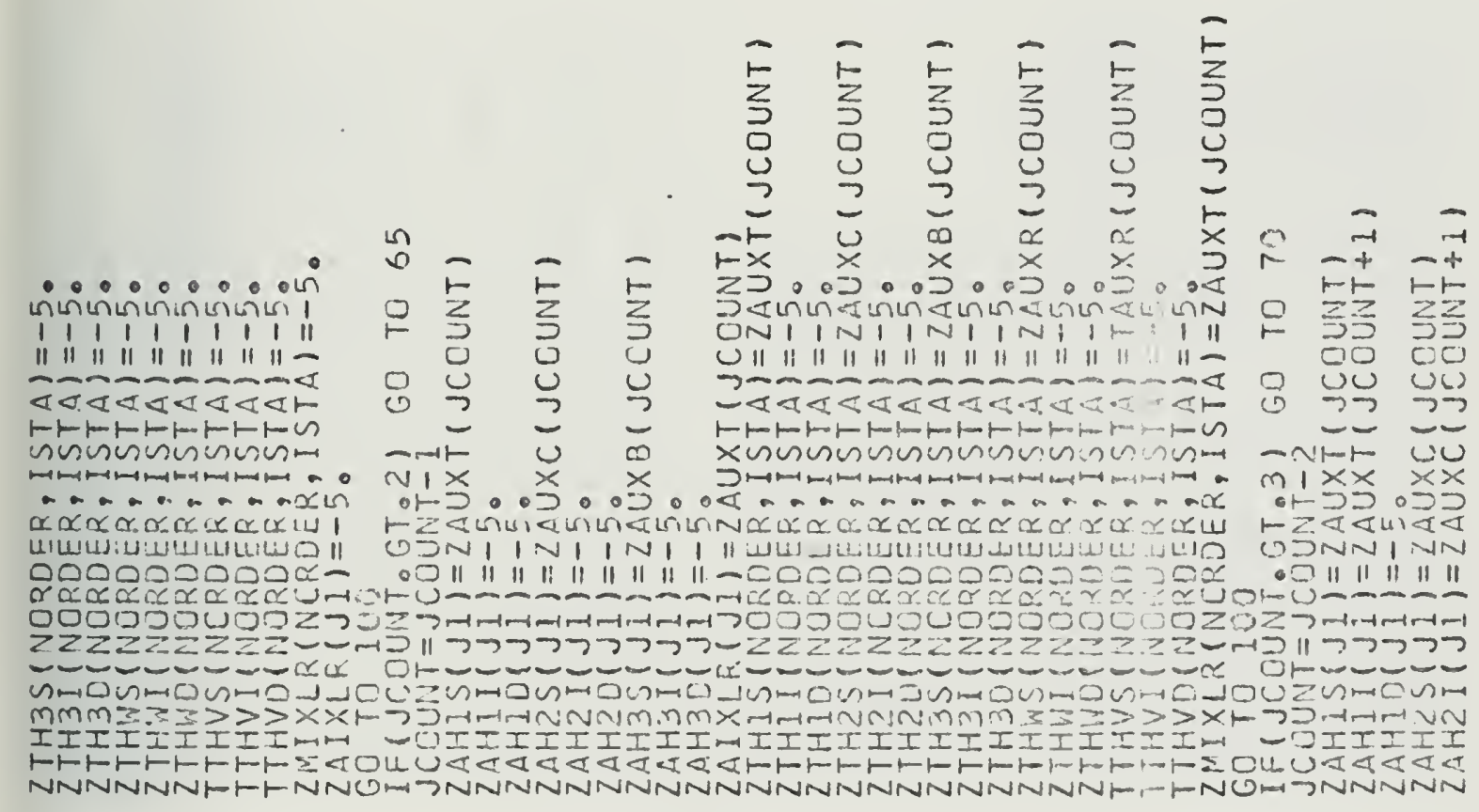




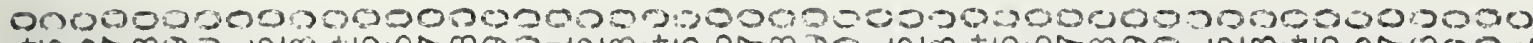

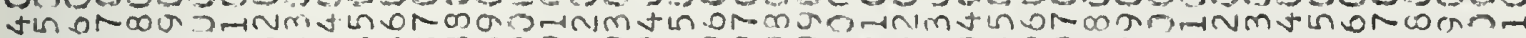

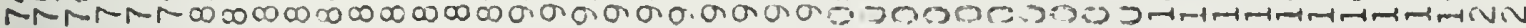

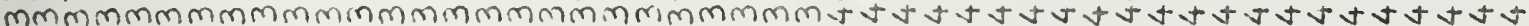

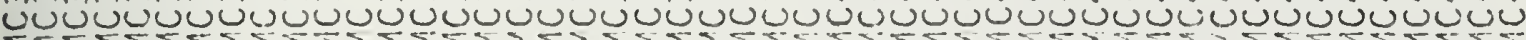

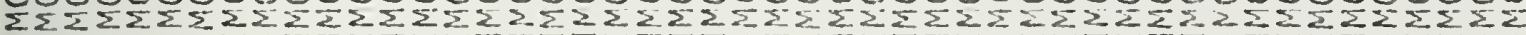
IIIIIIIIIIIIIIIIIIIIIIIIIIIIIIIIIIIIIIIIIIIIIIII

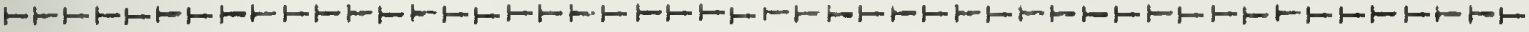

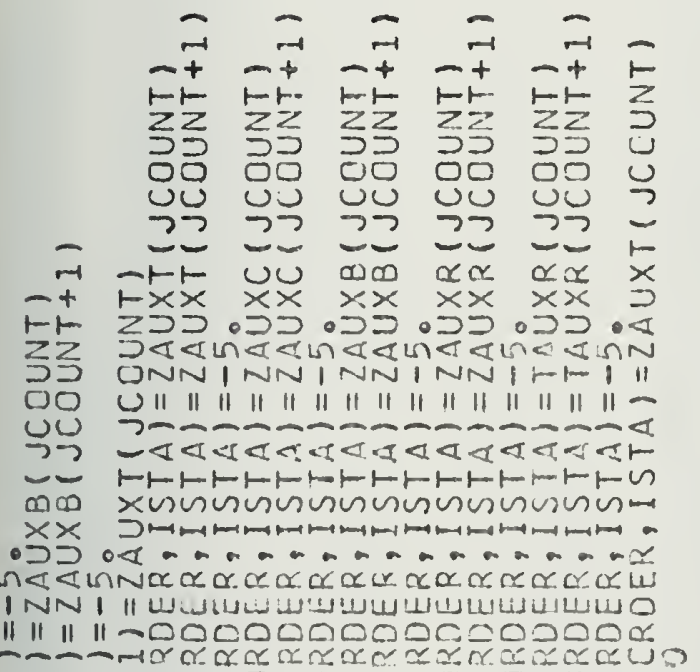

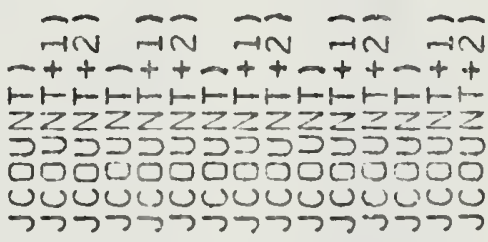

$-1-\infty$ - $-\infty$ -

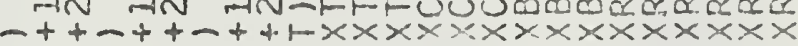

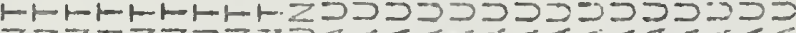

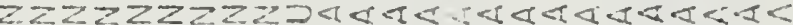
$5535555303 N N N N$ INNNNNNNFr-

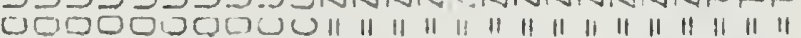

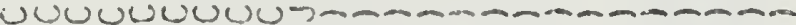

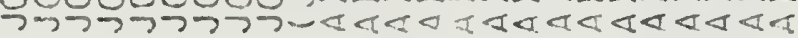
ーーーーーーーーートトトトトトトトトレトトトトトト -t Lư umm

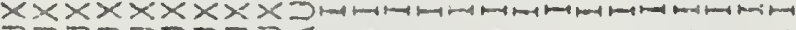

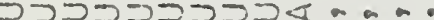
<

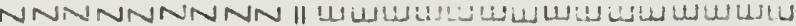
"| || || || |" || || || || -000CD0000000000

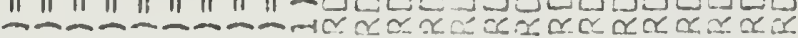

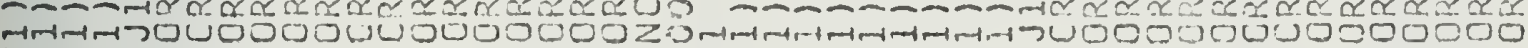

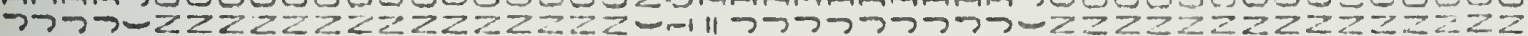

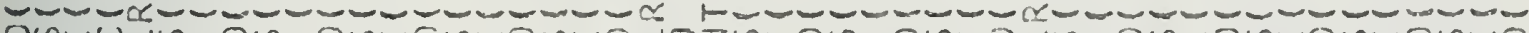

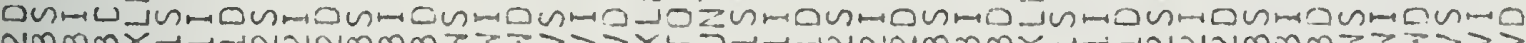

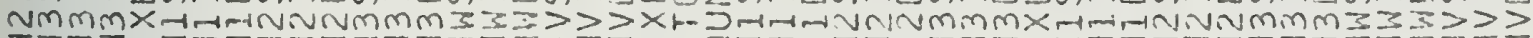
IIII

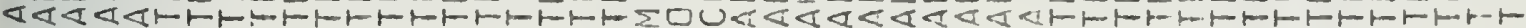

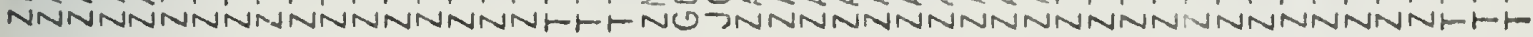

r 


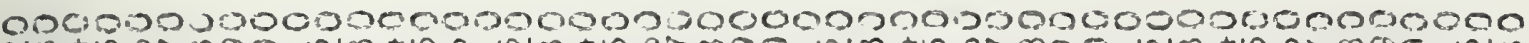

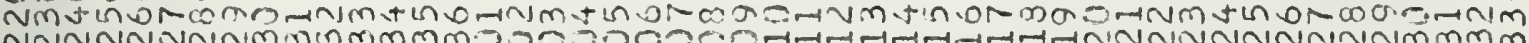

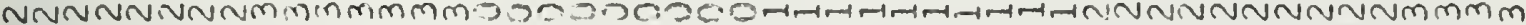

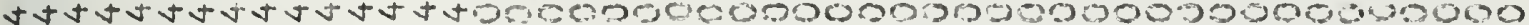

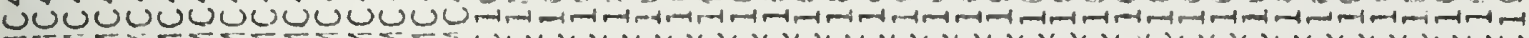

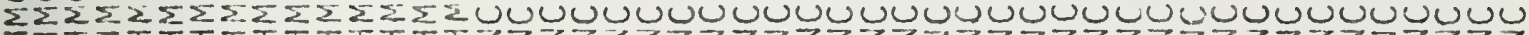
IIIIIIIIIIIIIIIZZZZZZZZZZZZZZZZZZZZZZZZZZZZZZZZZ

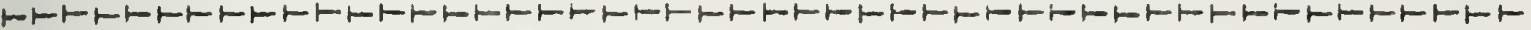

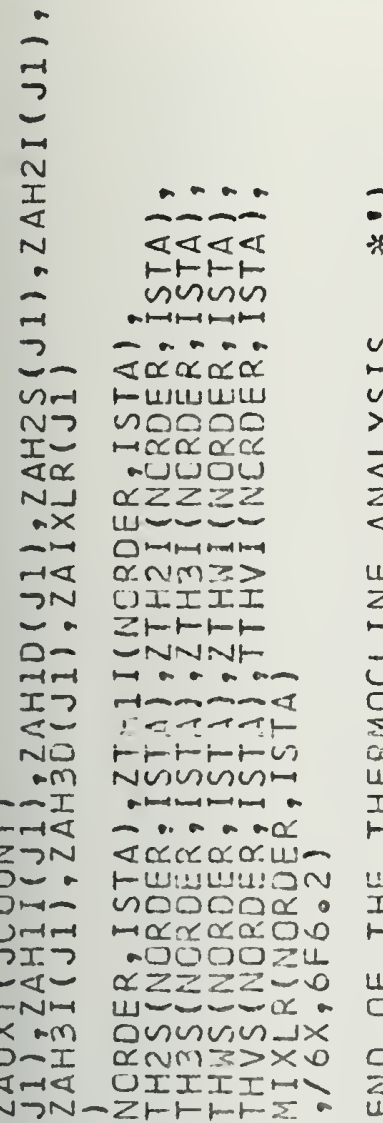

In

山・ロ

땀

Pun

$\varangle \propto .0$

$\alpha \vdash 0$

แU

a -12

$\sum \pi \omega$

Ut $>$

the

400

in 3

nut

$\mapsto I$

UI-س

$\infty I$

0

ะงกษ

$>Z u$

-4! : 2

owh

rw

$\varangle \mapsto \infty$

$\sim 2$

$-0 z$

व

$>z n$

$\alpha \leq z$

แ⿰口口

$1 \geq<$

$2 x=$

$\mapsto$ U

$0 \propto$

Int

it n

zZn

-แH

แU

$-30$

$z+\infty$

Uు

2. 01

u. $z$

or. $0<$

บ내

Z1 -

யम<つ

ตับบัน

$0,1] \alpha$

$a$

2 $\frac{4}{0}$

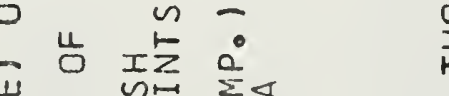

$\alpha \vdash$ ज० $\sum_{4}$

$\supset$ in $\Sigma a$ ru

$\leftleftarrows \ll$ सा

$\alpha \quad I 0<0$

네

드

w 00 טய

:

zZ ४

is i $\frac{1}{2}, \quad \overrightarrow{4}$

जun in $2 \pi \frac{1}{4} 5$

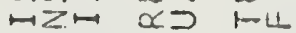

UOU Ut UO

$\because n \infty$ ZU \&

বा一⿺

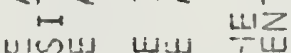

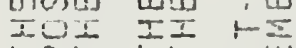

FQ.F FE W

UZU ज৩IZU

ZwZ ZZजm佂

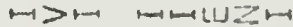

ZッZルZZร

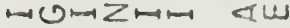

$<<0<\ll \omega h I$

-0!以トトIZ!

nスーストZZト0

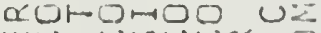

шо unuor 0

† 4 U OLU

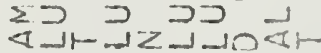

$q\langle\backsim\langle 1 \omega<\measuredangle 11\rangle \geqslant$

$\checkmark>\alpha>>>>$

0.

トUトートトメつ-

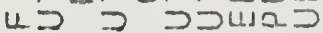

onua żZะZzแ⿻心

Zाम

$\omega$

a

एद

0

4 in

ज แ

$\rightarrow=10-1 m$ 00 0 $0 \sum^{\infty} \pm$

แบ

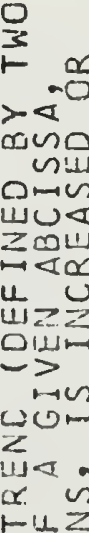

142

40

I:Ut

1 Dr-

in:

$0>0$

zแ山>

$0 x>0$

H下OU

- 01

$<-<Z$

$\vdash(v)$

ZZu

WOII

セセトル

$\alpha \vdash \quad u$

Q๓z:

Low

工O 프

- 15 لن

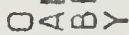

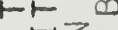

जएँ

$\angle \mapsto$ U.

$\mapsto 00$ N

Q $U<\pi$

× ZトW

$0 \gtrless \varangle \alpha$

Q $\square \cup \cup$

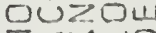

$I<x+10$

-

$\Sigma$ 


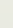




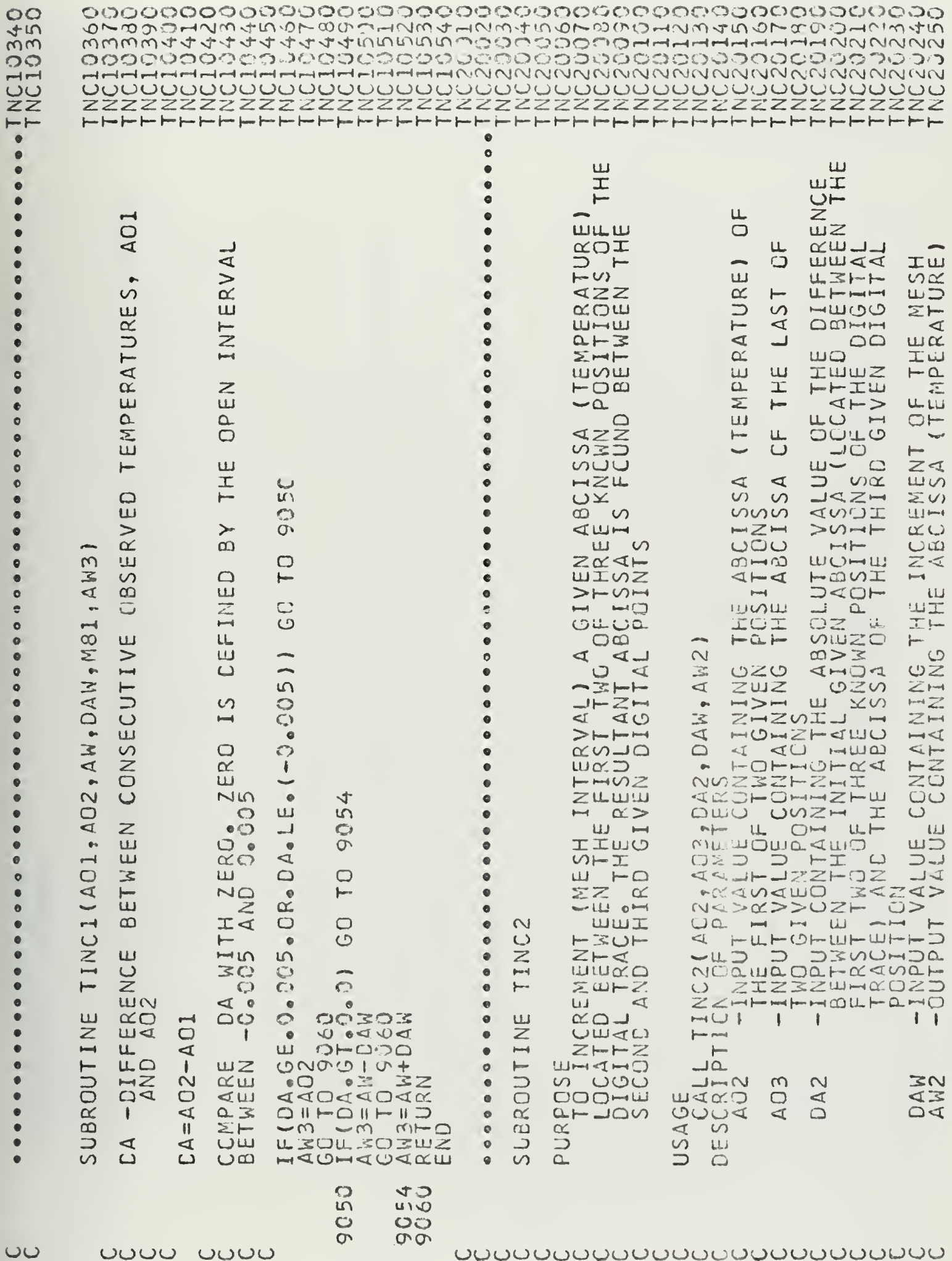

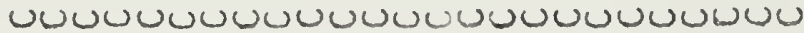


ODOODODOJO5030

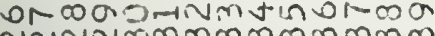
NNNNMMmmmmmmm

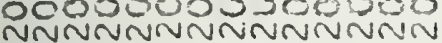
UUUUUUUUUUUUUUUU トレトトトトトトトトトトトト
000300000050006200000000000000.30

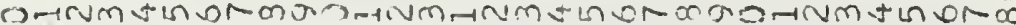

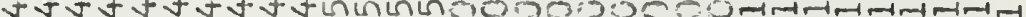

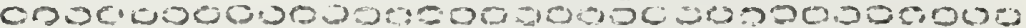

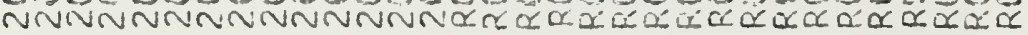

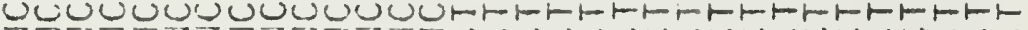

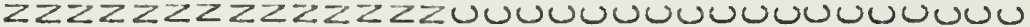

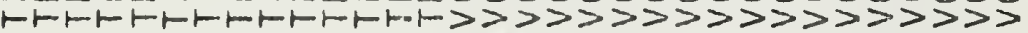

noún

onven

யいZいル ZnஈUZ ज小

U. $\backsim<5$ WZM

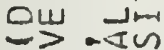
मUZठ w०Zस $\angle 04$

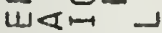
$\propto$ - $1<$ 늗 OजाI.แ०O 0 tw $\equiv$

$4.7 \leq \leftarrow$ $0>\vdash-\frac{1}{4}$ zแ) $>-1$ OI $\operatorname{In}$ mixuo - 다는 $\varangle-4 Z F$ -n ZZUU工出 แด.III ロツレルト

$0+2 \frac{1}{2} z$ งแ जоแயแ I $2 \geq I 3$ एu $1-\infty$ $\lim _{0 \rightarrow 0} Z$ Zn w moono ○顸山 व ZR-UL वर $<0$ 0ugưu I $x \geq 0$ w
U

$\vec{n}$

니

$\alpha$

$\widetilde{\alpha}$

แบ-

or

$-N=$

แ二

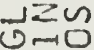

zus

व

UILU

山上

Iト

는

U

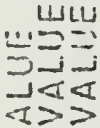

$\langle I\rangle>$

$>$

แบแ

UII

Ir

(o)

$0 z z$

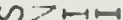

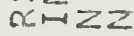

in

- 0 - o

प0 जी

$\alpha:<$

w口 o 0

$\therefore \propto$

Fa D UN N

\# 10 \& <

In

$0=0$

$=0 \frac{1}{3} \frac{1}{3}$

$N<0$ कर ब

wo De $\rightarrow$ ound

Zद 1 watr-ou-

$=1 \quad 000104$

tm uz on onvin

20

떠

(5) 닝 $0 Z$

$a \geq 0 \vdash 0\|1\| D$

IF - - N NTO

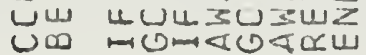

$$
\begin{array}{ll}
2 & +0 \\
0 & 00 \\
0 & 00
\end{array}
$$

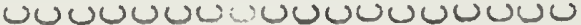

IZ 0 O- . 
0000 a. O.-1N HNVN 0000 axa トトト ưu $>>>$ $n$

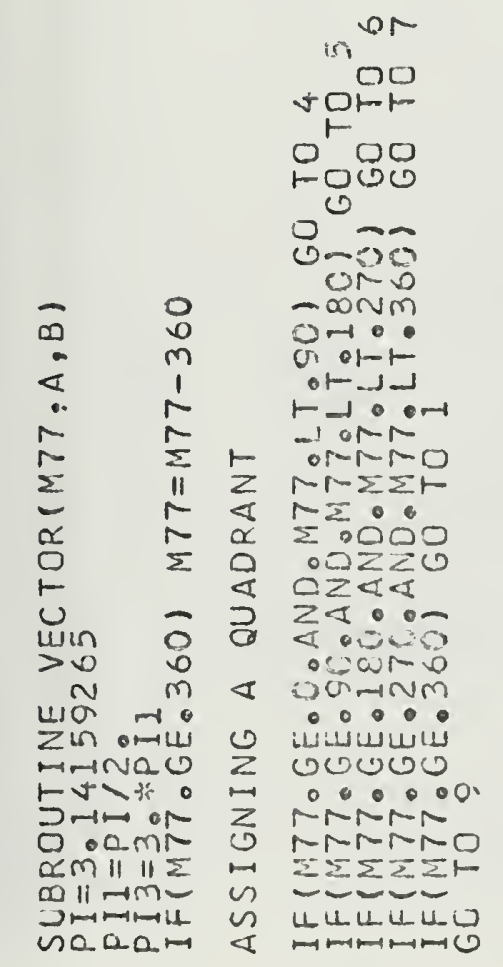

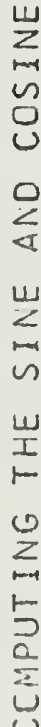

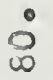

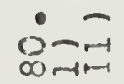
$=0$

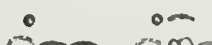

Con $0 \mathrm{~mm}$

$-1 \frac{1}{10}+10$ $>-a 1>11>10$

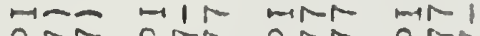

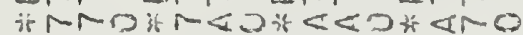

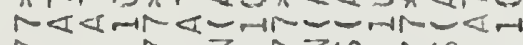
N-N N-z Nzn Nun

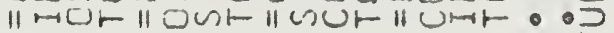

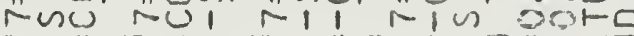

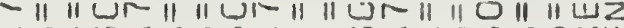
पदणण $\rightarrow$
5
in
○
N
or 0

\section{0

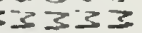

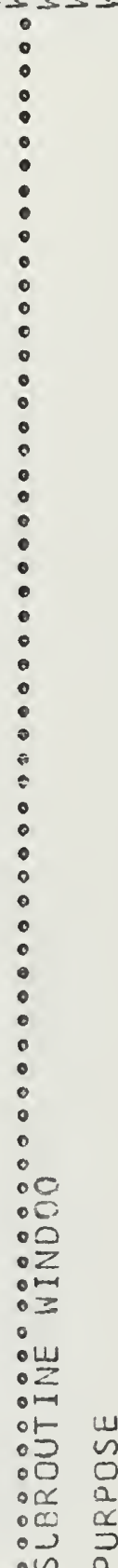

ưu

ư

טu

טuׁง 


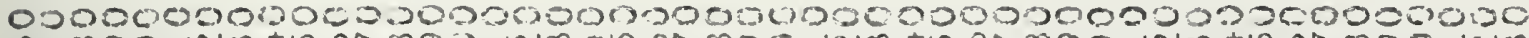

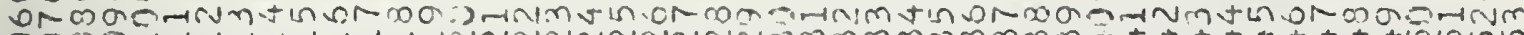

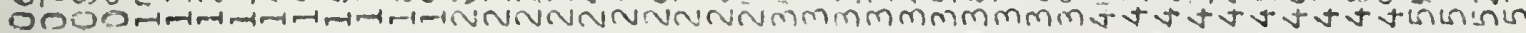
0000000000306.703000000500000005000001200500000 .20

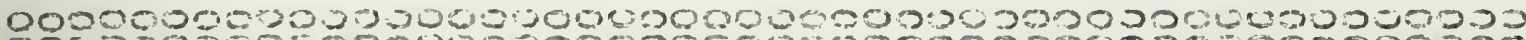
006.50002005000050500060500000000000000000000000

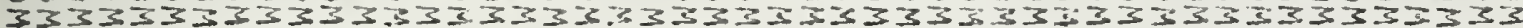

wr

IOQ

It

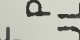

Dou

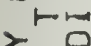

$4 \cdot 2$

oz⿺

ய๓口

ILW

inn

믈

I $<$

$I$

षण

$1-U z$

$4-10$

I

Wद

$\alpha \alpha-$

Dont

not-:?

बाuZर

แலயᄂ

$\Sigma \sum 0$

40

$>\sum a x$

- $4 \sum \ll$

an⿺

$\cup \cup Z$

Qw

JIvu

U尸匕

$>$ mb

$\vdash$

$0<04$

mo<

322 400

U 0 m

Iயน

FuU <แuس

$n x>\alpha$

जा म

แ 0

U.

0बा

$x \vdash 3 I$

$0 . n$ F OU

U以IO

HOFト

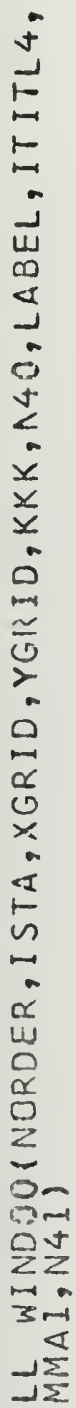

山区 oux is

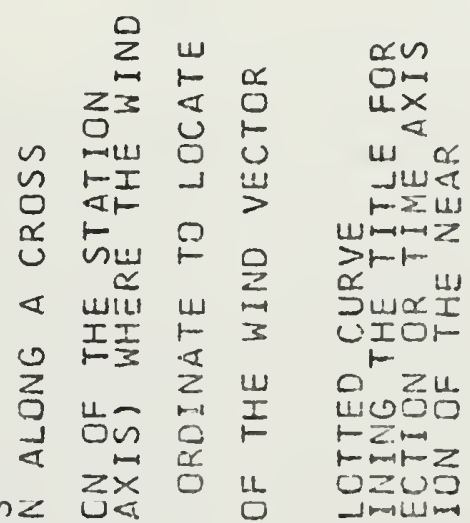

政

n

$\because 5$

Ur

Qr

un

ox ur

जis

$\sim 2$

U⿻

U>

04

맘

a!

แய

ज๐冋

$\propto$

110

-U⿺

$\sum \sum \infty$

व

$-10<2$ at of - GoবUUIL c. 0 0. বトロトz

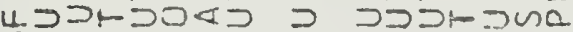

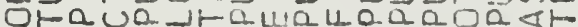

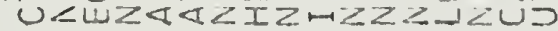

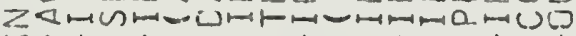
i. 111 $\rightarrow \infty$

सiय 0 Q

aç

orou 0

$\cup=\operatorname{x}$

is

แั

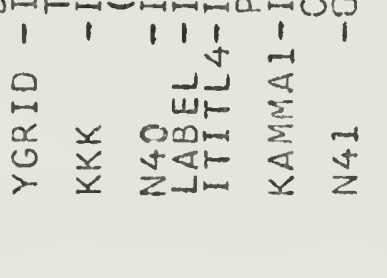

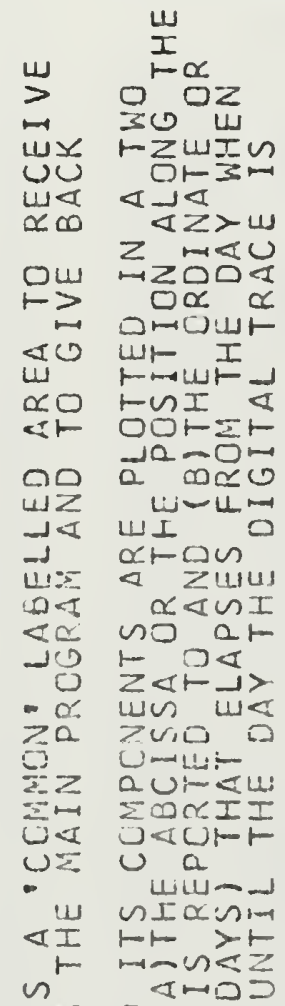

WS oO

ज七: 020

$\supset x \omega<* \sum m \| 1$

Un $\sum m-n$

LU $\mathrm{LC}_{2}<?$

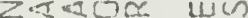

ルト

$-<\alpha U \leq I 1-11]$

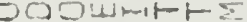

己 $u>0$

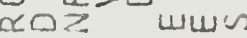

oum $0, \alpha>\&$

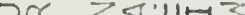

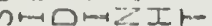
DU30<40

nov $\mapsto \quad \omega \leq$

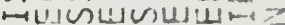

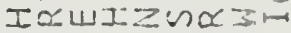

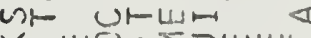

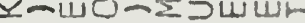

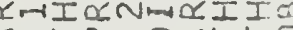

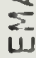

$\propto$
0
2
0
0
1
5
5
0
0

$\frac{u}{\alpha}$

또

0

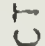

U

$\sim$

QD

$\sum-1$

zin

$Z$

w次

Fト

$<$

C)

$\approx$

$n$

-w

$z_{u \rightarrow}$

zin

U.L

용

$\sum U$

$\omega z$

ט०

0

$5-3$

und

$\alpha>0$

品

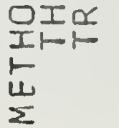

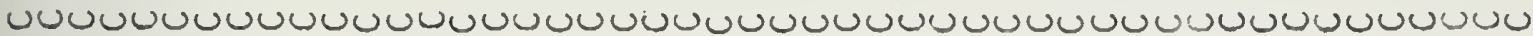




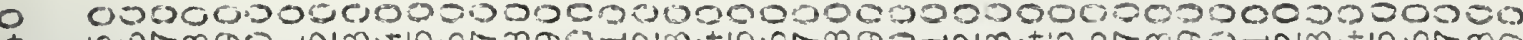

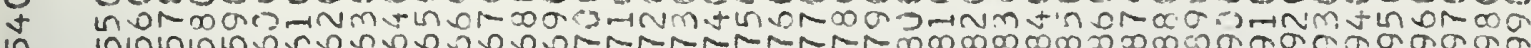

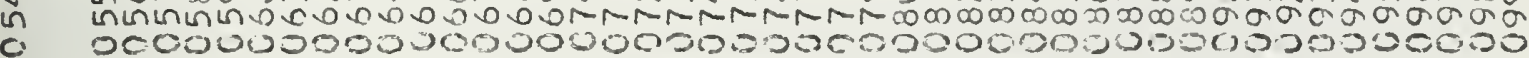

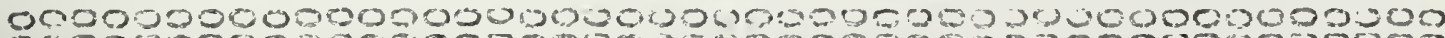
$00500000000030000005,000,200000000020000000000$

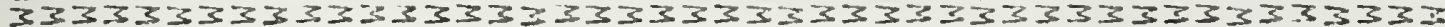

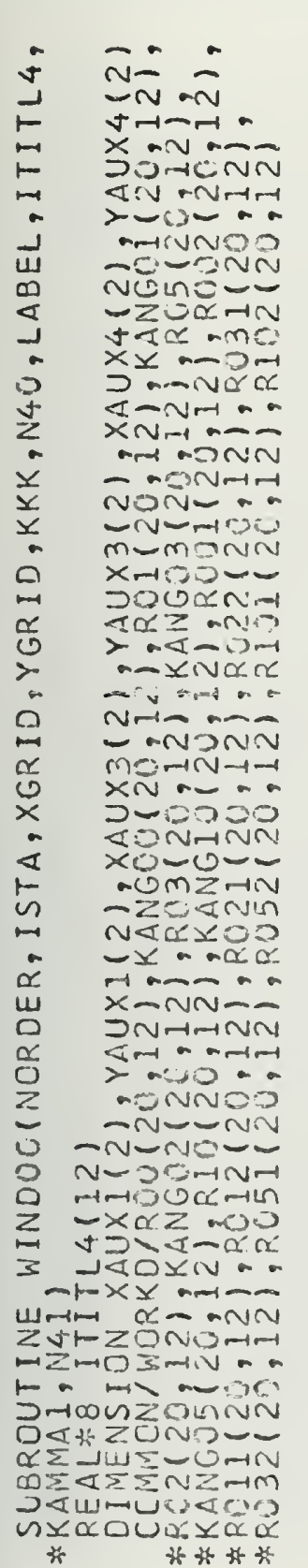

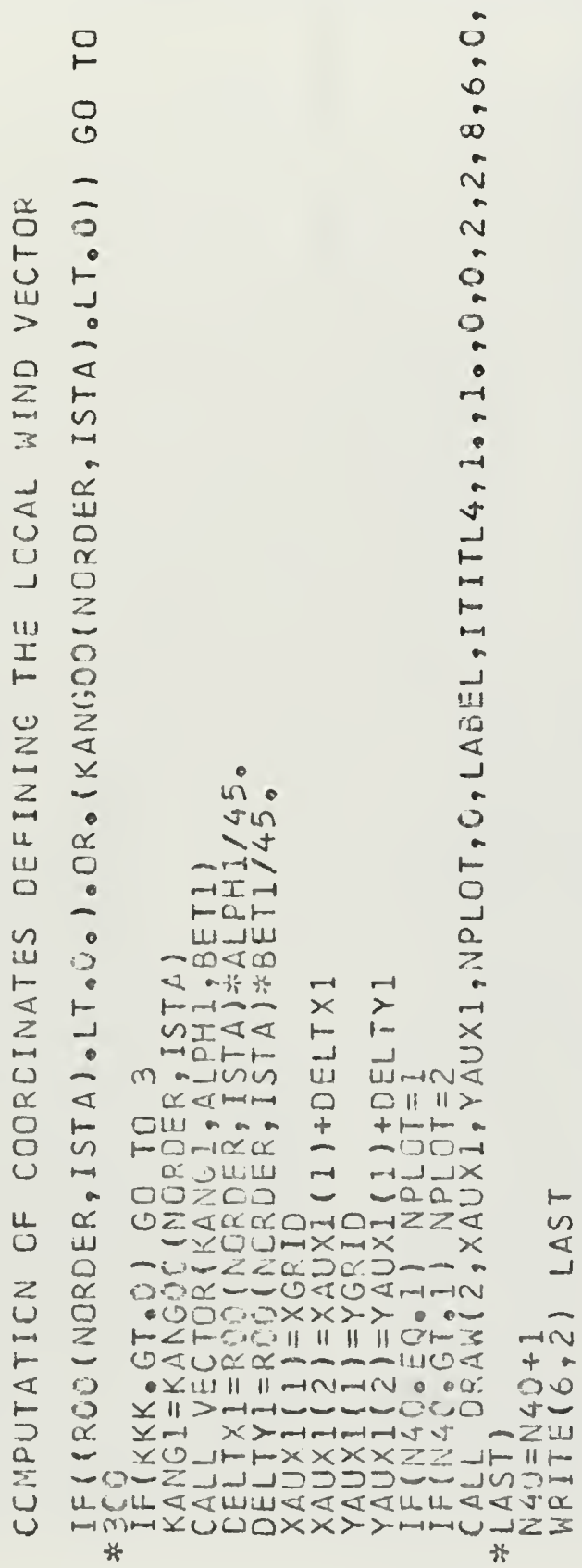

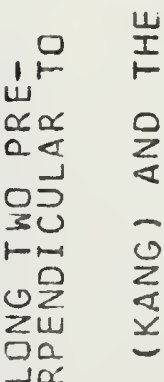

aロ

सU

Q

$\propto$

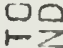

$\omega<$

U.]

is $\geq$

이

-

I

迹

OO

$-12$

世-

$E<$

14.

ن以

ond

2

un

32

n.

007

ulito

cren

Wh-

IOU

oć

Qivin

ZZO

円ロ

oun

Zயแ

HWI

$41 F$

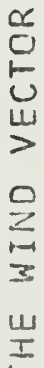

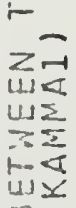

02

Utr

$\underset{i<}{1}$

$\geq \leq$

Yu

LI]

UL

4. I

0

$\propto 2$

$<0$

$5 \frac{1}{5}$

3

$\because 2$

40

..下

$<u$

$\sum w$

रा

$x 0$
5

D

$M$

$\rightarrow 2$

$4 \leq$

$\sum \sum$ ix $\leq \frac{1}{1}$

c) 11

OHe m<-

$0 \leq m$

$-<$ a 0

xa 0

(1) $4 !-0=$

$0-0<\ldots$

뜰 $-\infty \infty$

- 00 EOan

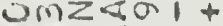

- ox $x$ on HWS-W or -josunts - a OM 0. -1- $<$ एᄂ <ட< $\sum \sum x\left\|\sum \sum\right\| m$

$\sum \sum \| n \sum \sum n$

$<<<<\varangle<<0$ $x x \geq 2 x \geq$ -

UU $4<U U<0$

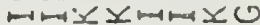





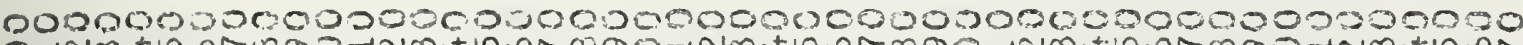

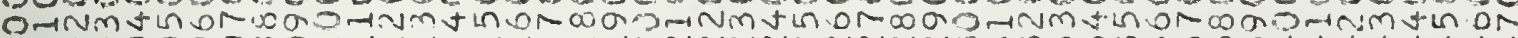

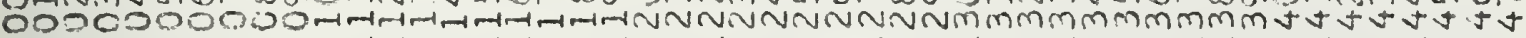

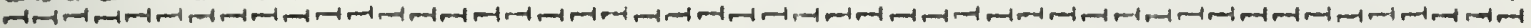

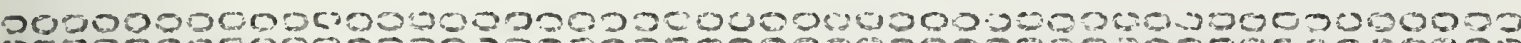

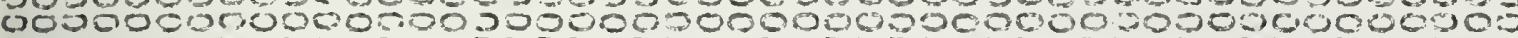

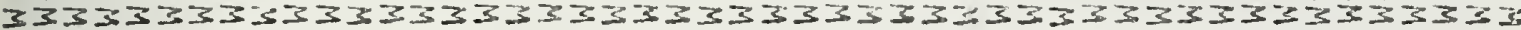

2

$\sum_{2}^{0}$

$0 \frac{1}{4}$

Zu

$\because 0$

西

$\pm 0$

0

a)

U.

$\rightarrow \infty$

$-2$

$>-1-$

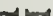

M $N$

$z+\frac{1}{2}$

wю แ」

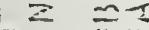

Z仯 部

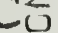

$a$

เs

15to $m$

กism

unt

ant

Uบ 1

विए

민 ज्ञा m=-

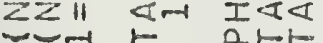

0 -

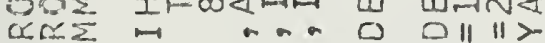

$11 \|<\quad 00+10 \alpha+10 \%$

- $00 \mathrm{~m}$

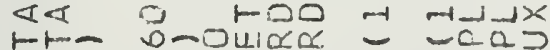

nuis moNIOOOMOMZZZ $\mapsto m$ ort 0 - $n Z \sum \sqcap \times \mapsto x$

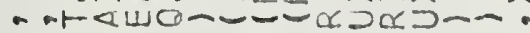

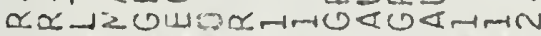

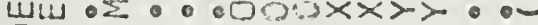

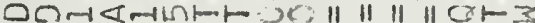
$\alpha \alpha<x<<00, \alpha \propto \ldots \ldots . . .60<$

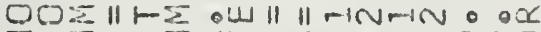
ZZ:

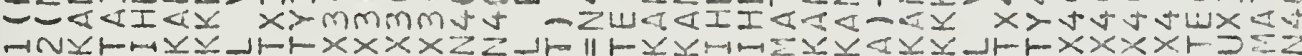

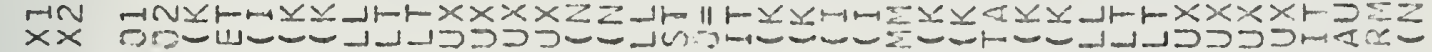

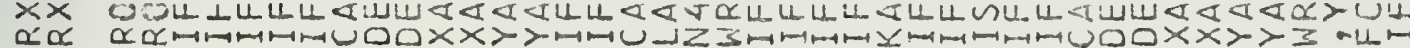

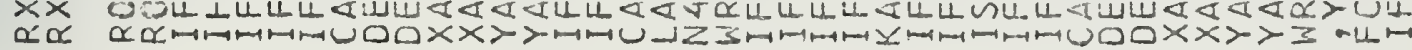
*

$\infty$

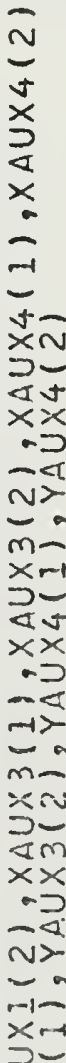

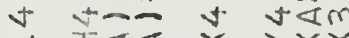

$\rightarrow$ I $4<x>x$ cotr r- $1-a$

Orisun a dनব

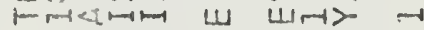

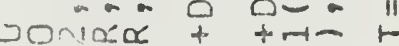
OraI HW - -xru tw0

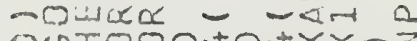
GUIOOON口, $x \times-z$

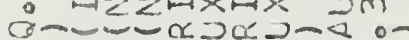

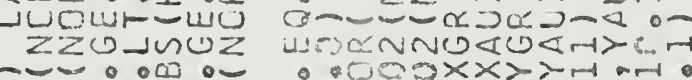

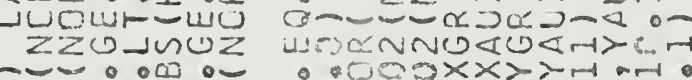
tNor

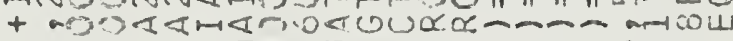

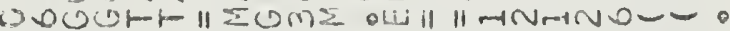
t-z ZU< $\infty$

* in

UUU 


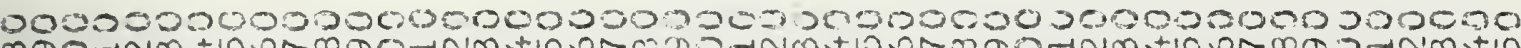

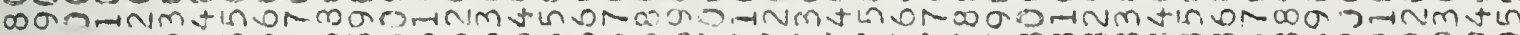

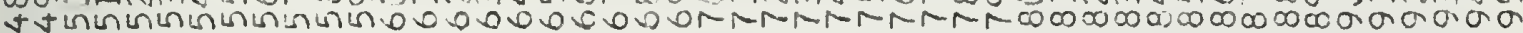

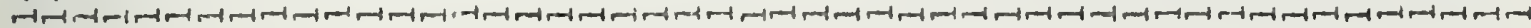

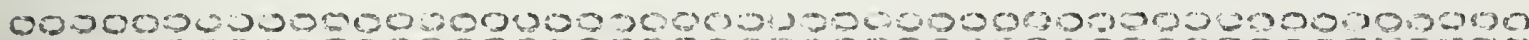

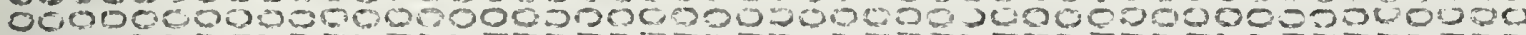

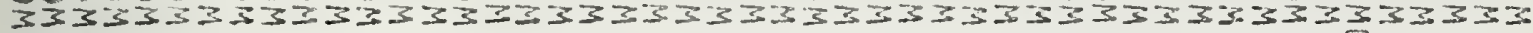
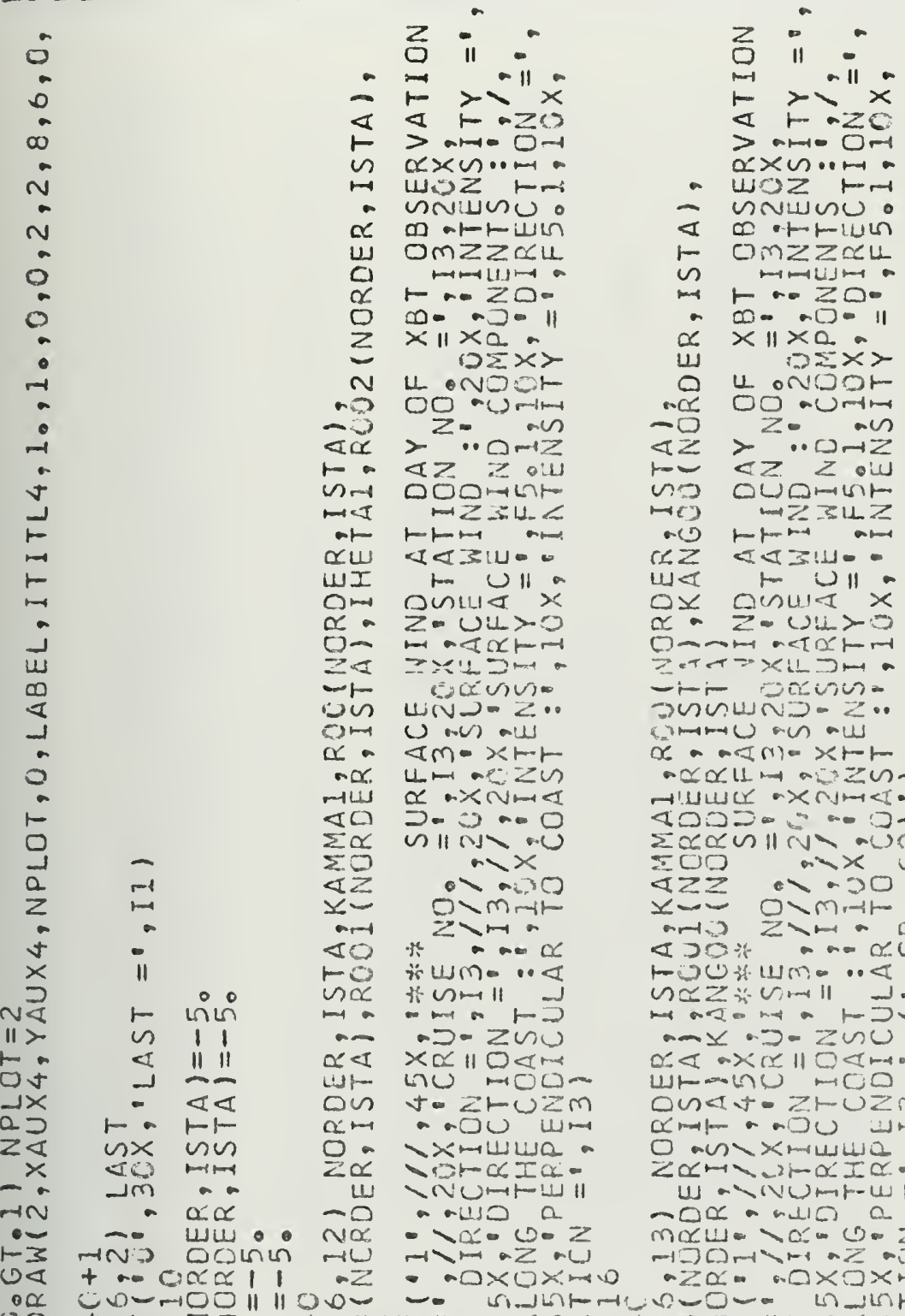
axu...

- $40 \mathrm{Z} Z$ H

क atトUा

U $O M Z Z \propto u$

C.

$\infty-\infty 50$

$\times 11 \times 0$.

$12 \sum x>$

4 ONDOL

m 00 aj

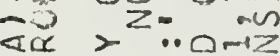

- $4 Z ?$ :

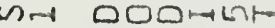

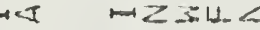

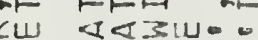

WI t Ull

inula $x$

$z-v_{L}>0$

$=$ a $x$ x

scivivio

UIV. 2 .

$U$ un als

$8 m-x+1$

Un aejzes

$\alpha$ ax $N$ mat

D. $0 \cdots 0$

n In $m x$

- $\backslash$ -

onmmit

Z\म

x $-\infty x$

ชัum - ..

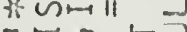

$-2-z \cos$

$x \propto \| 0<m$

Li $\rightarrow 00-$

\& on tratu

i $0 M z Z \ll L$

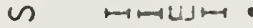

$\because \quad \cdots \geq 0$

$\alpha \times \| \times 0$

U.

- 4 onjar

ace 00 aum

¿Z >

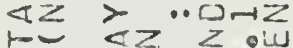

ज? OUDWLI

जe)

$\rightarrow 0$

rth ar

14< < 3140 -

to U

$0 x-2$ gnt $x-x$

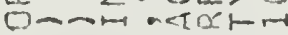

Z:T

-iri gecisis.

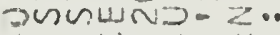

(intatu) an aw

$x$ a n m M $x+5$

axol1m azzon

-inuse ax

$400=\infty \cdots 0$

zecan $\|$ rv

Eup and in

दटz eर.

x-C ODMr-1

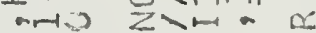

$<>0 \%$ - $20 \%$

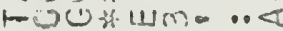

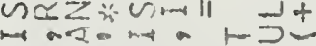

$\max x-2-z i n$

$0<n \times x \| \square<m+\infty$

Htwing $\rightarrow 00$.

onat-zトUZm

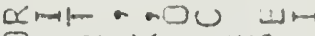

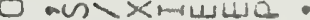

Z゙ロ -

U arvumR-w 11

- $0 x$ andio a

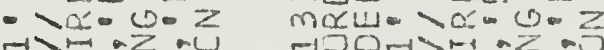

艺 $11 \frac{0}{2} \ldots$

$12-u x$

$>+10$

crenz min

- யOZमन्न

- जNUI 0.

क at Ln

- OmzuL

$\rightarrow 1 m b 00$

- D- a.L II

$a \times \| \times a$ or

un 1 orvist

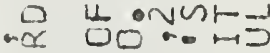

$=2>-. x \geq \cos$

$1-4 z$ riwa

no oucNtz-

जक $\mapsto z-z W m$

《)

$\alpha z<<3>\infty$ o

Uा 5 all-

Qx. Eum $x a \|$

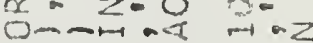

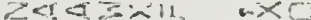

$-i-\infty=0$

avivintis

exrme an or

or man- trou

acrouma in ars

Hisuse ax द- म-

स०OJ-0 0-0

$\div \alpha \sim \| N$ U.

zioj a o.

$x \geq 2$ on $1 x$

$x-$ -

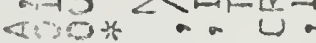
1- 00 : write 0 -

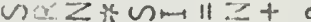

ad-

$\cdots x$ - $\cdots=2-14$ $c<a \times \bar{\alpha} \| n<\ldots$

1. -

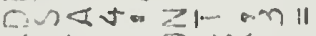

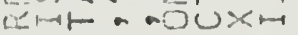

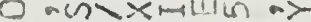

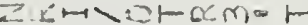

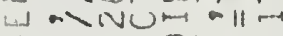

- 0 a - - wo $\backsim$

notu - > $x=-z z$

-130-1> ‥ cou

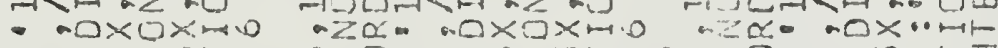

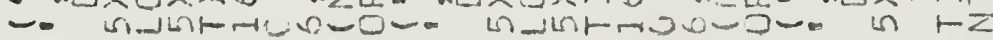

So tor ZZ-1NT-3Nt

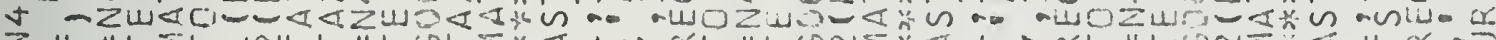

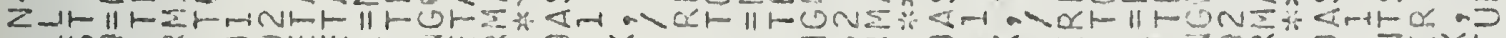

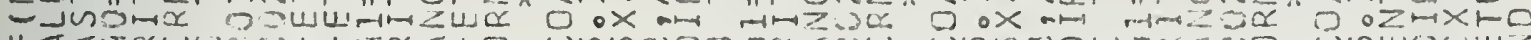

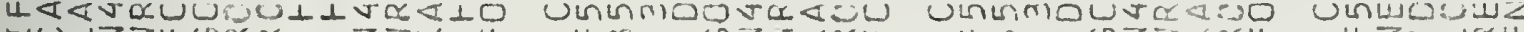
nU⿺廴⿻肀二𠃋 $\ddot{\mathrm{r}}$

N

0 h้
Uninagya

- Lurime

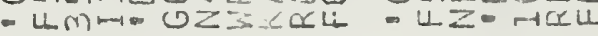

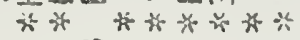

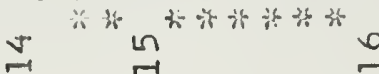


$000200=00000002000000000000000000000000000000000$

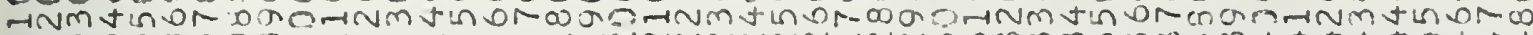

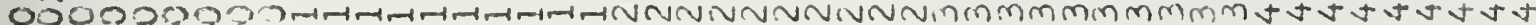
000000000900009000000000050000000000000030000000

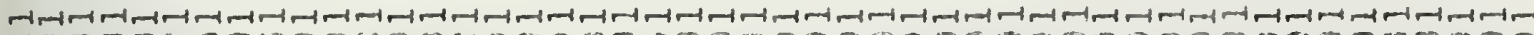

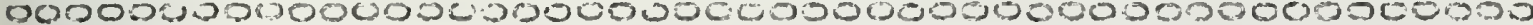

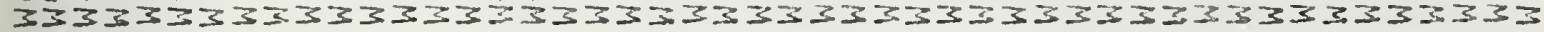

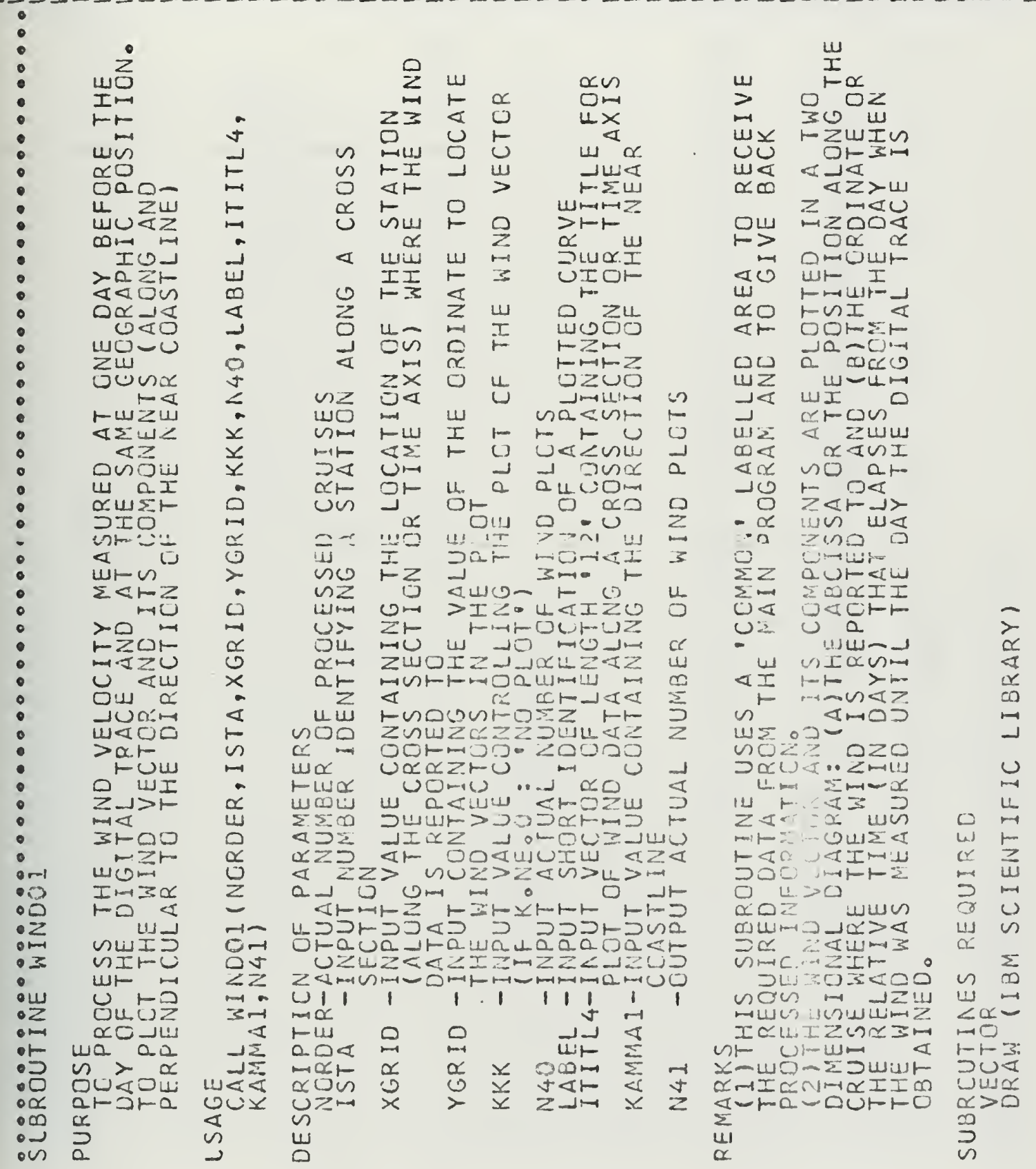


000000 $\arg \operatorname{ang}^{2}$ tonthininto 000000

मूलन $33=353$
0000000000000000000000000000000000000000

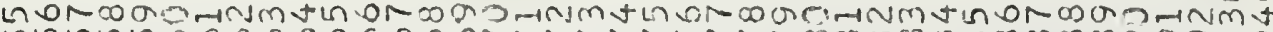

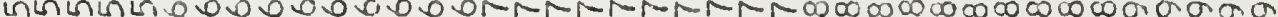
2000000000000.00000000000000003000000000

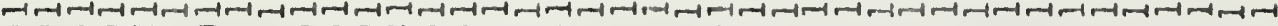
000000000000000000000000000000000000000

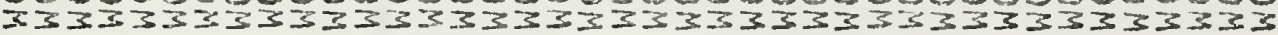

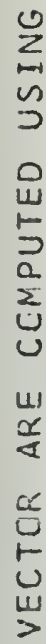

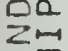

$i_{3=1}^{I}$

wo

In

Fr

U.

○ய

n

10

Zn

uxo

zr

as

$\sum \omega$

02

u

own

어쏘

Irth

-

4

2

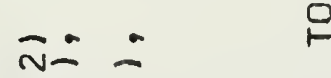

$-r v+r$

thr-

$\times-N$ -

JorenN

4 arvart or

amNOTO

- E-ONr u

NouIOS- U]

$-Z 030-1 \mathrm{~N}$

$t<r e$ amb?

$x y$ and

$\supset m-n x \alpha$

I-NA

$x$ Nat ment

- - un-

Nan-a.

- n-mescos

m-meanN

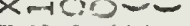

DOCORNir

$\varangle \alpha \geq$ ands

- $x$ Nox

antart.

$\mathrm{NH}-\mathrm{C}-\mathrm{C}$

- angrun

mejirvant

xiv-n -

$5-2 \sin 20$

Ionrinv

-3minr

- OMER zivin

Nat $\alpha y 00$

$-x+a \alpha$

X-NON-2

DNAHNO

In $\rightarrow$ -

rapes a

and

-NT-RN

- N-NOZ-

- $-30-4$ Nin

$-x \propto \geq 0$

$+5><-\alpha a$

- J<0xr.

$+x x$ and

UIm $x-m N$ Zงt-Z0nנe-t

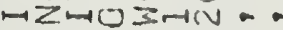

-. ma m- mo

DH⿻

$0 \leq 4$ it ZUNO$\propto \sum-\omega \Sigma-0-1 N$

$\infty \geq<\sum \sum N \geq-1 m$

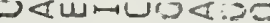

$\sim \geq \propto O \cup \times \simeq \alpha \alpha$

*x

0

$>\quad$

$0<$

$\approx n$

is

L

$\cup \alpha$

- 0

I $=$

$\sum_{-1}^{2}$

$2 \pm$

$u \alpha$

네 0

$\sim 2$

$\stackrel{4}{14}$

$\pm 1$

$2-1$

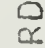

$0 \vdash$

0

$4 \propto$

C

$$
\begin{aligned}
& 2 \\
& 0 \\
& 5 \\
& 5 \\
& 5 \\
& 0 \\
& 0 \\
& 0 \\
& 0
\end{aligned}
$$

感

o
นึ่

$-1$

$-1 I-1$

แ) गس

- (i) $<0$

व

$1+12 \frac{1}{10}$

$n I<<$

$m a n t h$

anjus

CUW ...

$10-1 \alpha \alpha$

aiouس

ग0ะ0?

$0 z<\alpha \alpha 0-10 \mu z z \pi$

- $-100, \geq x+x$

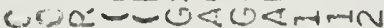

$000+10 \times<>>0$

$1-\angle 1-32011 \|$ \| $11 \mathrm{CH}=$

जबUव口

0xU\| \|नNAN •

$x \|>-11-\ldots-c 00$

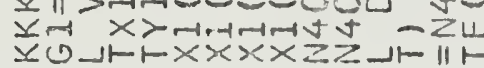

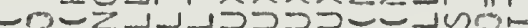

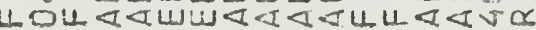
$\rightarrow$ (n)

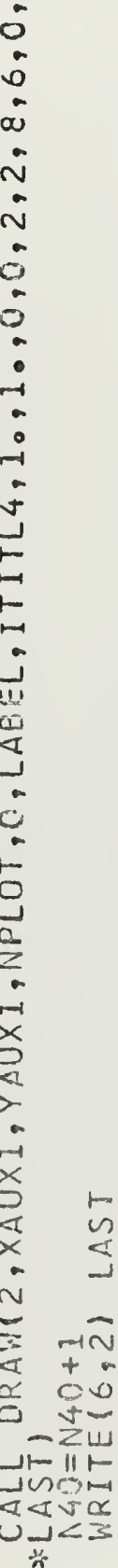

1

15

$\alpha \propto$

$0<\frac{2}{4}$

넌

10

02

2 L

$+x$

IU⿺

문

U< $>$

$>1$

$0 \frac{1}{2}$

10

3

!11

近

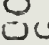

$+12$

แ-

$I<$

4 .

○u

sin

1

$z$

แun

Ziz

an

나

n ouz

ULi

Un-

IDU

F U

$a x$

ZZ2

$z \geq 0$

oun

ZแH

$+\pi$ QI

411

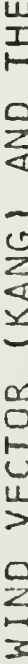

논

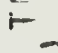

$2-1$

U⿺辶

니는

zख

wx

$\infty \simeq$

ur.

Uu

(2) -

Z4 mer

wo $\Sigma u$

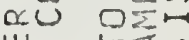

w 1 过。

แLW צू

UI O U

mit $0=0$

(D) -00

ㅈ $\$ m z$

-.

गय -100

0 - 0

$<2 \quad-1+12$

$<0<<<$

$m \sum \Sigma v$

uㅏㄹ

닌

$\leq \alpha-2$

सा UU U⿺辶े

Yo 
0032000000000000,0000000002000000000000000000000 inokrogh

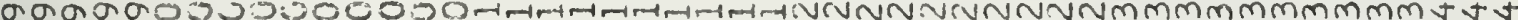

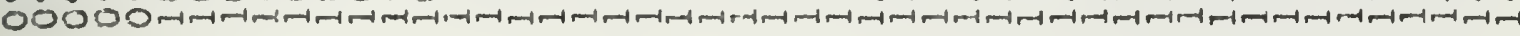

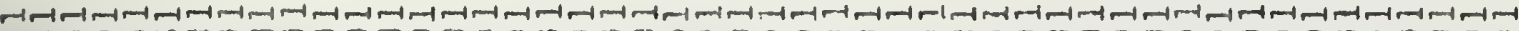
00000000000000000000000000000000000000000000000

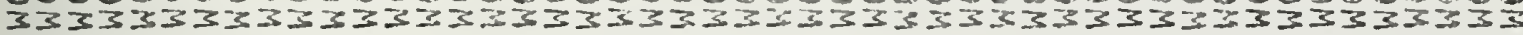

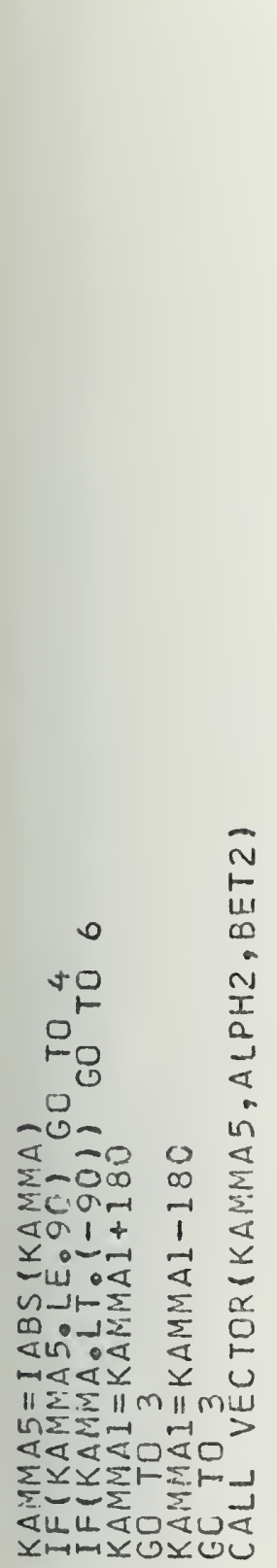

$\circ \leftarrow$

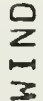

¿ख़

Ju

$\mathcal{U}$

Q-

$-2$

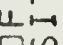

$<$

$>1-$

$-z$

nim NI

$\sum-1$

Wr- W-

$z=z$

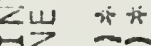

-

$1 \leq$

$<<0$

g

vesin

tath to 8

แU

z

a nor

$\alpha \propto<$ u

WIS:

$x \propto<1$

5

00

Uง

10

is

$\oslash \alpha$

U⿺

w3

IUU

$\mapsto m$

0

Z山

10

$-\alpha$

<ए

A

$\because \ddot{v}$

$x x$

$x x$

$\alpha \propto$

ZZII

$-2-1$

fros

oxus

II $\|<$
II $0<14$

$00-\alpha \alpha+4+1-10$

-0r<uய - o-00m tr-

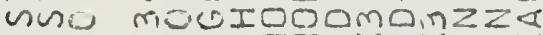
$\mapsto$ ord 00 a $=2 \pi x-4 x$

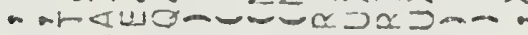

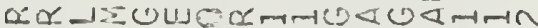

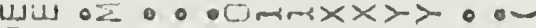
DU. $\alpha \propto<x<<0 \omega \alpha x-2-1,0<$ UOS $\| 1-\sum$ OU\| $\|$ IN

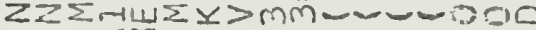

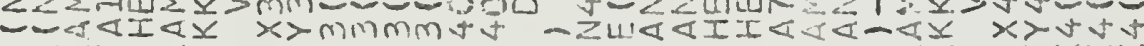

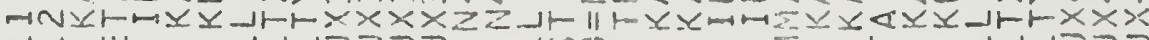

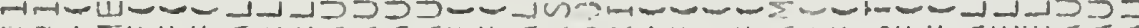

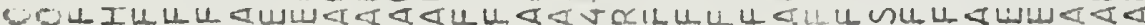
वर्Am x 


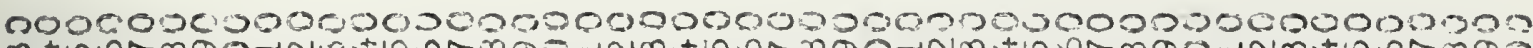

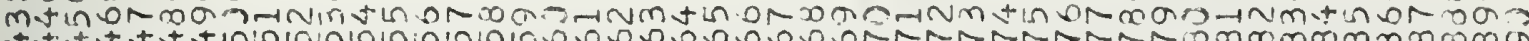

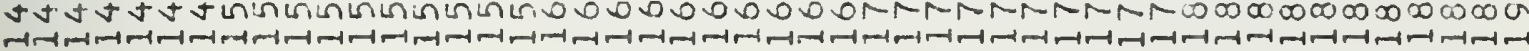

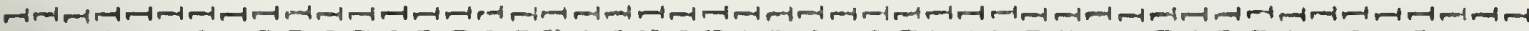
$00006000000500090000-3000000000040006000000000000$

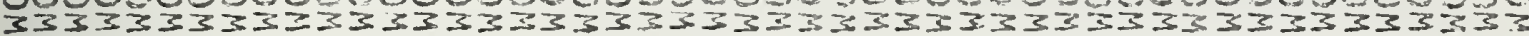

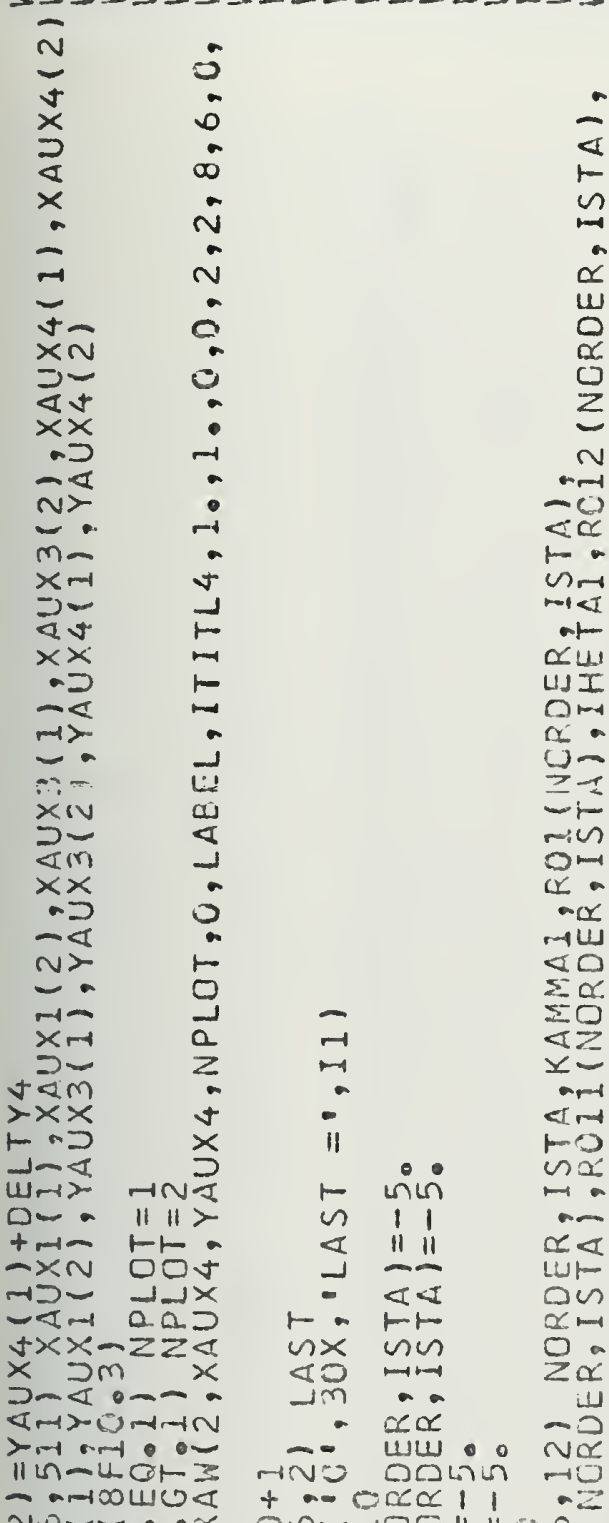

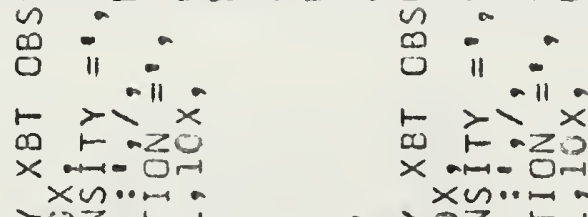

$>0,1+\infty$

<rvininu

$m z Z \propto u L$

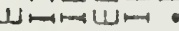

$\alpha$ ar $z \emptyset=$

$0-.0011$

Uा $\| x$.

u $\operatorname{cis} x$

$\infty$ on Dist

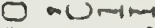

$2 z \cdots 0, \cdots$

$0 \geq z$

UC)

$r=Z \div Z$

rot ard

4i心山-

$\vdash \vdash \omega \|$

षजu⿺ $x$

$9 \cos \alpha+\infty$

$\geq x \|$ and

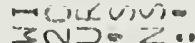

$$
\text { an ous }
$$

uim $x$ - $x 1=$

$\omega_{m \rightarrow}-3 \geq n$

\& ax $N m-1$

$4=0 \cdots 0$

a $\| N>-w$

$\Rightarrow$ ax

n.o 0.50

O) $(n+n)$

Zरtr $n$

* a a $\alpha$

सm - ..

* $\operatorname{man}^{-1}+5$

-

$x \propto \| 0<1$

แก

$\because z r u z m$

- 00 11!n-1

>x-muo.

Vuraxian

- -

- vecosz

$3-10 \geq-3$
- zoziuñon

\& $\omega$ atrui

r $\quad m Z Z \alpha u$

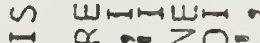

- 0- $-0-11$

$\alpha \quad \| x a$.

- $\infty$ ervour

$-x>0$ avin

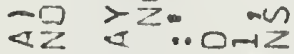

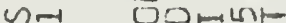

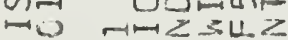

ni $\mapsto m$ an

자 $<34=$

U⿺ $\mapsto$ Uा

$0 x$ रisul $x$

$c \times-u u_{-}>-2$

U $\rightarrow \infty-\infty$ ard oct

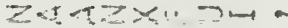

- Hrmaguna

mus $20 \mathrm{~N}=2$.

Compar nen alle

a. a

arcoum -OZZn-

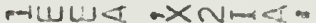

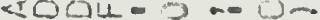
$\sum x x x \| v>-00$

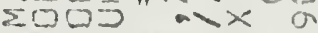
$\varangle \geq Z u$ oर asu।

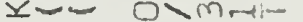
art Z>

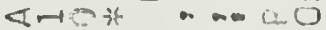
$50(0 \% \omega r)=\ldots<$ $n=z * n_{n} \rightarrow 11 \quad j+$ ज वरा मून max $=D-z ! n)$ $\alpha<a x a|l| 0<m$

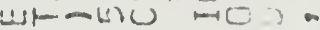

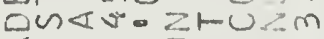
x๓r क a Onux-wula n

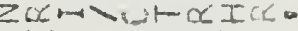

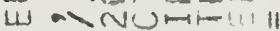
moc a $m \propto u=-\backslash \alpha-0 n z$

-1000 w w $02 x>$

$\because 0 \geq \geq 011$ $\infty-a$

$011<$

$r>0$ $\infty F$

$\times a n$ $x \backsim 2$

$\therefore$ 20z-

- 0 -

$\vdash \quad n z u$

$\backsim \quad 4 n m()$

- O. -

$\propto u \| x \in$

ш

- co orvar

$a x>0$ an

$-0<2 \cdots x$

I- 02

जn

$\rightarrow ?+12$.

on bots

$\alpha z<3$

1上< $\vdash$ -

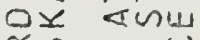

o. $U$

1200 m

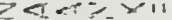

-i-in

muns $\leq N 2$

गमझ की

a a a wm -

$a \propto \alpha \cup m$. नाயu< $\rightarrow x$

$\varangle 004=0$

$\sum \alpha \propto \alpha \propto N$

¿ण0D .

4ZZn

$x=-0>m$

arta $2 \geq$

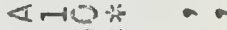

$100 \%$ แुल

nxzk nm II

- nदा मn

a $\simeq a-20$ $\alpha<a \times \infty 110$

untalno

OजषA Z

$\alpha \mapsto \models \cdot 0 u$

0 and x’matli

$z \alpha m<E, 1 \alpha$

แU a vivu-

- $0 x$ a mo

กㅁ․ $>x$

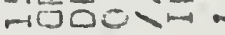
- $2 \alpha$ a ax

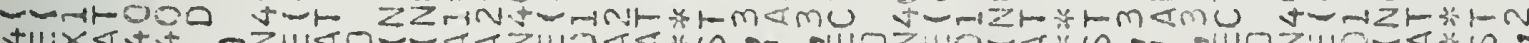

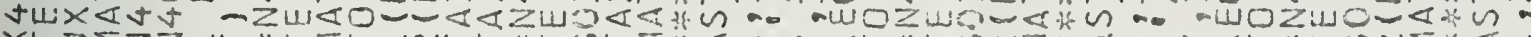

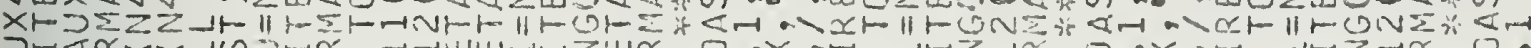

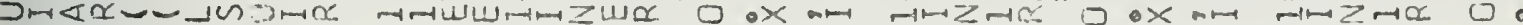

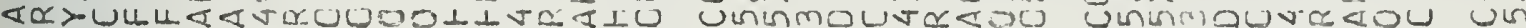

$>3$ *

$\rightarrow$

in

我

$\sim 0$

(i)

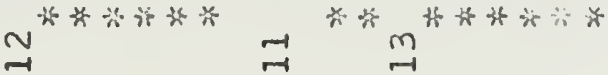

$\checkmark$ น 
0000000.20500000000000000000000050000006000000000

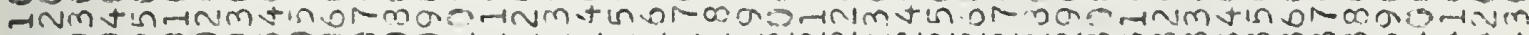

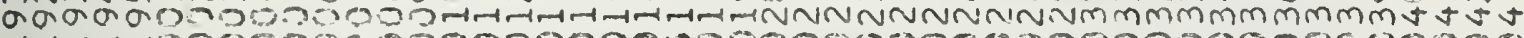

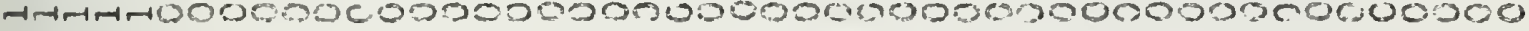

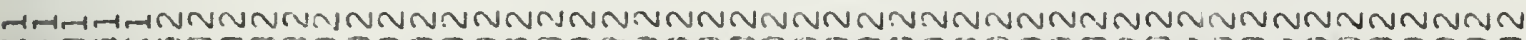

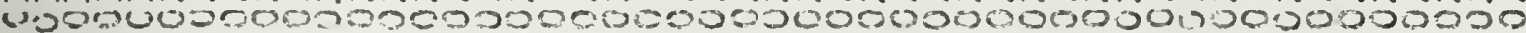

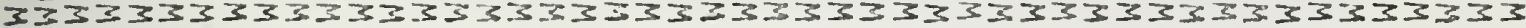

年

$\operatorname{lin}^{+\infty}$

as

$<$

(1)

$-0$

r

$\alpha$

$2 \frac{\alpha}{1-\lambda}$

$\rightarrow 2$

nu

U日

टயता

매

- $\alpha$.

-14]=

xall

0.

$-1+2$

करति

- แrit

. NT

-

is $\sim \alpha$

4.

$0-0$

on.

w $x$

I 3

$1 \alpha-1$

()

$z+0$

U๐

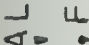

$\therefore \ldots$

am 11

$x \mapsto$

in $a$

$m=-$

- $n$

zz

- 口u

"rit

12

U⿺

งus. ㅇ

$\vdash \alpha \cdot 5$

Zix $x=0$

w0OU<

$Z=m \alpha u$

* $*$ * *
I

w Ju

$0 \leq$

แ<山ト

कutu

분

?

<过

$0 x \alpha \propto$

$\cos 4$

00011

×யலZ

10

जL

๒U上I $<$

onou

$4 \geq 0$

वுtபব

$5 x \geq$

든

Wr L上

E 40

แய

$>0>\alpha$

$1-2$ -

$5<0$

๑ய:

UU $U$ I

世<

$>0$ แII

0 FI

$2-$

$\sum \frac{\pi}{4} r \infty$

$31-0<$

正

Iロ

คㄴำ

जI

जI

()) $0 \alpha$

उU

cota

of wa mo

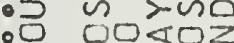

- a a tona

a) $x$

$\rightarrow 2$

- is a

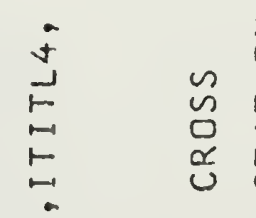

0

z

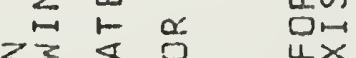

$2 \geq \& 5$

मய 0 U

呩

का

งा山 $1-9$ >

a.

SuxI

UIOH

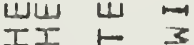

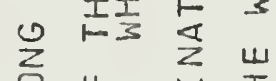

un 0 I

$0 \geq 4$

$12 m$

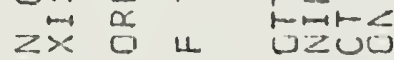

$n=$

แบ厂

$0 \ll$

nn

估

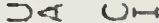

un

cor

4.

$d$ d

a 0 on

a

0 व

C.T 110 แ1 II 72014

山 I $20 ;$ :

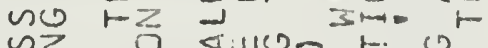

$\sim 2$

Un

$\cup>$

口u

a.t

(0,

r. 0

$>I Z-U<I Z 0$

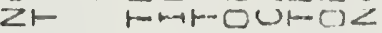

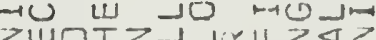

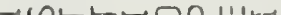

$z$ \& $x$ or $1<4$

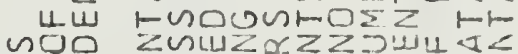

¿ $\mapsto 00$ -

$w \alpha$

$14 \alpha$

Uaczr-u UOחU … $\alpha 0$

und

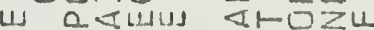

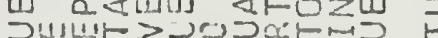

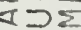

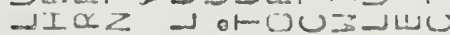

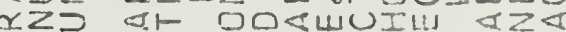

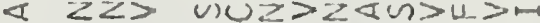

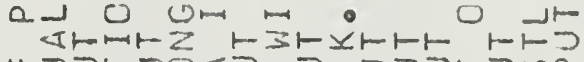

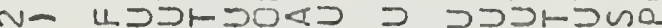

am ofoun Jtaulu

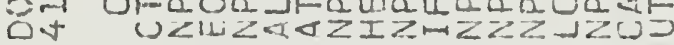

$z z$

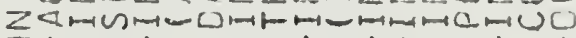

I. $\mapsto C$

$<$

$-1 \geq$

in

n
01

m他

$\rightarrow \alpha+\infty$

coñ

uzm $x$

n

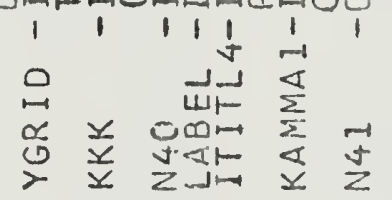

峞

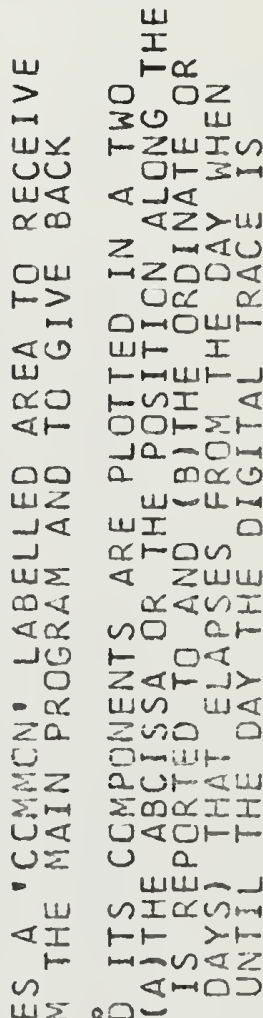

जUZZ 020

गूO

us 1 따 3

$z<Q 0 \times$ un

แト

$\vdash<\alpha \cup\{I m u$

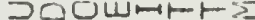

o $u>0$

o०z wives

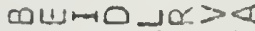

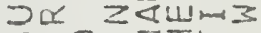

जिएव

จแ $30=500^{\circ}$

nav $n-1 \geq \mu$

ตแบนแบแผตZ

IฉUUIZno जF UトUा \&

$x-110-\Sigma \supset$ อง

$\alpha$ AICWNOII $D$ 【ートローのUトFல E

or 

Decocisos 27.0000:300

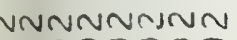
30033,333

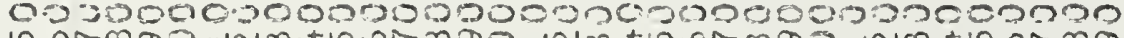

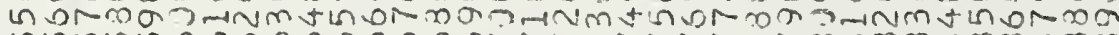

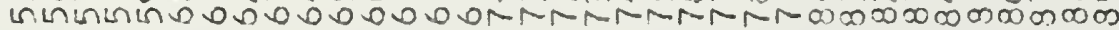

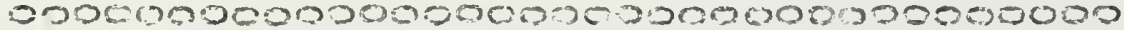

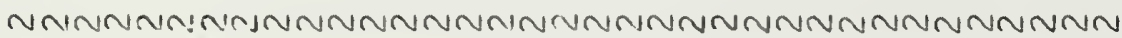

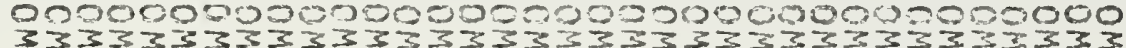

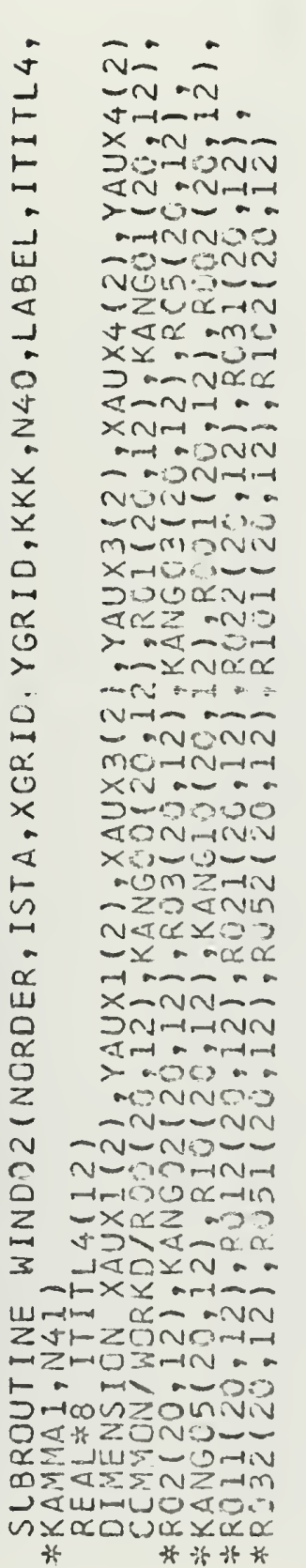

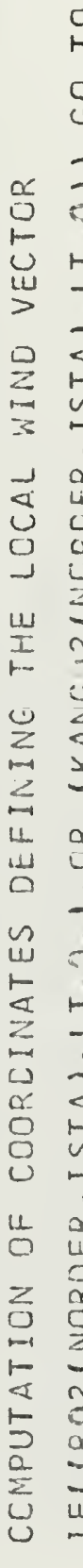

$\square$

$\underset{0}{0}$

-

2

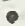

$+$

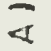

1

-

U

$\geq$

$\frac{w}{\alpha}$

$\propto$

$\omega$

岀

in

a

政

In

एण

In

-

$1-$

OL

$n$

$\leftarrow U$

$\geq m$

Zr

UแU

니

$\square z$

30

wn

OIC

I

ए



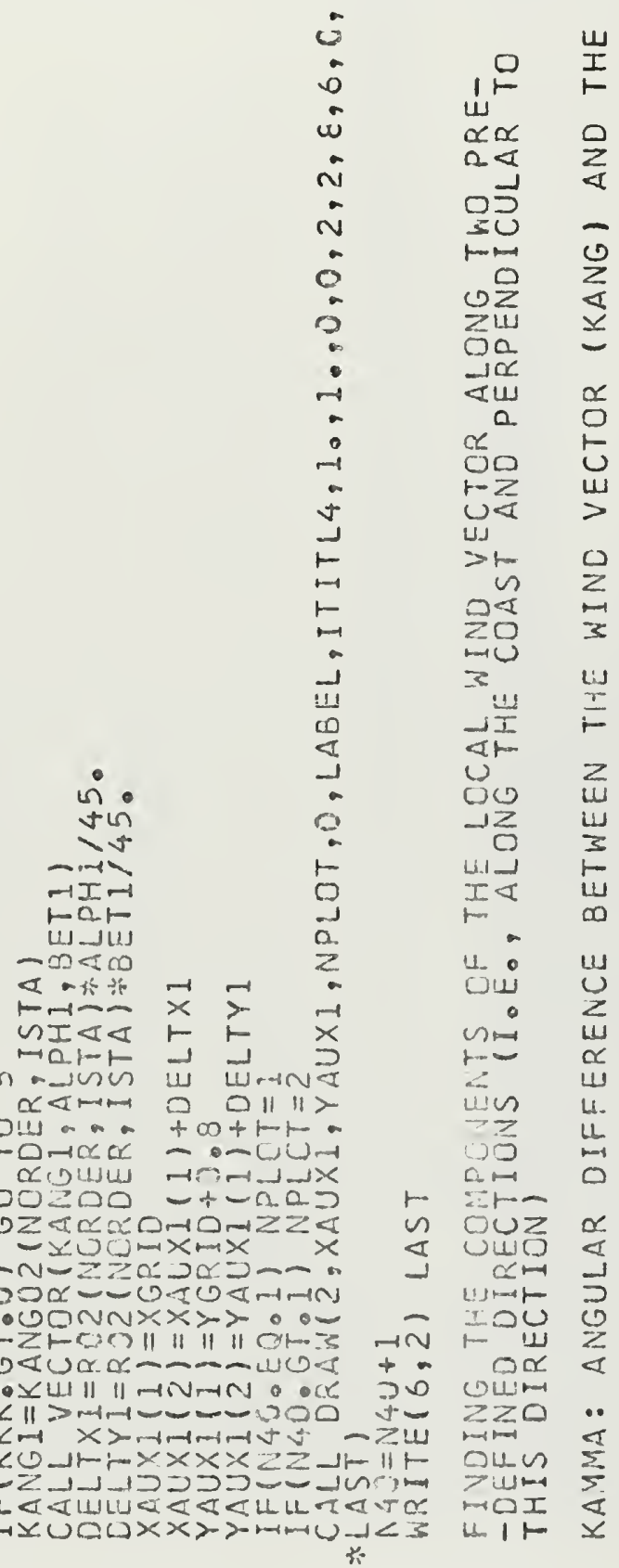

xưunus 


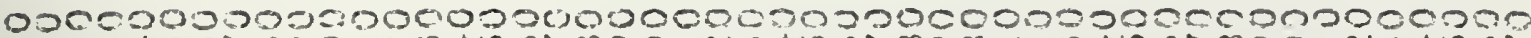

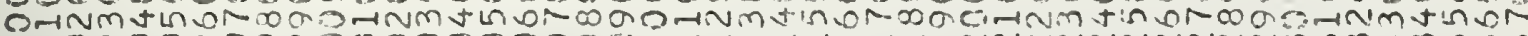
aबa

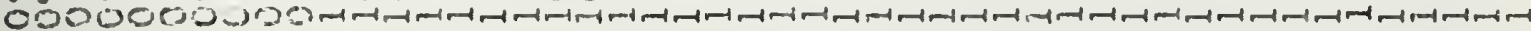

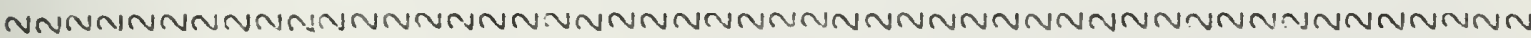

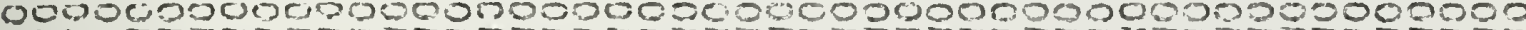

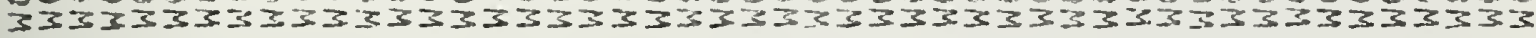

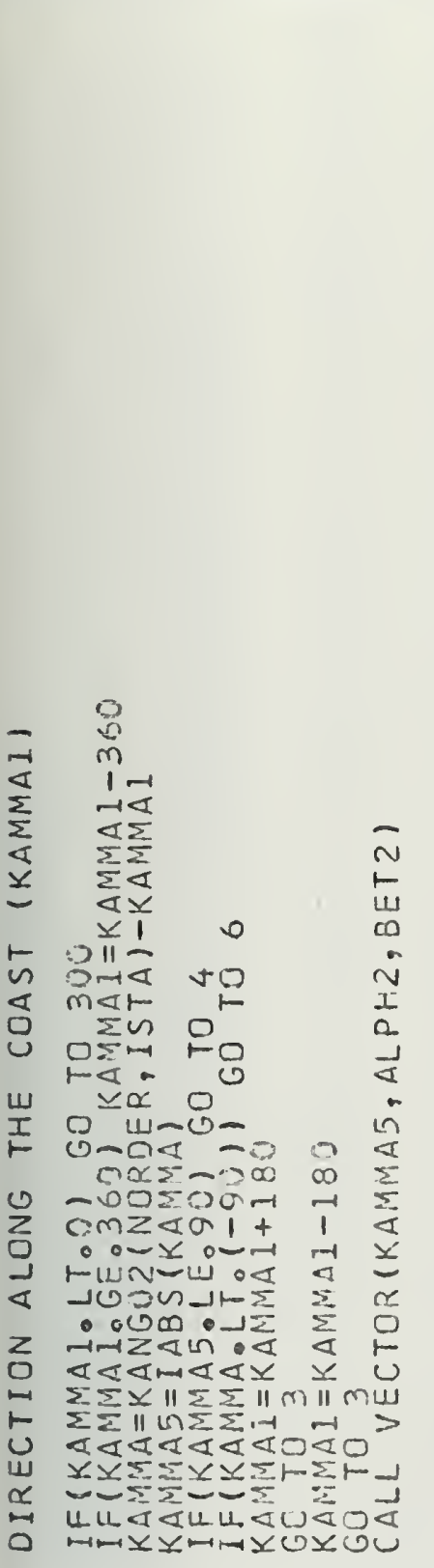

m

$0 \mathrm{H}$

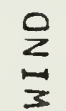

$\sum_{\substack{00 \\ 0}}^{0}$

$\mathbb{1}$

0

12

4

Z

>w

$1-z$

$4 m$

$n-N Z$

U上 $\omega_{-}$

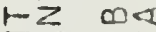

7 in को

$-0$

$-\sum$

isc, m

$1-+0$

bint

anm

$\alpha \propto \alpha$

แU⿺

$00=$

वर्s

$90 \frac{x}{211}$

-

to NNA

002

$\approx x \leq$

$0 \propto \quad 11114$

U⿺

แ5

IU

$<<$

$1+\infty$

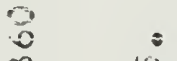

$m$ जा

สนกา

$\rightarrow$

$\pi$

a n)

Uis ram

I wsu

m mesco

II $\quad+x$

In $I \vec{L}$

- $a$ L

wo Jinin

If $\infty$ arme

$\rightarrow$ an

Q Onata

क

$m m$

$x>$

$+5 \quad m$

$\begin{array}{lll}-1 & -1\end{array}$

ये चातन

0 D\| II>

$+\infty+1-\infty$

- $-00 \mathrm{~m}$

$\rightarrow P+1-1 \%$

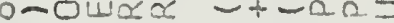

nus mPNIDLDMDMZZI

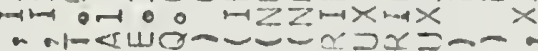

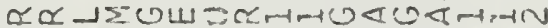

Wu: $0 \sum 00 N N \times x>>00$

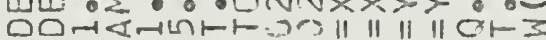

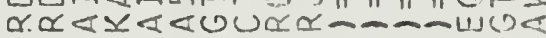

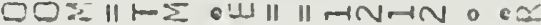

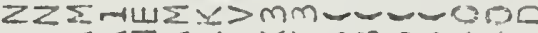

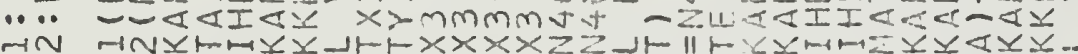

3

$\because$

$\infty$

N

N

3

5

$\because \quad 0$ or

a

$-1$

$\stackrel{5}{2}$

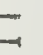

$-$

5

$\frac{1}{2}$

i

-

5

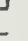

$\geq$

(1)

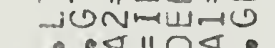

- $<110<$.

- ONNGE-

$<\varangle U<0 \leq \varangle$

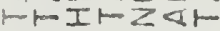

numui-xus

Hम IN

a-man-

$\because c r \quad, \quad B=\alpha$

1- แU10-Znil nomm

सCL : Ox or

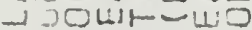
$\geq \geq 0$,

anc

$\circ 00$

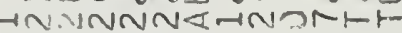
$+m 0<<n<00<00$ 0000-

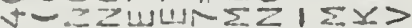
- Zu:

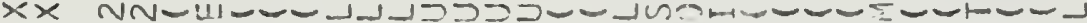

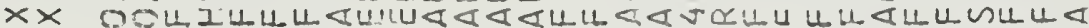

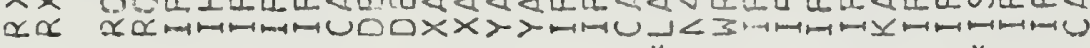




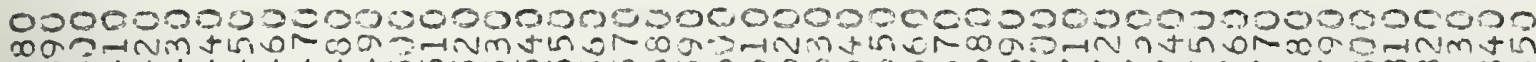

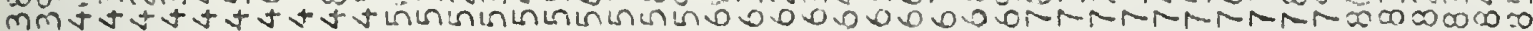

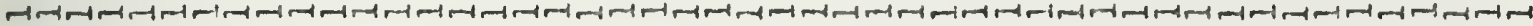

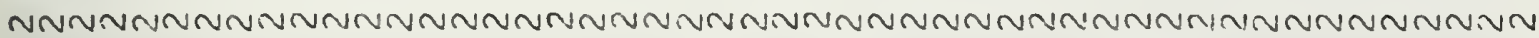
000000000200000000900000000000000900000200000003

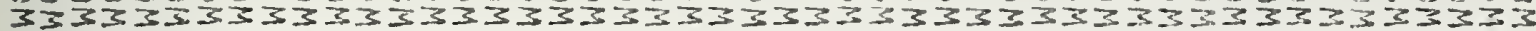

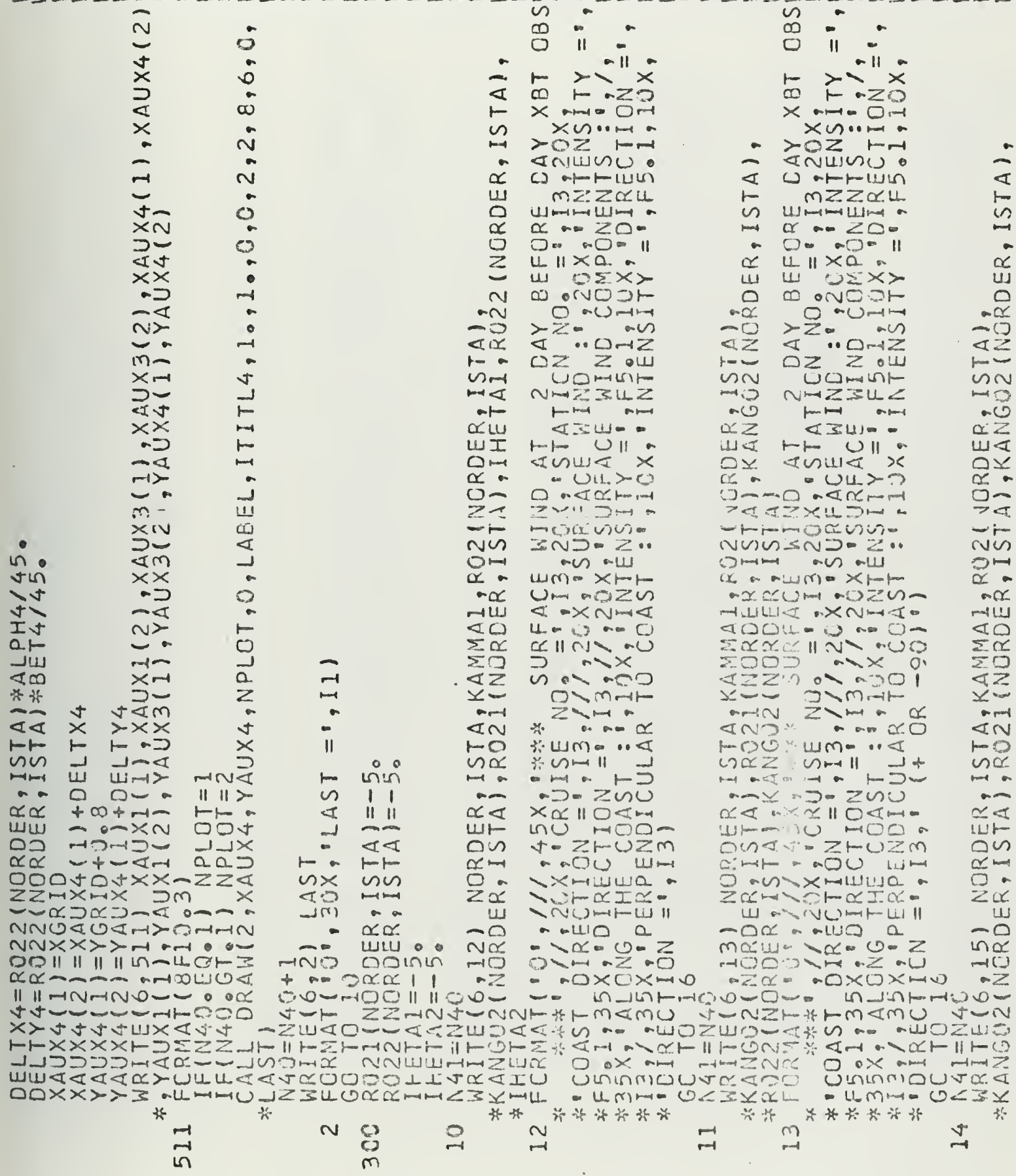





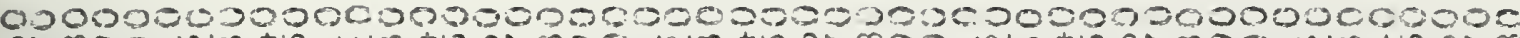

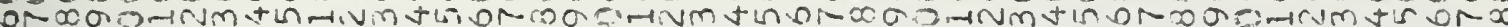

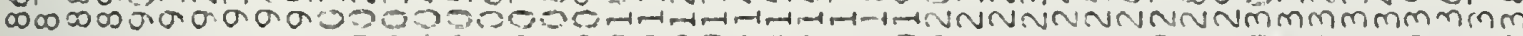

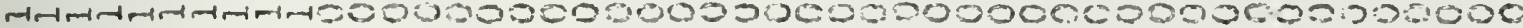

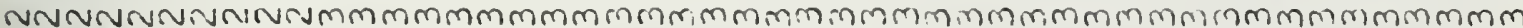
$00000 \% 000000000000000000020000000000000000003000$

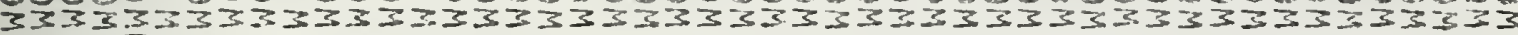

$\sim 20$

0 iा

$\sum_{0}$

$1>U \times$.

व 15

$x$ aromit

xuz …

- OZMम

QNISI of

0.1 เnu

mzunu

Unखण

$\alpha \ldots<-1$

0- - Lل $\|$

$u \| x \alpha \propto$

ш $0 د 2<$

$\infty$ orvusta

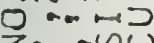

$<2: . x z=$

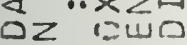

Wont-ZZ -

NHZ -Zयक

トッフルロே

$43>0 x$.

rt abe

<nu $\times a l 1$

- 0 -

-0잠

षExi⿺ 핀

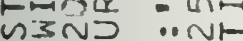

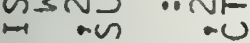

mघ)

qum $\sim \sim \alpha$

யय $x$ ष 4 -

Uu- $0 \quad 0-0$

ax $\|$ N

zu ox-uix

- $>$ \mI

$0^{2}=$

$0 *$ UMm $=0:-1$

Z*um\|z+。

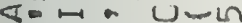

$x-J-z-1 u$

$-\times \alpha \| 0 \varangle-$

- inv na

டホーZト-m II

H. - 0 Uxम

$n>x$ - 4 (s) $n>$

m- Dot

- NuF* 11 -

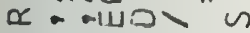

U- $\backslash \alpha-a z z$

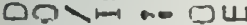

c. - $0 \times \cdot \cdot m$

U-. in 1 Z

Zト*

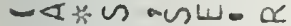

NE* $<-11-\alpha+2$

Non 0 or?

ciu Untiिare

वU - Uz- -

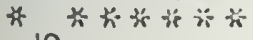

in

$\stackrel{0}{\rightarrow}$
U us 2

$\propto 0$

ㄴ. 는

$\omega$,

<nा

nutus

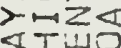

IIUD

0 a

wanc

แQ⿱一⿻上丨亅八

미

IUU⿺

nus

r-U上I

王什 $<$

๑ñ⿰冫欠

แ $Z 0$ 띠

고 z

< 0 -

Ur - r

$\sum \varangle \cup \cup$

$>0>\alpha$

$>0>\alpha$

जर००

U 2

OUயm

UU $\geq I$

जर

$>0$ w

トI0

$\sum-$

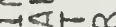

उन $0<$

$m-1-1$

แजव

om $I n$

- 0 Fón

19

जI ous

wr 20

U. $0 x$

or wata

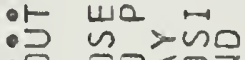

Dण

$\therefore \quad 0100<$

$-\infty \propto$

- 5

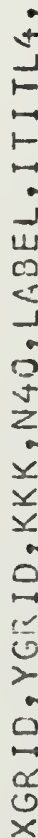

a

1

$\rightarrow$

a

แU

○

$\stackrel{0}{0}$

$\geq$

$m-$

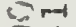

อง

$z z$

$3+1$

$\frac{1}{2}$

$J \sum$

फरद

Wus

\&

in

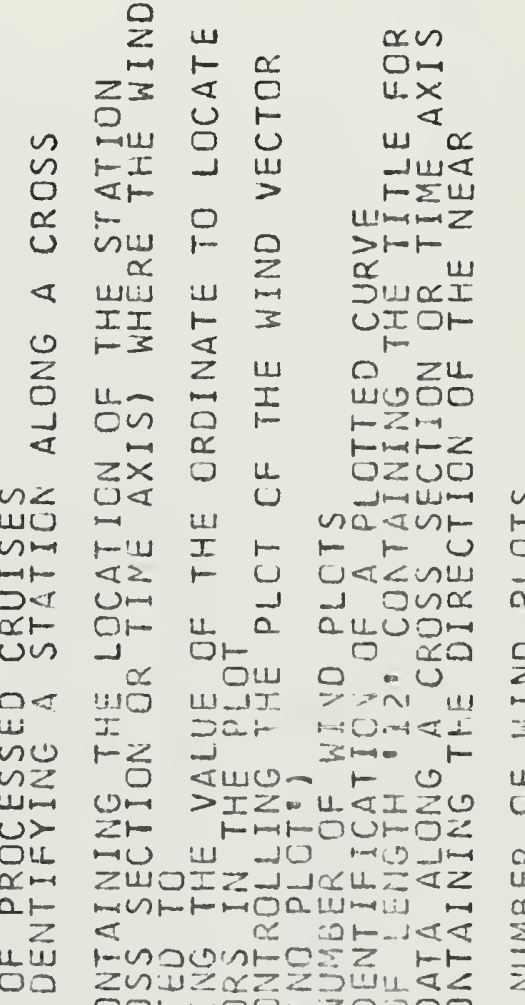

ce $\mapsto$ DOt.

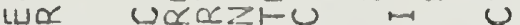

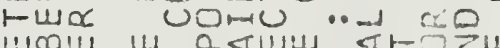

एकाओ แ

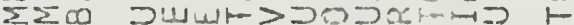

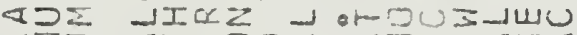

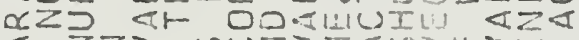

$<\bar{z} z\rangle \quad$ vuz $\rangle z\langle u>1>m$

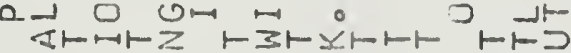

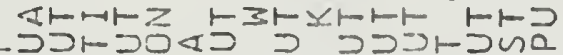
วR-aUa

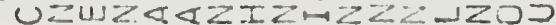

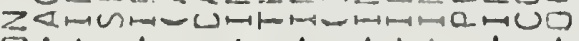

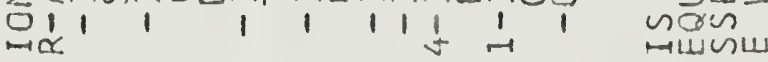

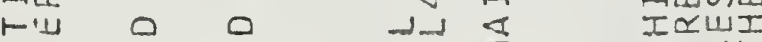

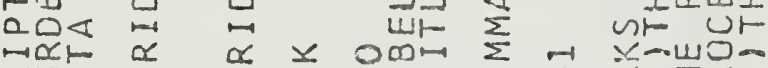

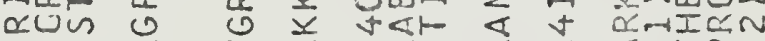

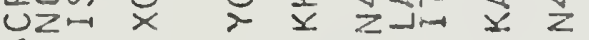

in

แึ

$\sum_{\text {U }}$

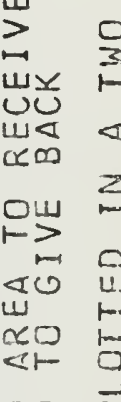

00

$\rightarrow<4$

w5 $\alpha$

का

सo. (v)

-

$=x \quad 4$

运

襍

UE

Us

$\checkmark I$

$\sim$

แ1山

गत्व

II $\operatorname{mat}$

(1) $1-\alpha$

$z<\varangle . C$

$-11-21$

500u

$4>$

$\alpha 0 \geq$

cuㅐ음 $20 \quad=$

Ju

Don

-1든

$\propto$

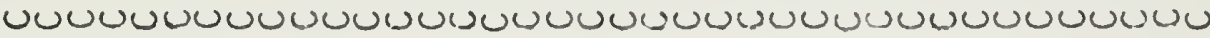




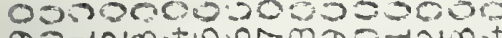

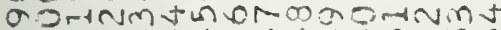
matatat.t.t.thinininin 0000050000002000

mmm.nmm 00000000090200900

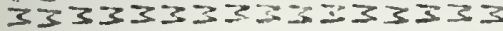

I

I

$0<$

(D) U

ZWIn

난

¿Z>W

ঋய

zon

aर $\propto$

1 I

जI

OrEL

$0-0$ re-

$\infty \propto 0$

$\mathrm{U}$
$\mathrm{L}$

Fou

zயu

$\alpha<\backsim I$

o ar

$0<$

एम $-1>$

$\operatorname{nic}=$

내

Uトष्य

$0 \subset I I$

বOFF

a

แเบ -

Ixum

- >1

-

षnOJ

$0 \geq 0$

. Żrall]

$\sum m-\alpha$

$\varangle \geq \supset$

OUI

区Iㄴ

円トト

0

แuns

$\triangle \propto ⿻ \leq$

पu山n=

ZIF

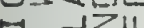

जuाया

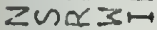

Uा

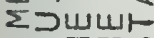

merIID

QLIF-O
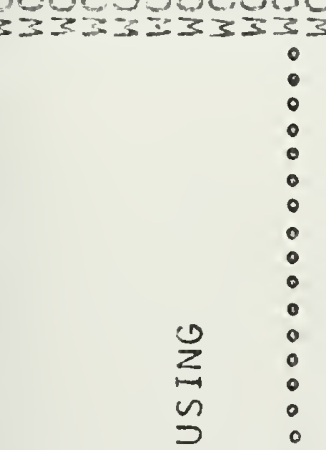

$\frac{5}{5}$

$\frac{w}{\alpha}$

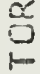

U

$>$

$n$

on

$\sum_{n=1}$

उi

แอ

I.

$\omega$

$0 \stackrel{2}{2}$

U

$\propto z$

m U

$\nabla 0$

u n

$\propto$

$\sim \sum_{\infty}$

U1

$z \propto-$

$-5=$

गUस

$\underset{\alpha>0}{0}$

क
200000050035002500060000000000

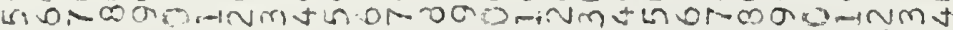

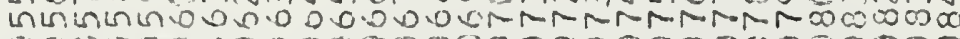

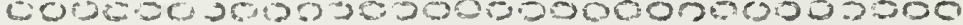

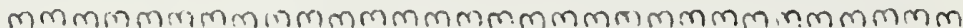

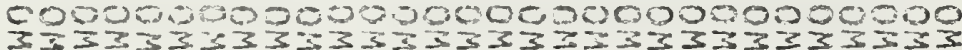

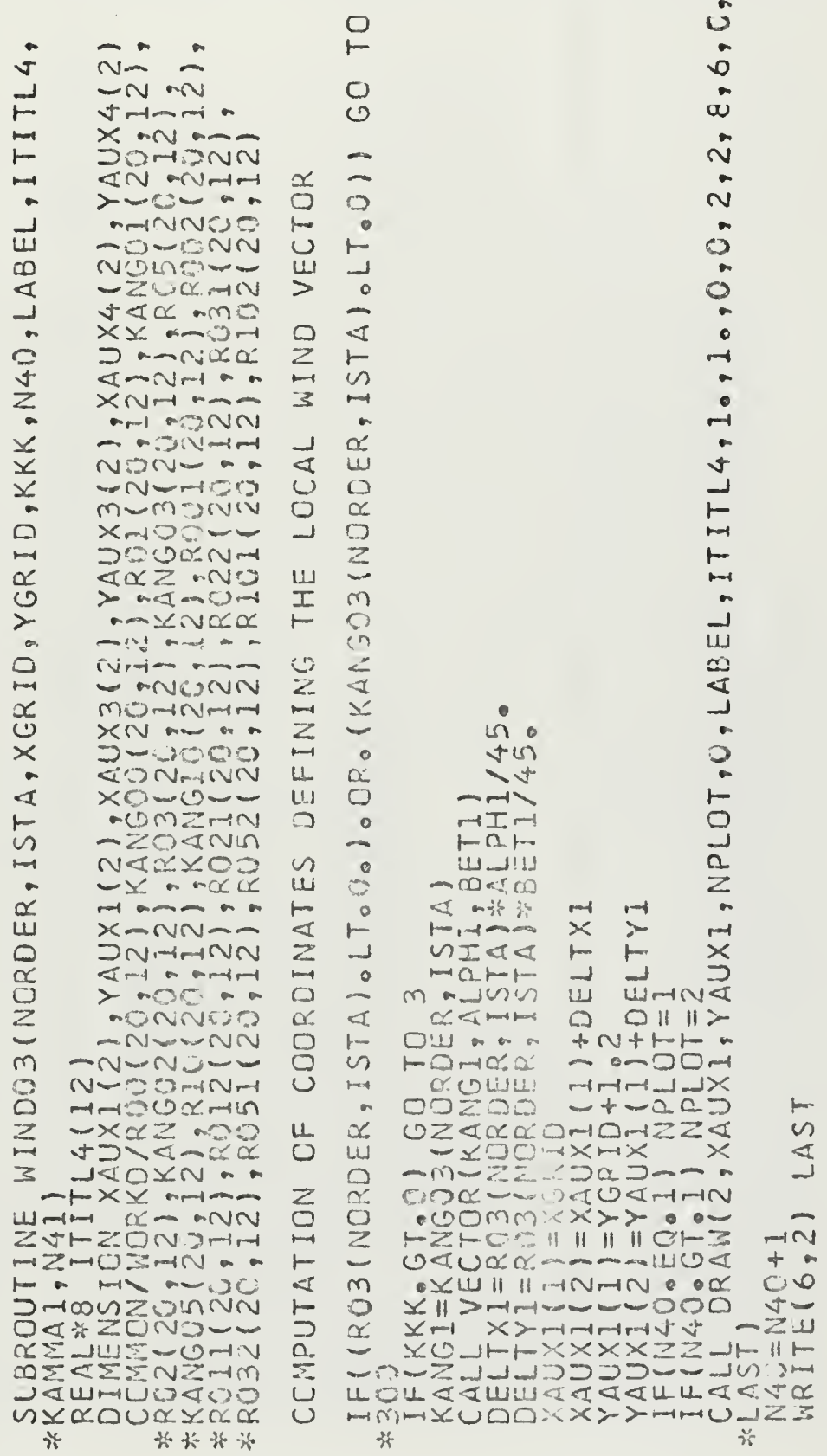




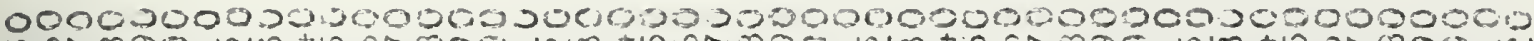

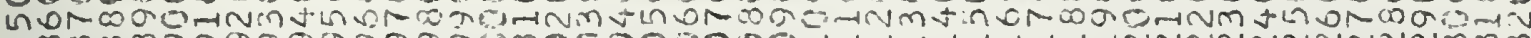

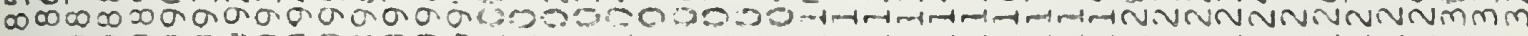

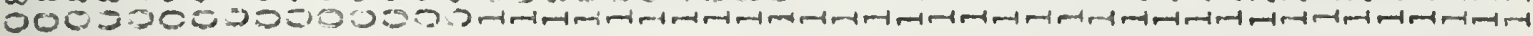

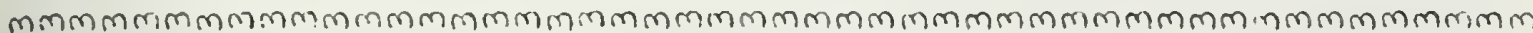

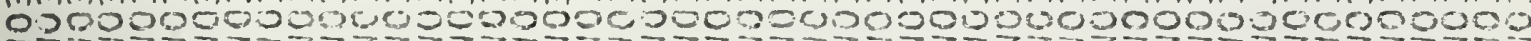

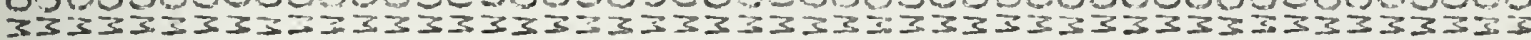

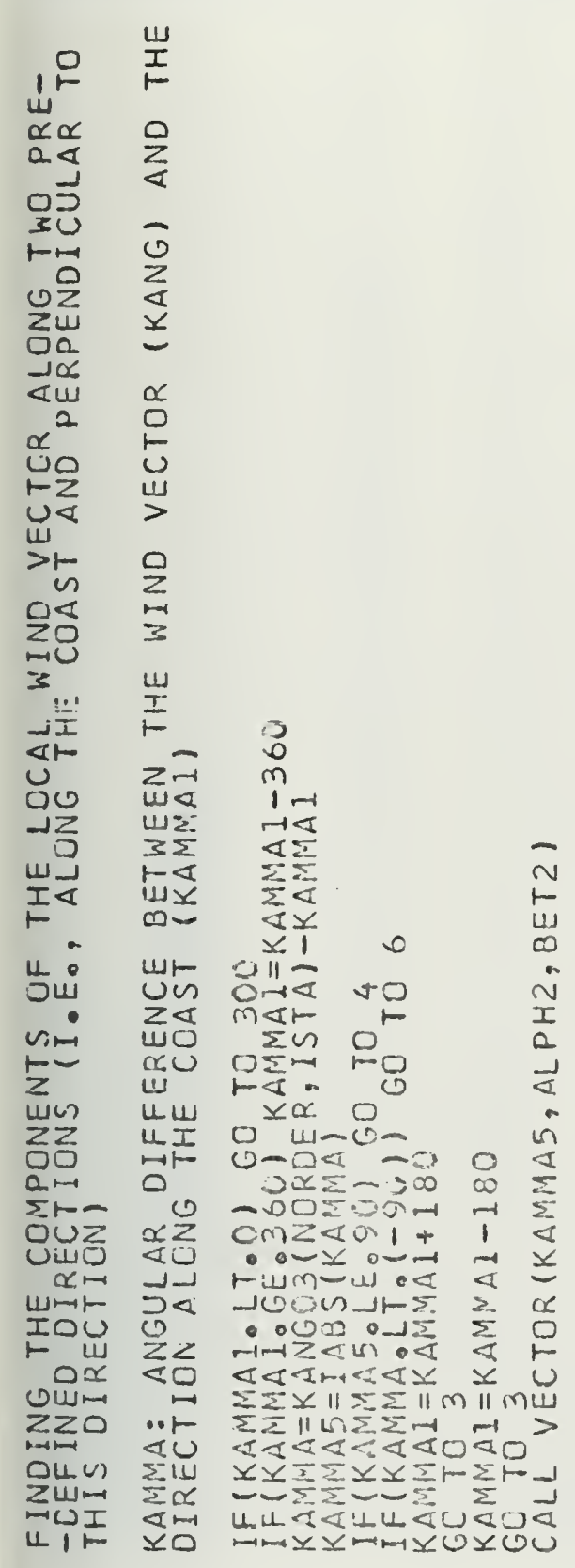

$m$ $\sum_{\longmapsto}^{0}$

$0<$

20

$=0$

JIL

$\circlearrowleft$

$\rightarrow>$

r

닌

2

$-4$

$>1$

$1-2$

sin

$z$

45

Ziji

$=z$ a.

$1-5$

20

4

01

an

$\geq 4$

0

10

int

$<$

$0 \alpha$

04

u. 5

IU

1

C)

07

Zu

$0 \mathrm{a}$

$-\alpha$

<U

a.

Mn

$x x$
$x x$

$\alpha \propto$

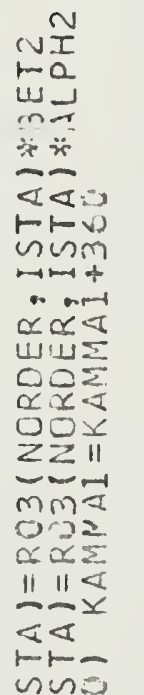

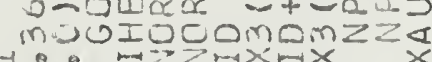
n.t. IU⿺辶- -

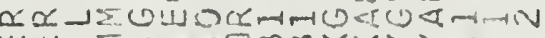
wu $: \frac{5}{2} \cdot 0 m m x<\langle>>0=$

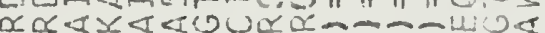

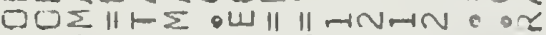

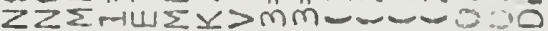
- $1<I<y \quad x>m m m m s$

0
0
0
0
0
0
0
0
0
0
0
0
0
0
0
0
0
0
0
0
0
0
0
0
0
0
0
0
0
0
0
0
0
0
0
0
0
0

02

or

$1+$

-7.1

$\leq \leqslant$

$\sum_{i}$

¿द

$x x$

IIII

NN

IL

분ㄷ

जロO घंब - meinm -1m|v । ILINM- $\sum \sum \varangle+\varangle \alpha$ $\sum \Sigma \mapsto N 1-\sum$

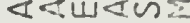
yメIトゅく o orris $1-\|I \simeq\|$ JONmtLr-1 - $0<110<1$ -nNos $\varangle<\omega<0$ ? troIt zजumLum⿻ IM an-m $=0$ $x \in=300$ $1-4 \omega 0-20$ noom. $24 m$ বूर्ट 1) ZZーDーNO - - 0.00. HNMmNCV F $+m 0<<m<$ c) COCE-1- $11 \Sigma$ ง ZZ11UN 5 - ZLU< III $I<<$

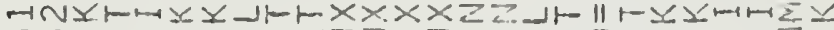

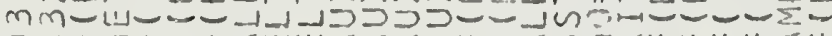

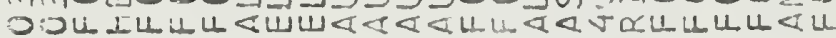

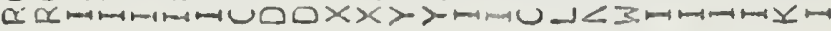
सx

$\infty$ 
000200000000000000000000000000000000060300005000

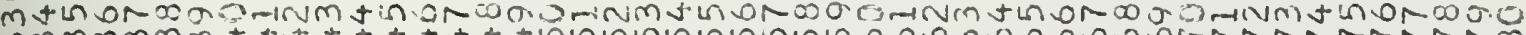
mmmmmmmง งナง

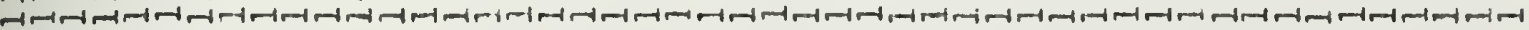

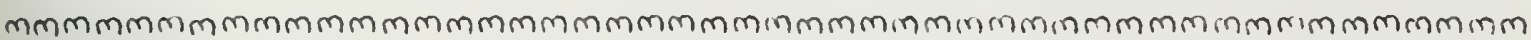

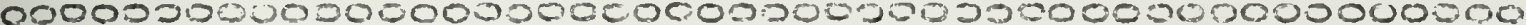

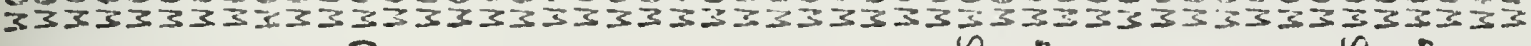

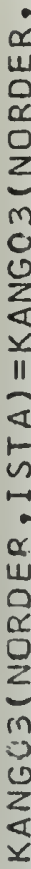

N

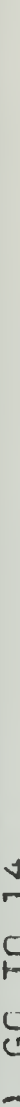

\section{เก่ $\sin$ $- \pm$

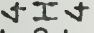 \\ tor \\ U- -14 \\ $\infty<\infty$

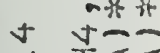 \\ 士ே< \\ $a+1-$}

DDajun ana

ODNar

0म<тயи

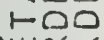

- כura

نே-

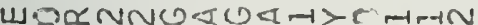

-00mmxx>> m-100Dण्डण

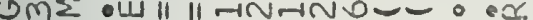

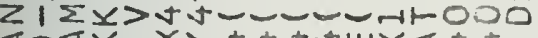

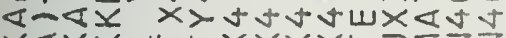
$x<x y+1-1-x \times x \times 1-3=$

0
0
0
0
0
0
0
0
0
0
0
0
0
0
0
0
0
0
0
0
0
0
0
0
0
0
0
0
0
0
0
0
0

$-1$

иง

?
*

at

in
$=$

1 เก้นก่

$\sim 11$

a II II

$\Rightarrow<<$

$1-r$

sx un

षण

- $\propto \propto$

HNE DOSIR
त्रह யய. $\infty$

11

$-r>^{n-x}$

a a $1-20$

$\mapsto \times$ an- $0=1$ xn・. $2025-1$

0 m.tun $m \geq Z \geq u$

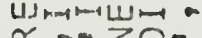

$30-0-11$

LI $11 \times a$.

U1 $0 \sum x>$

D ANOIS

r

$=m$

$\leftarrow \propto$

?.u-in

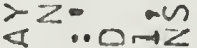

$0 \geq z$ ow

$\sim \pi \quad$ monint

$a+$

$\alpha \amalg$

UI

0

U-

$>1$

min

Ond

o.

1n an

र्जu -

th $\omega 11-$

Tnuse $x$

- טu. $>0$

$0 \cdots<\propto r-\pi$

-1ำกำ

IND- Z.・

$$
\text { an }-111
$$

um- xr-1

no un nozu

ना1 \& axnम

To $u=0 \cdots 0$

$\sum \propto$

$\sum 0$

$\varangle z$

on $N>$ N

ax

nox acos

D) Mn-

Z

$<m$

$-\infty$

xum-..4

nde

ㄴ.

는

aD. zusu

$\sim<$

$x \widetilde{1} \| 0<\mathrm{In}$

เाU

$\checkmark-z \mapsto \cup z m$

- nOU LUm

>xฺแயua

vitarix

- nயO 0

$->\alpha-0-2$

o>m-2 -2000 $\infty$ i.

$->v^{-11} x$

$\infty 1-20$

an=0न xun.tran

$>021-n$

- «nveno.

$\varangle$ ๑ 1 $m Z Z \simeq u$

แ๓raun

$\alpha \cdots 20$.

$0-\infty 0-11$

$4 \| x a$.

Li $0 \sum x>$

$\infty$ ond

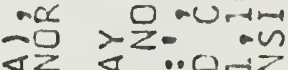

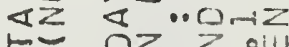

nm Uomint

mis $m=23 \omega-2$

n(5) tm am

ㄹ एदU -

U< $\vdash \vdash \omega \|$

ox बजियर $x$

c a $-U L>0$

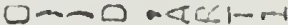

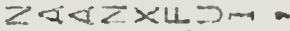
minis 3 in

Oमm on aly

c. a aum- X.t-

$-\alpha \propto U_{n-1}+2 \geq u$ -Uயयद axता-

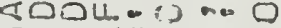

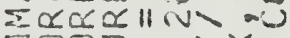
$=050$ ax

IZZn ol -00

$x$ - O>nnt $\min z=n$ बmot an -Oo Huma

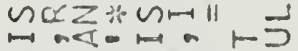
mx -D. zusu $\alpha<a \times c \mid \| 0<m$

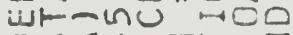

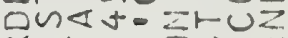
omt n-0u H ㅁos $x \times$ muna

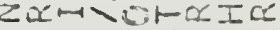
is a d NUntFu maL- $\backslash \alpha=0-$ m0OO\- az" -

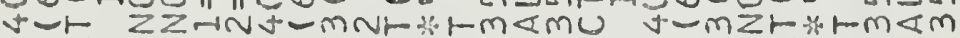

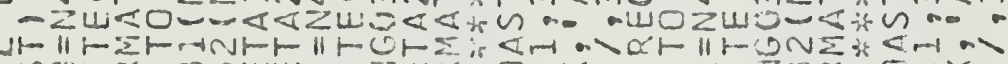

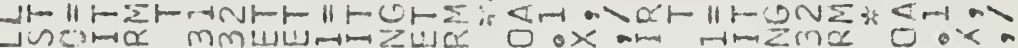

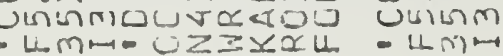

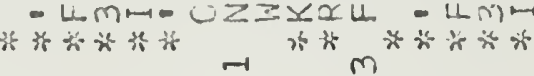




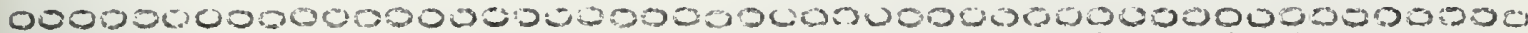
-INM 5 LI W

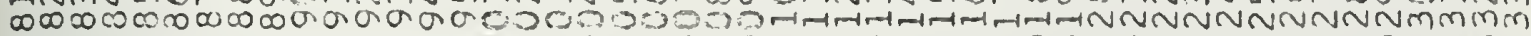

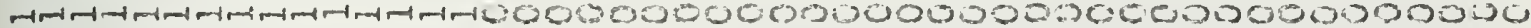

MmmmmMmm $0000000000400000000000000050500=005000000090003$

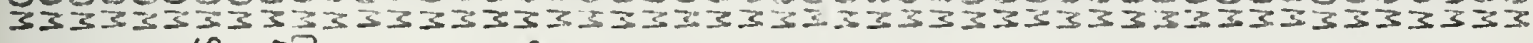

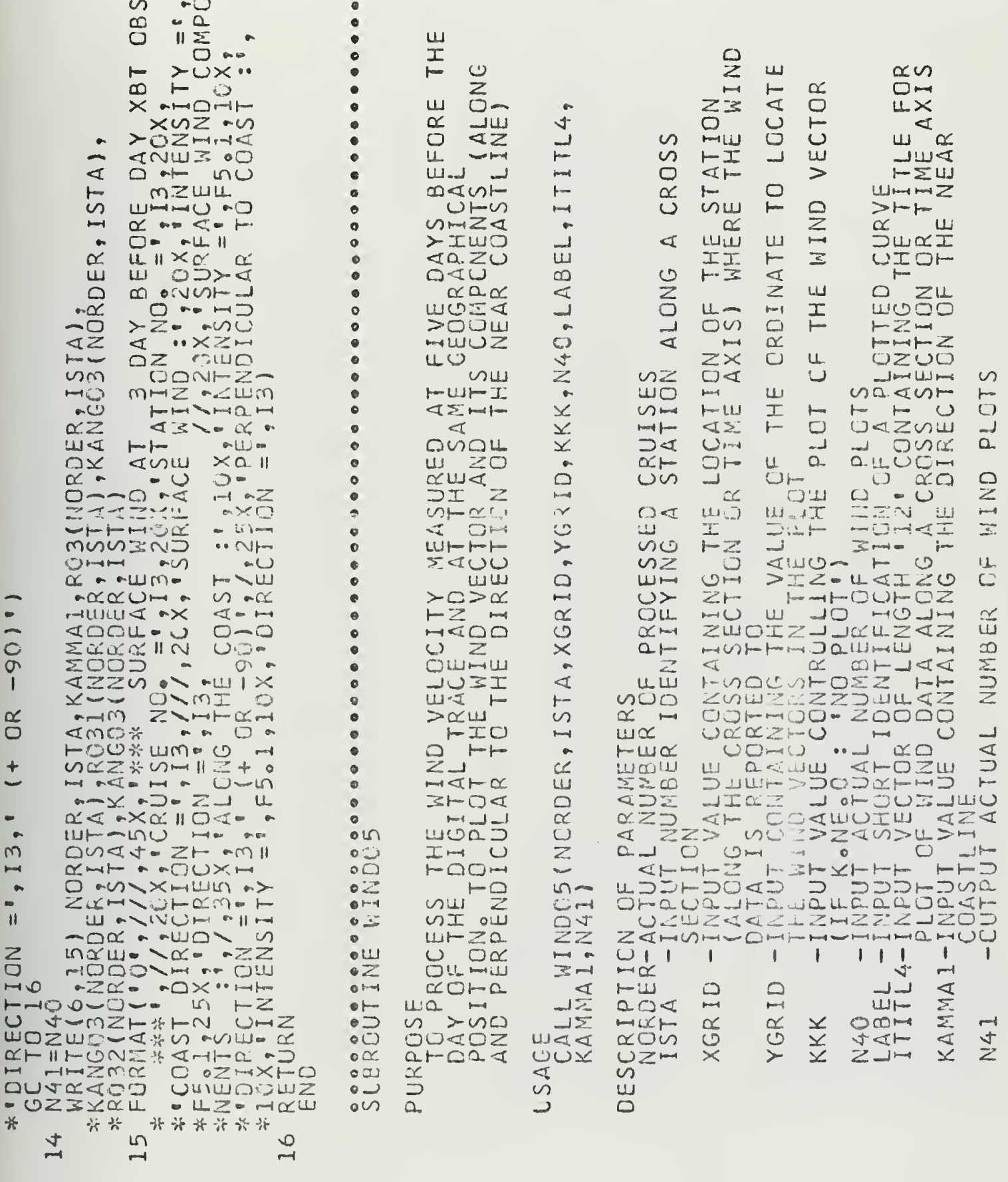


000000000000000030000

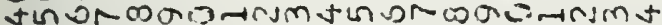
mmmermmatatatatatanisininis 0000000000000505000500

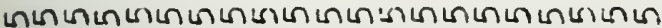
000000000000020000000

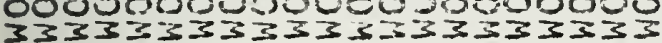

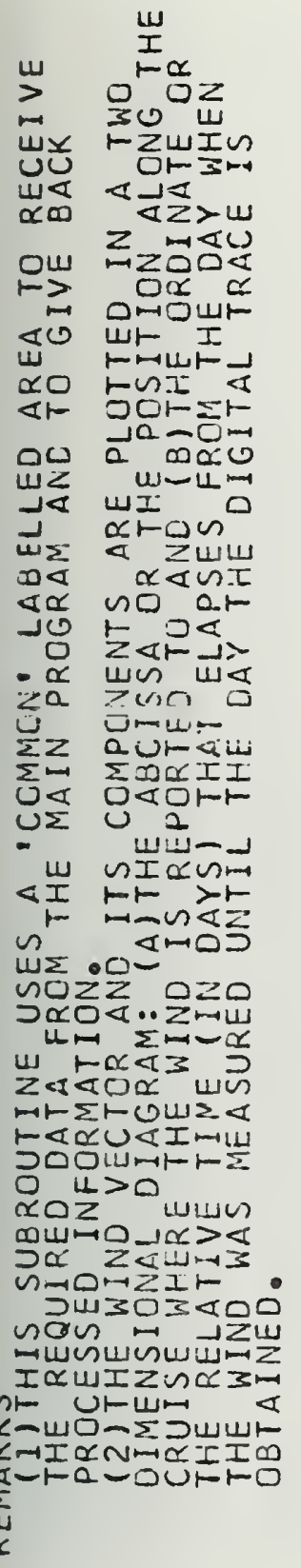

0000000000000003000000000

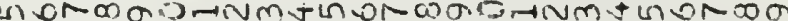

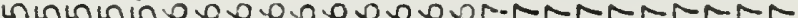
6000060040000.250 .200000000

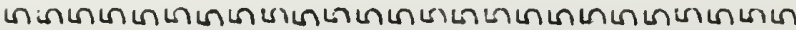
0000000030000000000000000

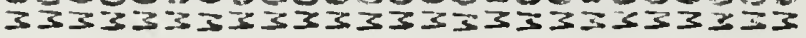

$\bar{v}=-$

$+\rightarrow-F$ $x$ an SOMONN \&Nan-trat $>-0-\cdots$ anNTID -ionina NOLIO -Zwarm tam $x$ $x x+\cdots$ $\supset \operatorname{mon} \alpha \alpha$ I-V V. . . $x+1-100$ $N=N-2$ NONON= $m-m$ and X-1 DCWarvm षQZ $-N=$ $>a<-3 \pi$ - XNII $x$ aval a. sim $=m \pi$ - nerara $m=1.1 N=1-1$ XN a- ... 2-0.200 CONmNN $\times \mathrm{CI}-\mathrm{O}-$ -0mzmN -Zu<rin N\&氏y0? $-x+-\alpha \alpha$ "r. X-NNAPNANN षा a - क्ना $>0.00=$ DONES an-niv - N-NOLN-O 3 mivm

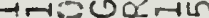
- xaz aOe $\checkmark 2><-x$ < $10 x$ a

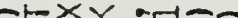
Wन ZงトZON JनI -ZnOIว-

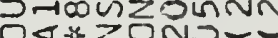
O\&*ZONDव $0 \sum \varangle \sum \sum N \geq \pi m$

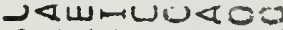

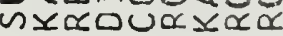

n. T2 $2+$ - 단 -II-

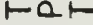
Uـان $-\infty<\infty$ \& $-4 x$

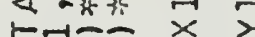
जI $\varangle \vdash \vdash$

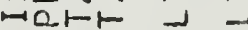
majus us unn वराम

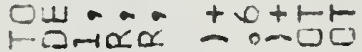
खणयं mनll $00 \geq 00-+-20$

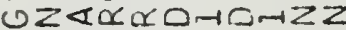
$-x 00-x-x$ - w-Z<0 $200-1-$

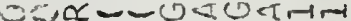

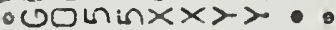
$1-Z F O 0\|\|\|\|$ "

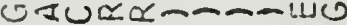
-Yu II II HNan 0 $x \|>-1-1-\backsim 0$

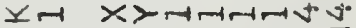
$x 0-1-x \times x \times 2 \geq 2$ -Z11 1 4.

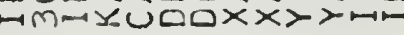
* 
0009000050000000020000000000000000000000000000013

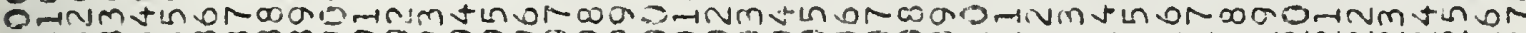

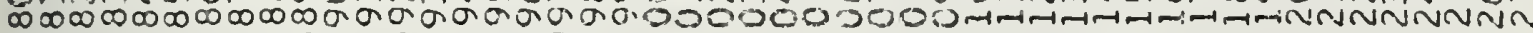
00000000000000000000 नान्न

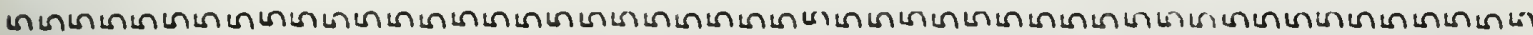

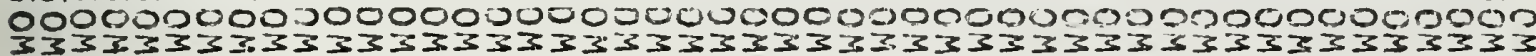

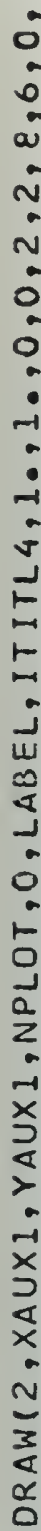

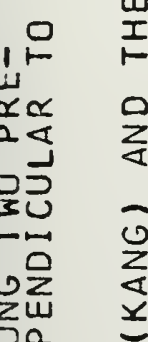

\lrcorner$\propto$

षा व

$\alpha^{0}$

믄은

$5 \geq \underset{1}{\longrightarrow}$

w

$>$ i 2

이눈

20

3

山

西

0

工 w⿺

피 《र

w-

w王

$F$

u.

ธய

n๓

卢

wn

Zz

an

is $\Sigma F$

○Uz 는

טயण <०

$\alpha$ ar

யrit

IDU

इय

गे

$-\pi \widehat{N}$

$+2$

00

t- லUn

Zu $z$

Trlit

deson

बरि

स $<x$

uㅢ

$\mapsto O I$

U1 1

$\sum \sum$

$\sum$

팜

至

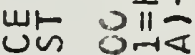

$\sum \leq 1 \leq t$

wO

w

$\sum_{i=1}$

Uw

UI $\sum_{3}^{0}$

0
20
20
$\geq 0$

깅

ن

0

ז

Un

$\sum_{\text {III }}$

r卡

-int $N$

n- NI

$z$

wi

m<

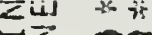

一O

a

tis 10

$0 \stackrel{x \propto 0}{0}$

0- $\begin{aligned} & 0 \\ & 0\end{aligned}$

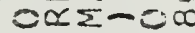

- 00:

omz<al

- o x เய山我

त.

-1 $\rightarrow$ Zरा

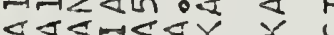

$\sum \sum x \| \sum \sum_{11} m$ II

$\sum \sum \| 4 \sum \sum-1+1>$

$<<<<<<<0<0$

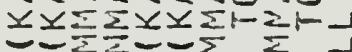

U.

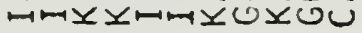

○

m

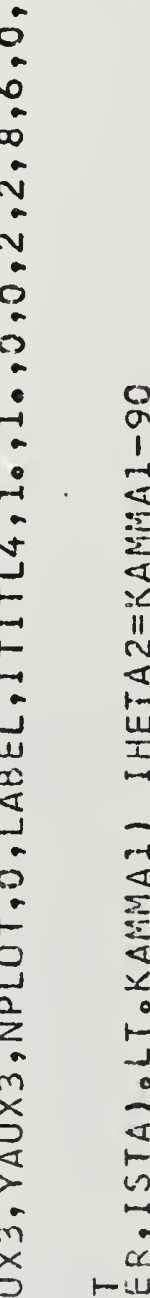

uड

IU vus moUIQOCMOMZZZ no

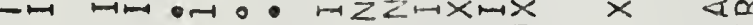

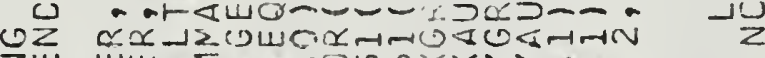

zw w山

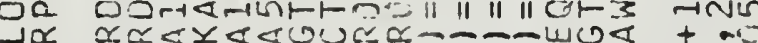

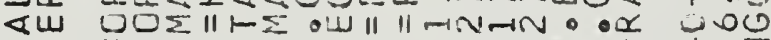

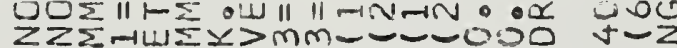

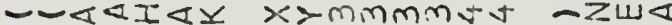
mivx: $-x y-1-1-x \times x \times z Z-1-11-x$ แnเแ-

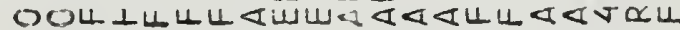

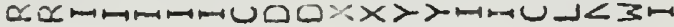


0000000000000000051200290000000000000303000000000

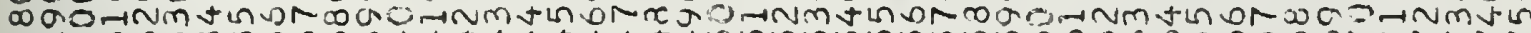

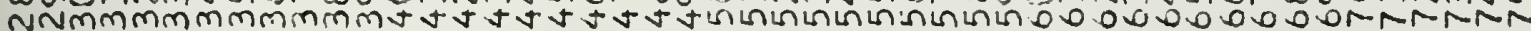

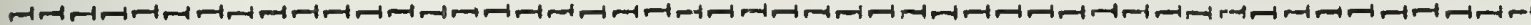

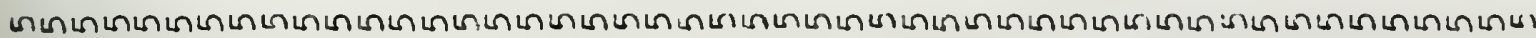
000000000000000000000000000000000000000000000000

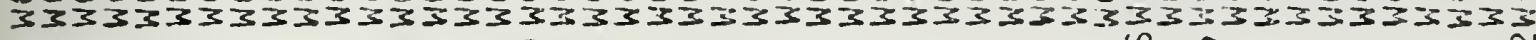

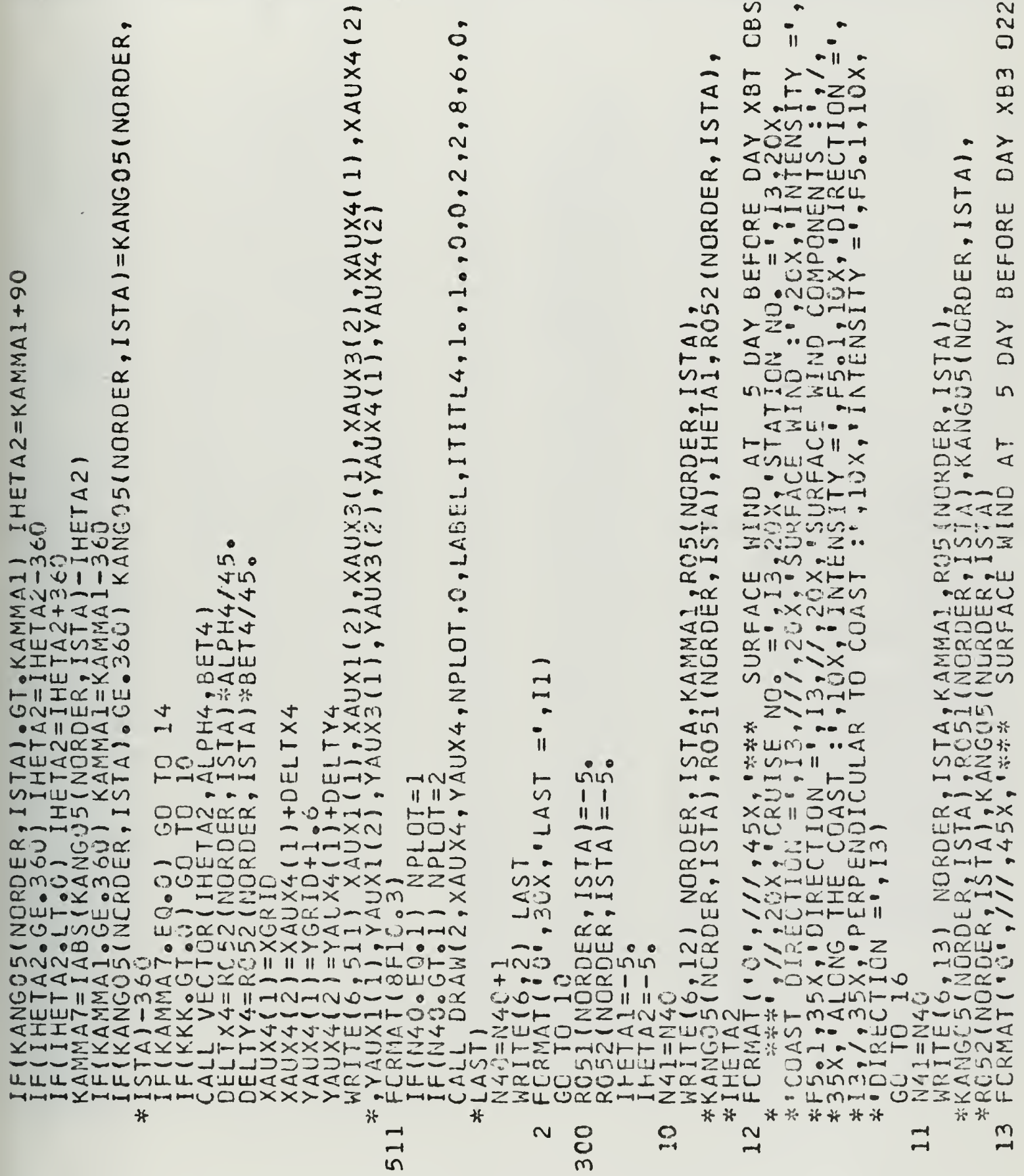




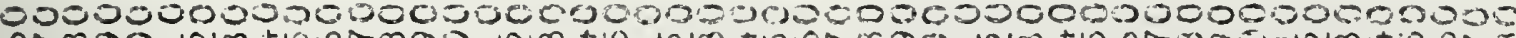

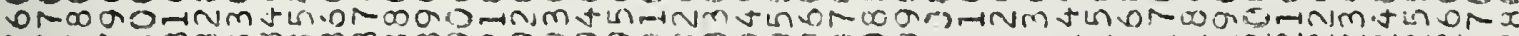

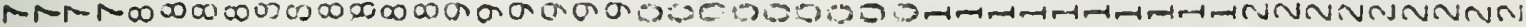

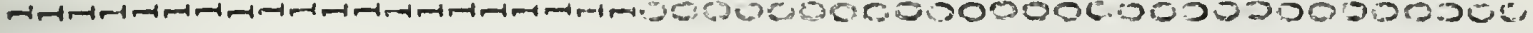

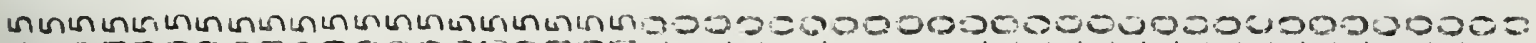

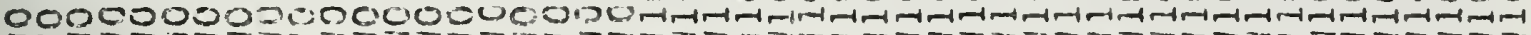

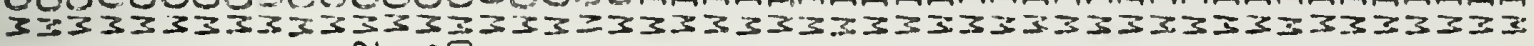

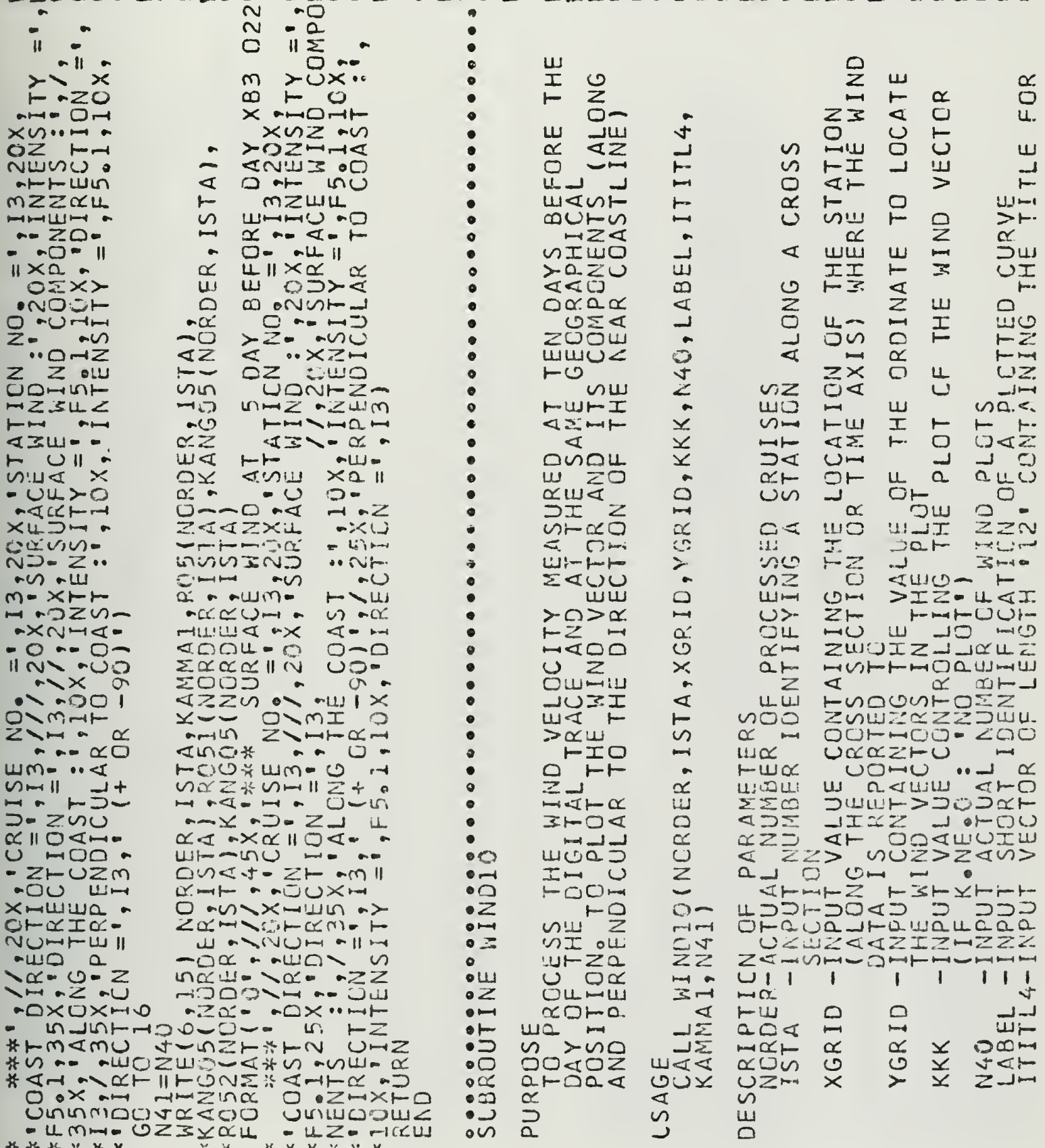


0000.3000602000300000000000

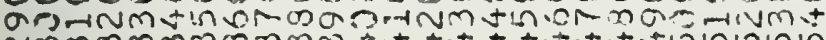

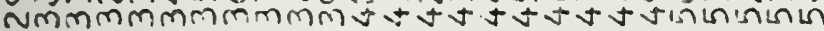
0000000.0500000000000010000

$000000200000000000020=0000$

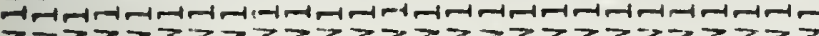

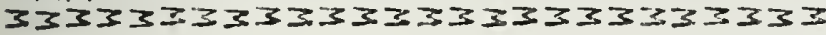

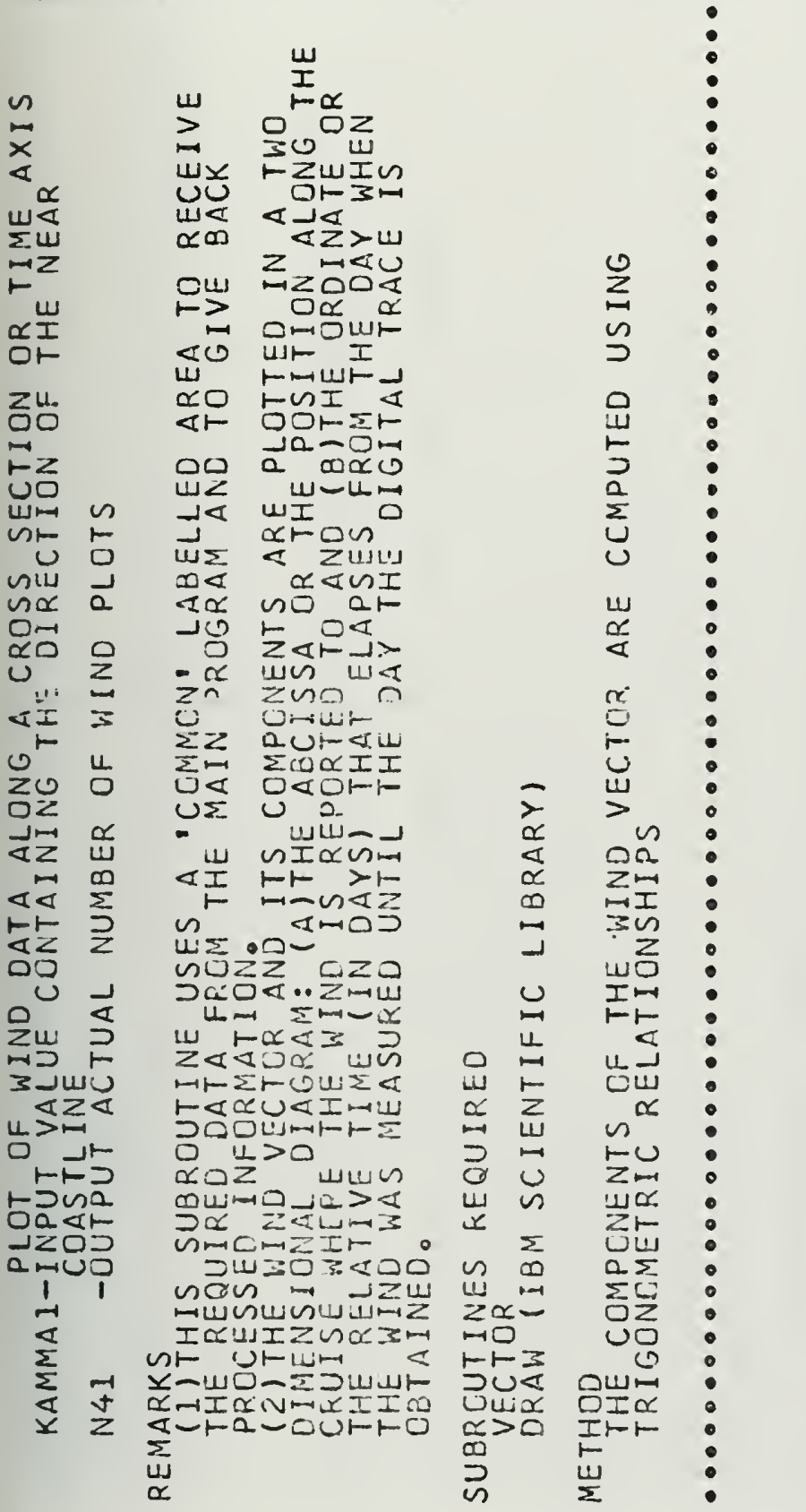

00000000500030000000

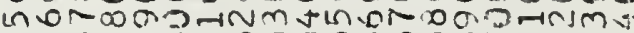
แกเก Q000.0000000000000000

$00000008000000.20000 \%$

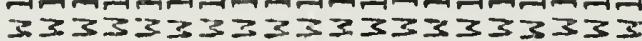

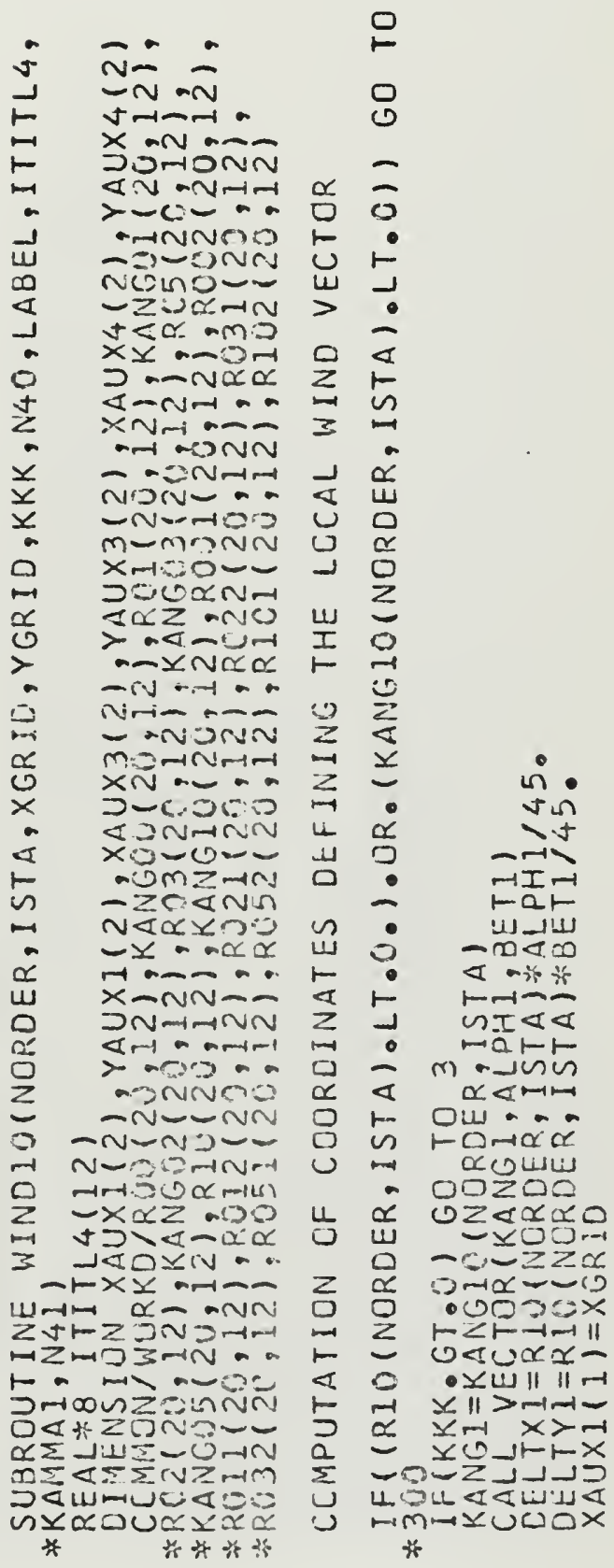


$00000002000000000000000002000000200000006020=300$

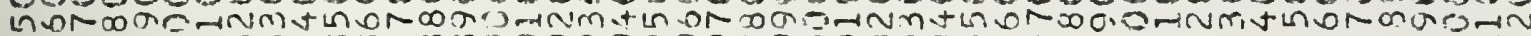

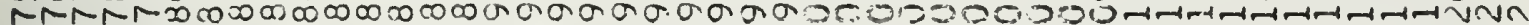

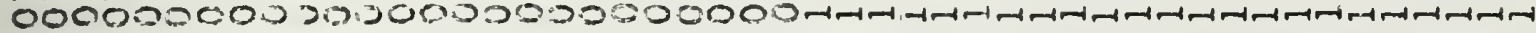

000000000000000000000000000000000000000000200003

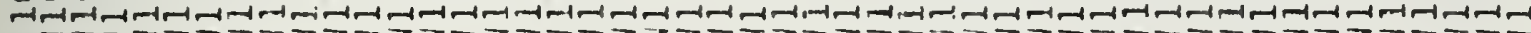

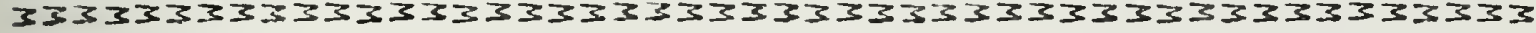

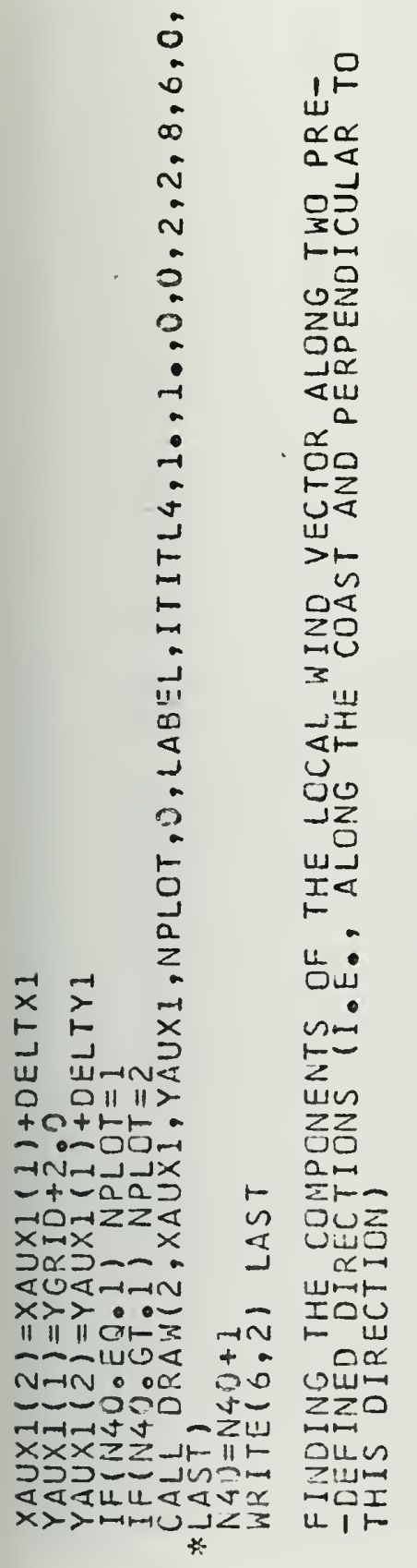

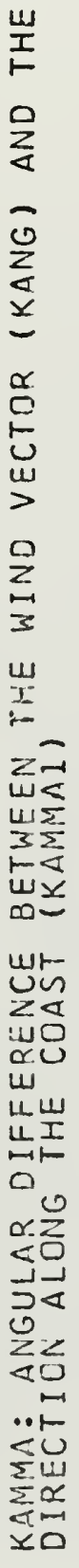

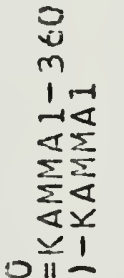

0112

$0_{n \rightarrow 1}+0$

m⿺𠃊

$0 \leq-\infty$

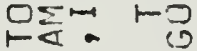

$x \propto 0$

0 แ1 0 -

acte-2:00

- Opserar

$O M \geq<\sigma 1+1$

$0-x$ o-

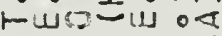

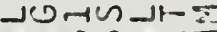

- 000 - J

-1नZZया

«Iखा

$\sum \sum Y\|5 \Sigma\| m \| m \omega$

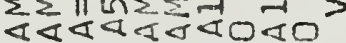

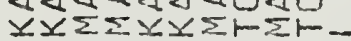

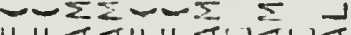

$4 U<\varangle U 山<0<0<$

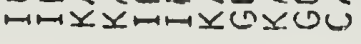

$\sum_{i=1}^{0}$

c)

$\sum u$

$=0$

$\frac{11}{10}$

కु

0

$-1$

cus

2

$>2$

$\mapsto \mapsto$

$\sum$

w.

$-2$

Zui

$\mapsto Z_{0}$

$-2$

$\mapsto \pm$

யง

Zn

an

$\leq 4$

วठ

us

10

n

$\alpha$

$0 \propto$

U⿺

13

IU

Fin

$\infty ?$

$\geq$

00

\lrcorner$\propto$

<I

a

$\ddot{m}$

$x x$

$\alpha \hat{\alpha}$

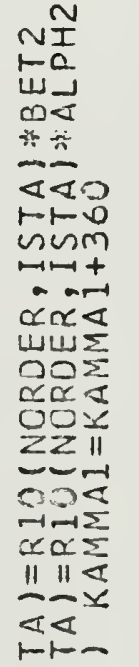

8

$m$ in.

Tn

$-m$

mIm

rar

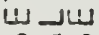

$\infty<\infty$

a t

$m-m m$

$I<<x>$

$a r b r$

Uొڤ

an a

0 Q 11 li

$00-1 \times \alpha x+5+h$

- 0 rousus - o¿ -00 -rvald

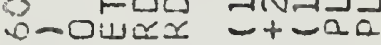
nus mesorooomomzz -1

-

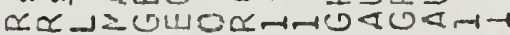
UIU $0 \leq 00000 \times x>>0$.

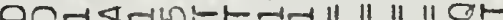
$\alpha \alpha<x<\alpha 0 \cup \alpha \alpha-\infty \ldots-m 0$ OOE II ZZE" - $<<I \varangle y \quad x>m m m m t t$

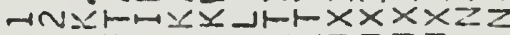

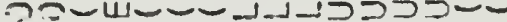

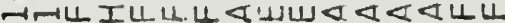

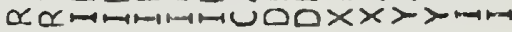




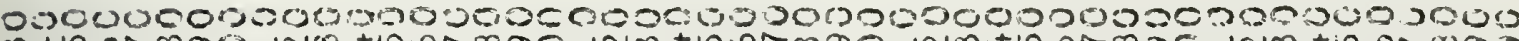

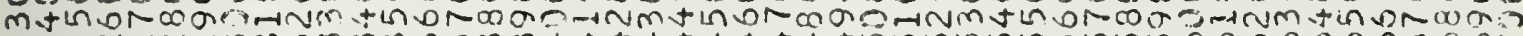

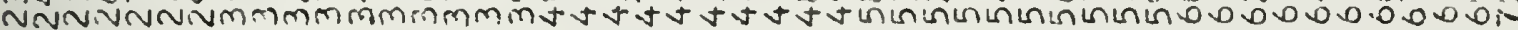

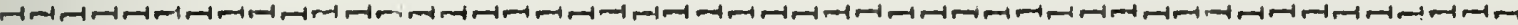

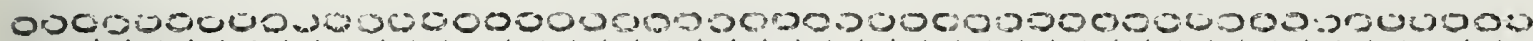

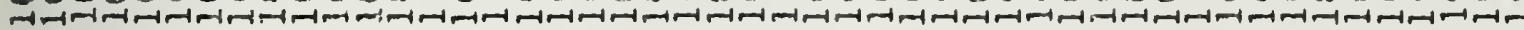

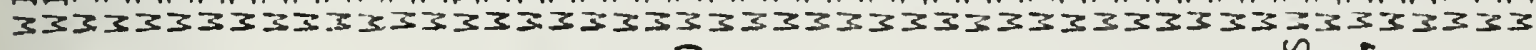

0

$\dot{0}$

$\infty$

$\stackrel{\infty}{\infty}$

?

N

í

0

$-1$

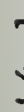

$\frac{5}{5}$

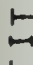

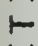

$m$

III

$\infty$

3

c)

.

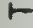

0

$\frac{1}{2}$

$z$

$m$

x

2

క

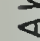

$\alpha$

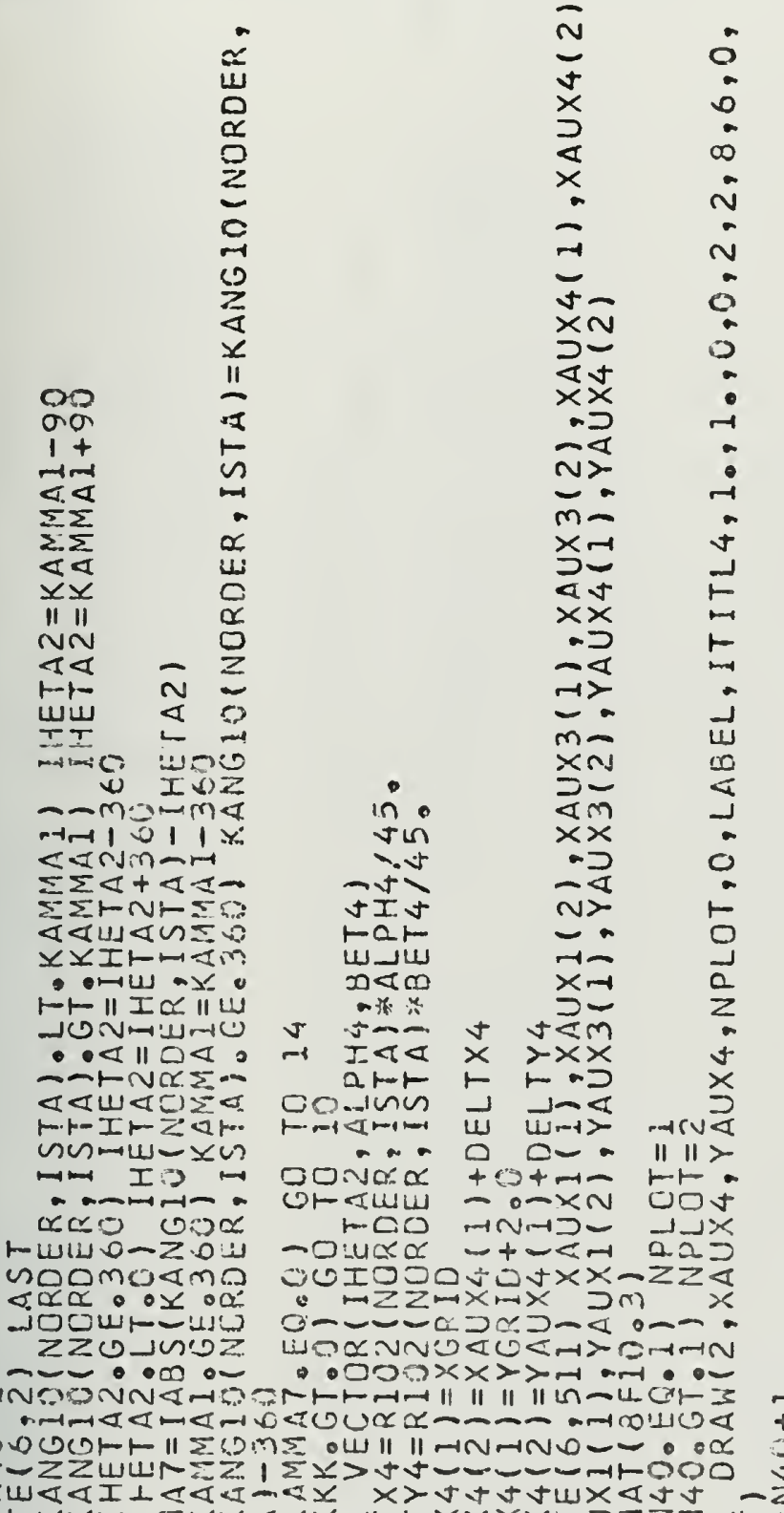

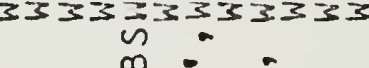

$\infty$ il -

m $2:-20$ $x \rightarrow-0=1$

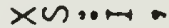
$>C 21-1$ aniesus c) $m \geq Z \sigma u$

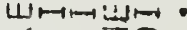
$\alpha \cdots \infty-$ O- n$4 \| x \Omega$. U $\mathrm{c}$. O NDJH C -Wनन$>2$-n $4 \cdots 0.1 z$ $0 \geq " \bar{z}$ 00-4Lก Criz311.2 -11 <IULI -1 LII पnus $x$ - vu $>-3$ $0 \cdot<\times 1-n$ Z洏つ以. $\rightarrow$ กำ $\geq \ldots$ in -14

un $x+1$ Un- $-2 \geq 2$ ? \& $\rightarrow x(\sqrt{1})=1$ $4-0-0$ $\alpha \| N\rangle=$

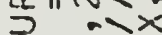
in ax $0>m+11=$ Z\$x<\infty$ *um- .. *らッ\| = $\therefore D a z \cos$ $x \propto \| Q<x$ In以 $\checkmark-z t \omega \vec{m}$ - - T65 Lidm >x-1UuR.

$>350 I \alpha$. >rvumbin - na a $-\alpha x-0-Z$ $0>n-2-0$ - $0 x 0 \times 10$

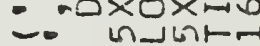
- Zu< 4 -

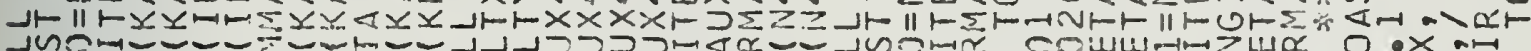

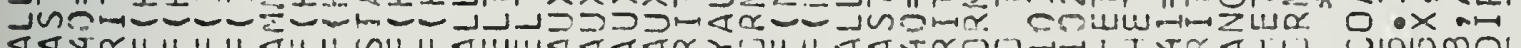

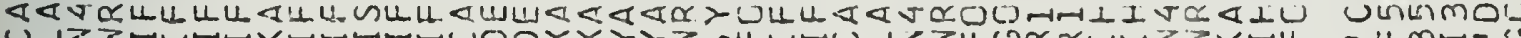

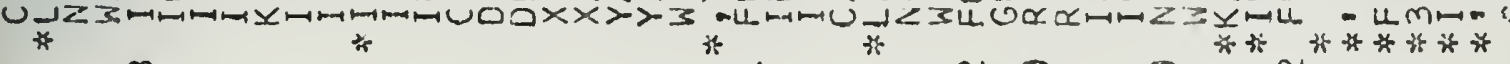

$\infty$ in
N 3 


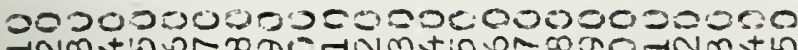
नNM

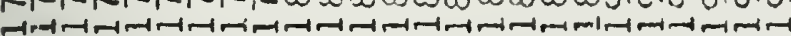

00000000000001000,005000000

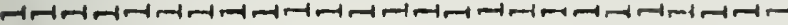

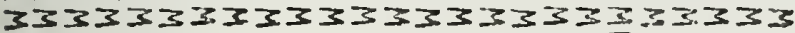

is a

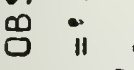

$r>>^{-11} \dot{x}$

$\infty \vdash-\geq 0$

am$\times \backsim \cdots+$ $>0 Z$ Inแ⿻上丨

\& 0 -r-tயu ค.) $Z Z \simeq 4$

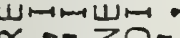

$\alpha \cdots \geq 0$ -

$0.0-1$

$\frac{11}{11} \times \dot{0} x>$

0 (1)

-Uता

$>$ Z

$<000$

口乙 z טornt Onz $3 u 2$ 나네 $\rightarrow$ < 3 LU - -

トLU\| \&نuse $x$

a. - Uu $>2$

$0=0+<\alpha+-$

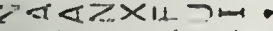

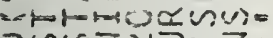

oทvinฺ- Z.

नHम नU -LU

a. - $-4 m-x \vdash$

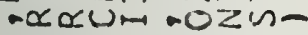

नயய< न:くNウட

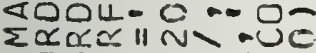

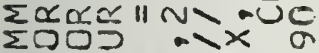

<zZu • >

$x=0$ orm $\rightarrow$

and Zरा a $\alpha$ <OनH : . 20

-

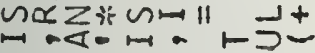
다-D- zजu

$\alpha \varangle a x \alpha \| 0<\mu$

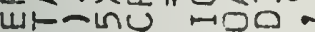

ou $4-Z \vdash U Z m$

वmt - - OU :11m

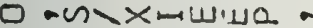

$z \alpha \mapsto s+\alpha \pm x$. W a vUnFU\|

-0\% - -40 Q

maw- $\propto ⿻-v=z$

-1000>m-z -

$-2 \alpha-a x 0 \times-10$ ษーOZト zWन-

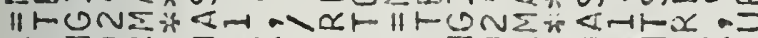

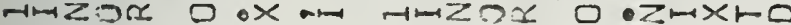

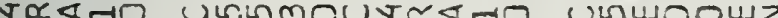

żyau - Umm- * *

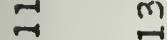

$\checkmark$ * * * 


\section{FORTRAN IV SUBPROGRAM TREND FOR IBM 360/OS}

$000000000000190000: 250000000=0000000000$

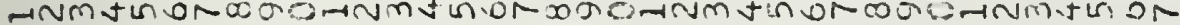

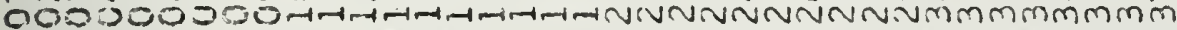

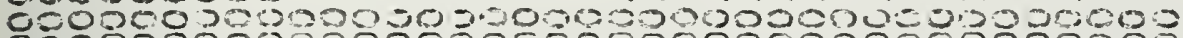


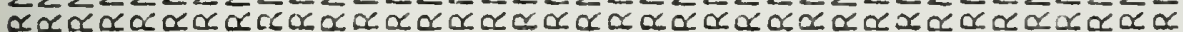

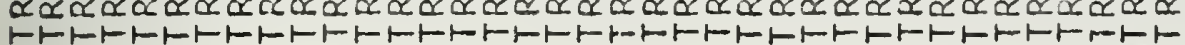

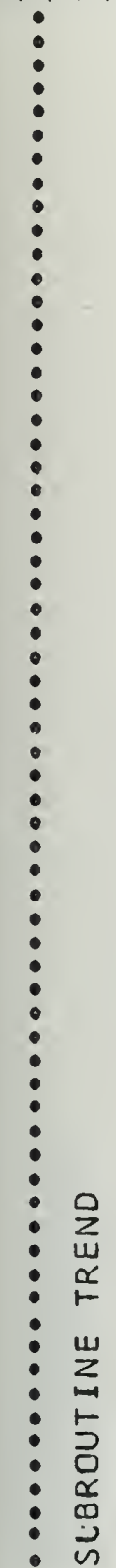

$w \Sigma$

纱

$\Sigma \propto$

$\propto$

U

-

피

05

10

QZ

$\leftarrow \alpha$

4

4

OU

जז

$\rightarrow$

$\sim>$

Ju-

它に

<仙

Unल

$>$

Ð0

0

ய-

วu․ㄴ

$\square<$

$0<$

$-\frac{\alpha}{2}$

nu

N\&Z

แ० נ心n

\& $n$

$z+\alpha$

○<س

$\rightarrow ?$

no

更

W曰

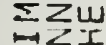

口曰-

w 0

이은

$11-$

Q

$\propto$

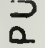

2

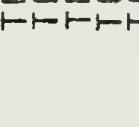

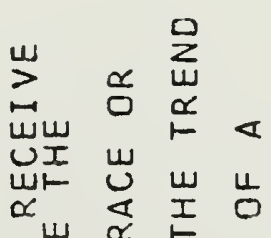

Ora $r-n$

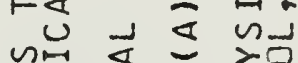

ய? $m$ แ..

$\propto \sum 0 \quad Z u \geq U$

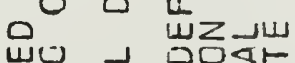

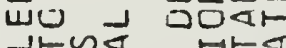

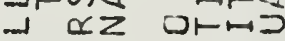

ய०Wm $1<10 D$

$\infty Z \geq 0 \quad 1-m<$

ব<Zm n-10x

\lrcorner$=\mapsto \alpha$ Z

$\sum \ldots 0$

Zazu rora

OUnI wUPAO

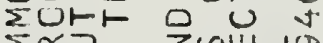

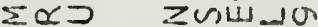

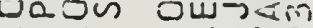

U वH $\cup \propto \infty>\sigma$

- zonar mós

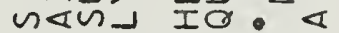

wड \& Fயन्म

ज एट $\alpha \pi n z$ गWI 4 and IF ID IO

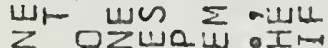
is -u $⺊$ ए

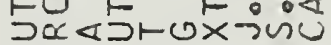
OUト

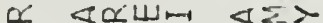
œZ0®-1

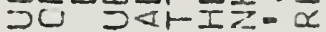
งmon>>யr 4 U山

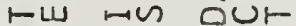
nবnuD - - $<z$ m

エ๙யயエயールト

जトCUト

즌

வーツローレーを

$\sum_{i=1}$

\&
4Z

षण $\rightarrow 0<\cup$

MD> แD.

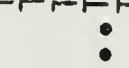

Domergonos cos-hnmalno

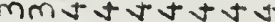
0.00000305

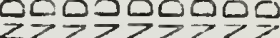
$\propto \alpha \propto \alpha \propto \alpha \propto \alpha \alpha$ ロトトトトートレト

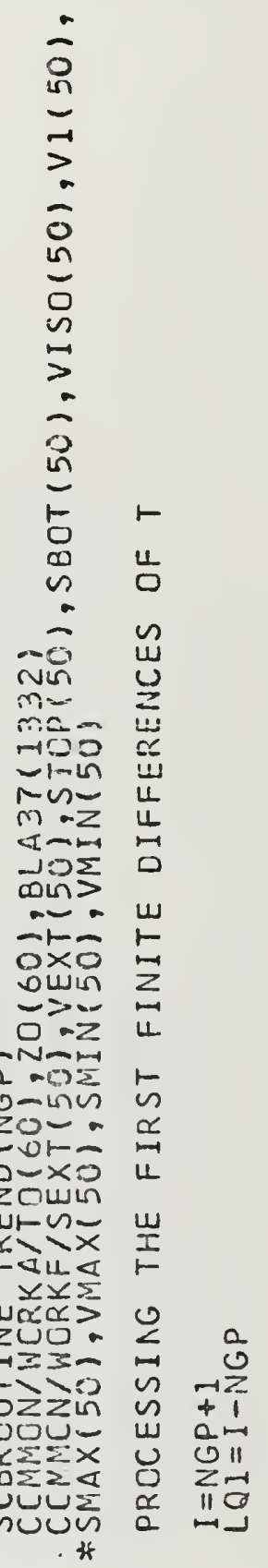


00000060000000060,00000000000000000000000000000 Torb.

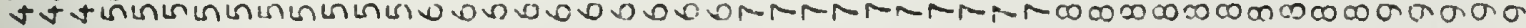

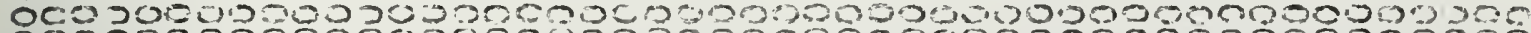

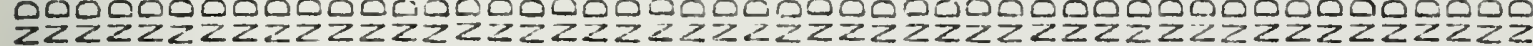

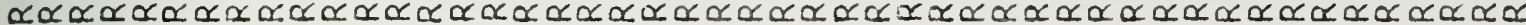

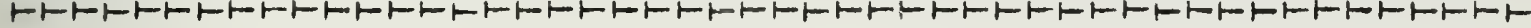

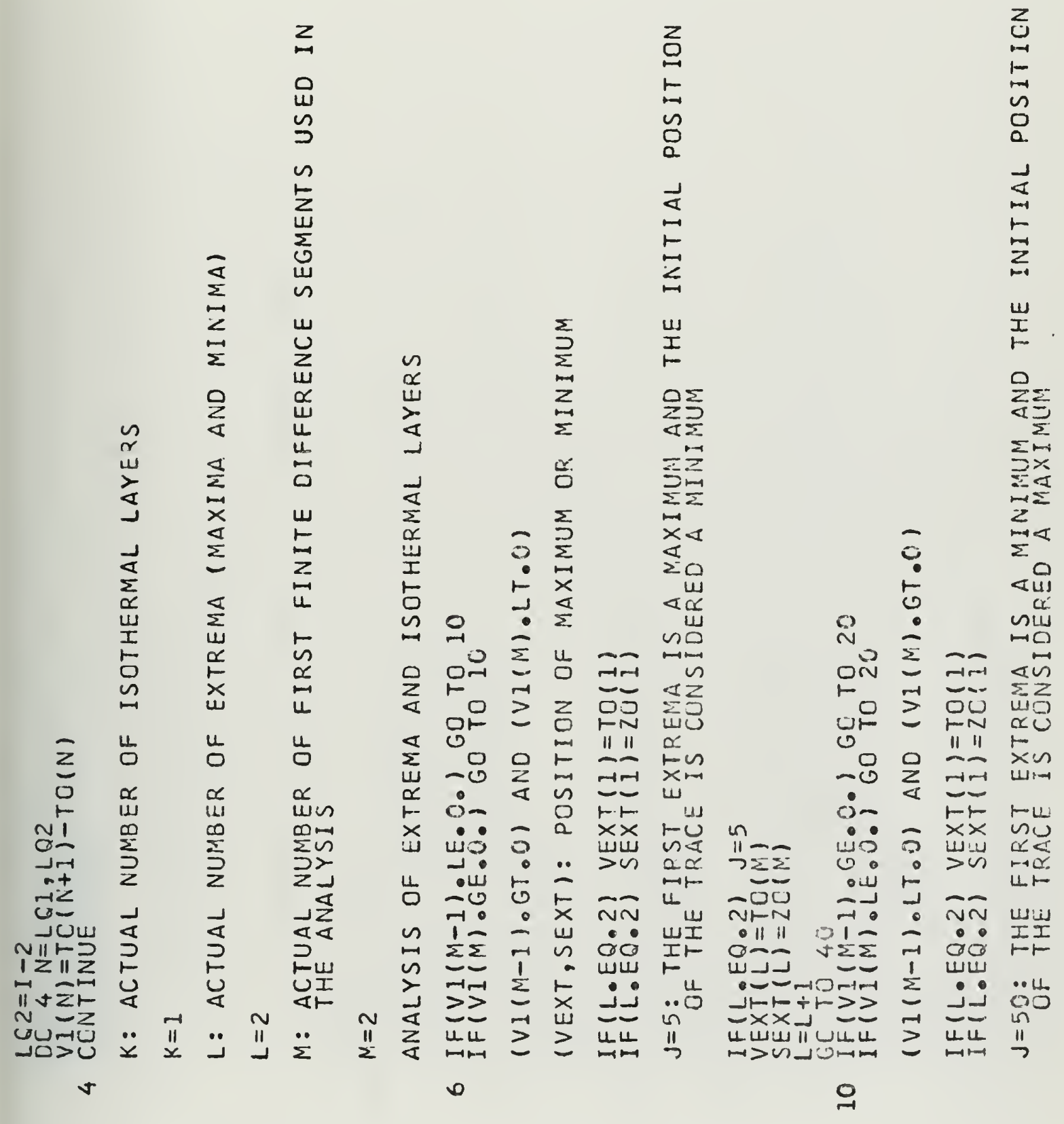

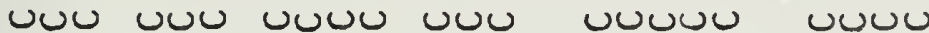

טuv טu৩ 
000000000000000000000000000000000000000000000000

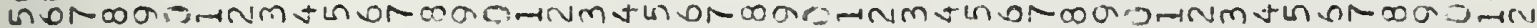

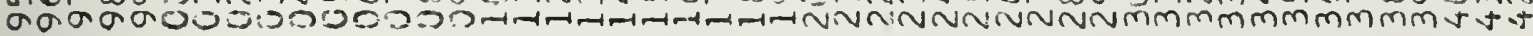

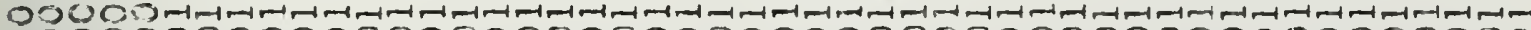
0000000000000000000000000000000000000000000000

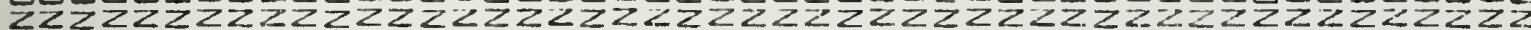

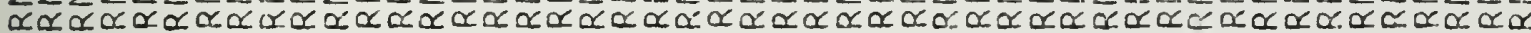

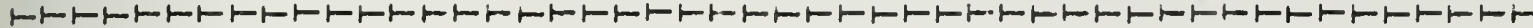

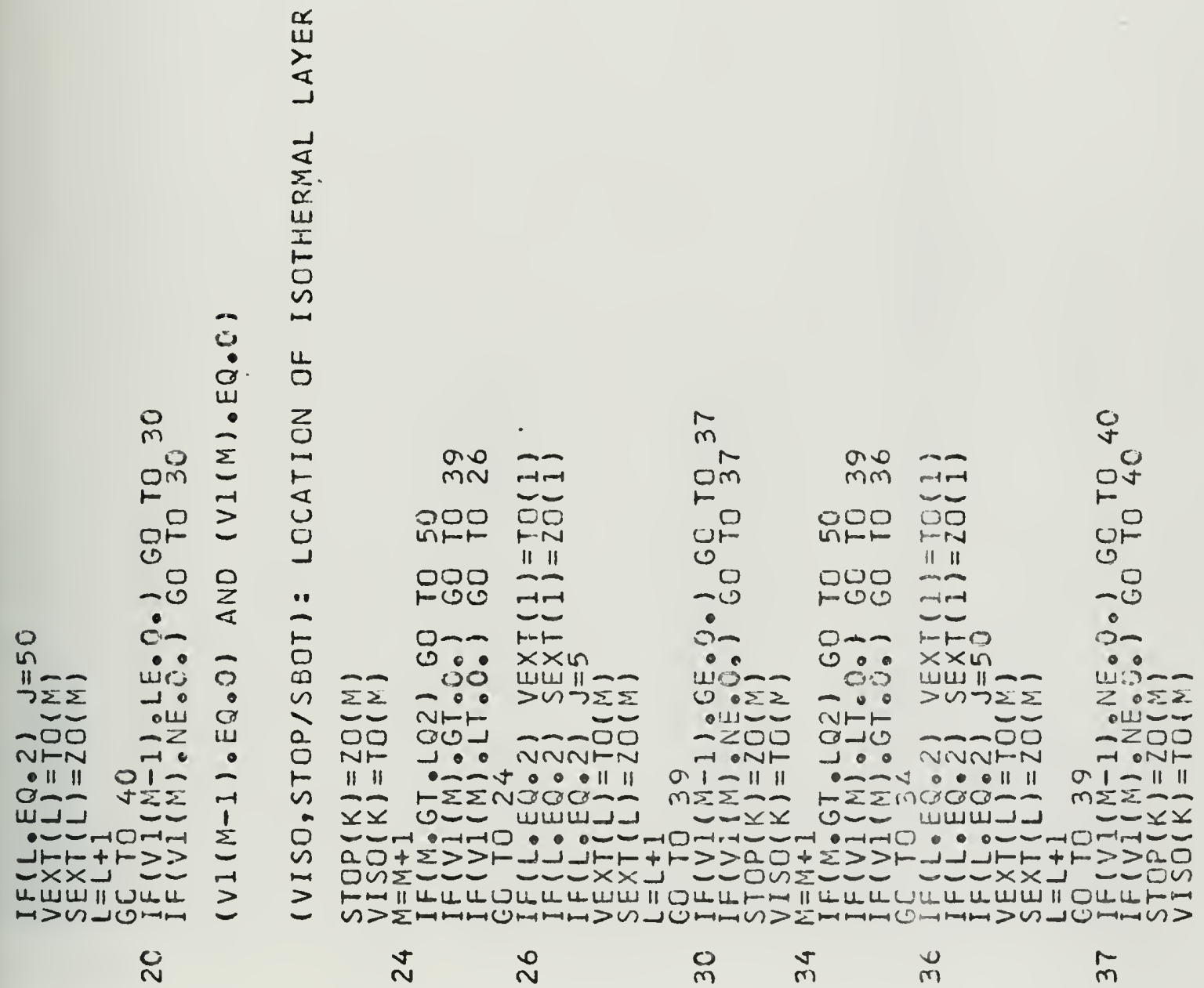





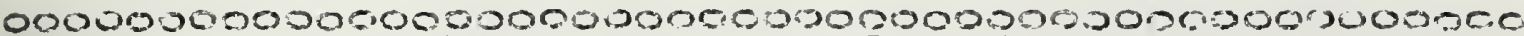

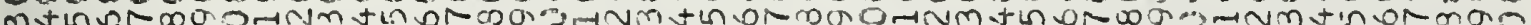
Ftง totง

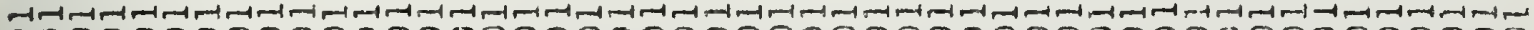
000000000000000000000000000000000000000000000000

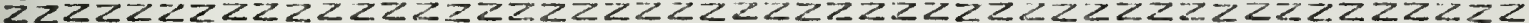

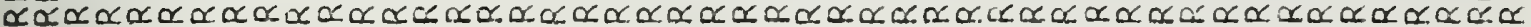

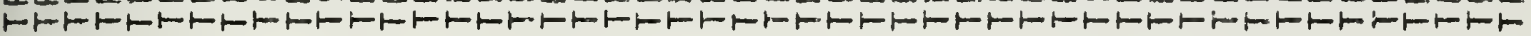

$\infty$

$m$

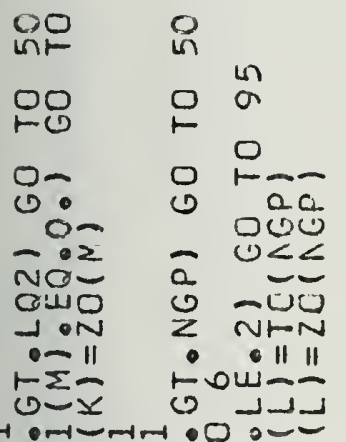

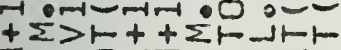

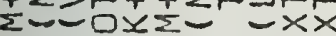

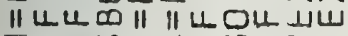
$\sum \mapsto \mapsto n x \Sigma+10 \mapsto>n$
$\infty$
m 0
in

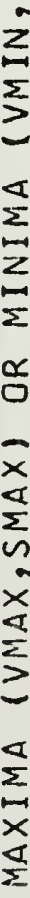

\section{Z}

$+4$

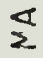

us

$\stackrel{a}{x}$

u

I

$I$

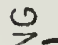

$z=$

tra

$\cup \Sigma$

UI

实

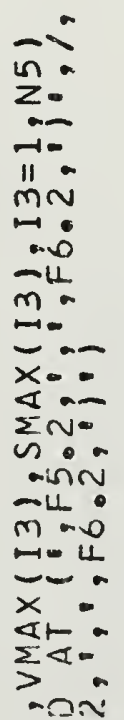

miss.

$m \rightarrow-6$

rराप

-u.

mo.

$\rightarrow-10$

$z-1$

$-1+4$

$\sum-$

जive

mat

$m=<$

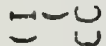

ZZル

$\because$ ind

$\sum^{2}=$

an

$-m \times N$

Nint

$\rightarrow-\infty$

an n-

$100 \pi-10-1$

- DS0. 00

$\rightarrow-0=$

- (n) 1.

Wt 00

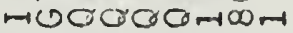
- เแயเบแ +

NUOCOOH

-

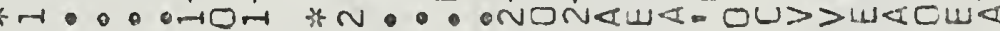

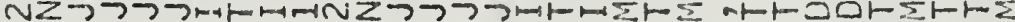

|| ||-C- i| || || || - - - || || || mrlL 4 LU

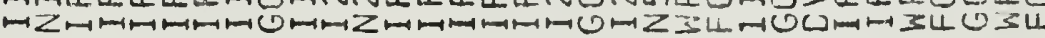
$\infty$
$\stackrel{\infty}{\infty} \infty$

o $\sigma^{*}$ in $\quad$ on 


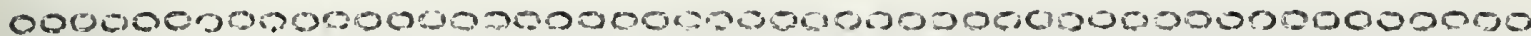

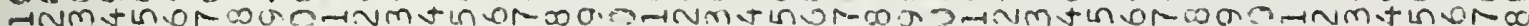

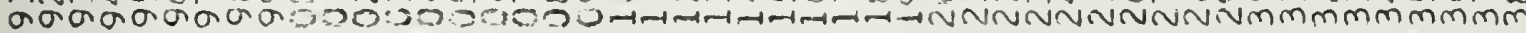

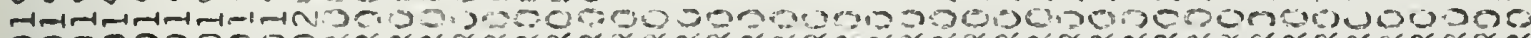

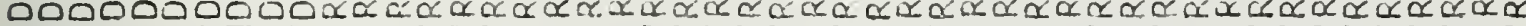

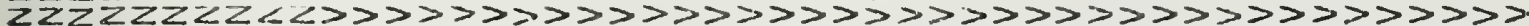

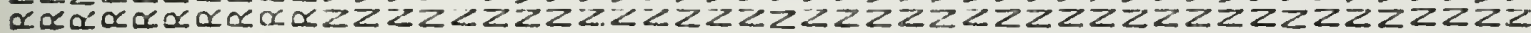

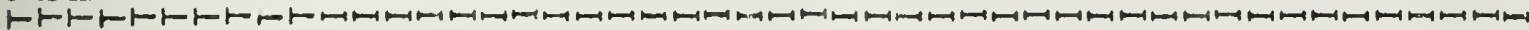

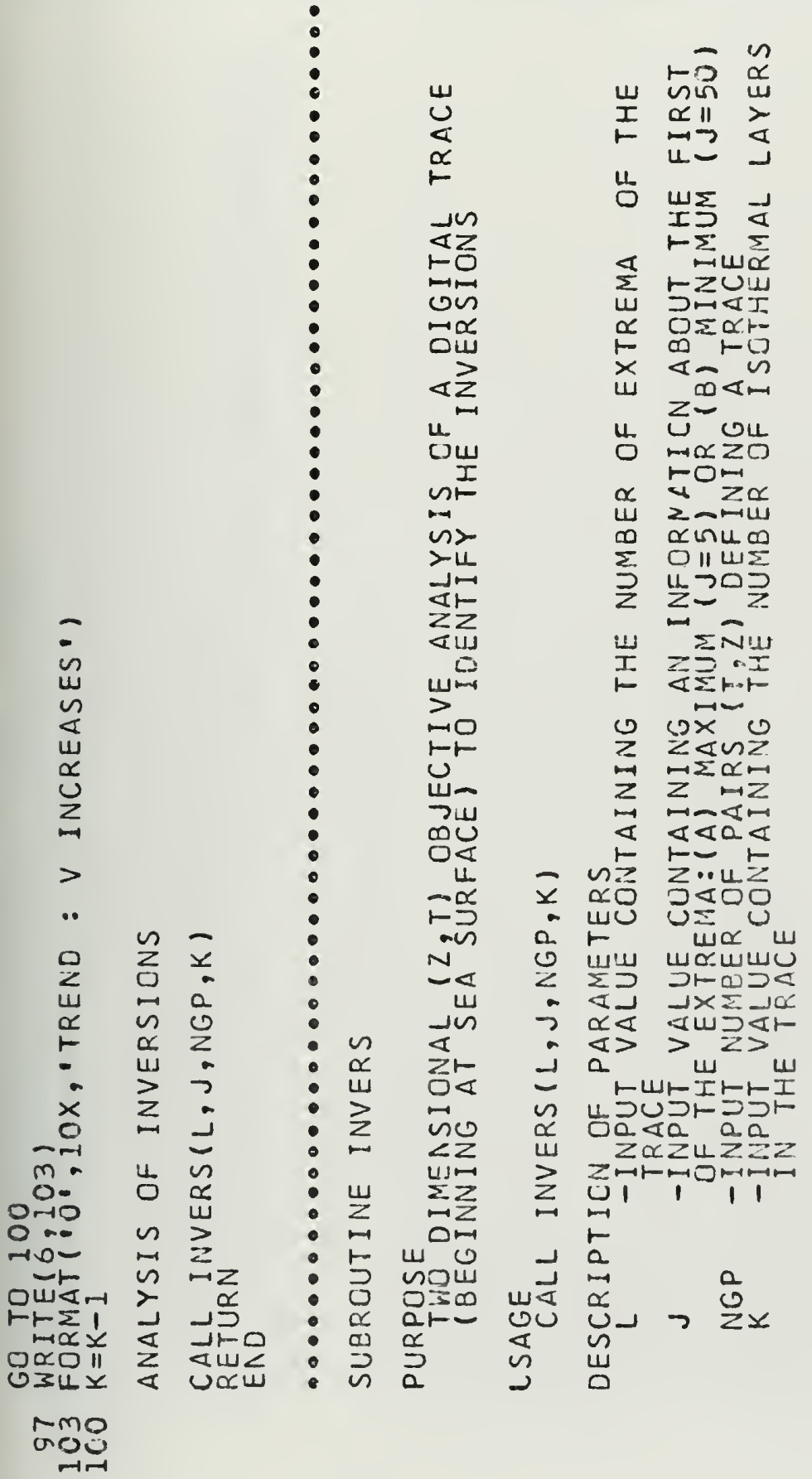

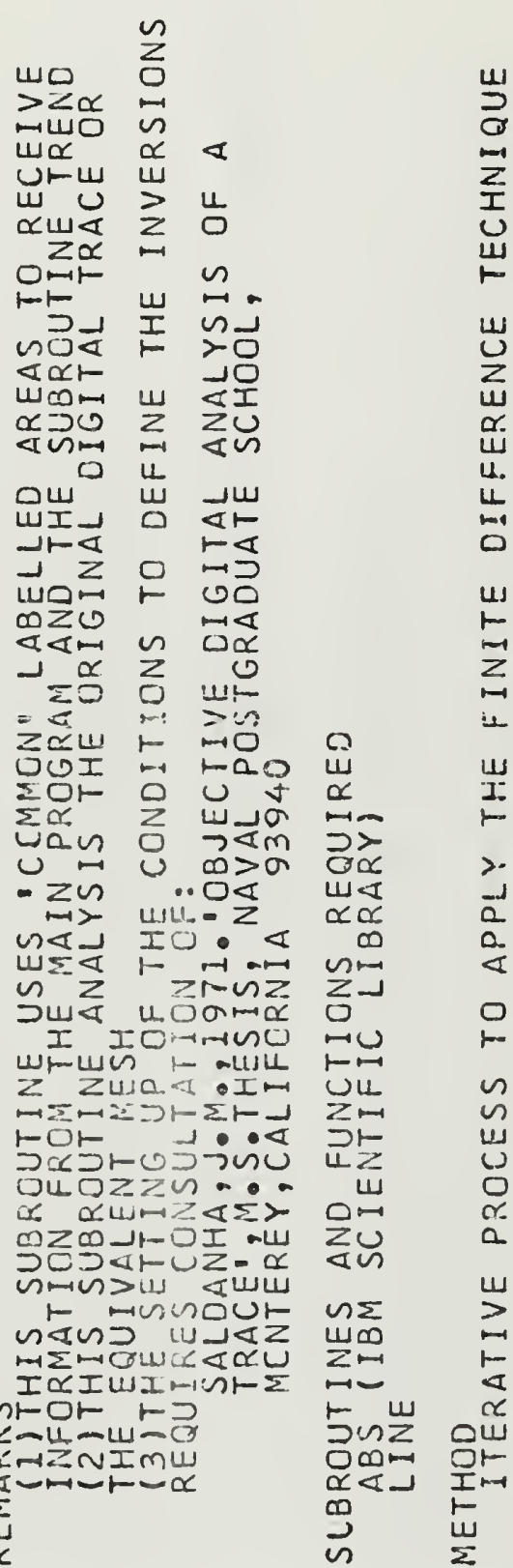


0000 $\min 2$ 0003 $\alpha \propto \alpha \propto$ $>>>>$ そ之之之

$\operatorname{lnth}$

000000002000030020000.0000000 .2000 .0000000012

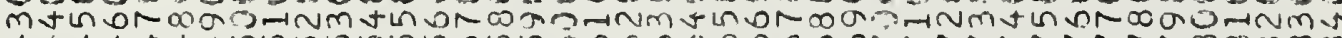

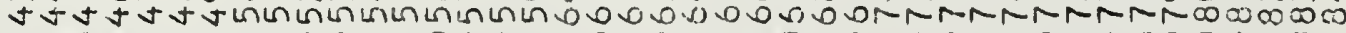

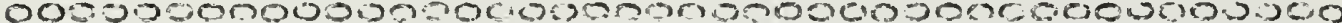
$\alpha \alpha \alpha \alpha \alpha \alpha \alpha \alpha \alpha \alpha \alpha \alpha \alpha \alpha \alpha \alpha \alpha \alpha \alpha \alpha \alpha \alpha \alpha \alpha \alpha \alpha \alpha \alpha \alpha \alpha \alpha \alpha \alpha \alpha \alpha \alpha \alpha \alpha \alpha \alpha \alpha \alpha$

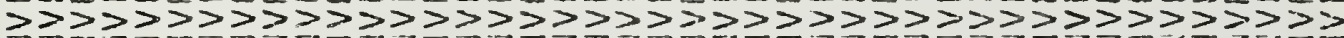

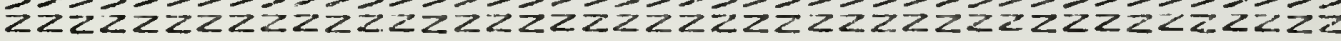

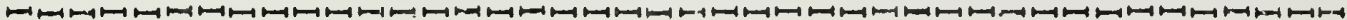
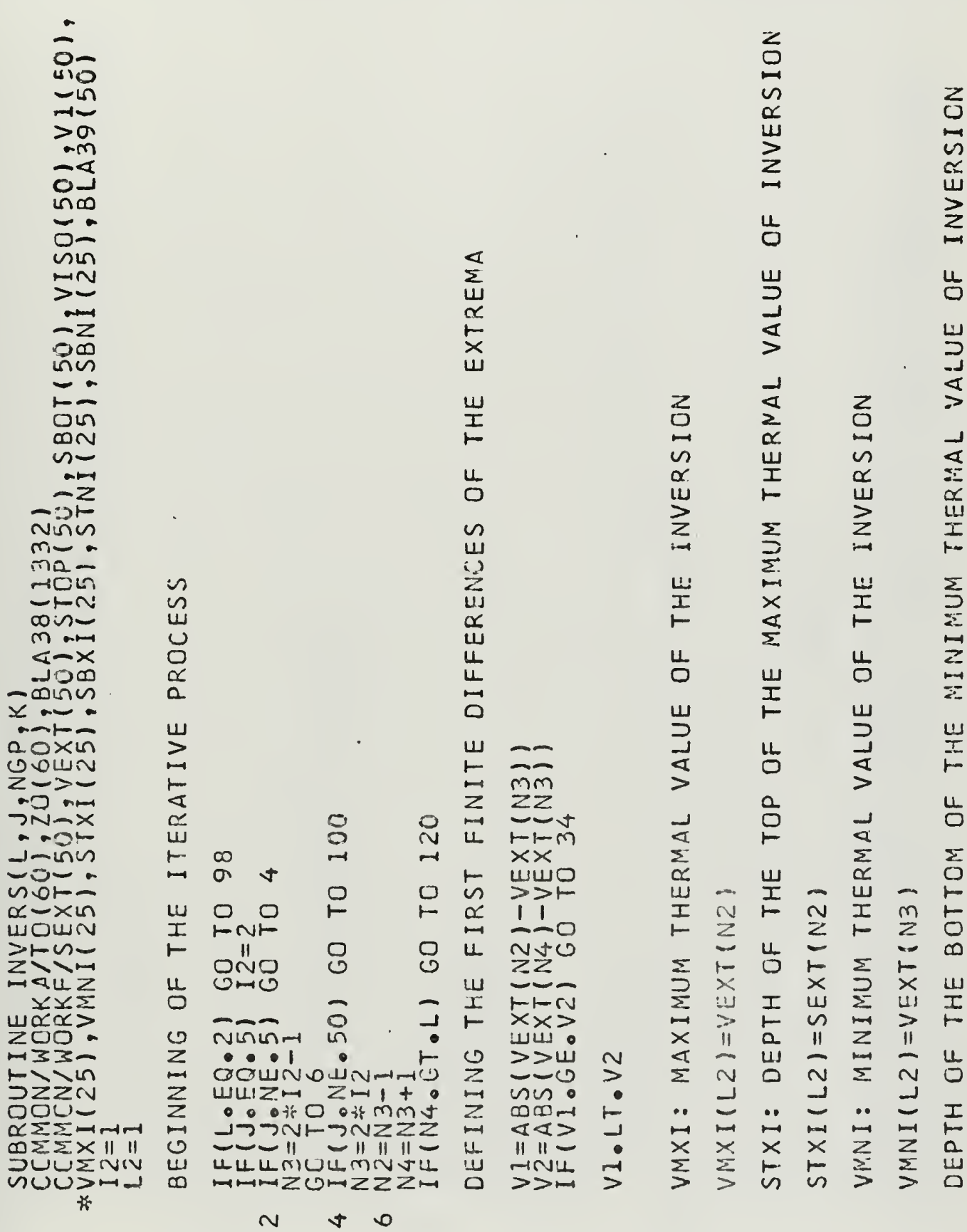


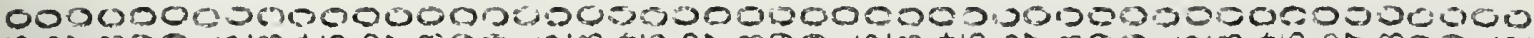

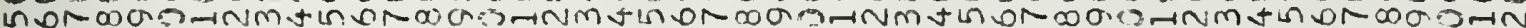

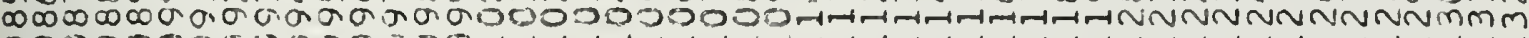

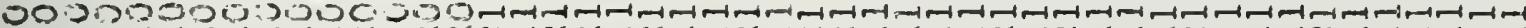

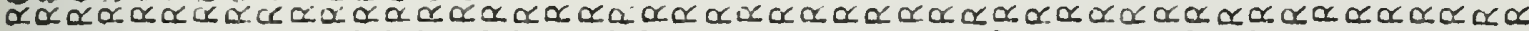

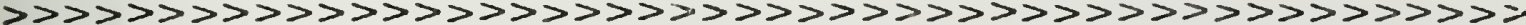

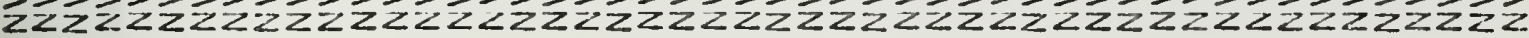

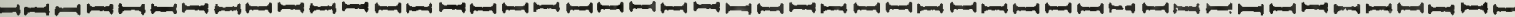

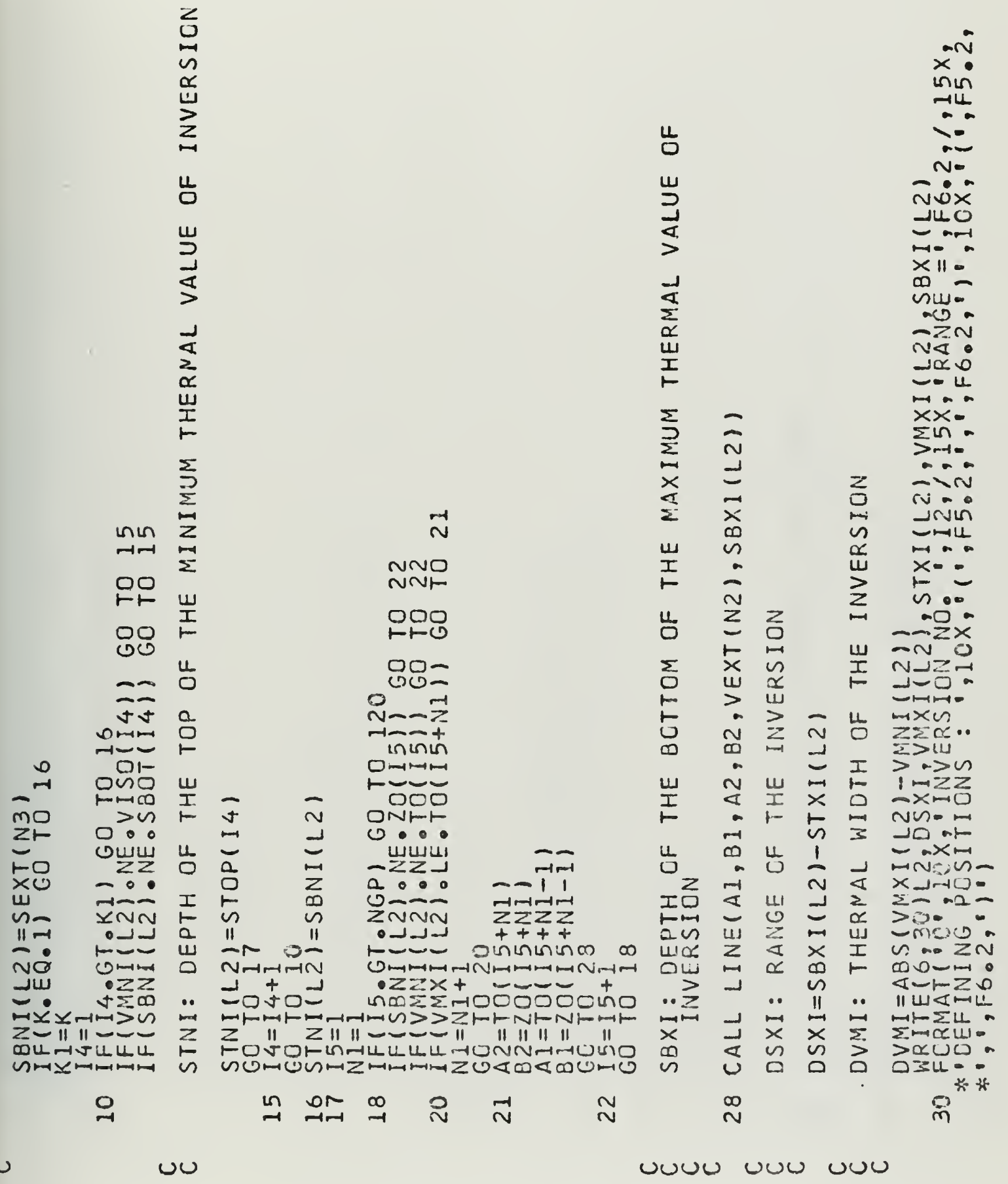


000000020500000200003000002023000000000000000000

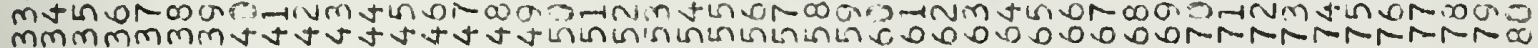

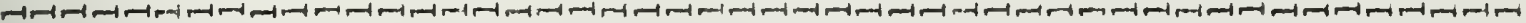

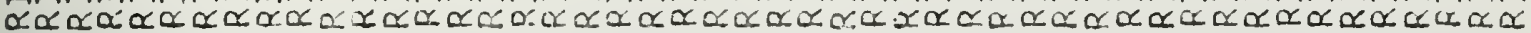

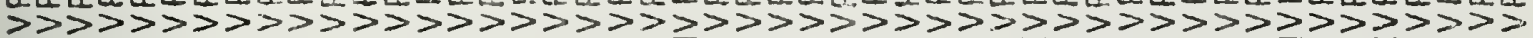

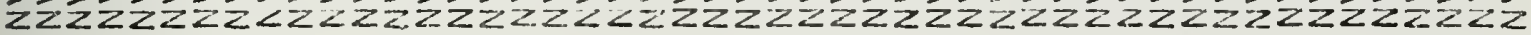

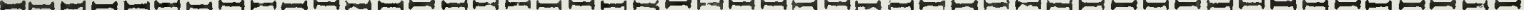
0

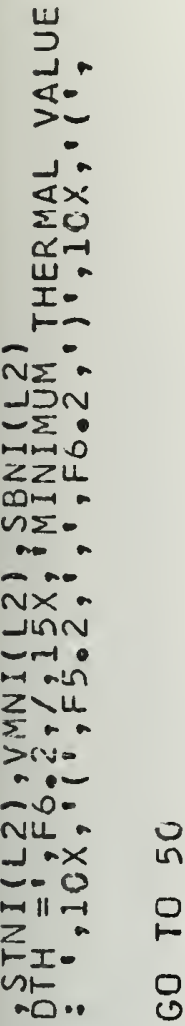

3

?

0

$\rightarrow-1$

NIn

$\sim, 0$

Z

$\sum \alpha-1$

ID -

$\rightarrow 100$ -

I-

$>$ alun

OXI.

- Int

N-1 4 I

a- $-\infty>$

$0-0 .+5$

யदय न

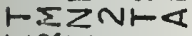

$\rightarrow \alpha$

๔Uนเก็น

зUயU1

$N^{i r * 6} \stackrel{5}{m}$

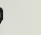

0
0
0
0
1
0
1
1
1
1

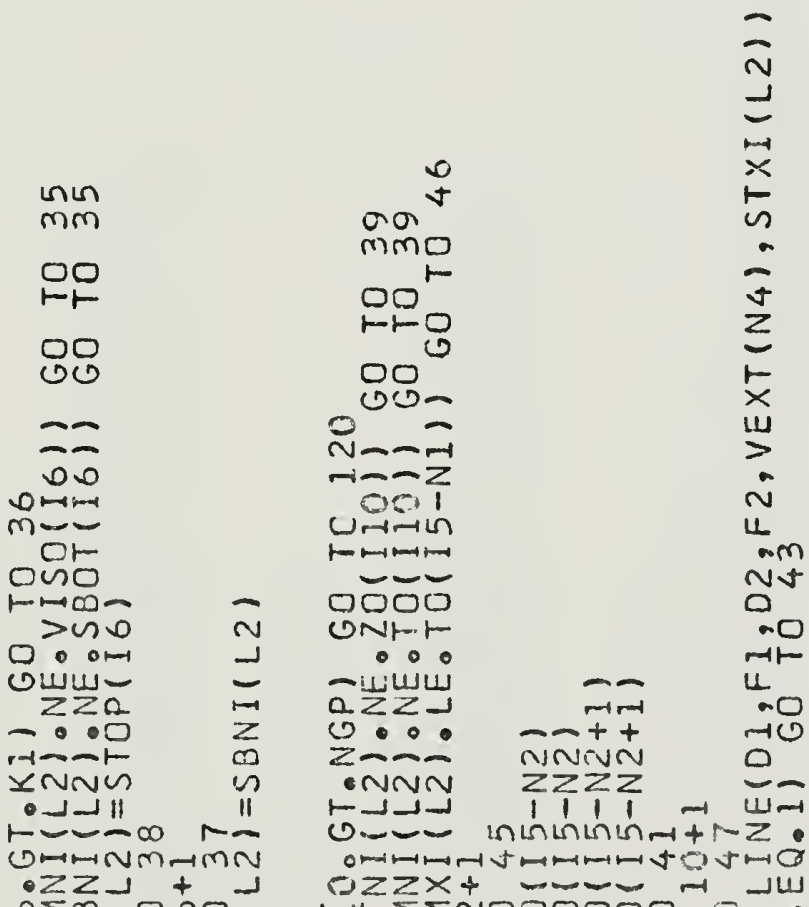

No

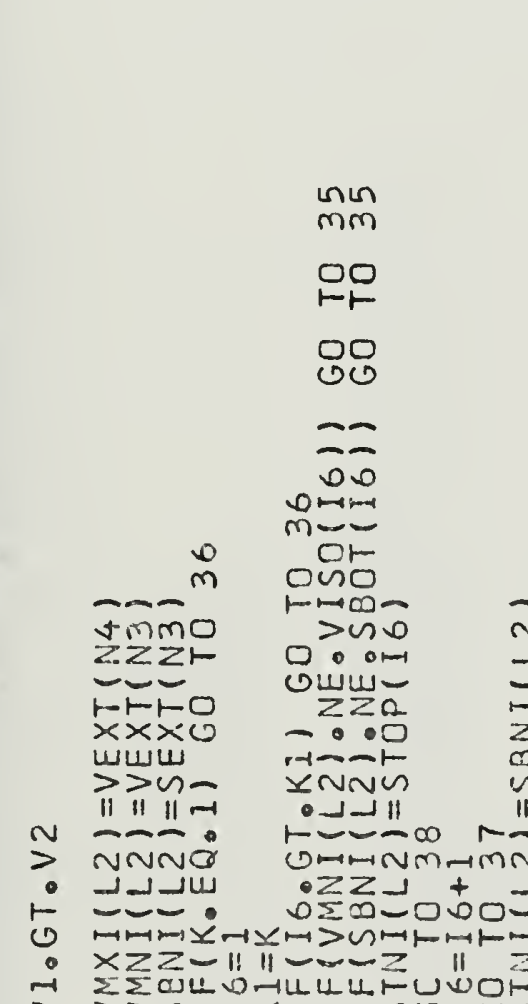

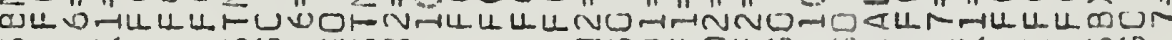
$m$
in $m m$ v

ษ $\begin{array}{lll}m & \text { ar } & \text { a }\end{array}$ $\underset{1-1}{1-1}$

잉

$\underset{m \rightarrow 1}{n-1}$

or

ono

$1 \rightarrow \infty-$

$>$ in

0 에

owi-

zza

근

$x \pm \pm n$

-ZZII

r- -5

Or-NAn $0 \times x+$ $+$ Nut

טuט 


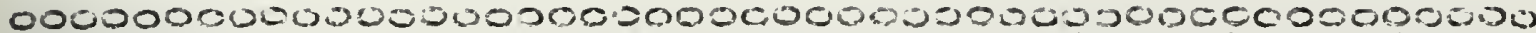

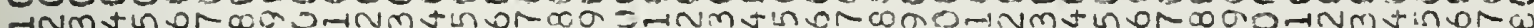

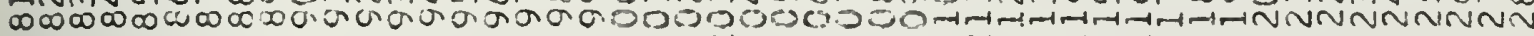

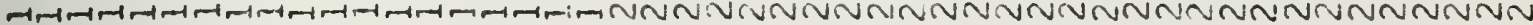

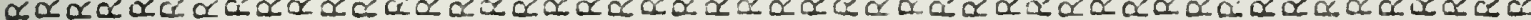

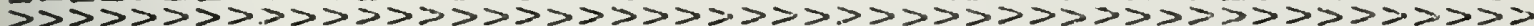

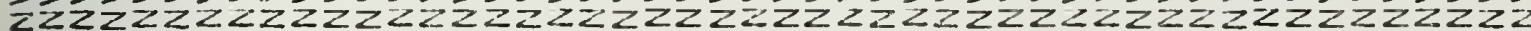

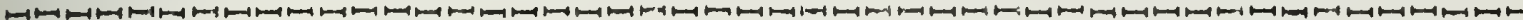

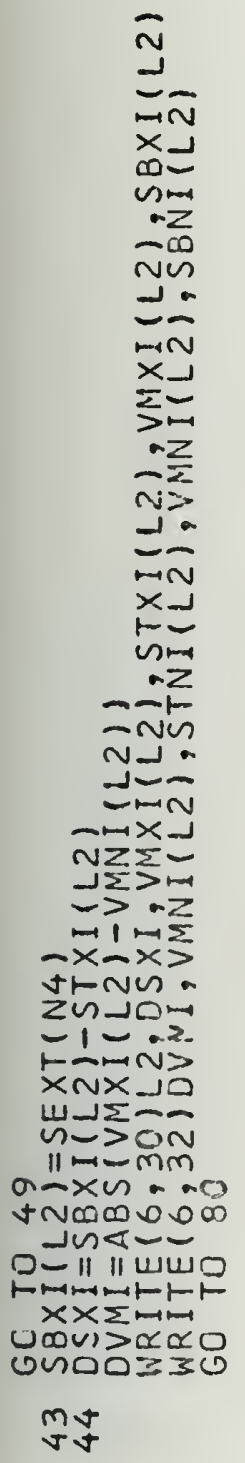

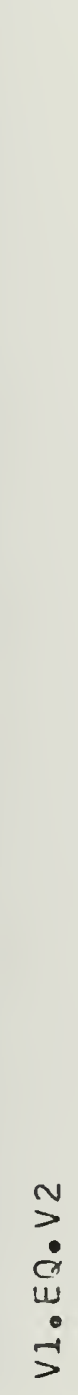

inin
inu

마

\section{0}

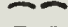

$\infty \infty$

○セセ

นก๊ュ

ดト

กับmตั

ZZZZF

-ニーヒー

$\times \times \times \times 0$

แயแயப

$>>>n-$

II II II II

$>$ Niñó

Nivive

-

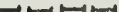

(1)

$x \times z Z=\|\|-Z-z\|z=\|\|x\|=-x \|$

$\sum-\sum \square L \infty-1 L L U$ -

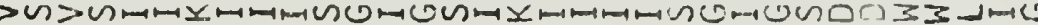
in
(i)
in inin
8
$\underset{w}{m}$
$\mathbf{Q}$ 
0000000

ob-tNms Ln

Nmmmmin

NNNNNNN

$\alpha \propto \alpha \propto \propto \propto \circ-$

之之之之之之之

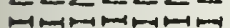

$=$

n

$\ddot{n}$

$\approx$

运

- z

-

$m \tilde{\alpha}$

w

崖之

崩

少u

0

$\sim$

, 足

$\rightarrow$

$\ddot{x}$

c)

an-

नित्ना

0.

Oन्मगन

no-ñ

-1 -

ดயयய<

HトEト

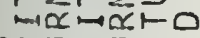

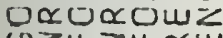

Uхu. $\leq 4 \square w$

Oमत्र

conv 

00000000000000000000000000000000000000

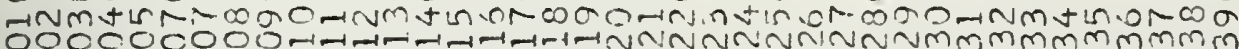
000000000000000000000000000000000000000

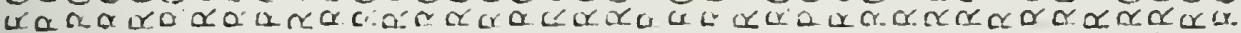

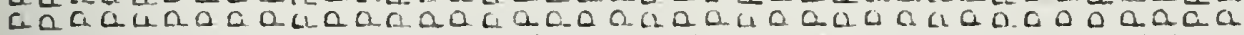

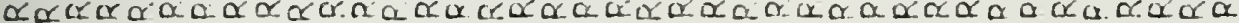

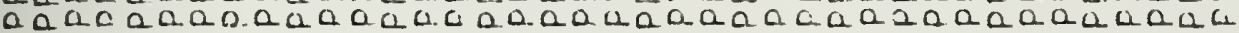

0000000 otinminting

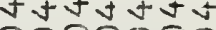
0000000

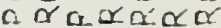
$\therefore 0.40 \cos 0$ $\alpha a \alpha \alpha c \alpha c r$ ananaa $\sum_{i}^{\subsetneq}$

actis

L:1 $\alpha$

$1=$

$\sum \frac{\pi}{4}$

$<\alpha$

$\alpha$ is

$x=$

$a \leq$

uL

is

iㅏㄴ

in

L!

Ea

a 4

Ii $\frac{5}{2}$

I

$>$

$>c$ :

- 11.

ca

ris

$\because 0$

$>2$

$z \geq$

‥ 5

is

-u

a

UI

l.'er

1 zas

F: $C$

$-6$

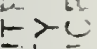

$=1-$

a. $-\frac{i}{2}$

CE:

:CuLle

$\sim<u$

c다드.

a c

5

(

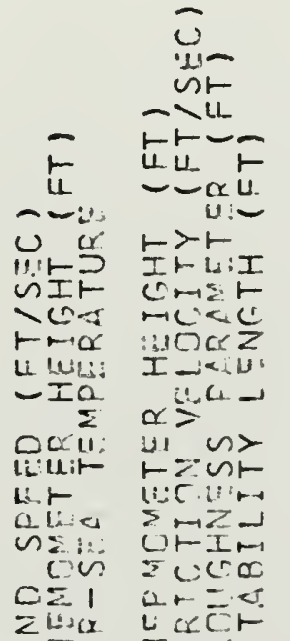

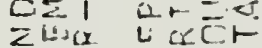

zZar Fuán

Luwur wiIIII

III IFFI

$r-r$

ere rese

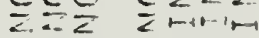

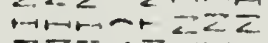

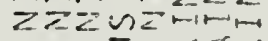

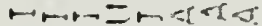

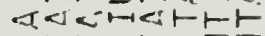

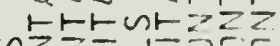

जZ

i.cicuse

ticcuse nisles

$y=-20,10=$

$\checkmark$ र」-

$a=\leq \leqslant \leq \leqslant>>>$

+5ン $>=: 1=$

ล.เトロ

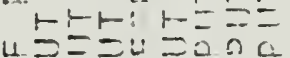

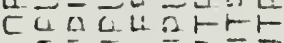

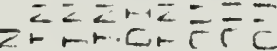

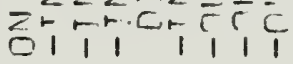

$\leftarrow$

a

c

(c) Givit $\quad$ Noñ

a) $\stackrel{\sim}{0}$

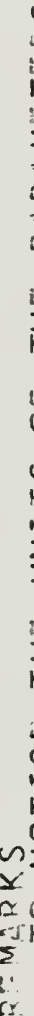

z

2

tic

巨家

$\infty=\frac{1}{a}$

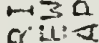

$r=\alpha$

n-

coz

zख

Eitis

$\operatorname{mos} 0$

34

uc

11.19:2

F远

U

वाis

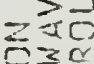

$D \geq \frac{c}{r}$

$1-2$

ฮL:

ULI : I

r.

$11 \mathrm{C}$.

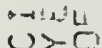

(i) $>-C$

o. - it-

$->$ is

eix

$\sim \cap \alpha$

$0.2-1$

$\because-1=0$

or: ars

$=$

in $=\angle-F$

arim

colsccu

<द:

$\sigma_{1}=$

C e? 2.

IUt

․

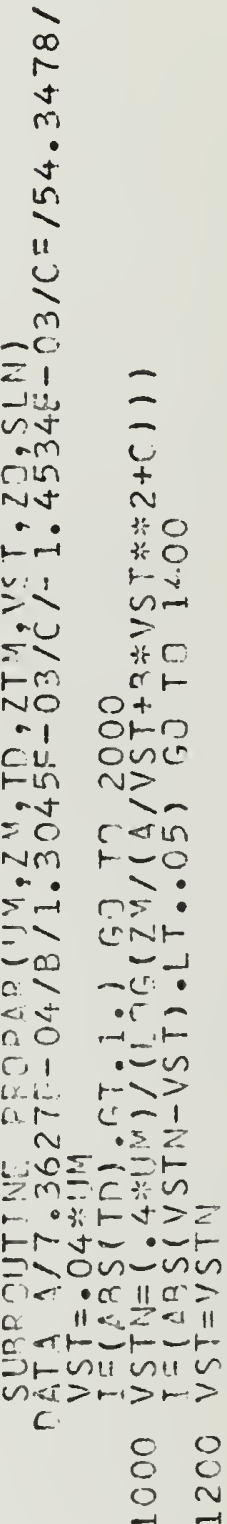

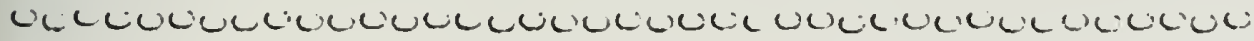


000000000000000000000

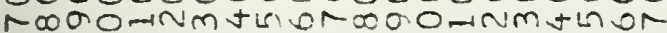
tatininininininininining 0000000 000000000000000000000

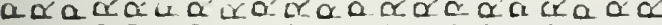

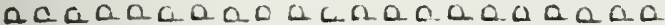
$\alpha \alpha \alpha \alpha \propto \alpha \alpha \alpha \alpha \propto \alpha \alpha \alpha \alpha \bar{\alpha} \alpha \alpha \alpha \alpha \alpha \alpha$ anarancararaugara

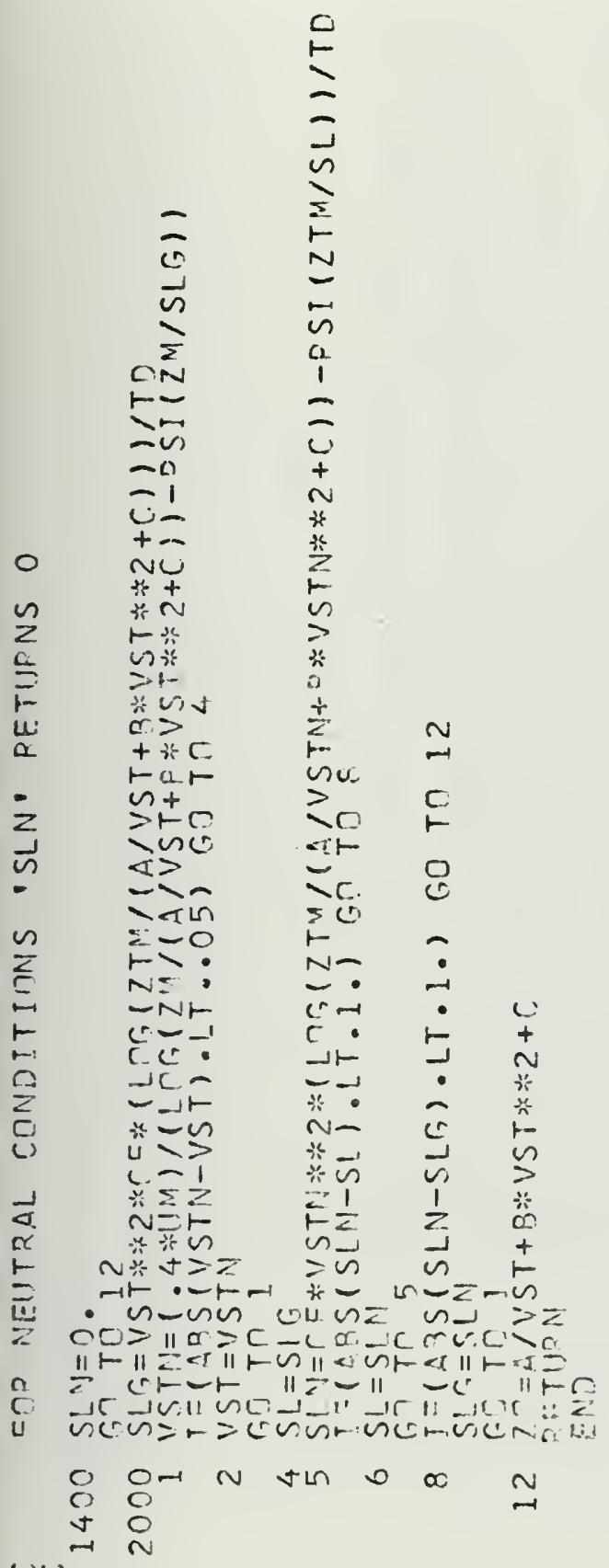




\section{FORTRAN IV SUBPROGRAM ITRATE FOR IBM 360/OS}

000000000000000000000000000000000000000000000000

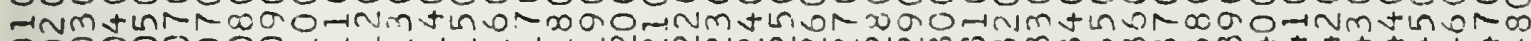
000000000 - 00 000000000000000000000000000000000000000000000000

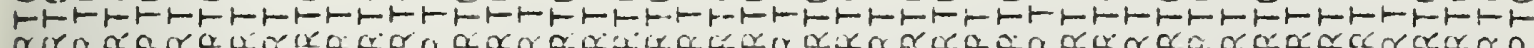

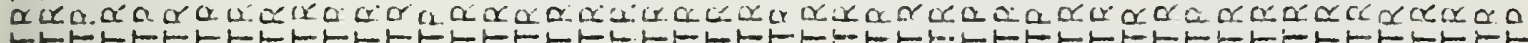

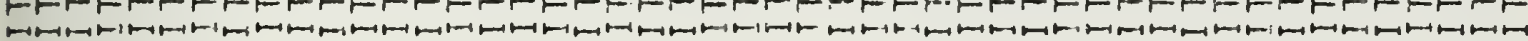

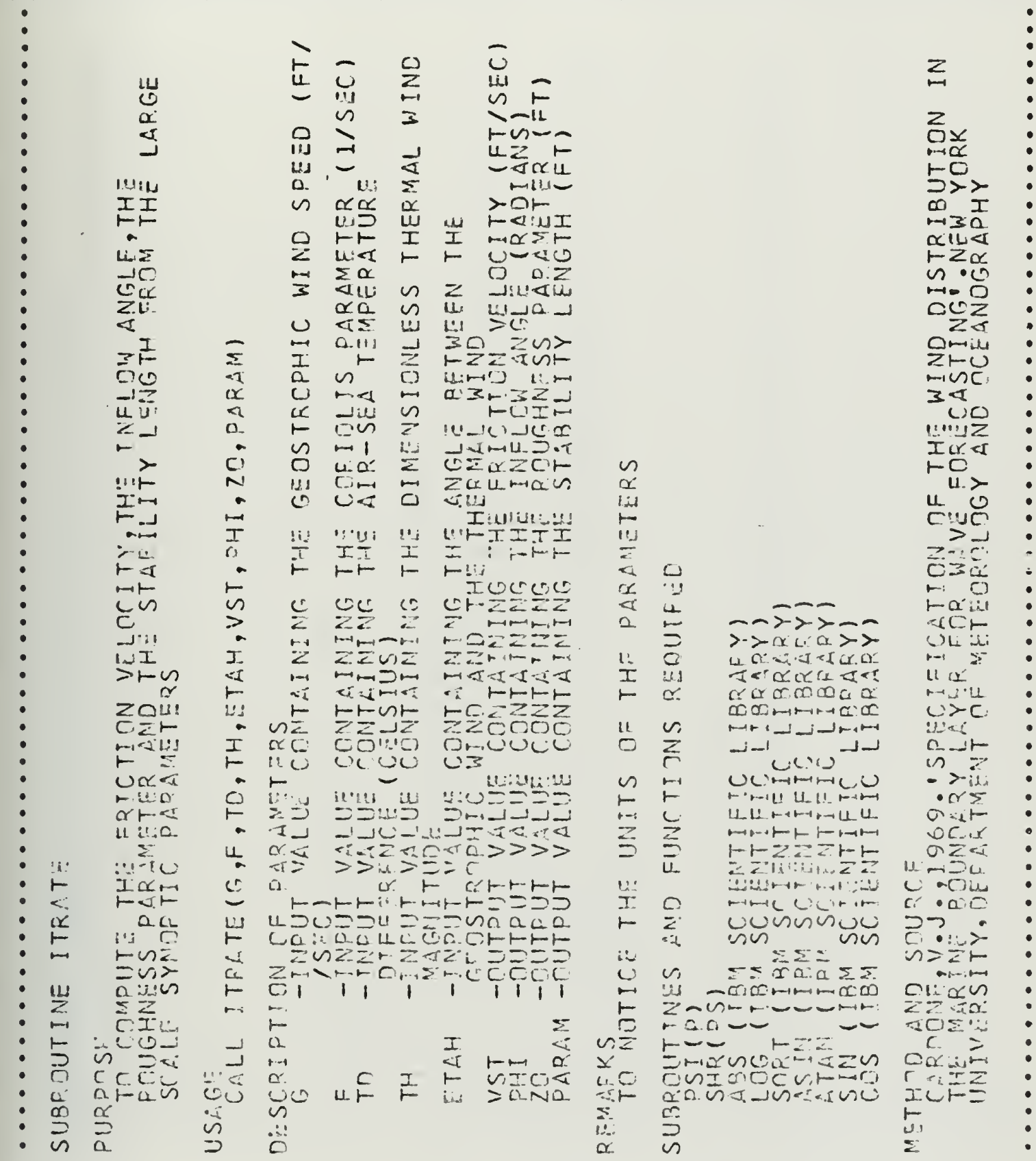


000000000000000000000000000000000000000000000

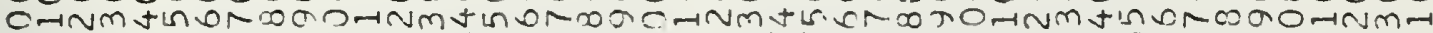

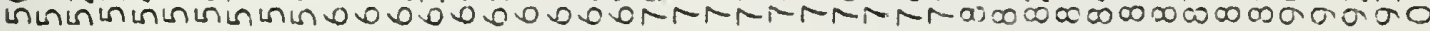
00000000000000000000000000000000000,0000000000

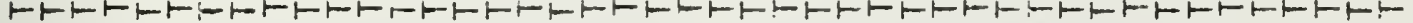

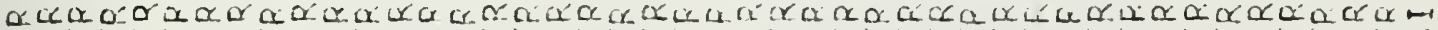

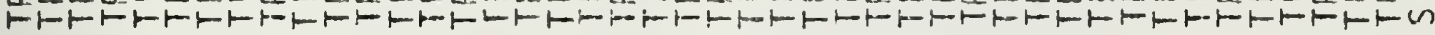

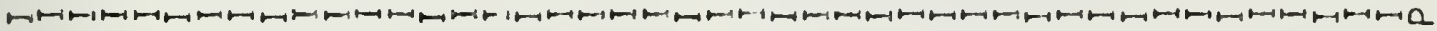

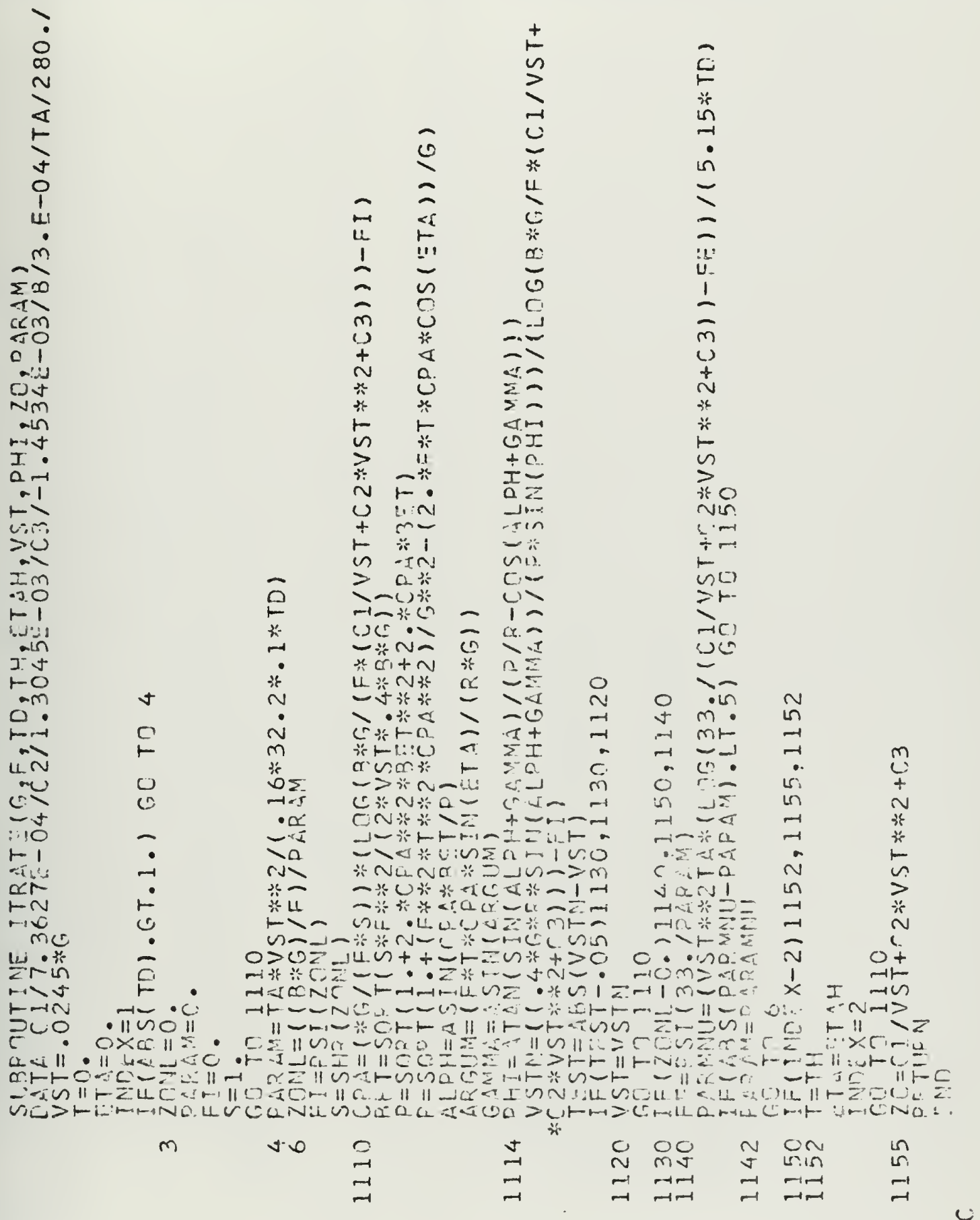




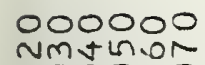
noodint 000000

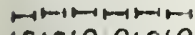
nusinas
0000000000000000

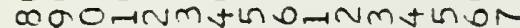
年 0000000000000000

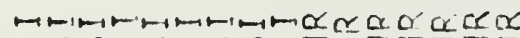
nunuinumincIIIIII acaradacañúnún

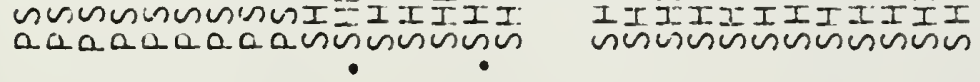

000000000000

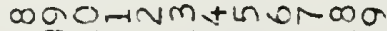
000000000000

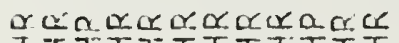
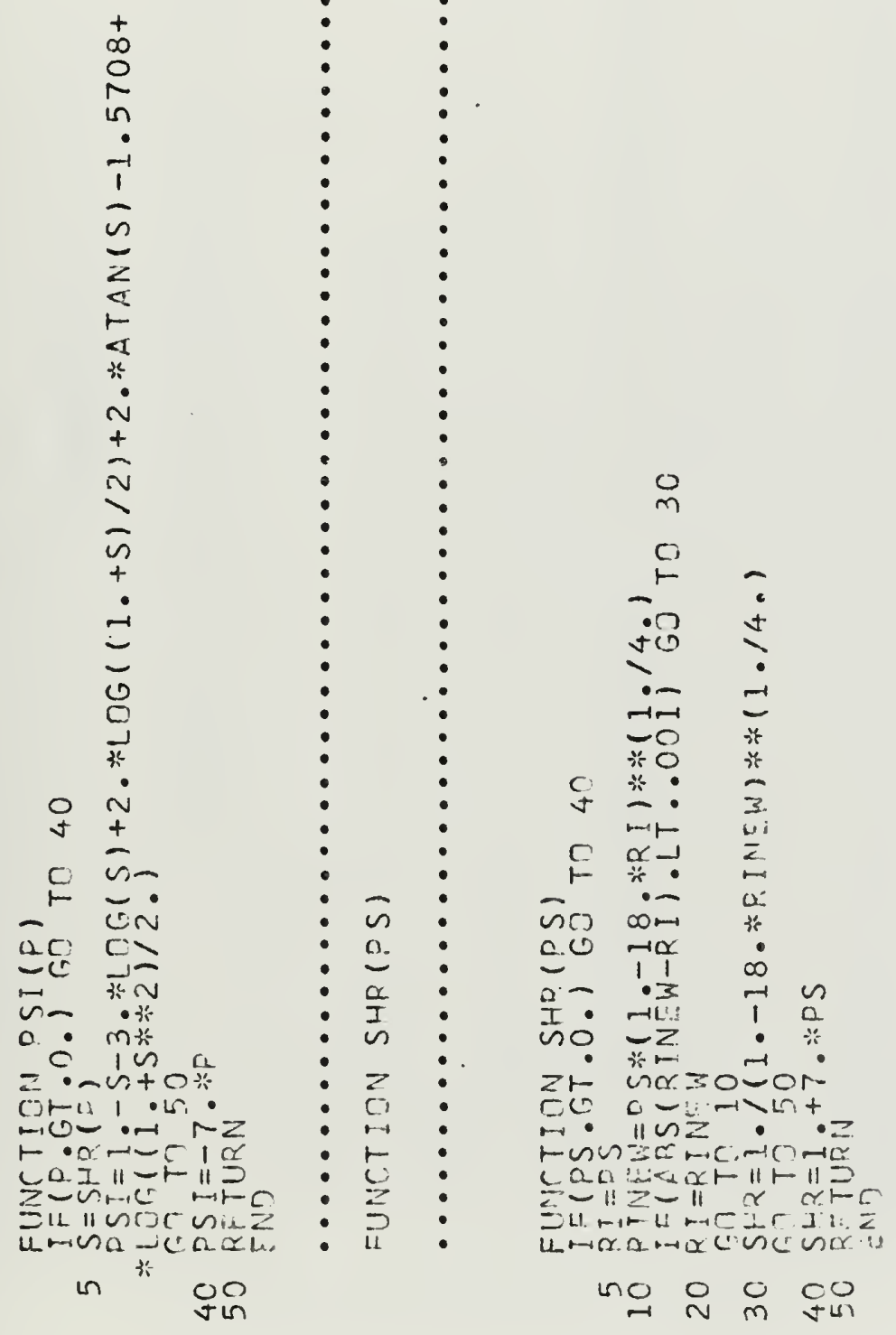


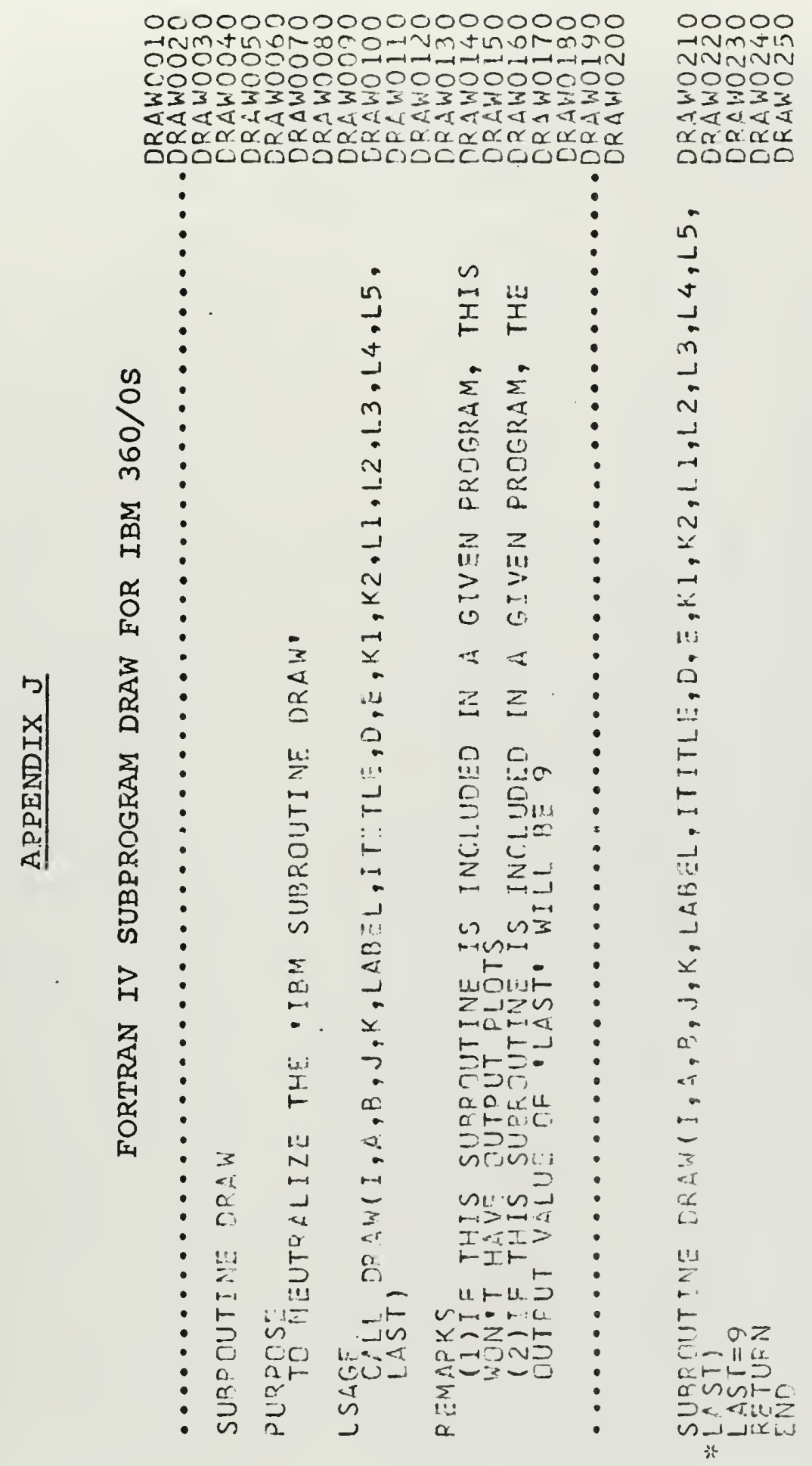

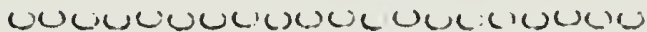


MODIFICATION TO THE FORTRAN IV BASIC COMPUTER PROGRAM (APPENDIX F) TO INCLUDE THE SUBPROGRAM PROPAR (APPENDIX I)

0000000 hinminion 0000000 żZZZZZZ

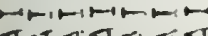
$<<\varangle<<<<$ $\sum \sum \sum \sum \sum \sum \Sigma$

$\begin{array}{ll}: & \\ : & \\ : & \\ : & \\ : & \\ : & \\ : & \\ : & \\ : & \\ : & \\ : & \\ : & \\ : & \\ : & \\ : & \\ : & \\ : & \end{array}$

-inma $\infty \infty \infty \infty \infty \infty \infty \infty$ 00000000

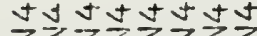
$z Z Z Z Z Z Z Z$

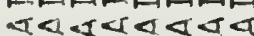
$\sum \sum \sum \sum \sum \sum \sum \sum$

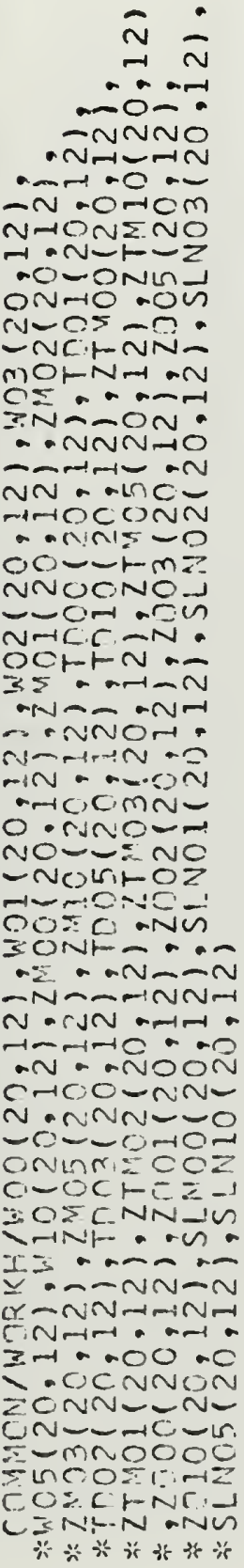

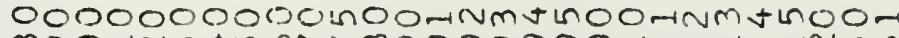

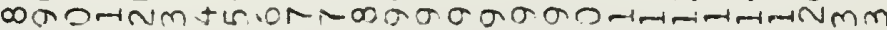

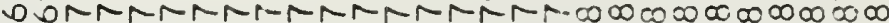

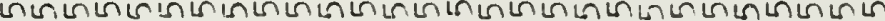
$Z Z Z Z Z Z Z Z Z Z Z Z Z Z Z Z Z Z Z Z Z Z Z Z Z Z Z Z$

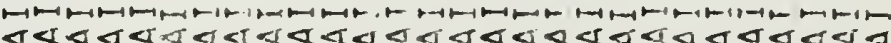

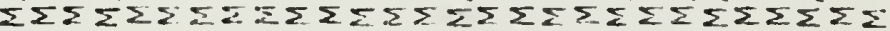

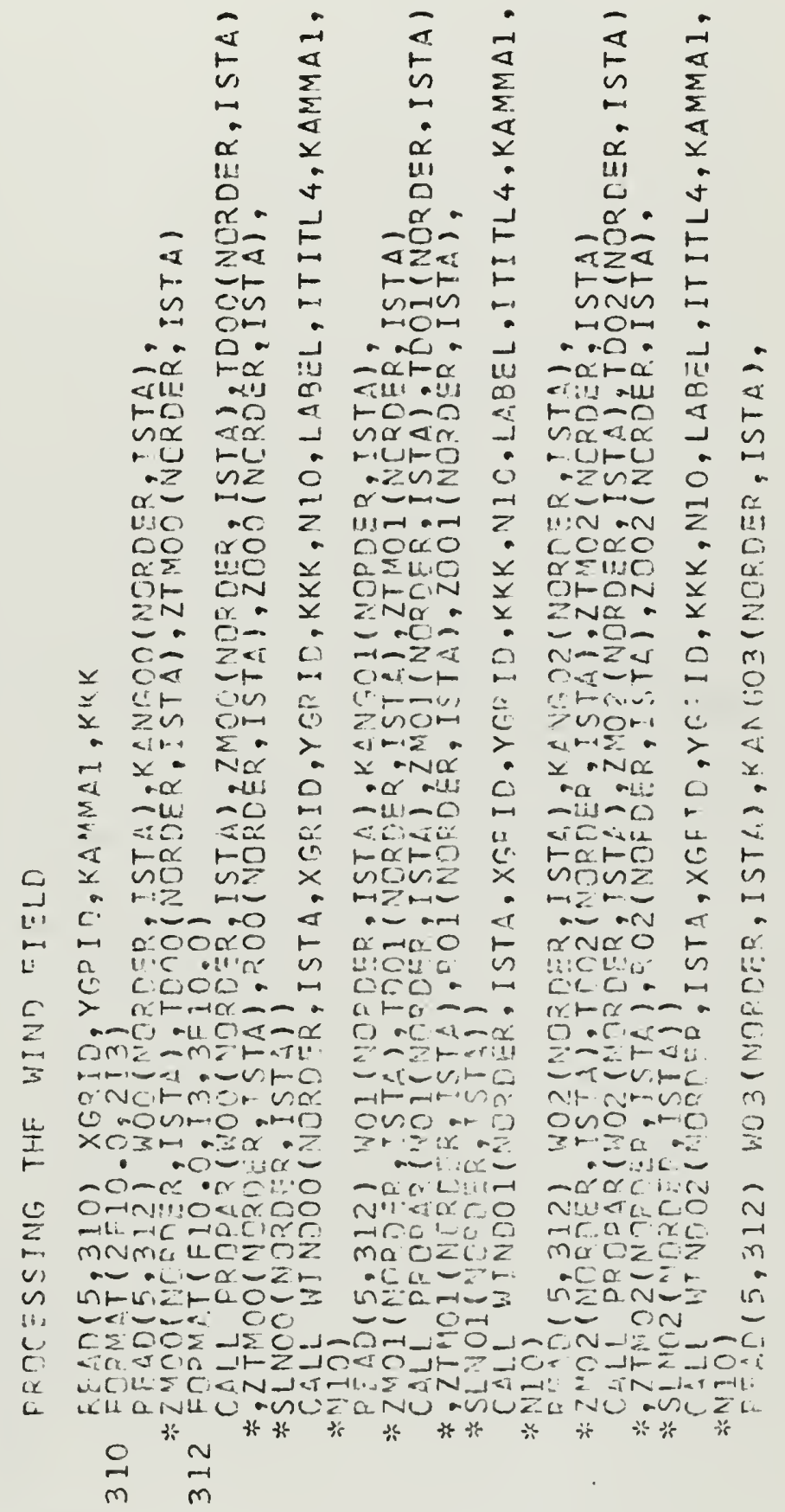




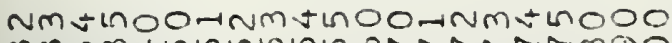

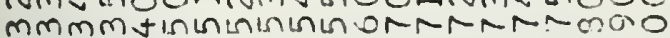
$\infty \infty 0000000 \infty 00000000 x \infty 2$

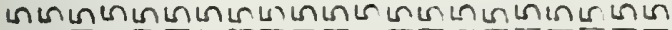
$Z Z Z Z Z Z Z Z Z Z Z Z Z Z Z Z Z Z Z Z Z$

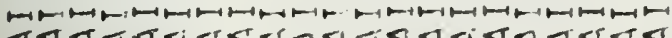

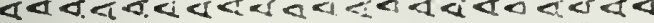

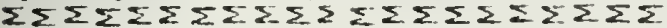

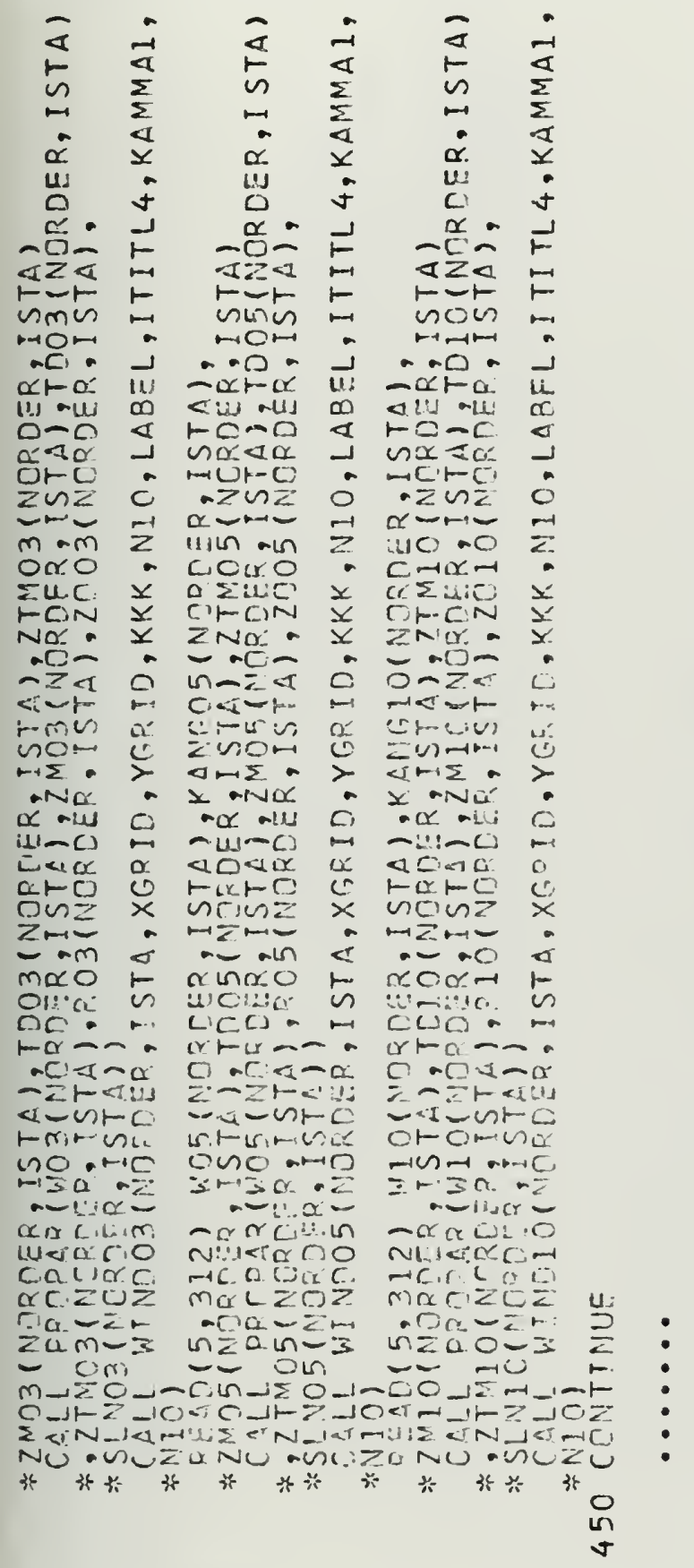

(u) 


\section{MODIFICATION TO THE FORTRAN IV BASIC COMPUTER PROGRAM (APPENDIX F) TO INCLUDE THE SUBPROGRAM ITRATE (APPENDIX J)}

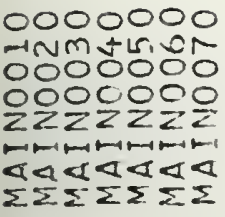

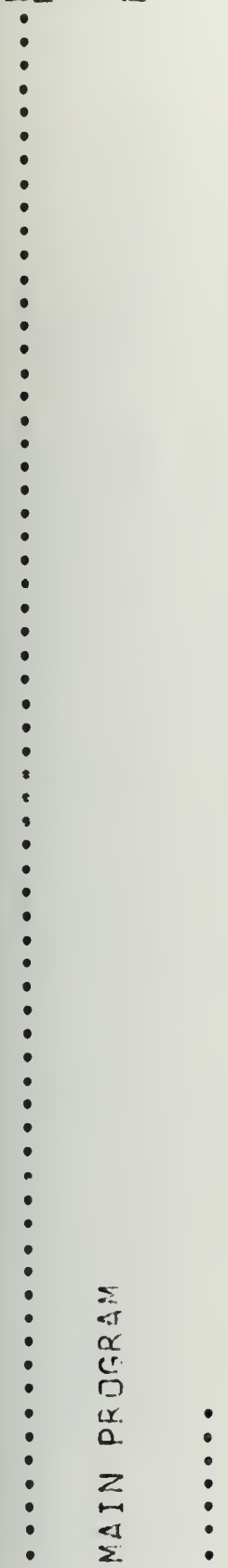
$\infty \infty \infty-0 \approx 0 \infty \infty \infty$ 000000000 A.t.7.t.7.t. $\angle<Z Z Z Z Z Z Z$

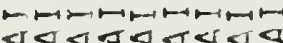
$\sum \sum \sum \sum \sum \sum \sum \sum \sum$

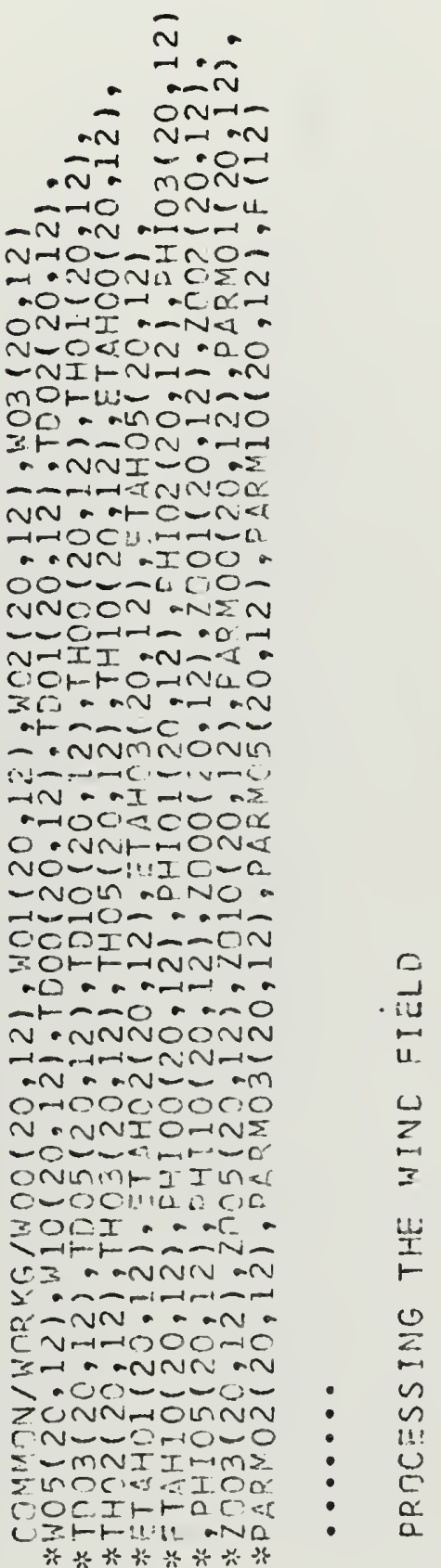

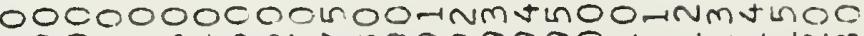

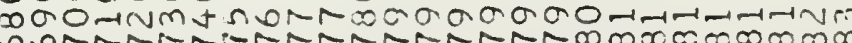

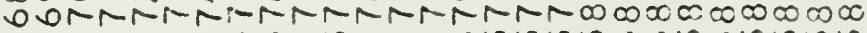

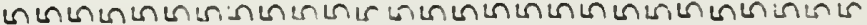

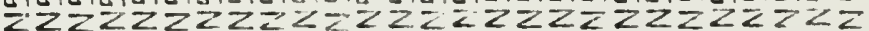

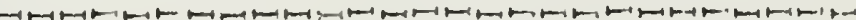

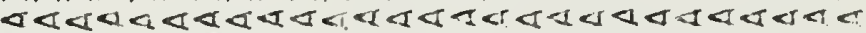

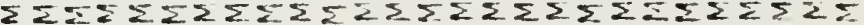

$\rightarrow \quad \alpha<$

- Zu!

11.

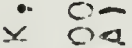

$\times \quad:$

$x<v$

$\rightarrow x$

- 1 -

$\sum^{1}-11$

$\sum_{i} \leq c$
in

U $-\vec{c}$

c.0 म- -0त $\mapsto$ rLCOR ICH

C. O:1:0 is:tou CHFITCLHMH >-L or

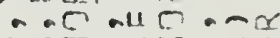
$n m=m \geq-k ! \cdot$ ๓⿴囗十 on $\mathrm{NH} m \mathrm{~m}$ - $\mathrm{CH}$

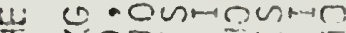
xc $3, \ldots+\ldots$ - 0 CHNL:OH:: 0

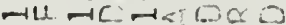
momreu ore $=$ m ar:-rit $\because$

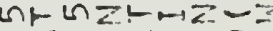
Camer $-C=-1$

$-i$

$\sum^{\infty}$

$\frac{1}{x}$

=i

Eara-

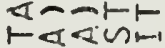

जil-rit

munar

$\rightarrow r-10 x$.

no. - - - is - J

- Liscacili

"L Cliwora

tccocies

$n 00 \times-1$

$n-2=-10$

a-ーニ0-

แ०ननः ह

CIOON.

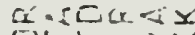

(I) - it

zus a -

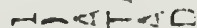

ocir Ur

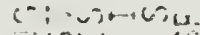

LUNA arc

$x-1-\alpha+>$

$x$ - lis is: a

no. a cuso

$\sim u-\alpha$ r

a. $\operatorname{cec} \alpha$

$-x+\Rightarrow C$

n $=\omega-2 x$

$\rightarrow=+-1=$

a $-0,1.7$

$\alpha-10-0$

ACliseron

CICILNH

rac: :

$-\infty-1-a U$

$-<-c^{3}-C$

-r thind

Oncurac

$=-2+1 \cdot \vec{a}$

$a-a-\alpha$

- cris c:ill

Nuit:LDO

HE स 2 Y $Y$ C

$m \propto \alpha r \mathrm{CZ}$

mpit

- $=1-$

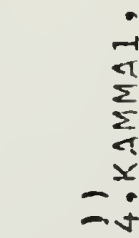

- $-a_{-1}$

- 1 an

untorth

mesen mat

atIFCY.

- cer - ats.

- ivia 0.E.tu

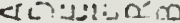

$-\alpha Q D C J \pi$

$\checkmark, 0 \alpha, x=\ldots$

- $<E$ E

$a N=-0$

11:ONNE?

CIOOA.

$C x=-\operatorname{cic} x=y$

OHF - C Y

ZLU $a-n$

- $-4<-4$

ก)

Ciertunt-

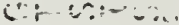

7 Conar ato

$\langle\pi+i-\alpha$ a $>$

$x-u$ u $x$.

ac a cullis

- Ui-n Cm

वE)तम(r)

- $25-5$

nous $\rightarrow x$

-

- mric

and I OH

11:0!ifer un

CICHN

or

a - c) $\cdots$

$\rightarrow-\geq-\ldots+1$

- 1 - 1 - 1

NT- Nt- Vict

ousonme

$-1-1 \pm 1+2=$ a no

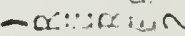

Nist $-1=0$

TC w C I C

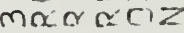

- nम-

nZー Zー

$-2-N$

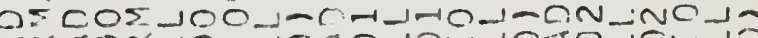

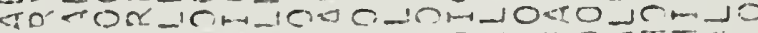
C ou $\alpha+u$ L

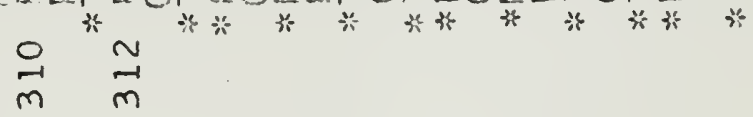




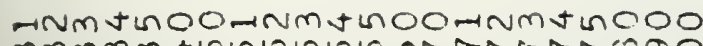

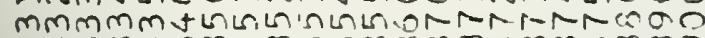

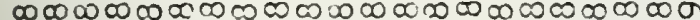

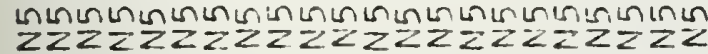

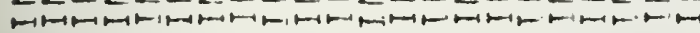

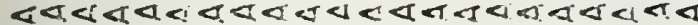

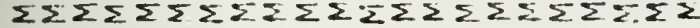

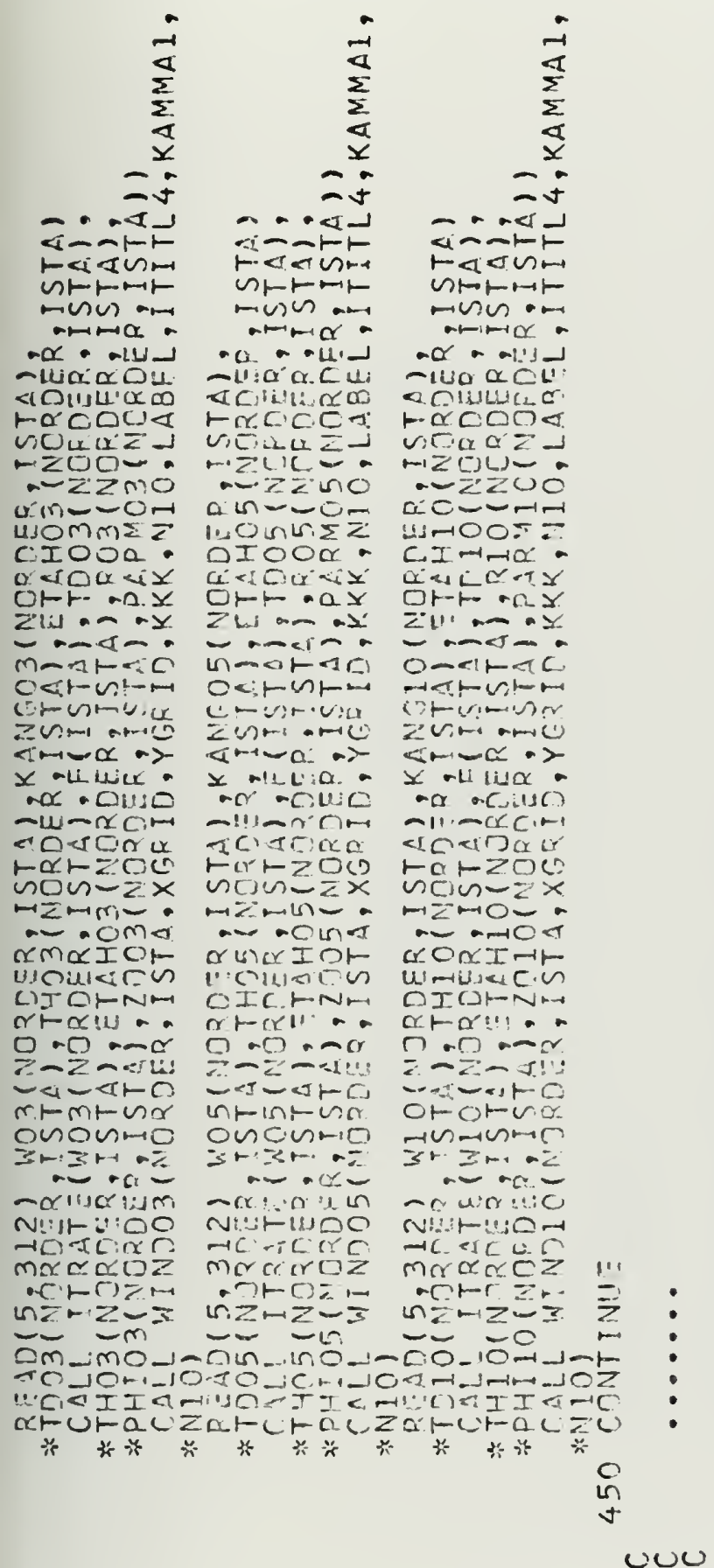




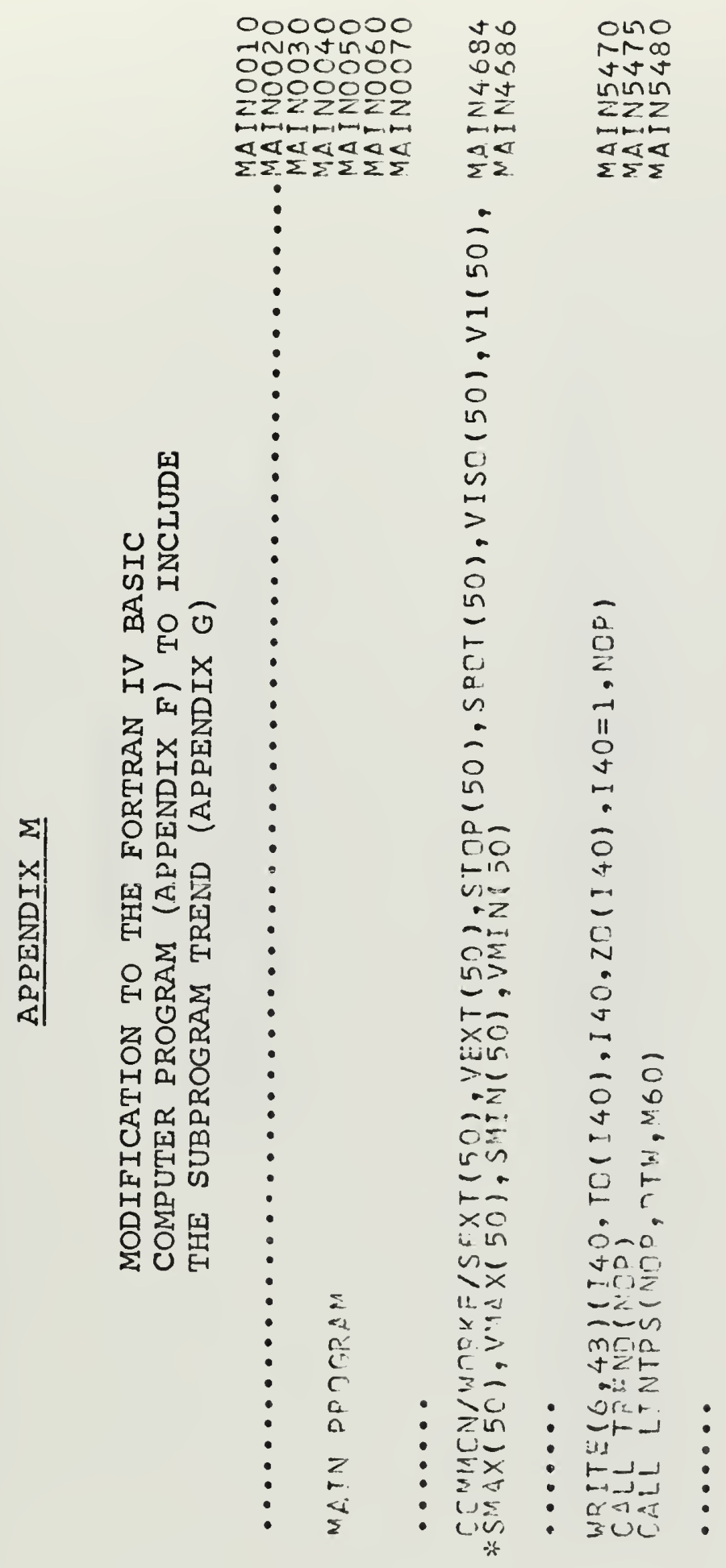

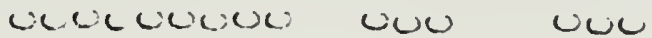




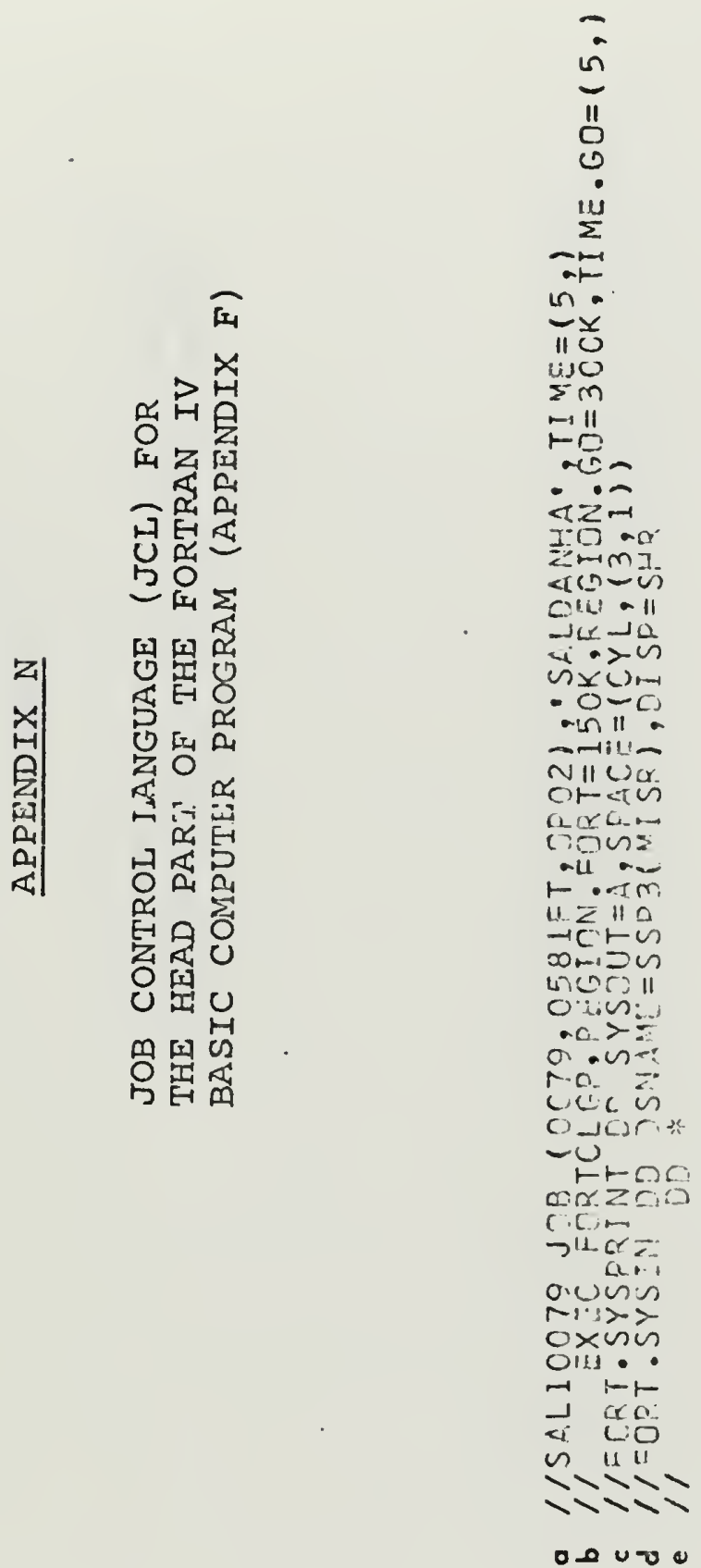



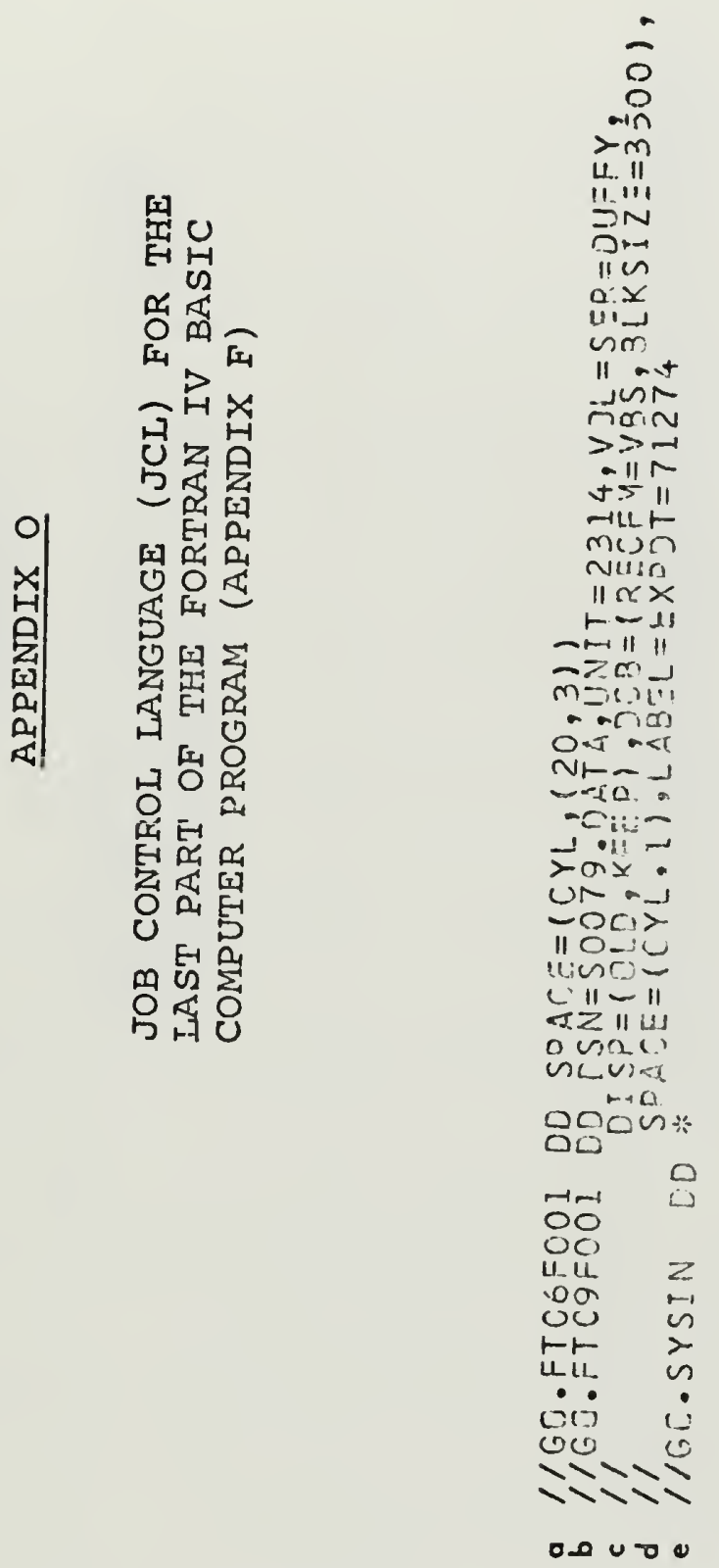


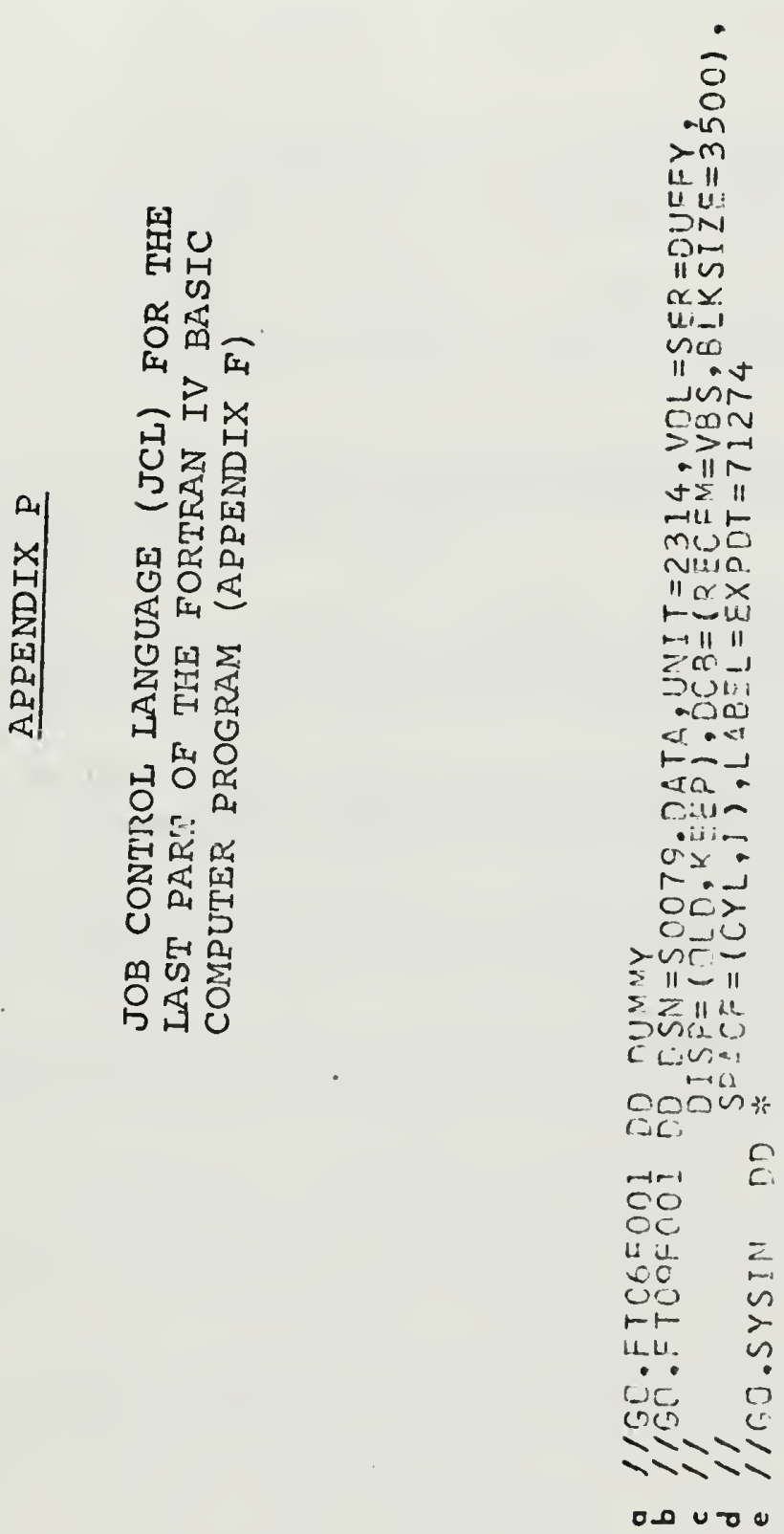



\section{BIBLIOGPAPHY}

Boston, N. E. J., 1966. Objective Definition of the Thermocline, Texas A\&M University, Dept. of Oceanog., Project 286 - D, Ref. $66-19 \mathrm{~T}$, p. $1-38$.

Cardone, V. J., 1969. Specification of the Wind Distribution in the Marine Boundary Layer for Wave Forecasting, New York University, Dept. of Meteorology and Oceanography.

Francis, S. A., and G. C. Campbell, 1965. "A Low Cost Expendable Bathythermograph", Marine Science Instr., Vol 3, Plenum Press, p. 85-89.

Grosfils, E. F., 1968. Objective Digital Analysis of Bathythermograph Traces, M.S. Thesis, Naval Postgraduate School, Monterey, California.

Hale, D. H., and P. D. Stevens, 1970. Computer Processing of Expendable Bathythermograph Traces, Fleet Numerical Weather Central, Tech. Note 61.

James, R. W., 1966. Ocean Thermal Structure Forecasting, Navozazno, $s p-i 05$, Aswfips ivanual, V. 5.

LaFond, E. C., 1951. Processing Oceanographic Data, U. S. Navy Hydrographic Office, H.O. Pub. No. 614, p. 1-187.

Magruder, Jr., P. M., 1970. Some Characteristics of Temperature Microstructure in the Ocean, M.S. Thesis, Naval Postgraduate School, Monterey, California.

Rossby, C. G., and R. B. Montgomery, 1935. "The Layer of Frictional Influence in Wind and Ocean Currents", Papers Phys. Oceanog. Meteorol., 3(3), p. 1-101.

Sauer, C. D., 1964. "Bathythermograph Data on Aperture Cards: a New Approach to an Old Problem", Journal of Fisheries Research Board Canada, 21 (3), p. 647-650.

Sauer, C. D., and E. L. V. Hope, 1967. "A System to Digitize Bathythermograph Aperture Cards", Journal of Fisheries Research Board Canada, $24(5), p .1155-1164$.

Spilhaus, A. F., 1938. "A Bathythermograph", Journal of Geophysical Research, v. 1, p. 95-100.

Sverdrup, H. U., Johnson, M. W., and Fleming, R. H., 1942. The Oceans, Prentice-Hall. 


\section{INITIAL DISTRIBUTION LIST}

No. Copies

1. Defense Documentation Center

Cameron Station

Alexandria, Virginia 22134

2. Library code 0212

Naval postgraduate school

Monterey, California 93940

3. Naval Weather Service Command

Washington Navy Yard

Washington, D. C. $203 y 0$

4. Commanding officer

Fleet Numerical weather Central

Monterey, California 93 y 40

5. Office of Naval Research, Code 480D

Department of the Navy

Washington, D. C. 20360

6. Department of cceanography, Ccie 58

Naval Postgraduate School

Monterey, California 93940

7. Department of Meteorology, Code 51

Naval Postgraduate School

Monterey, California 93940

8. National Oceanographic Data Center

Attn: Henry odom

Washington, D. C. 20390

9. Director-Geral

Instituto Hidrografico

Ministerio da Marinha

Lisboa, Portugal

10. Director

Instituto Hidrografico

Cadiz, Espana

11. Director

Instituto de Pesquisas da Marinha

Ministerio da Marinha

Ilha do Governador

Rio de Janeiro, Brasil 
Hidrografia e Navegacao

Ministerio da Marinha

Rio de Janeiro, Brasil

13. Biblioteca Central

Ministerio da Marinha

Lisboa, Portugal

14. Director

Servico Meteorologico Nacional

Lisboa, Portugal

15. Dr. Dale F. Leipper

Department of Oceanography, coce 58Lr

Naval Postgraduate School

Monterey, California 93940

16. Dr. Taivo Laevastu

Fleet Numerical weather Central

Monterey, California 93940

17. Dr. Noel Boston

Department of Oceanography, Code 58Bb Naval Postgraduate School

Monterey, California 93940

18. Dr. Kenineth Dâvidson

Department of Meteorology, Code 5lDs

Naval Postgraduate School

Monterey, California 93940

19. Dr. Robert Andrews

Department of Oceanography, Code 58Ad

Naval Postgraduate School

Monterey, California 93940

20. Dr. Douglas R. MCLain

National Marine Fisheries Service

Fleet Numerical weather Service

Monterey, California 93940

21. CDR Jose Ataide

Irstituto Hidrograrico

Ministerio da Marinha

Lisboa, Portugal

22. CDR Mauro Pereira

Rua Desembargador Isidro, n. 10, Apt. COl Tijuca, Rio de Janeiro, Brasil 

23. LCDR Almir Silva

Directoria de Hidrografia e Navegacao

Ministerio da Marinha

Rio de Janeiro, Brasil

24. LCDR Jaime Sanchez

ARC "San Andres"

Base Naval Cartagena

colombia, South America

25. LCDR Leopoldo Salas

9 Ralston Drive

Monterey, California 93940

26. LCDR Roberto Rodrigues

777 Newton Street

Monterey, California 93940

27. LT AIexander Maratos

Hydrographic office of Navy

Paparrigopoulou 2

Athens, Greece

27. LT Antonio Souto

261 Larkin street

Monterey, California 93940

29. IT Eric Grosfils

P. O. Box 502

Brewster, Massachusetts 02631

30. LT Jose Saldanha

Instituto Hidrografico

Ministerio da Marinha

Lisboa, Portugal 

Security classilication of thle, body of abstract and indexing annotalion nusf be onfered when the overall report is classified)

RIGINATING ACTIVITY (Corporale author)
Naval postgraduate school

Monterey, California 93940

Unclassified

2b. GROUP

An Objective Method to Analyze Oceanic Variclines and

Their Statistical Relation to Surface Winds

'ESCRIPTIVE NOTES (Type of reporl and,inclusive dates)
Master's Thesis; September 1971

UTHOR(S) (First name, middlo initial, last namo)

Jose Manuel Pinto Bastos Saldanha

\begin{tabular}{|c|c|c|}
\hline $\begin{array}{l}\text { EPOAT OATE } \\
\text { September } 1971\end{array}$ & $\begin{array}{l}\text { 7. TOTAL NO. OF PAGES } \\
210\end{array}$ & $\begin{array}{c}\text { 7b. NO. OF REFS } \\
13\end{array}$ \\
\hline \multirow[t]{2}{*}{ CONTRACT OR GRANT NO. } & \multicolumn{2}{|c|}{ 9A. ORIGINATOR'S REPOAT NUMBERIS) } \\
\hline & $\begin{array}{l}\text { Ob. OTHER REPORT NO(S) } \\
\text { this roport) }\end{array}$ & het numbers that may bo essigned \\
\hline
\end{tabular}

Approved for public release; distribution unlimited.

Naval Postgraduate School

Monterey, California 93940

oceanographic and meteorologic data, namely expendable or mechanical bathythermograph traces and wind reports have been systematically collected for years and every day more observations are made.

To handle such a large amount of information, it is advantageous to set up a computerized model which is: (I) automatic (2) performs an objective analysis of XBT/BT traces to identify the most significant thermal features, such as thermoclines and inversions, (3) restricts the human participation to an initial stage dealing with organization of data available and selection of proper values for the parameters of the model, and (4) preserves the initial information.

A FORTRAN IV program takes into consideration the above requirements to: (1) perform objective analyses of XBT/BT digital traces, (2) process wind information, (3) study the statistical characteristics of the original and processed data, and (4) plot the generated fields.

The digital model was tested and its app? ication may be extended to the study of any environmental $\mathrm{v}$ iable continucusly distributed along a vertical or horizontal axis. 
objective

Model

Analyze

Environmental

Variclines

Inversions

statistical

Relation

Surface

Winds 





\section{Thes is \\ S1527 Saldanha \\ 130772 \\ c. 1 \\ An objective method} to analyze oceanic variclines and their statistical relation to surface winds.

BIIIDERY

Thes is

S1527 Saldanha

c. 1

An objective method to analyze oceanic variclines and their statistical relation to surface winds. 
An objective method to analyze oceanic $v$ 